\title{
A RE-EVALUATION OF THE CHASMOSAURINE CERATOPSID CHASMOSAURUS (DINOSAURIA: ORNITHISCHIA) FROM THE UPPER CRETACEOUS (CAMPANIAN) DINOSAUR PARK FORMATION OF WESTERN CANADA
}

\author{
by \\ James Alexander Campbell \\ A thesis submitted to the Faculty of Graduate and Postdoctoral Affairs in partial \\ fulfillment of the requirements for the degree of
}

Master of Science

In

Department of Earth Sciences

Carleton University
Ottawa, Ontario

(C) 2014

James Alexander Campbell 


\begin{abstract}
The chasmosaurine ceratopsid Chasmosaurus is known from the Upper Cretaceous (Campanian) Dinosaur Park Formation (DPF) of southern Alberta and Saskatchewan. The two valid species, Chasmosaurus belli and Chasmosaurus russelli, are distinguished by differences in cranial ornamentation, and their purported stratigraphic segregation in the DPF, with $C$. russelli replaced by $C$. belli over time. In the DPF, Chasmosaurus is replaced by a third chasmosaurine, Vagaceratops irvinensis.

A specimen-based phylogenetic analysis of Chasmosaurus and Vagaceratops recovered Vagaceratops (CMN 41357 and TMP 1987.045.0001) as a sister taxon to Chasmosaurus, and not to Kosmoceratops as per previous analyses. All but two Chasmosaurus specimens formed a single, unresolved group in the 50\% majority rule analysis. AMNH 5402 and YPM 2016 were recovered within the 'Vagaceratops' clade. This clade shares anteroposteriorly shortened parietal fenestrae, and a straight posterior parietal margin, adorned on each side with five epiossifications. The remaining Chasmosaurus-like specimens all possess anteroposteriorly elongate parietal fenestra, and a variably embayed posterior parietal margin, adorned on each side with three epiossifications.
\end{abstract}

A stratophylogenetic analysis demonstrated that no stratigraphic separation exists between C. belli and C.russelli, or between Chasmosaurus-like and Vagaceratops-like specimens. These previously unreported temporal overlaps resulted from the recent rediscovery of the C. russelli holotype (CMN 8800) quarry in the uppermost DPF, and the assignment of YPM 2016 (middle DPF) to the Vagaceratops-like group.

An ontogenetic analysis demonstrated that $C$. belli and C. russelli do not form 
discrete, non-overlapping age classes, indicating that ontogeny cannot account for differences between these two putative taxa. The problematic taxa 'Eoceratops canadensis', 'Chasmosaurus kaiseni' and 'Chasmosaurus brevirostris' were recovered as a juvenile-subadult, subadult, and young adult, respectively, supporting their previous synonymization with Chasmosaurus. 'Mojoceratops perifania' was recovered as an old adult, but cannot be distinguished from Chasmosaurus due to its lack of diagnostic features. Based on the distribution of postorbital horncore sizes amongst Chasmosauruslike specimens, there appear to be two distinct ontogenetic trajectories culminating in relatively short, small circumference-based horncores, and relatively long, large circumference-based horncores, respectively. The "short horncore" trajectory encompasses all specimens of C. belli and one specimen of C. russelli, whereas the "long horncore" trajectory encompasses remaining $C$. russelli specimens. This indicates that postorbital horncore size is not taxonomic amongst Chasmosaurus-like specimens, and may correspond to sexual dimorphism.

A morphometric analysis demonstrated that $C$. belli and C. russelli were not consistently resolved as distinct morphotypes; however, the relative embayment of their posterior parietal margins differed significantly. Shallowly and deeply embayed margins are typically associated with relatively short and long postorbital horncores, respectively. The association between embayment angle and putatively sexually dimorphic postorbital horncores suggests that this former feature is also sexually dimorphic.

The results of the analyses conducted indicate that 1, C. belli and C. russelli are synonymous, with C. belli having taxonomic priority, and 2, the Vagaceratops-like specimens AMNH 5402 and YPM 2016 are referable to $V$. irvinensis, and may represent 
directed morphological change over time. This hypothesis may be tested with the recovery of additional specimens. 


\section{Acknowledgements}

I have many people who helped me at every step of this project who I would like to acknowledge. Thank you to Michael Ryan for providing me with such an exciting and stimulating project, and for introducing me to the timeless badlands of southern Alberta. I would like to thank Claudia Schröder-Adams for her guidance, support and mentorship, and for having lured me into studying Earth Sciences at Carleton University. I would also like to thank Robert Holmes, for his assistance and guidance in this project.

I would like to thank the following people for collections access at their respective institutions: Kieran Shepherd, Margaret Currie and Clayton Kennedy (Canadian Museum of Nature); David Evans, Kevin Seymour, Ian Morrison, Brian Iwama, and Caleb Brown (Royal Ontario Museum); Brandon Strilisky and Graeme Housego (Royal Tyrrell Museum of Palaeontology); Andrew Locock and Victoria Arbour (University of Alberta); Mark Norell and Carl Mehling (American Museum of Natural History); and Christopher Norris and Daniel Brinkman (Yale Peabody Museum).

Thank you to Caleb Brown, Nic Campione, Thomas Cullen, Kentaro Chiba, Kirstin Brink, Derek Larson, Mark Loewen, and Darren Tanke for insightful discussions; Philip Currie and Eva Koppelhus for their support; Margaret Currie for her skillful preparation of CMN 34829; David Eberth for providing me with revised radiometric dates for the Dinosaur Park Formation and locality data for TMP 2009.034.0009; Don Brinkman for helping me track down the uncollected Chasmosaurus specimen in Dinosaur Provincial Park; and Paul Barrett and the United Kingdom Natural History Museum for providing me with images of NHMUK R4948. Thank you as well to my friends in the Department of Earth Sciences. 
Thank you to Jordan and Angela Mallon for my stay in Calgary, and to Jordan for insightful discussions and for providing me with photos of Chasmosaurus specimens. For my stay in Drumheller, I would like to thank Joanna Northover, Graeme Housego and Dawna McLeod for offering me room and board.

For the 2012 field season in the Milk River region of southern Alberta, I would like to express my deep thanks to Michael Ryan and David Evans for inviting me along and making it a reality. I would like to thank Ian Morrison, Caleb Brown, Wendy Sloboda, Derek Larson, Nic Campione, Amanda McGee, Collin Van Buren, Kentaro Chiba, Don Cretin, Cathy Falls, Michel Haché, Gail Huckerby, Charise Currier, and Tamara Chau for field assistance and discussions. I would like to thank Cecil Nesmo for generously offering his farm as a site for our base camp.

Financial assistance was most generously made possible through two Ontario Graduate Scholarships, two Dr. George A. Jeletzky Memorial Scholarships, National Geographic Society's Young Explorer's Grant (for fieldwork), and René Vandervelde Travel Grant (for presenting my findings at the Society of Vertebrate Paleontology Annual Meeting in Los Angeles, California), for which I am deeply grateful. I would also like to thank Claudia Schröder-Adams for having funded my trip to Edmonton to the joint Canadian Palaeontology Conference and University of Alberta Laboratory for Vertebrate Palaeontology $50^{\text {th }}$ Anniversary Conference (for presenting the newly described chasmosaurine specimens), made possible by a Natural Sciences and Engineering Research Council Collaborative Research and Development Grant.

I would like to thank my girlfriend Celeste Vatcher for her support and equallyobsessive fascination with dinosaurs. At last, I would like to thank my family for their 
love and support, for immersing me in the natural world, and for having shown me Jurassic Park at the innocent and formative age of four. 
Table of Contents

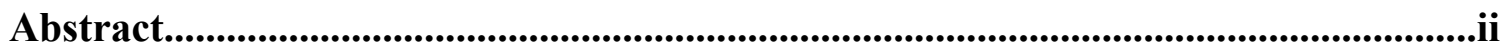

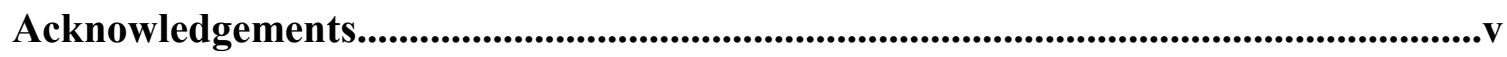

Table of Contents..........................................................................................................................viii

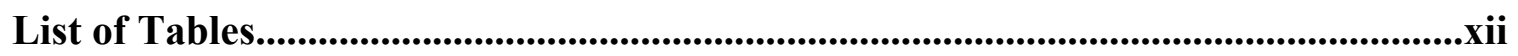

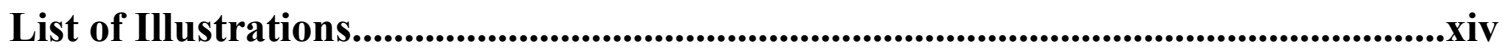

List of Anatomical Abbreviations........................................................................

List of Appendices................................................................................................xxiii

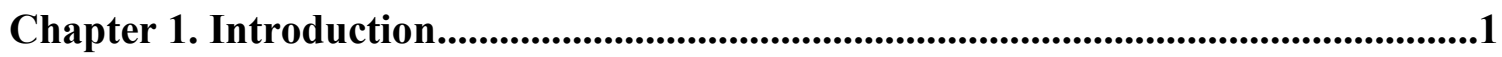

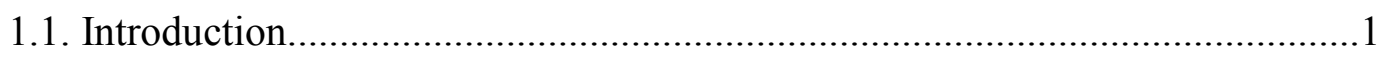

1.2. Rationale for examining Chasmosaurus ...............................................

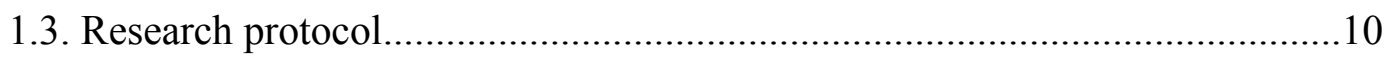

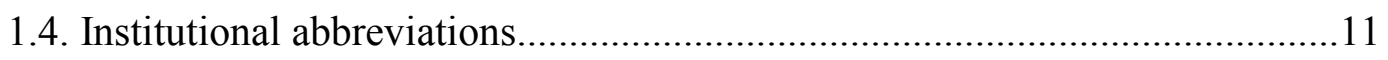

Chapter 2. Taxonomy of Chasmosaurus...........................................................................12

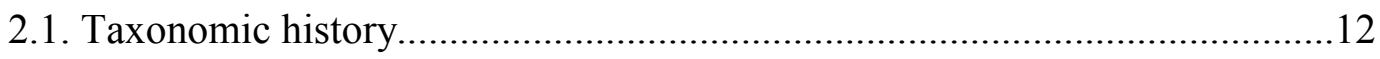

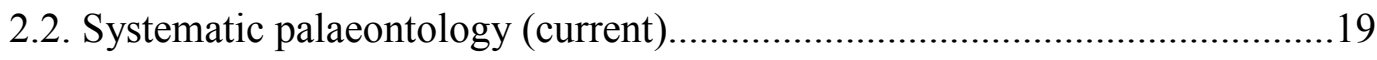

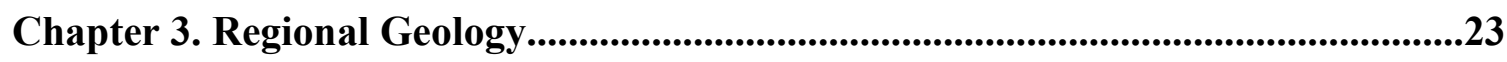

Chapter 4. Materials and Methods............................................................................32

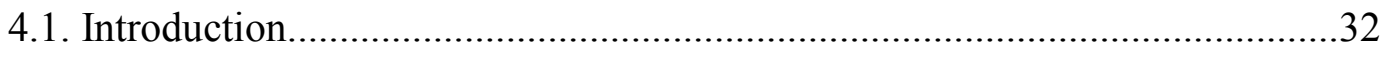

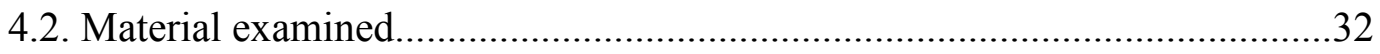

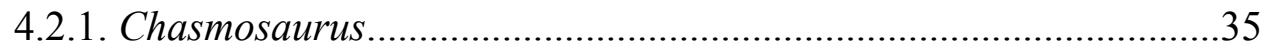

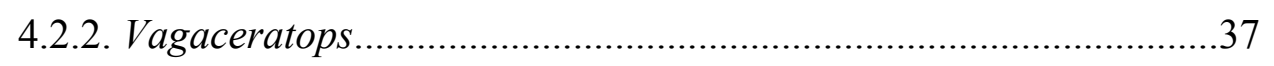




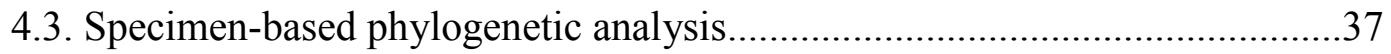

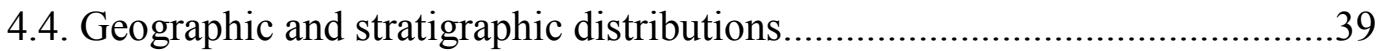

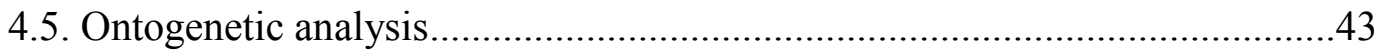

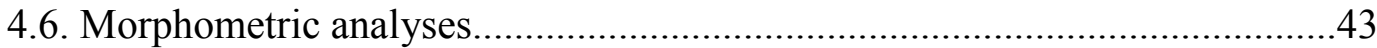

Chapter 5. Anatomical Description of Selected Chasmosaurus and Vagaceratops

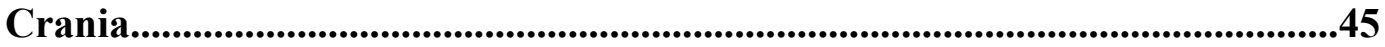

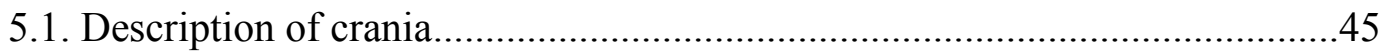

5.1.1. Chasmosaurus.....................................................................49

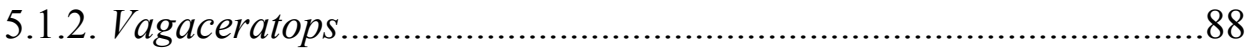

5.1.3. New chasmosaurine material................................................. 98

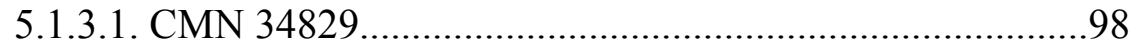

5.1.3.2. TMP 2011.053.0046...............................................110

5.1.3.3. Redescription of CMN 8802...................................120

5.2. Variation of cranial material within Chasmosaurus and related taxa...........149

Chapter 6. Phylogenetic Relationships of Chasmosaurus and Vagaceratops

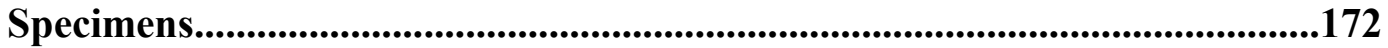

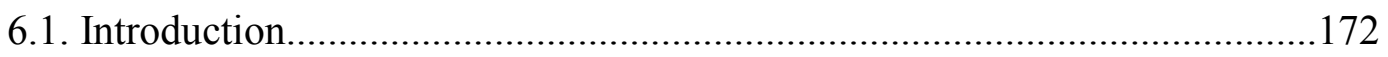

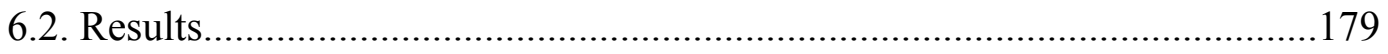

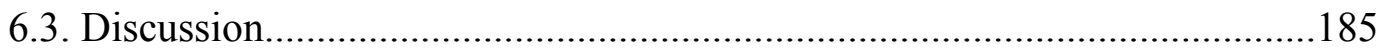

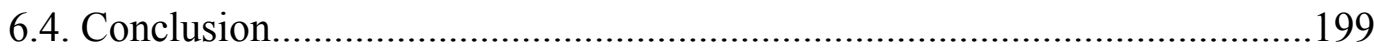

Chapter 7. Morphological Variation in Chasmosaurus and Vagaceratops due to their

Geographic and Stratigraphic Distribution...............................................201

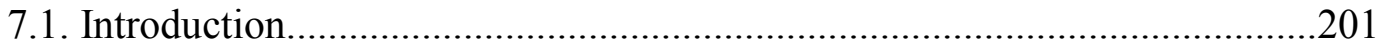




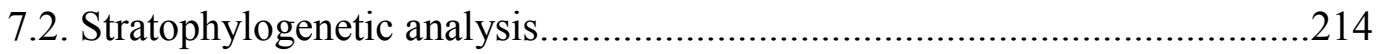

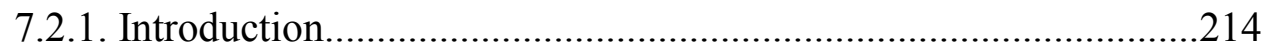

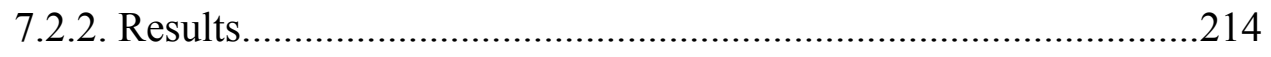

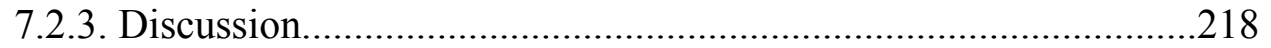

7.2.3.1. Stratophylogenetic analysis............................................... 218

7.2.3.2. Morphological change over time....................................222

7.2.3.3. Morphological variation due to geographic

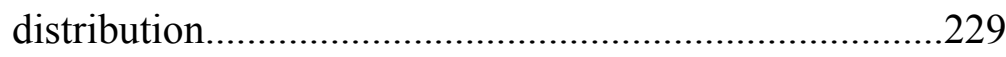

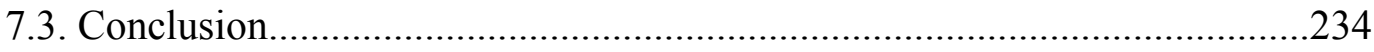

Chapter 8. Ontogenetic Analysis of Chasmosaurus........................................................236

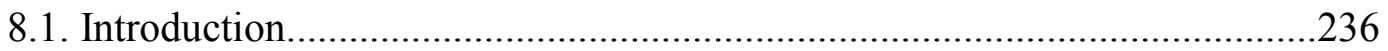

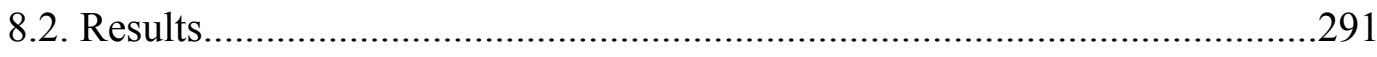

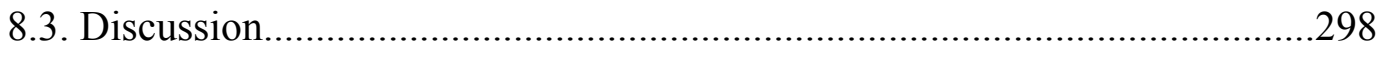

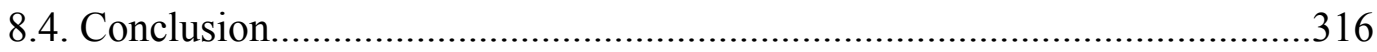

\section{Chapter 9. Morphometric Analysis of Chasmosaurus and Vagaceratops}

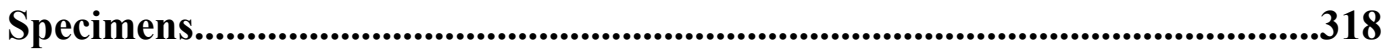

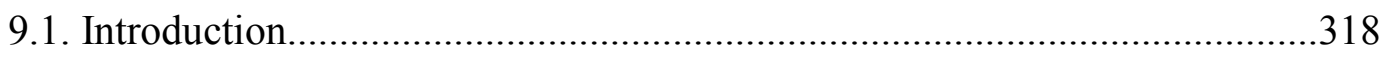

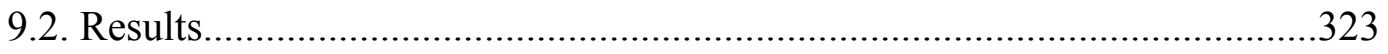

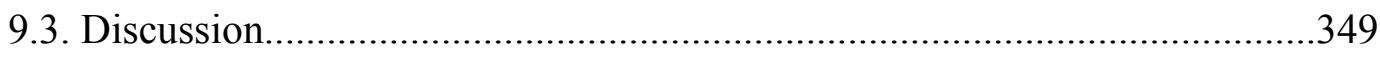

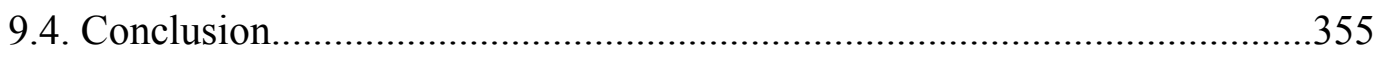

9.5. Addendum: revised morphometric analyses.................................................357

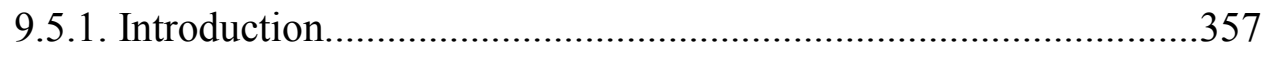

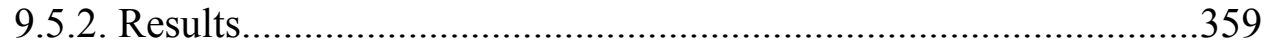


Chapter 10. Conclusions.

Appendix I. Locality data for Dinosaur Park Formation chasmosaurine specimens

Appendix II. Cranial parameter dataset for Dinosaur Park Formation chasmosaurine specimens

Appendix III. List of characters used in specimen-based phylogenetic analysis

Appendix IV. Supporting data for Chapter 9 (imputed specimen/parameter dataset, parameter loadings on principal component axes, and principal component loadings on discriminant function axes)

Appendix V. Ontogenetically variable phylogenetic characters .509 Appendix VI. Revised taxonomic diagnoses of Dinosaur Park Formation chasmosaurine taxa considered valid in this study.

References. .520 


\section{List of Tables}

Table 1. Selected cranial measurements of specimens referred to Chasmosaurus and

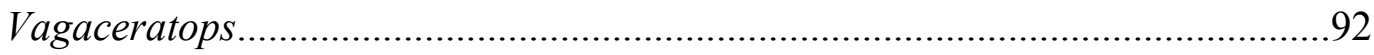

Table 2. Character-taxon matrix used in phylogenetic analysis.................................175

Table 3. Taxonomic referrals, geographic localities and stratigraphic elevations of Chasmosaurus-like and Vagaceratops-like specimens above the Oldman-

Dinosaur Park formational contact...........................................................2 212

Table 4. Cranial parameters with relationship to stratigraphy...................................224

Table 5. Potential regional differences amongst Chasmosaurus-like and Vagaceratops-

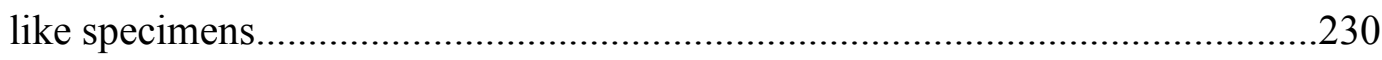

Table 6. Specimen-character matrix used in ontogenetic analysis............................273

Table 7. Postorbital horncore length and basal circumference, squamosal length/width ratio and continuity of lateral parietal bar in Chasmosaurus specimens.............274

Table 8. Results of non-parametric multivariate analysis of variance of postorbital

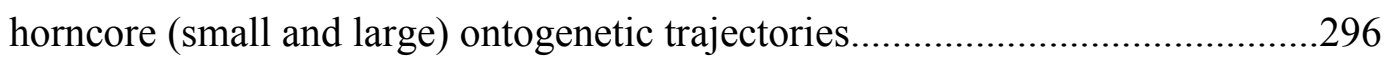

Table 9. Thickness of posterior end of medial parietal bar, and presence/absence of a shallow, longitudinal sulcus on the parietal fenestra margin. .297 
Table 10. Results of first non-parametric multivariate analysis of variance of 11 Vagaceratops-like, Chasmosaurus belli and Chasmosaurus russelli specimens. 330

Table 11. Results of second non-parametric multivariate analysis of variance of 11 Vagaceratops-like, Chasmosaurus belli and Chasmosaurus russelli specimens

Table 12. Results of third non-parametric multivariate analysis of variance of seven Chasmosaurus belli and Chasmosaurus russelli specimens 344

Table 13. Results of Mann-Whitney U-test of differences in posterior parietal embayment between Chasmosaurus belli and Chasmosaurus russelli. 347

Table 14. Results of Mann-Whitney U-test of differences in postorbital horncore basal circumference between Chasmosaurus belli and Chasmosaurus russelli.

Table 15. Results of fourth analysis of variance of 11 Vagaceratops-like, Chasmosaurus belli and Chasmosaurus russelli specimens 364

Table 16. Results of fifth analysis of variance of 11 Vagaceratops-like, Chasmosaurus belli and Chasmosaurus russelli specimens.

Table 17. Results of analysis of variance, testing for differences in posterior parietal embayment between Vagaceratops-like, Chasmosaurus belli and Chasmosaurus russelli specimens. 372 


\section{List of Illustrations}

Figure 1. Regional geology, Belly River Group of southern Alberta..............................4

Figure 2. Chasmosaurus russelli holotype quarry, at Onefour Research Station, AB.......6

Figure 3. Regional map of chasmosaurine fossil-bearing localities in southern Alberta and

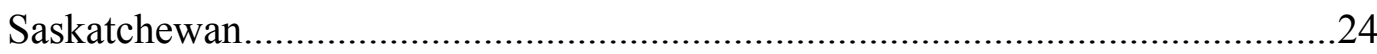

Figure 4. Stratigraphic relationships of the Belly River Group between Dinosaur Provincial Park (A) and the Onefour (B) area of southern Alberta......................26

Figure 5. Parameters of cranial measurements used in this study..................................34

Figure 6. Cranial elements referred to in this study.................................................46

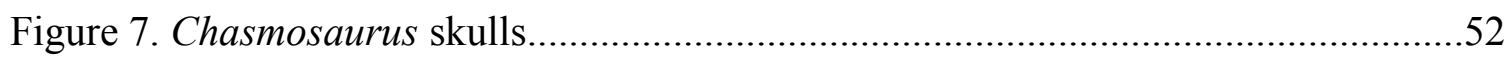

Figure 8. Frill of AMNH 5402 (Chasmosaurus belli)..............................................62

Figure 9. Frill of CMN 2245 (Chasmosaurus belli).................................................65

Figure 10. Frill of YPM 2016 (Chasmosaurus belli)................................................... 72

Figure 11. Chasmosaurus sp. postorbital horncores................................................. 76

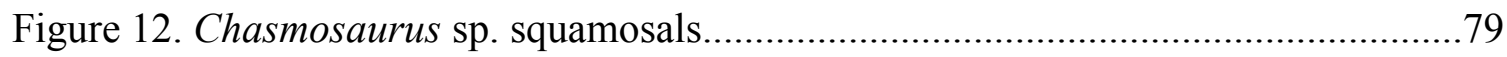

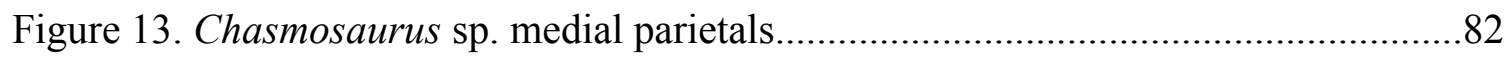

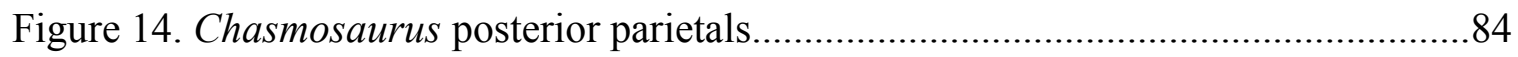

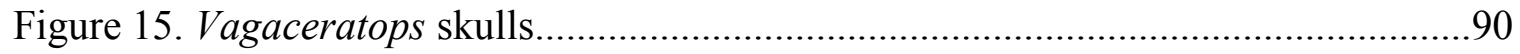


Figure 16. Partial Vagaceratops frills .96

Figure 17. Skull of CMN 34829 (Chasmosaurus sp.)...............................................100

Figure 18. Nasal of TMP 2011.053.0046 (Vagaceratops sp.).....................................112

Figure 19. Left quadrate of TMP 2011.053.0046 (Vagaceratops sp.)..........................114

Figure 20. Left squamosal of TMP 2011.053.0046 (Vagaceratops sp.)........................116

Figure 21. Parietal of TMP 2011.053.0046 (Vagaceratops sp.)..................................118

Figure 22. Premaxillae and rostral of CMN 8802 (Chasmosaurus sp.)........................122

Figure 23. Left postorbital of CMN 8802 (Chasmosaurus sp.)...................................125

Figure 24. Right postorbital of CMN 8802 (Chasmosaurus sp.).................................126

Figure 25. Left articulated jugal, quadratojugal and quadrate of CMN 8802

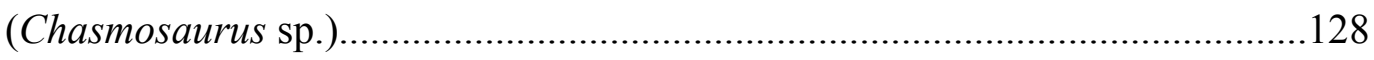

Figure 26. Right articulated jugal, quadratojugal and quadrate of CMN 8802

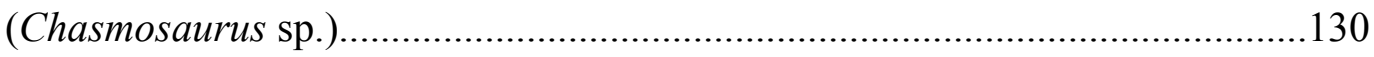

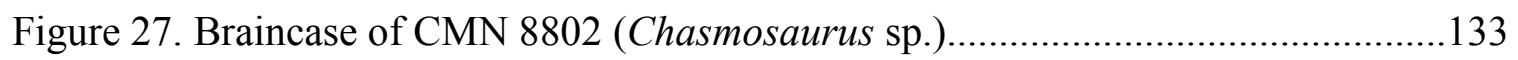

Figure 28. Left squamosal of CMN 8802 (Chasmosaurus sp.)..................................137

Figure 29. Right squamosal and jugal of CMN 8802 (Chasmosaurus sp.)...................138

Figure 30. Parietal of CMN 8802 (Chasmosaurus sp.)...........................................140

Figure 31. Left lower jaw of CMN 8802 (Chasmosaurus sp.)...................................143 
Figure 32. Right lower jaw of CMN 8802 (Chasmosaurus sp.)......

Figure 33. Chasmosaurus and Vagaceratops frills. .156

Figure 34. Left posterolateral corner of frill of AMNH 5402 (Chasmosaurus belli), YPM 2016 (C. belli) and CMN 41357 (Vagaceratops irvinensis holotype). 163

Figure 35. Chasmosaurus and Vagaceratops lower jaws 170

Figure 36. Schematic of an idealized chasmosaurine snout 174

Figure 37. Specimen-based phylogenetic analysis of Chasmosaurus and Vagaceratops specimens 180

Figure 38. Judiceratops tigris (holotype, YPM VPPU 022404). .194

Figure 39. Judiceratops tigris (referred specimen, YPM VPPU 023262).

Figure 40. Judiceratops tigris (referred specimen, YPM VPPU 023261). .198

Figure 41. Structural contour map of Dinosaur Provincial Park, with contours showing elevation of regional disconformity between Oldman and Dinosaur Park formations. 206

Figure 42. Stratigraphic positions of chasmosaurine specimens from Dinosaur Provincial Park with known data. .208

Figure 43. Stratophylogenetic analysis using ceratopsid taxa from Dinosaur Park Formation that were included in the phylogenetic analysis. 216 
Figure 44. Scatter plot showing squamosal length of Dinosaur Park Formation chasmosaurine specimens and their respective elevation above the regional disconformity separating the Dinosaur Park and Oldman formations. .225

Figure 45. Scatter plot showing dorsal temporal fenestra to parietal fenestra length of Dinosaur Park Formation chasmosaurine specimens and their respective elevation above the regional disconformity separating the Dinosaur Park and Oldman formations. 226

Figure 46. Scatter plot showing jugal notch to parietal fenestra length of Dinosaur Park Formation chasmosaurine specimens and their respective elevation above the regional disconformity separating the Dinosaur Park and Oldman formations. .227

Figure 47. Scatter plot showing jugal notch to back of frill of Dinosaur Park Formation chasmosaurine specimens and their respective elevation above the regional disconformity separating the Dinosaur Park and Oldman formations .228

Figure 48. Ontogenetic changes in specimens previously referred to Chasmosaurus articulation of rostral with premaxillae (character 1)

Figure 49. Ontogenetic changes in Chasmosaurus - articulation of epinasal with nasals (character 2) .246

Figure 50. Ontogenetic changes in Chasmosaurus - articulation of epijugal with jugal and quadratojugal (character 3) 
Figure 51. Ontogenetic changes in Chasmosaurus, squamosal - articulation of episquamosals with squamosal (character 4), remodeling of episquamosals (character 5) changing outline of lateral margin of squamosal (character 16), and increase in squamosal length/width ratio (character 17). .251

Figure 52. Ontogenetic changes in Chasmosaurus, parietal - articulation of epiparietals with parietal (character 6) and orientation of epiparietals (character 18)............254

Figure 53. Ontogenetic changes in Chasmosaurus - articulation between other cranial elements: premaxillae (character 7), premaxilla-nasal (character 8), nasals (character 9), nasal-frontal (character 10), frontals (character 11), and frontalpostorbital (character 12)

Figure 54. Ontogenetic changes in Chasmosaurus - proposed resorption of nasal horncore (character 13) .263

Figure 55. Ontogenetic changes in Chasmosaurus - proposed resorption of postorbital horncores (character 14). 265

Figure 56. Ontogenetic changes in Chasmosaurus - lateral parietal bar (character $15)$ .268

Figure 57. Scatter plot showing postorbital horncore length vs. basal circumference of Chasmosaurus specimens. 277

Figure 58. Scatter plot showing postorbital horncore length vs. basal circumference in all Dinosaur Park Formation chasmosaurine specimens .278 
Figure 59. Scatter plot showing postorbital horncore length vs. rostral-to-epijugal length of Dinosaur Park Formation chasmosaurine specimens 283

Figure 60. Scatter plot showing postorbital horncore basal circumference vs. rostral-toepijugal length of Dinosaur Park Formation chasmosaurine specimens. 284

Figure 61. Scatter plot showing postorbital horncore length vs. squamosal length of Dinosaur Park Formation chasmosaurine specimens 285

Figure 62. Scatter plot showing postorbital horncore basal circumference vs. squamosal length of Dinosaur Park Formation chasmosaurine specimens 286

Figure 63. Ontogenetic transformation series for Chasmosaurus specimens

Figure 64. Squamosal length vs. rostral-to-epijugal length of Dinosaur Park Formation chasmosaurine skulls 299

Figure 65. Postorbital horncores of Dinosaur Park Formation chasmosaurine skulls arranged by ontogenetic stage, and horncore development (long and short)......302

Figure 66. Principal component analysis (PCA 1) of 18 Vagaceratops-like, Chasmosaurus russelli, Chasmosaurus belli, and Chasmosaurus sp. specimens .325

Figure 67. Discriminant function analysis (DFA 1) of 11 Vagaceratops-like, Chasmosaurus russelli, and Chasmosaurus belli specimens. .331

Figure 68. Principal component analysis (PCA 2) of 11 Vagaceratops-like, Chasmosaurus russelli and Chasmosaurus belli specimens .333 
Figure 69. Discriminant function analysis (DFA 2) of 11 Vagaceratops-like,

Chasmosaurus russelli and Chasmosaurus belli specimens

Figure 70. Principal component analysis (PCA 3) of seven Chasmosaurus russelli and

Chasmosaurus belli specimens.

Figure 71. Discriminant function analysis (DFA 3) of seven Chasmosaurus russelli and

Chasmosaurus belli specimens.

345

Figure 72. Scatter plot of postorbital horncore basal circumference vs. posterior parietal embayment in Chasmosaurus belli and Chasmosaurus russelli specimens.......354

Figure 73. Principal component analysis (PCA 4) of 18 Vagaceratops-like, Chasmosaurus russelli, Chasmosaurus belli, and Chasmosaurus sp. specimens

Figure 74. Principal component analysis (PCA 4) of 18 Vagaceratops-like, Chasmosaurus russelli, Chasmosaurus belli, and Chasmosaurus sp. specimens (12 cranial parameters): principal component axes 1 and 2 versus parameter 17

Figure 75. Principal component analysis (PCA 5) of 18 Vagaceratops-like, Chasmosaurus russelli, Chasmosaurus belli, and Chasmosaurus sp. specimens 


\section{List of Anatomical Abbreviations}

\begin{tabular}{|c|c|}
\hline $\begin{array}{l}\text { V1-------foramen for exit of trigem- } \\
\text { inal nerve, ophthalmic } \\
\text { branch }\end{array}$ & $\begin{array}{l}\text { jn---------jugal notch } \\
\text { j-qj f-------facet for quadratojugal } \\
\text { on jugal }\end{array}$ \\
\hline V2\&3-----foramen for exit of trigem- & la-----------lacrimal \\
\hline $\begin{array}{l}\text { inal nerve, maxillary and } \\
\text { mandibular branches }\end{array}$ & $\begin{array}{l}\text { Itf------------lateral temporal fenestra } \\
\text { m------------maxilla }\end{array}$ \\
\hline VII------foramen for facial nerve & n------------nasal \\
\hline $\begin{array}{l}\text { X-XII---foramen for exit of vagus, } \\
\text { accessory and hypoglossal } \\
\text { nerves }\end{array}$ & $\begin{array}{l}\text { nhc---------nasal horncore } \\
\text { ns-----------nares } \\
\text { n sp----------narial spine }\end{array}$ \\
\hline a---------angular & n st---------narial strut \\
\hline add------adductor fossa & oc-----------occipital condyle \\
\hline ar--------articular & orb----------orbit \\
\hline bo--------basioccipital & p------------epiparietal \\
\hline bpt pr---basipterygoid process & p lc-------epiparietal loci \\
\hline bt--------basal tuber & pa-----------parietal \\
\hline cpr------coronoid process & pa af-------accessory fenestra in \\
\hline crp------crista prootica & parietal \\
\hline d---------dentary & paf----------parietal fenestra \\
\hline $\begin{array}{l}\text { d-d f----dentary facet on dentary } \\
\text { d-pd f---predentary facet on dentary } \\
\text { dtf o-----opening of the dorsal } \\
\text { temporal fenestra }\end{array}$ & $\begin{array}{l}\text { pa lb--------lateral bar of parietal } \\
\text { pa mb------medial bar of parietal } \\
\text { pa mp------medial platform of } \\
\text { parietal }\end{array}$ \\
\hline eo--------exoccipital & pa mp prt--protuberance on medial \\
\hline epij------epijugal & platform of parietal \\
\hline epin-----epinasal & pa-paf m---margin of parietal \\
\hline es--------episquamosal & fenestra on parietal \\
\hline es lc-----episquamosal loci & pa pb-------posterior bar of parietal \\
\hline f---------foramen & par---------prearticular \\
\hline fm-------foramen magnum & pa-sq f------squamosal facet on \\
\hline fo--------fenestra ovalis & parietal \\
\hline fpf-------frontoparietal fontanelle & pd------------predentary \\
\hline fr--------frontal & p-es-------epiparietosquamosal \\
\hline fr-n f----nasal facet on frontal & pm---------premaxilla \\
\hline gle--------glenoid fossa & pm-m f-----maxilla facet on \\
\hline ica-------internal carotid artery & premaxilla \\
\hline it pr-----infratemporal process & pm-n dc----premaxilla-nasal dorsal \\
\hline $\begin{array}{c}\text { it pr j----infratemporal process of } \\
\text { jugal }\end{array}$ & $\begin{array}{c}\text { contact } \\
\text { pm-n f------nasal facet on premaxilla }\end{array}$ \\
\hline j----------jugal & pm ome-----expanded oral margin \\
\hline j-epij f---facet for epijugal on jugal & of premaxilla \\
\hline
\end{tabular}




\section{List of Anatomical Abbreviations (continued)}

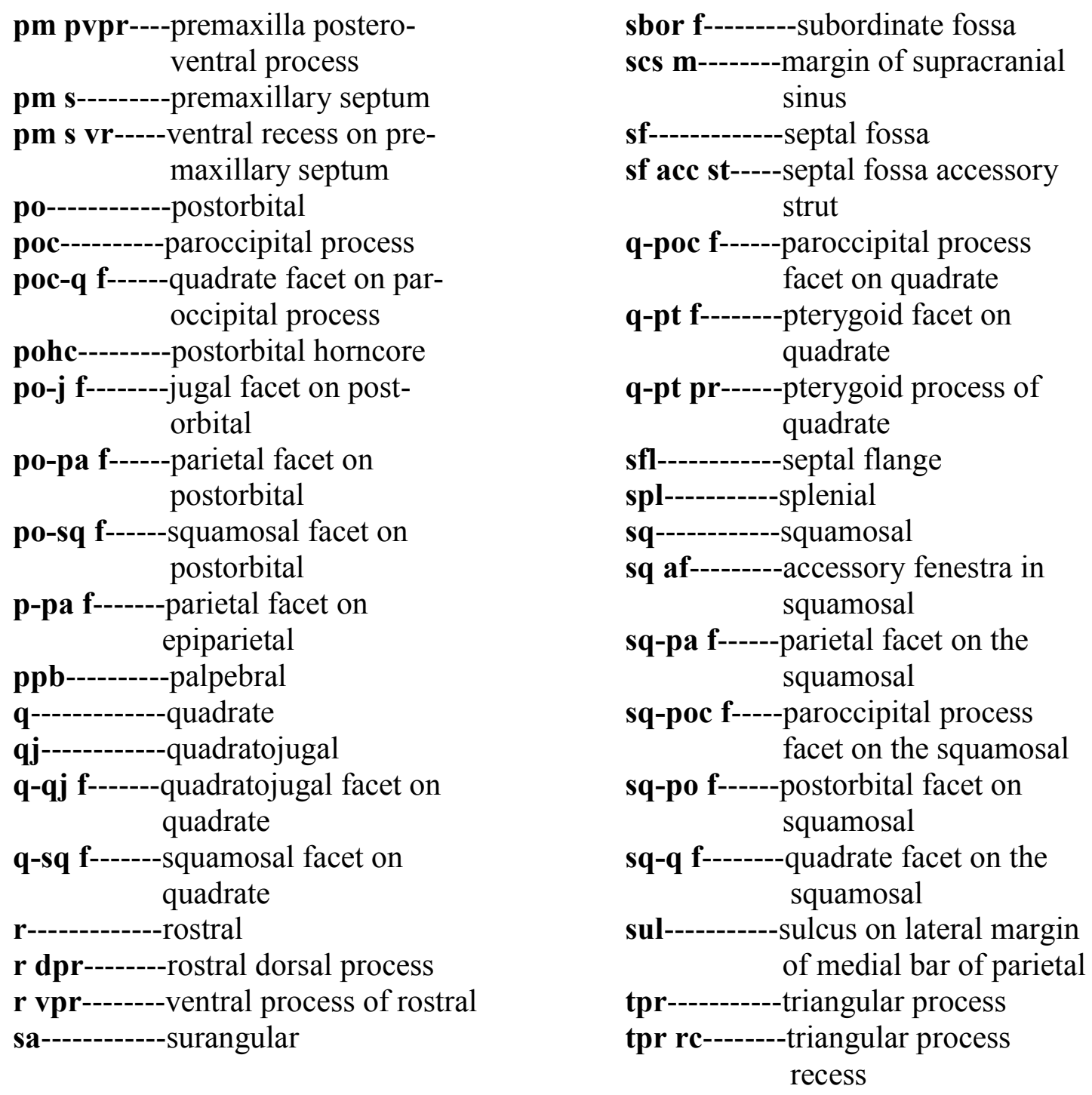




\section{List of Appendices.}

Appendix I. Locality data for Dinosaur Park Formation chasmosaurine specimens

Appendix II. Cranial parameter dataset for Dinosaur Park Formation

chasmosaurine specimens

Appendix III. List of characters used in specimen-based phylogenetic

analysis

Appendix IV. Supporting data for Chapter 9 (imputed specimen/parameter dataset, parameter loadings on principal component axes, and principal component loadings on discriminant function axes)

Appendix V. Ontogenetically variable phylogenetic characters

Appendix VI. Revised taxonomic diagnoses of Dinosaur Park Formation chasmosaurine taxa considered valid in this study. 


\section{Chapter 1. Introduction}

\subsection{Introduction}

Ceratopsidae is a group of herbivorous ornithischian dinosaurs known primarily from the Late Cretaceous sediments of North America (Sampson et al., 2010), and at least one locality in China (Xu et al., 2010). They are characterized by the presence of a rostral, the ornamentation of the parietosquamosal frill, and the morphology of the nasal and postorbital horncores. Two clades (subfamilies) are currently recognized within Ceratopsidae: Centrosaurinae and Chasmosaurinae (Lehman, 1990). Centrosaurines are characterized by a relatively short parietosquamosal frill, with a rectangular squamosal and a frill typically ornamented with epiossifications. Chasmosaurinae is characterized by a longer parietosquamosal frill, with an elongate, triangular squamosal and typically less ostentatious epiparietal ossifications.

Chasmosaurinae comprises the following currently recognized taxa:

Agujaceratops mariscalensis Lehman, 1989; Anchiceratops ornatus Brown, 1914;

Arrhinoceratops brachyops Parks, 1925; Bravoceratops polyphemus Wick and Lehman, 2013; Chasmosaurus belli Lambe 1902; Chasmosaurus russelli Sternberg, 1940;

Coahuilaceratops magnacuerna Loewen, Sampson, Lund, Farke, Aguillón-Martínez, De Leon, Rodríguez de la Rosa, Getty, and Eberth, 2010; Eotriceratops xerinsularis Wu, Brinkman, Eberth, and Braman, 2007; Judiceratops tigris Longrich, 2013; Kosmoceratops richardsoni Sampson, Loewen, Farke, Roberts, Forster, Smith, and Titus, 2010; Medusaceratops lokii Ryan, Russell and Hartman, 2010; Nedoceratops hatcheri Lull, 1905; Ojoceratops fowleri Sullivan and Lucas, 2010; Pentaceratops sternbergi Osborn, 1923; Tatankaceratops sacrisonorum Ott and Larson, 2010; Titanoceratops 
ouranos Longrich, 2011; Triceratops horridus Marsh, 1889; Triceratops prorsus Marsh, 1890; Torosaurus latus Marsh, 1891; Torosaurus utahensis Gilmore, 1946; Utahceratops gettyi Sampson, Loewen, Farke, Roberts, Forster, Smith, and Titus, 2010; and Vagaceratops irvinensis Holmes, Forster, Ryan, and Shepherd, 2001.

Chasmosaurines were historically characterized by long, robust postorbital horncores and a less pronounced nasal horncore. This pattern, however, is now recognized as being plesiomorphic for Ceratopsidae, being found in the outgroup taxon Zuniceratops (Wolfe and Kirkland, 1998) and the basal centrosaurines Albertaceratops, Diabloceratops and Xenoceratops (Ryan et al., 2013a).

Chasmosaurines are known exclusively from Late Cretaceous sediments of western North America (Sampson and Loewen, 2010). During the Late Cretaceous, the Western Interior Seaway connected the Boreal (present-day Arctic Ocean) and Tethyan (present-day Gulf of Mexico) seas and divided North America into two landmasses, Appalachia to the east and Laramidia to the west (Sampson and Loewen, 2010). Appalachia and Laramidia remained separate until the Maastrichtian, when the Western Interior Seaway regressed and united the two landmasses. Ceratopsids are distributed along a north-south corridor on the eastern side of Laramidia, with chasmosaurines ranging from present day northern Mexico (Coahuilaceratops) to southern Alberta, Canada (e.g., Chasmosaurus) (Sampson and Loewen, 2010). It has been proposed that chasmosaurines were latitudinally arrayed into distinct biomes, based on the geographically-restricted ranges of specific taxa (Sampson et al., 2010). Chasmosaurines are known from a 12 million year interval, spanning the Middle Campanian (77.5 Ma, Medusaceratops lokii; Ryan et al., 2010b) to Late Maastrichtian (65.5 Ma, Triceratops 
horridus; Sampson and Loewen, 2010).

The Upper Cretaceous (Campanian) sediments of the Dinosaur Park Formation (Fig. 1) of Alberta and Saskatchewan (especially in the region of Dinosaur Provincial Park) are arguably the best sampled Upper Cretaceous dinosaur-bearing unit in the world, and has produced a diverse assemblage of dinosaurs (Currie, 2005; Ryan et al., 2010a; Farke et al., 2011; Ryan et al., 2012). The Dinosaur Park Formation has produced the world's greatest diversity and number of ceratopsids (Ryan and Evans, 2005). It is noted for its abundance of centrosaurines including Centrosaurus apertus (from multiple bone beds), Styracosaurus albertensis, an indeterminate pachyrhinosaur (Ryan et al. 2010a), and Spinops sternbergorum (Farke et al, 2011). The Dinosaur Park Formation also produced three chasmosaurines: Chasmosaurus belli, Chasmosaurus russelli and Vagaceratops irvinensis (Holmes et al., 2001).

The first specimens of Chasmosaurus were collected by Lawrence Lambe of the Geological Survey of Canada in 1897 from the region that is now Dinosaur Provincial Park. Originally referred to Monoclonius (Lambe, 1902), Lambe (1914b) removed it from this taxon and created the genus Chasmosaurus to receive it. Since that time, dozens of partial-to-complete skulls have been collected from the Dinosaur Park Formation within the boundaries of Dinosaur Provincial Park, in the more southern exposures southeast of the village of Manyberries, Alberta, and in Saskatchewan. As new fossil specimens have been collected and prepared, the morphological variation of this genus has been recognized by some researchers as being greater than can be constrained within a single generic hypodigm. To accommodate this variation, some workers have erected new species of Chasmosaurus (e.g., Chasmosaurus kaiseni; Brown, 1933) or entirely new chasmosaurine genera (i.e., Mojoceratops perifania; Longrich, 2010; and V. irvinensis; 


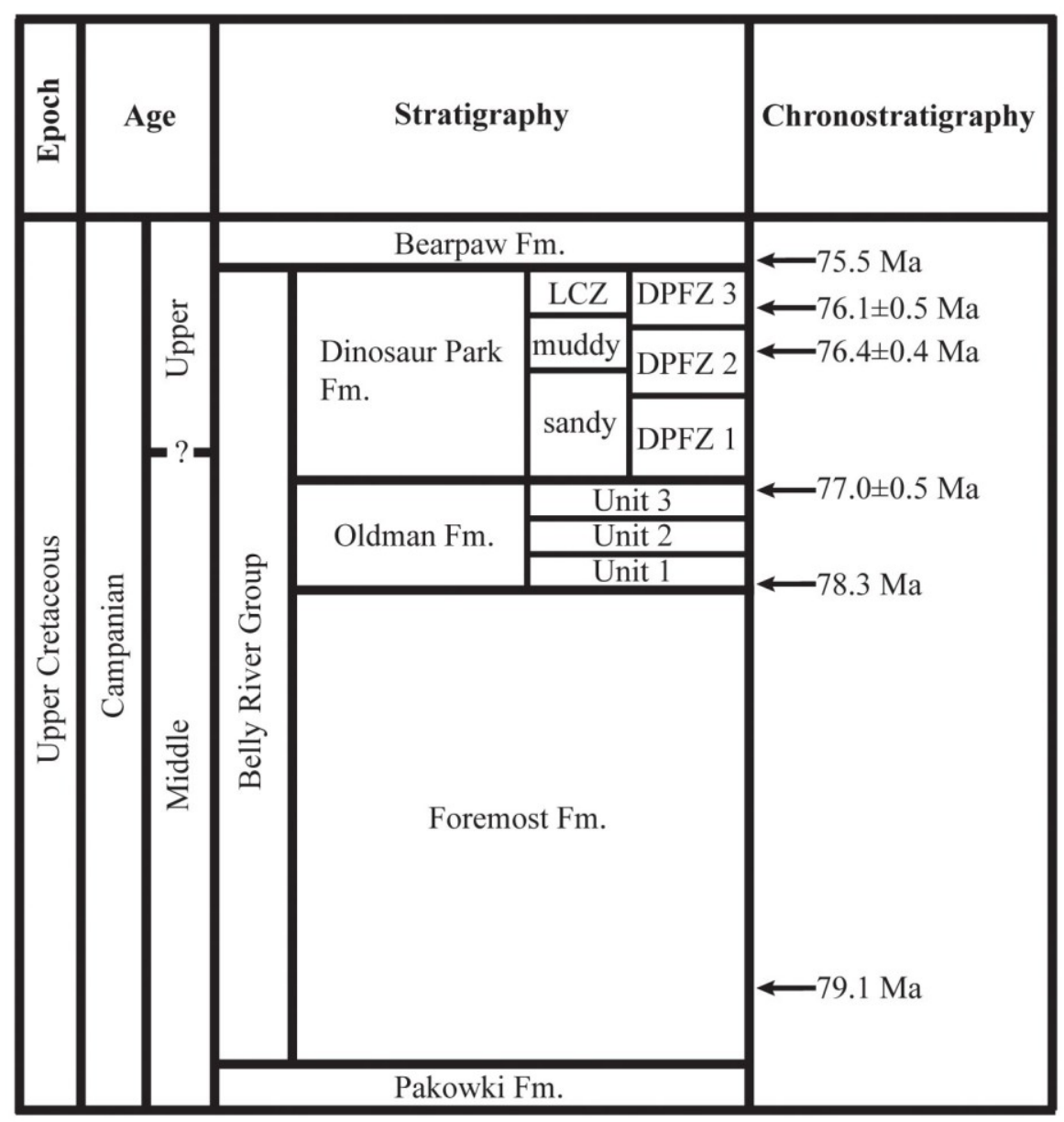

Figure 1. Regional geology, Belly River Group of southern Alberta (modified from Eberth, 2005: Fig. 3.1.); DPFZ 1 = Dinosaur Park Faunal Zone 1 (CentrosaurusCorythosaurus), DPFZ 2 = Dinosaur Park Faunal Zone 2 (Styracosaurus-Lambeosaurus lambei) and DPFZ 3 = Dinosaur Park Faunal Zone 3 (pachyrhinosaur-Lambeosaurus magnicristatus) (Ryan and Evans, 2005; Ryan et al., 2012); LCZ = Lethbridge Coal Zone. Chronostratigraphic ages of lower Foremost Formation from Eberth (2005), lowermost Oldman Formation from Eberth and Deino (1992), and uppermost Oldman Formation to lowermost Bearpaw Formation from David Eberth (pers. comm.). 
Sampson et al., 2010), or attributed the variation to sexual dimorphism (e.g., C. russelli specimens included in C. belli; Sternberg, 1927), ontogeny (e.g., Eoceratops canadensis considered to be a juvenile of C. kaiseni; Lehman, 1989) or individual variation within a smaller number of species (e.g., C. belli and C. russelli considered to be gradational forms of the same species; Lehman, 1998). As a result, Chasmosaurus has had a long and convoluted taxonomic history, with seven species associated with it. Two of those species (Chasmosaurus mariscalensis from Texas and Chasmosaurus irvinensis) have subsequently been assigned to new chasmosaurine genera (Agujaceratops mariscalensis; Lucas et al., 2006; and $V$.irvinensis; Sampson et al., 2010). The remaining five (C. belli, Chasmosaurus canadensis, Chasmosaurus brevirostris, C. kaiseni, and C. russelli) were re-examined by Godfrey and Holmes (1995) who supported their assignment to one of two valid Chasmosaurus species, C. belli and C. russelli. Their conclusions were supported and refined by Maidment and Barrett (2011).

The validity of $C$. belli and C. russelli is supported by their purported stratigraphic segregation within the Dinosaur Park Formation (Holmes et al., 2001; Ryan and Evans, 2005), with C. russelli being replaced stratigraphically by C. belli over time. However, recently conducted fieldwork indicates that the holotype (CMN 8800) of $C$. russelli was actually recovered from the uppermost strata of the Dinosaur Park Formation. This revision expands the $C$. russelli stratigraphic interval so that it overlaps with the range of $C$. belli (Fig. 2). The stratigraphic division between these species is further obscured by a significant degree of overlap in diagnostic cranial characters, mostly in the postorbital horncores, and morphology and ornamentation of the posterior margin of the parietal. 


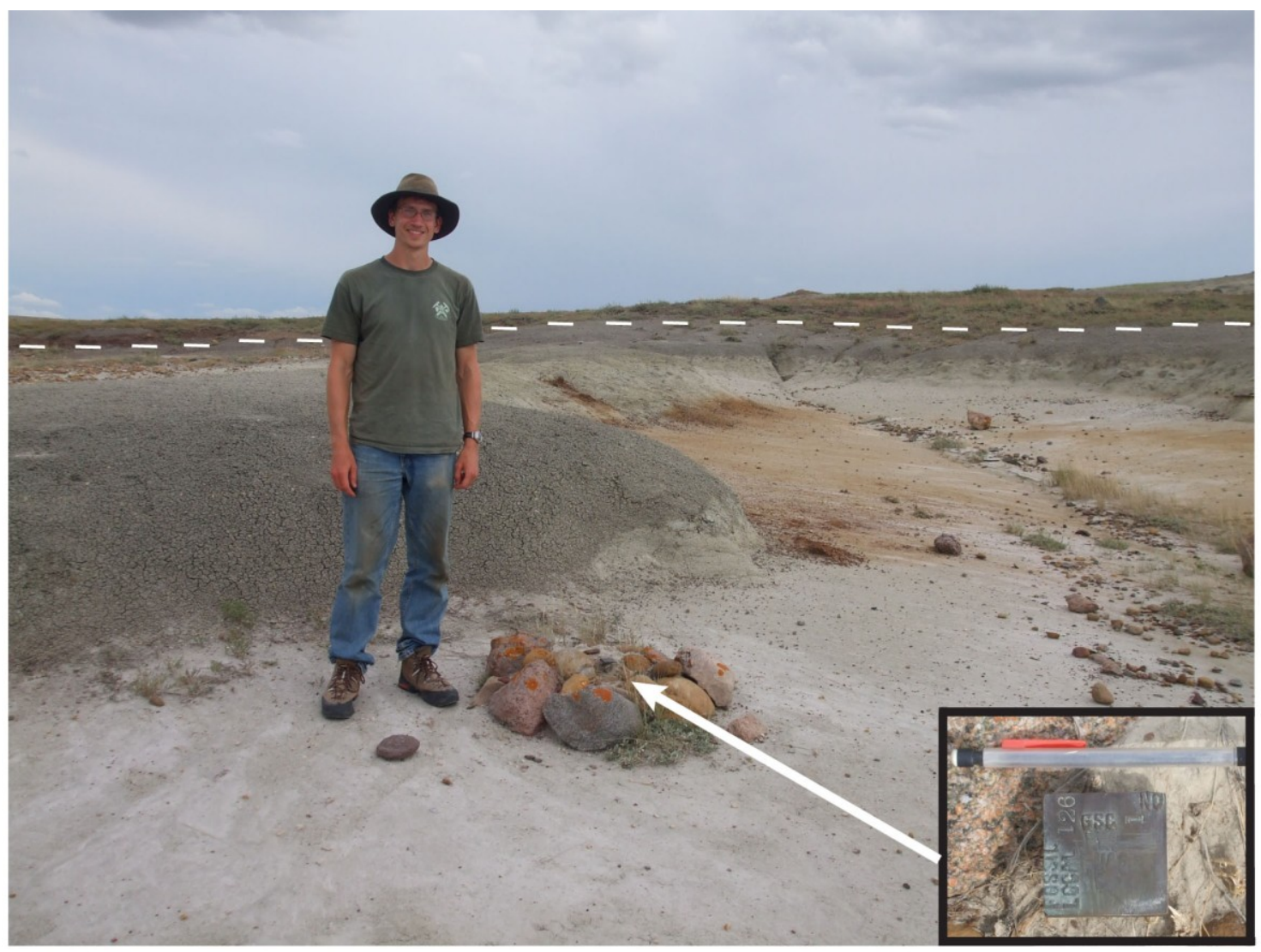

Figure 2. Chasmosaurus russelli holotype quarry, at Onefour Research Station, AB.

White dashed line in background represents base of Lethbridge Coal Zone. Inset shows quarry stake: "Fossil Local 126, GSC 1". Photo taken by David Evans. 
The first dinosaur material collected from the Belly River Group was not accurately mapped relative to modern methodologies (although many did subsequently have quarry stakes placed in them (Sternberg, 1950; Currie and Russell, 2005)), meaning that the precise stratigraphic and geographic localities of many quarries were poorly known. Currie and Russell (2005), and others, have recently used original field notes and photographs to successfully relocate many historic quarries (primarily within the Dinosaur Provincial Park region), including a number of Chasmosaurus specimens, and mapped them with precision GPS units. The Manyberries and Milk River regions in the southernmost portion of the province have also produced several significant Chasmosaurus specimens that are diagnosable to the species level, including the holotype of $C$. russelli (CMN 8800). However, this region is relatively understudied and the precise field locality data for some Chasmosaurus specimens collected from there is not currently documented, although they are being actively sought.

\subsection{Rationale for examining Chasmosaurus}

There was a tendency amongst early fossil taxonomists to name new species based on specimens that under modern criteria only differ slightly from each other, and would not be diagnosable. As more specimens have been collected over time, the recognition of the degree of morphological variation between supposed species has resulted in the synonymization of a large number of taxa (e.g., collapsing 16 species of Triceratops into Triceratops horridus; Ostrom and Wellnhofer, 1990).

The morphological features used to distinguish Chasmosaurus belli and Chasmosaurus russelli are similarly highly gradational. Although some workers have attributed these cranial differences to variation within a single species, these theories 
could not be thoroughly tested due to the lack of precise geographic and stratigraphic positions for many of the specimens that limited hypothesis testing of spatial and temporal relationships.

A detailed reappraisal of Chasmosaurus was conducted by Godfrey and Holmes (1995) who implicitly tested the null hypothesis of only one valid species. Although admitting that considerable gradational variation existed amongst Chasmosaurus specimens, they retained $C$. belli and $C$. russelli as separate taxa, and used the purported stratigraphic segregation of specimens assigned to these taxa in the Dinosaur Park Formation to support their conclusions. Since the time of their study, more chasmosaurine specimens have been collected from the Dinosaur Park Formation (Maidment and Barrett, 2011), and geographic and stratigraphic localities of earlier specimens have been better established.

Building off the work by Godfrey and Holmes (1995) and Maidment and Barrett (2011), I re-examined the hypothesis of one species of Chasmosaurus. I test the following null hypothesis, which must be satisfied if $C$. belli and C. russelli are synonymous: no morphological or phylogenetic distinction can be drawn between these taxa. I also test the null hypothesis that these taxa inhabited the same geographic region at the same time. If this latter hypothesis cannot be rejected, it does not necessarily mean that $C$. belli and C. russelli are synonymous, but it can be used to support their synonymy. I also test a third hypothesis, that these two 'species' represent different stages of maturity of a single taxon. The recognition of multiple taxa based on differing morphologies of a single ontogenetic series has been used to support the synonymization of previously well-supported taxa (e.g., Triceratops, Nedoceratops and Torosaurus forming a successive ontogenetic series; Scannella and Horner, 2010, 2011). Several 
ceratopsian species are currently represented by datasets including specimens representing a large range of putative ontogenetic stages: basal ceratopsians, e.g., Protoceratops andrewsi (Dodson, 1976) and Psittacosaurus (Meng et al., 2004); centrosaurines, e.g., Centrosaurus apertus (Ryan et al., 2001) and Pachyrhinosaurus lakustai (Currie et al., 2008); and chasmosaurines, e.g., Agujaceratops mariscalensis (Lehman, 1989) and Triceratops (Horner and Goodwin, 2006), that will be useful for investigating chasmosaurine ontogeny.

The potential synonymization of $C$. belli and $C$. russelli would have implications for hypotheses of ornithischian faunal turnover within the Dinosaur Park Formation. The Dinosaur Park Formation is currently divided into three faunal zones, with each characterized by a unique stratigraphically-restricted centrosaurine ceratopsid and lambeosaurine hadrosaurid zone (Ryan and Evans, 2005). In ascending stratigraphic order, they are the Centrosaurus-Corythosaurus Zone (lowest $30 \mathrm{~m}$ ), the StyracosaurusLambeosaurus lambei Zone, and the pachyrhinosaur-Lambeosaurus magnicristatus Zone. The latter two zones span the overlying $30 \mathrm{~m}$, with the latter zone at or including part of the Lethbridge Coal Zone (Fig. 1). The occurrences of C.russelli, C. belli and Vagaceratops irvinensis were previously thought to correspond to these lower, middle and upper faunal zones, respectively (Ryan and Evans, 2005).

The taxon Vagaceratops will also be reassessed based on its close phylogenetic relationship to Chasmosaurus and its recently demonstrated stratigraphic overlap with Chasmosaurus. 


\subsection{Research protocol}

The research conducted for this thesis project included both fieldwork in Alberta and museum-based specimen examination at institutions curating significant Chasmosaurus and related specimens.

Fieldwork was conducted during the summer of 2012 with the Southern Alberta Dinosaur Project (SADP) in the southern Milk River region of Alberta. The two accomplished goals of this fieldwork included the relocation of the Chasmosaurus russelli holotype (CMN 8800) quarry (Fig. 2) and the excavation of a partial chasmosaurine skull near the local town of Manyberries. Permits to access the C. russelli holotype quarry were issued to Dr. David Evans; the rest of the field work was carried out under permits issued to Drs. David Evans and Michael Ryan.

Museum collections visited included the American Museum of Natural History, the Canadian Museum of Nature, the Royal Ontario Museum, the Royal Tyrrell Museum of Palaeontology, the University of Alberta Laboratory for Vertebrate Palaeontology, and the Yale Peabody Museum where relevant material was photographed, measured, and character-coded. Only the Natural History Museum, London, UK, was not visited; information from the relevant material from this institution was obtained from Maidment and Barrett (2011).

This thesis is outlined as follows: Chapter 2 discusses the detailed taxonomy of Chasmosaurus and Vagaceratops; Chapter 3 discusses the geological setting of the Dinosaur Park Formation; Chapter 4 discusses the materials and methods; Chapter 5 provides a description of each specimen of Chasmosaurus and Vagaceratops, as well as descriptions of three previously undescribed specimens; Chapter 6 presents a specimenbased phylogenetic analysis of Dinosaur Park Formation chasmosaurines; Chapter 7 
examines the geographic and stratigraphic distributions of Chasmosaurus and Vagaceratops specimens; Chapter 8 examines the putative ontogenetic (growth) transformation series of Chasmosaurus specimens; Chapter 9 presents morphometric analyses of Chasmosaurus and Vagaceratops, using cranial linear and angular measurements; and Chapter 10 discusses the results of all analyses in light of the initial hypotheses. This thesis will comprise the most rigorous analysis of Chasmosaurus to date.

\subsection{Institutional abbreviations}

AMNH, American Museum of Natural History, New York, New York, U.S.A.; CMN, Canadian Museum of Nature, Ottawa, Ontario, Canada; MOR, Museum of the Rockies, Bozeman, Montana, U.S.A.; NHMUK, Natural History Museum of the United Kingdom, London, United Kingdom; ROM, Royal Ontario Museum, Toronto, Ontario, Canada; TMP, Royal Tyrrell Museum of Palaeontology, Drumheller, Alberta, Canada; UALVP, University of Alberta Laboratory for Vertebrate Palaeontology, Edmonton, Alberta, Canada; UCMP, University of California Museum of Paleontology, Berkeley, California, U.S.A.; UMNH VP, Utah Museum of Natural History Vertebrate Paleontology Collections, Salt Lake City, Utah, U.S.A.; USNM, National Museum of Natural History (formerly United States National Museum), Washington, D.C., U.S.A.; UTEP, University of Texas at El Paso, El Paso, Texas, U.S.A.; UW, University of Wyoming Museum of Geology, Laramie, Wyoming, U.S.A; YPM, Yale Peabody Museum, Yale University, New Haven, Connecticut, U.S.A; YPM VPPU, Division of Vertebrate Paleontology Princeton University Collection, Yale Peabody Museum, Yale University, New Haven, Connecticut, U.S.A. 


\section{Chapter 2. Taxonomy of Chasmosaurus}

\subsection{Taxonomic history}

Lambe (1902) erected the chasmosaurine species Monoclonius belli and Monoclonius canadensis, respectively, on the basis of a partial parietal (CMN 0491), and a fragmentary skull and single dorsal vertebra (CMN 1254). Both of these specimens were collected along the Red Deer River, near present-day Dinosaur Provincial Park, Alberta, from what is now referred to as the Dinosaur Park Formation (Fig. 1). M. belli was diagnosed as having a relatively straight posterior parietal bar, while $M$. canadensis was, in part, diagnosed by having relatively long postorbital horncores (Lambe 1902). Hatcher et al. (1907) reassigned these two species to the genus Ceratops, forming Ceratops belli and Ceratops canadensis, respectively. M. belli was assigned to Ceratops, based on the dissimilarity of its parietal to that of Monoclonius dawsoni. M. canadensis was assigned to Ceratops, based on the similarity of its postorbital horncore to that of Ceratops montanus. Ceratops canadensis was considered distinct from Ceratops montanus, based on differences in the cross-sectional shape and orientation of the postorbital horncore between these two species. Hatcher et al. (1907) noted the lack of comparative material between Ceratops belli and Ceratops canadensis, and suggested that future fossil discoveries may reveal that these two species are synonymous.

Lambe (1914a) assigned the relatively complete skull CMN 2245 to Ceratops belli. Based on this more complete specimen, he considered Ceratops belli to be distinct from both Monoclonius and Ceratops and referred it to its own genus, Protorosaurus, forming Protorosaurus belli. As Protorosaurus was discovered to be preoccupied, Lambe (1914b) erected the genus Chasmosaurus to receive it, forming Chasmosaurus 
belli. The following year, Lambe (1915) reassigned Ceratops canadensis to the genus Eoceratops, forming Eoceratops canadensis. This reassignment was made because $E$. canadensis (CMN 1254) was considered to be distinct from the fragmentary type material of Ceratops which consists of a non-diagnostic occipital condyle and pair of postorbital horncores. Gilmore (1923) referred the relatively small skull, UALVP 40, to $E$.

canadensis as well. Both CMN 1254 and UALVP 40 possess relatively long postorbital horncores considering their small skull size, and, partly for this reason, they were both considered adults of the 'pygmy' E. canadensis (Tyson, 1977).

Brown (1933) erected a second species of Chasmosaurus, Chasmosaurus kaiseni, based on the holotype skull AMNH 5401. C. kaiseni was diagnosed by its relatively long postorbital horncores. Later that year, Lull (1933) designated the skull ROM 839 as the holotype of a third species, Chasmosaurus brevirostris, diagnosed by a relatively short snout. After re-examining UALVP 40, Lull (1933) disagreed with Gilmore's (1923) assignment of the skull to E. canadensis, and argued instead that it more closely resembled C. kaiseni.

Sternberg (1940) erected a fourth species of Chasmosaurus, Chasmosaurus russelli, and designated the skull CMN 8800 as the holotype, and CMN 8801, 8802 and 8803 as paratypes. C. russelli was diagnosed by having a deeply embayed posterior parietal margin, differing from the relatively straight condition of this margin in C. belli. With the referral of CMN 8803 to C. russelli, the geographic range of Chasmosaurus previously restricted to Alberta - was expanded eastwards into Saskatchewan. CMN 8802 was supposedly destroyed during a museum move in 1941, with only the lower jaws salvaged (Godfrey and Holmes, 1995). However, in 2011, a large, fragmentary chasmosaurine skull labelled CMN 8798 was re-identified by Michael Ryan as the 
supposedly destroyed CMN 8802.

Following additional preparation of UALVP 40, Tyson (1977) agreed with Gilmore's (1923) original referral of this skull to E. canadensis. Lehman (1989) later agreed with Lull (1933) and proposed synonymising C. kaiseni with E. canadensis, based on their shared possession of elongate postorbital horncores. As Chasmosaurus has taxonomic priority over Eoceratops, Eoceratops became a junior synonym of Chasmosaurus, making Eoceratops canadensis the junior synonym of a fifth species of Chasmosaurus - Chasmosaurus canadensis.

Lehman (1989) erected a sixth species of Chasmosaurus, Chasmosaurus mariscalensis, based on material collected from a bonebed in the Upper Campanian Aguja Formation of Big Bend, Texas. He designated the partial skull UTEP P.37.7.086 as the holotype and referred several other specimens from the type locality and other nearby localities to this taxon. C. mariscalensis was diagnosed by Lehman (1989), in part, by its possession of short, broad squamosals with relatively large episquamosals. The referral of this species to Chasmosaurus greatly expanded the geographic range of this genus southwards, which until then had been restricted to southern Alberta and Saskatchewan.

Based on the ontogenetic variation present in C. mariscalensis, Lehman (1990) reasoned that the short snout of $C$. brevirostris was within the range of variation present in C. belli, and thus designated C. brevirostris a junior synonym of C. belli. Godfrey and Holmes (1995) conducted a systematic survey of all available Chasmosaurus specimens from the Dinosaur Park Formation to assess the validity of the three species present in that formation and valid as of that date (C. belli, C. canadensis and C. russelli). Godfrey and Holmes (1995:728) rediagnosed C. belli as: "parietal frill with nearly straight transverse posterior bars, each bearing one large triangular epoccipital on its 
posterolateral comer; other parietal epoccipitals variable in number and degree of coossification with the parietal, but always much smaller. The lateral bar of the parietal completely encloses the parietal fenestra". As these features were also present in $C$. canadensis (including C. kaiseni), they designated C. canadensis (including C. kaiseni) a junior synonym of C. belli. Godfrey and Holmes (1995) concurred with Lehman (1990) and retained $C$. brevirostris as a junior synonym of $C$. belli.

Godfrey and Holmes (1995:728) also rediagnosed C. russelli as: "posterior margin of parietal frill broadly arched on either side of median emargination. Each side bears three low triangular, roughly equal-sized epoccipitals. The lateral ramus of the parietal is reduced and does not completely encircle the fenestra in all but one specimen, permitting the squamosal to form a part of its lateral border". They concluded that there were two valid species of Chasmosaurus, C. belli and C. russelli, in the Dinosaur Park Formation; they also implied that $C$. mariscalensis of the Aguja Formation of west Texas was a distinct taxon.

Lehman (1998) proposed that the morphological variation within $C$. belli and $C$. russelli was within the realm of intraspecific, ontogenetic and sexual dimorphic variability within one species, $C$. belli, but he did not formally synonymize these taxa. He argued that these two species are end-members of a gradational morphological spectrum, and that no geographic or stratigraphic distinction could be drawn between them. However, he did not quantify the variation, and his statement of their stratigraphic overlap was unsupported at that time.

Holmes et al. (2001) erected a seventh species of Chasmosaurus, Chasmosaurus irvinensis, and designated the mostly complete skeleton CMN 41357 as the holotype, and also referred TMP 1987.045.0001 and TMP 1998.102.0008 to this species. C. irvinensis 
was diagnosed as: "chasmosaurine ceratopsid with transversely broad snout; nasal horn core short and transversely broad; brow horn absent, its normal position occupied by a low, raised, rugose boss, in one specimen bearing a large, smooth surfaced, hemispherical resorption pit; jugal notch on anterior squamosal broadly rounded and open (not parallelsided); squamosal tapers little posteriorly, subrectangular in outline, and projects almost directly laterally. Posterior parietal bar straight in anterior and dorsal aspects, projects only slightly posterior to the squamosal; maximum diameter of parietal fenestra less than length of preorbital region of the skull. Ten epoccipitals on posterior parietal bar, lateral epoccipital low and shield-shaped, the remaining eight nearly indistinguishably coossified together, and composed of flattened posteroventral laminae that wrap around the back of the bar, and larger laminae that curve strongly dorsally and anteriorly over the bar" (Holmes et al., 2001:1424).

C. irvinensis represented the third-known chasmosaurine to possess up to 10 epiparietals, after Torosaurus latus and Torosaurus utahensis. Following a phylogenetic analysis of chasmosaurines by Sampson et al. (2010), C. irvinensis was recovered outside the Chasmosaurus clade as a sister taxon to their newly described Kosmoceratops richardsoni, and moved it to the new genus, Vagaceratops, forming Vagaceratops irvinensis. The 10 epiossifications on the posterior margin of the frill of K. richardsoni were initially interpreted as epiparietals, as in V. irvinensis; however, this interpretation was based on adult specimens where the suture between the parietal and squamosal were not visible. Further examination of juvenile $K$. richardsoni specimens, where the parietalsquamosal suture was more visible, revealed that only six of these epiossifications are articulated with the parietal, resulting in an epiparietal count of six. Sampson et al. (2010) likewise reinterpreted the adult specimens of $V$. irvinensis as also having six epiparietals. 
Lucas et al. (2006) re-examined the material referred to C. mariscalensis and determined that it was sufficiently different from Chasmosaurus to be placed in a different genus, and, thus, erected Agujaceratops to receive it, forming Agujaceratops mariscalensis. Lucas et al. (2006:370) noted that Agujaceratops differs from Chasmosaurus in having "a relatively deep facial skeleton (at the orbit, though this varies somewhat ontogenetically), relatively short and broad squamosal (length/width $<2$ ), more than six relatively large epoccipitals on the squamosal (though some Chasmosaurus have as many as nine), lacking a parietal median bar that is deep or deeper dorsoventrally than wide with a rectangular/ subrectangular cross section and flat lateral sides, lacking a jugal flange that contacts the squamosal below the infratemporal fenestra, having a maxilla without a lateral horizontal shelf and a premaxilla without a posterodorsal extension, having relatively long supraorbital horncores and a relatively deep embayment of the posterior median parietal". This taxonomic modification restored Chasmosaurus to its former exclusively Canadian geographic range.

Longrich (2010) erected the new taxon, Mojoceratops perifania, from specimens previously referred to Chasmosaurus, and assigned TMP 1983.025.0001 as the holotype, CMN 8803 and TMP 1999.055.0292 as paratypes, and provisionally referred AMNH 5656, AMNH 5401, CMN 1254, CMN 34832, and TMP 1979.011.0147 to this species. M. perifania was diagnosed by the following features: "(1) lateral rami of the parietal posterior bar bearing a prominent sulcus on their anterior surface, (2) lateral rami of the parietal posterior bar with a thickened anterodorsal edge, bearing node-like swellings, (3) [epiparietal] 1 bearing a medial accessory process, (4) lateral rami of the parietal posterior bar anteroposteriorly narrow and dorsoventrally thickened, giving them a rodlike shape, (5) a strongly notched caudal margin of the parietals, with the lateral rami 
diverging at an angle of no more than $105^{\circ},(6)$ strongly arched lateral rami of the parietal posterior bar, giving the posterior bar a distinctive " $\mathrm{m}$ " shape, and extending the parietals well beyond the squamosals, (7) [epiparietals] project caudally from the parietal, (8) parietosquamosal frill erect, long axis of the parietal forming an angle of about $45^{\circ}$ with horizontal, (9) parietal; fenestrae anteriorly extended towards the [dorsotemporal fenestrae] to a degree not seen in Chasmosaurus, (10) triangular [frontoparietal] fontanelle with strong transverse expansion, (11) prominent [postorbital] horns, length exceeding $200 \%$ of basal diameter, (12) [postorbital] horns oriented dorsolaterally, being inclined away from the vertical plane by $45^{\circ}$ in anterior view, (13) epijugal ossification prominent and subconical" (Longrich, 2010:683). He also recognized that the seeminglystraight posterior parietal bar of AMNH 5401 was actually reconstructed. Based on these features, he reasoned that the Godfrey and Holmes' (1995) assignment of AMNH 5401 to C. belli was invalid. The validity of M. perifania was later reconsidered by Maidment and Barrett (2011). They referred M. perifania to a junior subjective synonym of $C$. russelli based on the fact that they were unable to confirm any of Mojoceratops' autapomorphies, or to identify any features distinguishing it from Chasmosaurus.

C. belli and C. russelli are currently the only valid species of Chasmosaurus. $C$. russelli, $C$. belli, and $V$. irvinensis represent the only known chasmosaurines from the Dinosaur Park Formation, and until recently have been believed to be stratigraphically separated and restricted to the lower, middle-to-upper, and uppermost intervals, respectively (Holmes et al., 2001; Ryan and Evans, 2005). This temporal separation has been used as support for distinguishing these three species (Holmes et al., 2001; Ryan and Evans, 2005). The most recent diagnoses for Chasmosaurus, C. belli and C. russelli by Maidment and Barrett (2011), and for V. irvinensis by Sampson et al. (2010) are 
presented in the next section.

\subsection{Systematic palaeontology}

\section{Ceratopsia Marsh, 1888}

Neoceratopsia Sereno, 1986

Ceratopsidae Marsh, 1888

Chasmosaurinae Lambe, 1915

Chasmosaurus Lambe, 1914b

Type species. Chasmosaurus belli Lambe, 1902:66

Diagnosis. "Chasmosaurus is diagnosed based on the following combination of characters: (1) Premaxillary flange along entire anterior margin of external naris; (2) supraorbital horns, when present, curve posteriorly along their length; (3) frill broadens posteriorly to form triangular shield with maximum width more than twice the skull width at orbits; (4) medial margin of squamosal, where it articulates with the lateral bar of the parietal, straight; (5) parietal fenestrae large, occupying most of the parietal, and being rounded or anteroposteriorly longer than transversely wide; (6) epiparietals triangular in shape and project posteriorly or dorsally but do not curve anteriorly. Characters 1-3 after Forster et al. (1993). All of these features are seen in other chasmosaurines and do not, therefore, represent autapomorphies, but this combination of features appears to be unique to Chasmosaurus." Maidment and Barrett (2011:4) 
Chasmosaurus belli Lambe, 1902

Monoclonius belli Lambe, 1902:59

Monoclonius canadensis Lambe, 1902:63

Ceratops belli Hatcher, Marsh and Lull, 1907:97

Ceratops canadensis Hatcher, Marsh and Lull, 1907:97

Protorosaurus belli Lambe, 1914a:131

Chasmosaurus belli Lambe, 1914b:149

Eoceratops canadensis Lambe, 1915:2

Chasmosaurus kaiseni Brown, 1933:2

Chasmosaurus brevirostris Lull, 1933:94

Chasmosaurus canadensis Lehman, 1989:139

Diagnosis. "Taxon displaying the combination of characters unique to the genus Chasmosaurus along with the following features: (1) parietal posterior bar bearing no median emargination and is nearly straight so that it forms a ' $\mathrm{T}$ ' shape with the parietal median bar; (2) lateral pair of epiparietals large and triangular; others, when preserved, are smaller (after Godfrey \& Holmes 1995). Both characters are autapomorphic for $C$. belli within Chasmosaurus." Maidment and Barrett (2011:4)

Holotype. "CMN 0491, a partial parietal. Although fragmentary, the holotype is diagnostic based on the combination of generic character 4 , and specific character 1 , a combination not observed in any other chasmosaurine." 
Distribution. Middle and upper beds of the Dinosaur Park Formation, Alberta, Canada (Ryan and Evans, 2005).

Referred specimens. AMNH 5402, CMN 2245, NHMUK R4948, ROM 843, and YPM 2016 (after Ryan \& Evans 2005).

\section{Chasmosaurus russelli Sternberg, 1940}

Mojoceratops perifania Longrich, 2010:683

Diagnosis. "Taxon displaying the combination of characters unique to the genus Chasmosaurus along with the following features: (1) parietal posterior bar bearing a median emargination and is broadly arched either side so that it forms an ' $\mathrm{M}$ ' shape with the parietal median bar; (2) each side of the posterior parietal bar bears three roughly equally sized epiparietals (after Godfrey \& Holmes, 1995). Both characters are autapomorphic for C. russelli within Chasmosaurus."

Holotype. "CMN 8800, comprising a partial skull lacking the lower jaw and part of the rostral, part of the jugals from both sides, part of the right quadrate, squamosal, and parietal (Sternberg 1940).” Maidment and Barrett (2011:5)

Distribution. Lower beds of the Dinosaur Park Formation, Alberta, Canada (Ryan and Evans, 2005).

Referred specimens. AMNH 5656, CMN 2280, CMN 41933, and TMP 1983.025.0001. 
Vagaceratops Sampson et al., 2010

Type and only species. Vagaceratops irvinensis Holmes, Forster, Ryan, and Shepherd, 2001:1424

Diagnosis. "Chasmosaurine ceratopsid diagnosed by the following autapomorphies": 1) “jugal notch on proximal squamosal broadly rounded and open (not parallel sided)"; 2) “transverse parietal bar straight"; 3) “epiparietals (ep1-ep3) and epiparietosquamosal (eps) short, forming recurved flat laminae"; and 4) "predentary length one half that of dentary”. Sampson et al. (2010:5)

Holotype. CMN 41357.

Distribution. Upper Dinosaur Park Formation, Alberta, Canada.

Referred specimens. TMP 1987.045.0001, TMP 1998.102.0008 and TMP 2009.034.0009. 


\section{Chapter 3. Regional Geology}

All specimens examined in this study were collected from Upper Cretaceous (Upper Campanian) sediments of southern Alberta and Saskatchewan (Fig. 3). The Belly River Group in these regions comprises three terrestrial formations which were deposited along the western margin of the Western Interior Sea (Eberth, 2005). In ascending stratigraphic order, these are the Foremost, Oldman and Dinosaur Park formations (Fig. $1)$.

The Belly River Group was originally referred to by Dawson (1883) as the Belly River Series, which also included the underlying Pakowki Formation and part of the older Milk River Group (Eagle Formation) (Eberth, 2005). Russell and Landes (1940) divided the part of the Belly River Series above the Pakowki Formation into the Foremost and Oldman formations. McLean (1971) had difficulty in distinguishing the Foremost and Oldman formations and merged them into the Judith River Formation. McLean's (1971) use of the name Judith River was based on the time equivalent Judith River Formation of north central Montana, where it had been in usage since 1915 (Bowen, 1915).

Eberth and Hamblin (1993) elevated the Judith River Formation to group status (Judith River Group), and subdivided into the Foremost, Oldman and Dinosaur Park formations. They retained the Foremost Formation, as defined by Russell and Landes (1940), and the Oldman Formation, but designated the upper portion of this latter unit as the Dinosaur Park Formation. The Oldman and Dinosaur Park formations were defined on the basis of differing lithologies (discussed below), environments (low-sinuosity, ephemeral fluvial system vs. high-sinuosity, fluvial-to-estuarine system, respectively), sediment sources and drainage directions (southern Cordillera of Canada and northern 


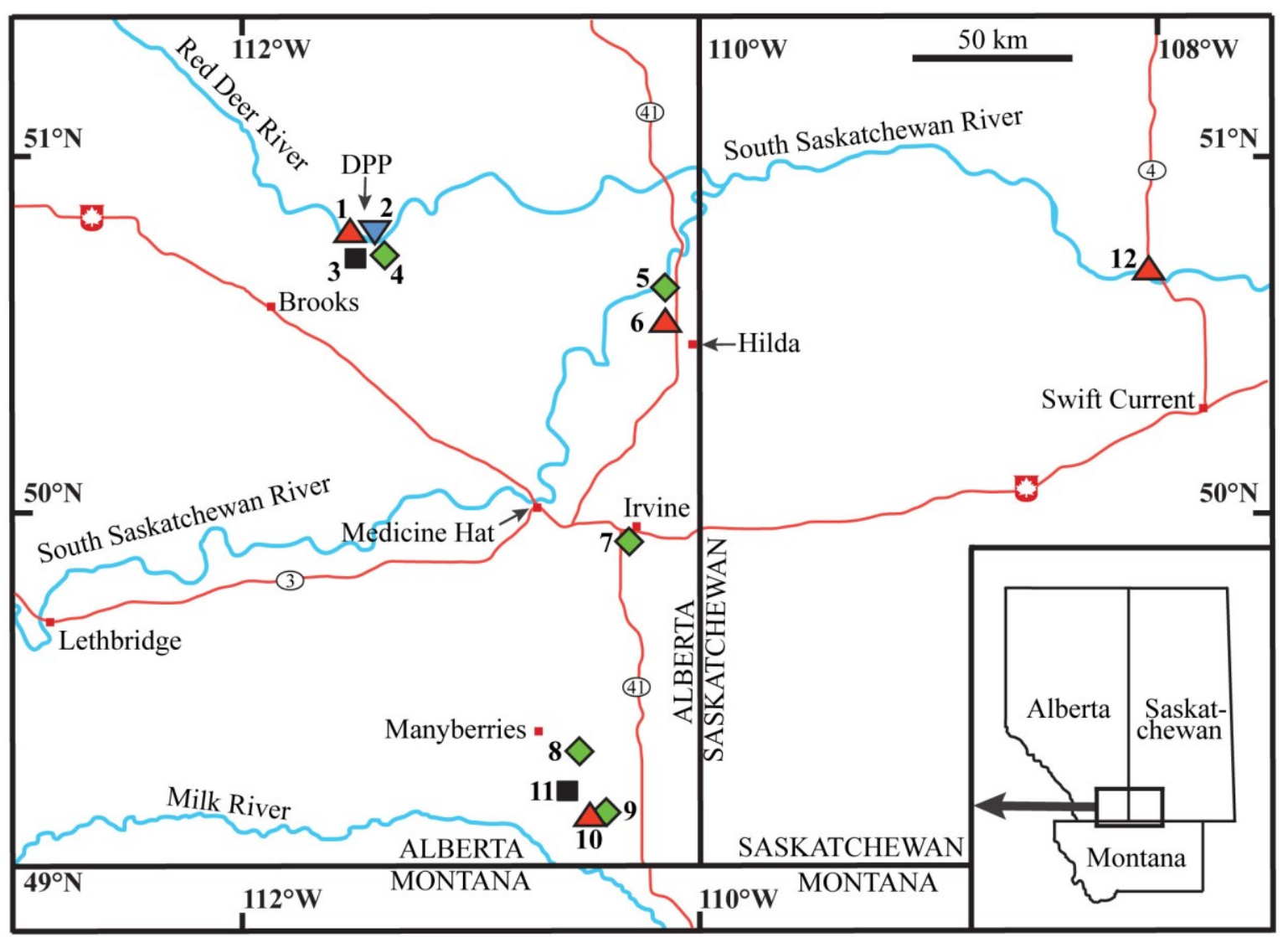

Figure 3. Regional map of Chasmosaurus russelli (red triangles), C. belli (blue inverted triangle), Chasmosaurus sp. (black squares), and Vagaceratops-like specimens (green diamonds): 1) AMNH 5401, AMNH 5656, CMN 0491, CMN 1254, CMN 2245, CMN 2280, CMN 8801, CMN 34829, CMN 34832, NHMUK R4948, ROM 839, ROM 843, TMP 1979.011.0147, TMP 1981.019.0175, TMP 1993.082.0001, TMP 1999.055.0292, and UALVP 40; 2) AMNH 5402; 3) YPM 2016; 4) TMP 1987.045.0001; 5) TMP 2009.034.0009; 6) TMP 1997.132.0002; 7) CMN 41357; 8) TMP 2011.053.0046; 9) TMP 1998.102.0008; 10) CMN 8800; 11) CMN 8802; and 12) CMN 8803. Specific locality data is not known for 6), but it may actually be closer to the South Saskatchewan River. DPP $=$ Dinosaur Provincial Park. 
Montana, draining northeastwards vs. north and central Cordillera and draining southeastwards, respectively), and their separation by a diachronous regional disconformity (Fig. 4). Jerzykiewicz and Norris (1994) later renamed Eberth and Hamblin's (1993) Judith River Group as the Belly River Group in the southwestern foothills region of Alberta. Hamblin and Abrahamson (1996) extended the use of Belly River Group into the southeastern prairie region of Alberta.

The Dinosaur Park and Oldman formations both have a wedge-shaped geometry, with the Oldman Formation thickening to the southwest, and the overlying Dinosaur Park Formation thickening to the northwest (Eberth and Hamblin, 1993: Fig. 19). The rising Cordillera to the west provided the sediment source for both of these formations, with sediments of the Oldman Formation originating along the eastern margin of the southern Cordillera in British Columbia and northern Montana, and sediments of the Dinosaur Park Formation originating along the north and central parts of the Canadian Cordillera (Eberth and Hamblin, 1993). These two sediment sources account for the differing lithology and relative thicknesses of the Oldman and Dinosaur Park formations. The Dinosaur Park Formation can be traced in outcrop and wire-line logs from Edmonton, Alberta, to Havre, Montana, and likely extends as far east as Saskatoon, Saskatchewan (Eberth and Hamblin, 1993). The Dinosaur Park Formation is approximately $120 \mathrm{~m}$ thick near Edmonton, and gradually thins out to the south, southeast and southwest. In the area of Dinosaur Provincial Park, the Dinosaur Park Formation has an exposed total thickness of $70 \mathrm{~m}$ (Eberth, 2005), versus only 30 m, or less, in the Milk River region (Eberth and Hamblin, 1993; Fig. 3). Exposed outcrop in Dinosaur Provincial Park reveals the uppermost $20 \mathrm{~m}$ of the Oldman Formation, the entire 70 m-thick Dinosaur Park Formation, and the lowermost $20 \mathrm{~m}$ of the Bearpaw Formation (Eberth, 2005). 


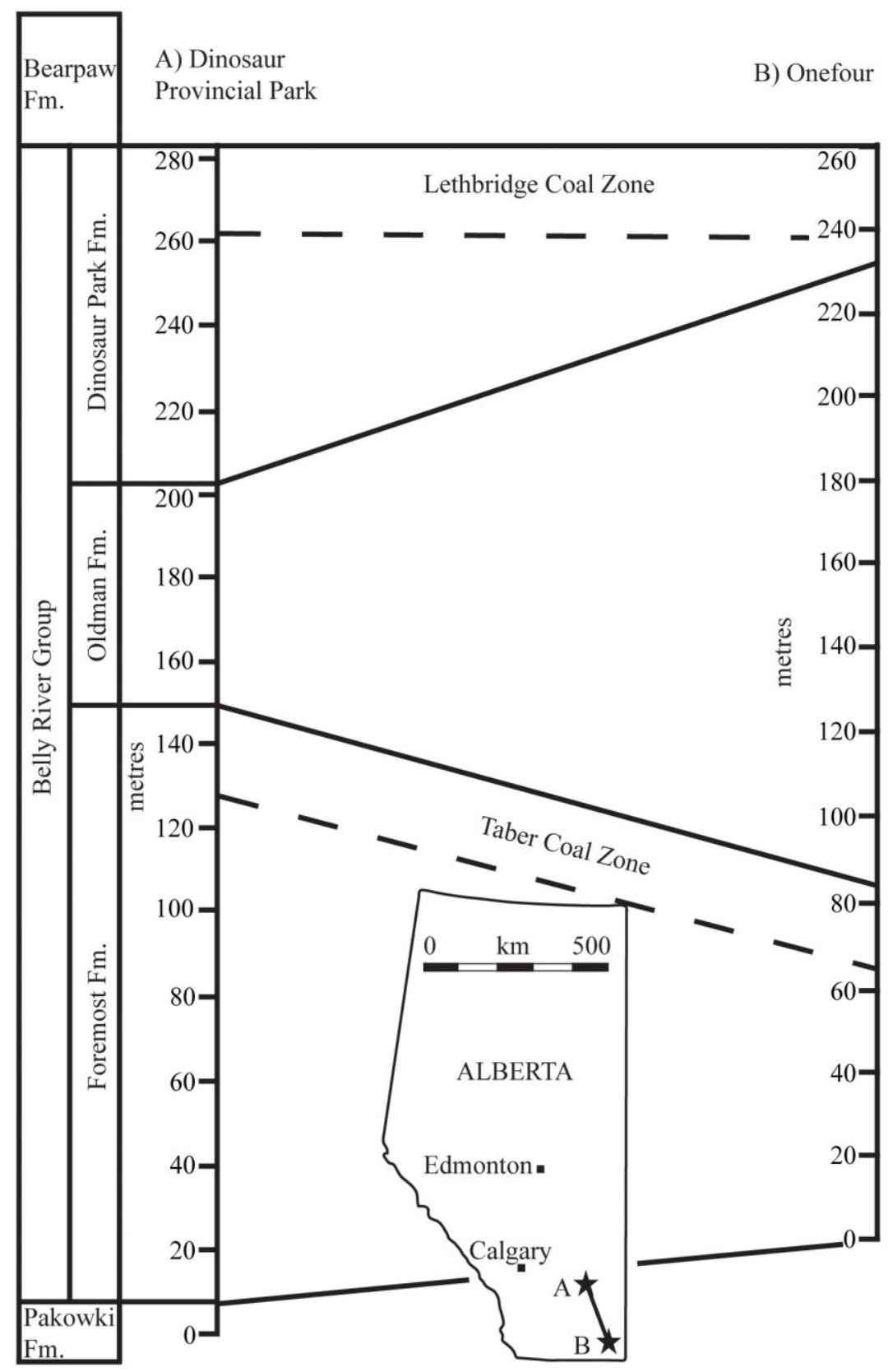

Figure 4. Stratigraphic relationships of the Belly River Group between Dinosaur

Provincial Park (A) and the Onefour (B) area of southern Alberta. Note the relative difference in thickness of the Dinosaur Park Formation above the regional disconformity in each area (Eberth and Hamblin, 1993: Figs. 19a, A1, modified); inset shows Province of Alberta and transect between (A) and (B). 
The Oldman and Dinosaur Park formations are separated by a time-transgressive regional disconformity, becoming progressively younger southwardly and westwardly (Eberth and Hamblin, 1993; Fig. 3). As a result of this diachronous contact, sediments of the upper portion of the Oldman Formation in the Milk River region are time equivalent to the lower portion of Dinosaur Park Formation as exposed in Dinosaur Provincial Park. Eberth (2005) divided the Oldman Formation into three informal stratigraphic units: the lower and upper units (units 1 and 3) exhibit isolated and lenticular palaeochannel-fills, and the middle unit (Unit 2; also referred to as the Comrey Sandstone Zone) displays amalgamated palaeochannel-fills and multimetre-thick sheets of sandstone (Fig. 1). The top of Unit 2 is exposed in Dinosaur Provincial Park.

The Dinosaur Park Formation is composed of a lower sandy interval composed of alluvial palaeochannel deposits, an upper muddy interval dominated by overbank deposits, and the Lethbridge Coal Zone in the uppermost (15 m thickness, as exposed in Dinosaur Provincial Park) interval (Eberth, 2005; Figs. 1 and 3). The Dinosaur Park Formation was deposited during the last major transgression of the Western Interior Sea (Bearpaw Sea), and transitions to the overlying marine Bearpaw Formation (Eberth, 2005). The Dinosaur Park-Bearpaw formational contact is conformable but timetransgressive, becoming progressively younger in a westward direction (Eberth, 2005). The westward migration of this contact over time is a result of the westward expansion of the Bearpaw Sea, which spanned the Late Campanian to Early Maastrichtian (He et al., 2005).

In Dinosaur Provincial Park, bentonites derived from the top of the Oldman Formation and near the base of the Bearpaw Formation yielded ${ }^{40} \mathrm{Ar} /{ }^{39} \mathrm{Ar}$ dates of 
77.0土0.5 Ma and 75.5 Ma, respectively (David Eberth, pers. comm.; Fig. 1). The Dinosaur Park Formation, therefore, spans approximately 1.44 Ma. Recently completed laser ablation-inductively coupled plasma-mass spectrometry (LA-ICP-MS) analyses of zircons from these same bentonites have yielded ${ }^{206} \mathrm{~Pb} /{ }^{238} \mathrm{U}$ dates which suggest that the Dinosaur Park Formation may have spanned an even shorter time interval (approximately 1.26 Ma; David Eberth, pers. comm.). Additional bentonites from the middle portion of the Dinosaur Park Formation and base of the Lethbridge Coal Zone in Dinosaur Provincial Park have also yielded ${ }^{40} \mathrm{Ar} /{ }^{39} \mathrm{Ar}$ dates of $76.4 \pm 0.4 \mathrm{Ma}$ and $76.1 \pm 0.5 \mathrm{Ma}$, respectively (David Eberth, pers. comm.; Fig. 1).

Assuming a constant rate of sedimentation between the upper Oldman Formation (5.5 m below Dinosaur Park Formation) and middle of the Dinosaur Park Formation (36.0 $\mathrm{m}$ above Oldman Formation), and between the middle of the Dinosaur Park Formation and the base of the Lethbridge Coal Zone (61.5 m above Oldman Formation) in Dinosaur Provincial Park, the average rate of accumulation for the lower and upper halves of the Dinosaur Park Formation are approximately $6.9 \mathrm{~cm}$ and $8.5 \mathrm{~cm} / 1000$ years, respectively (David Eberth, pers. comm.). It is important to note that the bedding surfaces of the Dinosaur Park Formation are not planar in some places, and can be incised by Ushaped, mud-filled fluvial channels up to $8 \mathrm{~m}$ deep in Dinosaur Provincial Park, and in an exceptional case up to $16 \mathrm{~m}$ deep near Onefour, Alberta (Eberth, 1996). Each channel was formed during a regressive-transgressive cycle, causing incision and subsequent fill of channels. As a result of such channel formation, a hypothetical specimen collected along a planar surface could have the same stratigraphic elevation as one collected at the base of a younger incised channel. 
The Judith River Formation of north central Montana was deposited between 79 and $74 \mathrm{Ma}$ (Goodwin and Deino, 1989; Rogers, 1995; Rogers and Swisher, 1996; Foreman et al., 2008; Rogers et al., 2010), the base of which is lithostratigraphically correlated to the base of the Oldman Formation in southern Alberta (Rogers, 1994), which is 78.3 Ma (Eberth and Deino, 1992). The Oldman and Dinosaur Park formations of Canada are lithostratigraphically equivalent to the Judith River Formation of the United States, differing only in name (Eberth and Hamblin, 1993). The Oldman and Dinosaur Park formations also correlate to portions of the Two Medicine Formation of western Montana (Rogers, 1994).

Specimens of Chasmosaurus and Vagaceratops have been collected from the Dinosaur Park Formation at several Albertan localities in or near Dinosaur Provincial Park, Manyberries, Onefour, Sandy Point, Hilda, and Irvine, and one site in Saskatchewan at Saskatchewan Landing Provincial Park (Appendix I). Collection sites are typically limited to outcrop in river valleys (e.g., Red Deer and South Saskatchewan rivers) and adjacent badlands, although outcrops can also occur in localized regions by erosion of the overlying younger sediments and soils. Although the source sediments for the Dinosaur Park Formation extends southwards into northern and western Montana as the Judith River and Two Medicine formations, respectively (Eberth and Hamblin, 1993), Chasmosaurus and Vagaceratops are not known from these regions.

The time-transgressive Dinosaur Park-Oldman formational contact (Fig. 3) is a complicating factor in stratigraphic correlations across Alberta and Saskatchewan. A hypothetical fossil collected from the lower portion of the Dinosaur Park Formation in Dinosaur Provincial Park is time equivalent to one collected from the upper Oldman 
Formation in the Milk River region. This contact is therefore a poor frame of reference when comparing the stratigraphic positions of specimens north-south across Alberta. The diachroneity of this contact, however, is negligible in relatively small geographic regions where specimens are densely concentrated (i.e., Dinosaur Provincial Park). In this latter case, the Dinosaur Park-Oldman formational contact can be used as a frame of reference for those specimens.

The Dinosaur Park-Bearpaw formational contact (rather, the underlying contact between the upper muddy interval and Lethbridge Coal Zone in the Dinosaur Park Formation) is a more reliable frame of reference for specimens arranged along a northsouth transect. This transect lies approximately parallel to the palaeoshoreline of the Bearpaw Sea, and sea level would have been consistent along its length at any given time during the deposition of the Dinosaur Park Formation (Eberth and Hamblin, 1993). The swampy environment represented by the Lethbridge Coal Zone (Braman and Koppelhus, 2005 ) is therefore an approximately isochronous frame of reference north-to-south; the Lethbridge Coal Zone also maintains a uniform thickness north-to-south, unlike the Dinosaur Park or Oldman formations (Eberth and Hamblin, 1993). The Dinosaur ParkBearpaw formational contact or Lethbridge Coal Zone are, however, poor frames of reference moving east-west across Saskatchewan and Alberta. Sediments from the upper portion of the Dinosaur Park Formational in Dinosaur Provincial Park are time equivalent to those of the Bearpaw Formation in Saskatchewan (Eberth, 2005).

The Taber Coal Zone is another regionally extensive frame of reference across Alberta, and lies at the top of the Foremost Formation, immediately below the Oldman Formation (Eberth and Hamblin, 1993; Fig. 3). In the Foremost Formation in the 
subsurface of Dinosaur Provincial Park, the Taber Coal Zone is overlain by a sandy zone referred to by Eberth (2005) as the Herronton Sandstone Zone. This sandy zone is exposed in outcrop $15 \mathrm{~km}$ northeast of Dinosaur Provincial Park (Jenner Bridge; Eberth, 2005), as well as the Milk River region.

The geographic distribution of Chasmosaurus and Vagaceratops specimens with known stratigraphy, which excludes a fragmentary Chasmosaurus specimen collected from Saskatchewan (Saskatchewan Landing Provincial Park), defines a longitudinally narrow north-south corridor spanning Dinosaur Provincial Park to the Onefour (Milk River) area in southeastern Alberta (Appendix I). As this corridor is twice as long as it is wide, the base of the Lethbridge Coal Zone is a better frame of reference to use than the Dinosaur Park-Oldman formational contact, when comparing specimen stratigraphy across Alberta. 


\section{Chapter 4. Materials and Methods}

\subsection{Introduction}

The analytical methods used in this thesis are outlined here and are organized based on the order of appearance of the analyses in subsequent chapters. This chapter begins by introducing the fossil specimens examined in this study, followed by the methods of analyses conducted on these specimens, including: specimen-based phylogenetic analysis, geographic and stratigraphic distributions, ontogenetic analysis, and morphometric analyses.

\subsection{Material examined}

All specimens currently referred to Chasmosaurus and Vagaceratops are curated in the collections of the AMNH, CMN, NHMUK, ROM, TMP, UALVP, and YPM (see Chapter 1 for institutional abbreviations). With the exception of NHMUK, I visited all of these institutions and examined, photographed, coded morphological characters, and measured their relevant specimens first-hand. The majority of photographs were taken using a FujiFilm FinePix A900 digital camera, although some were taken by other individuals, where noted. In addition to the specimens listed below, three chasmosaurine partial skulls (CMN 8802, CMN 34829 and TMP 2011.053.0046) were also described in detail for this project (Chapter 5). 


\section{Measurements}

Skull measurements followed Godfrey and Holmes (1995: Fig. 5), with additional new measurements added, for a total of 36 parameters (Fig. 5). A set of Fowler digital sliding callipers were used for measurements along a relatively flat surface and up to 300 $\mathrm{mm}$ in length. Measurements along a curved surface and/or exceeding $300 \mathrm{~mm}$ in length were made using a cloth measuring tape. All measurements were taken a minimum of three times and the average is reported in Appendix II. AMNH 5401 and 5402 are inaccessibly displayed behind glass panels, so the measurements published by Godfrey and Holmes (1995) were used. NHMUK R4948 is in the Collections of the Natural History Museum, London, which was not visited for this project; instead, the measurements published by Maidment and Barrett (2011) were used. The posterior (frill) half of the skull CMN 41357 is on display in the fossil gallery of the Victoria Memorial Museum Building (Ottawa, Ontario), and the disarticulated elements representing the anterior half of the skull are stored in drawers in the collections of the Natural History Building (Aylmer, Quebec). Measurements for this skull were instead obtained from a cast specimen (housed in Aylmer), as it incorporates all of these elements and better facilitated examination. All linear measurements taken are given in Appendix II. 


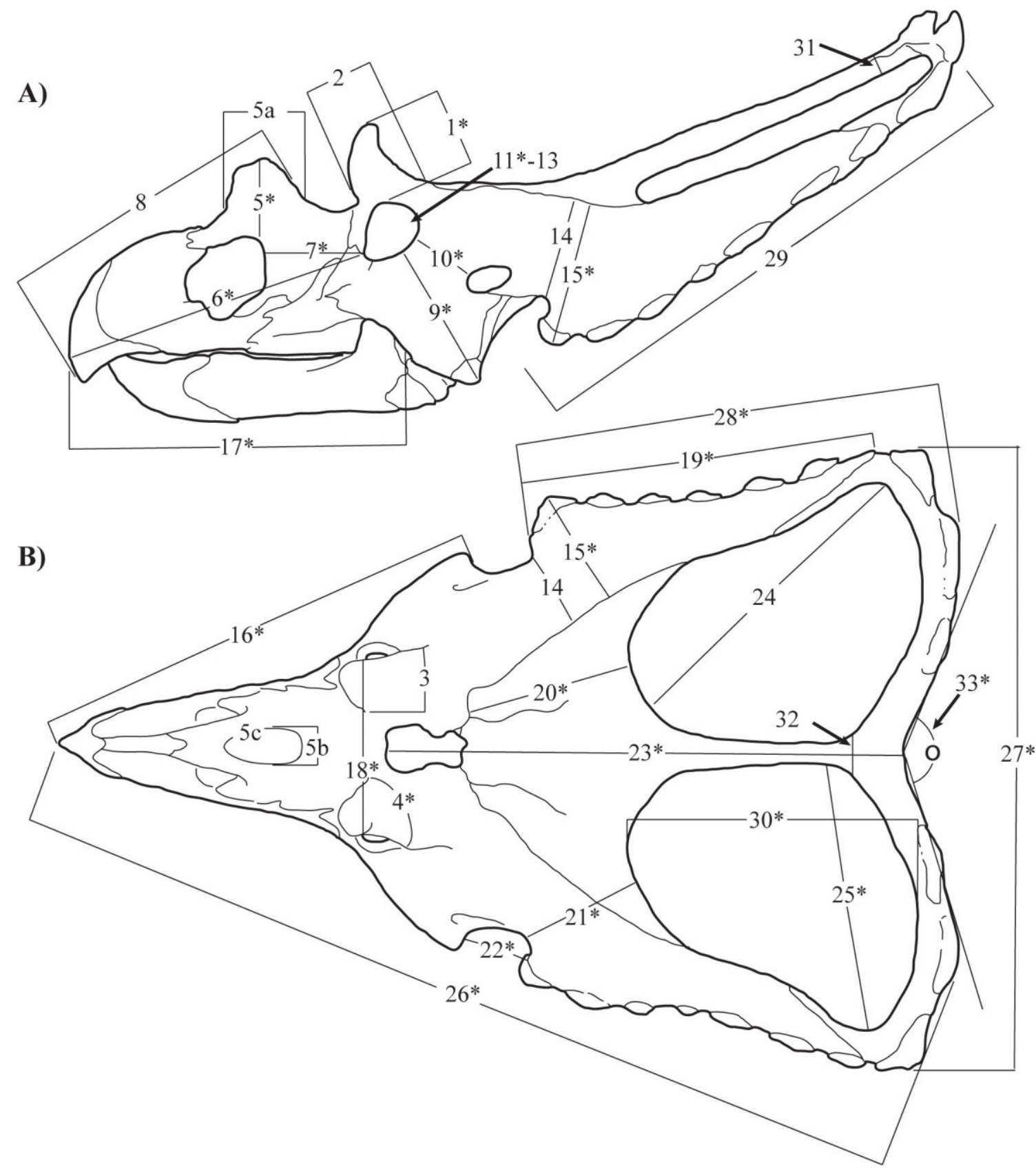

Figure 5. Parameters of cranial measurements used in this study: A) lateral view; and B) dorsal view (Modified from Godfrey and Holmes, 1995: Fig. 5). Asterisks denote parameters used in principal component analysis. 


\subsubsection{Chasmosaurus}

The following cranial specimens have been referred to Chasmosaurus. The original specific designation (if any) for each specimen is listed in Appendix I.

Complete and partial skulls:

AMNH 5401 (holotype, Chasmosaurus kaiseni), AMNH 5402, AMNH 5656, CMN 1254 (holotype, Eoceratops canadensis), CMN 2245, CMN 2280, CMN 8800 (holotype, Chasmosaurus russelli), CMN 8801, CMN 8802, CMN 34829, CMN 34832, NHMUK R4948, ROM 839 (formerly ROM 5436; holotype, Chasmosaurus brevirostris), ROM 843 (formerly ROM 5499), TMP 1979.011.0147, TMP 1981.019.0175, TMP 1983.025.0001 (holotype, Mojoceratops perifania), TMP 1993.082.0001, UALVP 40, YPM 2016.

Partial frills (including isolated parietals and squamosals):

CMN 0491 (holotype, Chasmosaurus belli), CMN 8803, CMN 41933, TMP

1981.018.0231, TMP 1981.023.0024, TMP 1981.030.0001, TMP 1982.016.0215, TMP

1986.036.0530, TMP 1991.046.0004, TMP 1993.075.0005, TMP 1993.086.0001, TMP

1994.012.0778, TMP 1996.029.0004, TMP 1997.132.0002, TMP 1998.093.0010, TMP

1998.128.0001, TMP 1999.055.0274, TMP 1999.055.0292, TMP 2007.020.0103, TMP 2008.012.0001, TMP 2009.032.0011.

Isolated postorbital horncores:

CMN 0186, CMN 0212, CMN 0275, CMN 0837, CMN 0838, CMN 0841 (with orbital rim, prefrontal and frontal), CMN 9673 (with orbital rim), CMN 9676, CMN 9808 (three 
horncores), CMN 9809, TMP 1967.017.0005 (with orbital rim), TMP 1979.014.0813, TMP 1979.014.0814, TMP 1981.016.0284, TMP 1981.016.0499, TMP 2008.012.0074.

Notes regarding specimen availability and cataloguing:

The following chasmosaurine specimens from the Dinosaur Park Formation housed in the collections of the Royal Tyrrell Museum of Palaeontology were unavailable for my examination, for the following reasons (Graeme Housego, pers. comm.): TMP 1981.003.0004 ("complete skull, Chasmosaurus sp."; in a warehouse in Red Deer, Alberta), TMP 1982.004.0008 ("complete skeleton, Chasmosaurus belli"; uncollected in Dinosaur Provincial Park), TMP 1982.016.0022 ("incomplete skull"; still unprepared), TMP 1989.007.0006 ("complete skeleton, Chasmosaurus belli"; sold to the Children's Museum of Indianapolis, Indiana), TMP 2009.032.0008 (“Chasmosaurus sp., squamosal"; still jacketed), and TMP 2011.053.0056 ("epoccipital"; still unprepared).

Longrich (2010) used the specimen number CMN 34832 interchangeably with CMN 34893 for a partial Chasmosaurus skull, with CMN 34893 actually being the specimen number for a fish fossil (Margaret Currie, pers. comm.).

One of the Chasmosaurus specimens listed in Currie and Russell (2005), ROM 632, has been found to have been previously deaccessioned from the ROM collections (David Evans and Kevin Seymour, pers. comm.). Although it was collected in Dinosaur Provincial Park, no stratigraphic or quarry data is associated with it (Currie and Russell, 2005).

An unnumbered $C$. belli specimen listed in Currie and Russell (2005:545) appears to be located in an undetermined location in Rio de Janeiro, Brazil. This specimen was 
collected from the Dinosaur Park Formation in what is now Dinosaur Provincial Park by Levi Sternberg in 1930 (Currie and Russell, 2005: CD ROM). The precise quarry and stratigraphic elevation have been documented, allowing the specimen to be placed into its proper stratigraphic context with other Chasmosaurus specimens (Appendix I).

An unnumbered RTMP Chasmosaurus specimen listed in Currie and Russell (2005:545) still remains uncollected in Dinosaur Provincial Park, quarry U172 (Don Brinkman, pers. comm.). Described simply as "edge of skull" (Currie and Russell, 2005: CD ROM), it was assigned to Chasmosaurus sp., but further excavation has yet to reveal whether the specimen can be diagnosed to species level.

\subsubsection{Vagaceratops}

The following cranial specimens have been referred to Vagaceratops. The original specific designation (if any) for each specimen is listed in Appendix I.

Skulls:

CMN 41357 (holotype, Vagaceratops irvinensis), TMP 1987.045.0001, TMP 2009.034.0009.

Partial frills:

TMP 1998.102.0008, TMP 2007.020.0039.

\subsection{Specimen-based phylogenetic analysis}

A specimen-based phylogenetic analysis for Chasmosaurus and Vagaceratops specimens $(\mathrm{n}=17)$ was conducted using the character-taxon matrix of Mallon et al. 
(2011). This matrix was chosen as it includes a number of representative chasmosaurine and centrosaurine species, as well as more basal ceratopsians, all of which serve as outgroup taxa to Chasmosaurus and Vagaceratops. The chasmosaurines Triceratops horridus, Torosaurus prorsus, Torosaurus latus, Torosaurus utahensis, and Nedoceratops hatcheri were all included in this analysis, despite their recent synonymization into one putative species, Triceratops horridus by Scannella and Horner (2010, 2011). These five species were coded as separate taxa (sensu Sampson et al., 2010; Mallon et al., 2011; Wick and Lehman, 2013), as their synonymization is still debated (Longrich and Field, 2012). Two new chasmosaurines were added to the matrix: Judiceratops tigris (Longrich, 2013) and Bravoceratops polyphemus (Wick and Lehman, 2013).

The Mallon et al. (2011) matrix consists of 149 morphological characters, of which 127 pertain to the skull and the remaining 22 are postcranial. Some changes, detailed in Appendix III, were made to their 2011 character state scores based on my reevaluation of their characters. Wick and Lehman (2013) also used the Mallon et al. (2011) matrix, and included two new characters, 150 and 151; the former character was used in this study, but the latter was not, as it was found to be parsimony uninformative; character 150 describes the shape of the contact between the quadratojugal and squamosal (Fig. 6). Three additional characters (151-153) were added to this matrix for a total of 153 characters (Chapter 6); characters 151-153 describe the shape and orientation of epiparietals 4 and 5. The complete list of characters is given in Appendix III.

Some characters in this study describe the presence or absence of a feature, and are followed by other characters that describe that feature in species or specimens that 
possess that feature. Species or specimens coded as 'absent' for the former characters were coded as '?' in the latter characters. Species or specimens in which a given feature is not preserved were also coded as'?' for characters relating to that feature. Therefore, species or specimens in which a given feature is either absent or not preserved were coded as "?".

A parsimony analysis was conducted on the character-taxon matrix using the program TNT 1.1 (Tree analysis using New Technology; Goloboff et al., 2003) with the tree bisection reconnection swapping algorithm using 1000 replicates, and the basal ceratopsian Leptoceratops gracilis specified as the outgroup taxon. Ten trees were saved per replication and a maximum of 10,000 trees stored per replicate. Relative support via bremer (decay) and bootstrap values for the recovered nodes were determined in TNT. Bootstrap values were obtained by conducting a bootstrap analysis using 1000 replicates.

\subsection{Geographic and stratigraphic distributions}

Geographical data

Only cranial material with diagnostic features and precise geographic and stratigraphic locality data were used in this study. Geographic locality data was obtained from several sources, including Currie and Russell (2005: CD ROM), the CMN fossil collections database (Margaret Currie, pers. comm.), the RTMP online collections database (HeRMIS, 2012), David Eberth (pers. comm.), and Michael Ryan and David Evans (pers. comm.). Stratigraphic data (elevations above sea level) was obtained from Currie and Russell (2005), except for TMP 1979.011.0147, which I collected first-hand in the field using a Garmin ETrex 30 handheld GPS unit. Stratigraphic data for specimens 
outside of Dinosaur Provincial Park were obtained from Michael Ryan (pers. comm.) for CMN 41357 and TMP 1998.102.0008, from David Eberth (pers. comm.) for TMP 2009.034.0009, from David Evans (pers. comm.) for TMP 2011.053.0046, and personally recorded with David Evans and Michael Ryan for CMN 8800. Locality data for specimens examined are given in Appendix I.

Stratigraphic data

Stratigraphic elevation relative to the Oldman-Dinosaur Park formational disconformity was calculated using the following method. The geographic locations of specimens collected in Dinosaur Provincial Park were plotted onto a structural contour map (Eberth, 2005) of that region. The contours of this map mark the approximate elevation (metres above sea level) of the regional disconformity separating the Oldman and Dinosaur Park formations within the Dinosaur Provincial Park region; the elevation of this disconformity varies by $30 \mathrm{~m}$ between the northwest and southeast corners of the Dinosaur Provincial Park region (Eberth, 2005). By subtracting a given specimen's elevation above sea level from the contact's elevation above sea level in a given locality on the map, that specimen's elevation above the contact could be determined with the caveat that specimens located further from the original mapped transect of the contact have progressively less accurate stratigraphic elevations above the disconformity. Error bars for each specimen is taken as $\pm 5 \mathrm{~m}$ following the methods of Ryan (2003) which take into account the potential problem of specimens preserved in palaeochannels.

For specimens collected in regions outside of Dinosaur Provincial Park where the Oldman-Dinosaur Park formational contact is younger than in Dinosaur Provincial Park 
(Figs. 3-4), the stratigraphic positions of these specimens were determined relative to the base of the Lethbridge Coal Zone. These specimens are:

CMN 8800 (Chasmosaurus russelli holotype), collected approximately $1 \mathrm{~m}$ below the Lethbridge Coal Zone, or approximately $54 \mathrm{~m}$ above the Oldman-Dinosaur Park formational contact in Dinosaur Provincial Park; TMP 2011.053.0046 collected approximately $2 \mathrm{~m}$ below the Lethbridge Coal Zone, or approximately $53 \mathrm{~m}$ above the Oldman-Dinosaur Park formational contact in Dinosaur Provincial Park; CMN 41357 (Vagaceratops irvinensis holotype) was collected at the base of, or within, the Lethbridge Coal Zone, or approximately $55 \mathrm{~m}$ above the Oldman-Dinosaur Park formational contact in Dinosaur Provincial Park; and TMP 1998.102.0008 was collected in an incised valley infilled by the Lethbridge Coal Zone, and is therefore at least $55 \mathrm{~m}$ above the OldmanDinosaur Park formational contact in Dinosaur Provincial Park. TMP 2009.034.0009 was also collected outside of Dinosaur Provincial Park, along the South Saskatchewan River, where the Oldman-Dinosaur Park formational contact is exposed. The stratigraphic position of this latter specimen was measured relative to this contact, which is lithostratigraphically and chronostratigraphically approximately equivalent to that of Dinosaur Provincial Park (David Eberth, pers. comm.).

Analyses

A stratophylogenetic analysis was conducted using the 153 unordered cranial and postcranial characters as used in Chapter 6, as well as an ordered stratigraphic character (character 154). The states for this stratigraphic character correspond to the three faunal zones in the Dinosaur Park Formation, as identified by Ryan and Evans (2005); 
consequently, only Dinosaur Park Formation chasmosaurines with known stratigraphy, and a Dinosaur Park Formation outgroup taxon (Centrosaurus apertus), were included in this analysis.

A parsimony analysis was conducted on the resulting character-taxon matrix using the program TNT 1.1 (Tree analysis using New Technology; Goloboff et al., 2003) with the tree bisection reconnection swapping algorithm using 1000 replicates. Ten trees were saved per replication and a maximum of 10,000 trees stored per replicate. Relative support via bremer (decay) and bootstrap values for the recovered nodes were obtained in TNT. Bootstrap values were obtained by conducting a bootstrap analysis using 1000 replicates. The resulting strict consensus tree was then examined to determine whether Dinosaur Park Formation chasmosaurine specimens formed stratigraphically constrained clades, each of which were inferred as representing an evolutionary lineage corresponding to a single taxon.

Once evolutionary relationships amongst Dinosaur Park Formation chasmosaurine specimens have been inferred, specimens within each lineage (if sample size was sufficiently large) were analyzed to determine whether any cranial features changed significantly over time. This was done by plotting each relevant cranial parameter (Fig. 5) against elevation above the Oldman-Dinosaur Park formation contact.

To evaluate morphological differences amongst specimens of Chasmosaurus and of Vagaceratops on the basis of geographic placement, approximately time equivalent specimens from different regions were compared. 


\subsection{Ontogenetic analysis}

To conduct a growth transformation series for Chasmosaurus specimens $(\mathrm{n}=14)$, a total of 18 cranial characters that have been suggested to vary with age (Ryan and Russell, 2005; Horner and Goodwin, 2006; Longrich and Field, 2012) were used. These 18 characters and the rationale for using them are explained in Chapter 8.

A parsimony analysis was conducted on the resulting character-specimen matrix using the program TNT 1.1 (Tree analysis using New Technology; Goloboff et al., 2003) with the tree bisection reconnection swapping algorithm using 1000 replicates. Ten trees were saved per replication and a maximum of 10,000 trees stored per replicate. Relative support via bremer (decay) and bootstrap values for the recovered nodes were obtained in TNT. Bootstrap values were obtained by conducting a bootstrap analysis using 1000 replicates. Six of the 18 characters have three states that describe an ontogenetic sequence; therefore, these characters were ordered in the analysis. An artificial embryo, coded " 0 " for all characters was used as the outgroup to root the tree and polarize the characters.

\subsection{Morphometric analyses}

A principal component analysis (PCA) was conducted using the R statistical programming language software (R Development Core Team, 2011). Of the 36 cranial parameters (Fig. 5) recorded for this project, 25 were appropriate for testing via PCA because they capture the overall shape of the skull, while minimizing redundant measurements. As is typical of fossil datasets, I encountered a substantial amount of missing data in my measurements. Unfortunately, when conducting a PCA with 
morphometric data, missing data is not tolerated (Stacklies et al., 2007). I used the Bayesian PCA (BPCA) missing data estimator method (Oba et al., 2003), using the "bpca" function in the package "pcaMethods" (Stacklies et al., 2007); a PCA was then run on this estimated, complete dataset.

Statistical comparisons between morphometric clusters of specimens corresponding to previously named taxa were made using the scores of principal components (PCs) collectively accounting for a significant majority ( $\sim 95 \%)$ of the total variance, and performed using the statistical program PAST 3.01 (Hammer et al., 2001). Significance was determined using a non-parametric multivariate analysis of variance (NPMANOVA), measured by permutation across groups using 10,000 replicates, and based on the Mahalanobis distance measure (Mahalanobis, 1936). Posthoc pairwise comparisons were also conducted using NPMANOVA with both Bonferroni correction and uncorrected probabilities.

A discriminant function analysis was also performed using the scores of PCs collectively accounting for a significant majority ( $\sim 95 \%)$ of the total variance. A MannWhitney U test was also conducted using 10,000 replicates. These were both performed using PAST 3.01 (Hammer et al., 2001). 


\section{Chapter 5. Anatomical Description of Selected Chasmosaurus and Vagaceratops Crania}

\subsection{Description of crania}

A brief description of each specimen currently referred to Chasmosaurus and Vagaceratops is given in this section, followed by a full description of the two newlyprepared partial skulls (CMN 34829 and TMP 2011.053.0046), and redescription of the Chasmosaurus russelli paratype, CMN 8802. Appendix II contains a complete list of quantitative measurements for each chasmosaurine specimen from the Dinosaur Park Formation included in this study. Cranial elements referred to in this chapter are illustrated schematically in Figure 6.

The skulls of most ceratopsids have elaborate ornamentation derived from the postorbital and nasal horncores, and the parietosquamosal frills, jugals and nasals adorned with dermally derived marginal epiossifications (Sampson et al., 1997). These ossifications articulated during maturity with the underlying bone, usually modifying both in the process, and, in some cases, developing into species-specific structures (Horner and Goodwin, 2008). There are five types of epiossifications in ceratopsids: epinasal, epijugals, episquamosals, epiparietals, and epiparietosquamosals (Fig. 6). The diagnostic rostral that forms the upper half of the ceratopsid beak is also probably of dermal origin, but because it is well-developed embryologically in ceratopsians (e.g., Protoceratops; Fastovsky et al., 2011) it will not be considered further. In this chapter, epiossifications are organized and discussed with their respective skull element(s) upon which they articulate. 
A)

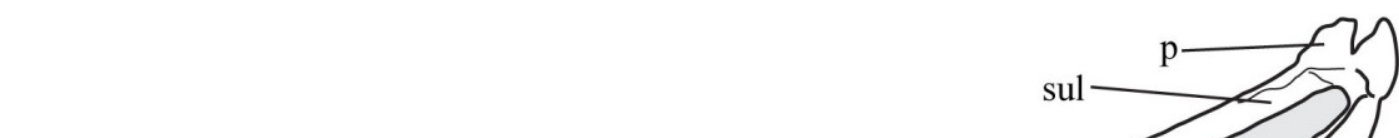

B)

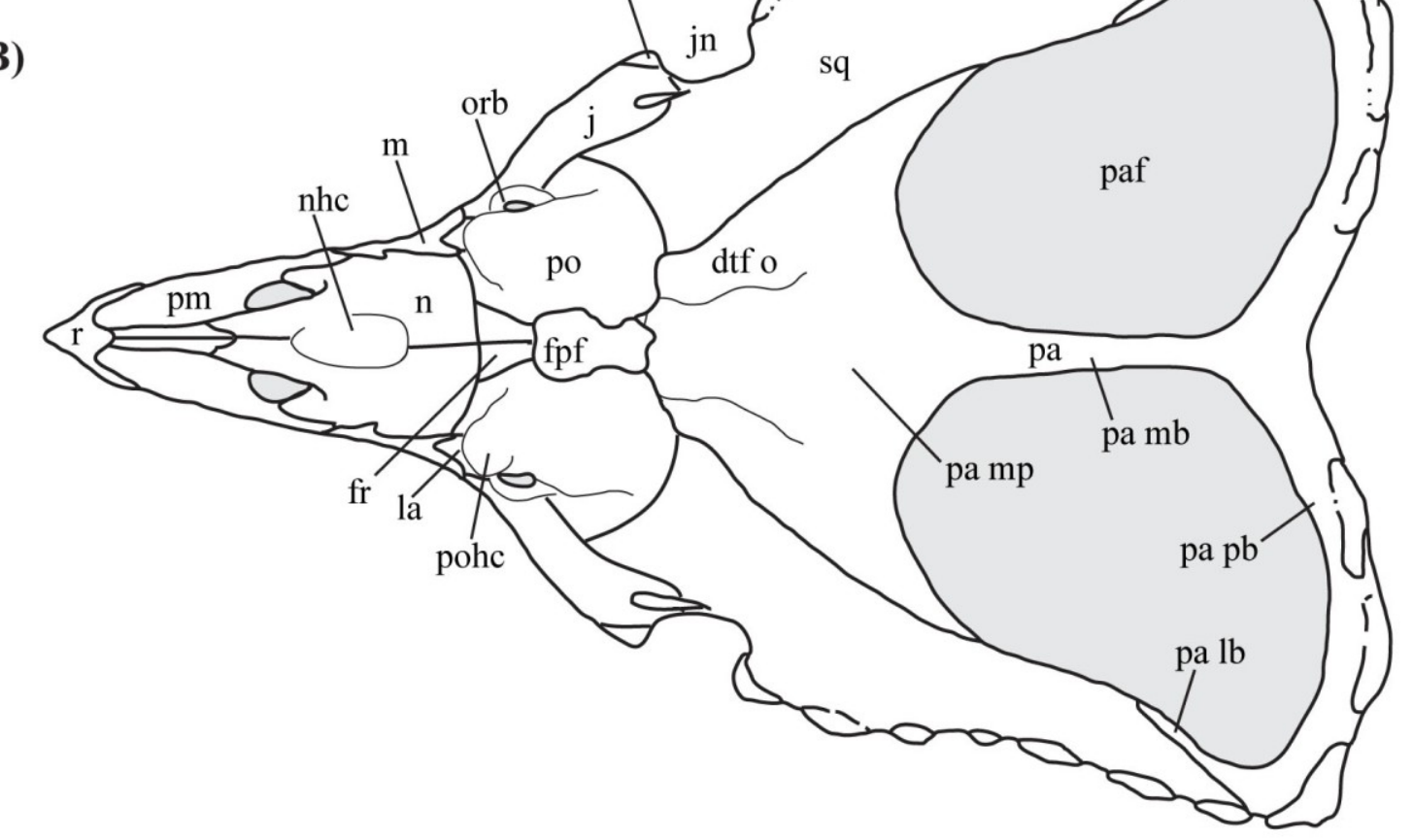

Figure 6. Cranial elements referred to in this study: A) lateral view; and B) dorsal view (Modified from Godfrey and Holmes, 1995: Fig. 5). See "List of anatomical abbreviations". 
The epinasal is a single, medial ossification that articulates on the dorsum of the paired nasals, usually on the surface that has formed a horncore. It has only been recognized in chasmosaurines, but not in all taxa, possibly due to fusion with the horncore in mature adult specimens. The typically pyramidal or conical epijugal articulates with the distal end of the jugal, as well as to the underlying quadratojugal in some chasmosaurines. The initially dorsoventrally compressed, tab-shaped episquamosals and epiparietals articulate with the margins of the squamosal and parietal, respectively. The epiossification that articulates across the contact between the parietal and squamosal is the epiparietosquamosal. Frill epiossifications were originally referred to as epoccipitals (e.g., Lambe, 1914b; Sternberg, 1940), regardless of whether they were on the parietal or squamosal, despite the fact that they are not related to the occipital. This term was replaced by ‘epiossification’ by Horner and Goodwin (2008).

A numbering system has been developed to describe the positions of epiossifications on the frill. Epiparietals are numbered sequentially $(\mathrm{P} \#)$ starting at the midline of the parietal and moving anteriorly towards the squamosal (Sampson et al., 2010). A midline epiossification, if present, is designated P0. Episquamosals are numbered sequentially (ES\#) starting at the posterior end of the squamosal, and progressing anteriorly to the jugal notch (Sampson et al., 2010). Direction of epiossification articulation has been determined to be anterior to posterior in chasmosaurines and the reverse in centrosaurines based on the ontogenetic pattern of articulation seen repeated across related taxa (Sampson et al., 1997).

The parietal and squamosals typically possess a gently undulating margin when viewed dorsally; the midpoint of these undulations serve as the attachment loci for 
epiossifications, if they develop (Horner and Goodwin, 2008; Currie et al., 2008). The undulating morphology is visible early in ontogeny (e.g., Chasmosaurus specimens CMN 1254 and TMP 1998.128.0001), but can be obscured by the overlying epiossifications during later ontogenetic stages. As ceratopsids mature, the epiossifications can become highly modified (e.g., developing from a low, D-shaped tab in dorsal profile to elongate robust spikes on the parietal of Styracosaurus, or changing from sharp-edged, triangular shapes in dorsal profile to elongate, low, indistinct masses on Triceratops) and have their basal sutures obliterated.

In early ceratopsid ontogeny, the posterior margin of the parietal is dorsoventrally thin and oriented in the plane of the frill, but thickens later in ontogeny as loci become more developed (e.g., Centrosaurus, Ryan et al., 2001; Triceratops, Horner and Goodwin, 2006; Styracosaurus, Ryan et al., 2007). In Centrosaurus apertus, loci 1 recurves during ontogeny into an anterodorsal orientation (Ryan et al., 2001), while the associated epiparietal develops into an elongate, anteriorly oriented hook. Anterodorsally oriented epiparietals are present in some other ceratopsids, e.g., P1-3 of Kosmoceratops and P1 of Utahceratops, Sampson et al., 2010; P1 of Anchiceratops, Mallon et al., 2011; P1-2 and P1-4 in some specimens of Chasmosaurus and P1-4 in Vagaceratops; the orientation and ontogenetic remodeling of the loci below the epiparietals in these taxa (except for Chasmosaurus) are unknown, as immature specimens lacking epiparietals are unknown. 


\subsubsection{Chasmosaurus}

Skulls:

Skulls are defined here as specimens comprising at least one of the following cranial regions: snout, braincase, or skull roof including postorbital horncores; 18 Chasmosaurus skulls were included in this study. Specimens were binned as small or large based on the following criteria: small specimens are skulls with (or estimated as having) rostral-toepijugal lengths of up to $628 \mathrm{~mm}$ (e.g., UALVP 40); large skulls have (or are estimated as having) rostral-to-epijugal lengths of $680 \mathrm{~mm}$, or greater (e.g., AMNH 5402).

Postorbital horncores were classified as short or long based on the following criteria: short horncores are (or are estimated as having been, before putative bone resorption) up to $132 \mathrm{~mm}$ in length (e.g., CMN 2280); long horncores are (or estimated as having been, before putative bone resorption) $176 \mathrm{~mm}$ in length, or greater (e.g., UALVP 40).

AMNH 5401 (holotype, Chasmosaurus kaiseni; Fig. 7A) is a skull (rostral-to-epijugal length $=730 \mathrm{~mm}$; squamosal length $=710 \mathrm{~mm}$ ), missing only the posterior margin of the frill. The rostral is articulated with the underlying premaxillae. The postorbital horncores in this specimen are among the longest known in Chasmosaurus (left $=350 \mathrm{~mm}$, right $=$ $370 \mathrm{~mm}$; right, estimated). Both epijugals are articulated. Each squamosal bears six episquamosals, except for the distal tip, where an unoccupied episquamosal locus is present. The degree of continuity of the lateral parietal bars cannot be determined due to incomplete preservation. The parietal fenestrae are anteroposteriorly elongate in shape, although their posterior extent cannot be determined. Although most of the posterior parietal bar is reconstructed, two epiparietals are preserved on the right posterolateral 
corner. These are embedded in plaster, so their original positions cannot be confirmed.

AMNH 5402 (Figs. 7B, 8A-E) is an almost complete skull (rostral-to-epijugal length = $680 \mathrm{~mm}$; squamosal length $=685 \mathrm{~mm}$ ). The rostral is articulated with the underlying premaxillae. The postorbital horncores are relatively short $($ left $=70 \mathrm{~mm}$, right $=65 \mathrm{~mm})$. Both epijugals are articulated. Each squamosal bears eight episquamosals, except for the distal tip, where an unoccupied episquamosal locus is present; therefore, a total of nine episquamosals would have been present. The lateral parietal bars are continuous. The frill is similar to AMNH 5401, although the parietal fenestrae are anteroposteriorly short, with approximately equal width and length (length/width $=1.04)$. The right squamosal has been distorted mediolaterally and pushed posteriorly, distorting the posterior parietal bar such that the right lateral corner extends further posteriorly than the left corner. A straight posterior bar (i.e., lacking a medial embayment) is inferred based on the undistorted right posterior bar. The left side of the posterior parietal bar is adorned with three epiparietals oriented in the plane of the frill. The first epiparietal adjacent to the midline on the left side is missing its central portion; its preserved, intact counterpart on the right side of the parietal confirms that the former is incomplete. On the right side, the third and part of the second epiparietals from the midline are reconstructed in plaster, patterned off those on the left side. There is a notably large gap between the first and second epiparietals on both sides of the parietal, unlike the pattern seen in all other Chasmosaurus frills. On each gap, there are two low-relief undulations which may represent epiparietal loci (Fig. 8C-E). The third epiparietal from the midline is the largest of the three, and partly overhangs the parietal-squamosal contact. Had this epiossification articulated completely 
with the frill, it would likely have articulated partly with the squamosal; for this reason, this epiossification is here on referred to as an epiparietosquamosal.

AMNH 5656 (Fig. 7C) is a small skull, missing the roof and the portion of the skull anterior to the jugals. Both epijugals are articulated. Each squamosal bears five episquamosals, except for the distal tip, where an unoccupied episquamosal locus is present; therefore, a total of six episquamosals would have been present. The squamosal length $(569 \mathrm{~mm})$ is intermediate to CMN 1254 and UALVP 40. AMNH 5656 has a wider frill than the transversely-compressed UALVP 40. The lateral parietal bars are continuous. The parietal fenestrae are anteroposteriorly elongate in shape (parietal fenestral length/width ratio $=1.89$ ). The posterior parietal bar is embayed medially, and each side possesses three marginal undulations oriented in the plane of the frill. On each side of the posterior bar, only the two medialmost undulations are adorned with epiparietals.

CMN 1254 (holotype, Eoceratops canadensis; Fig. 7D) is a partly disarticulated skull, consisting of a right nasal, right postorbital with a long, well-developed horncore (216 $\mathrm{mm}$ ), right squamosal, right lateral parietal bar, and left lower dentary. The right half of the epinasal is present, but detached from the underlying nasal. The squamosal is $500 \mathrm{~mm}$ in length, approximately 2.5 times longer than TMP 1998.128.0001. CMN 1254 lacks episquamosals and possesses seven pronounced marginal undulations. The lateral parietal bar is continuous, excluding the squamosal from the parietal fenestra. 
Figure 7. Chasmosaurus skulls: A), AMNH 5401 (Chasmosaurus kaiseni holotype) in dorsal (top) and right lateral (bottom) views; B), AMNH 5402 (Chasmosaurus belli) in dorsal (top) and left lateral (bottom) views; C), AMNH 5656 (Chasmosaurus russelli) in dorsal (top and right anterolateral (bottom) views; D), CMN 1254 (Eoceratops canadensis holotype) in dorsal (top) right lateral (bottom) views; E), CMN 2245 (C. belli) in dorsal (top) and left lateral (bottom) views; F), CMN 2280 (Chasmosaurus russelli) in dorsal (top; cast) and left lateral (bottom) views; G), CMN 8800 (C. russelli holotype) in left lateral view; H), CMN 8801 (C. sp.) in right lateral view; I), CMN 34832 (C. sp.) in medial view of left squamosal (left) and dorsal view of braincase (right); J), NHMUK R4948 (C. belli) in ventral view of left squamosal (top left), dorsal view of frill (top right) and right lateral view of skull (bottom); K), ROM 839 (Chasmosaurus brevirostris holotype) in dorsal (top) and right lateral (bottom) views; L), ROM 843 (C. belli; cast) in dorsal (top) and right lateral (bottom) views; M), TMP 1979.011.0147 (C. sp.) in dorsal view; N), TMP 1981.019.0175 (C. sp.) in dorsal (top) and right lateral (bottom) views; O), TMP 1983.025.0001 (Mojoceratops perifania holotype) in dorsal (top) and left lateral (bottom) views; P), TMP 1993.082.0001 (C. sp.) in ventral view; Q), UALVP 40 (E. canadensis) in dorsal (top) and left lateral (bottom) views; and R), YPM 2016 (C. belli) in dorsal (top) and left lateral (bottom) views. Plaster reconstruction shown in grey half-tone. Images of UALVP 40 courtesy of Jordan Mallon; images of NHMUK R4948 modified from Maidment and Barrett (2011). See "List of anatomical abbreviations”. Scale bar equals $10 \mathrm{~cm}$. 
Figure 7.

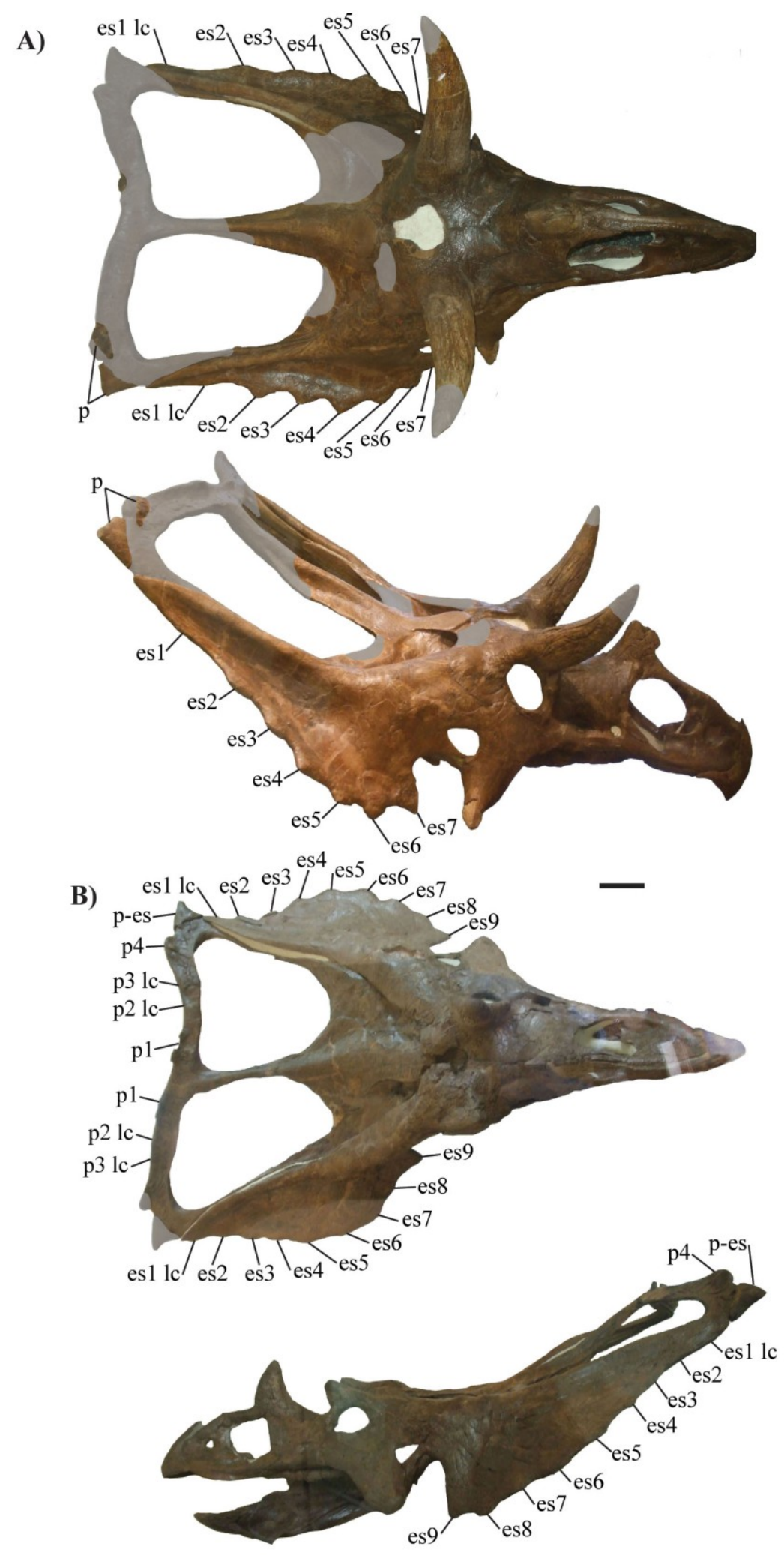


Figure 7. (continued).
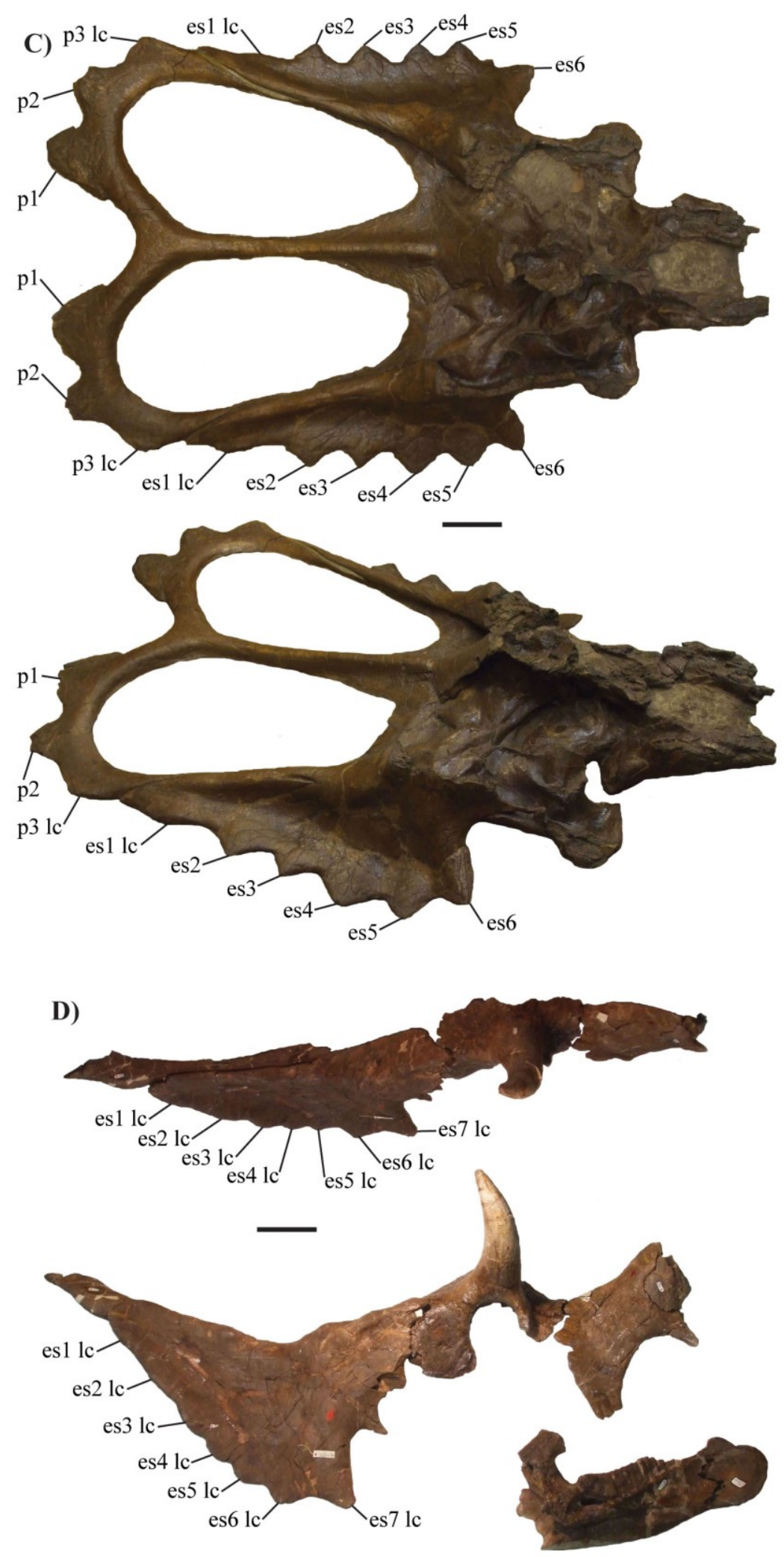
Figure 7. (continued).

E)
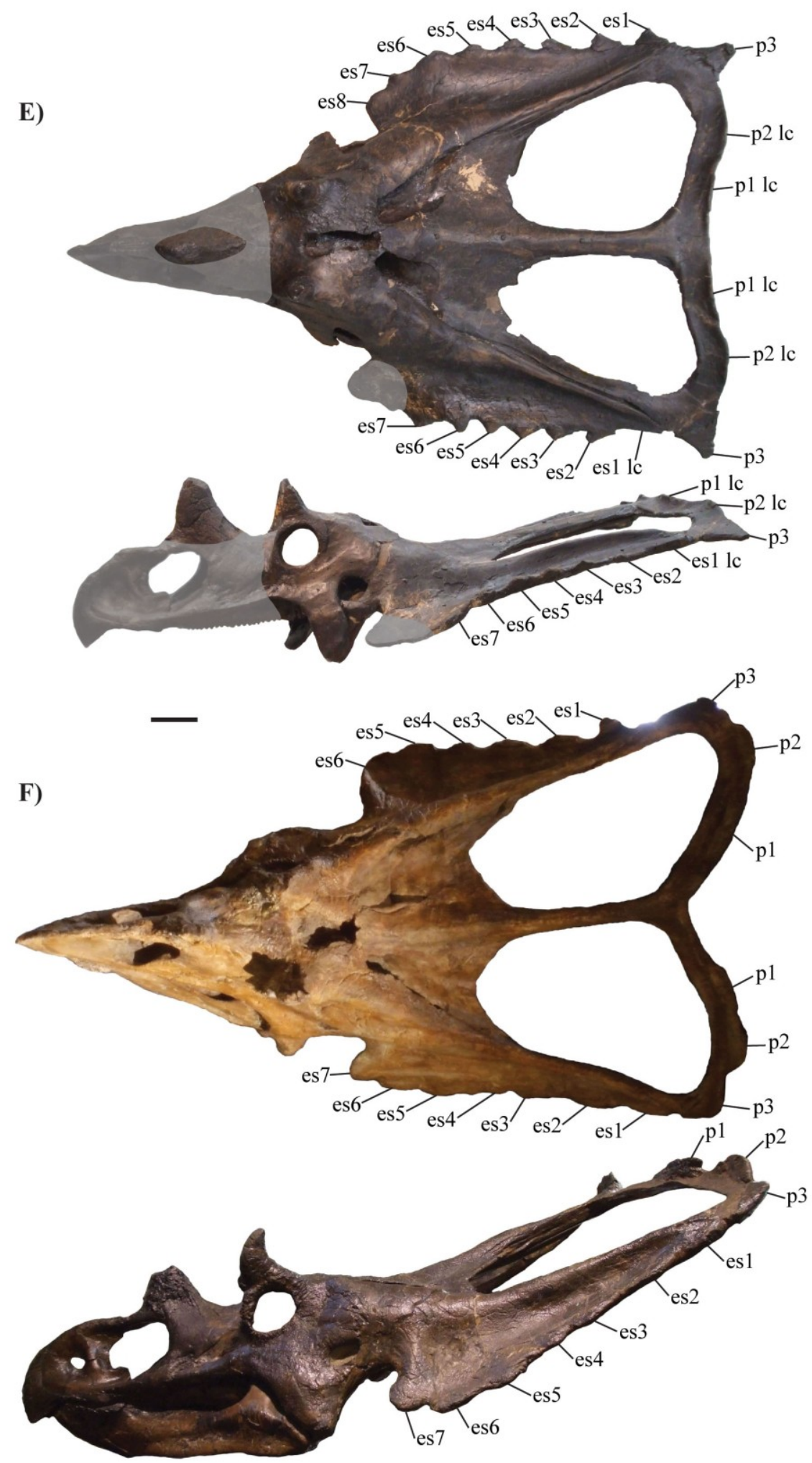
Figure 7. (continued).
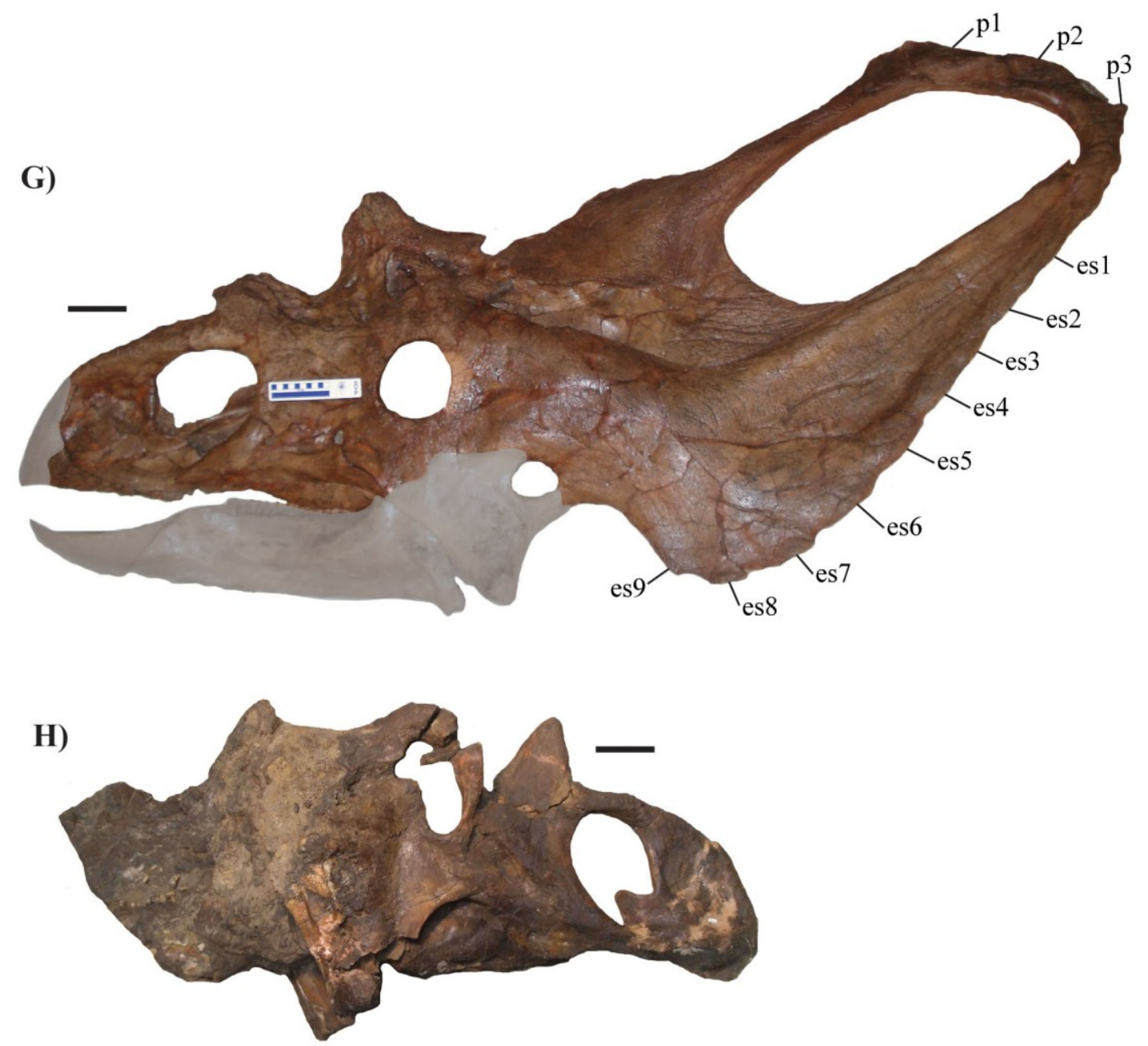
Figure 7. (continued).

I)
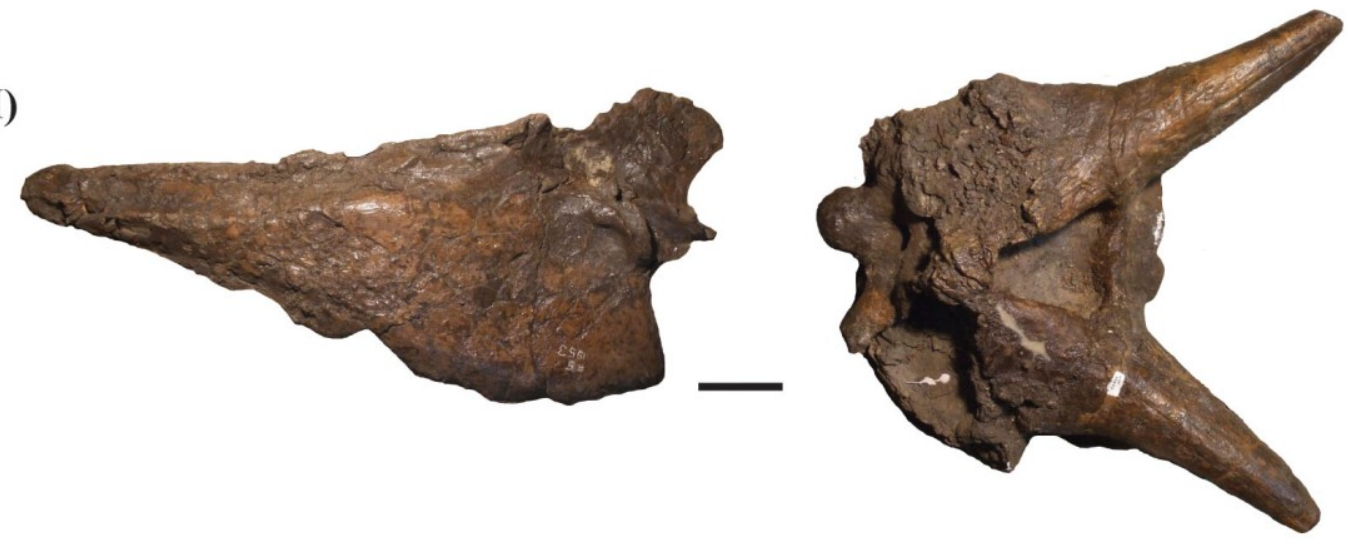

J)
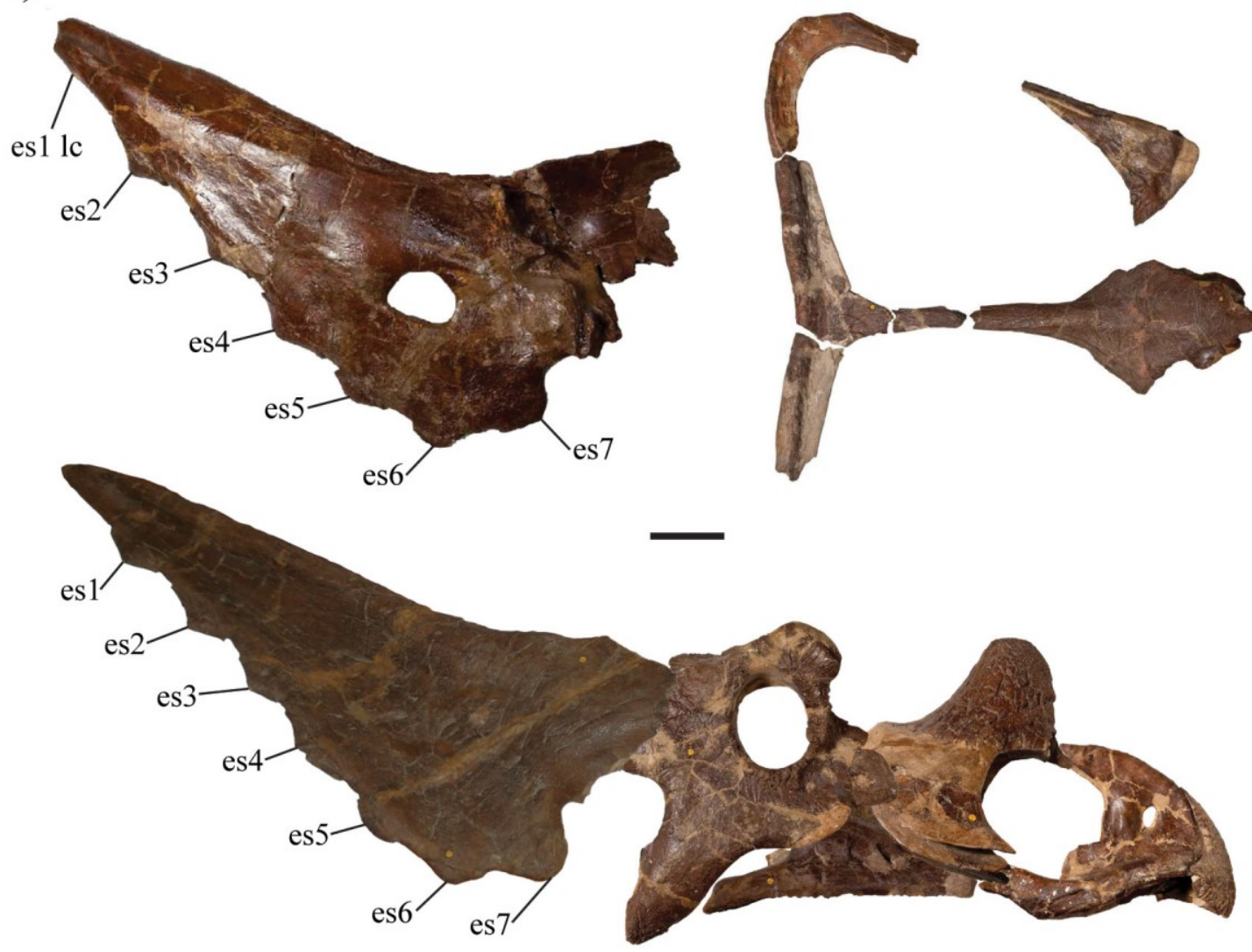
Figure 7. (continued).

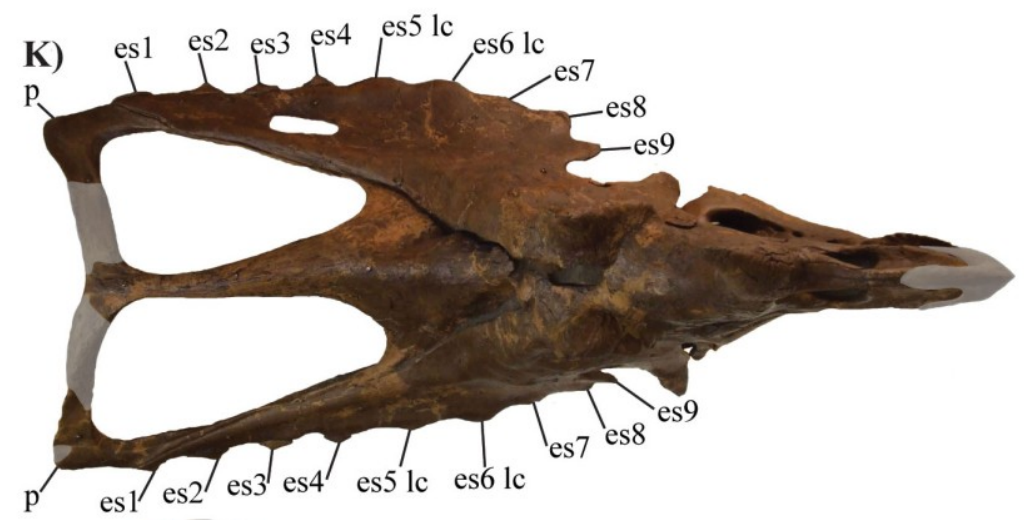

L)
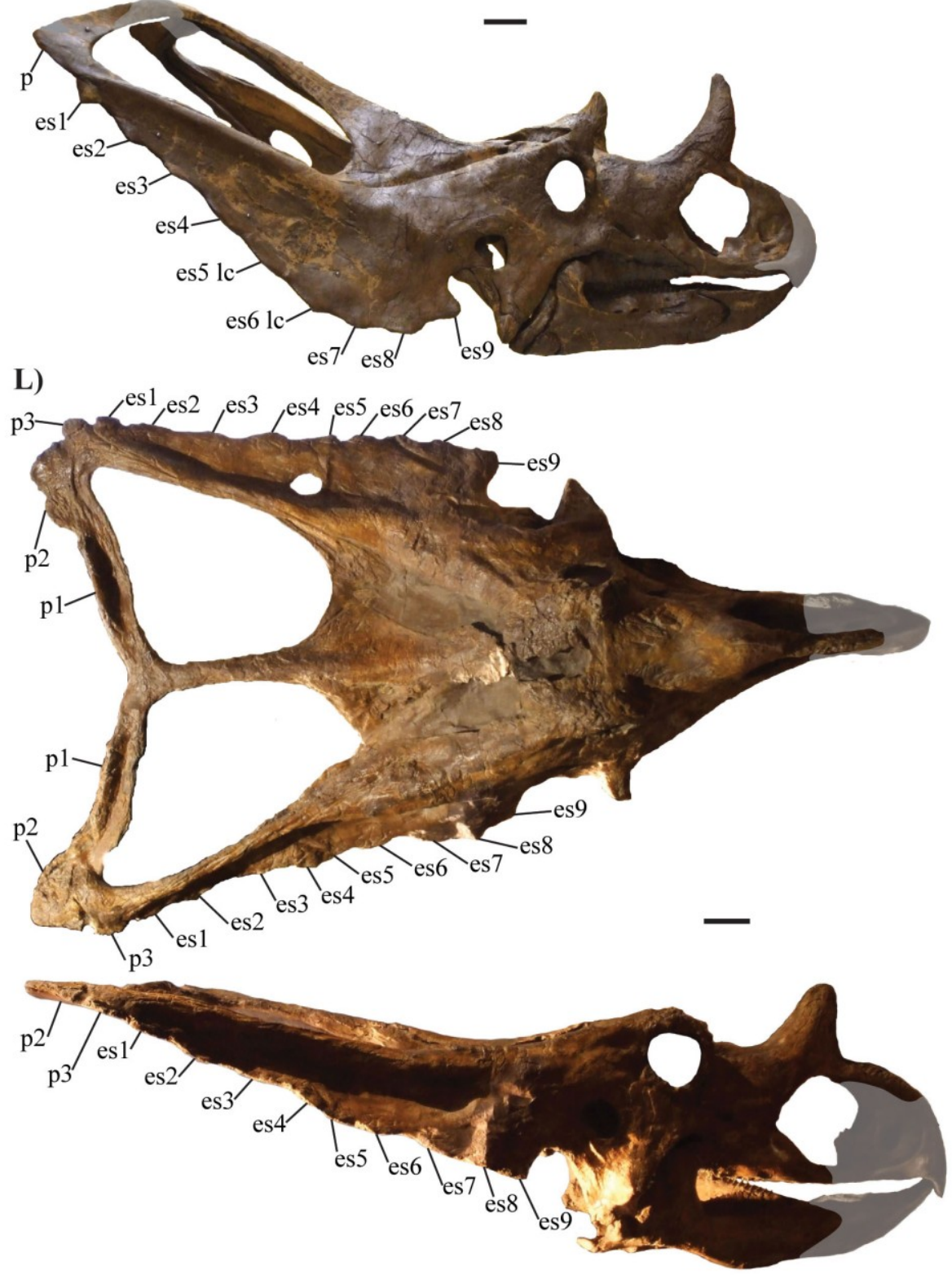
Figure 7. (continued).
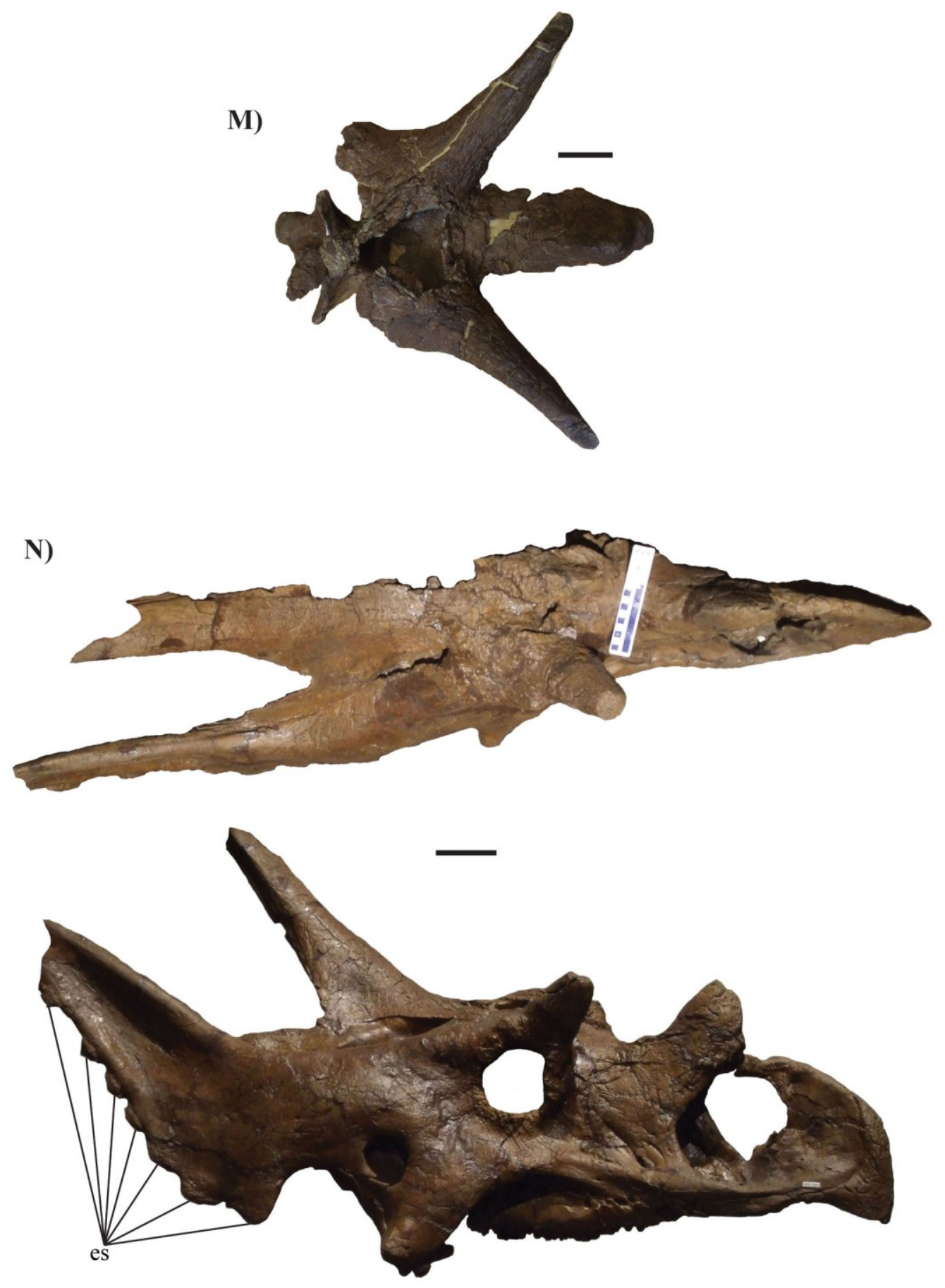
Figure 7. (continued).

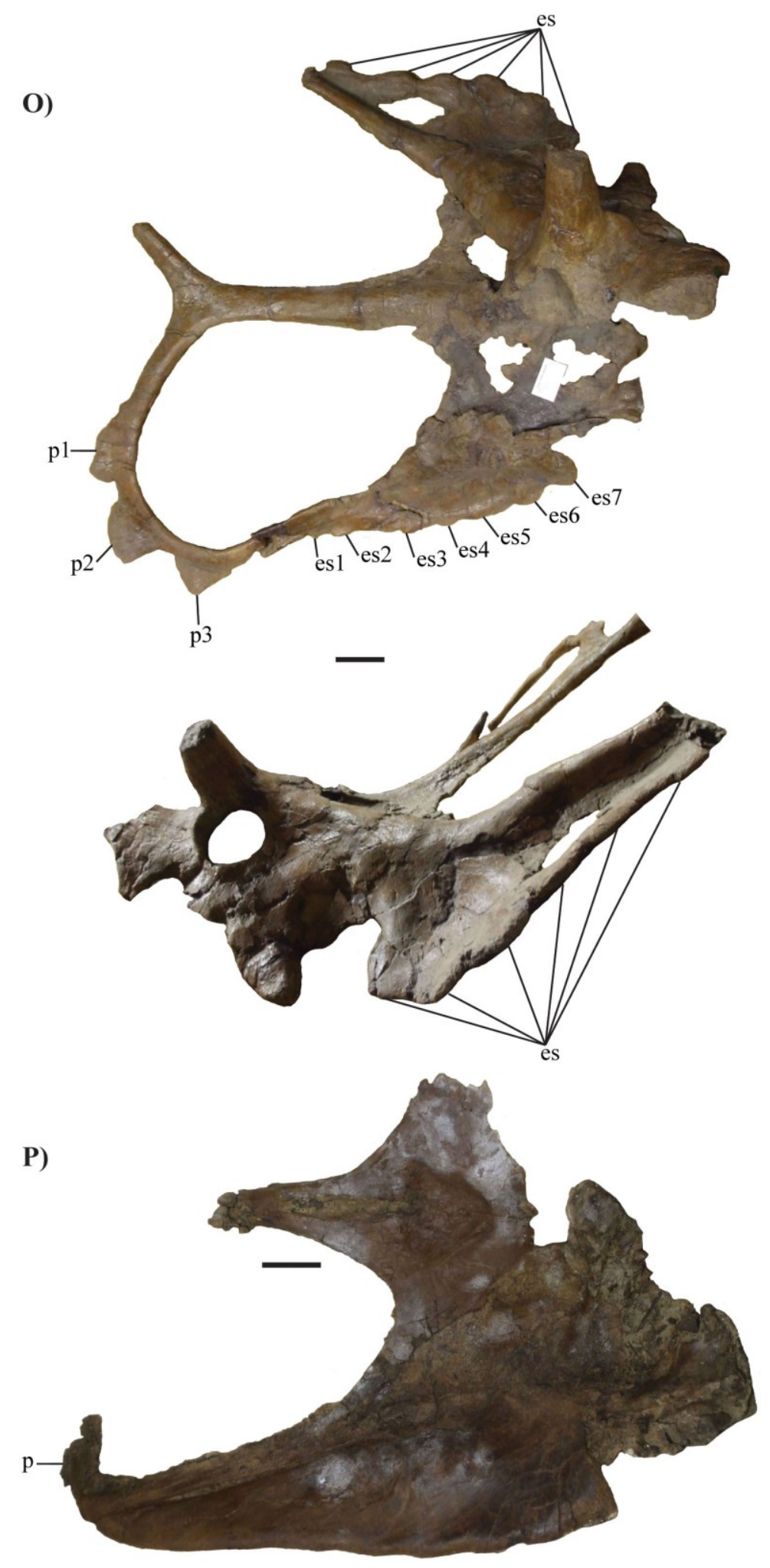


Figure 7. (continued).
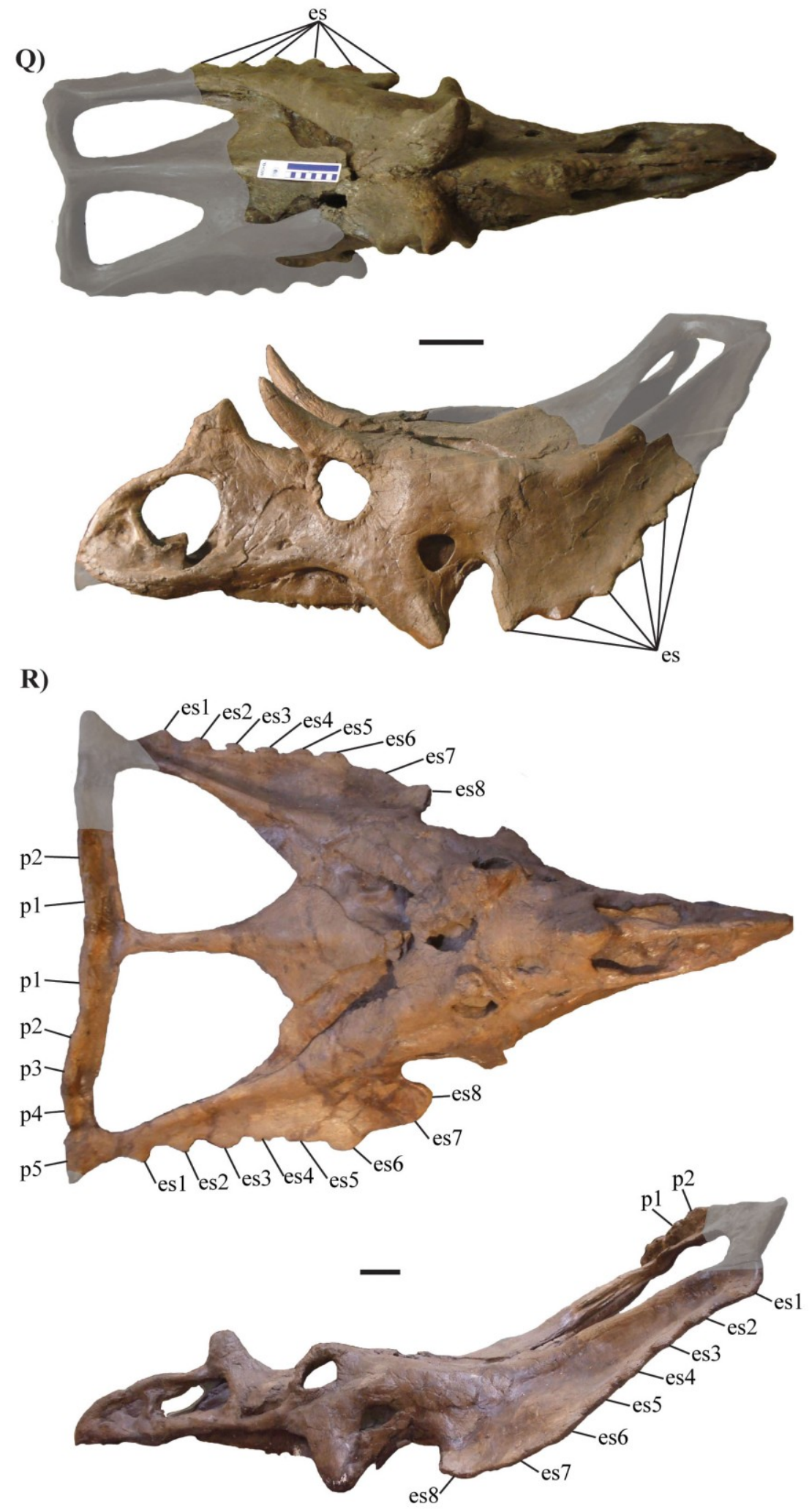
Figure 8. Frill of AMNH 5402 (Chasmosaurus belli) in: A) dorsal; B) dorsal (left side); C) oblique dorsal (left side); D) posterior (left side); and E) dorsal (right side) views. Brackets delimit size of epiossifications; dashed line shows parietal-squamosal contact. Arrows denote inferred epiparietal attachment sites (low-relief undulations); note transverse groove below each site. Plaster reconstruction shown in grey half-tone. See "List of anatomical abbreviations". Scale bars equal $10 \mathrm{~cm}$. 
Figure 8.
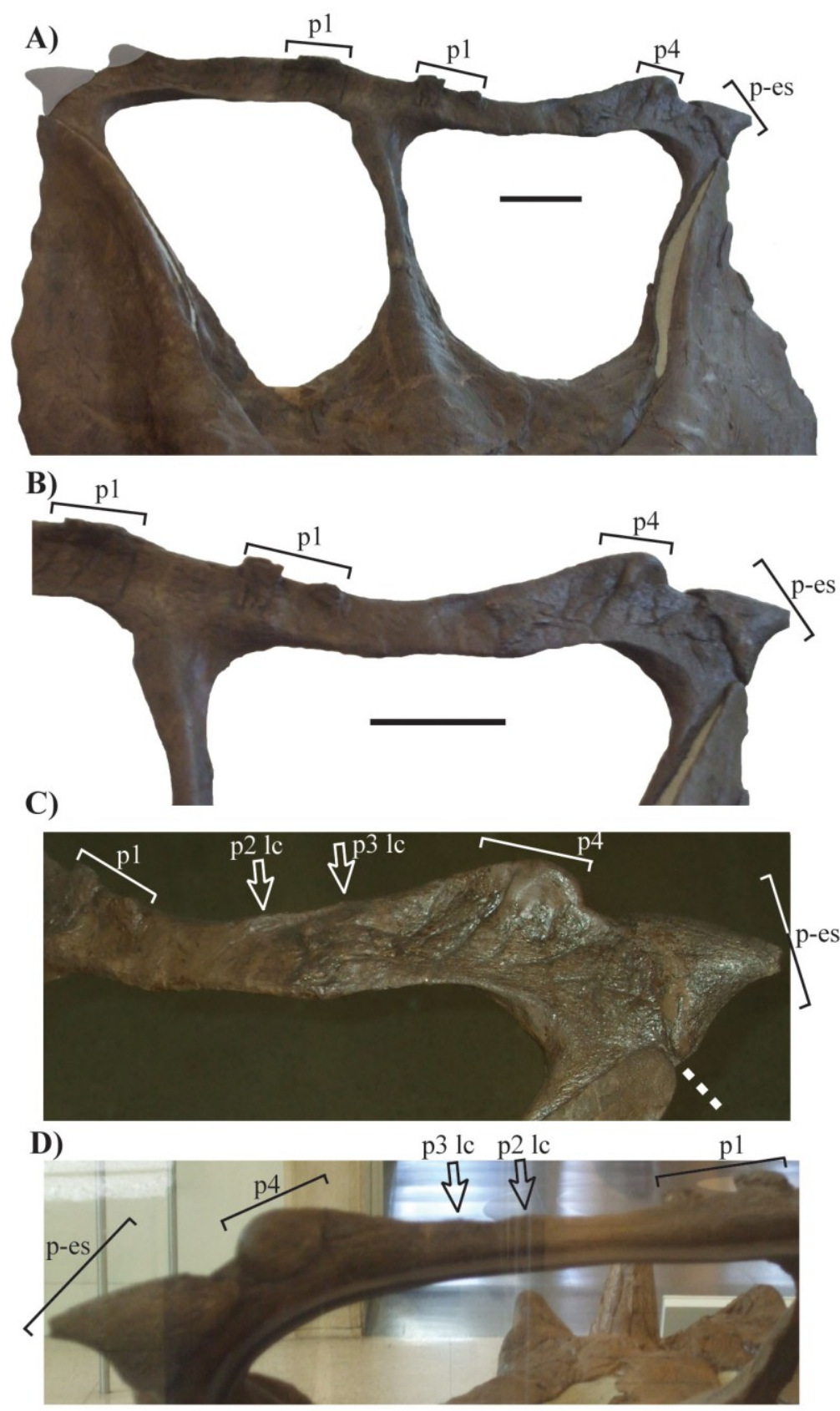

E)

p3 lc p3 lc

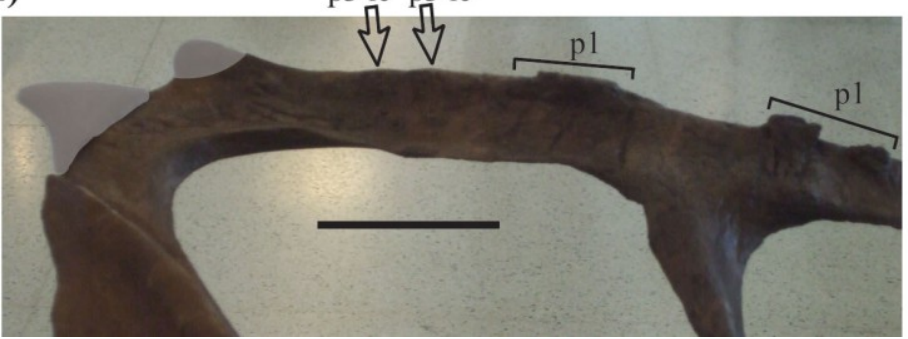


CMN 2245 is a skull (squamosal length $=779 \mathrm{~mm}$; Fig. 7E), missing the face anterior to the postorbital horncores (except for the nasal horncore), and the anterior margin of the left squamosal. Both epijugals are articulated. The postorbital horncores are relatively short $($ left $=102 \mathrm{~mm}$, right $=100 \mathrm{~mm})$. The right squamosal bears eight episquamosals; an episquamosal count for the left squamosal cannot be determined, as the anterior end of this element has been reconstructed in plaster. An unoccupied episquamosal locus is present on the distal tip of the left squamosal. The lateral parietal bars are continuous and the parietal fenestrae are anteroposteriorly elongate (parietal fenestra length/width $=$ 1.31). The posterior parietal bar has a shallow medial embayment. The posterolateral corners of the parietal each bear a large, posterolaterally-directed epiparietal. Medial to the preserved epiparietal are two wide undulations, oriented slightly anterodorsally, that are either epiparietal loci, or represent modified epiparietals (Fig. 9A-C).

CMN 2280 (Fig. 7F) is a complete skull (rostral-to-epijugal length $=750 \mathrm{~mm}$; squamosal length $=709 \mathrm{~mm}$ ). The rostral is articulated with the underlying premaxillae. The left postorbital horncore is relatively short $(132 \mathrm{~mm})$, although the right horncore is truncated $(88 \mathrm{~mm})$ and pitted across the apex. Both epijugals are articulated with the jugals. The left and right squamosals bear seven and six episquamosals, respectively. The lateral parietal bars are discontinuous and the parietal fenestrae are anteroposteriorly elongate (parietal fenestra length/width $=1.26$ ). The posterior parietal bar is shallowly embayed and each side is adorned with three approximately equal-sized epiparietals. The two medialmost epiparietals (P1 and P2) have a slight anterodorsal inflection; $\mathrm{P} 3$ is directed in the plane of the frill. 
A)

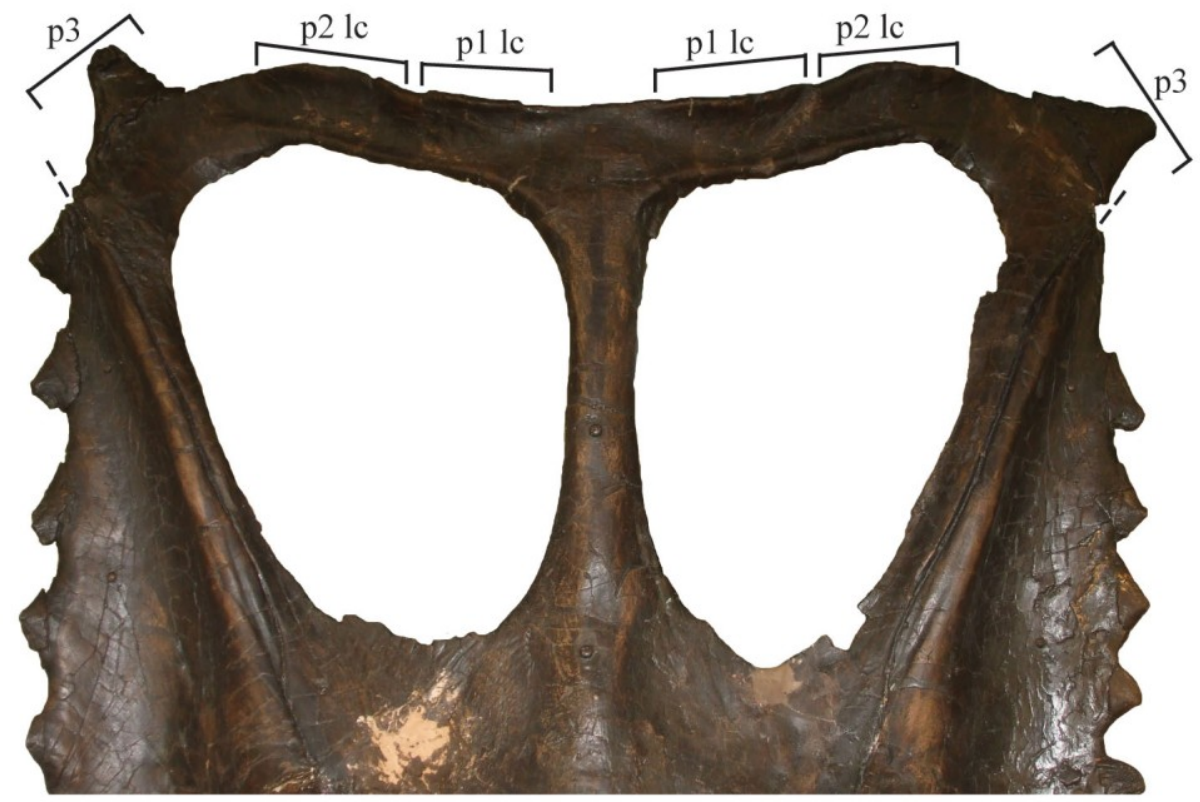

B)

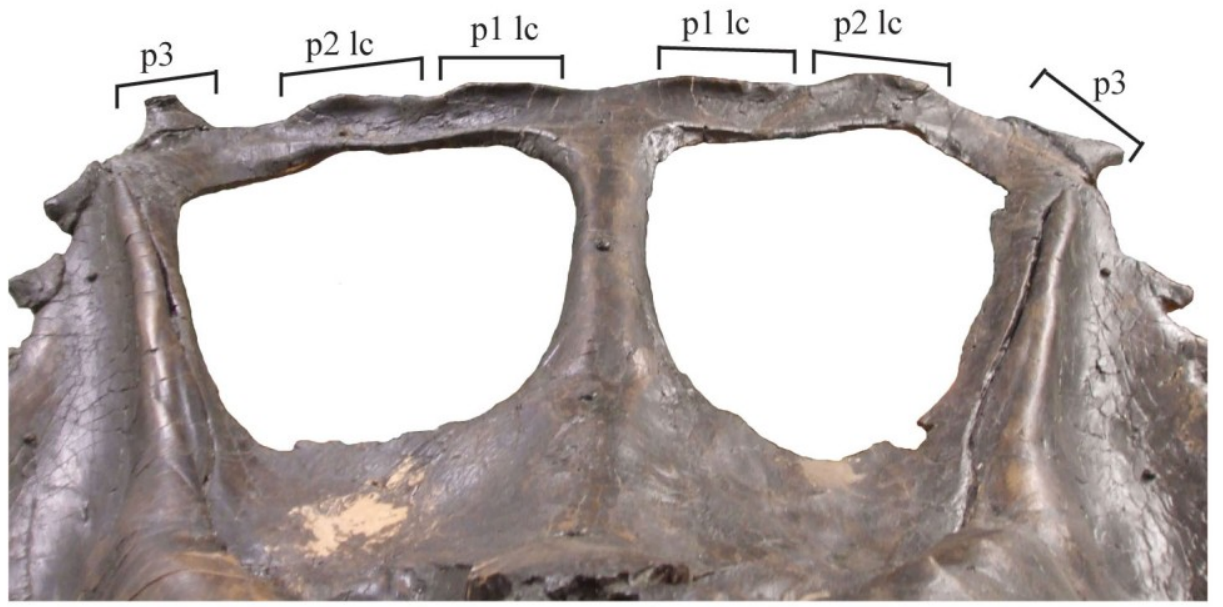

C)

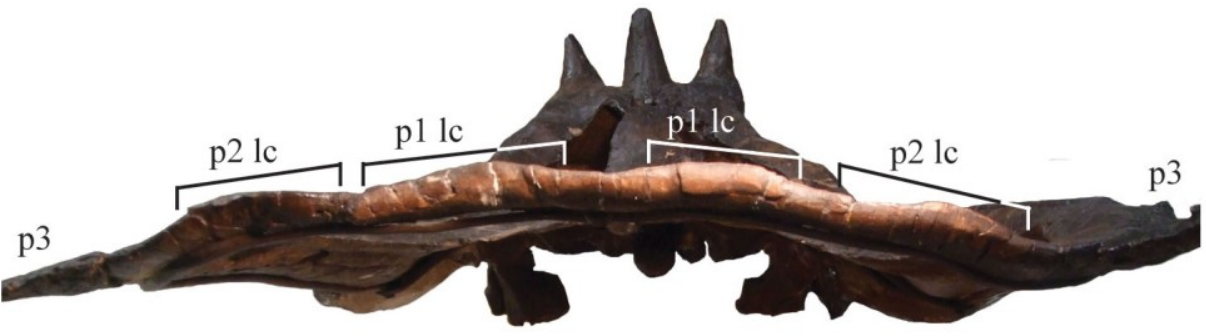

Figure 9. Frill of CMN 2245 (Chasmosaurus belli) in: A) dorsal; B) anterior; and C) posterior views. Brackets delimit size of epiossifications; dashed line shows parietalsquamosal contact. See "List of anatomical abbreviations". Scale bar equals $10 \mathrm{~cm}$. 
CMN 8800 (holotype, Chasmosaurus russelli; Fig. 7G) is a skull (rostral-to-epijugal length $=880 \mathrm{~mm}$; squamosal length $=997 \mathrm{~mm}$ ), missing the right side of the skull; the rostral and left jugal are also missing, and have been reconstructed with plaster. The nasal horncore is rugose and pitted, suggestive of bone resorption. Nasal horncore pitting has not been previously documented for chasmosaurines; however, Tanke and Farke (2007) noted pitting on a cf. Centrosaurus nasal (TMP 1988.050.0113). The similarly rugose and pitted postorbital horncores have large bases (left basal circumference $=412 \mathrm{~mm}$; right side inaccessible) and are inferred to have been long before modification (preserved lengths: left $=40 \mathrm{~mm}$, right $=108 \mathrm{~mm}$ ). The preserved left squamosal bears ten episquamosals, which is the highest count for any Chasmosaurus specimen. The preserved left lateral parietal bar is discontinuous and the preserved left parietal fenestra is anteroposteriorly elongate (parietal fenestra length/width $=1.81$ ). The posterior parietal bar is the most deeply embayed of all Chasmosaurus frills examined. Three epiparietals are present on the preserved left side, with P1 being the largest. P1 and P2 are both oriented slightly anterodorsally, while what remains of $\mathrm{P} 3$ is oriented in the plane of the frill, as in CMN 2280.

CMN 8801 (Fig. $7 \mathrm{H}$ ) is a skull (rostral-to-epijugal length $=835 \mathrm{~mm}$ ) that is missing most of the frill. Only the right side of the skull is accessible. The rostral and right preserved epijugal are both articulated with their respective underlying elements. The lower jaws are also preserved. The dorsal surface of the right postorbital preserves a large, pitted mound (length $=57 \mathrm{~mm}$ ). 
CMN 34832 (Fig. 7I) is a partial skull (squamosal length $=682 \mathrm{~mm}$ ), comprising frontals, postorbitals including long postorbital horncores $(\mathrm{left}=236 \mathrm{~mm}$, right $=237$ $\mathrm{mm}$ ), frontoparietal fontanelle, and the left squamosal. Due to poor preservation, it cannot be determined whether the episquamosals are present or disarticulated.

NHMUK R4948 (Fig. 7J) is a skull (squamosal length $=840 \mathrm{~mm}$ ) that is largely disarticulated, but missing only the right posterolateral corner of the parietal. The rostral is articulated with the underlying premaxillae. The preserved right postorbital horncore is short $(55 \mathrm{~mm})$ with a sharply truncated apex, suggesting that it was modified during ontogeny. Both epijugals are articulated with the jugals. The right squamosal bears seven episquamosals. The left squamosal bears six episquamosals, with an additional unoccupied locus adjacent to the distal tip. There is a large perforation in the left squamosal. Tanke and Farke (2007) noted that the bone surrounding this fenestra is recessed and thin compared to the rest of the squamosal, and not swollen as in inferred pathological cases. The continuity of the lateral parietal bars in this skull cannot be determined due to incomplete preservation. The parietal fenestrae are anteroposteriorly elongate (parietal fenestra length/width $=1.28$; estimated for the left side). The posterior parietal bar has a very shallow embayment. No epiparietals can be discerned, although there is a distinct, dorsally-thickened ridge spanning the entire posterior parietal margin.

ROM 839 (holotype, Chasmosaurus brevirostris; Fig. 7K) is a skull (rostral-to-epijugal length $=782 \mathrm{~mm}$; squamosal length $=917 \mathrm{~mm}$ ), missing most of the posterior parietal bar, which has been reconstructed in plaster. ROM 839 has the longest nasal horncore 
(237 mm) of any Chasmosaurus specimen. The postorbital horncores are relatively short (left $=107 \mathrm{~mm}$, right $=93 \mathrm{~mm}$ ). Only the right epijugal is articulated. Each squamosal bears seven episquamosals, although each possesses two unoccupied loci (5 and 6); therefore, a total of nine episquamosals would have been present. On the better preserved right side of the frill, the lateral parietal bar is very thin, but complete. Each posterolateral corner of the posterior bar bears a large, articulated epiparietal. There is a large perforation on the left squamosal.

ROM 843 (Fig. 7L) is a skull (rostral-to-epijugal length $=897 \mathrm{~mm}$; squamosal length $=$ $893 \mathrm{~mm}$ ) missing only the rostral and most of the premaxillae, which have been restored with plaster. The dorsal postorbital horncores are capped by low-relief, pitted mounds (lengths: left $=67 \mathrm{~mm}$, right $=55 \mathrm{~mm}$ ). Both epijugals are articulated with the jugals. The left and right squamosals bear nine and eight episquamosals, respectively. There is a large perforation in the left squamosal, as in NHMUK R4948 and ROM 839. The lateral parietal bars are continuous. The parietal fenestrae are slightly anteroposteriorly longer than wide (parietal fenestra length/width $=1.09$ ). The posterior parietal bar has a deep embayment. Two small, posteriorly-directed P3s are positioned at the posterolateral corner of the parietal. The one on the left is oriented posterolaterally, while the one on the left is oriented laterally. P2 is approximately four times larger than P3. Of note, is that the left P2 has a bifurcated dorsal outline, which is due to breakage. Adjacent to the midline bar, there is a distinct anterodorsally-recurving ridge, traditionally inferred to be P1, whose basal width is approximately 1.5 times that of $\mathrm{P} 2$. 
TMP 1979.011.0147 (Fig. 7M) consists of a braincase, nasal horncore, and a skull roof with long postorbital horncores (left $=263 \mathrm{~mm}$, right $=298 \mathrm{~mm}$ ), and a transversely wide frontoparietal fontanelle.

TMP 1981.019.0175 (Fig. 7N) is a skull (rostral-to-epijugal length $=768 \mathrm{~mm}$ ) missing its left side and posterior half of the frill. The rostral is articulated with the underlying premaxillae. More than half of the right postorbital horncore is preserved (length, 134 $\mathrm{mm}$ ); however its full length cannot be determined. This horncore is inferred as having been relatively long, based on its large preserved base and truncated dorsal surface. The left postorbital horncore is missing entirely and represented by a rugose and pitted surface. The preserved right epijugal is articulated. The preserved right squamosal is relatively short (squamosal length $=600 \mathrm{~mm}$, estimated) given the size (rostral-toepijugal length) of the skull. It is comparable in total estimated length to the small UALVP 40 skull. The right squamosal of TMP 1981.019.0175 bears seven episquamosals, but likely would have had more, as the distal tip of the element is missing. The preserved right lateral parietal bar thins medially, allowing the squamosal to form part of the margin of the parietal fenestra.

TMP 1983.025.0001 (holotype, Mojoceratops perifania; Fig. 7O) is a skull (squamosal length $=650 \mathrm{~mm}$ ), missing the snout, the right side of the face, and the left posterolateral portion of the frill. The left preserved postorbital horncore is relatively long based on its large preserved base; more than half of the horncore is preserved (length, $142 \mathrm{~mm}$ ). The preserved left epijugal is articulated with the jugal. The left and right squamosals bear six 
and seven episquamosals, respectively; the posterior end of the left squamosal is missing, and it would likely have had at least seven episquamosals in total. An accessory fenestra is present on the left squamosal. The right lateral parietal bar is discontinuous and the parietal fenestrae anteroposteriorly elongate in shape (parietal fenestra length/width = 1.32). The posterior parietal bar is embayed and each side is adorned with three roughly equal-sized epiparietals that are oriented in the plane of the frill.

TMP 1993.082.0001 (Fig. 7P) is a frill (squamosal length $=879 \mathrm{~mm}$ ), consisting of part of the skull roof (still embedded in matrix), the left squamosal, an uninterrupted left lateral parietal bar, the anterior portion of the midline parietal bar, and the posterolateral corner of the left lateral parietal bar with only one epiparietal possibly preserved. This skull could only be viewed ventrally as it was too fragile to flip over. Due to poor preservation, it cannot be determined whether the episquamosals are present or disarticulated. This specimen was referred to Vagaceratops by Ryan (2003). This assignment may have been based on the specimen's relatively high stratigraphic elevation in the Dinosaur Park Formation, within the Vagaceratops interval. However, given the specimen's large and anteroposteriorly elongate parietal fenestrae, and the recentlydemonstrated upward stratigraphic range extension for Chasmosaurus, this specimen is likely referable to this latter taxon.

UALVP 40 (Fig. 7Q) is a skull (rostral-to-epijugal length $=628 \mathrm{~mm}$ ) missing the posterior half of the frill and rostral bone, which have been reconstructed in plaster. In UALVP 40, only the left epijugal is articulated with the jugal, while the right one is 
disarticulated. The postorbital horncores are long $($ left $=164 \mathrm{~mm}$, right $=176 \mathrm{~mm})$, similar to CMN 1254. UALVP 40 is similar in size to that of CMN 1254, although the squamosal is slightly longer $(600 \mathrm{~mm}$ (estimated) vs. $500 \mathrm{~mm})$. The estimated squamosal length was obtained by extrapolating the preserved straight medial and lateral margins of the element until they converged posteriorly; I believe that the plaster reconstruction is a reasonable approximation of the element's original length. Six episquamosals are articulated on the preserved squamosal. UALVP 40 appears to have undergone some transverse taphonomic compression and was most likely wider in life.

YPM 2016 (Fig. 7R) is a skull (rostral-to-epijugal length $=800 \mathrm{~mm}$; squamosal length $=$ $730 \mathrm{~mm}$ ) missing only the left posterolateral corner of the frill which has been restored with plaster. The rostral is articulated with the underlying premaxillae. The dorsal surfaces of the postorbitals are low-relief, pitted mounds, suggestive of postorbital horncore resorption. Both epijugals are articulated with the jugals. Each squamosal bears eight episquamosals. The lateral parietal bars are discontinuous. The parietal fenestrae are relatively short and nearly uniform in anteroposterior length and width (parietal fenestra length/width $=1.03$ ), resembling those of AMNH 5402. The posterior parietal bar (Fig. 10A-D) is straight and adorned with five epiparietals on the right side; this specimen has not previously been interpreted as having such a high epiparietal count. Only P1 and 2 are preserved on the left side. The preserved medialmost epiparietals (P1-P4) have a slight anterodorsal orientation, while P5 is oriented in the plane of the frill. 
Figure 10. Frill of YPM 2016 (Chasmosaurus belli) in: A) dorsal view; B) dorsal view of right half; C) posterior view of right posterior parietal bar; and D) posterior view of medial portion of right posterior parietal bar. Brackets delimit size of epiossifications; dashed line shows parietal-squamosal contact. Plaster reconstruction shown in grey halftone. See "List of anatomical abbreviations". Scale bar equals $10 \mathrm{~cm}$. 
Figure 10.

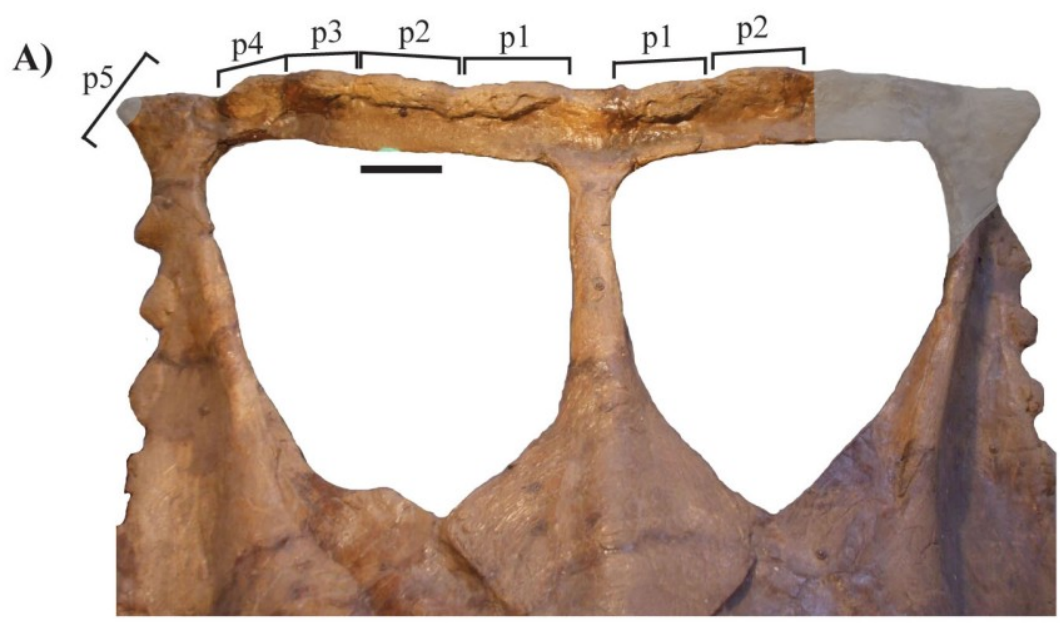

B)

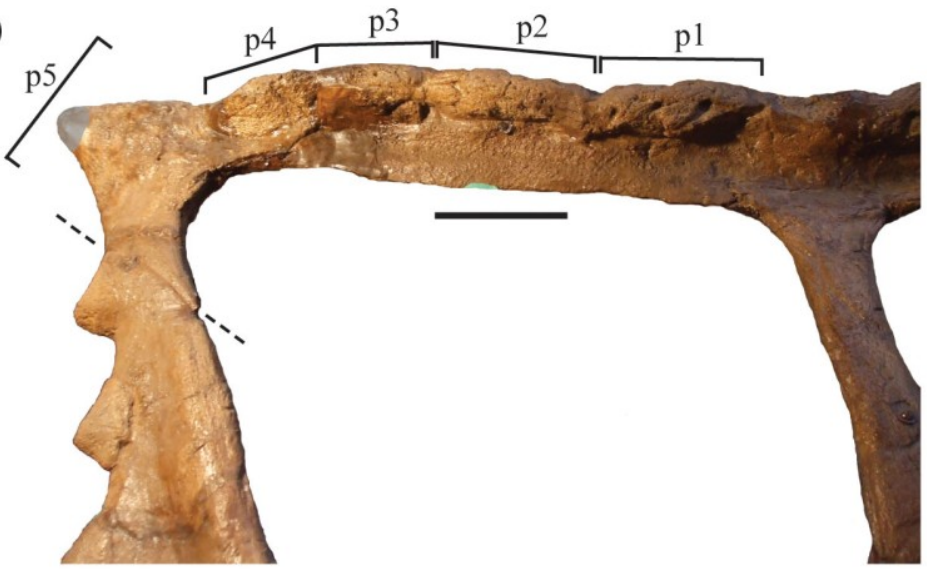

C)

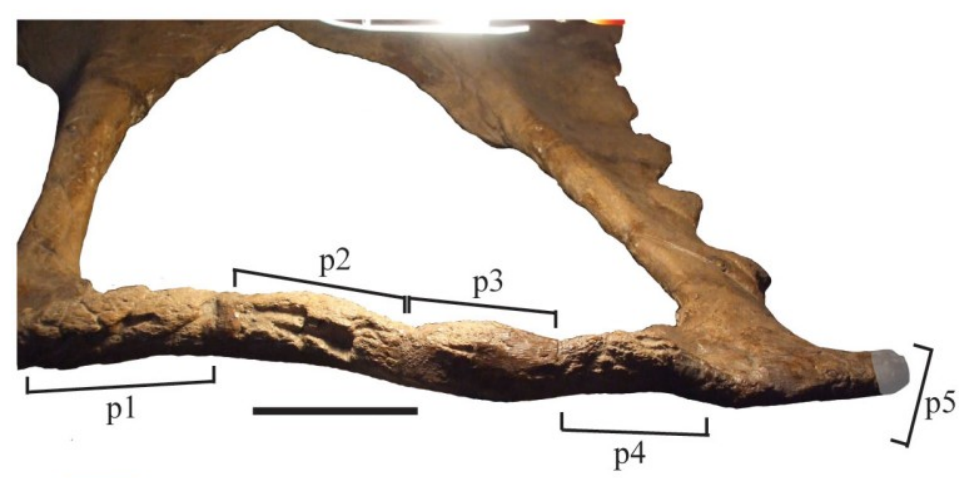

D)

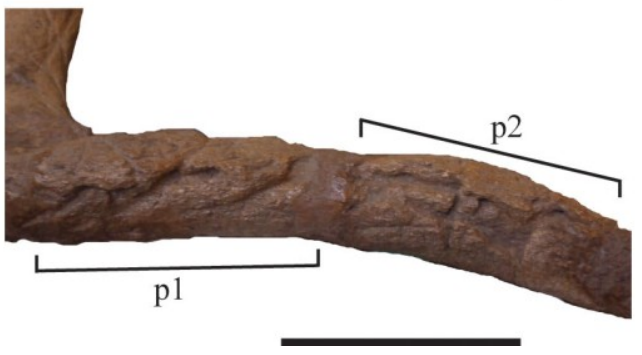


Postorbital horncores:

The circumorbital region of ceratopsids is bordered by the lacrimal anteriorly, the palpebral anterodorsally, the postorbital dorsally and posteriorly, and the jugal dorsoventrally (Sampson et al., 2010; Fig. 6). In adult-sized specimens, these elements typically articulate together into a unit called the supraorbital (Sampson et al., 2010). The postorbital horncore is a dorsal outgrowth of the postorbital and is present in most genera. The possession of short or long postorbital horncores was historically considered to be a centrosaurine and chasmosaurine trait, respectively; however, long postorbital horncores are also present in the outgroup taxon, Zuniceratops (Wolfe and Kirkland, 1998), and the basal centrosaurines Albertaceratops (Ryan, 2007), Diabloceratops (Kirkland and DeBlieux, 2010) and Xenoceratops (Ryan et al., 2013a). All adult-sized chasmosaurines have long postorbital horncores, except for some specimens of Chasmosaurus which have relatively short horncores compared to related taxa, and Vagaceratops where the pitted dorsal surface of the postorbital of this genus has been interpreted as a natural feature in a taxon that lacked postorbital horns, or the remodelled base of a horncore destroyed by bone resorption (Holmes et al., 2001). Chasmosaurine postorbitals form most of the lateral and dorsal margins of the supracranial sinus situated on the dorsum of the skull between the postorbitals (Lehman, 1990).

Isolated circumorbital elements referred to Chasmosaurus are shown in Figure 11. These specimens consist only of the postorbital unless otherwise indicated, and are arranged in order of horncore length, from shortest to longest. These specimens and their associated side of the skull (where known) are as follows: TMP 1981.016.0499 (right side; Fig. 11A); TMP 2008.012.0074 (left side; Fig. 11B); CMN 9808c (Fig. 11C), 
missing the upper portion of the horncore; TMP 1979.014.0813 (left side; Fig. 11D); CMN 0275 (left side; Fig. 11E); CMN 9808b (likely from the right side; Fig. 11F); CMN 9809 (likely from the left side; Fig. 11G); CMN 0838 (left side; Fig. 11H), also preserving a palpebral, but missing the upper portion of the horncore; TMP 1967.017.0005 (left side; Fig. 11I), also preserving a palpebral; CMN 0212 (Fig. 11J); CMN 9673 (left side; Fig. 11K), also preserving a palpebral; TMP 1981.016.0284 (Fig. 11L); TMP 1979.014.0814 (right side; Fig. 11M); CMN 0837 (Fig. $11 \mathrm{~N}$ ), but missing the apex of the horncore; and CMN 9676 (left side; Fig. 11O), also preserving a palpebral.

The relatively short and postorbital horncores in Figure 11A and C-G are consistent in length (TMP 1981.016.0499 $=50 \mathrm{~mm}$ (approximately) to $93 \mathrm{~mm}=\mathrm{CMN}$ 9809) with those on the more complete Chasmosaurus skulls discussed previously in this chapter (e.g., CMN 2245, and ROM 839); however, their length fall into the range of centrosaurines in the Dinosaur Park Formation (i.e., Centrosaurus, Styracosaurus and Spinops). The horncores in Figure 11B and H-J are more elongate in shape, similar to some Chasmosaurus skulls (e.g., CMN 1254, CMN 2280 and UALVP 40), but also to a basal long-horned centrosaurines (Ryan et al., 2013b). The postorbital CMN 9673 (Fig. $11 \mathrm{~K})$ is also short as preserved, but the presence of a longitudinally extensive medial margin (lateral margin of supracranial sinus) supports its referral to Chasmosaurinae. The dorsal surface of CMN 9673 is deeply rugose and pitted, and may represent a remodelled horncore which has undergone extensive bone resorption. The horncores in Figure 11I-J and L-O are elongate (111 mm, TMP 1967.017.0005, Fig. 11I; to 440 mm, CMN 9676; 
A)

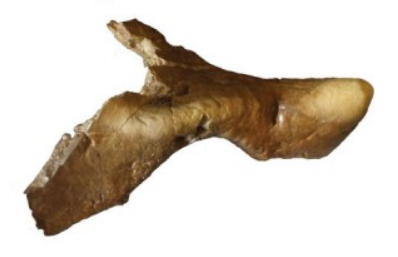

E)

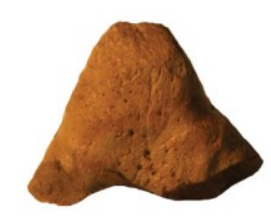

H)

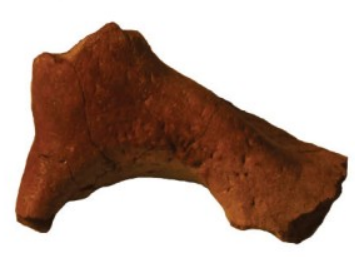

B)

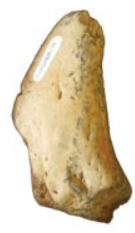

C)

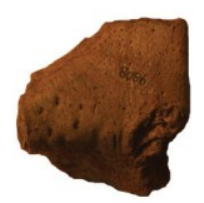

F)

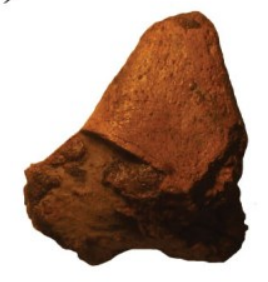

I)

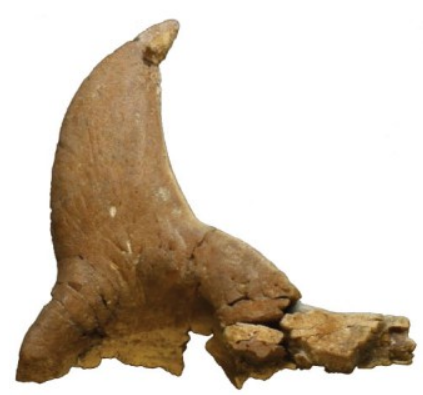

D)

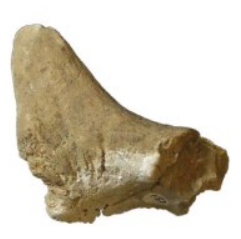

G)

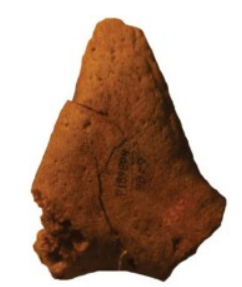

J)

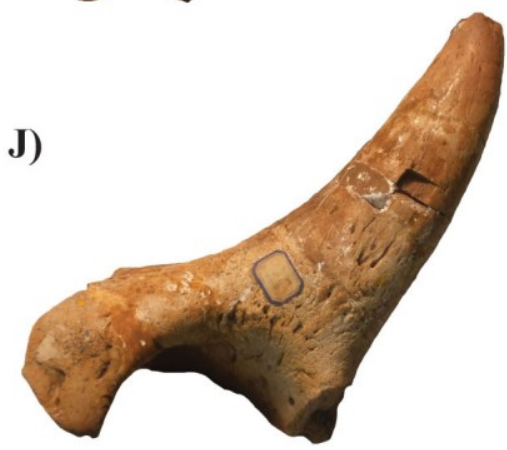

Figure 11. Chasmosaurus sp. postorbital horncores in lateral view: A, TMP 1981.016.0499; B, TMP 2008.012.0074; C, CMN 9808c; D, TMP 1979.014.0813; E, CMN 0275; F, CMN 9808b; G, CMN 9809; H, CMN 0838; I, TMP 1967.017.0005; J, CMN 0212; K, CMN 9673; L, TMP 1981.016.0284; M, TMP 1979.014.0814; N, CMN 0837; and O, CMN 9676 (dorsal view). Scale bar equals $10 \mathrm{~cm}$. 
Figure 11. (continued).

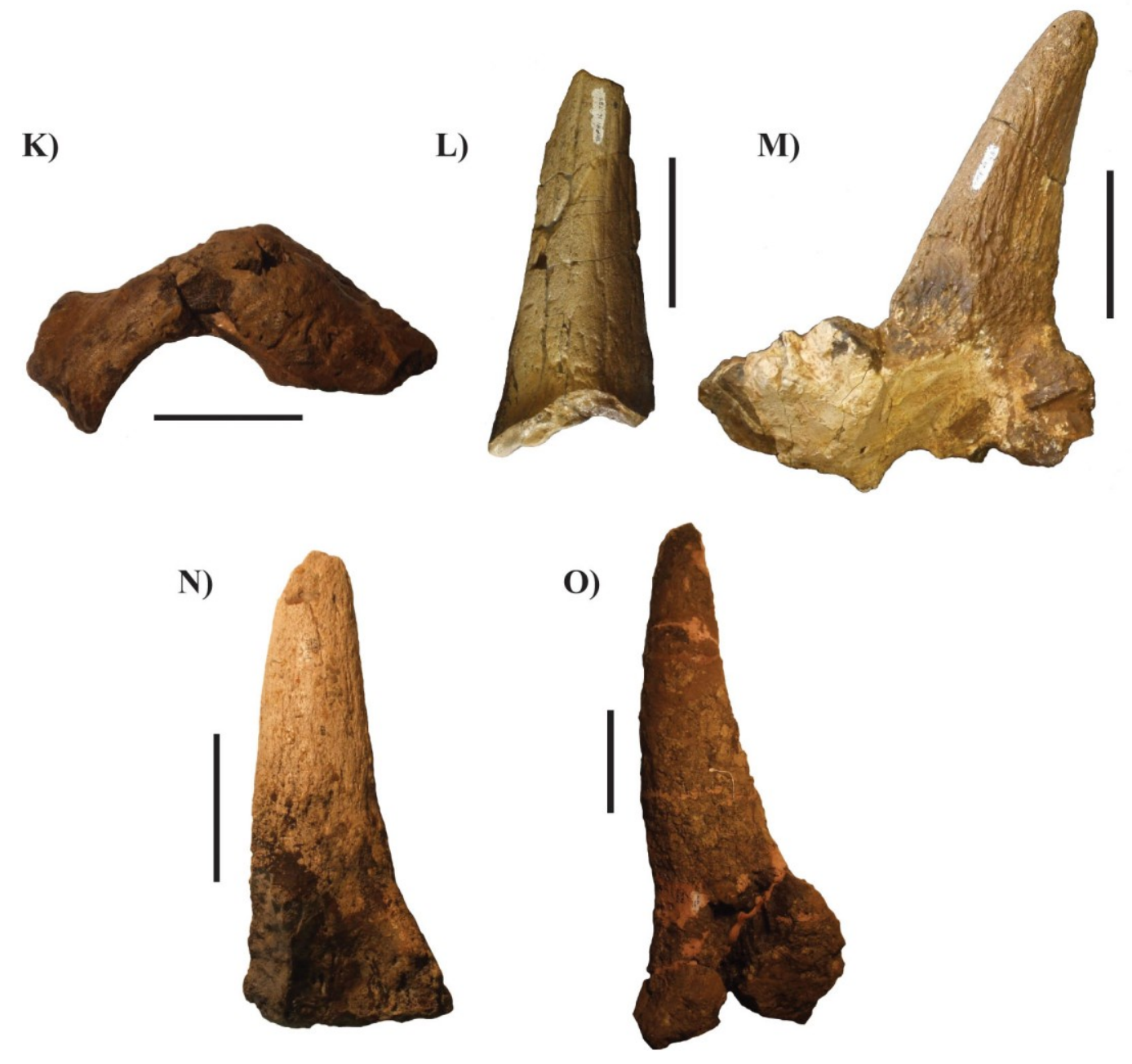


Fig. 110) relative to centrosaurine postorbital horncores in the Dinosaur Park Formation, and are most likely chasmosaurine.

Isolated frills (squamosals):

TMP 1991.046.0004 (Fig. 12A) consists of the distal end of a right squamosal; the posteriormost episquamosal is preserved, and anteriorly there is a loci for another episquamosal.

TMP 1998.128.0001 (Fig. 12B) is a left squamosal, $208 \mathrm{~mm}$ in length. This specimen is the smallest chasmosaurine specimen examined; the next smallest squamosal (CMN 1254) is much larger, measuring $500 \mathrm{~mm}$ in length. TMP 1998.128.0001 has nine loci on the lateral margin. No episquamosals are present. 
A)
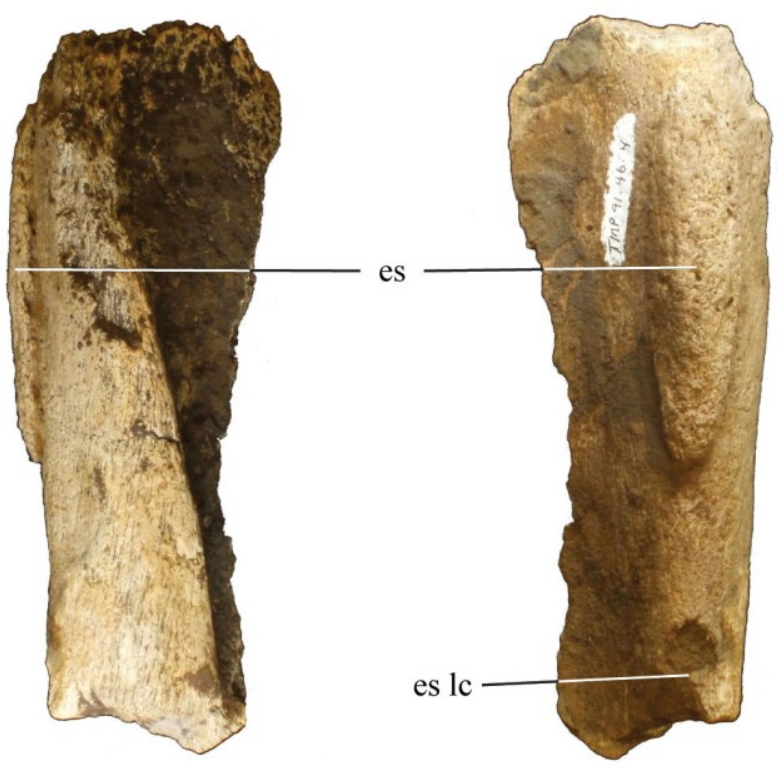

B)
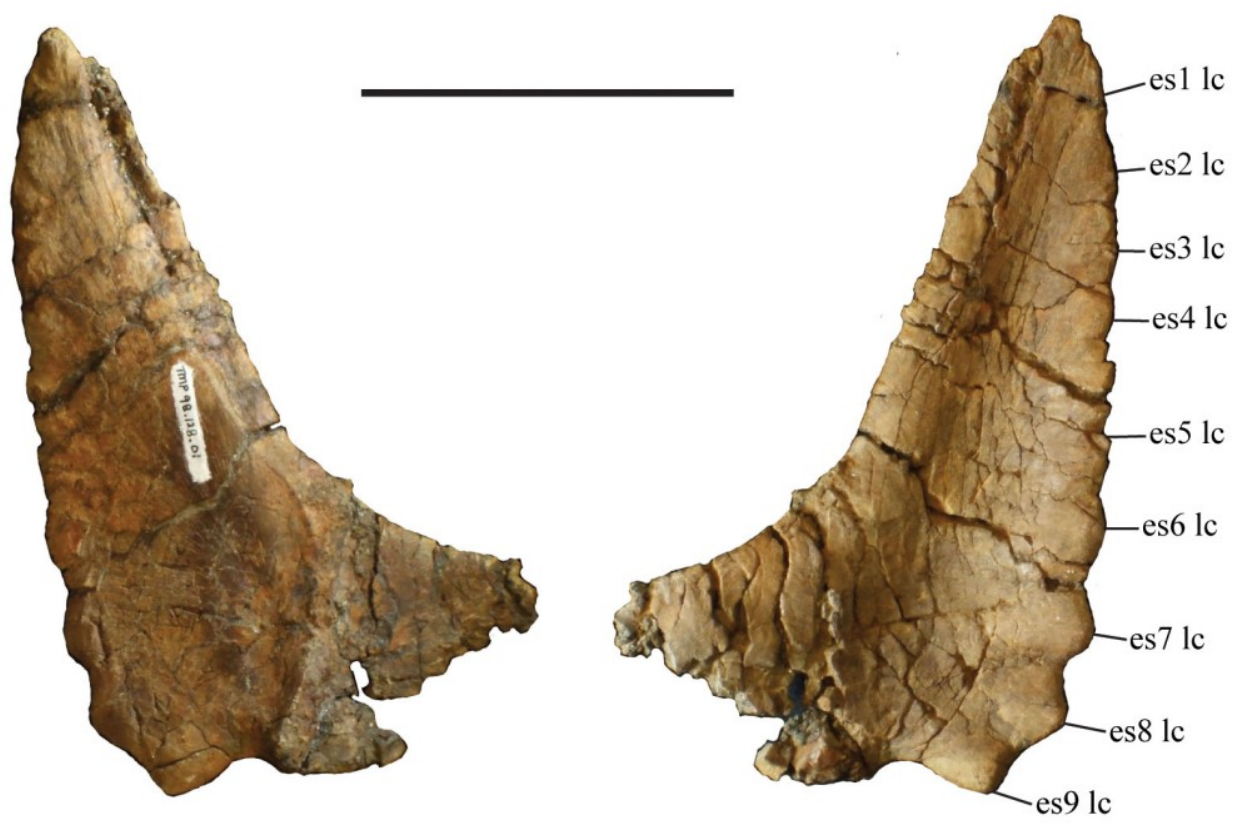

Figure 12. Chasmosaurus sp. squamosals: A), TMP 1991.046.0004 in dorsal (left) and ventral (right) views; and B), TMP 1998.128.0001 in dorsal (left) and ventral (right) views. See "List of anatomical abbreviations". Scale bar equals $10 \mathrm{~cm}$. 
Isolated frills (parietals)

Partial medial parietal bars:

TMP 1981.023.0024 (Fig. 13A) consists of the mid-section of a medial parietal bar. TMP 2007.020.0039 (Fig. 13B) consists of most of a medial parietal bar; based on its length (approximately $430 \mathrm{~mm}$ ), it would have had relatively long parietal fenestrae. TMP 2009.032.0011 (Fig. 13C) is an almost complete medial parietal bar; based on its length (approximately $400 \mathrm{~mm}$ ), it also had relatively long parietal fenestrae.

TMP 1999.055.0274 (Fig. 13D) consists of three fragments comprising the medial parietal bar and medial platform (the raised surface anterior to the parietal fenestrae, and between the dorsal temporal fenestrae; termed "anterior expansion" in Lambe, 1902:58); the partial medial bar is relatively long and correspondingly would have had relatively anteroposteriorly long parietal fenestrae. TMP 1982.016.0215 (Fig. 13E) consists of the right side of a medial platform of the parietal and anterior end of the medial bar.

Partial posterior parietal bars:

CMN 0491 (holotype, Chasmosaurus belli; Fig. 14A) consists of most of a medial parietal bar and short sections of posterior bars adjacent to it, each of which preserve a dorsallythickened ridge. The preserved posterior bar has a modest embayment. No epiparietals can be discerned, although the ridge on each side may represent a highly modified P1.

TMP 2008.012.0001 (Fig. 14B) is almost identical in form and in degree of preservation 
as CMN 0491, but it has a shorter and narrower medial bar. It displays a similar degree of embayment, although no epiparietals or thickened ridges are present.

CMN 8803 (Fig. 14C) consists of most of a posterior parietal bar and what is inferred to be approximately the posterior one-half of the medial bar. There is a deep groove lining the parietal fenestrae margin. This groove, together with a dorsoventrally thickened medial bar, gives the posterior bar a distinct I-shape in cross-section. The posterior bar resembles an inverted $\mathrm{W}$ in dorsal profile, with a very deep embayment adjacent to the midline parietal bar, and the short preserved portions of the rest of the posterior bar being reflected slightly anteriorly. The left and more complete right side of the posterior bar each possess two epiparietals. The epiparietals adjacent to the midline (P1) are small and tab-like, with a posteromedial orientation due to their positions on the edge on the embayment. The broad-based, low P2 (present only on left side) straddles the embayment angle, and has a less distinct base than that of P1. P3 (present only on right side) is broken through its midline so its size and extent cannot be determined. All epiparietals are oriented in the plane of the frill.

TMP 1999.055.0292 (Fig. 14D) consists of the left side of a posterior parietal bar. The curved posterior bar suggests that it had a pronounced medial embayment similar to CMN 8803, but the depth of this embayment cannot be determined. The deep groove on the medial margin of the posterior bar is also similar to that of CMN 8803. It is adorned with three nearly equal-sized epiparietals oriented in the plane of the frill, although P1 
Figure 13. Chasmosaurus sp. medial parietals: A), TMP 1981.023.0024 in dorsal (top) and ventral (bottom) views; B), TMP 2007.020.0039 in dorsal (left) and ventral (right) views; C), TMP 2009.032.0011 in dorsal (left) and ventral (right) views; D), TMP 1999.055.0274 in dorsal (top) and ventral (bottom) views; and E), TMP 1982.016.0215 in dorsal (top) and ventral (bottom) views. See "List of anatomical abbreviations". Scale bar equals $10 \mathrm{~cm}$. 
Figure 13.

A)

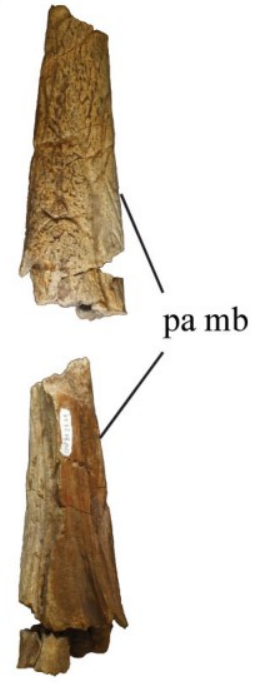

B)

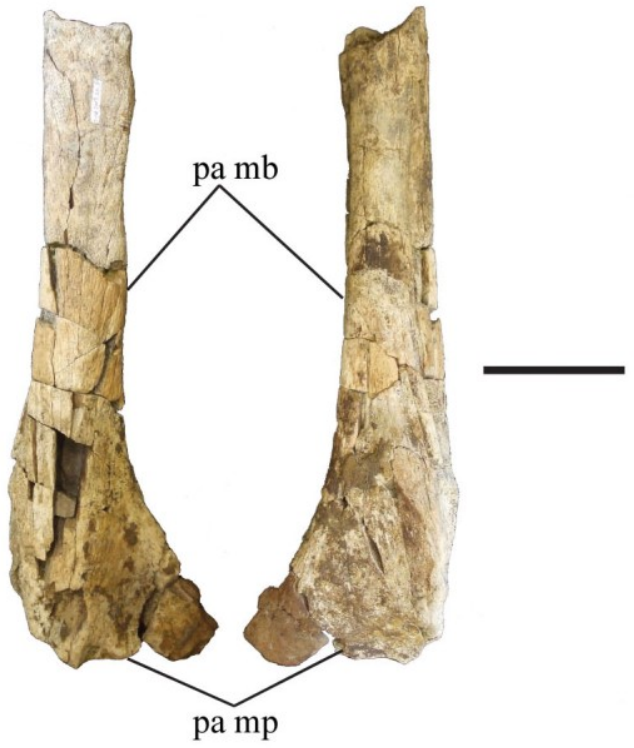

C)

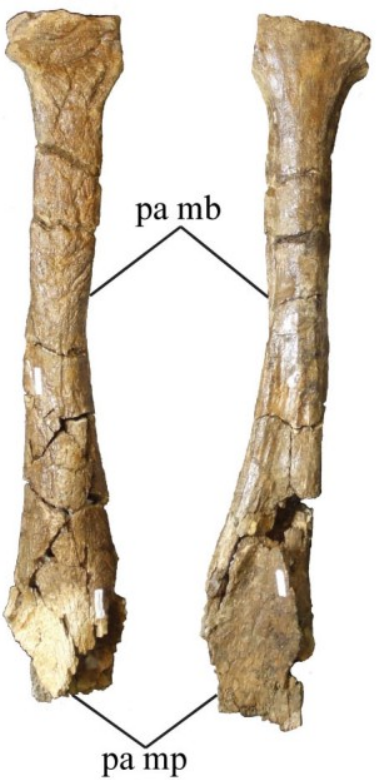

D)

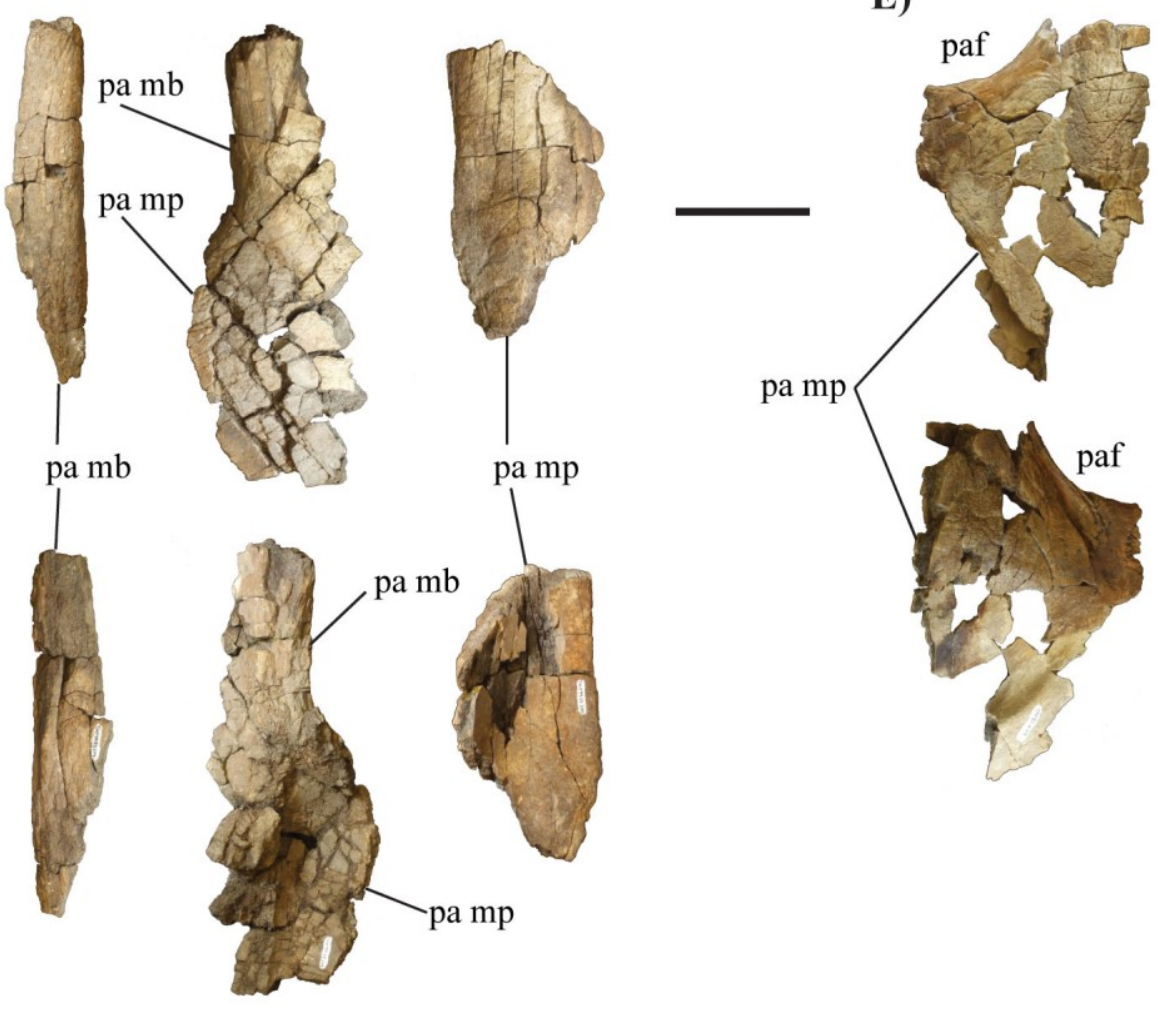


Figure 14. Chasmosaurus posterior parietals: A), CMN 0491 (Chasmosaurus belli holotype) in dorsal (left) and ventral (right) views; B), TMP 2008.012.0001 (C. sp.) in dorsal (left) and ventral (right) views; C), CMN 8803 (Chasmosaurus russelli) in dorsal (left) and ventral (right) views; D), TMP 1999.055.0292 (C. russelli) in ventral (left) and dorsal (right) views; E), TMP 1997.132.0002 (C. russelli) in dorsal (left) and ventral (right) views; F), CMN 41933 (C. sp.) in dorsal (left) and ventral (right) views; G), TMP 1981.030.0001 (C. sp.) in dorsal (left) and ventral (right) views; H), TMP 1994.012.0778 (C. sp.) in dorsal (left) and ventral (right) views; I), TMP 1993.086.0001 (C. sp.) in dorsal (left) and ventral (right) views; J), TMP 1993.075.0005 (C. sp.) in dorsal (left) and ventral (right) views; K), TMP 1996.029.0004 (C. sp.) in dorsal (left) and ventral (right) views; L), TMP 1998.093.0010 (C. sp.) in dorsal (left) and ventral (right) views; M), TMP 1986.036.0530 (C. sp.) in dorsal (left) and ventral (right) views; N), TMP 2007.020.0103 (C. sp.) in dorsal (left) and ventral (right) views; and O), TMP 1981.018.0231 (C. sp.) in dorsal (left) and ventral (right) views. See "List of anatomical abbreviations". Scale bar equals $10 \mathrm{~cm}$. 
Figure 14.

A)

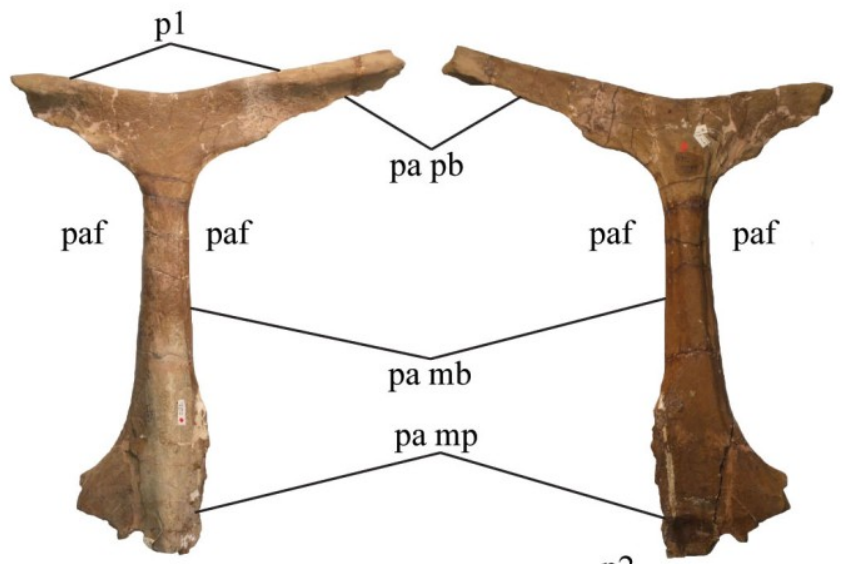

C)

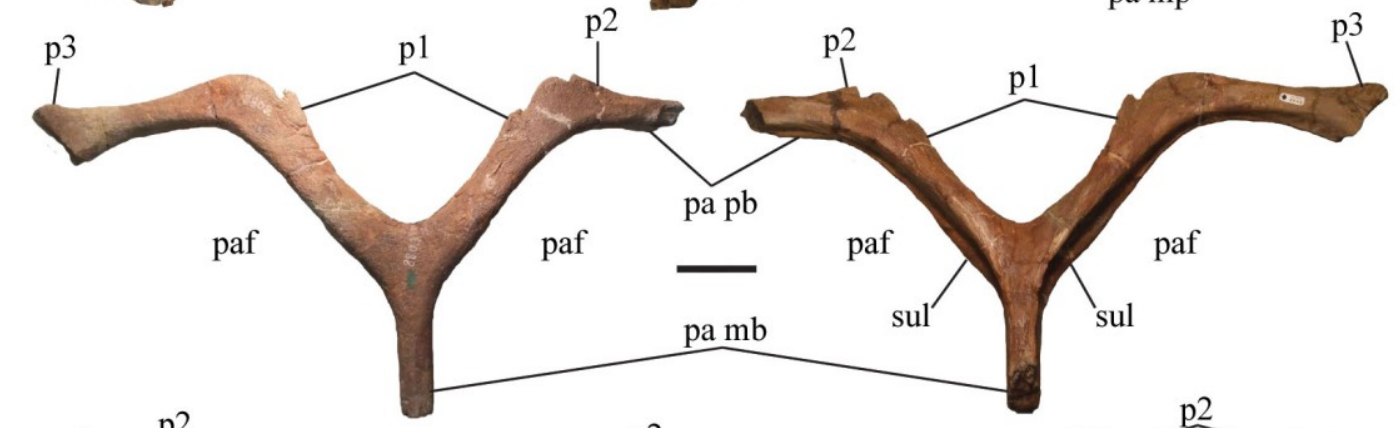

B)

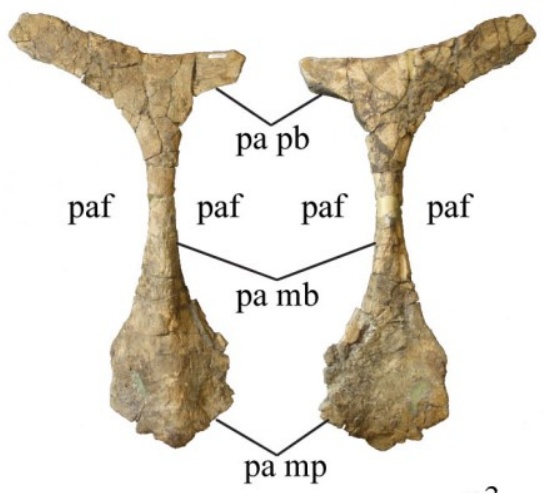

D)

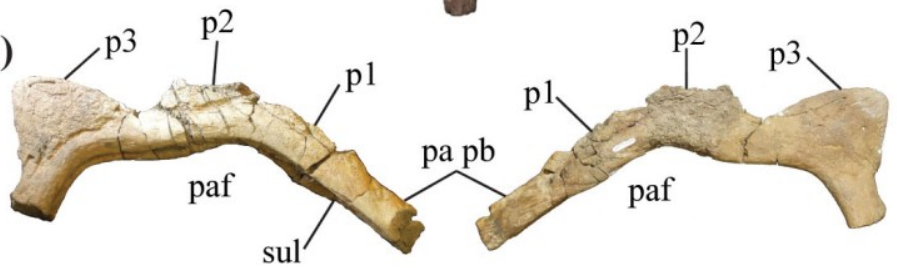

E)

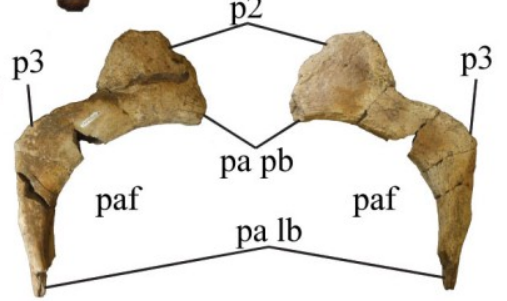


Figure 14. (continued).
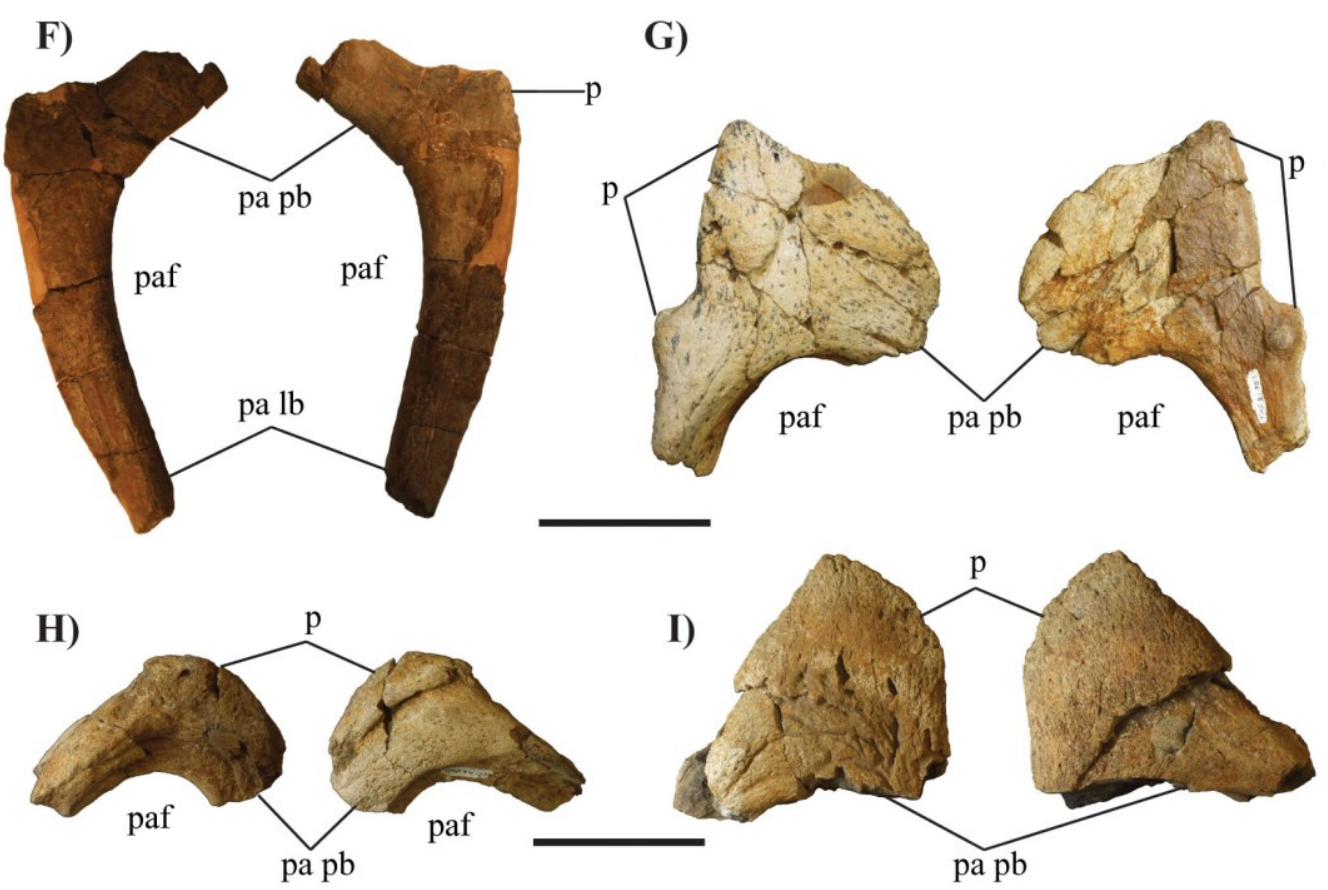

J)
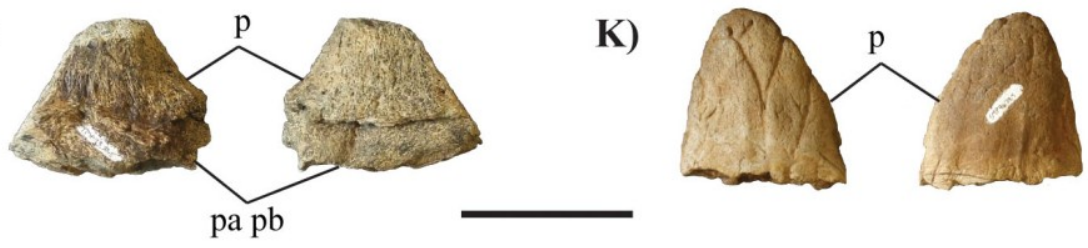

L)

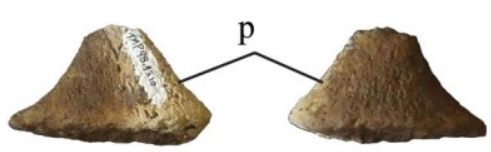

M)

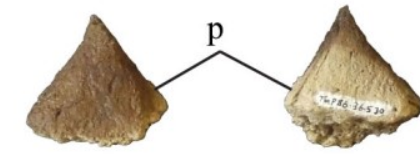

N)

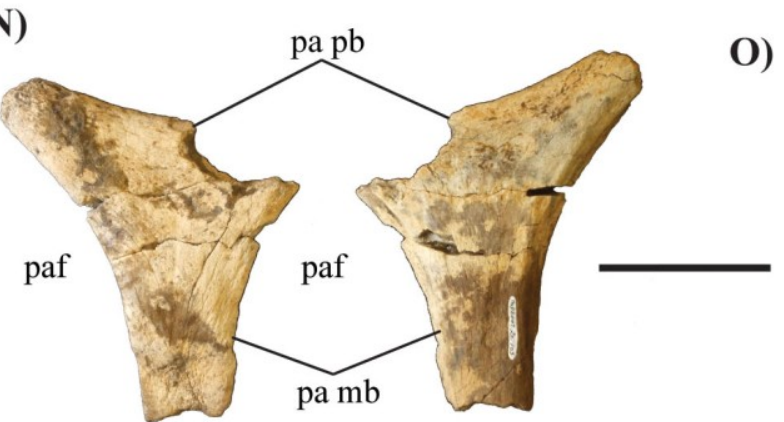

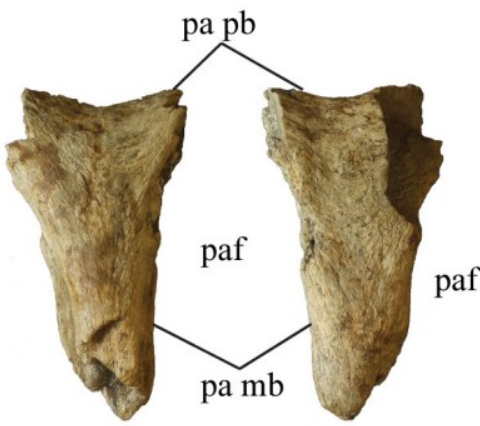


and $\mathrm{P} 2$ are only partially preserved.

TMP 1997.132.0002 (Fig. 14E) consists of the left posterolateral corner of a parietal. This specimen has two equal-sized epiparietals, with the lateral-most epiparietal (or loci) located close to the parietal-squamosal contact.

CMN 41933 (Fig. 14F) consists of the posterolateral corner (side unknown) of a parietal. There is a pronounced knob on the corner which either represents a modified, articulated epiparietal or an epiparietal locus.

TMP 1981.030.0001 (Fig. 14G) consists of the right posterolateral corner of a parietal. The specimen is adorned with two epiparietals that differ greatly in size. The relative positions of these epiparietals on the posterior bar and their discrepancy in size is reminiscent of the epiparietals on ROM 843.

TMP 1994.012.0778 (Fig. 14H) consists of the left posterolateral corner of a parietal with a single articulated epiparietal. TMP 1993.086.0001 (Fig. 14I) consists of the right posterolateral corner of a parietal with a single articulated epiparietal. TMP 1993.075.0005 (Fig. 14J) consists of a large epiossification with a portion of underlying bone; it likely represents an epiparietal based on its large size. TMP 1996.029.0004 (Fig. $14 \mathrm{~K})$ is a large, disarticulated epiossification, most likely representing an epiparietal. TMP 1998.093.0010 (Fig. 14L) and 1986.036.0530 (Fig. 14M) are small, disarticulated epiossifications, probably representing episquamosals. 
TMP 2007.020.0103 (Fig. 14N) consists of the junction between medial and posterior parietals bars; it has a slight median embayment, although its extent cannot be determined. TMP 1981.018.0231 (Fig. 14O) also consists of the junction between medial and posterior parietal bars. It has a deep longitudinal groove similar to those of CMN 8803 and TMP 1999.055.0292.

\subsubsection{Vagaceratops}

Skulls:

CMN 41357 (holotype, V, irvinensis; Fig. 15A) is an almost complete skeleton originally referred to Chasmosaurus irvinensis by Holmes et al. (2001). A phylogenetic analysis by Sampson et al. (2010) found that CMN 41357, and the other material referred to Chasmosaurus irvinensis, did not group with Chasmosaurus, but rather was the sister taxon to their newly described Kosmoceratops; therefore, they erected the new taxon, Vagaceratops, to receive it. The skull is disarticulated, but preserves the entire frill and most of the right facial region and lower jaws. The skull was described in detail by Holmes et al. (2001), and only the information relevant to this project will be discussed here.

Vagaceratops differs from Chasmosaurus in the following features: anteroposteriorly-shortened parietal fenestrae with length/width ratios ranging from 0.72 (CMN 41357) to 0.87 (TMP 1987.045.0001); in Chasmosaurus, the ratio is between 1.03 (YPM 2016) and 1.89 (AMNH 5656) (Table 1); straight posterior parietal bar (in Chasmosaurus, this bar ranges from being deeply embayed (CMN 8800) to straight (YPM 2016)) (Table 1); and eight to 10 epiparietals, with four (TMP 1987.045.0001 and 
TMP 1998.102.0008, both inferred; a fifth epiossification lateral to these straddles the parietal-squamosal contact) to five (CMN 41357) on each side, respectively. In CMN 41357, the parietal-squamosal suture is difficult to see posteriorly, but is thought to be situated directly laterally adjacent to P5. Chasmosaurus has previously been diagnosed as having only three per side (Godfrey and Holmes, 1995), although YPM 2016 and AMNH 5402 are interpreted here as having five and four (inferred) per side, respectively; AMNH 5402 and its epiparietal configuration are discussed further in this chapter. The four medialmost pairs of epiparietals in Vagaceratops are elongate, anterodorsally recurved and coossified at their bases, while the fifth lateral-most pair is oriented posterolaterally in the plane of the frill.

The rostral of CMN 41357 is articulated with the premaxillae. The surface of the right postorbital is rugose, pitted, and perforated by a small hole. The preserved right epijugal is articulated. Each squamosal bears eight episquamosals. The left lateral parietal bar is continuous, while the right bar is not.

TMP 1987.045.0001 (Fig. 15B) is an almost complete skull, lacking only the central and right portions of the frill. The skull has undergone severe dorsoventral taphonomic compression. The rostral is articulated with the underlying premaxillae. The dorsal surface of each postorbital is rugose and pitted. The preserved left squamosal bears eight episquamosals. The left preserved lateral parietal bar is continuous. Two epiossifications are preserved on the left dorsolateral corner; the medialmost one is articulated with the parietal (epiparietal), while the lateral one bridges the parietosquamosal contact (epiparietosquamosal). The elongate and anterodorsally recurved medialmost epiossification preserved in TMP 1987.045.0001 (Fig. 15B) is similar in size, orientation 
Figure 15. Vagaceratops skulls: A), holotype CMN 41357 (cast) in dorsal (top) and lateral (bottom) views; and B), TMP 1987.045.0001 in dorsal (top) and lateral (bottom) views. Plaster reconstruction shown in grey half-tone. See "List of anatomical abbreviations". Scale bar equals $10 \mathrm{~cm}$. 
Figure 15.
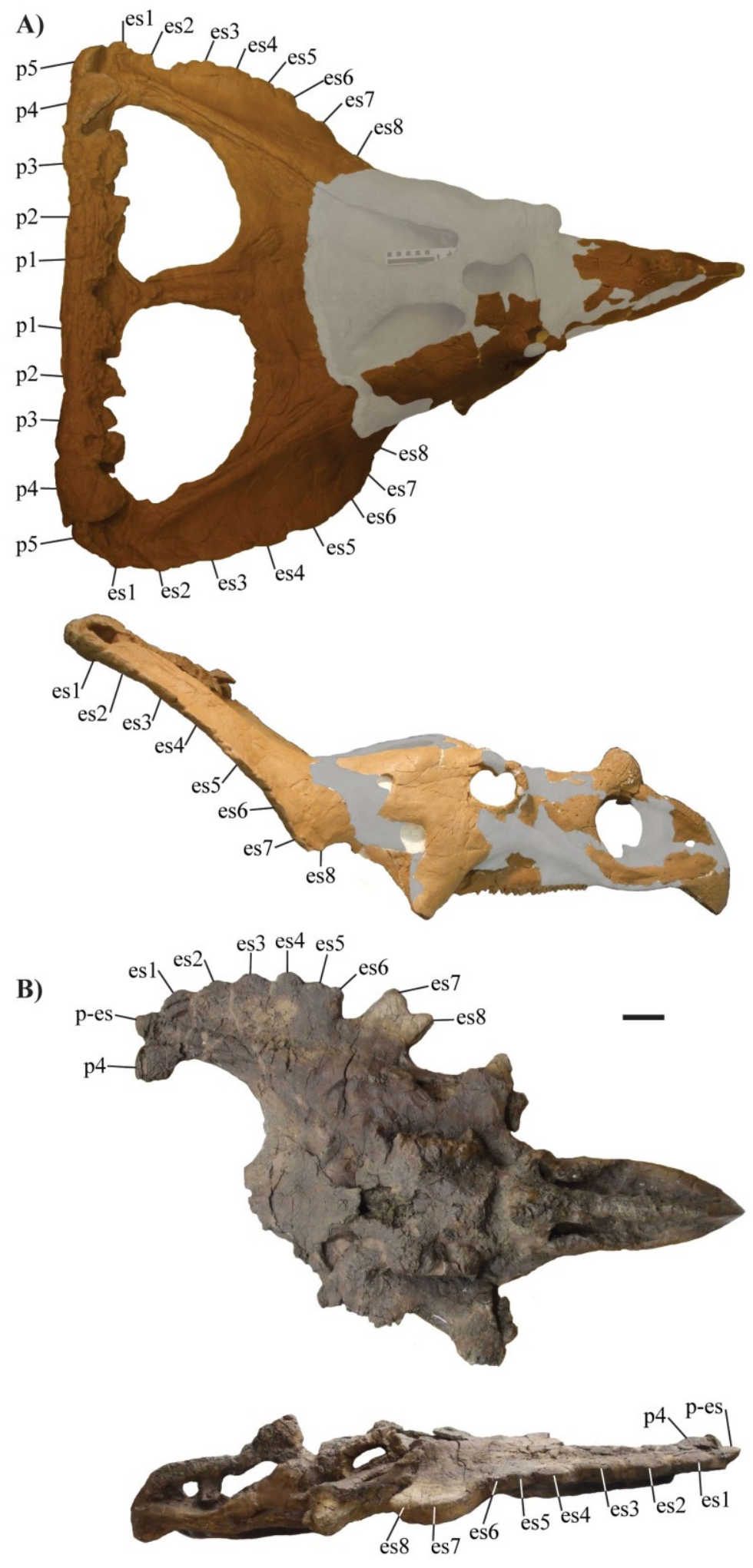
Table 1: Selected cranial measurements of specimens referred to Chasmosaurus and Vagaceratops (see Fig. 5 for description of measurements). Single asterix $(*)$ represents inferred incomplete feature (i.e., incomplete, resorbed postorbital horncore); double asterix $(* *)$ represents estimated measurement; measurements taken off best preserved side of skull are shown in bold.

\begin{tabular}{|c|c|c|c|c|c|}
\hline Specimen number & $\begin{array}{l}\text { Rostral-to-epijugal length } \\
\text { (left/right side; mm) }\end{array}$ & $\begin{array}{l}\text { Postorbital horncore length } \\
\text { (left/right side; } \mathrm{mm} \text { ) }\end{array}$ & $\begin{array}{c}\text { Squamosal length } \\
\text { (left/right sides, mm) }\end{array}$ & $\begin{array}{l}\text { Parietal fenestra, } \\
\text { length/width ratio }\end{array}$ & $\begin{array}{l}\text { Embayment angle of } \\
\text { posterior parietal bar }\end{array}$ \\
\hline \multicolumn{6}{|l|}{ Chasmosaurus } \\
\hline AMNH 5401 & $670 / 730$ & $350 / 370$ & $710 / 695$ & $\begin{array}{ll}---- \\
\end{array}$ & $\begin{array}{l}---- \\
\end{array}$ \\
\hline AMNH 5402 & 680/----- & $70 / 65$ & $640 / 685$ & 1.04 & $180 * *$ \\
\hline AMNH 5656 & ------/----- & ------/----- & $569 / 560$ & 1.89 & 115 \\
\hline CMN 0491 & -----/----- & -----/----- & -----/----- & $\begin{array}{ll}---- \\
--\end{array}$ & 158 \\
\hline CMN 1254 & -----/----- & $-----/ 216$ & $-----/ 500$ & $\begin{array}{ll}---- \\
\end{array}$ & $\begin{array}{ll}---- \\
--1\end{array}$ \\
\hline CMN 2280 & $\mathbf{7 5 0} / 700$ & $132 / 88^{*}$ & 709/668 & 1.26 & 124 \\
\hline CMN 8800 & 880/----- & $40 * / 108 *$ & 997/----- & 1.81 & 89 \\
\hline CMN 8801 & $\mathbf{8 3 5} / 802$ & $-----/ 57 *$ & -----/----- & $\begin{array}{ll}---- \\
\end{array}$ & $\begin{array}{ll}---- \\
\end{array}$ \\
\hline CMN 8802 & ------/----- & 71/----- & ------/----- & ----- & $\begin{array}{l}---- \\
\end{array}$ \\
\hline CMN 8803 & -----/----- & -----/----- & -----/----- & $\begin{array}{ll}---- \\
\end{array}$ & 101 \\
\hline CMN 34829 & ------/----- & $56 / 59$ & $\mathbf{8 5 0} * * / 810 * *$ & ----- & $\begin{array}{l}---- \\
\end{array}$ \\
\hline CMN 34832 & -----/----- & $236 / 237$ & 682/----- & ----- & $\begin{array}{ll}---- \\
\end{array}$ \\
\hline ROM 843 & $\mathbf{8 9 7 / 8 5 2}$ & $67 / 55$ & $\mathbf{8 9 3} / 965$ & 1.09 & 136 \\
\hline TMP 1979.011.0147 & & $263 / 298$ & ------/----- & $\begin{array}{ll}---- \\
\end{array}$ & $\begin{array}{l}---- \\
\end{array}$ \\
\hline TMP 1981.019.0175 & $-----/ 768$ & $64 * / 134 *$ & $----/ 600 * *$ & $\begin{array}{ll}---- \\
--\end{array}$ & $\begin{array}{ll}---- \\
--1\end{array}$ \\
\hline TMP 1983.025.0001 & -----/----- & $142 * /-----$ & -----/650 & 1.32 & 128 \\
\hline TMP 1993.082.0001 & -----/----- & ------/----- & 879/----- & $\begin{array}{ll}---- \\
\end{array}$ & $\begin{array}{ll}---- \\
\end{array}$ \\
\hline TMP 1998.128.0001 & ------/----- & ------/----- & 208/----- & $\begin{array}{ll}---- \\
-\end{array}$ & $\begin{array}{ll}---- \\
--1\end{array}$ \\
\hline UALVP 40 & 628/645 & $164 / 176$ & $600 * * /-----$ & $\begin{array}{ll}---- \\
\end{array}$ & $\begin{array}{ll}---- \\
\end{array}$ \\
\hline YPM 2016 & $735 / 800$ & $37 / 41$ & 750/730 & 1.03 & 171 \\
\hline \multicolumn{6}{|l|}{ Vagaceratops } \\
\hline CMN 41357 & $-----/ 795$ & ------/----- & $680 / 700$ & 0.72 & 180 \\
\hline TMP 1987.045.0001 & $617 / 637$ & ------/----- & 633/----- & 0.87 & $180 * *$ \\
\hline
\end{tabular}


and position as P4 in CMN 41357 (Fig. 15A). The epiparietosquamosal of TMP 1987.045.0001 is interpreted as being homologous to that of P5 of CMN 41357, as they are both positioned at the posterolateral corner of the frill and oriented in the plane of the frill. Based on the two above inferred homologies, and possession of an anteroposteriorly shortened parietal fenestra, TMP 1987.045.0001 is inferred as having a straight posterior parietal margin.

Like Vagaceratops, Kosmoceratops has relatively small parietal fenestrae and 10 elongate, anterodorsally recurving epiossifications on the posterior frill margin. The posterior epiossifications of UMNH VP 17000 (holotype, Kosmoceratops) show no indication of attachment sutures so they were initially interpreted as being five epiparietals per side, following the model of Vagaceratops proposed by Holmes et al. (2001). The discovery of an undescribed, subadult-sized Kosmoceratops revealed that the three medialmost pairs of epimarginals are three epiparietals, while the fourth and fifth pairs are epiparietosquamosals and episquamosals, respectively, and argued that the sister taxon, Vagaceratops, probably followed the same pattern (Sampson et al., 2010).

The parietal-squamosal suture is difficult to discern on the holotype of Vagaceratops (CMN 41357; Fig. 15A), but appears to be situated lateral to the fifth epiossification from the midline of the parietal, suggesting an epiparietal count of five per side for this specimen. In the referred Vagaceratops specimen TMP 1987.045.0001 (Fig. 15B), the two preserved epiossifications on the posterior margin of the frill are interpreted in this study as being homologous to P4 and P5 of CMN 41357, based on their size, orientation (anterodorsally and in plane of frill, respectively), and posterolateral positioning on the frill. In TMP 1987.045.0001, the parietal-squamosal 
suture is situated beneath the "P5", making this epiossification an epiparietosquamosal. The homology between the two preserved posterior epiossifications of TMP 1987.045.001 and the fourth and fifth posterior epiossifications in CMN 41357 indicates that Vagaceratops possessed four (TMP 1987.045.0001, inferred) or five (CMN 41357) epiparietals (sensu Holmes et al., 2001; contra Sampson et al., 2010).

Partial frills:

TMP 1998.102.0008 (Fig. 16A) consists of two fragments representing most of each side of a posterior parietal bar, but the central portion is missing. Each side has a relatively straight posterior margin and three anterodorsally projecting, abutting epiparietals. These epiparietals appear to be homologous in position and form to $\mathrm{P} 2-\mathrm{P} 4$ in CMN 41357, as the P4 is positioned directly posterior to the distal end of the lateral parietal bar in both specimens. In TMP 1998.102.0008, the parietal-squamosal contact is situated lateral to P4. Based on the specimen's similarity to CMN 41357, a fifth epiossification from the midline is inferred as having been present, but not preserved. Based on the close abutting nature of the preserved epiparietals in TMP 1998.102.0008, this fifth epiossification is inferred to have been originally positioned over the parietalsquamosal contact, making it an epiparietosquamosal.

TMP 2009.034.0009 (Fig. 16B) is a partial, fragmentary skull, consisting of the anterior half of the left squamosal (Fig. 15, left), part of the posterior and medial parietal bars (Fig. 15, right), and most of the braincase (Fig. 15, bottom). The posterior parietal bar, although missing the lateralmost ends, has a relatively straight posterior margin. Along this margin, there is a thickened ridge on the dorsal surface. Immediately anterior 
to this ridge on the right side of the frill, there are three short, wide-based, equal-sized epiparietals. These epiparietals appear to have been broken off and displaced from the ridge, possibly during burial, or because the epiparietals had not yet fused with the parietal at the time of death. Given their positions, it appears that the epiparietals would have been in close contact with each other when articulated. The possession of a thickened ridge, straight posterior margin, and wide, equal-sized epiparietals nearly in contact with each other is characteristic of both Chasmosaurus (i.e., YPM 2016) and Vagaceratops (i.e., CMN 41357, TMP 1998.102.0008). Unfortunately, the parietal fenestrae are incomplete, so their anterior extent cannot be determined. 
Figure 16. Partial Vagaceratops frills: A), TMP 1998.102.0008 in posterior (top), dorsal (middle) and anterior (bottom) views; and B), TMP 2009.034.0009 in medial view of left squamosal (left), dorsal view of parietal (right), and posterodorsal view of braincase (bottom). See "List of anatomical abbreviations". See "List of anatomical abbreviations". Scale bar equals $10 \mathrm{~cm}$. 
Figure 16.

A)
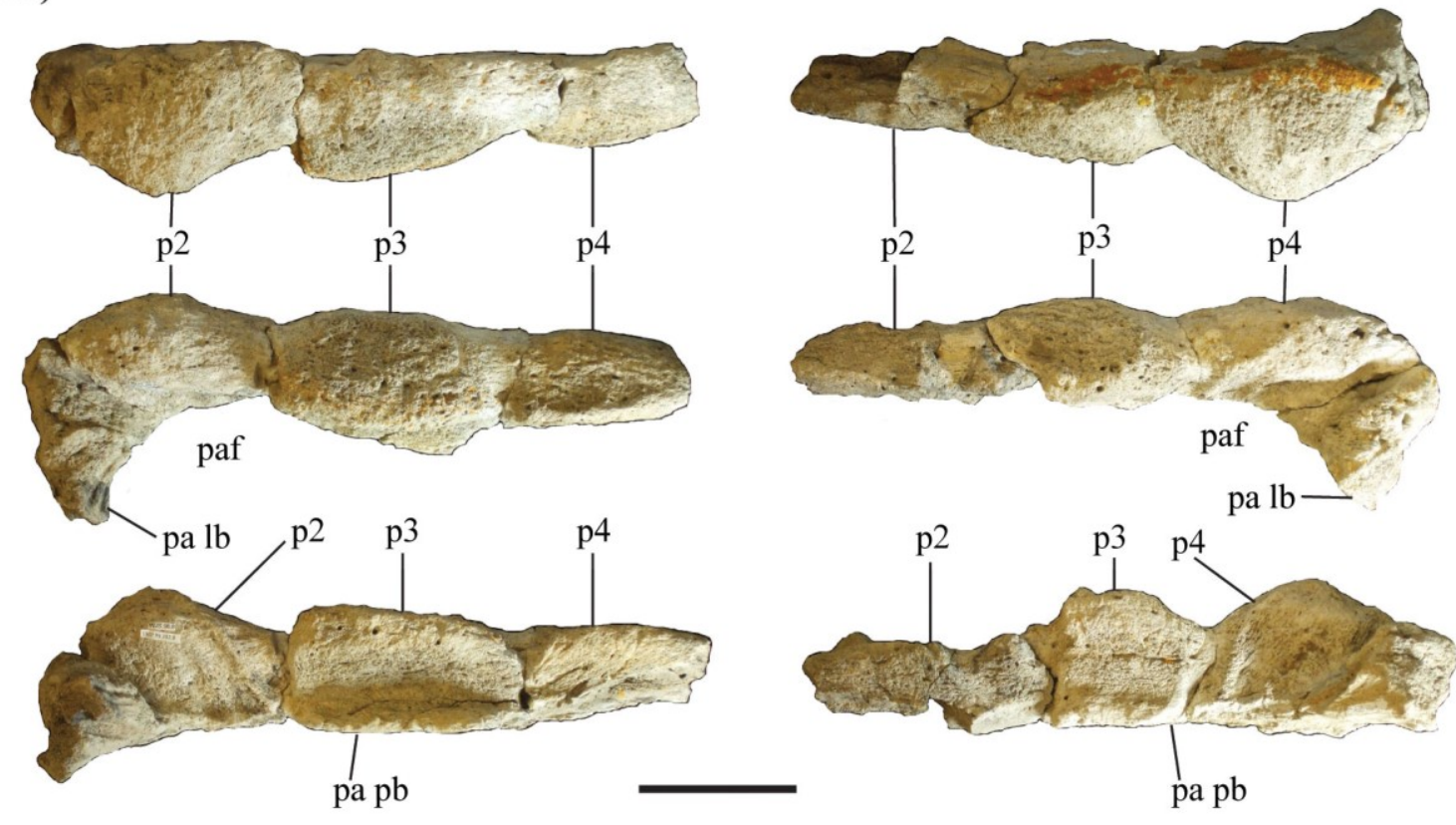

B)
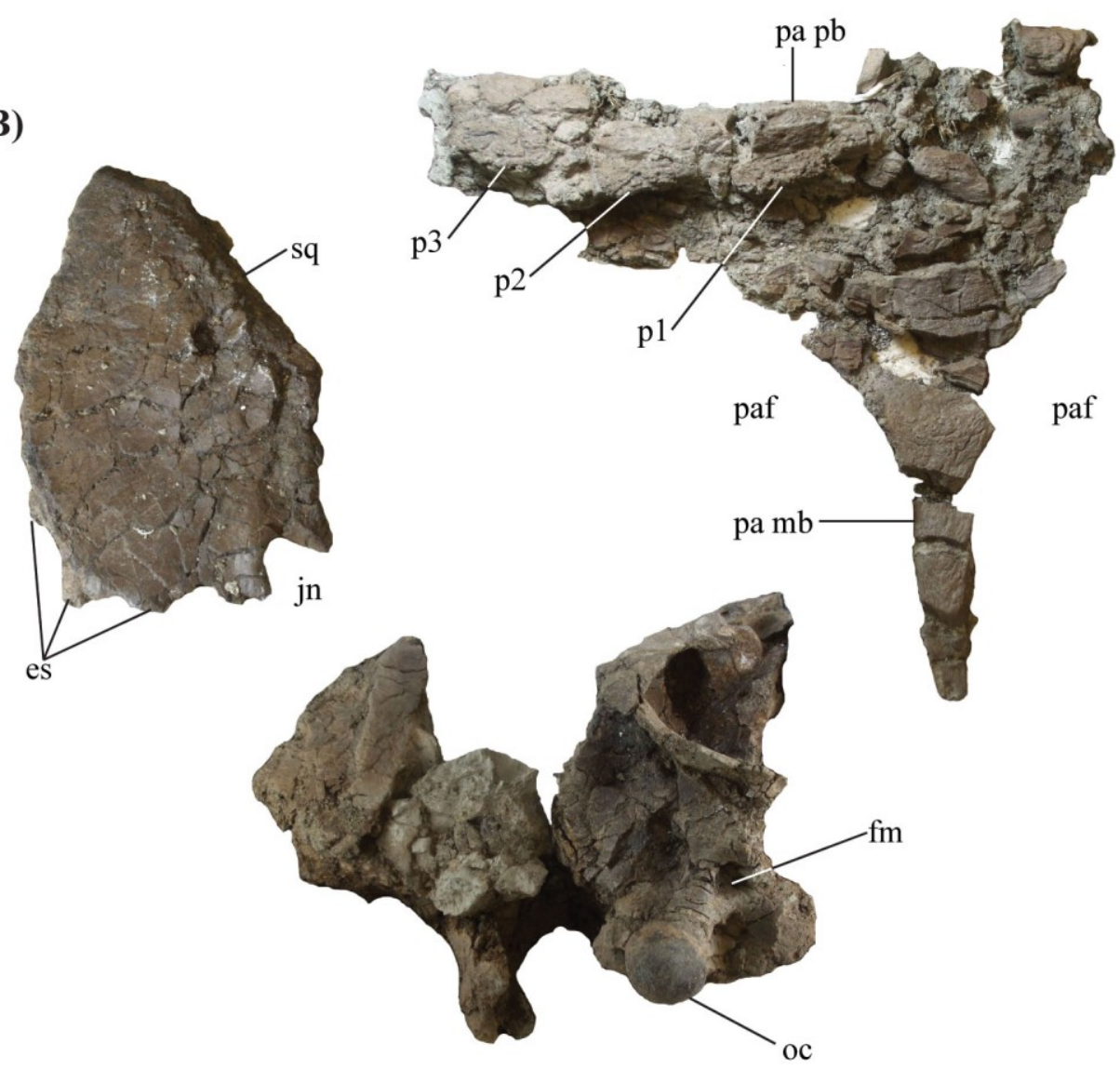


\subsubsection{New chasmosaurine material}

\subsubsection{CMN 34829}

CMN 34829 (Fig. 17) is a highly fragmented, partial skull that was collected by C.H. Sternberg in 1915, from CMN fossil site P-1532. The locality description is as follows: "near lower end of Deadlodge Canyon, on the east side of the Red Deer River, almost east of Cravath Corners Post Office" (Sternberg, 1915), placing the specimen approximately within the boundaries of Dinosaur Provincial Park. When discovered by L. H. Sternberg, the skull was "in a slide and the crest [was] in pieces" (Sternberg, 1915).

The skull includes the skull roof and braincase, the right circumorbital region, and the anterior portions of the parietal and both squamosals. Small, associated fragments of this skull are present with the specimen, but they cannot be reattached to the skull and appear to be non-diagnostic.

CMN 34829 was previously noted as an unprepared specimen in Godfrey and Holmes (1995), but it has recently (2011-2012) undergone re-preparation at the Canadian Museum of Nature, and is now available for a complete description. Of note, it was misidentified as field number 11, rather than field number 10, in Sternberg's records of the 1915 field season. Godfrey and Holmes (1995) also mention another skull (field number 10 , but actually 11) collected during this field season, but conclude that it was likely destroyed in a fire in the CMN Collection facility in the 1930's.

CMN 34829 is referred to Chasmosaurus sp. based, in part, on the supracranial sinuses walled mostly by the postorbitals, the presence of long triangular squamosals, a transversely narrow and strap-like parietal medial bar, a parietal (as measured from the posterior end of the medial bar) that does not extend far posteriorly beyond the 
squamosals, and anteroposteriorly elongate parietal fenestrae that differentiates it from Vagaceratops.

Circumorbital region Postorbital

Both postorbitals (Fig. 17A-H), with their associated orbital margins, are preserved. The right side of the skull is more complete and preserves the sutures for the palpebral and the jugal at the anterodorsal and posteroventral margins. The postorbital articulates with the squamosal posteriorly, although the suture is difficult to discern (as is typical for most adult-sized ceratopsids). The postorbitals form most of the lateral margins of the frontoparietal fontanelle on the skull roof, as is typical of chasmosaurines (Lehman, 1990). The postorbitals also form the anteromedial margins of the dorsal temporal fenestrae.

The supracranial sinus is present in all ceratopsids, and is bordered by the frontal, postorbital, parietal, laterosphenoid, supraoccipital, and exoccipital (Forster, 1996). The frontoparietal fontanelle, resulting from incomplete closure of the secondary roof over the sinus in most ceratopsids, is bordered mostly by the frontal (and postorbital to a lesser degree) in centrosaurines, and mostly by the postorbital (and frontal to a lesser degree) in chasmosaurines (Lehman, 1990; Farke, 2010). Ontogenetically, the frontal sinus begins as depression on the skull roof, a condition seen in some basal neoceratopsians such as Protoceratops and Zuniceratops (Farke, 2010).

The frontoparietal fontanelle is transversely expanded in some taxa (e.g., 
Figure 17. Skull of CMN 34829 (Chasmosaurus sp.) in A-B, dorsal; C-D, left lateral; EF, right lateral; G-H, anterior; and I-J, centro-dorsal views. Note large accessory fenestra on right side of parietal and large protuberance above left dorsal temporal fenestra. See "List of anatomical abbreviations". Scale bar equals $10 \mathrm{~cm}$. 
Figure 17.
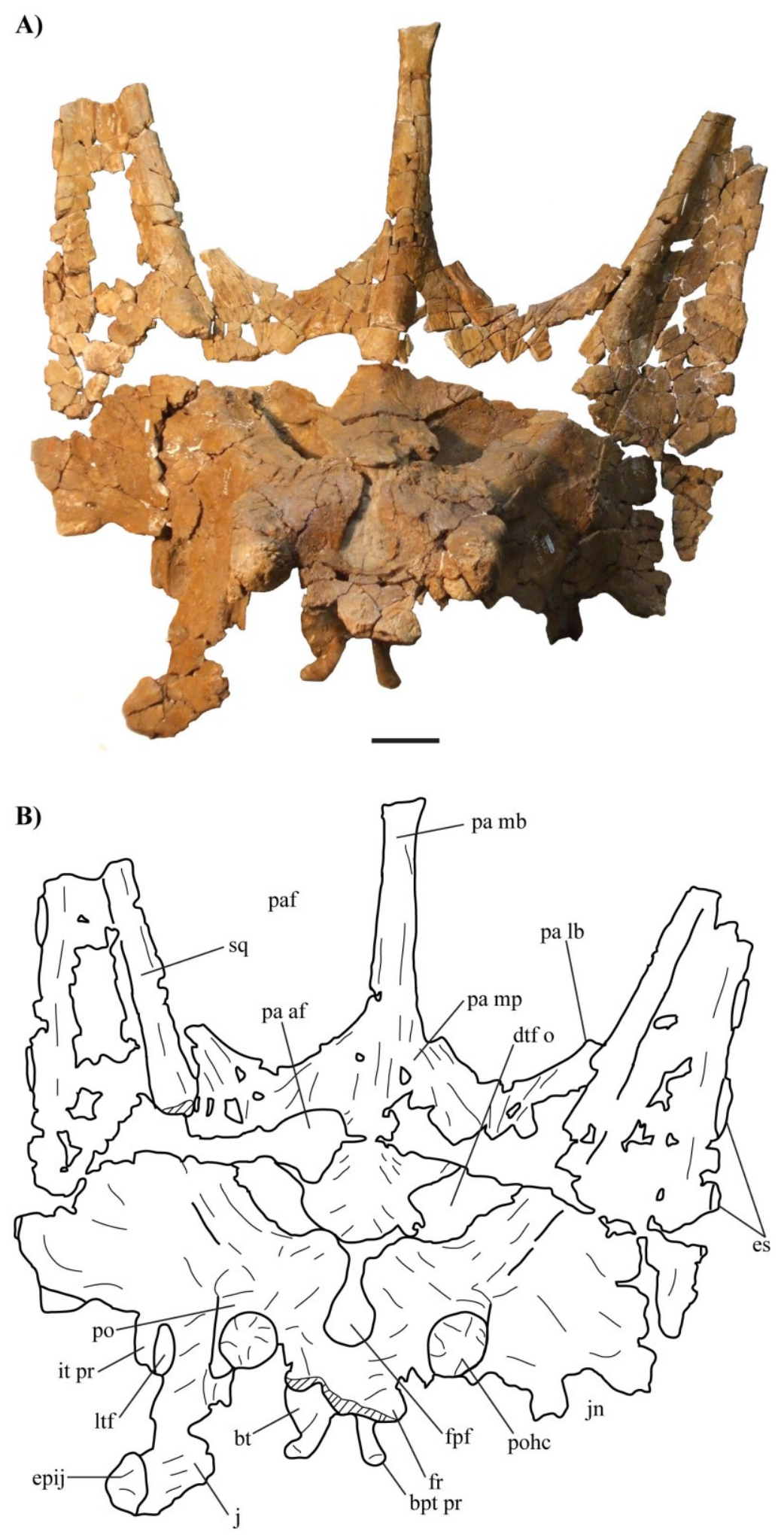
Figure 17. (continued).

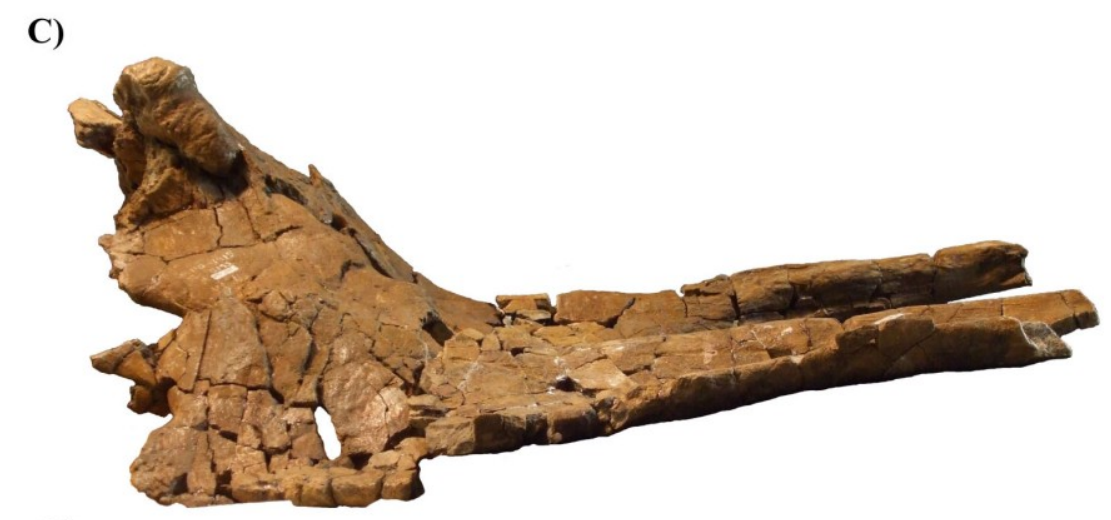

D)
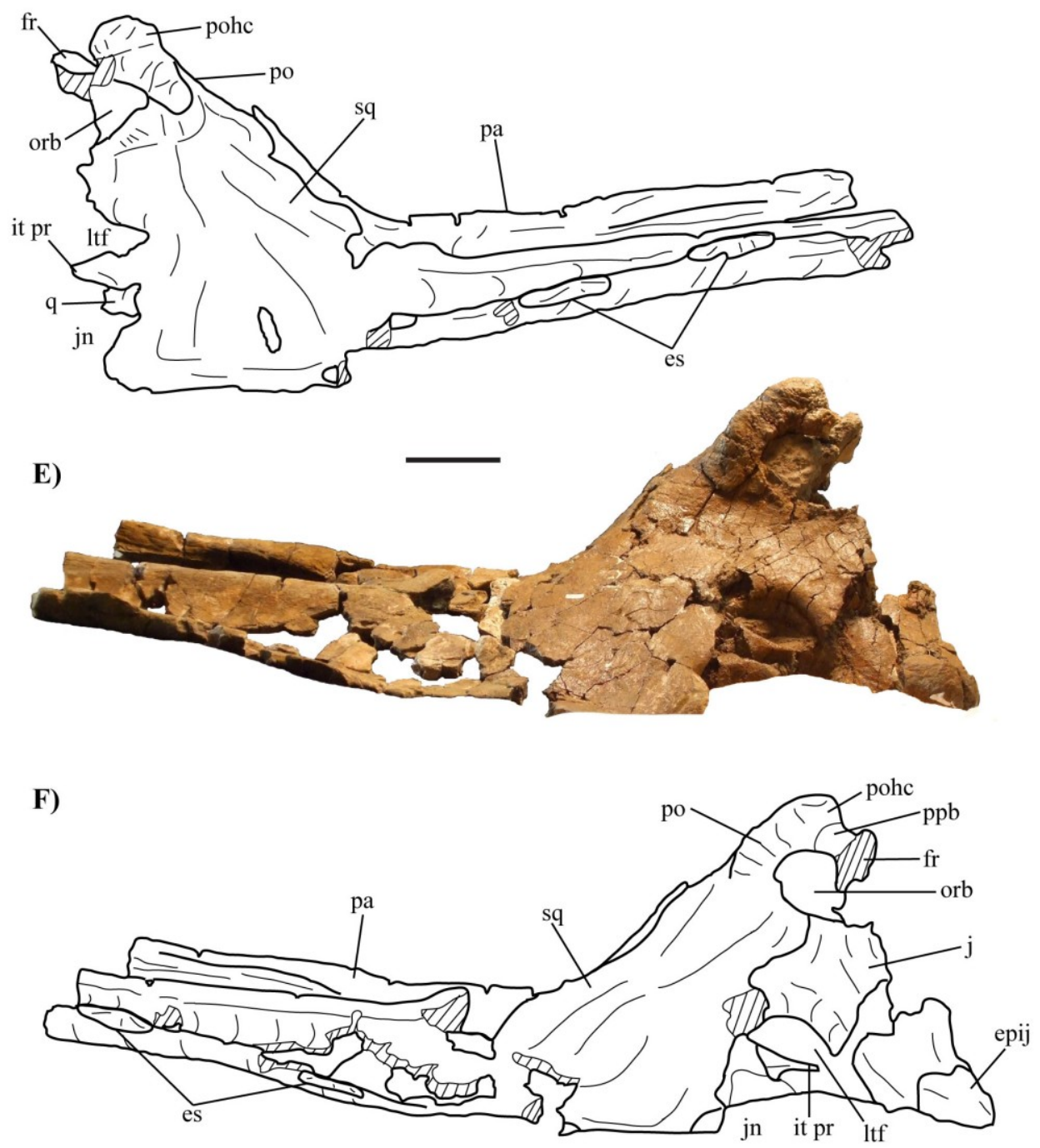
Figure 17. (continued).

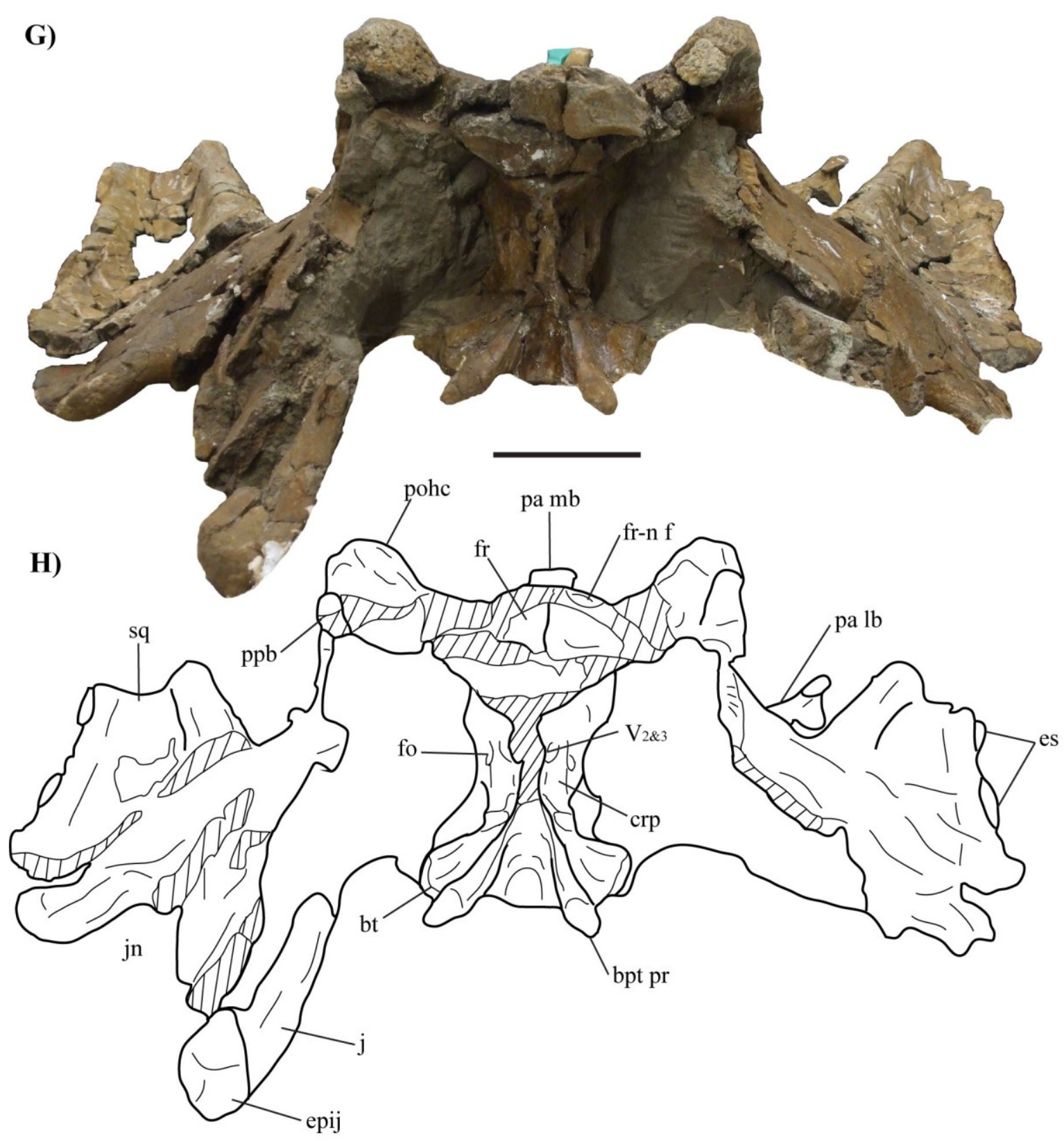


Figure 17. (continued).

I)
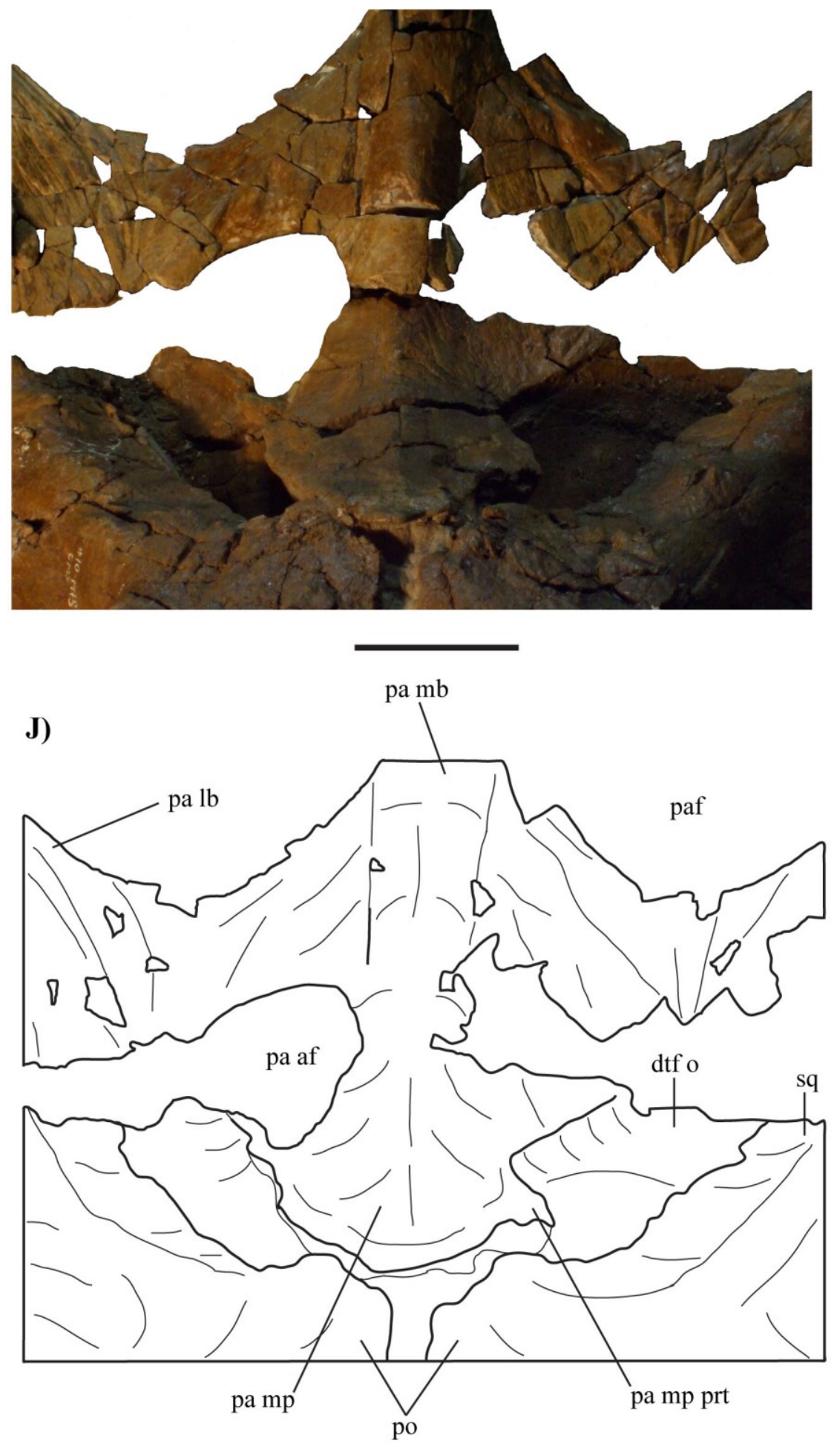
Chasmosaurus, Vagaceratops, and Agujaceratops), posteriorly-restricted and reduced in others (e.g., Anchiceratops), and completely roofed over in others (e.g., Arrhinoceratops, Kosmoceratops, Triceratops prorsus and some specimens of Torosaurus latus; Farke, 2010), although this latter condition is probably directly related to ontogeny. In CMN 34829 (Fig. 17A-B), the margin of the fontanelle is keyhole shaped, similar to Chasmosaurus (e.g., AMNH 5402, ROM 843) and Vagaceratops (e.g., TMP 1987.045.0001). In CMN 34829, the sinus is mostly infilled with matrix, making it difficult to determine the extent of the sinus. The postorbitals contact the anteromedial end of the parietal, in the region between the frontoparietal fontanelle and dorsal temporal fenestra.

The postorbital horncores (Fig. 17C-F) are positioned anterior to the dorsal midline point of the orbit. They are short, rounded, and oriented dorsally, with heavily vascularisation and small pits covering the dorsal surface. The pitted surfaces suggest that the horncores were originally longer and underwent remodelling prior to death. Their preserved height $(59 \mathrm{~mm}$ ) is within the range of Chasmosaurus (YPM $2016=41 \mathrm{~mm}$ to $440 \mathrm{~mm}=\mathrm{CMN} 9676)$, but shorter than the low-relief mounds in Vagaceratops (CMN $41357=36 \mathrm{~mm}$ to $44 \mathrm{~mm}=\mathrm{TMP} 1987.045 .0001)$.

Palpebral

The dorsal portion of the right palpebral is preserved. The suture with the postorbital is visible (Fig. 17E-F). It has a thickened, rugose surface like the postorbital.

Frontal

Both frontals are preserved (Fig. 17A-H), except for their lateral margins that 
would have articulated with the postorbitals. The posterior margin of the paired frontals form the anterior margin of the frontoparietal fontanelle, and articulate with the postorbitals, although the suture cannot be clearly seen. The midline suture between the frontals appears to be obliterated dorsally; however, this suture is visible ventrally (Fig. $17 \mathrm{G}-\mathrm{H})$. On the anterior margin of the left frontal, there is a cup-shaped facet where the left nasal would have articulated.

Jugal

The right jugal (Fig. 17A-B and E-H) is partially preserved, but its ventral region is crushed and displaced from the rest of the skull. Although the maxillae are not preserved, it is likely that the ventrally-directed jugal would have extended below the maxillary toothrow, as in all ceratopsids. The jugal forms most of the ventral margin of the orbit, although the element's anterior extent is missing. This element also forms the anterodorsal margin of the lateral temporal fenestrae. The suture between the jugal and squamosal is visible dorsal to the lateral temporal fenestra. A large, well-developed, laterally-oriented epijugal is articulated with the ventral end of the jugal. The epijugal is similar in size to those seen on most specimens of Chasmosaurus and Vagaceratops where it is preserved, but relatively small compared to larger chasmosaurines such as Pentaceratops.

Quadratojugal

The right quadratojugal (Fig. 17E-F) is either absent or crushed beyond recognition. The anteriorly-directed process on the right squamosal projects beyond the 
midpoint of the lateral temporal fenestra, suggesting that the quadratojugal was excluded from the fenestra.

Quadrate

Only the dorsal end of the crushed quadrate is preserved on the left side of the skull (Fig. 17C-D). Its contact with the anteroventral surface of the squamosal is obscured by matrix.

Braincase

The braincase is visible only in anterior view (Fig. 17G-H); the posterior and ventral sides are still inaccessibly embedded in matrix and plaster. The anteriormost portion of the braincase is not preserved. The basal tubera are present ventrally. The posteriorly positioned paroccipital processes are not visible, but are likely still encased within the matrix above the basal tubera. The occipital condyle and foramen magnum are not visible. Few ceratopsids have braincases from which any of the sutures are visible, and much of the description that follows is based on the published interpretations of material that I did not examine personally.

The fenestra ovalis is dorsal and anterior to the visible portion of the basal tubera is on the lateral surface. The fenestra ovalis is bordered anteriorly by the exoccipital, posteriorly by the sphenoid, and ventrally by the basisphenoid (e.g., Triceratops, Hatcher et al., 1907). Anterior to the fenestra ovalis is the trigeminal foramen (V2\&3), which transmits the maxillary (V2) and mandibular (V3) parts of the trigeminal nerve (Maidment and Barrett, 2011). In Triceratops, the trigeminal foramen perforates the 
prootica (Forster, 1990). The crista prootica forms a thin, vertically-oriented ridge that separates the fenestra ovalis and trigeminal foramen (Forster, 1996). Anterior to these foramina, the braincase becomes transversely compressed. Anterior to the basal tubera, the basipterygoid processes are present, and project anteroventrally and gently laterally from the basisphenoid. These processes originate dorsally as rounded, thickened ridges which descend ventrally and protrude downwards. The distal ends of these processes taper in width and are rounded, where they would articulate with the pterygoid.

Frill

Squamosal

Both squamosals (Fig. 17A-F) are mostly preserved, except for the distal margins. The squamosals are long and triangular, as is typical of Chasmosaurus, with an estimated total length for both of $850 \mathrm{~mm}$. In Chasmosaurus, squamosals range up to $997 \mathrm{~mm}$ (CMN 8800), while the few known for Vagaceratops do not exceed $700 \mathrm{~mm}$ (CMN 41357) (Table 1). The squamosals have a concave dorsal surface, which becomes more pronounced posteriorly. The squamosal articulates with the postorbital anteriorly. The anteromedial margin forms the lateral margins of the dorsal temporal fenestrae, while the rest of the medial margin preserves a shallow and narrow facet for the lateral margin of the lateral parietal bar; however, the continuity of this bar cannot be determined.

There are five preserved episquamosals on the left squamosal, and four on the right. Given the amount missing from each squamosal, and the spacing frequency of the preserved episquamosals, I estimate that each element probably had either six or seven episquamosals per side. The episquamosals are anteroposteriorly wide-based, low, and 
roughly equal in size except for the anteriormost one, which is slightly longer with a more pronounced triangular shape. The squamosal-episquamosal sutures become increasingly more obvious posteriorly, supporting the pattern of anterior-to-posterior direction of articulation that has been postulated for chasmosaurines (Sampson et al., 1997).

Parietal

More than half of the parietal is preserved (Fig. 17A-F), missing the posterior and most of the lateral bars. The parietal extends anteromedially to form the posterior margin of the frontoparietal fontanelle, laterally to form the medial margins and floor of the upper temporal fenestrae, and posteriorly to form the anterior margins of the parietal fenestrae. The medial platform of the parietal extends posterolaterally forming the narrow lateral bars which form the lateral margins of the parietal fenestrae and contact the squamosals medially. There are several vascular grooves that radiate posteriorly towards the parietal fenestrae. Only a portion of the left lateral bar is preserved, in articulation with the ventromedial facet of the squamosal. The medial platform has a wide, dorsallythickened crest that transitions into the medial bar posteriorly.

The medial bar (Fig. 17C-F) is very robust, ranging in dorsoventral thickness from approximately $66 \mathrm{~mm}$ near the anterior end of the parietal fenestrae to approximately $40 \mathrm{~mm}$ at its distal-most preserved end. The posterior end of the preserved medial bar (Fig. 17A-B) is slightly expanded suggesting that it is near its posterior terminus. The medial bar is nearly square in cross-section at this point. The lateral sides of the medial bar have a wide, longitudinal groove that is mediolaterally shallow on the 
left side, but deep on the right side (approximately $15 \mathrm{~mm}$ at the deepest point).

The parietal fenestrae are estimated to have been at least $410 \mathrm{~mm}$ in anteroposterior length. This length is consistent with the range seen in Chasmosaurus (354 mm on AMNH 5402 to $627 \mathrm{~mm}$ on NHMUK R4948), but longer that those reported for Vagaceratops (260 mm on TMP 1987.045.0001 to $265 \mathrm{~mm}$ on CMN 41357).

There is a relatively large accessory parietal fenestra (Fig. 17A-B and I-J) between the parietal and dorsal temporal fenestra on the right side of the parietal. It is nearly circular in shape with a smooth, rounded margin, measuring approximately 101 $\mathrm{mm}$ long and $82 \mathrm{~mm}$ wide. No previously-described specimen of Chasmosaurus or Vagaceratops possess this fenestra, although they are known for other chasmosaurines, e.g., Nedoceratops (Tanke and Farke, 2007) and Anchiceratops (UW 2419 and AMNH 5273; Mallon et al., 2011).

CMN 34829 has a large protuberance on the medial platform (Fig. 17A-B and I$\mathrm{J})$, which projects almost halfway across the left upper temporal fenestra. This type of feature is unknown in Chasmosaurus or Vagaceratops; however, irregular bone growth is known in Chasmosaurus, e.g., the accessory bridge of bone over the frontoparietal fontanelle in CMN 8800 (Fig. 7G) and YPM 2016 (Fig. 7R).

\subsubsection{TMP 2011.053.0046}

A disarticulated partial skull (TMP 2011.053.0046) (Figs. 18-21) was collected by the Southern Alberta Dinosaur Project during the 2011 field season in the Milk River region, approximately $14.5 \mathrm{~km}$ southeast of the village of Manyberries (UTM $12 \mathrm{U}$ 0534562, 5463885). It was collected approximately $2 \mathrm{~m}$ below the Lethbridge Coal Zone 
(973 metres above sea level in the uppermost Dinosaur Park Formation). The specimen consists of most of the left squamosal, fragments of the parietal (anterior end of the midline bar, part of a lateral bar, and the left posterolateral corner of posterior bar), the left quadrate, most of the nasal ornamentation, and a fragment which may represent the margin of an accessory fenestra in the left squamosal. TMP 2011.053.0046 was prepared at the Royal Ontario Museum.

Snout

Nasal

Parts of the articulated nasals (Fig. 18A-F), including the nasal ornamentation, are preserved, but represented mostly by the right side. The anterior process of the nasals is not preserved, but would normally articulate with the dorsal process of the premaxillae. The ventral surface of the articulated nasals form the dorsal margin of the nares. The descending lateral flanges of the nasals are not preserved. The nasals (Fig. 18E-F) extend posteriorly where they would articulate with the frontals; however, the articulating facets for the frontals are not preserved. The base of a large horncore is centred dorsally and posterior to the midpoint of the nares, as in both Chasmosaurus and Vagaceratops. The rostral and upper half of the horncore is missing, so the original height cannot be determined. It has an estimated basal length of at least $120 \mathrm{~mm}$. The horncore is covered with vascular grooves converging towards the apex. No epinasal can be discerned, but this may be due to the lack of preservation; it is also possible that part of an epinasal is preserved, but its sutures with the underlying nasals have become obliterated. 
A)

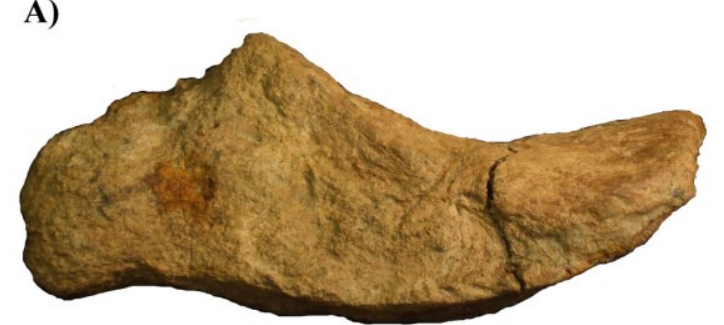

C)

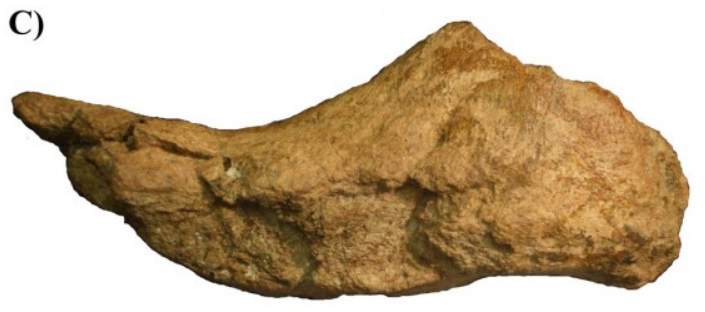

E)

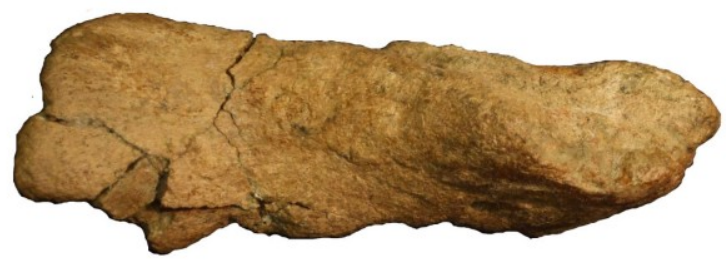

B)

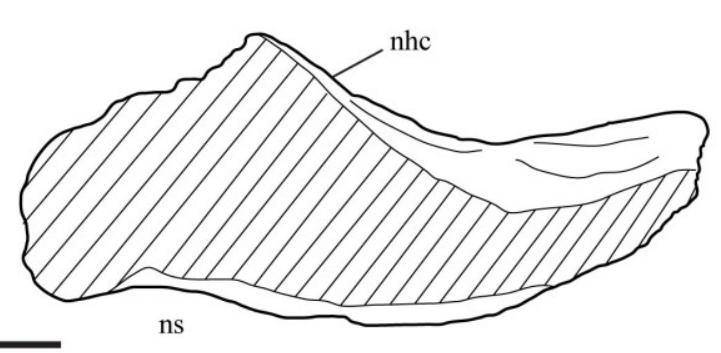

D)

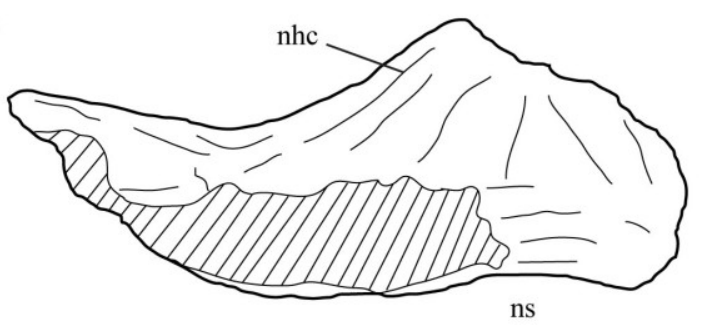

F)

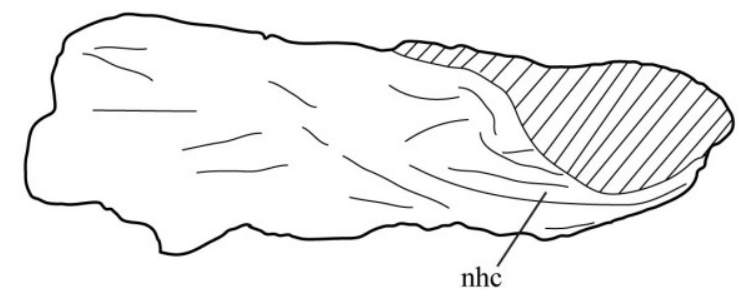

Figure 18. Nasal of TMP 2011.053.0046 (Vagaceratops sp.) in A-B, left lateral, C-D, right lateral, and E-F, dorsal views. See "List of anatomical abbreviations". Scale bar equals $10 \mathrm{~cm}$. 


\section{Cheek region}

Quadrate

The preserved left quadrate (Fig. 19A-D) is missing most of the palatine flange, but is otherwise complete. It is anteroposteriorly flattened, roughly rectangular in outline, and is slightly wider on the dorsal end compared to the ventral end. The dorsal end would originally have had a sub-rectangular outline and would have been more medially extensive in lateral view than is preserved, as suggested by the chipped medial margin (palatine flange) of the element. Below this broken margin, there is a well-developed pterygoid process (Fig. 19A-D). There is a small facet for the pterygoid immediately above this process. This facet is defined by a ridge that lines the medial margin and continues onto the pterygoid process. On the lateral margin, adjacent to the pterygoid process, there is an elongate facet for the quadratojugal. Superior to this facet, there is a small rugose area which represents the articulating facet for the squamosal. Posterior to the squamosal facet, there is a facet for the paroccipital process (Fig. 19C-D). The rounded ventral end of the element would have articulated with the glenoid fossa on the lower jaw. 

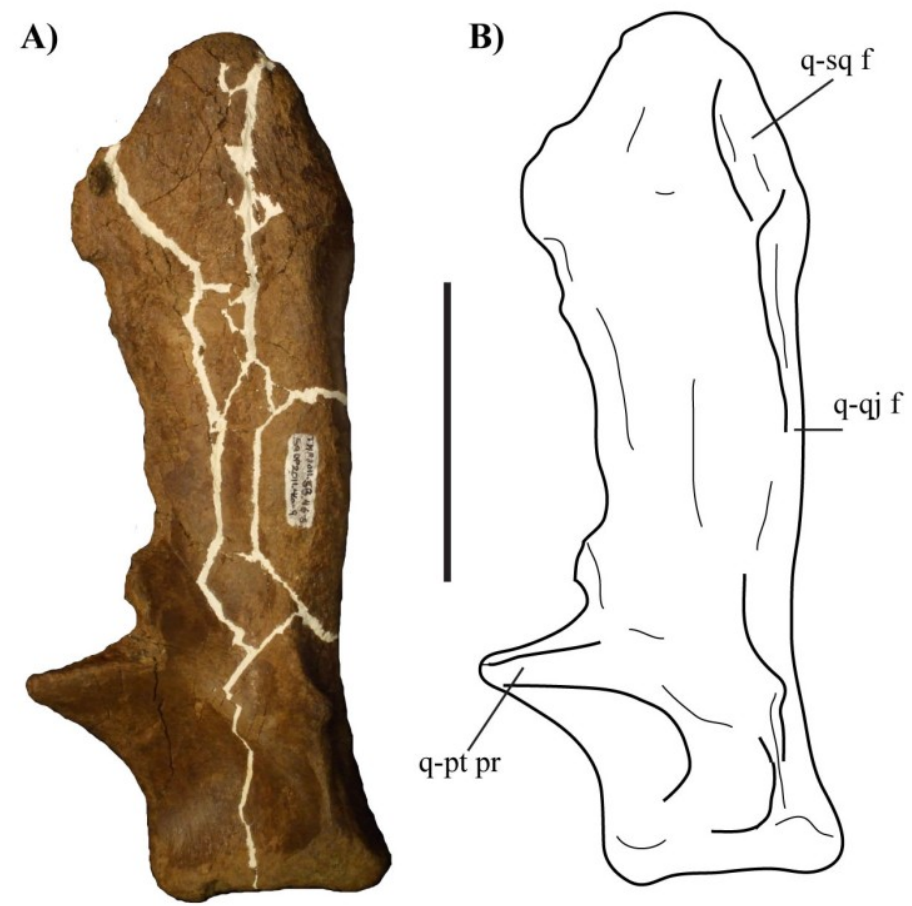

C)

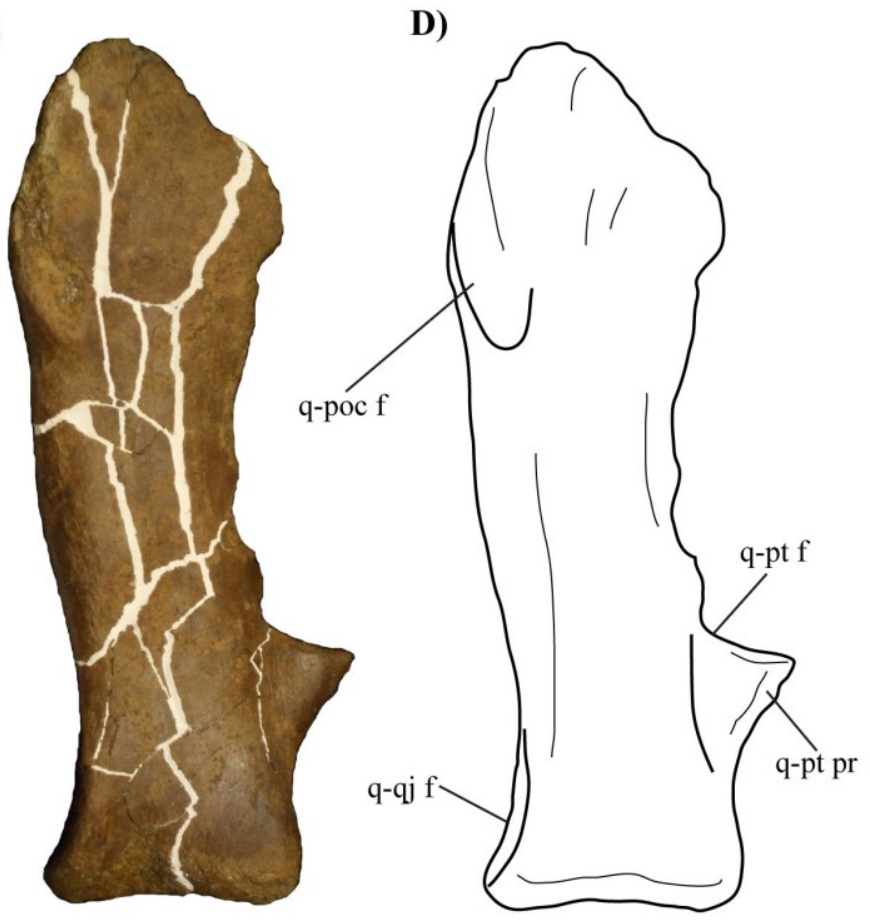

Figure 19. Left quadrate of TMP 2011.053.0046 (Vagaceratops sp.) in A-B, anterior and C-D, posterior views. See "List of anatomical abbreviations". Scale bar equals $10 \mathrm{~cm}$. 
Frill

\section{Squamosal}

The anterior half of the long, left squamosal is preserved (Fig. 20A-D). The concavity of the dorsal surface increases posteriorly. There are two equal-sized, wellarticulated episquamosals on the lateral margin (Fig. 20A-B). The anterior end of the squamosal forms the posterior margin of the jugal notch. Anteromedially to this margin, the squamosal infratemporal process extends towards the jugal and separates the jugal notch from the lateral temporal fenestra. Only the posteroventral margin of the lateral temporal fenestra is preserved. On the ventral side of the squamosal, an elongate facet to receive the quadrate is present immediately above the jugal notch (Fig. 20C-D). There is a narrow and shallow facet where the lateral parietal bar would attach on the ventromedial margin.

At least two other fragments of a squamosal are preserved. The first (Fig. 20E-H) represents part of the lateral margin of the squamosal with one articulated episquamosal and a locus for a second one adjacent to it. The unadorned neighbouring episquamosal locus suggests that an episquamosal would have occupied this site, but had not articulated with the squamosal at the time of death. The dorsal surface is rugose and vascularised (Fig. 20E-F), while the ventral side (Fig. 20G-H) is smooth.

The second fragment (Fig. 20I-L) is a flattened and curved, with a well-defined inner, and a less well-defined, outer margin. The outer margin is adorned by what appears to be an episquamosal. The inner margin may represent the edge of an accessory squamosal fenestra. 
A)

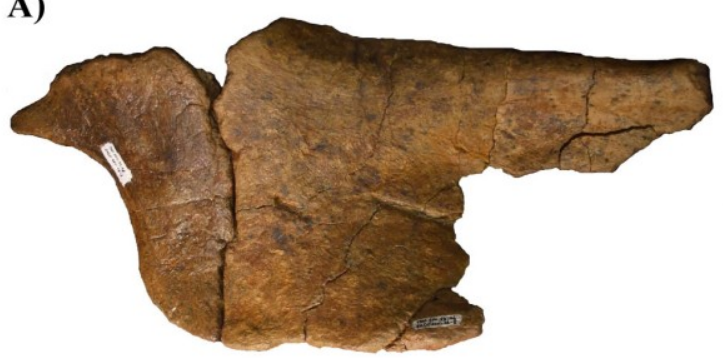

C)

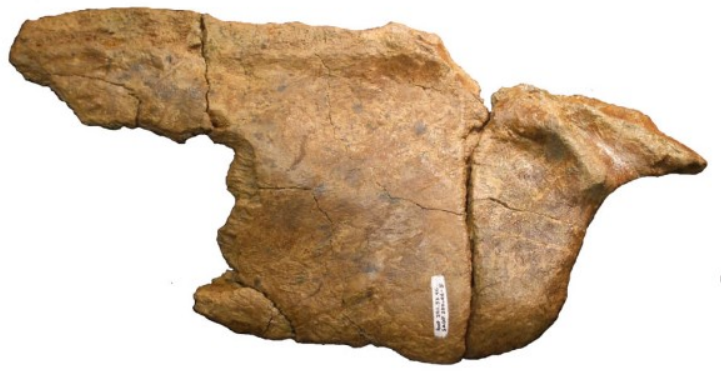

E)

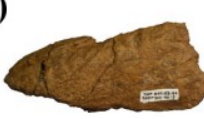

I)

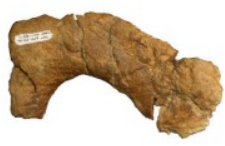

F)

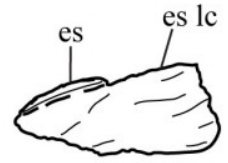

J)

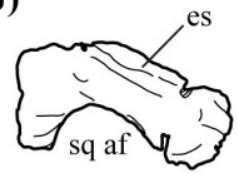

B)
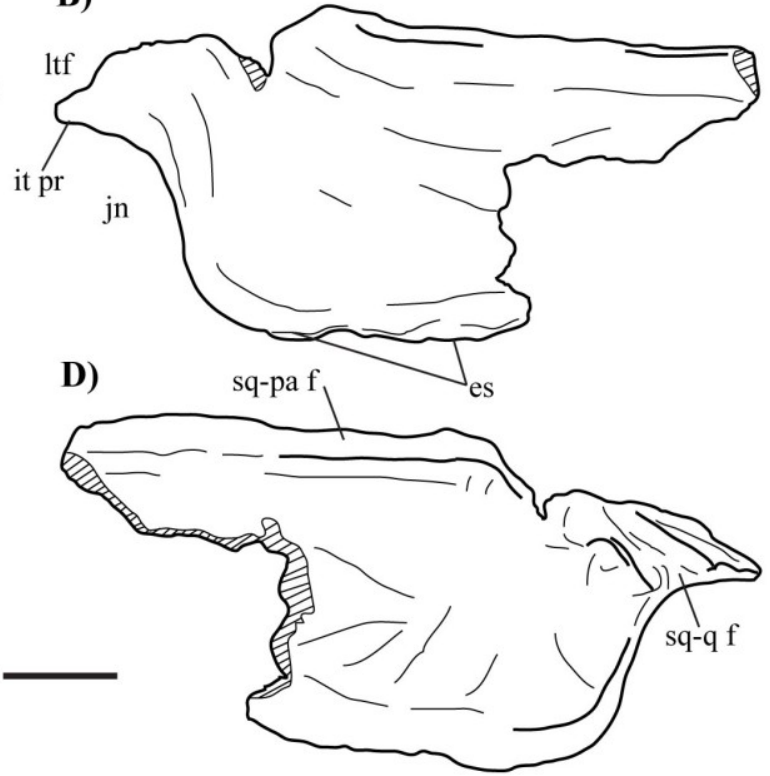

G)

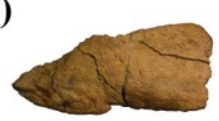

H)

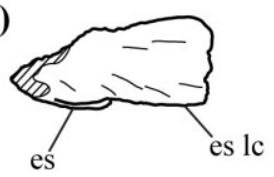

K)

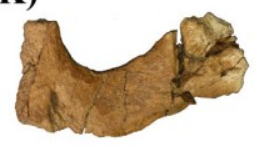

L)

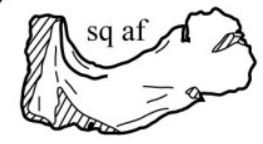

Figure 20. Left squamosal of TMP 2011.053.0046 (Vagaceratops sp.) in A-B, dorsal and C-D, ventral views. Second squamosal fragment in $\mathbf{E - F}$, dorsal and $\mathbf{G}-\mathbf{H}$, ventral views. Third squamosal fragment in I-J, dorsal and K-L, ventral views. See "List of anatomical abbreviations". Scale bar equals $10 \mathrm{~cm}$. 


\section{Parietal}

The parietal (Fig. 21) is represented by at least three fragments; the left posterolateral corner of the posterior bar, a short segment of the lateral bar (side unknown) and the anterior end of the medial bar and platform.

The posterior bar segment (Fig. 21A-H) preserves an anterodorsally-recurving margin and is adorned by a short, broad-based epiparietal. The suture is obliterated on the anterior side, but can be faintly discerned on the posterior side. Anterior to the anterodorsally-recurving margin of the parietal is a wide, relatively thin flange that defines the posterolateral margin of the parietal fenestra. Lateral to the epiparietal, on the posterior margin, there is a smooth, well-defined, bulbous mass which is broken anteriorly. The mass is situated on the posterolateral corner of the parietal, and the posterior margin of the mass is oriented posterolaterally (Fig. 21C-D and G-H). This mass likely represents the base of a second, well-articulated epiparietal. Based on its position and morphology, this second epiparietal would have been oriented posterolaterally. Laterally adjacent to this second epiparietal is the posterior end of the lateral parietal bar.

On the lateral margin of the lateral bar is the rugose facet for the distal end of the squamosal (Fig. 21E-H), suggesting that the squamosal extended approximately as far posteriorly as the posterior margin of the frill. The anterodorsally recurving posterior margin of the parietal is situated adjacent to the angled margin separating it from the lateral parietal bar, similar to that seen in Vagaceratops (CMN 41357, TMP 1987.045.0001 and TMP 1998.102.0008). In Chasmosaurus, epiparietals situated adjacent to the parietal-squamosal contact are oriented in, or close to, the plane of the 
A)

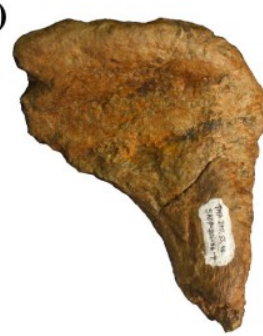

B)

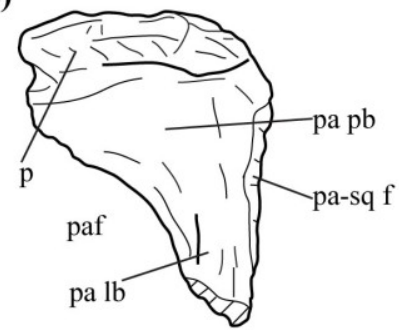

I)

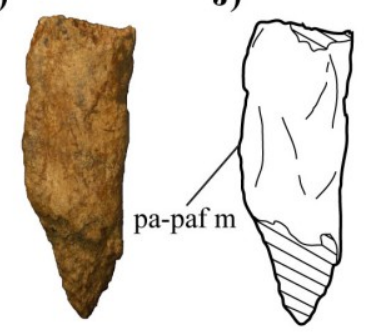

C)

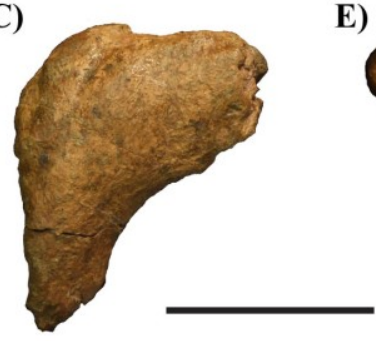

D)

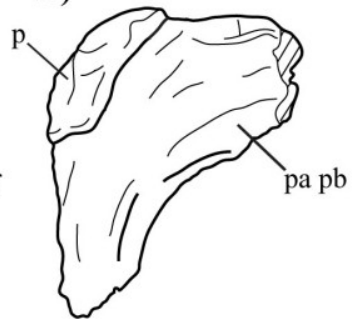

K)

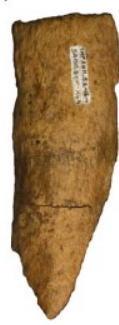

E)

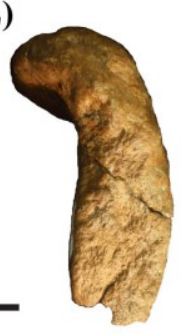

F)

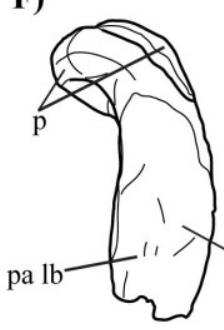

L)

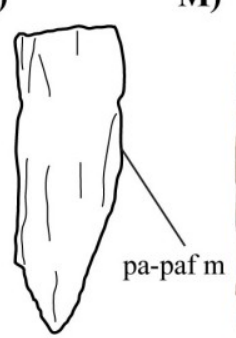

M)
G)

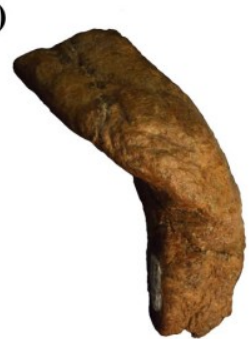

H)
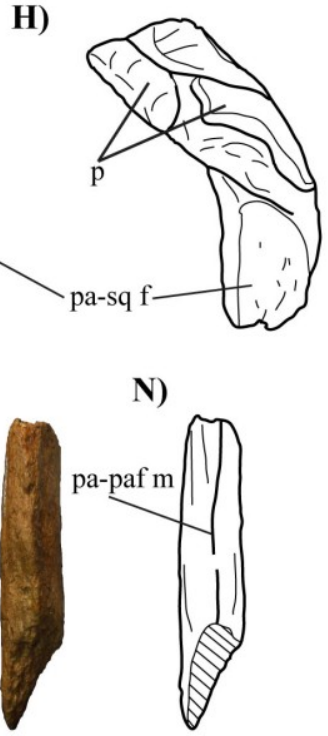

O)

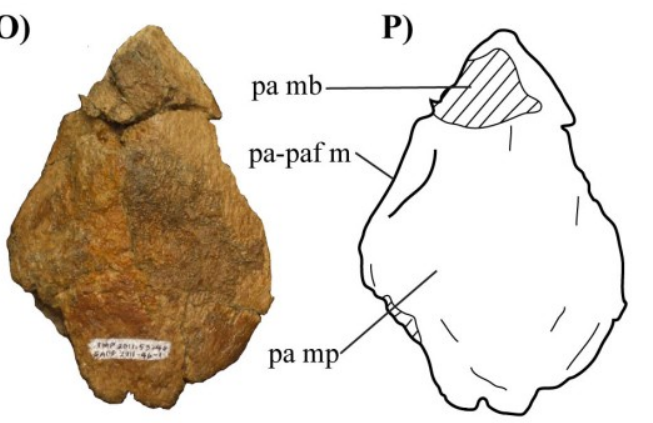

Q)

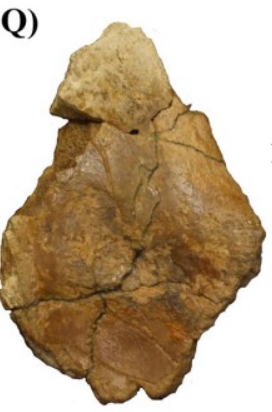

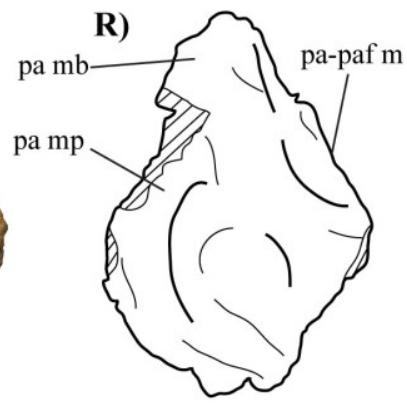

Figure 21. Parietal of TMP 2011.053.0046 (Vagaceratops sp.). Posterior parietal bar in A-B, dorsal; C-D, ventral; E-F, lateral; and G-H, posterolateral views. Lateral parietal bar in I-J, dorsal; K-L, ventral; and M-N, medial views. Medial parietal platform in $\mathbf{O}-\mathbf{P}$, dorsal; and Q-R, ventral views. See "List of anatomical abbreviations". Scale bar equals $10 \mathrm{~cm}$. 
frill. The anterodorsally-recurving epiparietal on the preserved medial end of the specimen is relatively short.

The lateral bar segment (Fig. 21I-N) is elongate and approximately oval in crosssection. The rugose surface (Fig. 21I-J) likely represents the dorsal sutural surface for the squamosal, while the opposite ventral side is smoother (Fig. 21K-L). The element tapers from a rounded margin towards what probably represents the lateral margin of the parietal fenestra (Fig. 21M-N). Due to poor preservation, it cannot be determined whether the lateral parietal bar was continuous around the margin of the parietal fenestra.

The medial fragment (Fig. 21O-R) represents the anterior end of the medial bar and part of the medial platform. The dorsal surface is rugose and slightly convex (Fig. 21O-P), while the ventral side is smoother and slightly concave (Fig. 21Q-R). The right posterolateral margin represents the anteromedial margin of the parietal fenestra. The element thickens posteriorly to form the anterior base of the rod-like median bar.

TMP 2011.053.0046 is diagnosed as chasmosaurine based on its inferred long triangular squamosals and anteroposteriorly narrow and strap-like parietal posterior bar. This skull was collected within $2 \mathrm{~m}$ of the Lethbridge Coal Zone, placing it within the same stratigraphic interval as the C. russelli holotype (CMN 8800) and V. irvinensis. The presence of an anterodorsally-recurved posterior parietal margin in TMP 2011.053.0046 is also present in mature specimens of Chasmosaurus and Vagaceratops. However, the anterodorsally-recurved ridge in TMP 2011.053.0046 is situated directly adjacent to the parietosquamosal contact, as in Vagaceratops (e.g., TMP 1998.102.0008). The length-towidth ratio of the parietal fenestrae, which would assist in assigning the specimen to either Vagaceratops or Chasmosaurus, cannot be determined due to incomplete 
preservation. However, based on the above observations, TMP 2011.053.0046 is

tentatively referred to Vagaceratops sp.

\subsubsection{Redescription of CMN 8802}

CMN 8802 (Figs. 22-32) was collected by C.M. Sternberg in 1937 south of Manyberries, Alberta, although there is some uncertainty as to the specimen's precise collection locality. Sternberg (1940:478) stated that he had collected the skull "near the same horizon as [CMN] 8800 in the NE 1/4, Sec. 10, T. 2, R. 5, W of 4th Meridian, or about $13 \frac{1}{2}$ miles south and 31/4 miles east of Manyberries". However, according to the CMN collections database, CMN 8802 is listed as having been collected at site "P-3708, Leg. subdiv. 14, in N.W. 1/4 of Sec. 10, Twp. 3, R. 5, W. of 4th Meridian, on south side of west branch of creek that runs past Joe Gilchrist's Ranch House, approximately 10 feet below prairie (marker \# 118 in quarry)". These two sites are separated by approximately $10 \mathrm{~km}$. The latter locality is, however, thought to be the correct one (David Evans, pers. comm.). This specimen is also thought to have been collected from the top of the Oldman Formation, immediately below the contact with the Lethbridge Coal Zone (David Evans, pers. comm.).

CMN 8802 was previously assigned by Sternberg (1940) as a paratype of $C$. russelli. Not long after this, the specimen was severely damaged during a museum move in 1941, and reported destroyed, except for the lower jaws (Godfrey and Holmes, 1995). However, this specimen was rediscovered in the CMN collections by Michael Ryan in 2011 as a skull that had been renumbered as CMN 8798, a pre-existing Centrosaurus apertus skull (Brown et al., 2009). Although fragmented, far more of the skull survived 
the incident than was previously reported.

CMN 8802 consists of both lower jaws, part of the rostral, most of the premaxillae, postorbitals, the jugal-quadratojugal-quadrate complex, braincase, and the anterior half of the parietosquamosal frill. Additional small fragments of this skull were also found, but most are non-diagnostic and cannot be reattached to the rest of the skull. CMN 8802 was briefly described by Sternberg (1940), but it was not figured.

Snout

Rostral

Only the ventral processes of the pitted rostral are preserved in CMN 8802 (Fig. $22 \mathrm{~A}-\mathrm{F}$ ), and articulated with the anteroventral surface of the premaxillae. The elongate process is typical of chasmosaurines. The ventral margin of ventral process is gently concave and would have originally terminated in a gently downturned beak.

\section{Premaxilla}

Both premaxillae are preserved and almost complete (Fig. 22A-F). These elements are tightly adpressed along their midline, flare posteriorly, and form the anterior and ventral margins of the nares. On the dorsal surface of these elements, the broken base of the transversely compressed premaxillary septum is preserved. The posterior end of this septum is thickened (Fig. 22E-F), and probably represents the base of a narial strut, which would have extended up to the dorsal margin of the premaxillae. The ventral margin (Fig. 22A-B) forms an articulation facet for the maxillae posteriorly. The flattened facets for the nasals are preserved on the dorsal margin of the posterior 
Figure 22. Premaxillae and rostral of CMN 8802 (Chasmosaurus sp.) in A-B, left lateral, C-D, right lateral, and E-F, dorsal views. See "List of anatomical abbreviations". Scale bar equals $10 \mathrm{~cm}$. 
Figure 22.
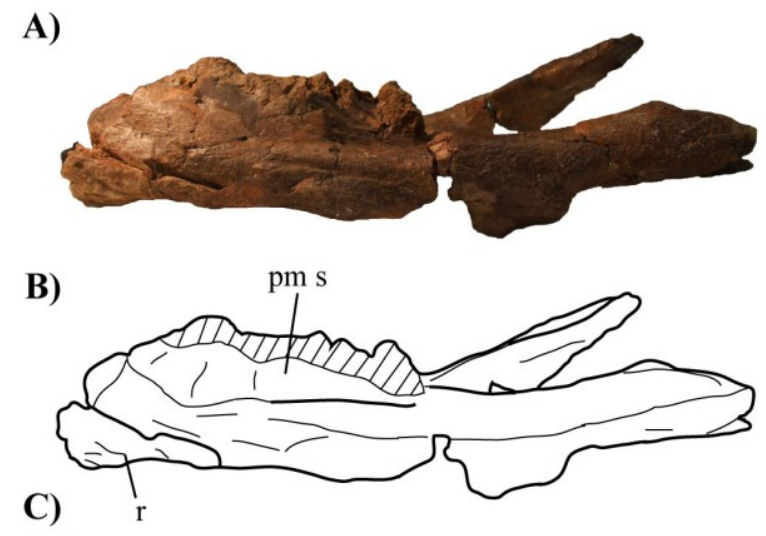

D)
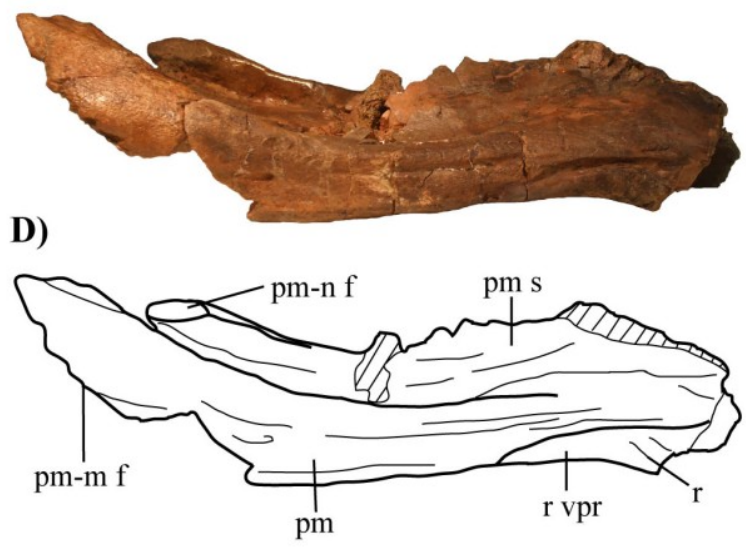

E)

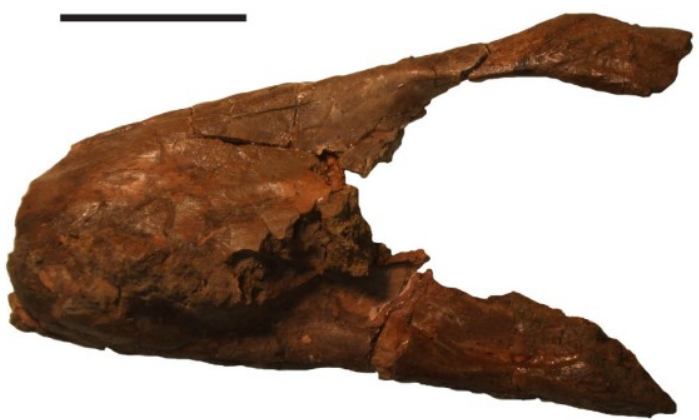

F)

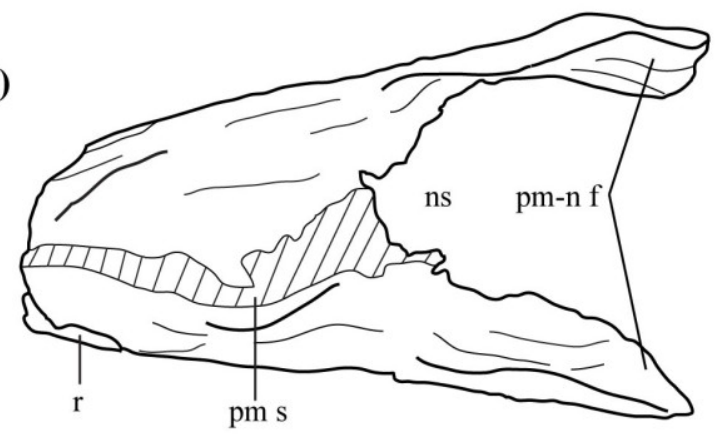


processes of the premaxillae (Fig. 22A-B and E-F).

The snout is insufficiently complete to ascertain its length-to-height ratio, and it is difficult to say whether it had a relatively low and elongate, chasmosaurine-like snout or if it was relatively high and abbreviated and centrosaurine-like (Maidment and Barrett, 2011).

Circumorbital and cheek regions Postorbital

Both postorbitals are present (Figs. 23-24), differing in their degree of preservation. The preserved portions adhere to typical chasmosaurine morphology and display the usual contacts for the other circumorbital elements. The more complete left horncore (Fig. 23A-D) is short (approximately $71 \mathrm{~mm}$ in length), with a rounded apex. It has a vascularised, rugose surface. Medial and ventral to this horncore is the supracranial sinus (Fig. 23C-D), which forms an excavation into the postorbital.

The more complete right postorbital (Fig. 24A-D) preserves most of the posterior half of the orbital margin, and articulating facet for the jugal and squamosal. Although incomplete, the height of the orbit is approximately $100 \mathrm{~mm}$. On the dorsal surface of the right postorbital, the bone thickens to form the posterior half of the postorbital horncore. 
A)

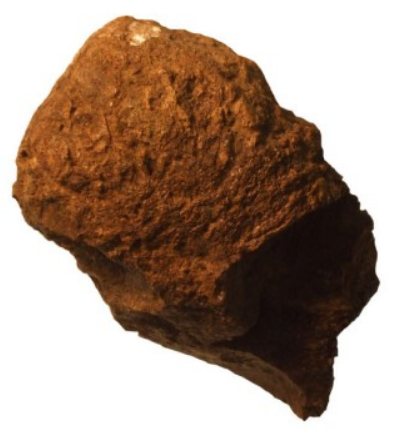

B)

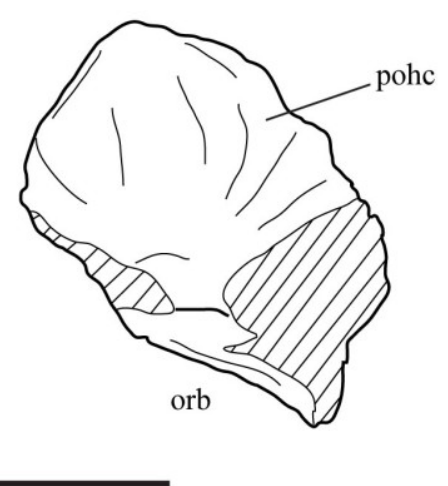

C)

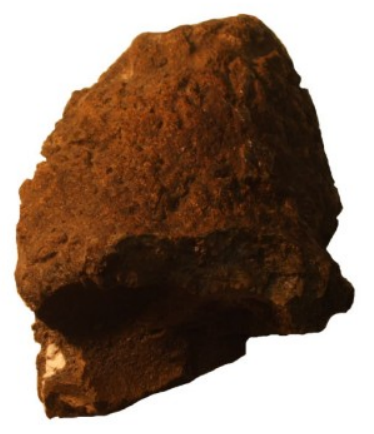

D)

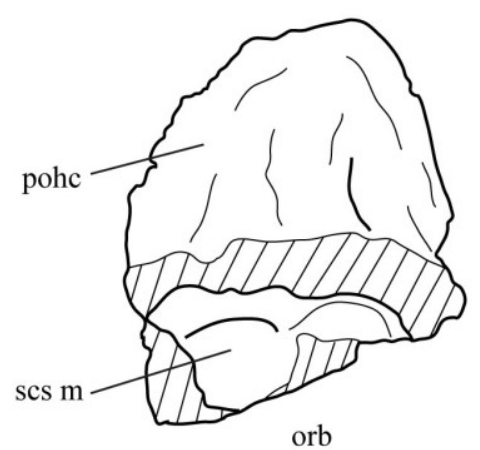

Figure 23. Left postorbital of CMN 8802 (Chasmosaurus sp.). A-B, lateral; and C-D, medial views. See "List of anatomical abbreviations". Scale bar equals $10 \mathrm{~cm}$. 
A)
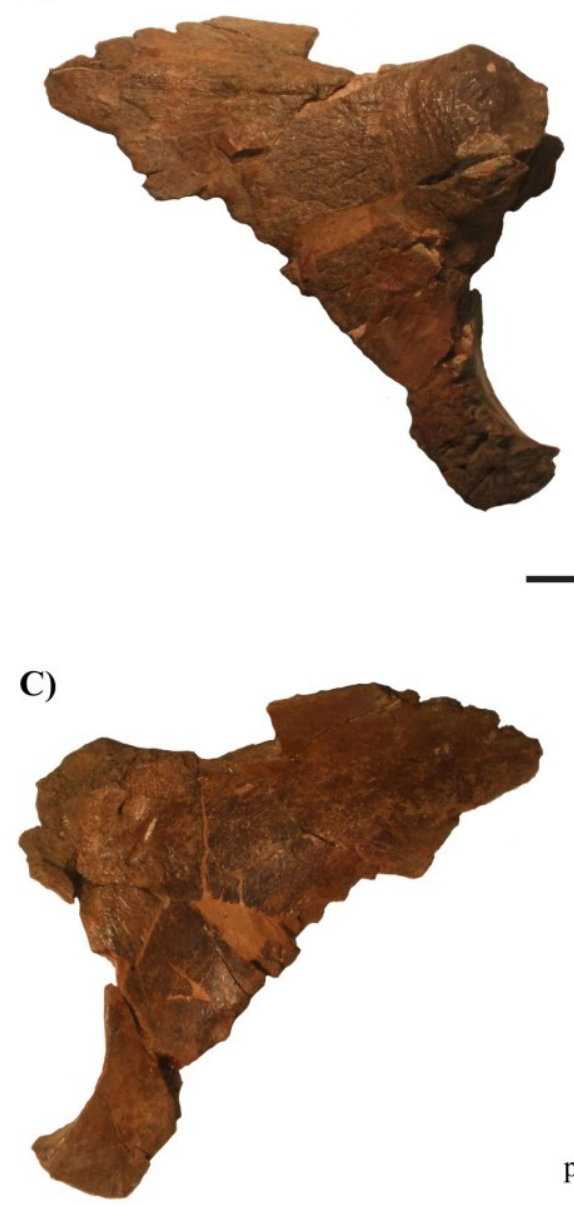

B)
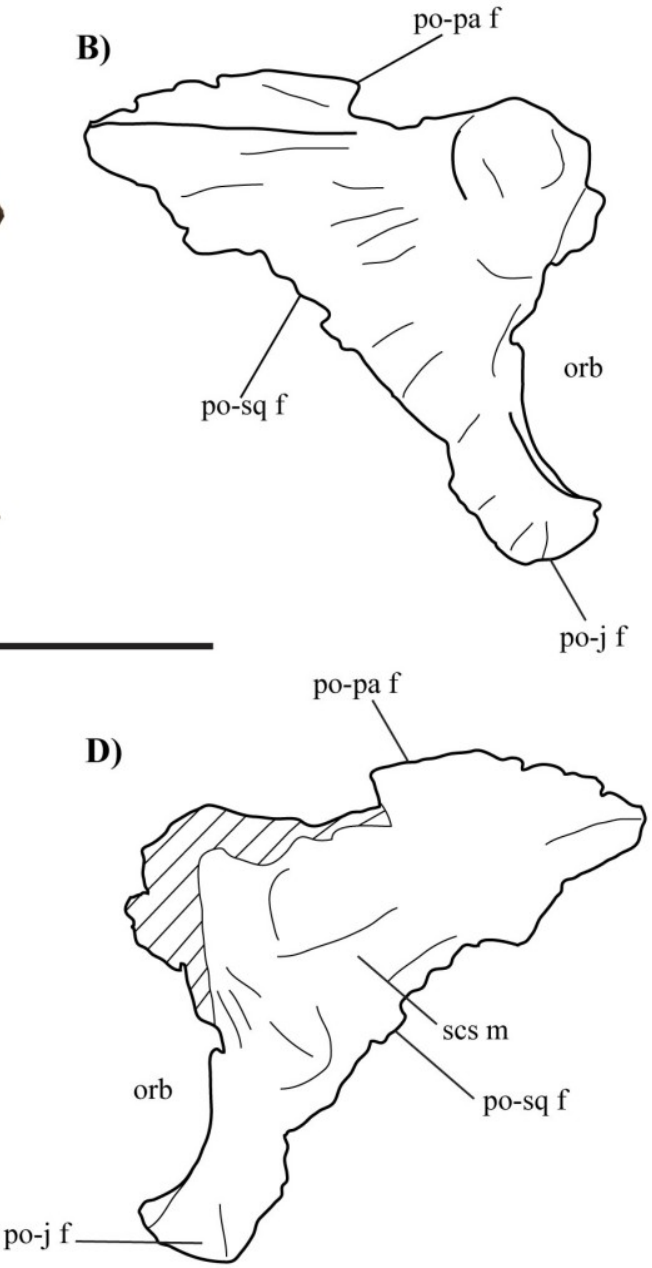

Figure 24. Right postorbital of CMN 8802 (Chasmosaurus sp.). E-F, lateral; and G-H, medial views. See "List of anatomical abbreviations". Scale bar equals $10 \mathrm{~cm}$. 
Jugal

Both jugals are partly preserved (Figs. 25, 26 and 29). The right jugal and squamosal (Fig. 29) preserve most of the margin of the lateral temporal fenestra and the jugal notch. The proximal portions of both jugals are not preserved, but would have formed the ventral margin of the orbit, as in all ceratopsids. Dorsally (Fig. 29A-B), there are vascular grooves which appear to radiate from where the orbital margin would be, and are consistent with those seen on the ventral end of the right postorbital (Fig. 24A-B). The epijugal (Figs. 25G-H and 26G-H) is articulated with the ventral ends of the jugal and quadratojugal. The epijugal is large and laterally oriented, with vascular grooves directed towards its apex. It is comparable in size to that of CMN 8801 (Chasmosaurus).

Quadratojugal

Portions of both the left and right quadratojugals are preserved (Figs. 25-26). Each quadratojugal is articulated with the lateral jugal and medial quadrate obscuring most of their features. The medial surface of the quadratojugal has a cup-like socket anteroventrally (Figs. 25C-D and 26C-D) which receives the ventrolateral end of the quadrate. The quadratojugal articulates with the ventral portion of the epijugal posteroventrally (Figs. 25G-H and 26G-H). Due to incomplete preservation, it cannot be determined whether the quadratojugal participated in the margin of the lateral temporal fenestra (Fig. 25A-B).

Quadrate

The proximal portions of the left and right quadrates are preserved (Figs. 25-26) 
Figure 25. Left articulated jugal, quadratojugal and quadrate of CMN 8802

(Chasmosaurus sp.). A-B, lateral; C-D, anterior; E-F, medial; and G-H, posterior views. See "List of anatomical abbreviations". Scale bars equal $10 \mathrm{~cm}$. 
Figure 25.

A)

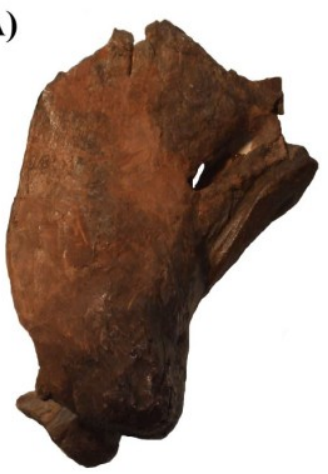

B)

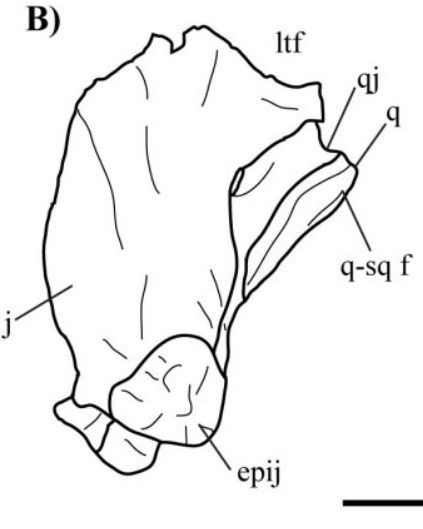

E)

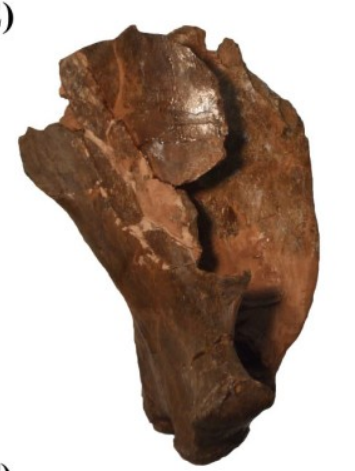

C)

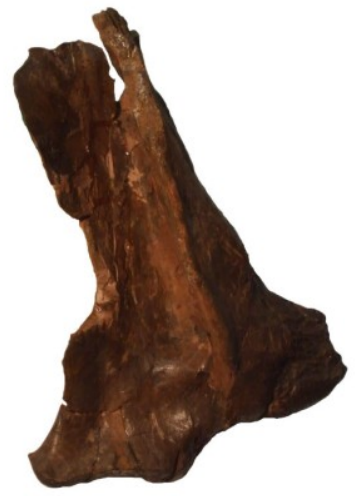

D)

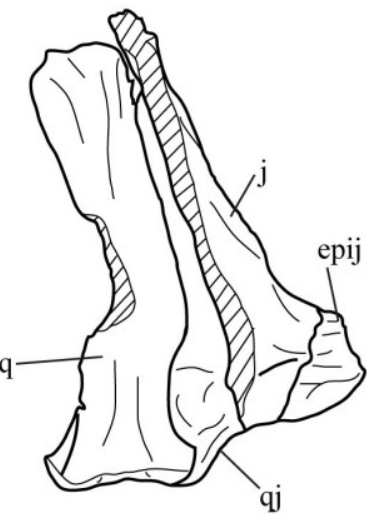

G)

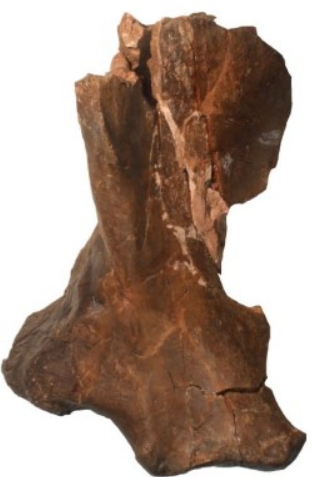

H)

F)

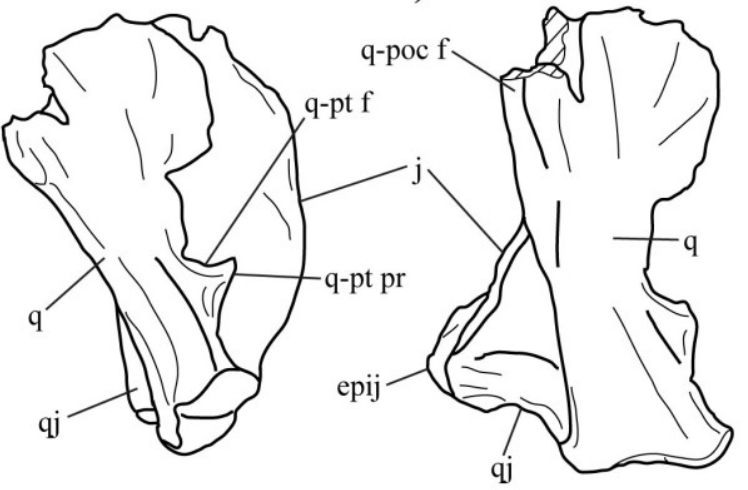


Figure 26. Right articulated jugal, quadratojugal and quadrate of CMN 8802 (Chasmosaurus sp.). A-B, lateral; C-D, anterior; E-F, medial; and G-H, posterior views. See "List of anatomical abbreviations". Scale bar equals $10 \mathrm{~cm}$. 
Figure 26.

A)

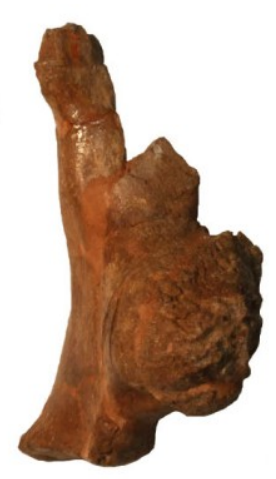

C)
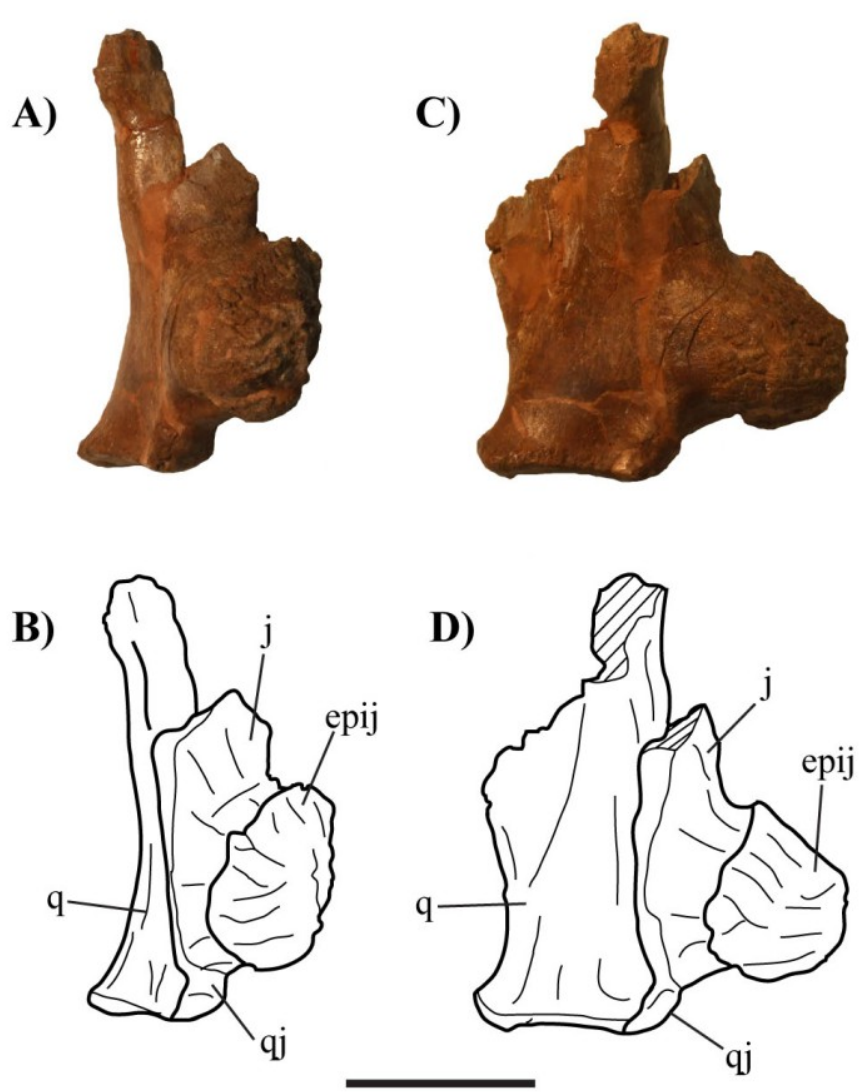

E)

G)
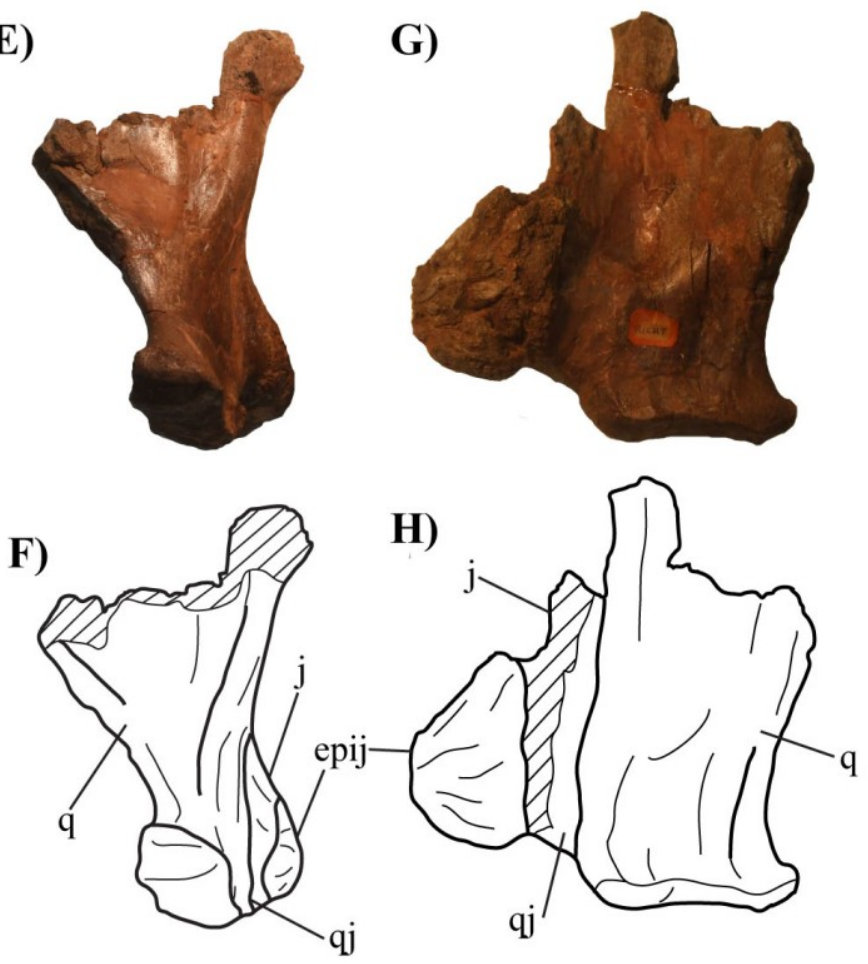

131 
and are articulated with their corresponding quadratojugal. The ventral end of each quadrate is smooth and rounded (Figs. 25E-F and 26E-F) and would have articulated with the glenoid fossa on the lower jaw (Figs. 31C-D and 32C-D). The pterygoid process (Fig. $25 \mathrm{E}-\mathrm{F})$ is present on the medial margin of the more complete left quadrate. Dorsal to this process is the pterygoid facet, which is bordered by a ridge that lines the medial margin of the quadrate and extends onto the process. The dorsal half of the quadrate is wide relative to the ventral half (Fig. 25G-H).

\section{Braincase}

The braincase (Fig. 27) is almost completely preserved, missing only the anterior portion and the distal ends of the paroccipital processes. The smooth, rounded occipital condyle has a circular outline in posterior view (Fig. 27A-B), and projects posteroventrally (Fig. 27I-L). The neck of the condyle has a uniform width in dorsal view (Fig. 27E-F), but it tapers distally in lateral view (Fig. 27I-L). The condyle is quite large, measuring $76 \mathrm{~mm}$ in mediolateral thickness. The only specimen of Chasmosaurus known to have a larger occipital condyle is that of ROM 843, which measures $79 \mathrm{~mm}$ in the same dimension. Despite its size, the suture between the basioccipital and the exoccipital can be faintly discerned (Fig. 27A-B and I-L). The basioccipital forms the bottom third of the condyle, while the two exoccipitals form the upper two. The suture between the two exoccipitals is not visible (Fig. 27A-B and E-F).

The foramen magnum lies directly above the base of the occipital condyle (Fig. 27A-B and E-F). The foramen is taller (approximately $30 \mathrm{~mm}$ ) than wide (approximately18 $\mathrm{mm}$ ). 

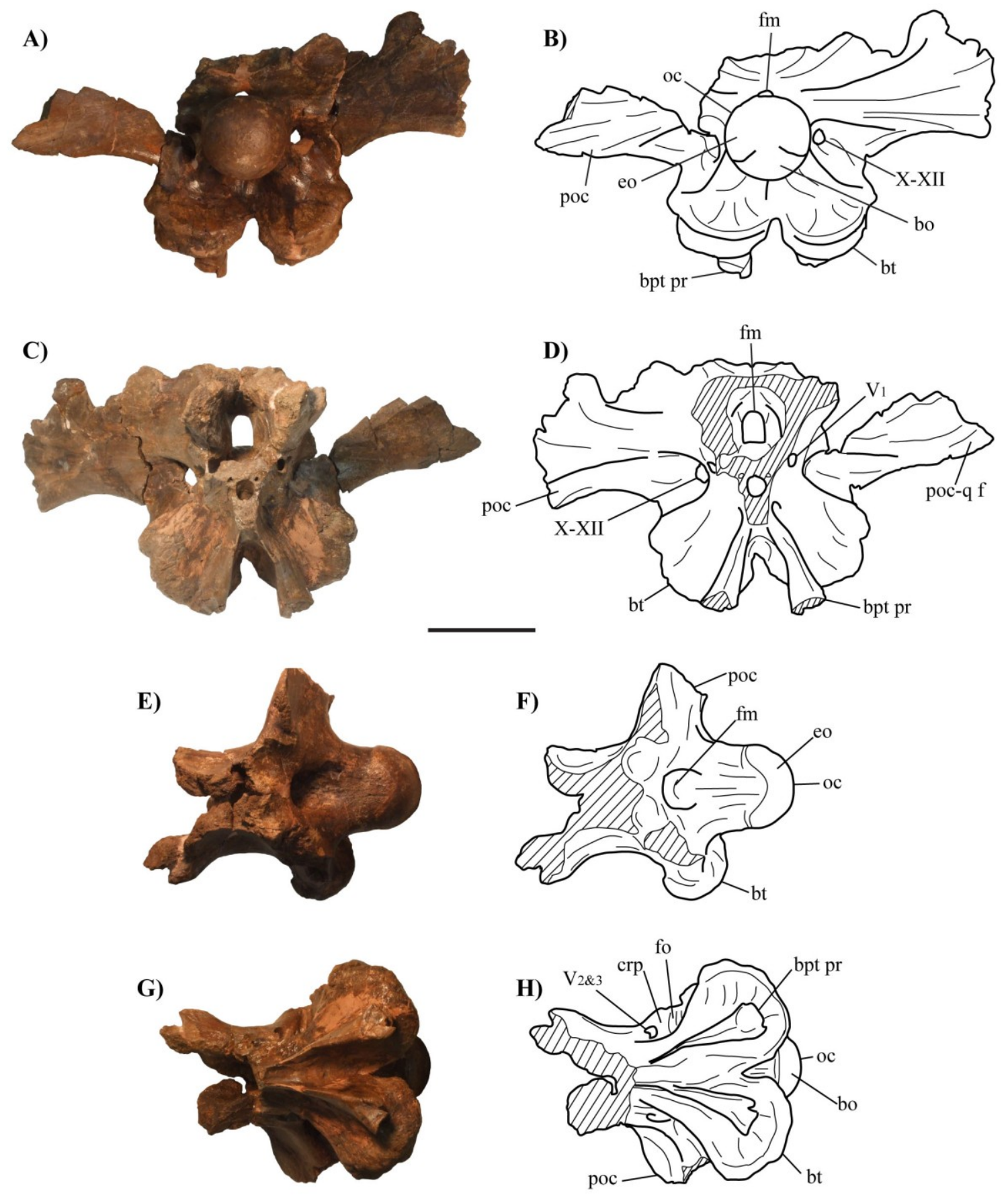

Figure 27. Braincase of CMN 8802 (Chasmosaurus sp.) in A-B, posterior, C-D, anterior, $\mathbf{E - F}$, dorsal, G-H, ventral, I-J, left lateral, and K-L, right lateral views. See "List of anatomical abbreviations". Scale bar equals $10 \mathrm{~cm}$. 
Figure 27. (continued).

I)

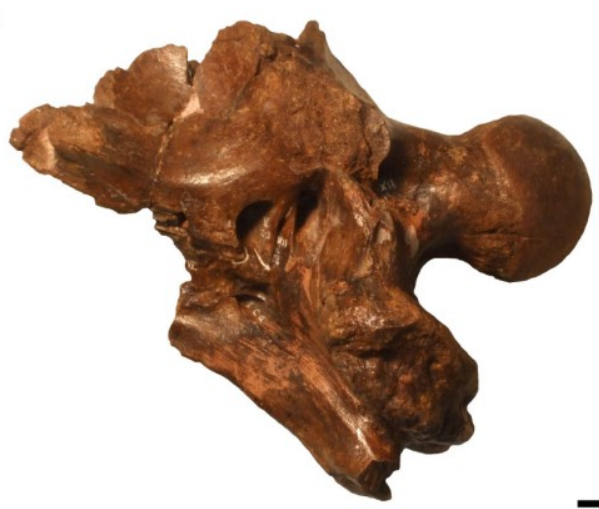

K)

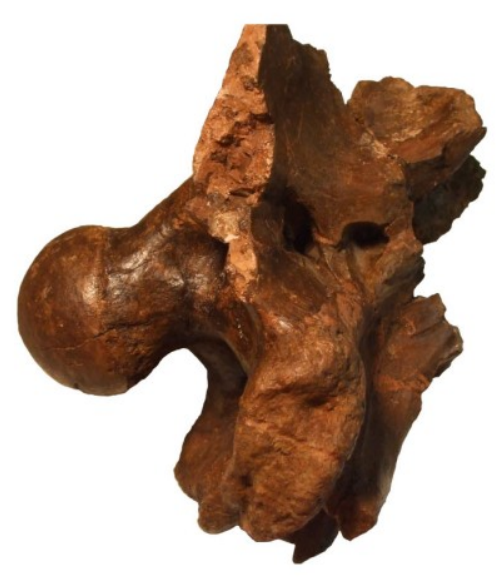

J)

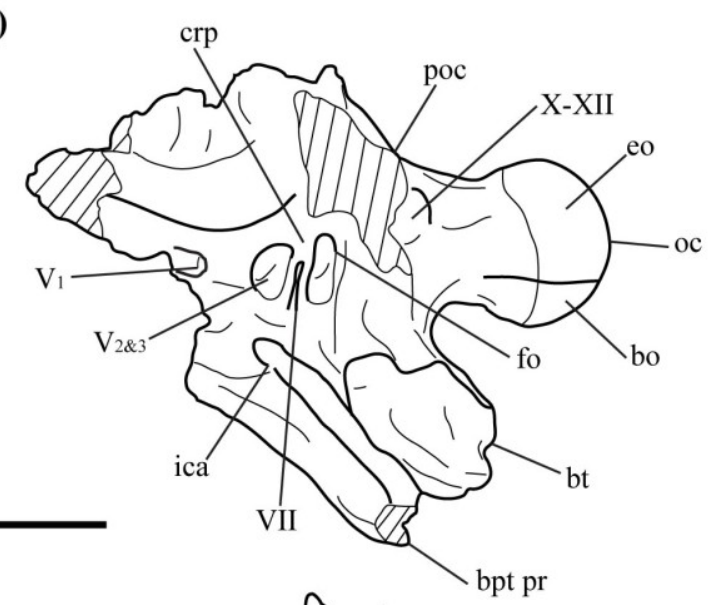

L)

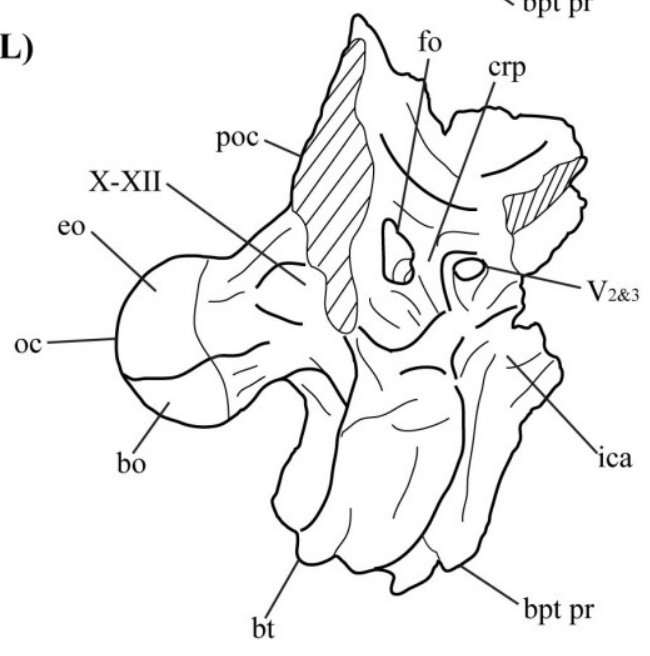


The basal tubera (Fig. 27A-D and G-H) flare out ventrolaterally below the occipital condyle and are separated by a deep medial embayment. Above the basal tubera, and laterally adjacent to the neck of the occipital condyle, the X-XII foramen for the exit of the vagus, accessory, and hypoglossal nerves is present (Fig. 27A-B). This foramen perforates the braincase on the right side, but cannot be discerned on the left side. On the anterior side of the tubera, the two basipterygoid processes project anteroventrally from the basisphenoid (Fig. 27C-D, G-L), although their ends are broken off and missing. Between these processes, the bone has a smooth and concave surface (Fig. 27G-H).

The anteroposteriorly compressed paroccipital processes (Fig. 27A-D) protrude laterally from the braincase. The distal ends of these processes are not preserved, but would have articulated with the anteroventral surface of the squamosals, as in all ceratopsids. Anterior to the base of each paroccipital process, the fenestra ovalis is present, and anterior to that is the trigeminal foramen (Fig. 27I-L). Posteriorly, these two foramina channel medially into the much larger foramen magnum (Fig. 27C-D). These two foramina are separated by the thin ridge of the crista prootica (Fig. 27I-L). On the left side of the braincase (Fig. 27I-J), a vertically-narrow, slit-like opening, the VII foramen, is present directly below the crista prootica. Although this foramen is not present on the right side of the braincase (Fig. 27K-L), there is a shallow depression where it would normally be located.

Directly ventral to the trigeminal foramen, the opening for the internal carotid artery is present (Fig. 27I-L). A channel that lies laterally adjacent to the basipterygoid process leads into this opening. 
Squamosal

Each squamosal is missing their approximately distal one-third (Figs. 28-29). The left squamosal (Fig. 28A-D) preserves five approximately equal-sized episquamosals, while the right (Fig. 29A-D) preserves four. The total episquamosal count cannot be determined due to incomplete preservation. The sutural contact between the episquamosals and the squamosal become increasingly pronounced progressing anterior to posterior, indicative of the order of articulation. The more complete right squamosal has a maximum width (as measured from the lateral margin of the first episquamosal to the medial margin of the squamosal) of $436 \mathrm{~mm}$, wider than any known Chasmosaurus $($ ROM $843=395 \mathrm{~mm})$ or Vagaceratops skull $(\mathrm{CMN} 41357=360 \mathrm{~mm})$.

The elongate facet for the parietal lateral bar (Figs. 28C-D and 29C-D) is present on the ventromedial surface of each squamosal. The more complete left facet (Fig. 28CD) is concave anteriorly, but gradually flattens and disappears posteriorly. The dorsal surface of the squamosal (Figs. 28A-B and 29A-B) is thickened laterally adjacent to this facet. Anterior to the lateral parietal bar facet, the medial margin defines the lateral margin of the dorsal temporal fenestra. It cannot be determined if the lateral parietal bar was continuous along the parietal fenestra.

The fluted sutural surface for the postorbital (Figs. 28C-D and 29C-D) is present on the anteroventral margin of each squamosal. The facets for the paroccipital and quadrate processes (Figs. 28C-D and 29C-D) are present on the medioventral surface of the squamosal. The more complete right squamosal (Fig. 29A-B) forms the posterior margin of the lateral parietal fenestra, although the squamosal infratemporal process that once bordered the ventral margin of this opening is not preserved. 

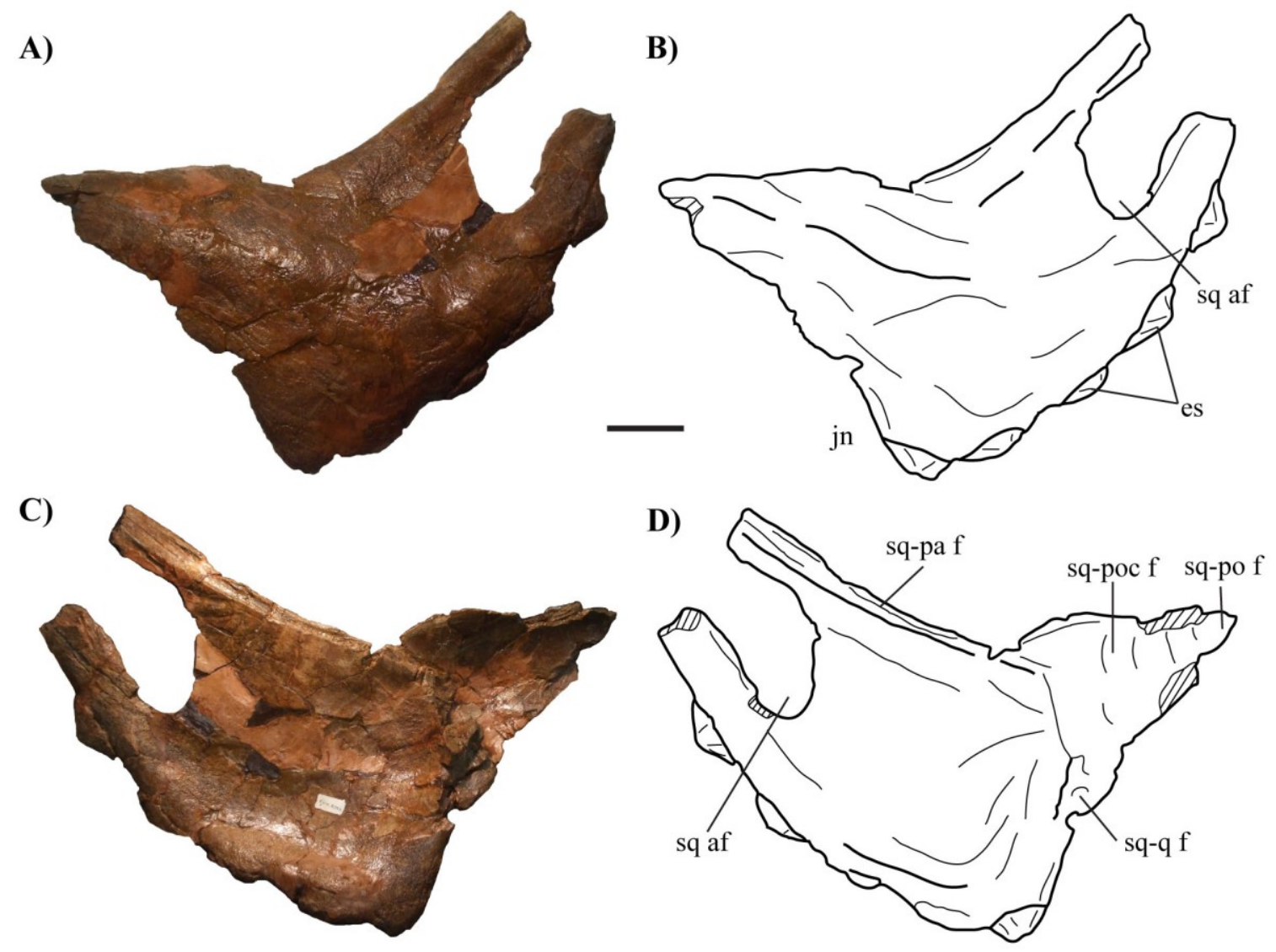

Figure 28. Left squamosal of CMN 8802 (Chasmosaurus sp.). A-B, dorsal; and C-D, ventral views. See "List of anatomical abbreviations". Scale bar equals $10 \mathrm{~cm}$. 

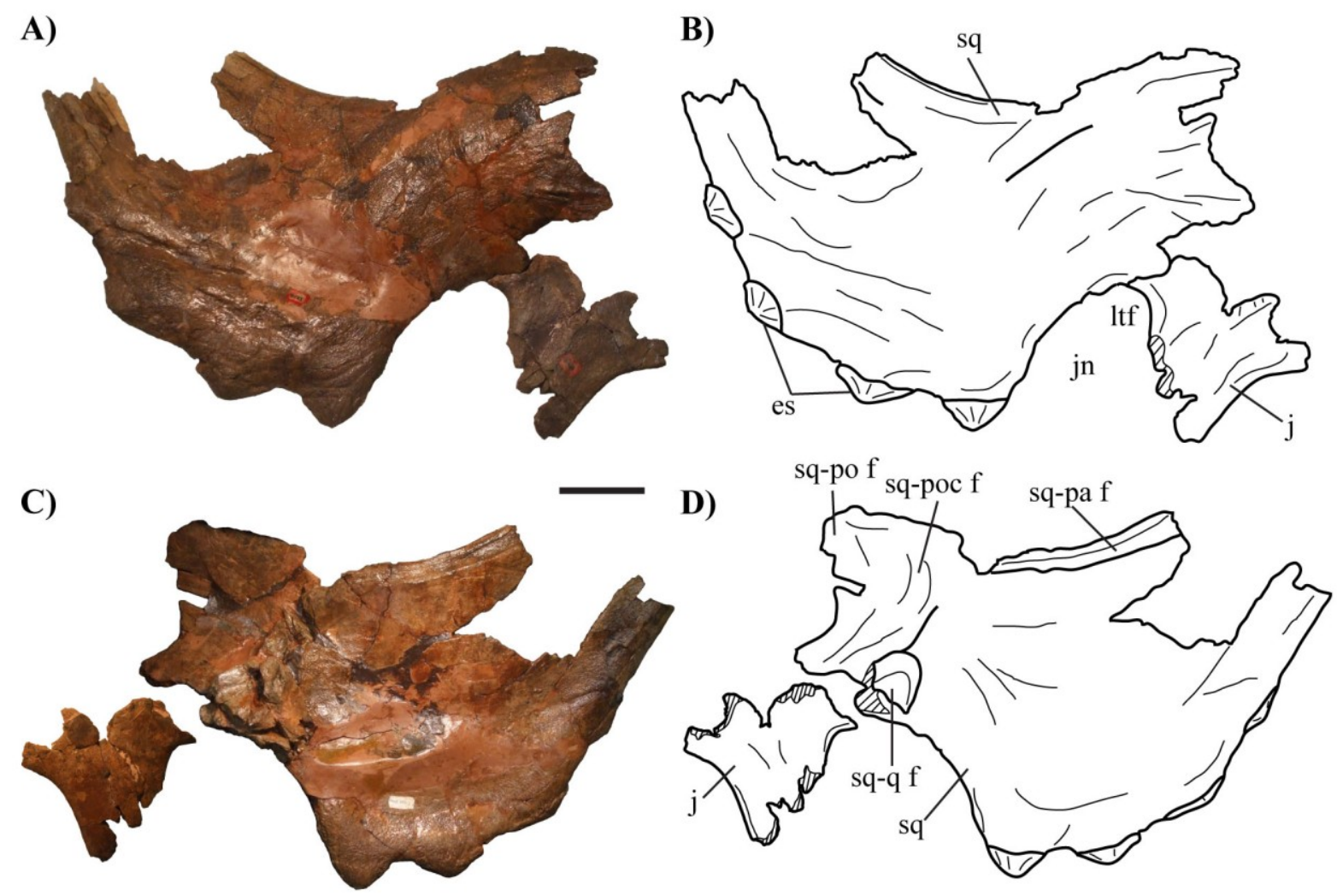

Figure 29. Right squamosal and jugal of CMN 8802 (Chasmosaurus sp.). A-B, dorsal; and C-D, ventral views. See "List of anatomical abbreviations". Scale bar equals $10 \mathrm{~cm}$. 
An accessory squamosal fenestra (Fig. 28A-D) is present on the left squamosal, and resembles that of NHMUK R4948 (Maidment and Barrett, 2011: Fig. 12E-F), ROM 839 (Fig. 7K), ROM 843 (Fig. 7L), and TMP 1983.025.0001 (Fig. 7O). The posterior margin of this accessory fenestra is not preserved in CMN 8802. Notably, in all of these specimens, the accessory squamosal fenestra occurs on the left side of the frill.

Parietal

The parietal (Fig. 30) is represented by most of the medial bar and the anterior medial platform. The dorsal surface of the parietal (Fig. 30A-B) is highly vascularised, while the ventral surface (Fig. 30C-D) is relatively smooth. The anteriormost end of the parietal (Fig. 30A-B) forms a raised platform that borders the dorsal temporal fenestrae laterally, and the posterior margin of the frontoparietal fontanelle anteriorly. Portions of the articulating facets for the squamosals (Fig. 30A-B) are preserved on the dorsolateral margin of the parietal. The anteromedial margin of the parietal fenestrae are partly preserved to the right of the medial bar. The distalmost preserved portion of the medial bar is rectangular and transversely compressed in cross-section. As in Chasmosaurus, Vagaceratops, Agujaceratops, and Pentaceratops, the medial bar is narrow and strap-like relative to the width of the frill. Although incomplete, the parietal fenestrae would have been large, as for Chasmosaurus. The ventral midline surface is concave.

Three isolated epiparietals (Fig. 30) are also preserved. One epiparietal is large (approximately $125 \mathrm{~mm}$ in length) and straight (Fig. 30A-F), and has a convex surface in dorsal and ventral views (Fig. 30E-J). This epiparietal is broken, permitting a cross- 

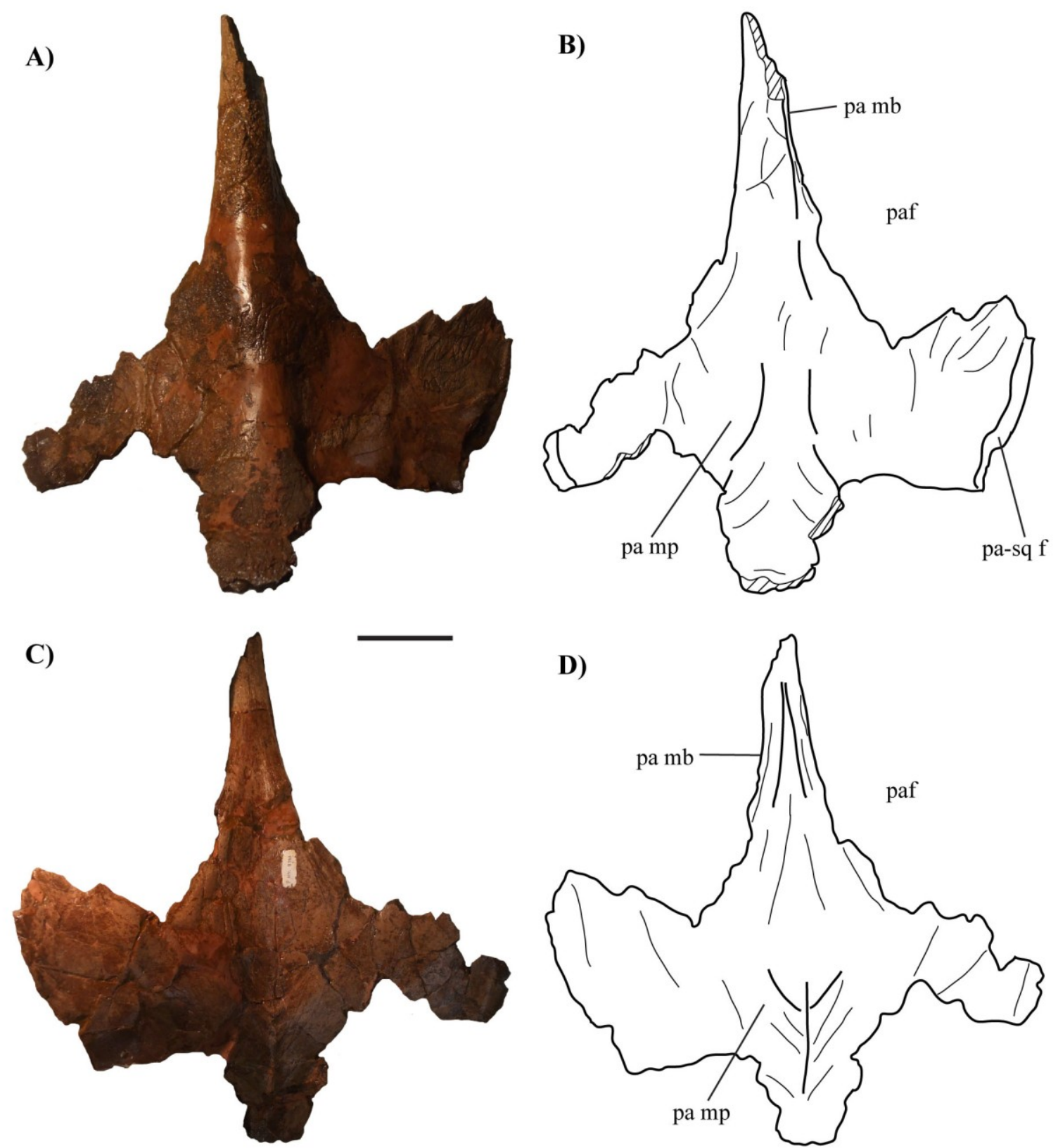

Figure 30. Parietal of CMN 8802 (Chasmosaurus sp.) in A-B, dorsal; and C-D, ventral views. Epiparietals: first epiparietal, E-J, in E-F, dorsal (?), G-H, ventral (?), and I-J, cross-sectional views; second epiparietal, K-P, in K-L, dorsal (?), M-N, ventral (?), and $\mathbf{O}-\mathbf{P}$, cross-sectional views; and third epiparietal, $\mathbf{Q - V}$, in $\mathbf{Q - R}$, dorsal (?), S-T, ventral (?), and U-V, cross-sectional views. See "List of anatomical abbreviations". Scale bar equals $10 \mathrm{~cm}$. 
Figure 30. (continued).

E)

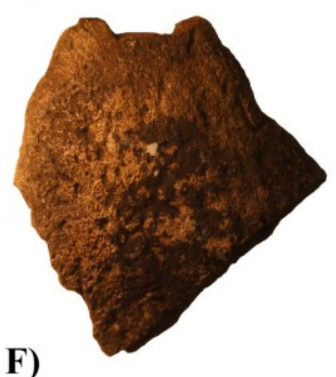

F)

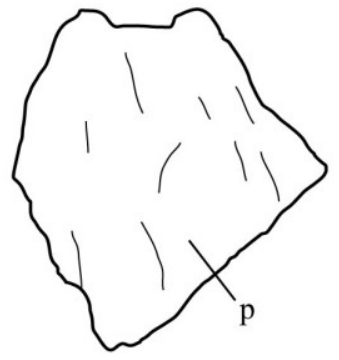

K)

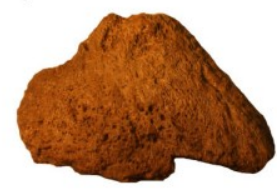

L)

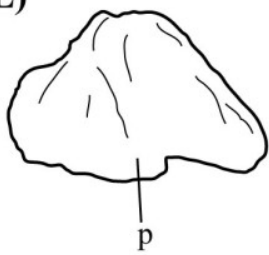

Q)

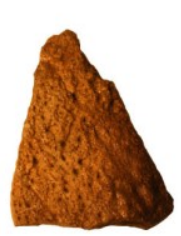

R)

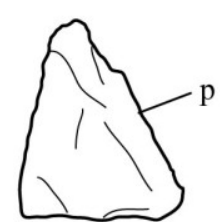

G)

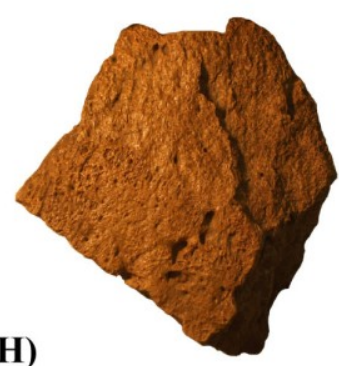

H)

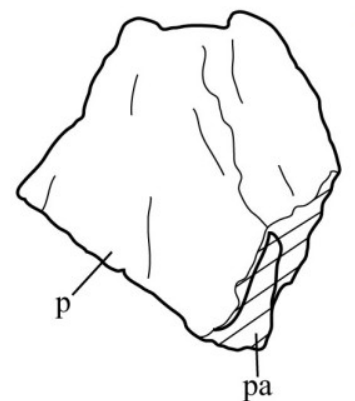

M)

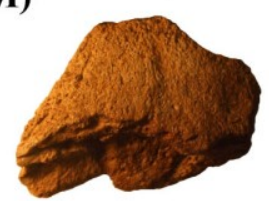

N)

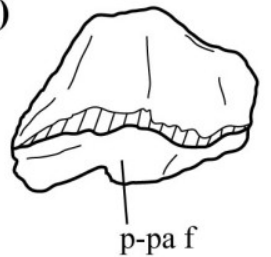

S)

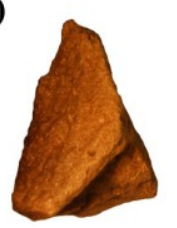

T)

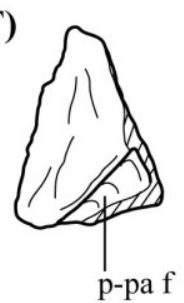

I)

J)

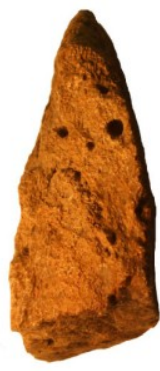

O)
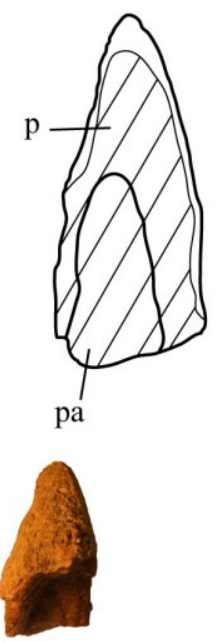

P)

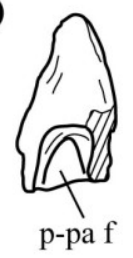

U)

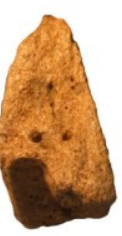

V)

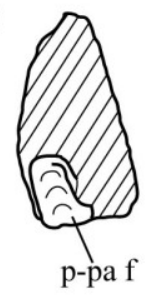


sectional view of the epiparietal-parietal articulation (Fig. 30I-J); the epiparietal overlaps (approximately $60 \mathrm{~mm}$, anteroventrally) the dorsal and ventral surfaces of the parietal, forming a parabolic sutural contact.

The next epiparietal (Fig. 30K-P) is shorter (approximately $60 \mathrm{~mm}$ ). The unmodified base of the epiparietal contact surface is distinct and the element does not appear to have been articulated to the parietal.

A partial third epiparietal (Fig. 30Q-V) has a preserved length of approximately $75 \mathrm{~mm}$, although would have been longer. Its overall shape and thickness suggests that it may have been as large as the first one described (Fig. 30E-J). The contact with the parietal (Fig. 30S-V) does not appear to be articulated.

Lower jaw

Both of the lower jaws (Figs. 31-32) are nearly complete, preserving the dentary, splenial, angular, surangular, articular, and prearticular. The predentaries are missing, although the smooth articulating facets for it are preserved on the dentaries. The ventral margin of each dentary is relatively straight, as in some Chasmosaurus specimens (e.g., CMN 2280, CMN 8801) and Vagaceratops (CMN 41357). Dentaries with convex ventral margins are also known in some Chasmosaurus specimens (e.g., CMN 1254, ROM 843). The shape of the ventral margin of the dentary is unlikely related to ontogeny, as convex margins are present in both relatively small $(\mathrm{CMN} 1254, \mathrm{SL}=500 \mathrm{~mm})$ and large (ROM 843, $\mathrm{REL}=897 \mathrm{~mm} ; \mathrm{SL}=893 \mathrm{~mm}$ ) Chasmosaurus skulls. 
Figure 31. Left lower jaw of CMN 8802 (Chasmosaurus sp.). A-B, lateral, C-D, dorsal, and E-F, medial views. See "List of anatomical abbreviations". Scale bar equals $10 \mathrm{~cm}$. 
Figure 31.
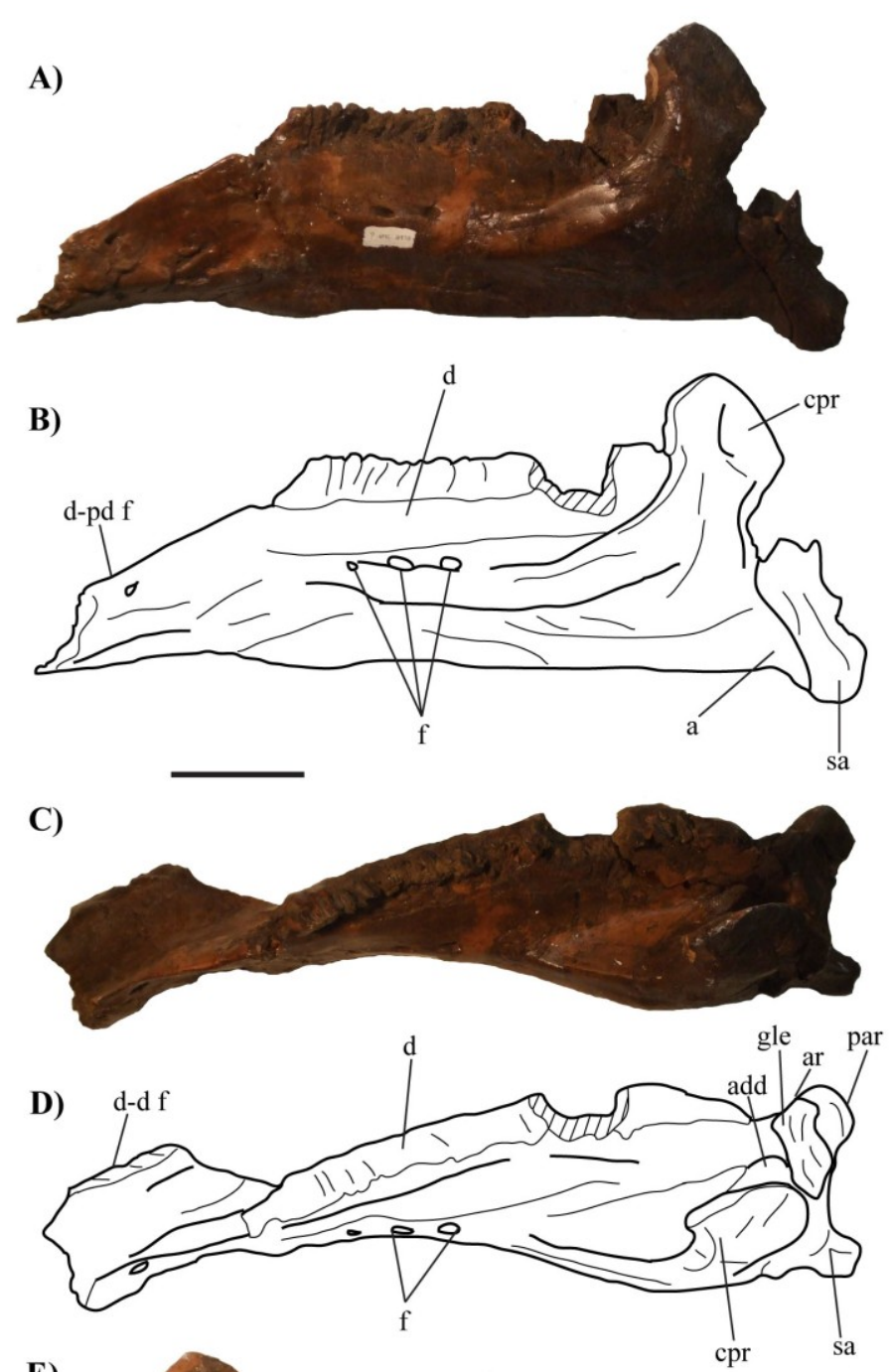

E)
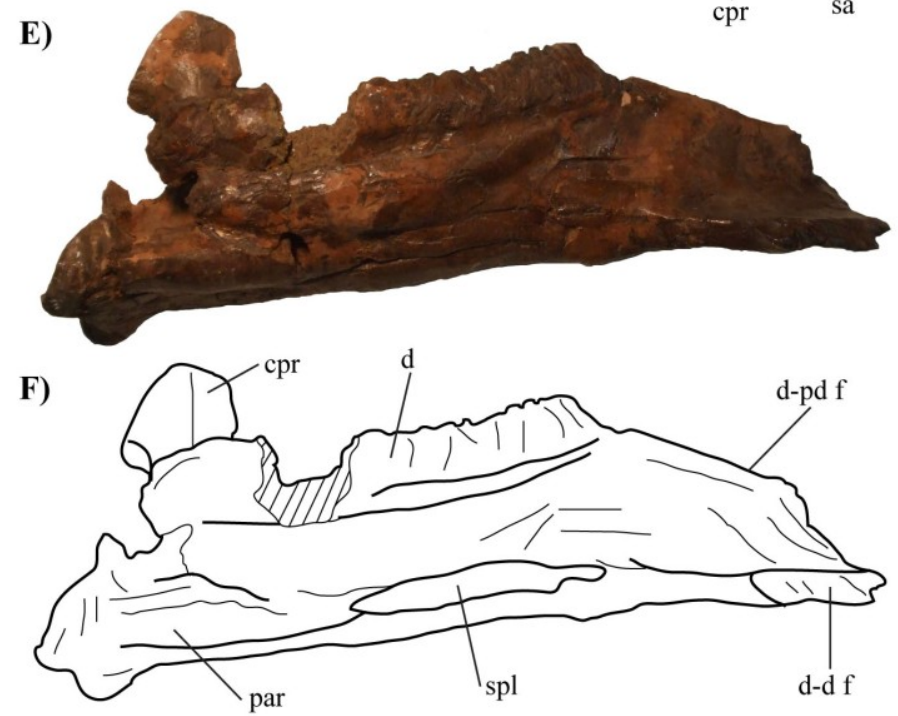
Figure 32. Right lower jaw of CMN 8802 (Chasmosaurus sp.). A-B, lateral, C-D, dorsal, and E-F, medial views. See "List of anatomical abbreviations". Scale bar equals $10 \mathrm{~cm}$. 
Figure 32.

A)
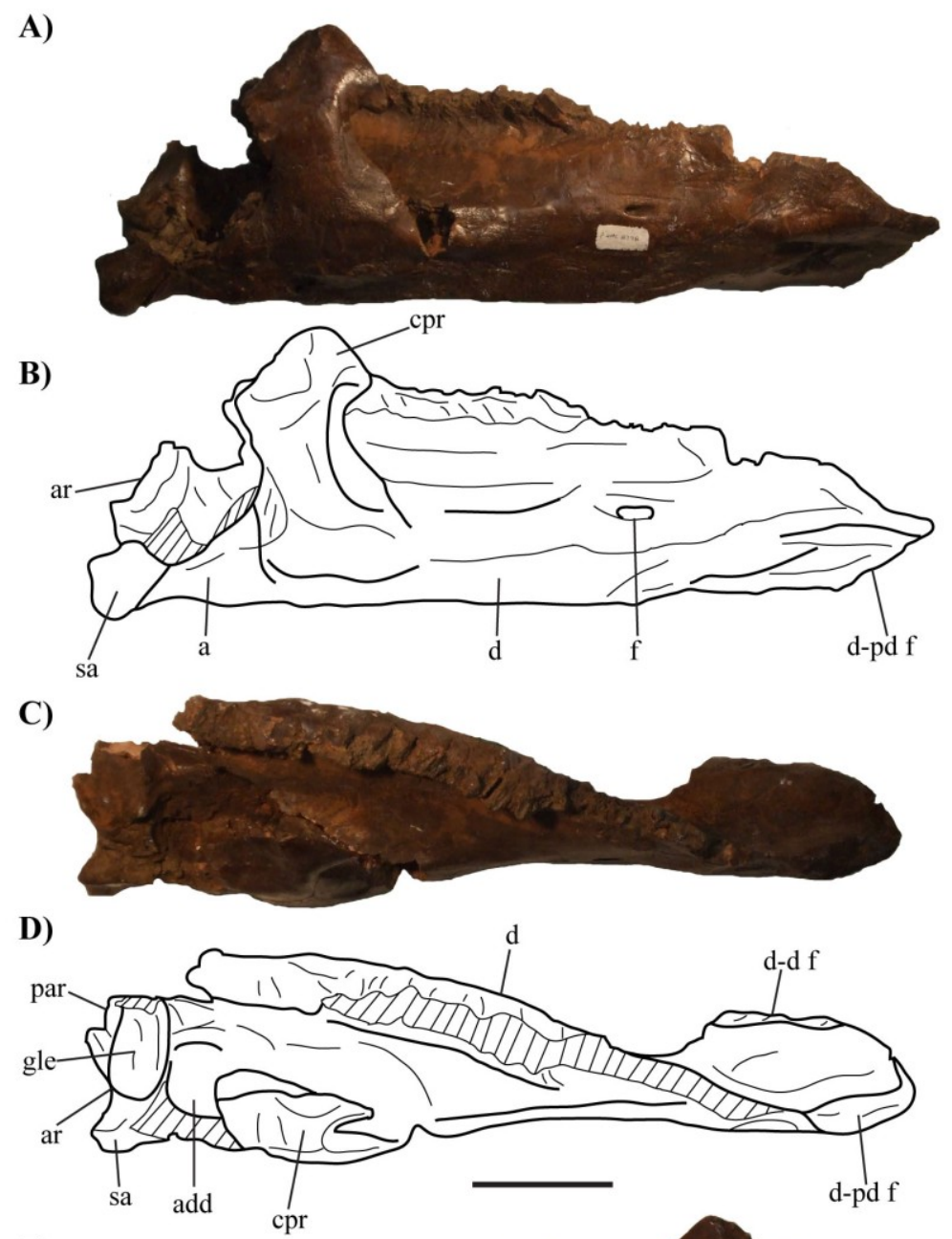

E)
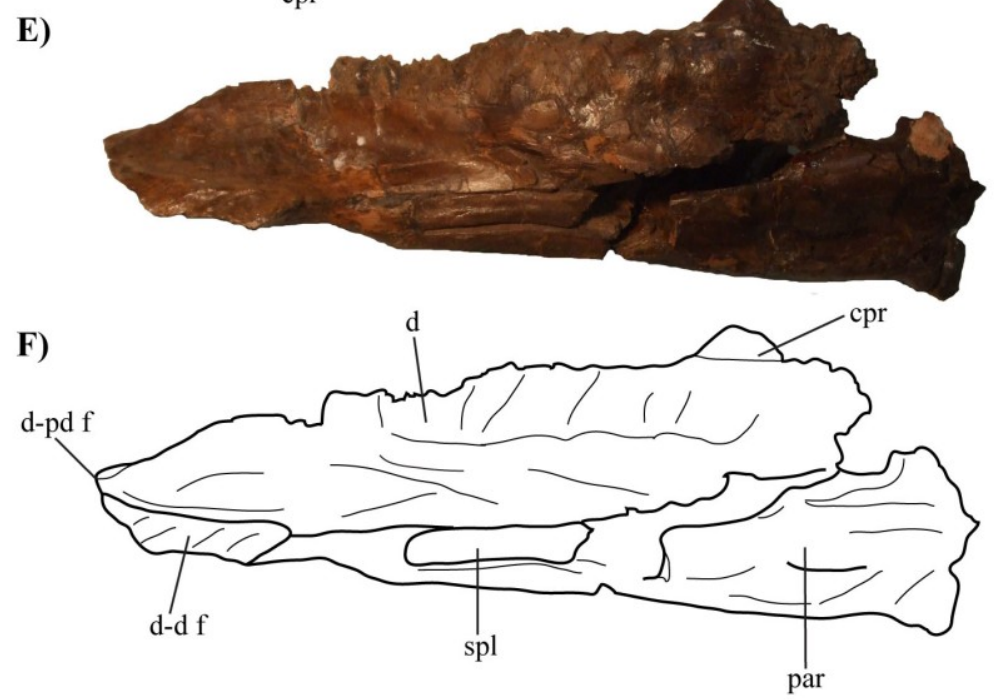
The anterior margin of each dentary (Figs. 31A-B, E-F, and 32A-B, E-F) is curved medially where they contact at the symphysis. In dorsal view (Figs. 31C-D and 32C-D), the dentaries are strongly divergent posteriorly. The poorly preserved toothrow occupies most of the length of each dentary. Three foramina are aligned anteroposteriorly on the lateral side of each dentary, below the anterior half of the toothrow. These foramina are more pronounced on the left (Fig. 31A-B). On the right side (Fig. 32A-B), only the middle foramina is visible while the other two are identifiable as small fossae. At the posterior end of each dentary, the transversely-compressed coronoid process extends above and lateral to the toothrow, such that the posterior end of the toothrow is obscured in lateral view (Figs. 31A-B and 32A-B). The anterior margin of the coronoid process is a sharp ridge (Figs. 31A-B and 32A-B) which extends as far anteriorly as the three foramina, but becomes less pronounced.

A shallow, narrow splenial groove is oriented anteroposteriorly on the ventromedial surface of each dentary (Figs. 31E-F and 32E-F). The splenial tapers at each end and extends the length of the toothrow.

The angular has a flat ventral margin that is confluent with posterior margin of the dentary. The angular is visible in lateral view and articulates with the dentary and surangular immediately below the base of the coronoid process (Figs. 31A-B and 32AB). Dorsal and posterior to the angular the surangular is visible in lateral view and extends below the ventral margin of the dentary. The surangular (Figs. 31 A-D and 32AD) forms most of the lateral margin of the adductor fossa, as well as a small portion of the glenoid fossa.

The articular is medial to the surangular is (Figs. 31C-D and 32C-D) and forms a 
concave dorsal surface to receive the ventral end of the quadrate. The articular forms the medial wall and most of the floor of the adductor fossa, as well as most of the glenoid fossa.

The prearticular (Figs. 31E-F and 32E-F) is situated posteriorly and below the coronoid process and the articular, and is visible medially. Although the anterior extent of the prearticular cannot be determined, it extends rostral to the coronoid process. No teeth are present in CMN 8802 and the poor preservation of the alveoli makes a tooth count impossible.

CMN 8802 is diagnosed as chasmosaurine based on its possession of a rostral with anteroposteriorly elongate ventral processes, no ventral expansion of the posteroventral margin of the premaxilla, long triangular squamosals, and a transversely narrow and strap-like parietal medial bar. Although incomplete, the parietal fenestrae of CMN 8802 are relatively large and more typical of Chasmosaurus than of Vagaceratops. Sternberg's (1940) original referral of CMN 8802 to C. russelli was based on his original diagnosis for this species; "skull large, relatively high and short in front of orbits; rostral straight inferiorly, not hooked downward at tip; nasal horncore massive; broad between orbits; no brow horncores; well-developed epijugal; parietals deeply indented posteriorly; squamosal border not strongly scalloped; epoccipitals small; mandible massive" (Sternberg 1940:478). The presence of an indented/embayed posterior parietal margin is the only one of the above features that has remained part of the diagnosis for $C$. russelli (Maidment and Barrett, 2011), and this feature is not preserved in CMN 8802. Based on the above observations, CMN 8802 is referred to Chasmosaurus sp. 


\subsection{Variation of cranial material within Chasmosaurus and related taxa}

This section discusses the variation within characters that have putative diagnostic utility within Chasmosaurinae, with specific reference to Chasmosaurus. These include characters of the parietal (thickness of medial parietal bar and lateral sulci, continuity of lateral parietal bars, embayment of posterior parietal bar), squamosal (length of squamosal), and cranial ornamentation (rostral, jugal, nasal, postorbital, epiparietals and episquamosals). The ontogenetic origins of these variations, if plausibly inferred, will be discussed in Chapter 8 (Ontogeny).

Snout

Rostral

The shape of the rostral is variable amongst Chasmosaurus specimens, ranging from anteroposteriorly short (e.g., ROM 839, Fig. 7K) to elongate (e.g., CMN 2280, Fig. 7F). Such variation in rostral shape occurs in other chasmosaurine taxa (e.g., Anchiceratops ornatus; Mallon et al., 2011). The anteroposteriorly short snout of ROM 839 is accentuated by its short rostral, both of which features were used to diagnose Chasmosaurus brevirostris (Lull, 1933). The exterior surface of the rostral in ROM 839 is covered in plaster, however, making it difficult to determine how much of the rostral is preserved. Similarly short rostra are known in other Chasmosaurus specimens as well, i.e., AMNH 5401 (Fig. 7A) and TMP 1981.019.0175 (Fig. 7N). The ventral margin of the rostral is also variable amongst Chasmosaurus specimens, ranging from relatively straight (e.g., TMP 1981.019.0175, Fig. 7N) to gently concave (e.g., AMNH 5401, Fig. 7A) to strongly concave (i.e., ROM 839; Fig. 7K). 
Nasal horncore

In chasmosaurines, the nasal horncore is formed by the articulation of the epinasal with the underlying nasals (Horner and Goodwin, 2006). The size and shape of the nasal horncore is variable in Chasmosaurus. The epinasal is disarticulated from the nasals in CMN 1254, but articulated in all other specimens, where preserved. The suture between the epinasal and nasals is not always visible in Chasmosaurus specimens, but the epinasal is inferred as being present in specimens possessing a horncore that is triangular in lateral view; without an articulated epinasal, the preserved right nasal of CMN 1254 has a concave dorsal margin in lateral view. In specimens, the nasal horncore varies from being positioned almost entirely posterior to the nares (e.g., CMN 8801), to almost entirely above it (e.g., ROM 843). The nasal horncore also varies from being tall and pointed (e.g., ROM 839), to flat-topped and pitted (e.g., YPM 2016), to an irregular and rugose mound (CMN 8800).

Circumorbital and cheek regions

Postorbital horncores

In Chasmosaurus, postorbital ornamentation takes the form of either a low-relief mound (e.g., YPM 2016, right side $=41 \mathrm{~mm}$ ) to an elongate horncore up to $370 \mathrm{~mm}$ in length (AMNH 5401, right side). Horncore length does not appear to be correlated to skull length, e.g., the postorbital horncore length in the relatively small skull CMN 1254 is $216 \mathrm{~mm}$ (right side), while those of the much larger skull ROM 839 are only $107 \mathrm{~mm}$ (left side) and $93 \mathrm{~mm}$ (right side). AMNH 5401 and CMN 2280 are nearly identical in skull size (rostral-to-epijugal length $=730 \mathrm{~mm}$ and $750 \mathrm{~mm}$, respectively), yet the 
postorbital horncores are $350 \mathrm{~mm}$ (left side) and $370 \mathrm{~mm}$ (right side), and $132 \mathrm{~mm}$ (left side) and $88 \mathrm{~mm}$ (right side), respectively.

In Chasmosaurus, postorbital horncore length is also known to vary within a single individual, e.g., CMN 8800 (Fig. 7G). In this skull, the right horncore is widebased, but very short as preserved, with a highly irregular and pitted dorsal surface. In contrast, the left horncore in CMN 8800 has a rugose pit in place of the horncore. In CMN 2280 (Fig. 7F), the left horncore is well-developed and pointed, while only the base of the left horncore is preserved, and has a pitted dorsal surface. In TMP 1981.019.0175 (Fig. $7 \mathrm{~N}$ ), the right horncore has a truncated apex (suggesting that it was longer), while the left horncore is reduced to a relatively flat surface. The orientation of the postorbital horncores also varies in Chasmosaurus, ranging from being straight and anterolaterallydirected in a sub-horizontal plane relative to the skull roof (e.g., TMP 1979.011.0147) to being near-vertical, recurved, and posteriorly-directed (e.g., CMN 1254).

Postorbital horncores have been shown to lengthen over ontogeny in centrosaurines (e.g., Centrosaurus apertus, Ryan et al., 2001; and Coronosaurus brinkmani, Ryan and Russell, 2005) and chasmosaurines (e.g., Agujaceratops mariscalensis, Lehman, 1989; Pentaceratops sternbergi, Lehman, 1993; and Triceratops horridus, Horner and Goodwin, 2006). It has also been noted that postorbital horncores change their orientation over ontogeny. Ryan and Russell (2005) noted that in Coronosaurus brinkmani, the postorbital horncores are initially short and vertically oriented, but become longer and laterally curved later in maturity. In their study on Triceratops ontogeny, Horner and Goodwin (2006) noted that postorbital horncores are initially straight, recurve in juveniles, straighten in subadults, and curve anteriorly in 
adults. Using evidence from extant bovids, Ostrom and Wellnhofer (1990) concluded that the differences in postorbital horncore orientation in Triceratops was attributable to ontogenetic, individual and/or sexual dimorphism.

Frontoparietal fontanelle

The shape and size of the frontoparietal fontanelle is highly variable in Chasmosaurus, ranging from being relatively narrow (ROM 839, ROM 843 and YPM 2016), to transversely expanded (AMNH 5401, CMN 8800, CMN 34832, and TMP 1979.011.0147). Transversely expanded fontanelles are generally associated with relatively large and long postorbital horncores (e.g., AMNH 5401, CMN 34832 and TMP 1979.011.0147). The presence of a wide frontoparietal fontanelle was previously used by Longrich (2010) as a diagnostic character of M. perifania. However, as it is also present in the Chasmosaurus russelli holotype (CMN 8800) it is not a valid diagnostic character for the former taxon. This feature is also known to vary ontogenetically in Triceratops, with putatively old, mature skulls having the fontanelle secondarily closed (Farke, 2010).

In both TMP 1981.019.0175 and UALVP 40, transverse compression has obscured the frontoparietal fontanelle, such that if it was present, it is not visible in dorsal view. In some specimens (CMN 8800 and YPM 2016), the fontanelle is partially covered by a bridge of bone formed by the postorbitals, but this is not as developed as in some other chasmosaurines (e.g., Kosmoceratops and Arrhinoceratops), where it is completely roofed over. 
Epijugals

In Chasmosaurus, epijugals have trihedral bases and vary from being relatively short (e.g., CMN 2280) to long and pointed (AMNH 5401). Chasmosaurus epijugals are not as long as the 'hyperlong' condition seen in Pentaceratops (Lehman, 1993) and Bravoceratops (Wick and Lehman, 2013). It has been postulated that epijugals undergo resorption with age, which may account for the variation in the length of this feature (e.g., Pachyrhinosaurus lakustai, Currie et al., 2008). The orientation of the epijugal is also variable in Chasmosaurus, ranging from posteroventrolaterally, i.e., AMNH 5401 (Fig. 7A), ROM 839 (Fig. 7K), NHMUK R4948 (Fig. 7J), to posterolaterally, e.g., CMN 2280 (Fig. 7F), TMP 1981.019.0175 (Fig. 7N).

Frill

Lateral parietal bars

The continuity of the lateral parietal bar adjacent to the squamosal is variable among Chasmosaurus skulls. Godfrey and Holmes (1995) noted that specimens with relatively straight posterior parietal bars (e.g., C. belli) have continuous lateral bars, while specimens with embayed posterior bars (e.g., C. russelli) have discontinuous lateral bars, but they did not offer an explanation for this pattern. They did, however, note that AMNH 5656 had an embayed posterior bar with continuous lateral bars. Regardless of this exception, Godfrey and Holmes (1995) used the above pattern, in part, to distinguish between $C$. belli and C. russelli. YPM 2016 has discontinuous lateral bars, and a relatively straight posterior parietal bar, supporting the recommendation of Maidment and Barrett (2011) that the relative continuity of the lateral parietal bar should not be used as 
a diagnostic character within Chasmosaurus. The degree of continuity of the lateral parietal bar also seems to vary in Vagaceratops irvinensis, which has a continuous left bar and discontinuous right bar in the holotype, CMN 41357 (Holmes et al., 2001).

Thickness of medial parietal bar and lateral sulci

In Chasmosaurus specimens, the posterior end of the medial parietal bars varies in thickness from $26 \mathrm{~mm}$ (ROM 839) to $65 \mathrm{~mm}$ (CMN 8803). Some specimens, e.g., CMN 8803, have a deep groove that indents the length of the lateral margins of the midline bar, giving it a distinct I-shape in cross-section. In some other specimens, e.g., TMP 1983.025.0001 and TMP 1999.055.0292, this groove continues along the anterior margin of the posterior bar. The function of these grooves is uncertain. The combination of a thick, I-shaped in cross section midline bar and a deeply embayed posterior parietal bar makes CMN 8803 seemingly unusual when compared to other Chasmosaurus specimens; however, none of these features are unique to CMN 8803, as they are also present in TMP 1983.025.0001 and TMP 1999.055.0292.

Shape of parietal fenestrae

Expansion of parietal fenestrae through ontogeny has been noted in ceratopsians (e.g., Centrosaurus, Brown et al., 2009 and Protoceratops, Fastovsky et al., 2011). Expansion of the parietal fenestra was also proposed in Scannella and Horner's (2010) Triceratops-Torosaurus synonymy, but this synonymy is still debated (Farke, 2011; Longrich and Field, 2012). The shape of the parietal fenestrae has been used, in part, to differentiate Chasmosaurus and Vagaceratops, with the former being relatively elongate 
and oriented anteroposteriorly, and the latter being relatively short and transversely elongate (Holmes et al., 2001). Although there is variation between specimens, the two taxa do not overlap in morphologies (Table 1).

Embayment of posterior parietal bar

The frills of Chasmosaurus and Vagaceratops are shown in Figure 33. They are arranged by the relative embayment of the posterior parietal bar, in order from deeply embayed to straight. This arrangement was chosen to illustrate the gradational nature of the embayment across specimens of the same approximate size. The angle of the embayment is defined by the angle between two lines that originate at the midpoint of the posterior margin of the posterior parietal bar, and diverge tangently parallel to the posterior margin of the posterior bar (i.e., not tangent to the medialmost epiparietal, but the underlying parietal) (see Fig. 5, parameter 33). An angle of $180^{\circ}$ would represent a perfectly straight posterior bar, while a smaller angle would represent a more pronounced embayment. In Chasmosaurus, this angle ranges gradationally from $89^{\circ}$ to $171^{\circ}$ (Table 1). In Figure 33, skulls A through $G$ have been referred to C. russelli (Maidment and Barrett, 2011), H through $\mathrm{N}$ to C. belli (Maidment and Barrett, 2011), and O through $\mathrm{R}$ to V. irvinensis (Longrich, 2010; Maidment and Barrett, 2011). Skulls A through G range from $89-128^{\circ}$, while $\mathrm{H}$ through $\mathrm{N}$ range from $136-171^{\circ}$, and $\mathrm{O}$ through $\mathrm{R}$ are both approximately $180^{\circ}$ (inferred for Q). Notably, the degree of embayment of the posterior frill margin can be used to easily distinguish the specimens used in this study into their current taxonomic designations. The utility of this feature in phylogenetic analysis will be tested in Chapter 6. 
Figure 33. Chasmosaurus (A-N) and Vagaceratops (O-R) frills: A) CMN 8800 (C. russelli holotype); B) CMN 8803 (C. russelli); C) TMP 1999.055.0292 (C. russelli); D) TMP 1997.132.0002 (C. russelli); E) AMNH 5656 (C.russelli); F) TMP 1983.025.0001 (Mojoceratops perifania); G) CMN 2280 (C. russelli); H) ROM 843 (C. belli; cast); I) TMP 2008.012.0001 (C. sp.); J) CMN 2245 (C. belli); K) CMN 0491 (C. belli holotype); L) NHMUK R4948 (C. belli); M) AMNH 5402 (C. belli); N) YPM 2016 (C. belli); O) TMP 2009.034.0009; P) TMP 1998.102.0008; Q) TMP 1987.045.0001; and R) CMN 41357 (holotype; cast). Brackets delimit size of epiossifications. The only skulls whose epiparietals are not labelled are I and L, as either the posterior bar is insufficiently preserved (I) or the posterior margin of the bar is too difficult to interpret (L). In both C) and D), the real right fragment is reflected on the left to help reconstruct the frill. Plaster reconstruction shown in grey half-tone. See "List of anatomical abbreviations". Scale bars equal $10 \mathrm{~cm}$. 
Figure 33.

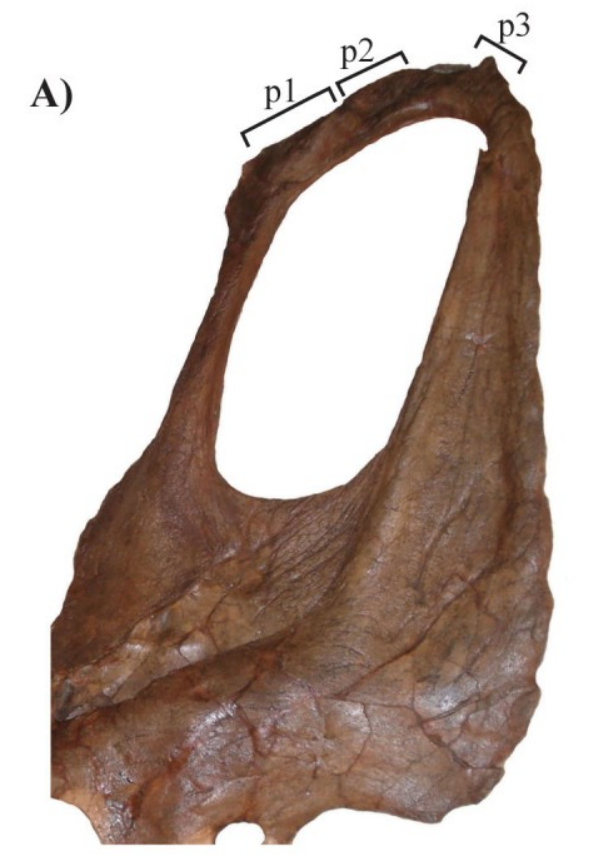

B)

C)

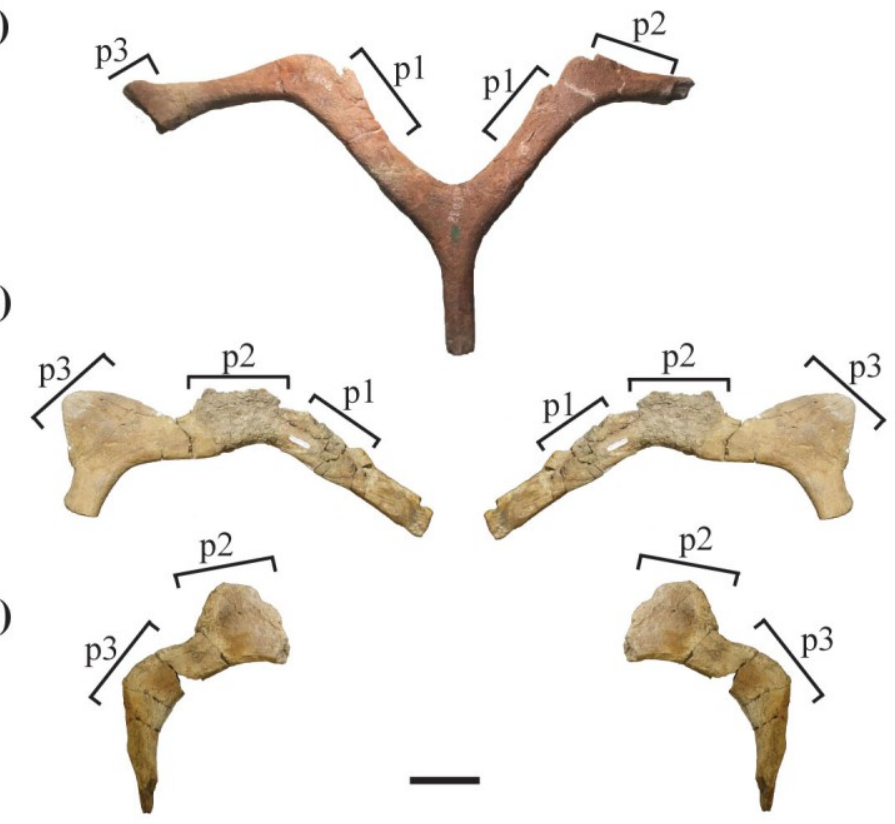

E)
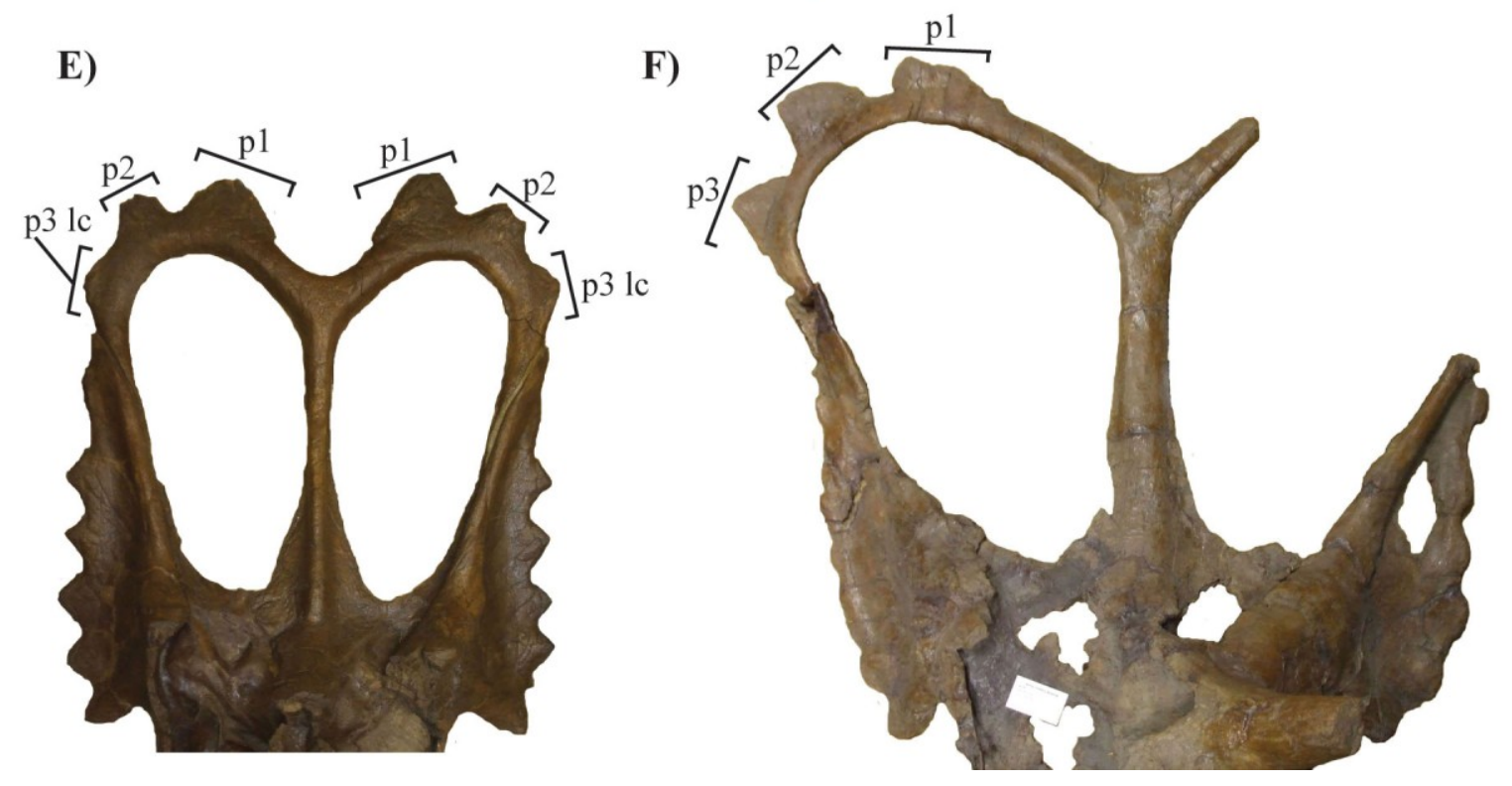
Figure 33. (continued).
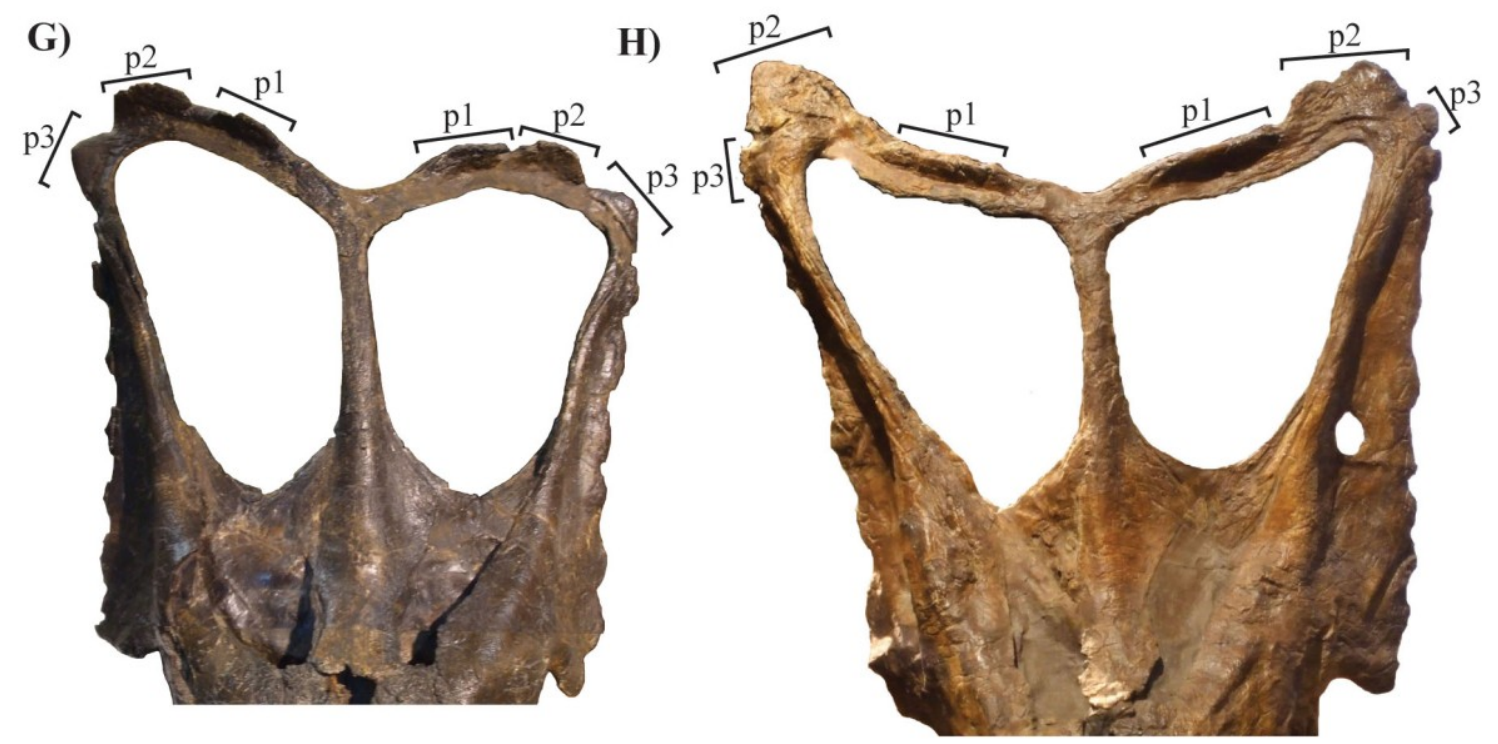

I)

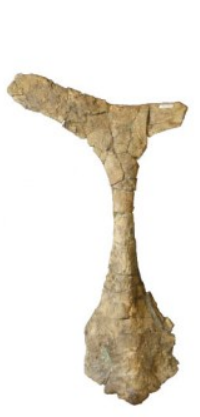

J)

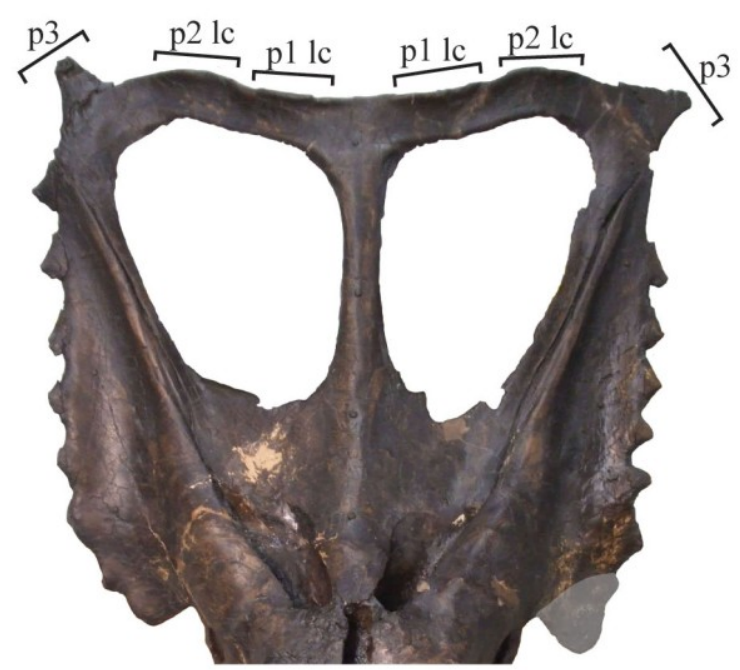

K)

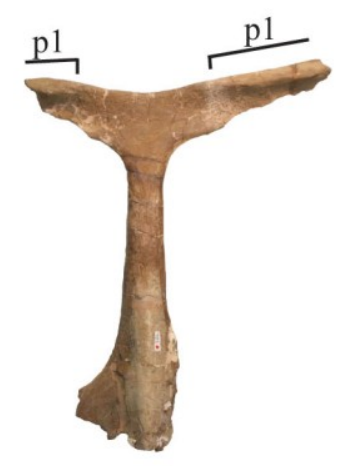

L)

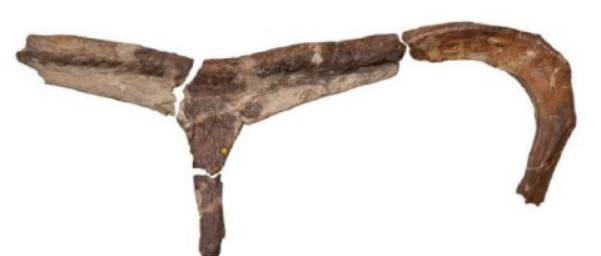


Figure 33. (continued).

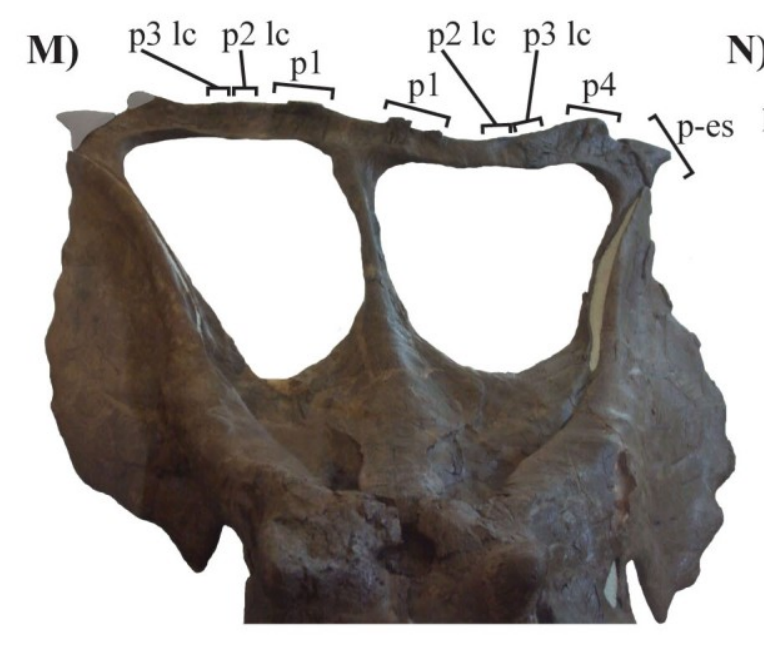

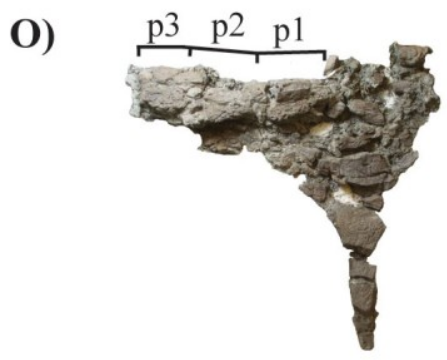

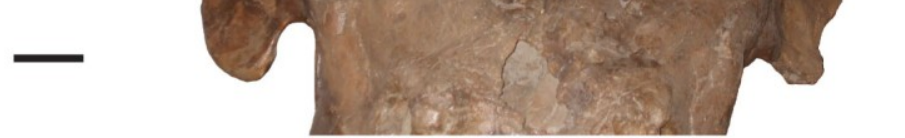

Q)

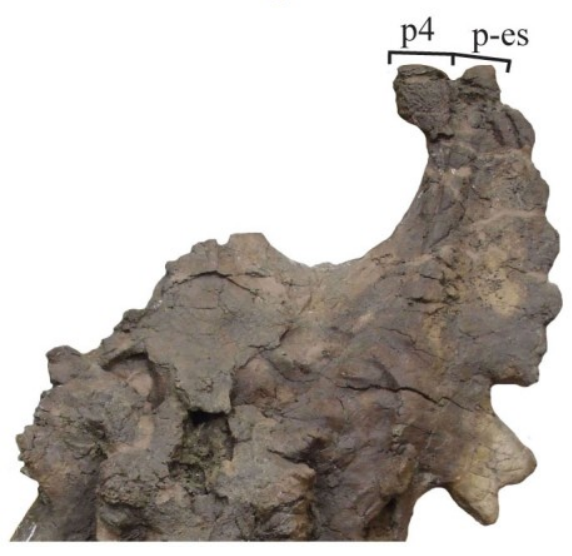

P)

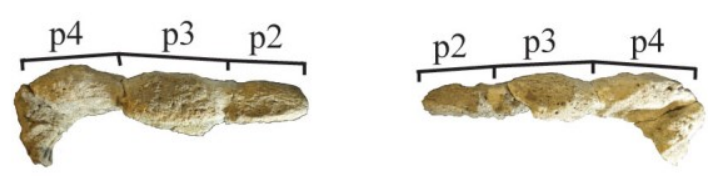

R)

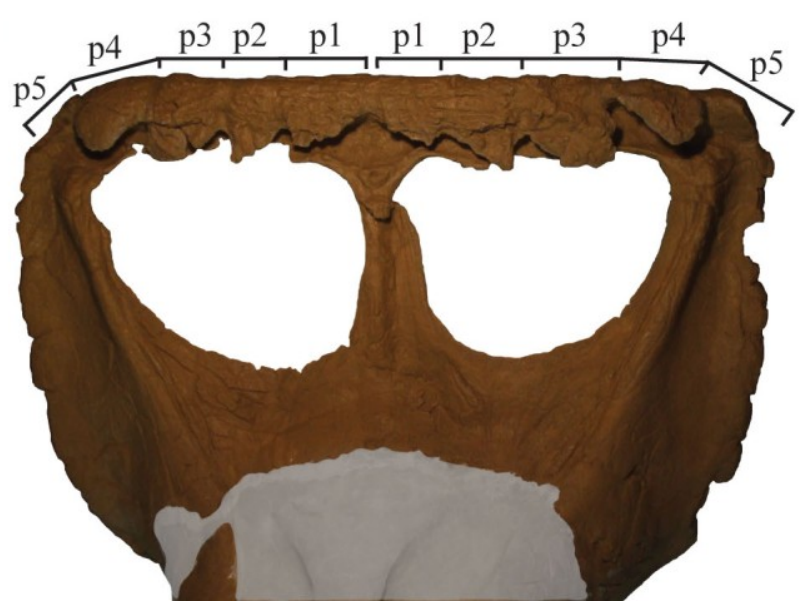


Frill epiossifications

In addition to the relative embayment of the posterior parietal bar, the transverse widths of the associated epiparietals and their position in relationship to the midpoint of the posterior parietal margin are also indicated in Figure 33. Skulls with, and those inferred as having, a total of three epiparietals per side are associated with both deeply and shallowly embayed (nearly straight) posterior bars, while skulls with, and those inferred as having, a total of five epiparietals per side are associated only with relatively straight posterior bars.

Almost every skull previously referred to Chasmosaurus have, or are assumed to have, three epiparietals on each side of the posterior parietal bar, for a total of six (Fig. 33). In these specimens, epiparietals vary in size both within and between individuals. For example, in TMP 1983.025.0001 (Fig. 33F), epiparietals are nearly identical in size, while in others (e.g., AMNH 5656, CMN 8800 and ROM 843), there is a large discrepancy in epiparietal size. Furthermore, the positioning of relatively small or large epiparietals on a given skull is also inconsistent, e.g., in AMNH 5656 (Fig. 33E), P1 has the largest basal width, while P2 is approximately half that width; the basal width of the P3 locus exceeds that of $\mathrm{P} 2$, indicating that $\mathrm{P} 3$ would have been larger than $\mathrm{P} 2$ and more similar in size to P1. In ROM 843 (Fig. 33H), however, the basal width of P1 and P2 are similar in size, but both are at least twice the width of P3; the ridge on the medial portion of the posterior bar in ROM 843 is here interpreted as a well-articulated P1 that was remodeled in life. 
As previously noted, CMN 2245 (Fig. 9) has three marginal undulations on the posterior parietal bar, with only the lateralmost position occupied by an epiparietal. Given the similarity in width and appearance amongst the three, the two medialmost undulations are here interpreted as unoccupied epiparietal loci. If this interpretation is accurate, then their equal widths suggest that the two medialmost epiparietals had basal widths similar in size to that of the preserved lateral-most one. Given the open suture between the lateral epiparietal and underlying loci, it is reasonable to assume that the two inferred medialmost epiparietals were also in early stages of articulation with the parietal, allowing them to fall off during burial. The pattern of epiossification in CMN 2245 suggests a lateral-to-medial direction of articulation for epiparietals across the posterior parietal bar, as has been inferred for other chasmosaurines (Sampson et al, 1997). If the above argument is correct, than CMN 2245 differs from both $C$. belli (relatively straight posterior bar) and C. russelli (three roughly equal-sized epiparietals), as diagnosed by Godfrey and Holmes (1995).

The only Chasmosaurus skull that has in excess of three epiparietals preserved on each side (five) is YPM 2016 (Fig. 10). The only chasmosaurines with more than three epiparietals per side of the posterior parietal bar are Anchiceratops (four; Mallon et al., 2011), Vagaceratops (TMP 1987.045.0001 = four to five $=\mathrm{CMN} 41357)$, and Torosaurus (five to six; Scannella and Horner, 2010). The P1 to P4 of YPM 2016 (Fig. 10) are low, anterodorsally oriented rugosities. Although YPM 2016 and CMN 41357 ( $V$. irvinensis) are identical in epiparietal count, the epiparietals in the latter specimen are curved more anteriorly and overlie the posterior parietal bar. However, given the rugose nature of the epiparietals in YPM 2016, it is possible that they were once longer and have 
been modified ontogenetically as seen on the cranial ornamentation of other ceratopsids. In CMN 41357 (Fig. 33R), P5 is positioned medial to the parietosquamosal contact, but closer to it than in YPM 2016 (Fig. 34). Another shared feature between CMN 41357 and YPM 2016 is the presence of a relatively straight posterior bar, $\left(180^{\circ}\right.$ and $171^{\circ}$, respectively; Fig. 33N).

The only other Chasmosaurus skull inferred as having in excess of three epiparietals per side is AMNH 5402 (Fig. 8). The large gap between the medial-most epiparietal (P1) and next lateral epiparietals in this specimen is unusual, because the spacing between epiparietals is nearly uniform in other Chasmosaurus skulls (Fig. 33). The gap in AMNH 5402 is large enough such that two additional epiparietals equal in size to the preserved ones could fit onto this margin. If two additional epiparietals were available at some point in ontogeny to occupy the gap in AMNH 5402, this would be consistent with the uniform inter-epiparietal spacing present in all other Chasmosaurus skulls. Two low-relief parietal undulations occupy the gap on each side of the posterior parietal bar (Fig. 8C-E). These undulations are separated from each other and from the two neighbouring epiparietals (P1 and "P4") at approximately equal intervals. These undulations also possess shallow, transverse grooves at their bases in dorsal view, making the undulations dorsoventrally pinched (Fig. 8C); the presence of such grooves is consistent with the presence of epimarginal loci in other Dinosaur Park Formation chasmosaurine specimens. The low-relief nature of each of these epiparietal loci is consistent with that of the P1 loci on the left side; this epiparietal is missing the middle portion, exposing the underlying parietal margin. The two inferred disarticulated 

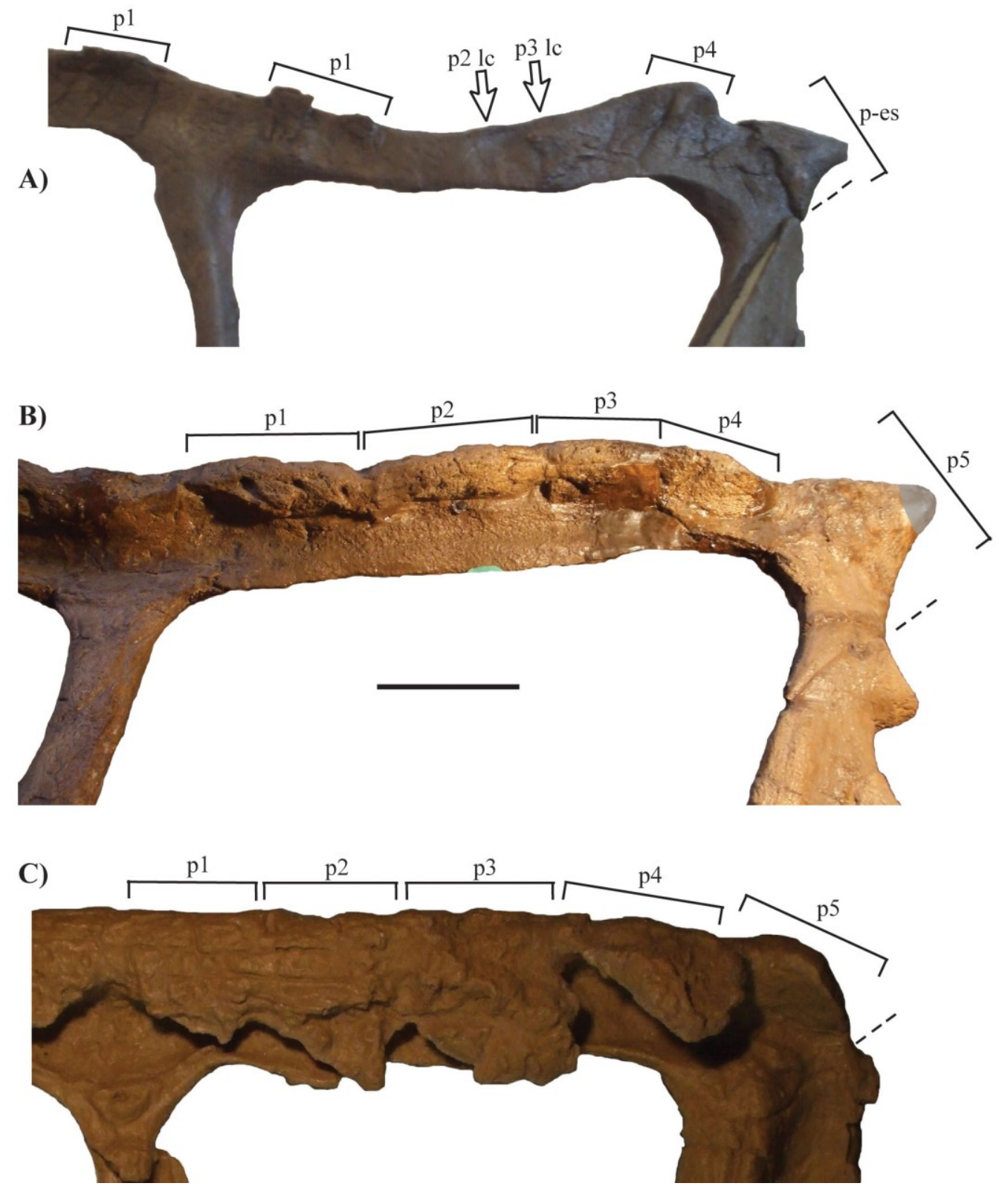

Figure 34. Left posterolateral corner of frill of A) AMNH 5402 (Chasmosaurus belli), B) YPM 2016 (C. belli; reflected) and C) CMN 41357 (Vagaceratops irvinensis holotype; cast). Brackets delimit size of epiossifications; arrows denote inferred epiparietal attachment loci (low-relief undulations). Plaster reconstruction shown in grey half-tone. See "List of anatomical abbreviations". Scale bar equals $10 \mathrm{~cm}$. 
epiparietals on each side of the parietal in AMNH 5402 are similar to the morphology of ROM 839 where all episquamosals are articulated except for two medially positioned (episquamosals 5 and 6) on both squamosals (Fig. 7K). The three preserved epiparietals in AMNH 5402 are situated exactly in the same positions as P1, P4 and P5 in YPM 2016 (Fig. 34), except the "P5" in AMNH 5402 overlies the squamosal and would likely have articulated with this element later in ontogeny, making it an epiparietosquamosal. Finally, both the nearly straight posterior parietal bar and anteroposteriorly shortened parietal fenestrae in AMNH $5402($ ratio = 1.04) are nearly identical to those of YPM $2016(1.03)$ - two features not found in combination in any other Chasmosaurus skull. Based on this reasoning, I infer that AMNH 5402 either had, or would have had, a total of eight epiparietals, but with $\mathrm{P} 2$ and $\mathrm{P} 3$ being absent probably due to lack of articulation prior to death; this epiparietal count is also inferred for TMP 1987.045.0001 (Fig. 33Q).

The hypothesis that AMNH 5402 would have possessed five epiossifications on each side of the parietal (with the fifth lateralmost one straddling the parietal-squamosal contact) will be further tested phylogenetically (Chapter 6) and ontogenetically (Chapter 8). The precise stratigraphic position of AMNH 5402 is not known, so its stratigraphic relationship to Vagaceratops and other Chasmosaurus specimens cannot be determined.

Scannella and Horner $(2010,2011)$ theorized that epiparietal count increases through maturity in their proposed Triceratops-Nedoceratops-Torosaurus synonymy, increasing from three to six per side. One problem with this theory is that there is little room between epiparietals in the putatively immature Triceratops, leaving limited space for more epiparietals to articulate in the putatively mature Torosaurus (Farke, 2011). 
Scannella and Horner (2011) addressed this issue by proposing that epiparietals instead divided into two during late ontogeny; however, they did not process a mechanism (unknown in any other ceratopsian) that would cause this to happen. They did, however, note the existence of a Triceratops episquamosal possessing two peaks (Scannella and Horner, 2011: Fig. 6). They suggested that the base of this episquamosal would have continued to lengthen over ontogeny (sensu Horner and Goodwin, 2006), and would have likely divided into two episquamosals. The right squamosal of NHMUK R4948 (Chasmosaurus) also possesses a double-peaked episquamosal (episquamosal 4; Fig. 7J), as noted by Maidment and Barrett (2011).

Scannella and Horner (2011) extrapolated their proposed splitting of epiossifications to epiparietals, and suggested that the six epiparietals of Triceratops (excluding midline epiparietal) could theoretically develop into the twelve epiparietals of Torosaurus. They also considered the possibility that epiparietal count may be related to stratigraphy, as 10 and 12 epiparietal-bearing specimens are restricted to the base of the Hell Creek Formation, and six-epiparietal bearing (excluding midline epiparietal) specimens occur higher in section. In this study, the stratigraphic distribution of Dinosaur Park Formation chasmosaurines will be examined in Chapter 7 in order to test whether epiparietal count changes consistently over time, transitioning from three (Chasmosaurus-like specimens) to four or five (Vagaceratops-like specimens).

Unlike epiparietal loci, the number of episquamosal loci in Chasmosaurus is not as conservative, ranging from seven (e.g., AMNH 5656 and $\mathrm{CMN} 2280)$ to 10 (CMN 8800), with intermediates known as well (e.g., YPM 2016, eight; AMNH 5402, nine). Scannella and Horner (2010) noted that episquamosal loci count also varies in their 
Triceratops-Torosaurus synonymy, ranging from four to seven, and proposed that this count increases through ontogeny. Farke (2011) disagreed with Scannella and Horner's (2010) proposal, noting that some juvenile Triceratops specimens possess five to seven loci; he instead attributed episquamosal loci count to individual variation. Farke (2011) also noted that in Agujaceratops, some juvenile or subadult specimens possess six episquamosal loci, while one putative adult specimen has ten; he suggested that, given the small sample size, ontogenetic and individual variation are equally possible in accounting for loci count. The small Chasmosaurus squamosal TMP 1998.128.0001 (squamosal length $=208 \mathrm{~mm}$ ) possesses nine loci (Fig. 12B); this count is similar to, and in some cases exceeds that of larger specimens (e.g., YPM 2016, CMN 8800; and AMNH $5656, \mathrm{CMN} 2280$, respectively). Therefore, there is no evidence to support the notion that episquamosal loci count increases through ontogeny in Chasmosaurus.

Articulation of episquamosals varies in Chasmosaurus, e.g., with the successively larger skulls CMN 1254 and TMP 1998.128.0001 having none articulated, AMNH 5656 and AMNH 5401 having all but the posteriormost one articulated, and CMN 2280 and CMN 8800 have all articulated. This distribution of episquamosals indicates that their articulation is size-related, as in other ceratopsids (Sampson et al., 1997; Horner and Goodwin, 2006). When episquamosals are preserved, their sutural contact with the squamosal becomes increasingly distinct towards the posterior margin (e.g., AMNH 5656), suggesting an anterior to posterior direction pattern of articulation for episquamosals, as in other chasmosaurines (Sampson et al., 1997).

While the episquamosal direction of articulation is predictable in Chasmosaurus, the same cannot be said of the epiparietals. While the traditionally accepted pattern of 
articulation (lateral to medial; Sampson et al., 1997) can be inferred for most Chasmosaurus skulls, e.g., CMN 2245, several examined in this study deviate from this pattern. The epiparietals of AMNH 5656 (Fig. 33E) appear to have articulated in a medial-to-lateral pattern, because $\mathrm{P} 1$ and $\mathrm{P} 2$ are articulated, but the $\mathrm{P} 3$ loci is unoccupied. The same direction of articulation seems to occur in some other specimens, such as CMN 2280 (Fig. 33G) and ROM 843 (Fig. 33H). In these two latter specimens, all three epiparietals have articulated, but their basal sutures become increasingly obvious progressing laterally. The orientation of the medialmost epiparietals also varies amongst chasmosaurine skulls. Amongst preserved Chasmosaurus posterior parietal bars (Fig. 33A-N), about half of them (AMNH 5402, AMNH 5656, CMN 8803, TMP 1983.025.0001, TMP 1997.132.0002, TMP 1999.055.0292) have epiparietals or loci that are all oriented in the plane of the frill. The medialmost epiparietals are oriented gently to strongly anterodorsally in all other preserved specimens (CMN 0491, CMN 2245, CMN 2280, CMN 8800, NHMUK R4948, ROM 843, and YPM 2016). Amongst specimens with the recurved epiparietals, those with three epiparietals (CMN 2245, CMN 2280, CMN 8800, ROM 843, and possibly CMN 0491 and NHMUK R4948) exhibit recurving in P1 and sometimes P2, while those with five (YPM 2016, as well as Vagaceratops CMN 41357 and likely TMP 1987.045.0001) exhibit recurving in P1-P4 (Fig. 34).

The modification and articulation of adjacent epiparietals can form a thickened ridge on each side of the posterior parietal bar in some ceratopsids. In Chasmosaurus, where preserved, these either take the form of an anterodorsally-thickened ridge on either side of the midline bar (e.g., Chasmosaurus, CMN 0491, CMN 2280, CMN 8800, and ROM 843), or one confluent ridge bordering the entire posterior margin, e.g., NHMUK 
R4948 and YPM 2016. A continuous ridge is also present in Vagaceratops (CMN 41357). Continuous ridges only occur in specimens with 10 epiparietals; interpreted ridges only occur on specimens with three epiparietals. Unfortunately, the number of epiparietals in CMN 0491 and NHMUK R4948 cannot be reliably determined.

Accessory frill fenestrae and cranial pitting

In Chasmosaurus, accessory fenestrae in the squamosals occur in several specimens (NHMUK R4948, ROM 839, ROM 843, and TMP 1983.025.0001). In all four of these skulls, the fenestra occurs in the left squamosal. The potential causes of this feature in ceratopsids were evaluated by Tanke and Farke (2007) and they concluded that either non-pathological bone resorption or a pathological process resulting from some form of bone disease were the most likely causes for the feature.

Other apparent abnormalities in Chasmosaurus skulls include pitting on nasal and postorbital horncores. In Chasmosaurus, both accessory fenestrae and pitted nasal and postorbital horncores are found only in relatively large skulls, and therefore may be related to ontogeny. These features are discussed further in Chapter 8.

Lower jaw

Chasmosaurus predentaries vary in relative lengths, ranging from approximately $28 \%(\mathrm{CMN} 2245)$ to $35 \%$ (AMNH 5402) of the total length of the lower jaw (Fig. 35AD). Holmes et al. (2001) noted that the Vagaceratops holotype (CMN 41357) had a relatively long predentary, comprising approximately $45 \%$ of the total length of the predentary; however, upon personal examination, this percentage is approximately $40 \%$ 
(Fig. 35E). While Vagaceratops has a larger predentary/lower jaw length ratio than any specimen of Chasmosaurus, it is not as large as previously reported. 
Figure 35. Chasmosaurus and Vagaceratops lower jaws: A) left side of CMN 2245 (Chasmosaurus belli) in lateral view; B) left side of CMN 2280 (Chasmosaurus russelli) in lateral view; C) right side of ROM 839 (Chasmosaurus brevirostris holotype) in lateral view (flipped); D) right side of AMNH 5402 (C. belli) in medial view; and E) right side of CMN 41357 (Vagaceratops irvinensis holotype) in lateral view (flipped). Image of CMN 2245 and ROM 839 courtesy of Jordan Mallon; image of CMN 41357 modified from Holmes et al. (2001: Fig. 2B). Dashed lines represent outline of lower jaw and predentary (pd) within it. Checkered line below each jaw shows ratio of predentary length vs. lower jaw length. Scale bars (superimposed over lower jaws) equal $10 \mathrm{~cm}$. 
Figure 35.

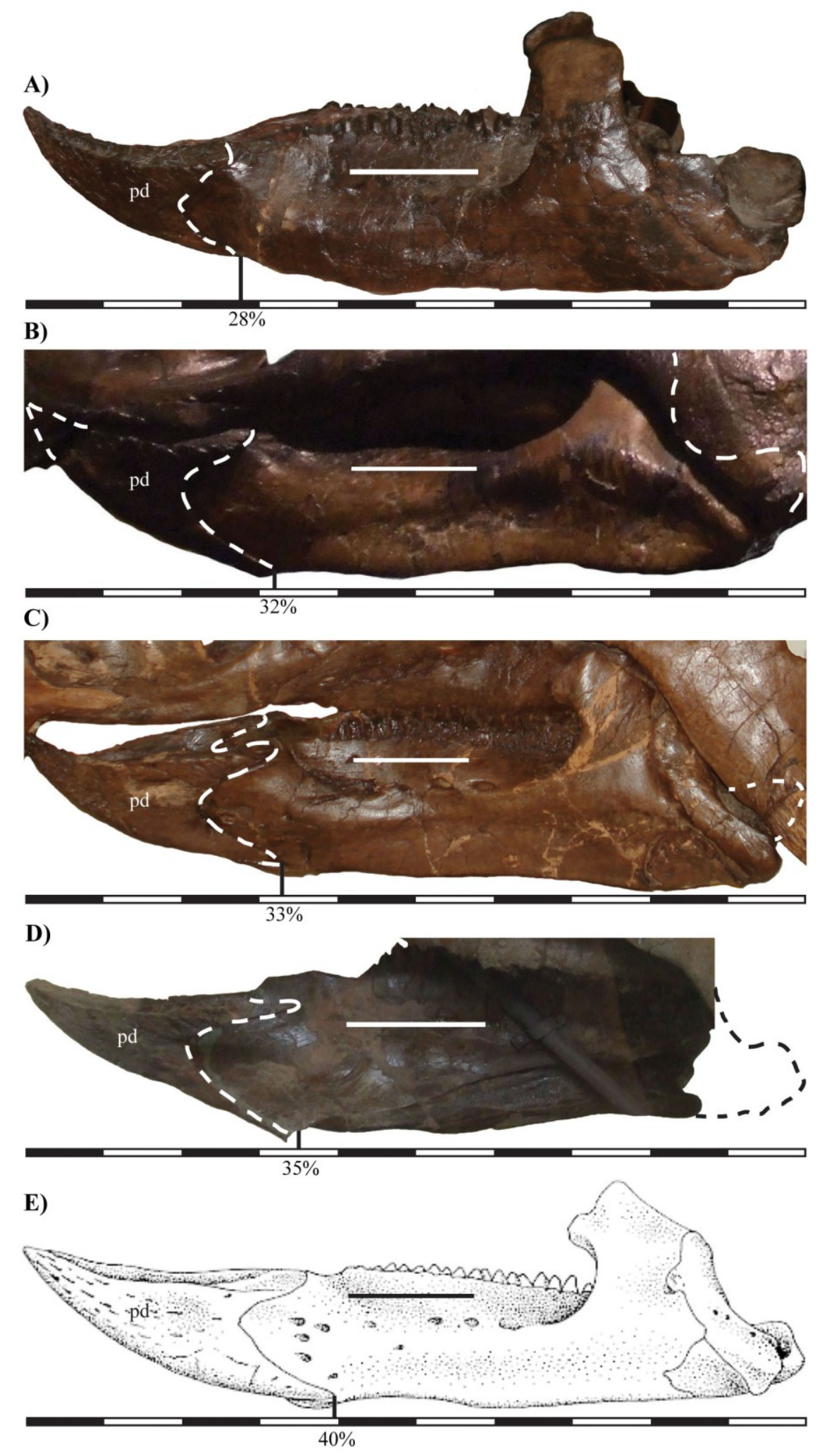




\section{Chapter 6. Phylogenetic Relationships of Chasmosaurus and Vagaceratops Specimens}

\subsection{Introduction}

This chapter discusses the phylogenetic relationships within and between chasmosaurine specimens from the Dinosaur Park Formation. The character-taxon matrix of Mallon et al. (2011) was used, modified from Sampson et al. (2010) by the addition of one character (149), consisting of 149 (127 cranial, 22 postcranial) characters for 14 chasmosaurines (Agujaceratops mariscalensis, Anchiceratops ornatus, Arrhinoceratops brachyops, Coahuilaceratops magnacuerna, Eotriceratops xerinsularis, Kosmoceratops richardsoni, Nedoceratops hatcheri, Ojoceratops fowleri, Pentaceratops sternbergi, Torosaurus latus, Torosaurus utahensis, Triceratops horridus, Triceratops prorsus, and Utahceratops gettyi), three centrosaurines (Albertaceratops nesmoi, Centrosaurus apertus and Pachyrhinosaurus lakustai) and four outgroup taxa (Leptoceratops gracilis, Protoceratops andrewsi, Turanoceratops tardabilis, and Zuniceratops christopheri). The two recently described chasmosaurines Judiceratops tigris (Longrich, 2013) and Bravoceratops polyphemus (Wick and Lehman, 2013) were also included in this analysis.

Seventeen specimens with complete or relatively complete frills or skulls, or with historical taxonomic significance (e.g., holotypes) were used to conduct a specimenbased phylogenetic analysis on these specimens. Included in the analysis were:

Chasmosaurus belli, AMNH 5402, CMN 0491, CMN 2245, NHMUK R4948, ROM 843, YPM 2016; Chasmosaurus russelli, AMNH 5656, CMN 2280, CMN 8800, TMP 1983.025.0001; Chasmosaurus brevirostris, ROM 839; Chasmosaurus kaiseni, AMNH 
5401; Chasmosaurus canadensis, AMNH 5401, CMN 1254, UALVP 40; Chasmosaurus sp., AMNH 5401, CMN 1254, ROM 839, TMP 1981.019.0175, UALVP 40; Eoceratops canadensis, CMN 1254, UALVP 40; Mojoceratops perifania, AMNH 5401, AMNH 5656, CMN 1254, TMP 1983.025.0001; and Vagaceratops irvinensis, CMN 41357, TMP 1987.045.0001.

Wick and Lehman (2013) used two additional characters in their study, only one of which (character 150) was retained for this study. Their character 151 (morphology of the nasal bridge in dorsal view) was determined to be parsimony uninformative, and was therefore excluded.

The Mallon et al. (2011) matrix includes nine characters (95-103) that describe epiparietals 0-3, but none that describe epiparietals 4 and 5. I reasoned that adding characters for epiparietals 4 and 5 (151-153) was justified because they do not describe autapomorphies, and are therefore parsimony informative.

The 153 characters and their descriptions are listed in Appendix III. Additionally, several character descriptions were modified from their original descriptions resulting in the recoding of some characters for some taxa. The rationales for these changes are given in Appendix III. The snout region of the skull is shown schematically in Figure 36; several characters refer to this region. The complete character-taxon matrix is shown in Table 2.

This analysis was performed in TNT 1.1 (Goloboff et al., 2003) using the tree bisection reconnection swapping algorithm using 1000 replicates, with 10 trees saved per replication, and the maximum number of trees stored set at 10,000. 


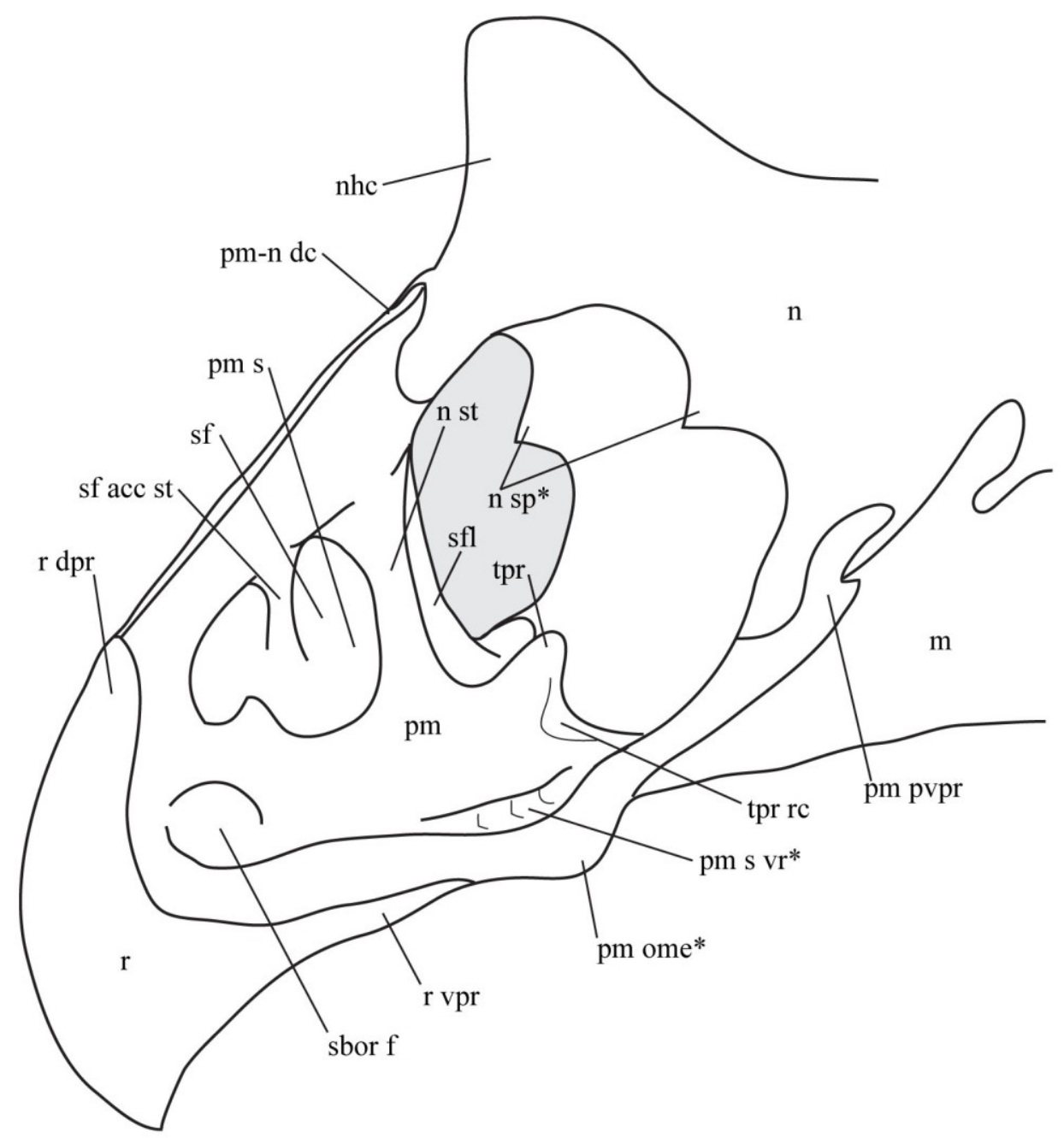

Figure 36. Schematic of an idealized chasmosaurine snout (Lehman, 1998: Fig.8, modified). Centrosaurine features (i.e., not present in chasmosaurines) are indicated by asterisks (*) (Lehman, 1990: Fig. 16.1). See "List of anatomical abbreviations". 
Table 2: Character-taxon matrix used in phylogenetic analysis (modified after Mallon et al., 2011). Dimorphic states for a given character, where present, are enclosed by brackets.

\begin{tabular}{|c|c|c|c|c|}
\hline Taxon & 10 & 20 & 30 & 40 \\
\hline Leptoceratops gracilis & $100 ? ? 0$ ???0 & ??0??01000 & 1 ?001?0?? 0 & 00000 ????? \\
\hline Protoceratops andrewsi & 000 ??0???0 & ??0??01000 & 1100001000 & 00000 ????? \\
\hline Zuniceratops christopheri & 111000 ???0 & ??0??0?010 & $000 ? 100 ? ? 0$ & $? 111100010$ \\
\hline Turanoceratops tardabilis & ?????????? & ?????????? & ???0?0???? & ????1?0110 \\
\hline Albertaceratops nesmoi & ?111?0???0 & ??0??????0 & $1 ? 1011110 ?$ & ?11?1?0011 \\
\hline Centrosaurus apertus & $011110 ? 0 ? 0$ & ??0??11100 & $1 ? 11111101$ & 0111100002 \\
\hline Pachyrhinosaurus lakustai & $011110 ? 0 ? 0$ & ??0??11100 & $1 ? 11111201$ & 0111111 ??? \\
\hline Kosmoceratops richardsoni & 1110011111 & 0011000010 & 0011111100 & 0111110111 \\
\hline Agujaceratops mariscalensis & 1110010101 & 1010000011 & $1 ? 11111100$ & 0111110110 \\
\hline Utahceratops gettyi & 1110010111 & $0 ? 11000011$ & 0011111100 & 0111111102 \\
\hline Pentaceratops sternbergi & 1110010111 & 1111000011 & 0011111100 & 0111110011 \\
\hline Coahuilaceratops magnacuerna & $11100 ? 1 ? ? 1$ & 0????00??1 & ??11111110 & ??1?11??11 \\
\hline Anchiceratops ornatus & $1110011(01) 11$ & 0011000100 & $1 ? 11111100$ & 0111111111 \\
\hline Arrhinoceratops brachyops & $1110011 ? ? 1$ & $001 ? 0001 ? 0$ & $1 ? 111 ? 1100$ & $01111 ? 1111$ \\
\hline Ojoceratops fowleri & 11 ? ?0????? & ?????????0 & ??????111? & ?1???????? \\
\hline Torosaurus latus & $1110010 ? ? 1$ & $11 ? ? ? 0010 ?$ & $1 ? 11111110$ & $0111111(01) 11$ \\
\hline Torosaurus utahensis & ?????????? & ?????????? & ?????????? & ???11?1011 \\
\hline Eotriceratops xerinsularis & $11100100 ? 1$ & $1011000 ? ? 0$ & ??111111?? & 011?111?11 \\
\hline Nedoceratops hatcheri & $11100100 ? 1$ & $1111100 ? 0 ?$ & $1 ? 111 ? 1110$ & $011 ? 1 ? 1011$ \\
\hline Triceratops horridus & $11100100 ? 1$ & 1111100100 & $1 ? 11111110$ & 0111111011 \\
\hline Triceratops prorsus & $11100100 ? 1$ & 1111100100 & $1 ? 11111110$ & 0111111011 \\
\hline Judiceratops tigris & ?????????? & ?????????? & ?????????? & ????1101?0 \\
\hline Bravoceratops polyphemus & ?1???????? & ?????????? & ??????111? & ????111?1? \\
\hline AMNH 5401 & 1110010101 & 0010000010 & $00111 ? 1100$ & $11111 ? 0110$ \\
\hline AMNH 5402 & 1110010101 & $? 010000 ? 10$ & $1 ? 111 ? 1100$ & $11111 ? 000 ?$ \\
\hline AMNH 5656 & ?????????? & ?????? ???? & ?????????? & 1????????? \\
\hline CMN 0491 & ?????????? & ?????????? & ?????????? & ?????????? \\
\hline CMN 1254 & ?1 ? ?0? ???? & ????????? 0 & ??????1100 & ?11?110010 \\
\hline CMN 2245 & ?????????? & ?????????? & ??????11?? & ?1111?0002 \\
\hline CMN 2280 & 1110010101 & $? 010000010$ & $1 ? 11111100$ & 1111110000 \\
\hline CMN 8800 & 1110010101 & ?010000?1? & $00111 ? 1100$ & $1111110 ? ? ?$ \\
\hline CMN 41357 & $111001 ? 101$ & 0010000010 & ??1?111100 & 1111?????? \\
\hline NHMUK R4948 & 1110010101 & ?010000??0 & ??11111100 & ?111110??? \\
\hline ROM 839 & 1110010101 & 0010000010 & $1 ? 11111100$ & $11111 ? 0000$ \\
\hline ROM 843 & ?1100??1?? & ??????0010 & $1 ? 11111100$ & 11111 ?0??? \\
\hline TMP 1981.019 .0175 & 1110010101 & 0010000010 & 1111111100 & $111111010 ?$ \\
\hline TMP 1983.025 .0001 & ?1 ???????? & ?????????? & ?????????? & ?11111011? \\
\hline TMP 1987.045 .0001 & $111001 ? 101$ & $0010000 ? ? 0$ & $1 ? 11111100$ & ?11??????? \\
\hline UALVP 40 & 1110010101 & 0010000010 & $1 ? 11111100$ & $11111 ? 0100$ \\
\hline YPM 2016 & 1110011101 & $0010000 ? 10$ & $1 ? 11111100$ & $1111110 ? ? ?$ \\
\hline
\end{tabular}




\section{Table 2 (continued).}

\begin{tabular}{|c|c|c|c|c|c|}
\hline Taxon & 50 & 60 & 70 & 80 & 90 \\
\hline L. gracilis & $? 000 ? 00000$ & $00000 ? 00 ? ?$ & $000000 ? 000$ & 00000 ????? & ?000?????? \\
\hline P. andrewsi & $? 000 ? 00000$ & $00000 ? 0100$ & $110000 ? 000$ & 0010000000 & 0000 ?????? \\
\hline z. christ... & 2111011?0? & ?11?0????? & ??0??????? & ?01?01000? & ???0?????? \\
\hline T. tardabilis & 2????????? & ??1 ?0????? & ?????????? & ?????????? & ?????????? \\
\hline A. nesmoi & $2110 ? 11112$ & $111 ? ? ? 1100$ & $111001011 ?$ & 1011110011 & $11110 ? 0$ ??? \\
\hline C. apertus & $2110 ? 11112$ & 1111101100 & 1110010110 & 1011110011 & $11110 ? 0000$ \\
\hline P. lakustai & ?111111112 & ?111101100 & 1110010110 & 1011110011 & 1111??0??? \\
\hline K. richardsoni & 1111110110 & 1111011112 & 0010111211 & 1011100010 & $01(01) 1111321$ \\
\hline A. maris... & 0111110110 & 1111??111? & $1011112 ? 11$ & ??1110?201 & 11011?111? \\
\hline U. gettyi & 2111110110 & ?111111111 & 1011112111 & 1011101201 & 1101111221 \\
\hline P. sternbergi & $211111(01) 111$ & 1111111111 & 1011112211 & 1011101201 & 1101111221 \\
\hline C. magna... & ?1 ???? ???? & ???????11? & ?01?1????? & ?01?1??201 & 1101??1??? \\
\hline A. ornatus & 1111110110 & 1111111112 & $101110 ? 111$ & 1011110011 & 0101111220 \\
\hline A. brachyops & 1111110110 & 1111011112 & ?01110?111 & 1011110011 & $01011 ? 1110$ \\
\hline o. fowleri & ??111????? & ???????112 & 0 ?101????? & ????1100?? & ?1011?1110 \\
\hline T. latus & 2111110112 & $111 ?(01) 11111$ & $101110 ?(01) 12$ & 1010110312 & $01011 ? 1110$ \\
\hline T. utahensis & 2111110112 & ?1??1??11? & $101110 ? 012$ & 1010110312 & $01011 ? 1111$ \\
\hline E. xerin... & $21 ? 111 ? 110$ & ???????11? & 1???1 ????? & ?????????? & ?1011?1??? \\
\hline N. hatcheri & $2 ? 11110 ? 12$ & ?1?1111111 & 1?101???02 & $1 ? 11110311$ & 01011?1110 \\
\hline T. horridus & 2111110112 & 1111111111 & $101010 ? 002$ & 11001 ????? & ?1011?1110 \\
\hline T. prorsus & 2111110112 & 1111011111 & $101010 ? 002$ & 11001 ????? & ?1011?1110 \\
\hline J. tigris & 2?11??1?1? & ??1????111 & 1 ?11?0???? & ?011100??? & ?1011?1110 \\
\hline B. polyphemus & ??????0?11 & 1?????111? & ??1?1???1? & ?01?1??201 & 11011?1??? \\
\hline AMNH 5401 & 2111111110 & 1111111111 & $10 ? 11$ ???12 & $101110 ? 101$ & $11011 ? 1 ? 10$ \\
\hline AMNH 5402 & 2111111112 & 1111111111 & $10 ? 110 ? ? 12$ & 1011100102 & $11010 ? 1 ? 10$ \\
\hline AMNH 5656 & ?????1??12 & 1???1?1111 & $10 ? 1111112$ & ?011100101 & $11011 ? 1 ? 10$ \\
\hline CMN 0491 & ?????????? & ?????????? & ?????11??? & ?01?10010? & 1101 ?????? \\
\hline CMN 1254 & 21111? ???? & ??1111?111 & 10111???1? & ?01 ??????? & 1101?????? \\
\hline CMN 2245 & 211111111? & 1111111111 & 1011111112 & 1011100101 & $11010 ? 1110$ \\
\hline CMN 2280 & 2111111112 & 1111111111 & 1011111110 & 1011100101 & $11011 ? 1110$ \\
\hline CMN 8800 & ?1111??1?? & ?111111111 & $10 ? 1112112$ & 1011100101 & $11011 ? 1110$ \\
\hline CMN 41357 & ?111111?12 & 1111111111 & 101110??12 & $? 011100000$ & $11011 ? 1110$ \\
\hline NHMUK R4948 & ?111111112 & $11111 ? 1111$ & 1011111112 & ?01?100101 & $11011 ? 1110$ \\
\hline ROM 839 & 2111111110 & 1111111111 & 10111???12 & 1011100101 & $11011 ? 1110$ \\
\hline ROM 843 & ?111111110 & 1111111111 & 1011111210 & 1011100102 & $11011 ? 1110$ \\
\hline TMP 81.19 .175 & 2111111112 & 111111111? & ?0111???1? & 10111??10? & 11010 ?1??? \\
\hline TMP 83.25 .1 & 2111111112 & 1111111111 & 1011111110 & 1011100101 & $11010 ? 1110$ \\
\hline TMP 87.45 .1 & ?111111112 & 1111111111 & 10111 ???12 & 101110???0 & $11011 ? 1110$ \\
\hline UALVP 40 & 2111111112 & 11111?111? & ?0111???1? & ?0111????? & ?1010?1??? \\
\hline YPM 2016 & ?111111112 & 1111111111 & $101110 ? ? 12$ & 1011100102 & $11011 ? 1110$ \\
\hline
\end{tabular}




\section{Table 2 (continued).}

\begin{tabular}{|c|c|c|c|c|c|}
\hline Taxon & 100 & 110 & 120 & 130 & 140 \\
\hline L. gracilis & ?????????? & ???000?000 & 0000000000 & $000001 ? 000$ & $000000000 ?$ \\
\hline P. andrewsi & ?????????? & ???00000000 & 0000000000 & 0000010000 & 0000000000 \\
\hline Z. christopheri & ?????????? & ????11?1?? & ?1?0011011 & ??001????1 & 100 ??????? \\
\hline T. tardabilis & ?????????? & ?????????? & ????0????? & ??1 (01) 11???? & ?????????? \\
\hline A. nesmoi & 101000 ???? & ?10??1?1?? & ?1?11011?1 & ?? ?111???? & ?????????? \\
\hline C. apertus & 1010011132 & 1001111111 & 1111101111 & 1111111111 & 1101111111 \\
\hline P. lakustai & 1 ?1000???2 & 1101011111 & $1 ? 11101111$ & $11111111 ? 1$ & ?10??11111 \\
\hline K. richardsoni & 1101012132 & 221??????? & ?11?011?11 & 1 ?1?11???? & ????1????? \\
\hline A. mariscalensis & 0???01???? & ???111111? & ??110?1111 & ??1?111111 & 110 ???? 111 \\
\hline U. gettyi & $0 ? 00013133$ & 030 ???1111 & ?111011111 & 111111???1 & ?10??????1 \\
\hline P. sternbergi & $0 ? 00013133$ & $030 ? 111111$ & 1111011111 & 1111111111 & $110 ? 111111$ \\
\hline C. magnacuerna & ?????????? & ?????????? & ??1?011111 & 1???11???? & ?10??????? \\
\hline A. ornatus & $(01) 2(01) 0013133$ & 0301111111 & $11 ? 1 ? ? 1 ? 11$ & ???111???? & ?????????? \\
\hline A. brachyops & $0 ? 00014004$ & 0401111111 & 1 ?? ??????? & ????11???? & ?????????? \\
\hline o. fowleri & ??0011400? & ? 40 ? ? ????? & ?111011?11 & 1???11???? & ??0 ? ? ? ? ?? \\
\hline T. latus & $0 ? 10014004$ & 0401111111 & 11 ?1?????? & ??1111???1 & ?10??????? \\
\hline T. utahensis & 1210114004 & 040 ? ? ?? ??? & ?111?11111 & ??1?111?11 & ?? ???????? \\
\hline E. xerinsularis & 12? ??? ???? & ?? ?? ? ? ? ?? & ??????? ? ?? & ????1111?? & ?? ?? ?? ? ?? \\
\hline N. hatcheri & 1????1 ???? & ??????111? & ?????????? & ?? ?? ?1 ?? ?? & ?????????? \\
\hline T. horridus & 1200114004 & 0401111111 & 1111011111 & 1111111111 & 1111111111 \\
\hline T. prorsus & 1200114004 & 0401111111 & 1111011111 & 1111111111 & ? ?1 ? ? ? ? ? ? \\
\hline J. tigris & ??00 ?? ?0? 4 & 0 ??1 ?????? & ?????????? & ?????????? & ?????? ? ??? \\
\hline B. polyphemus & ????1????? & ???1111??? & ????????11 & ?????????? & ?????????? \\
\hline AMNH 5401 & 0????????? & ????11?1?1 & ??? ??????? & ????11???? & ?? ????? ??? \\
\hline AMNH 5402 & $121001000 ?$ & ????11?1?1 & ?1110?1111 & 11??11???? & ?????????? \\
\hline AMNH 5656 & ??00013003 & 0 ?0?????1? & ?????????? & ????11???? & ?????????? \\
\hline CMN 0491 & ????01010? & ?????????? & ?????????? & ?????????? & ?????????? \\
\hline CMN 1254 & ?????????? & ?????????? & ?11???1?11 & 111??????? & ?????????? \\
\hline CMN 2245 & $0 ? 0001 ? 1 ? ?$ & ?30111???? & ?111011111 & 1111111111 & $11001 ? ? 1 ? 1$ \\
\hline CMN 2280 & $0 ? 00013103$ & $030 ? 11 ? ? ? 1$ & ?1110?1111 & 1111111111 & $110 ? 1 ? ? 111$ \\
\hline CMN 8800 & $0 ? 00010100$ & 030 ?????? 1 & ??? ? ?? ? ?? ? & ?????????? & ?? ?? ?? ? ? ? \\
\hline CMN 41357 & $0 ? 11012132$ & 221?????? 1 & ?111011111 & $11 ? 1111111$ & ?10?1111?? \\
\hline NHMUK R4948 & 0????????? & ??????1 ??1 & ??110???11 & ??1?111111 & $11 ? 01 ? ? ? ? 1$ \\
\hline ROM 839 & 0??0 ?????? & ???111?111 & ?111011111 & 111111???? & ?????????? \\
\hline ROM 843 & $0 ? 00010103$ & ?30?11?111 & ?11???1111 & $11111111 ? 1$ & ?1001111?1 \\
\hline TMP 81.19 .175 & ?????????? & ???111?111 & ?????????? & ??1111???? & ?? ????? ??? \\
\hline TMP 83.25 .1 & $0 ? 00013003$ & 030??????? & ?????????? & ?????????? & ?????????? \\
\hline TMP 87.45 .1 & 12???????? & ????11???1 & ?????????? & ???111???? & ?????????? \\
\hline UALVP 40 & ?????????? & ???111?1?1 & ??110?1?11 & ??1111???? & ?????????? \\
\hline YPM 2016 & $0 ? 10010100$ & 001111?1?1 & ?????????? & ??1111???? & ?????? ?? ?? \\
\hline
\end{tabular}




\section{Table 2 (continued).}

$\begin{array}{lll}\text { Taxon } & 150 & 153 \\ \text { L. gracilis } & 0 ? 000000(01) \text { ? } & \text { ??? } \\ \text { P. andrewsi } & 000000000 ? & \text { ??? } \\ \text { Z. christopheri } & \text { ??01?0??1? } & \text { ??? } \\ \text { T. tardabilis } & \text { ????????? } & \text { ??? } \\ \text { A. nesmoi } & \text { ?????????0 } & 000 \\ \text { C. apertus } & 11011111(01) 0 & 000 \\ \text { P. lakustai } & 110111 ? ?(01) 0 & 000 \\ \text { K. richardsoni } & \text { ????????10 } & \text { ??? } \\ \text { A. mariscalensis } & \text { ?1111??1? } & \text { ??? } \\ \text { U. gettyi } & \text { ??11111?1? } & \text { ??? } \\ \text { P. sternbergi } & \text { 1111?11??0 } & \text { ??? } \\ \text { C. magnacuerna } & \text { ????????1? } & \text { ??? } \\ \text { A. ornatus } & \text { ????????00 } & \text { 20? } \\ \text { A. brachyops } & \text { ?????????0 } & \text { ??? } \\ \text { O. fowleri } & \text { ????????1? } & \text { ??? } \\ \text { T. latus } & \text { ?????????(01) } & \text { 302 } \\ \text { T. utahensis } & \text { ????????10 } & \text { 302 } \\ \text { E. xerinsularis } & \text { ?????????? } & \text { ??? } \\ \text { N. hatcheri } & \text { ?????????0 } & \text { ??? } \\ \text { T. horridus } & \text { 111111111(01) } & \text { ??? }\end{array}$

$\begin{array}{lll}\text { Taxon } & 150 & 153 \\ \text { T. prorsus } & \text { ???????11(01) } & \text { ??? } \\ \text { J. tigris } & \text { ?????????? } & \text { ??? } \\ \text { B. polyphemus } & \text { ????????11 } & \text { ??? } \\ \text { AMNH } 5401 & \text { ?????????0 } & \text { ??? } \\ \text { AMNH } 5402 & \text { ????????10 } & \text { 001 } \\ \text { AMNH } 5656 & \text { ?????????? } & \text { ??? } \\ \text { CMN 0491 } & \text { ?????????? } & \text { ??? } \\ \text { CMN 1254 } & \text { ????????1? } & \text { ??? } \\ \text { CMN 2245 } & \text { ?1??111110 } & \text { ??? } \\ \text { CMN 2280 } & \text { 11111???10 } & \text { ??? } \\ \text { CMN 8800 } & \text { ?????????? } & \text { ??? } \\ \text { CMN 41357 } & \text { ?11??11?10 } & \text { 111 } \\ \text { NHMUK R4948 } & \text { ????111??0 } & \text { ??? } \\ \text { ROM 839 } & \text { ????????10 } & \text { ??? } \\ \text { ROM } 843 & \text { ?111111?10 } & \text { ??? } \\ \text { TMP 81.19.175 } & \text { ?????????0 } & \text { ??? } \\ \text { TMP 83.25.1 } & \text { ?????????0 } & \text { ??? } \\ \text { TMP 87.45.1 } & \text { ?????????0 } & \text { 111 } \\ \text { UALVP 40 } & \text { ????????10 } & \text { ??? } \\ \text { YPM 2016 } & \text { ?????????0 } & \text { 011 }\end{array}$




\subsection{Results}

In the analysis, 5790 most-parsimonious trees were recovered, and the resulting strict consensus and 50\% majority rule consensus trees (Fig. 37) had a tree length $=283$ steps, a consistency index $=0.63$ and a retention index $=0.78$. Bootstrap and Bremer values for each node are shown in Figure 37; only Bootstrap values of 50\% or higher are given.

The strict consensus tree recovered resembled that of Mallon et al. (2011) in the recovery of the basal position of successively more derived outgroup taxa (Leptoceratops, Protoceratops, Zuniceratops, and Turanoceratops) and the monophyletic clades Centrosaurinae and Chasmosaurinae. The relationships between outgroup taxa was better resolved, with Leptoceratops situated basal to Protoceratops; these two taxa formed a polytomy in Mallon et al. (2011). Centrosaurinae was not as well resolved as in Mallon et al. (2011), forming a polytomy. Resolution within Chasmosaurinae was more robust than in Mallon et al. (2011) with the recovery of fewer polytomic taxa; however, only the genera Torosaurus, Triceratops, Kosmoceratops, and Agujaceratops were not polytomic.

In the strict consensus tree (Fig. 37, left side), Agujaceratops is an outgroup to a clade comprised of the 17 Dinosaur Park Formation chasmosaurine skulls that have all been referred to Chasmosaurus at some time in their history; this latter clade also includes Judiceratops. The members within the Dinosaur Park Formation chasmosaurines + Judiceratops clade form a polytomy, although one clade is present: YPM 2016+(CMN 41357+TMP 1987.045.0001); YPM 2016 is the sister operative taxonomic unit to CMN 41357+TMP 1987.045.0001. 
Figure 37. Specimen-based phylogenetic analysis of Chasmosaurus and Vagaceratops specimens: Strict consensus (left) and 50\% majority rule consensus (right) of 5790 mostparsimonious trees $(\mathrm{TL}=283$ steps, $\mathrm{CI}=0.63, \mathrm{RI}=0.78)$ using 153 cranial $(131)$ and postcranial (22) characters. Bootstrap replicate frequency and Bremer support values are shown below each node; only Bootstrap values of $50 \%$ or higher are given. 
Figure 37.

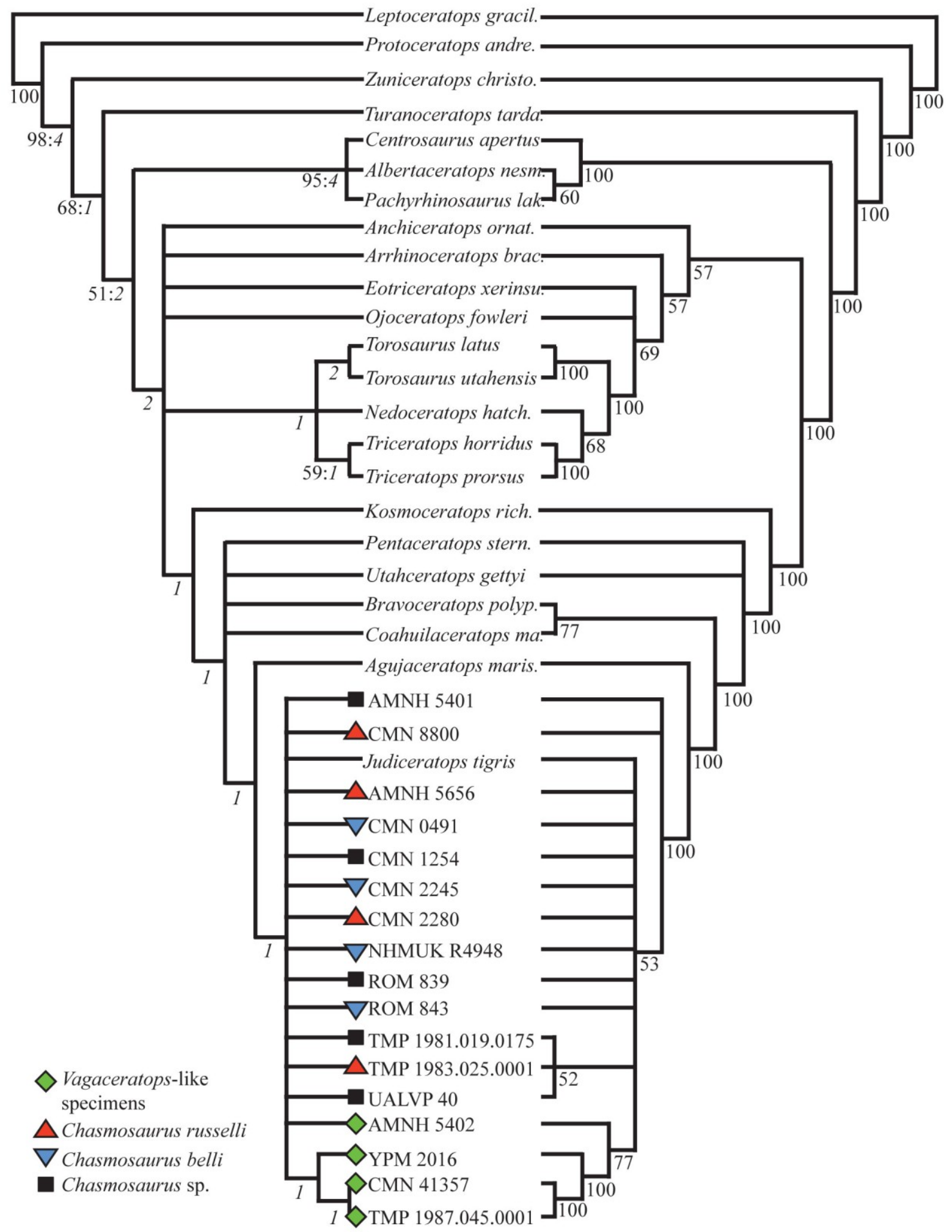


The topology of the $50 \%$ majority rule tree (Fig. 37 , right side) is similar to that of the strict consensus tree, although the former is more resolved. The differences between these two trees are as follows: Centrosaurus is the sister taxon to Albertaceratops and Pachyrhinosaurus (majority rule) versus an unresolved relationship (strict); Anchiceratops, Arrhinoceratops and Eotriceratops + Ojoceratops are successive outgroups to the Torosaurus $+[$ Triceratops + Nedoceratops] clade (majority rule) versus an unresolved relationship (strict); Nedoceratops is the sister taxon to Triceratops horridus + Triceratops prorsus (majority rule) versus an unresolved relationship (strict); and Bravoceratops + Coahuilaceratops are more derived than the unresolved (strict) Pentaceratops and Utahceratops clade. AMNH 5401 and CMN 8800 form a polytomic sister clade to the polytomic clade containing all other members of the Dinosaur Park Formation chasmosaurines + Judiceratops. Within this latter clade is the unresolved polytomic clade of TMP 1981.019.0175+TMP 1983.025.0001+UALVP 40 and the unresolved Vagaceratops-like clade of [AMNH 5402+[YPM 2016[CMN 41357+TMP 1987.045.0001]]].

In the strict consensus analysis, the inclusion of Mojoceratops perifania (TMP 1983.025.0001 and referred specimens AMNH 5401, AMNH 5656 and CMN 1254) and Vagaceratops irvinensis (CMN 41357 and referred specimen TMP 1987.045.0001) within the Dinosaur Park Formation chasmosaurine clade (Fig. 37) differs from Mallon et al. (2011) in which M. perifania formed a polytomy with Chasmosaurus, while Vagaceratops was recovered as the sister taxon to Kosmoceratops. The inclusion of Judiceratops within the Dinosaur Park Formation chasmosaurine clade differs from 
Longrich (2013), where Judiceratops formed a polytomic relationship with Chasmosaurus.

Synapomorphic character states for each clade in the strict consensus tree (Fig. 37, left side) are given below (character number, with the state at the node indicated after the $\operatorname{dot}):$

Protoceratops $+[$ Zuniceratops $+[$ Turanoceratops + Ceratopsidae] $]: 58.1$

Zuniceratops $+[$ Turanoceratops + Ceratopsidae]: 2.1, 3.1, 25.1, 32.1, 33.1, 34.1, 35.1, 42.1, $43.1,46.1,52.1,53.1,106.1,108.1,112.1,117.1,119.1,120.1,125.1,130.1,131.1,144.1$

Turanoceratops + Ceratopsidae: 123.1

Ceratopsidae: 23.1, 26.1, 27.1, 48.1, 49.1, 51.1, 54.1, 57.1, 63.1, 71.1, 75.1, 82.1, 84.1, 104.1, 107.1, 109.1, 110.1, 111.1, 113.1, 114.1, 118.1, 121.1, 122.1, 124.1, 127.1, 128.1, 129.1, 132.1, 135.1, 136.1, 137.1, 138.1, 139.1, 140.1, 141.1, 142.1, 145.1, 146.1, 147.1, 148.1

Centrosaurinae: $1.0,4.1,5.1,6.0,8.0,10.0,13.0,16.1,17.1,18.1,19.0,20.0,21.1,29.0$, 30.1, 31.0, 47.1, 50.2, 55.1, 56.0, 59.0, 60.0, 61.1, 62.1, 64.0, 65.0, 66.1, 67.0, 68.1, 69.1, 70.0, 72.0, 73.1, 74.1, 76.1, 77.0, 78.0, 79.1, 80.1, 81.1, 83.1, 85.0, 87.0, 91.1, 92.0, 93.1, 94.0, 95.0, 103.0, 115.1, 116.0, 133.0, 143.0, 150.0, 151.0, 152.0, 153.0

Chasmosaurinae: $1.1,4.0,5.0,6.1,10.1,13.1,16.0,17.0,24.1,28.1,30.0,36.1,44.1$, $45.1,59.1,62.0,65.1,87.1,96.1,105.1,115.0,116.1,143.1$ 
Triceratops + Nedoceratops + Torosaurus: 7.0, 8.0, 11.1, 12.1, 14.1, 15.1, 18.1, 19.0, 21.1, 29.1, 31.0, 37.1, 39.1, 40.1, 41.2, 47.0, 50.2, 56.1, 60.1, 61.1, 66.0, 70.2, 76.1, 83.0, 85.1, 88.1, 89.1, 94.0, 97.4, 98.0, 99.0, 100.4, 101.0, 102.4, 103.0, 149.1

Torosaurus: 64.1, 69.1, 72.0, 73.1, 74.0, 77.0, 78.3, 79.1, 80.2, 81.0, 93.1, 151.3, 152.0, 153.2

Triceratops: 20.0, 38.0, 64.0, 68.0, 69.0, 72.1, 73.0, 74.0, 90.0, 91.1, 92.2, 93.0, 95.1, 133.1

Kosmoceratops $+\left[\right.$ Bravoceratops + Coahuilaceratops + Pentaceratops + Utahceratops ${ }^{+}$ $[$ Agujaceratops $+[$ Judiceratops + Chasmosaurus $+[$ Vagaceratops + YPM 2016]]]]: 8.1, 15.0, $18.0,19.1,69.1,72.0,73.1,74.1,76.0,149.1$

Bravoceratops + Coahuilaceratops + Pentaceratops + Utahceratops $+[$ Agujaceratops $+[$ Judic eratops + Chasmosaurus $+[$ Vagaceratops + YPM 2016]]]: 55.1, 56.1, 60.1, 61.1, 64.1, 79.0, $81.1,83.0,133.0$

Agujaceratops $+[$ Judiceratops + Chasmosaurus $+[$ Vagaceratops + YPM 2016]]: 9.0, 12.0, $14.0,29.0,37.0,88.1,89.1,95.0$

Judiceratops+Chasmosaurus+[Vagaceratops+YPM 2016]: 11.0, 20.0, 31.1, 41.2, 47.1, $77.0,78.1,90.0,134.0,150.0$

Vagaceratops+YPM 2016: 50.2, 66.0, 70.2, 85.1, 93.1, 98.1, 103.1, 152.1, 153.1

Vagaceratops: 80.0, 151.1 


\subsection{Discussion}

In this analysis, all of the Dinosaur Park Formation chasmosaurine specimens are recovered (strict consensus) as the polytomic clade Chasmosaurus (plus Judiceratops) being the sister clade to the more derived Vagaceratops-like clade (Fig. 37), supporting the hypothesis that previously diagnosed Dinosaur Park Formation Chasmosaurus taxa are not taxonomically distinct from each other. This group is also poorly resolved in the majority rule tree, except for AMNH 5401 (holotype, Chasmosaurus kaiseni) and CMN 8800 (holotype, Chasmosaurus russelli) forming a sister group polytomy to all other more derived chasmosaurine specimens.

The results of the analysis confirms the null hypothesis that the chasmosaurine specimens examined in this analysis (excluding the Vagaceratops+YPM 2016 clade, and AMNH 5402, discussed later) are best referable to a single species with Chasmosaurus belli having historical priority. Specimens referred to the other previously-named Dinosaur Park Formation chasmosaurine species, C. russelli (AMNH 5656, CMN 2280, CMN 8800, and TMP 1983.025.0001) Chasmosaurus brevirostris (ROM 839), C. kaiseni (AMNH 5401), C. canadensis (AMNH 5401, CMN 1254 and UALVP 40), Eoceratops canadensis (CMN 1254 and UALVP 40), and Mojoceratops perifania (AMNH 5401, AMNH 5656 and TMP 1983.025.0001) also cannot be resolved as distinct taxa. These six latter taxa are here considered to be junior synonyms of $C$. belli.

The sequence of successively more derived chasmosaurines in this analysis is arranged in reverse stratigraphic order, with the stratigraphically higher Eotriceratops, Ojoceratops and Triceratops-like clade recovered as the most basal taxa, and the 
stratigraphically lower Dinosaur Park Formation chasmosaurines + Judiceratops clade as the most derived group of taxa (strict consensus, Fig. 37). This phylogenetic sequence is contradictory to most other chasmosaurine studies, where chasmosaurines are arranged roughly in accordance with their stratigraphic positions, from the least derived Chasmosaurus to the most derived Triceratops (Lehman, 1996; Dodson et al., 2004; Sampson et al., 2010). The reverse stratigraphic order of taxa observed in this study was similar, however, to the majority rule tree of Mallon et al. (2011), and is due, in part, to the unelaborated parietosquamosal epiossifications of the Triceratops-like clade that resembles basal centrosaurines and other non-ceratopsid ceratopsians.

The recovery of the Dinosaur Park Formation chasmosaurines + Judiceratops clade as a sister clade to Agujaceratops is consistent with other phylogenetic studies (Holmes et al., 2001; Sampson et al., 2010; Mallon et al., 2011) and is not unexpected, given that Agujaceratops was previously referred to Chasmosaurus (Lehman, 1989).

Majority rule analysis: The shared possession of primitive states (0) for characters 21 (accessory antorbital fenestra present) and 22 (fenestra allowing visibility of narial cavity in lateral view) in AMNH 5401 and CMN 8800 likely explains their basal positions relative to other Chasmosaurus specimens, which do not possess an accessory antorbital fenestra. The variable size of this fenestra in AMNH 5401 and CMN 8800 suggests that the expression of this feature is inconsistent and unlikely to be of phylogenetic significance within Chasmosaurus. The TMP 1981.019.0175+TMP 1983.025.0001+UALVP 40 clade consists of one specimen with a complete frill (TMP 1983.025.0001; holotype, M. perifania) and two other specimens missing the posterior halves of their frill (TMP 1981.019.0175 and UALVP 40). It is notable that TMP 
1981.019.0175 and UALVP 40 have not been previously referred to M. perifania; AMNH 5401, AMNH 5656 and CMN 1254 have, however, but do not cluster with the holotype, TMP 1983.025.0001.

CMN 1254 and UALVP 40 were originally considered as representing adult forms of the putative pygmy E. canadensis (Tyson, 1977), but later considered to be relatively immature individuals of Chasmosaurus (Lehman, 1989). The recovery of these two specimens in the Dinosaur Park Formation chasmosaurine clade supports their referral to Chasmosaurus (sensu Lehman, 1989). The hypothesis that these two specimens are relatively immature individuals will be tested later in Chapter 8 .

The null hypothesis of one species of Chasmosaurus will be further tested by examining the stratigraphic (Chapter 7), ontogenetic (Chapter 8) and morphometric (Chapter 9) distributions and relationships between Chasmosaurus specimens. The phylogenetic relationships of these specimens will be re-discussed in Chapter 10, following the results of Chapters 7-9.

"Vagaceratops-like" clade: The recovery of a distinct Vagaceratops-like clade ([AMNH 5402+[YPM 2016+[Vagaceratops irvinensis (CMN 41357 and TMP 1987.045.0001)]]]) as the sister taxon to Chasmosaurus (sensu Holmes et al., 2001; contra Sampson et al., 2010) is not unexpected given the well-supported autapomorphies for Vagaceratops. The inclusion of AMNH 5402 and YPM 2016 is, however, unique to this study. The Chasmosaurus skull YPM 2016 (Figs. 7R and 10) and Vagaceratops (CMN 41357+TMP 1987.045.0001) (Fig. 37) share a unique combination of four characters, previously thought to be unique to Vagaceratops (Holmes et al., 2001) that 
suggests YPM 2016 is more similar to Vagaceratops than Chasmosaurus and, is thus, best removed from Chasmosaurus. These characters are:

1. Anteroposteriorly shortened parietal fenestrae, with the fenestra transverse width equal to or exceeding its anteroposterior length; parietal fenestra length/width ratio between $0.72(\mathrm{CMN} 41357)$ and 1.03 (YPM 2016), versus 1.09 (ROM 843) and 1.89 (AMNH 5656) in Chasmosaurus.

2. A straight posterior parietal margin (inferred for TMP 1987.045.0001, but coded as “?”" in analysis).

3. Four anterodorsally oriented epiparietals on each side (P1-4; inferred for TMP 1987.045.0001).

4. A fifth epiossification oriented in the plane of the frill, either articulated with the parietal (P5; CMN 41357 and YPM 2016) or straddling the parietal-squamosal contact (epiparietosquamosal; TMP 1987.045.0001).

YPM 2016 (Fig. 10) differs from CMN 41357 and TMP 1987.045.0001 in that its four medialmost epiparietals (P1-4; P4 of TMP 1987.045.0001) are greatly reduced in length and do not appear to be co-fused at their bases. YPM 2016 also has a significantly larger parietal fenestra length/width ratio (1.03) compared with TMP 1987.045.0001 (0.87) and CMN 41357 (0.72).

The recovery of AMNH 5402 (Fig. 8) in the majority rule tree within the Vagaceratops-like clade is based on its possession of an anteroposteriorly shortened parietal fenestrae (length/width ratio $=1.04)$, a relatively straight posterior parietal 
margin (factoring in taphonomic distortion), and five inferred epiossifications on each side of the parietal, with the putative fifth straddling the parietal-squamosal contact (i.e., P1-4 and an epiparietosquamosal). AMNH 5402 possesses epiparietals (interpreted here as P1 and P4, Fig. 8) that are similar in length to those of YPM 2016, but not CMN 41357 and TMP 1987.045.0001 (P4) (Fig. 34). It is worth noting that the preserved P1 and P4 of AMNH 5402 are oriented in the plane of the frill (Figs. 8 and 34), and not anterodorsally as in CMN 41357, TMP 1987.045.0001 (P4) and YPM 2016 (Fig. 34). Although AMNH 5402 is interpreted here as having (or would have) had four epiparietals, $\mathrm{P} 2$ and $\mathrm{P} 3$ are missing and their shape, size and orientations cannot be determined or coded other than "?" in the analysis.

The predentary of AMNH 5402 comprises approximately $35 \%$ of the length of the lower jaw (Fig. 35D), which is larger than that of other Chasmosaurus specimens (CMN $2245=28 \%$ to $33 \%=$ ROM 839 ; Fig. $35 \mathrm{~A}-\mathrm{C})$, but smaller than that of Vagaceratops (CMN $41357=40 \%$; Fig. 35E). Given the range of this ratio amongst Chasmosaurus and the small sample size of specimens examined, it is difficult to unequivocally state whether the relative length of the predentary is a valid diagnostic character to separate Chasmosaurus and Vagaceratops.

Although AMNH 5402 (Fig. 8) and YPM 2016 (Fig. 10) are similar to Vagaceratops, it is premature to say whether they are referable to this taxon. The stratigraphic position of YPM 2016 (unknown for AMNH 5402) relative to Vagaceratops will be examined in Chapter 7, and the ontogenetic relationship between AMNH 5402 and YPM 2016 will be examined in Chapter 8. 
The taxonomic validity of Judiceratops tigris: The recovery of Judiceratops within the Chasmosaurinae clade supports Longrich's (2013) referral of this taxon to this clade. However, the recovery of this taxon within the polytomic Dinosaur Park Formation Chasmosaurus in both the strict consensus and majority rule trees suggests that Judiceratops cannot be distinguished from the Chasmosaurus specimens used in this study. Longrich (2013) diagnosed Judiceratops on the basis of three fragmentary specimens with little overlapping cranial material amongst them: YPM VPPU 022404 (holotype; Fig. 38), YPM VPPU 023262 (referred specimen, Fig. 39) and YPM VPPU 023261 (referred specimen, Fig. 40).

Although Longrich (2013) identified at least four non-Chasmosaurus-like features in Judiceratops (1, episquamosals that are imbricated in lateral view; 2, an anterolateral squamosal corner that projects posterolaterally; 3 , anteriormost episquamosals large relative to other episquamosals; and 4, a relatively wide medial parietal bar with a concave ventral surface), I believe that these features have been misdiagnosed and, thus, have recoded Judiceratops according to the following reinterpretations:

1. Longrich (2013) interpreted the episquamosals as being imbricated in lateral view, similar to, but not as extreme as, the condition in centrosaurines (e.g., Pachyrhinosaurus lakustai, Currie et al., 2008). However, the episquamosals display no signs of imbrication in lateral view, but are instead not inflected from the axis of the squamosal as in all chasmosaurines (Fig. 38G-H; contra Longrich, 2013).

2. Longrich (2013) interpreted the embayment between the two anteriormost marginal undulations of the squamosal YPM VPPU 023262 as the posterior margin of the jugal 
notch (Longrich, 2013: Fig. 4). Based on this interpretation, he reasoned that the anterolateral corner of the squamosal projected posterodorsally. However, the anteriormost undulation is similar in shape and size to the second anteriormost undulation, and appears to be adorned by the preserved anterior portion of an episquamosal (Fig. 39A-B). This undulation projects anterolaterally, resulting in an anterolaterally directed squamosal corner, as in all chasmosaurines (Fig. 39C; contra Longrich, 2013). The jugal notch therefore lies anterior to the anteriormost undulation (Fig. 39C).

3. Longrich (2013) also interpreted the two anteriormost episquamosals of YPM VPPU 023262 (i.e., episquamosals adorning the second and third anteriormost loci of Fig. 39AC) on the squamosal as large relative to other episquamosals (Longrich, 2013: Fig. 4). However, the laterally projecting loci underlying these episquamosals are large, giving the false illusion that the episquamosals themselves are large (contra Longrich, 2013). The size (i.e., height) of episquamosal loci tend to increase towards the anterior end of the squamosal in Chasmosaurus (e.g., CMN 1254, Fig. 7D).

4. The lateral margins of the medial parietal bar of YPM VPPU 023262 (Longrich, 2013: Fig. 7; Fig. 39D) are broken, and the element's lateral extent cannot be determined. The medial portion of the lateral margin of this element appears to have a gently convex left lateral margin in dorsal view; the lesser preserved right lateral margin also appears to possess portions of this convex margin. These convex margins are interpreted here as being the original, unbroken lateral margins of the element; this interpretation is supported by their smooth edges in dorsal view, and their symmetry to each other. The 
presence of a convex margin of a medial parietal bar is unknown in any other ceratopsian possessing parietal fenestrae, where the medial bar is instead concave anteriorly and posteriorly, and straight medially (e.g., Chasmosaurus, Torosaurus and Centrosaurus). The convex lateral parietal margin of Judiceratops is reinterpreted here as being the laterally projecting ridge that forms the medial margin of the dorsal temporal fenestrae opening on the parietal medial platform; such a ridge is convex in ceratopsids.

Progressing posteriorly (i.e., upwards) on the specimen in Figure 39D, two transitions occur in the interpreted parietal medial platform: 1 , the element's crosssectional shape transitions from concave to flat; and 2, the bone texture transitions from parallel and oriented anteroposteriorly to radiating laterally at the posteriormost (i.e., bottom) margin of the element. The flat cross-sectional shape and laterally radiating bone texture of the posteriormost end of this element suggests that this end actually represents the anteriormost portion of the platform (immediately posterior to supracranial sinus), which possesses both of these features in ceratopsids (compare with CMN 34829, Fig. 17I-J; CMN 8802, Fig. 30A-B; and CMN 2280, Fig. 33G). This element is therefore consistent with being a parietal medial platform, and not a medial parietal bar (contra Longrich, 2013); this interpretation is supported by the element's concave ventral surface which is typical of parietal medial platforms in ceratopsids. Based on this evidence, I reinterpret the anteriormost preserved margin in Figure 39D (Longrich, 2013: Fig. 7) as being the posteriormost margin (Fig. 39E-F, rotated $180^{\circ}$ ).

Despite the close phylogenetic relationship between Judiceratops and Dinosaur Park Formation chasmosaurine skulls (Chasmosaurus and Vagaceratops), Judiceratops is known from considerably older strata. Longrich (2013) reported Judiceratops as being 
collected from exposures of the Judith River Formation in Hill County, northern Montana, and cited a radiometric date of approximately $78 \mathrm{Ma}$ for these beds (Goodwin and Deino, 1989). These beds correspond to sediments of the upper Foremost and lower Oldman formations (Longrich, 2013). This indicates that Judiceratops is at least one million years older than Chasmosaurus, as the top of the Oldman Formation in Dinosaur Provincial Park is approximately $77 \mathrm{Ma}$ (David Eberth, pers. comm.). Given the high rate of turnover of ceratopsid taxa within the Belly River Group of Canada, it is probable that material referred to Judiceratops does represent a new chasmosaurine species; however, the fragmentary, composite J. tigris lacks autapomorphies or any unique combination of diagnosable characters, and should be considered nomen dubium, pending the discovery of diagnostic material. 
Figure 38. Judiceratops tigris (holotype, YPM VPPU 022404). Left postorbital horncore in: A) anterior; B) ventrolateral; and C) dorsal views (modified from Longrich, 2013:

Fig. 1). Right postorbital horncore in: D) anterior; E) ventrolateral; and F) dorsal views (modified from Longrich, 2013: Fig. 1). Fragments of lateral margin of right squamosal in: G) dorsal; and H) lateral views (modified from Longrich, 2013: Fig. 3). Posterior parietal bar in: I) dorsal; and $\mathbf{J})$ ventral views (modified from Longrich, 2013: Fig. 5). Close-up of right posterolateral portion of posterior parietal bar in: $\mathbf{K}$ ) dorsal; L) ventral; and M) posterior views (modified from Longrich, 2013: Fig. 6). See "List of anatomical abbreviations". Scale bar equals $10 \mathrm{~cm}$. 
Figure 38.

A)

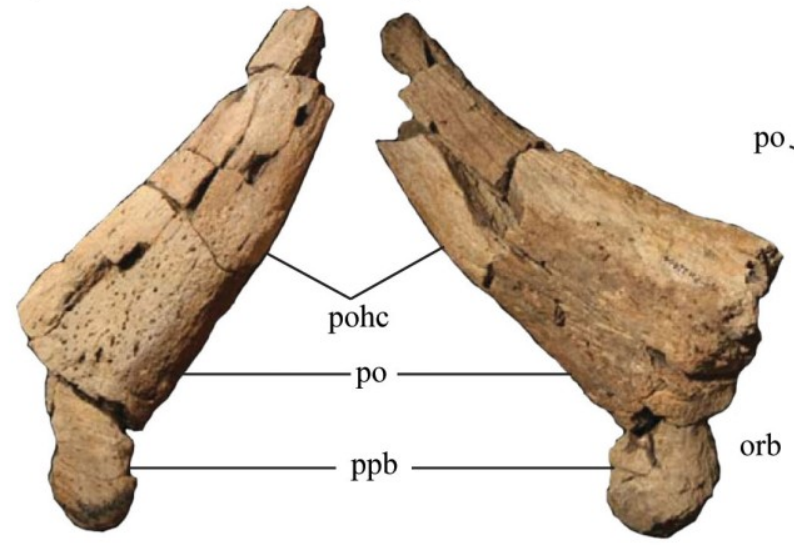

D)

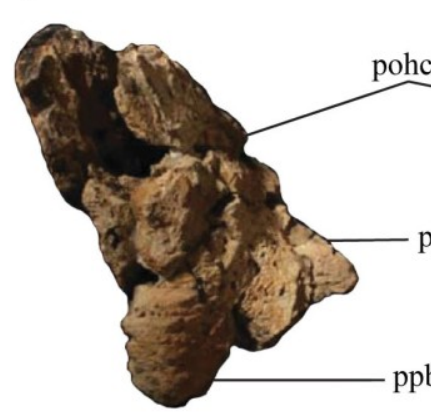

E)
C)

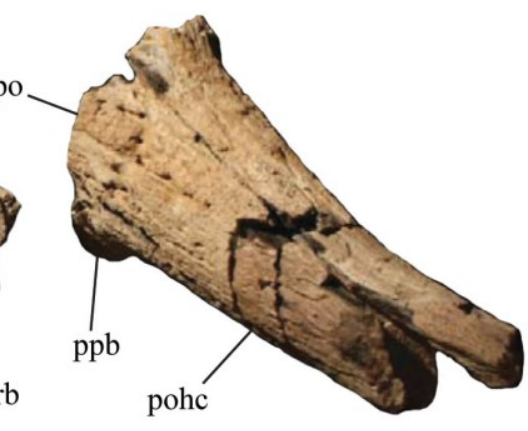

F)

G)

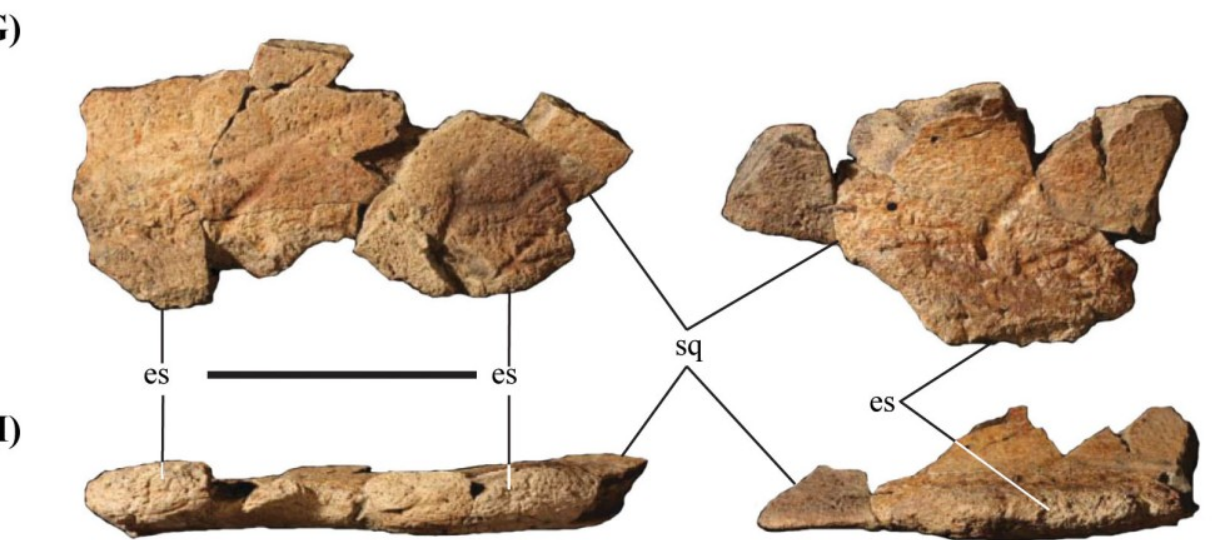


Figure 38. (continued)
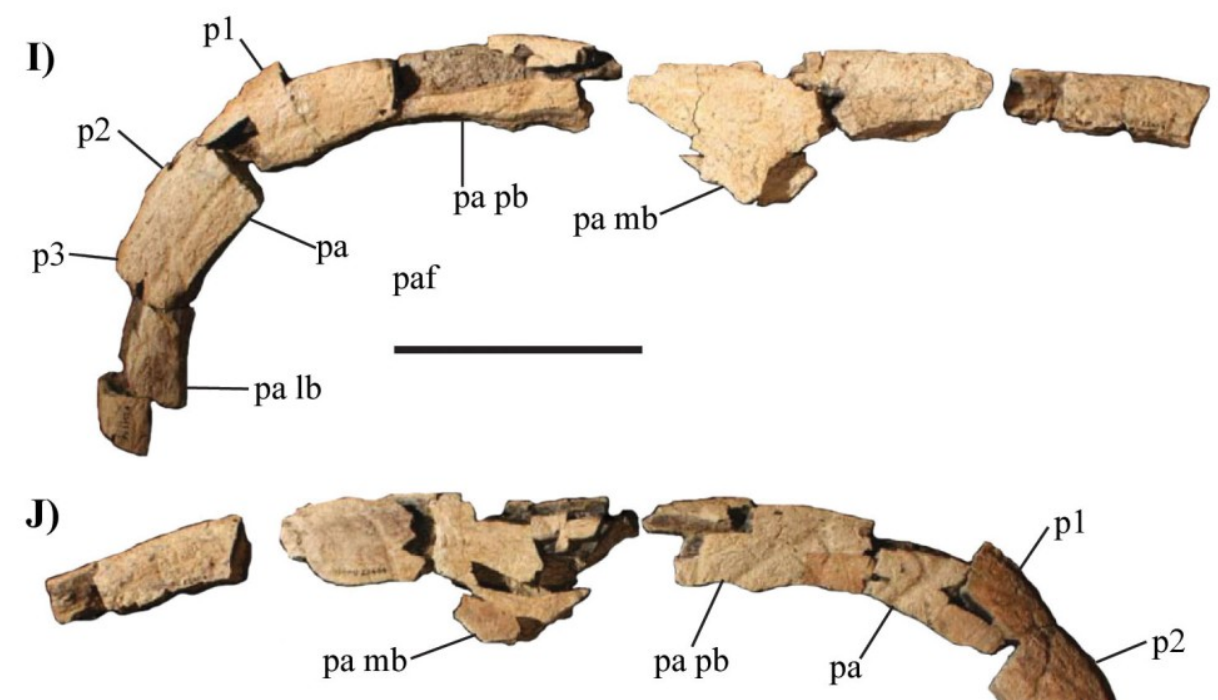

K)

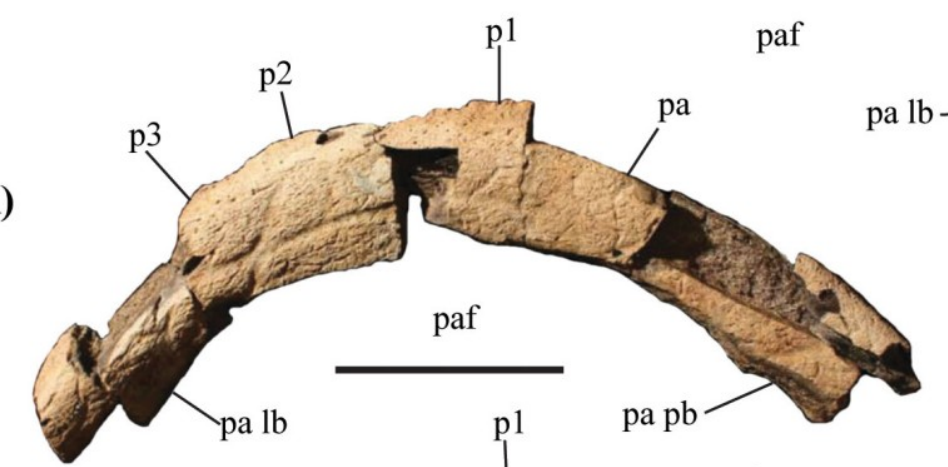

L)

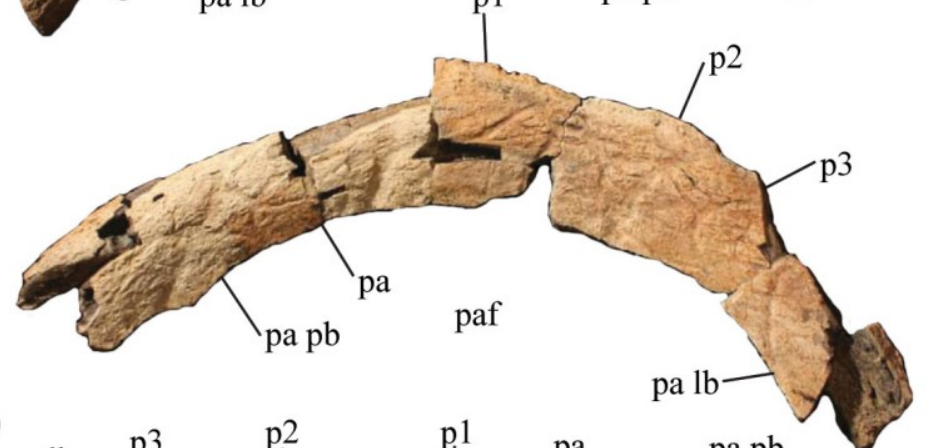

M)

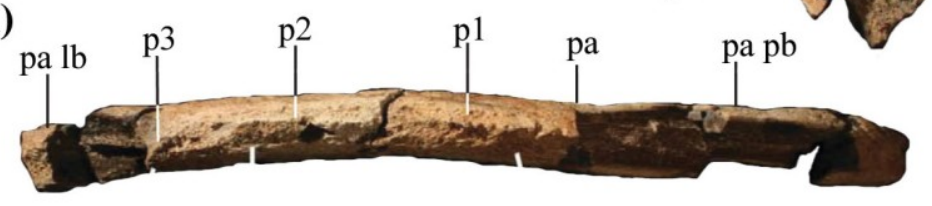


A)

B)
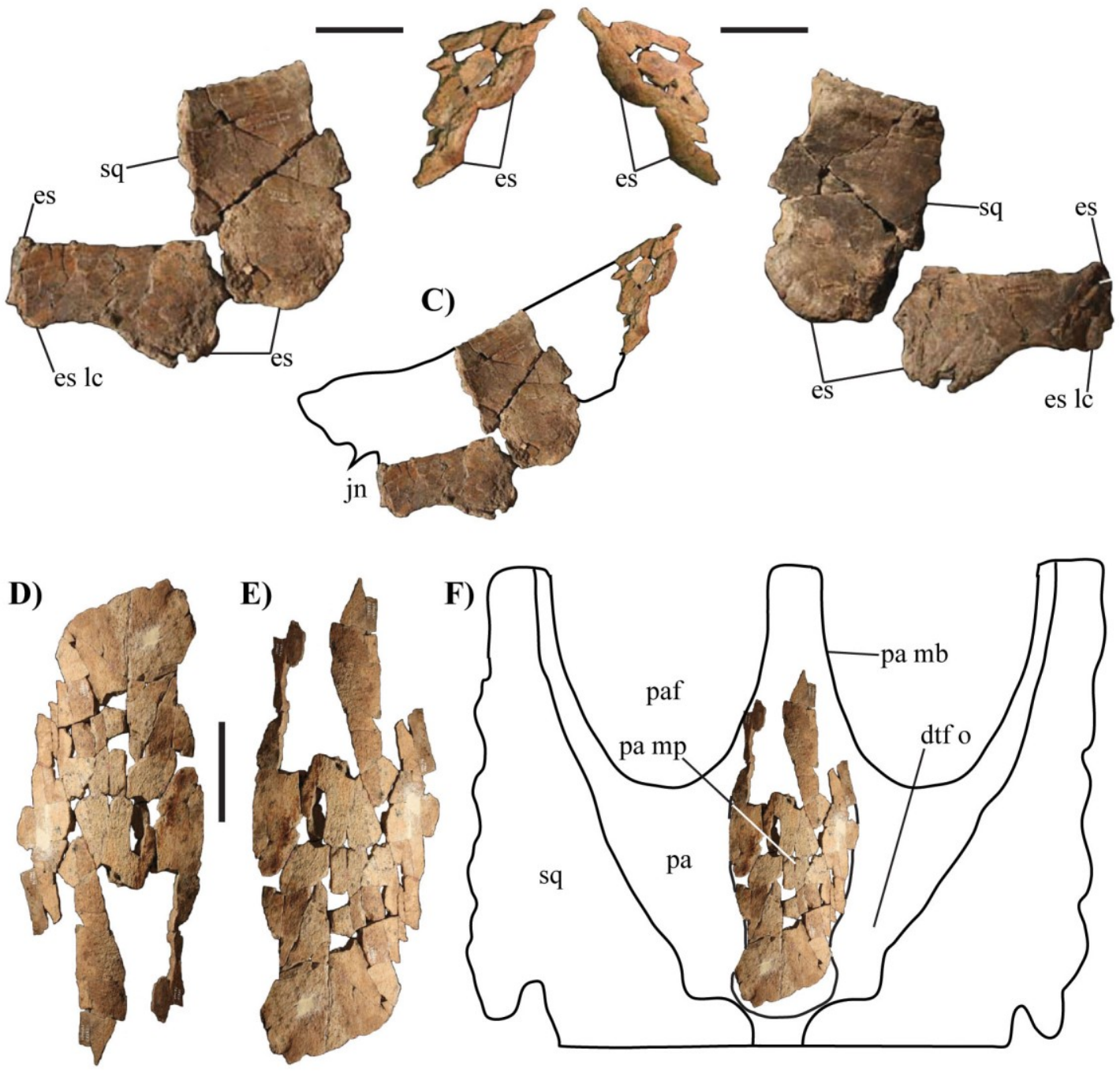

Figure 39. Judiceratops tigris (referred specimen, YPM VPPU 023262). Left squamosal in: A) dorsal; and B) ventral views (modified from Longrich, 2013: Fig. 4); and C) dorsal view of reconstructed squamosal, as interpreted in this study. Dorsal view of medial platform of parietal: D) as figured in Longrich (2013: Fig. 7, 'medial parietal bar'); E) rotated $180^{\circ}$; and $\mathbf{F}$ ) dorsal view of reconstructed medial platform, as interpreted in this study. See "List of anatomical abbreviations". Scale bar equals $10 \mathrm{~cm}$. 
A)

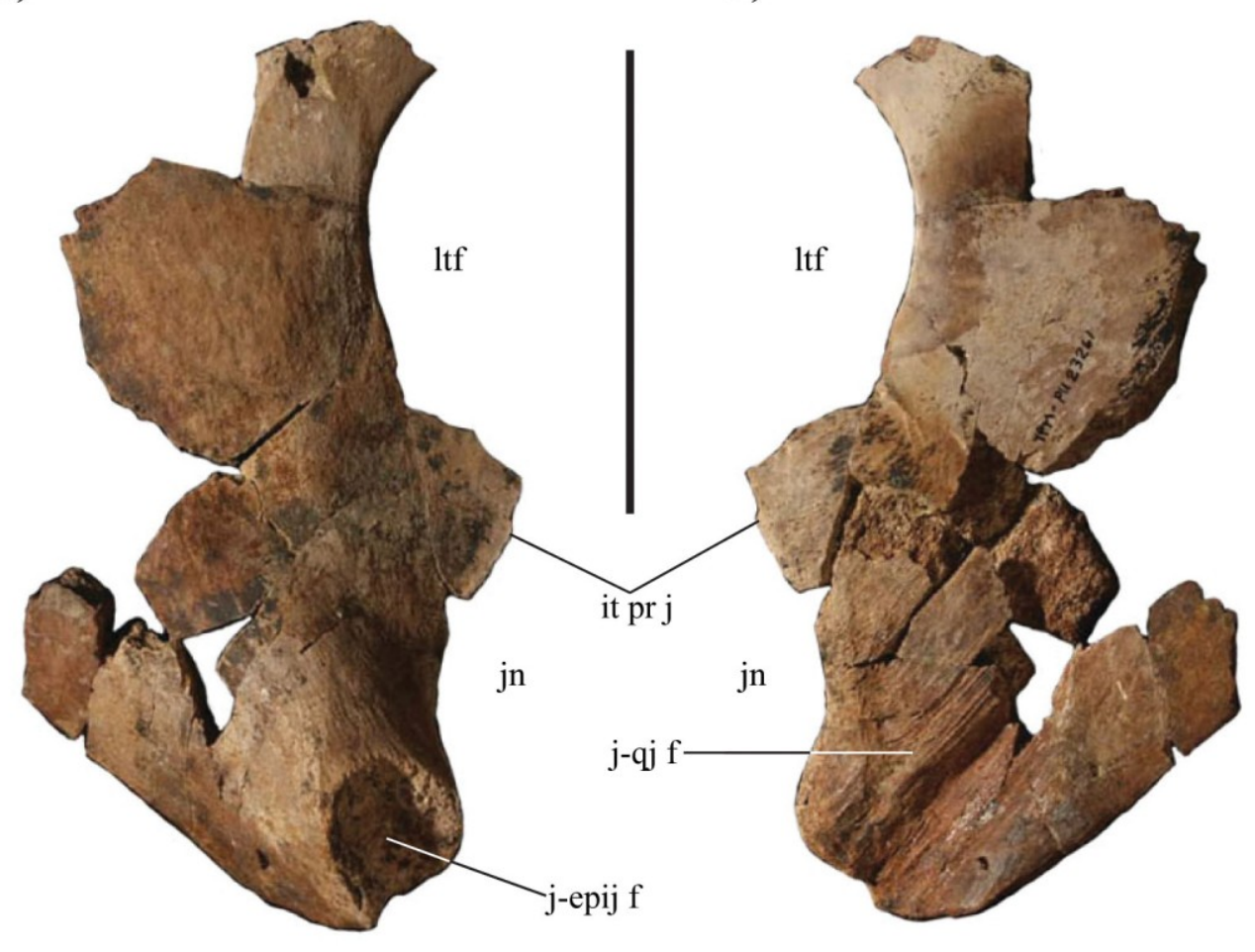

Figure 40. Judiceratops tigris (referred specimen, YPM VPPU 023261). Left jugal in: A) dorsal; and B) ventral views (modified from Longrich, 2013: Fig. 2). See "List of anatomical abbreviations". Scale bar equals $10 \mathrm{~cm}$. 


\subsection{Conclusion}

The 17 best preserved chasmosaurine skulls from the Dinosaur Park Formation form a monophyletic clade within Chasmosaurinae. This clade includes the polytomic Chasmosaurus that comprises all specimens previously referred to Chasmosaurus belli, Chasmosaurus russelli, Chasmosaurus brevirostris, Chasmosaurus canadensis, Chasmosaurus kaiseni, Eoceratops canadensis, and Mojoceratops perifania, as well as Judiceratops tigris from the Judith River Formation of northern Montana, and the Vagaceratops-like clade including YPM 2016, previously referred to Chasmosaurus. In this analysis, Vagaceratops is recovered as the sister taxon to Chasmosaurus, and not to Kosmoceratops (contra Sampson et al., 2010), which has implications for the origins of chasmosaurines within Laramidia.

All previously referred species contained within the Dinosaur Park Formation chasmosaurines + Judiceratops clade cannot be resolved as distinct taxa and are best subsumed into Chasmosaurus belli; these specimens are here on referred to as “Chasmosaurus-like" in this study. Vagaceratops (CMN 41357 and TMP 1987.045.0001) shares a suite of characters with the "Chasmosaurus" skulls AMNH 5402, YPM 2016, suggesting that they are closely related or possibly even synonymous; these specimens are here on referred to as "Vagaceratops-like" in this study. Judiceratops is phylogenetically indistinguishable from Dinosaur Park Formation chasmosaurines and does not appear to possess any characters, or unique combination of characters, to support its validity as a distinct species, despite it being at least one million years older than Dinosaur Park Formation chasmosaurines. Further complicating the matter is the fact that Judiceratops is a composite species based on several non- 
associated specimens with relatively few overlapping features. The fragmentary Judiceratops is therefore here considered nomen dubium, pending the discovery of diagnostic material.

The null hypothesis of only one valid species of Chasmosaurus will be further tested by examining Chasmosaurus-like specimens in the context of: 1, stratigraphy, determining whether previously referred species overlap temporally, supporting their synonymy (Chapter 7); 2, ontogeny, determining whether some previously referred species actually represent relatively immature forms of another species, supporting their synonymy (Chapter 8); and 3, morphometrically, determining whether previously referred species are morphometrically distinct, or indistinguishable and supporting their synonymy (Chapter 9). Vagaceratops-like specimens will also be included in Chapters 79 to determine whether their stratigraphic, ontogenetic and morphometric distributions support their referral to the same genus or species therein. 


\section{Chapter 7. Morphological Variation in Chasmosaurus and Vagaceratops due to their Geographic and Stratigraphic Distribution}

\subsection{Introduction}

This chapter examines whether the morphological variation in cranial material referred to Chasmosaurus and Vagaceratops can be explained by their geographic or stratigraphic distribution; accordingly, only specimens with precise locality data are included. By arranging these specimens in their proper stratigraphic sequence, morphological trends over time can be evaluated for each of these taxa. Furthermore, the known temporal range of Chasmosaurus russelli, Chasmosaurus belli and Vagaceratopslike specimens can be established and compared to determine whether they were separated in time or lived contemporaneously. Morphological variation between fossil taxa can only be attributed to temporal differences if their respective specimens are stratigraphically segregated. If this condition is met, and if the specimens representing those taxa collectively display a directed pattern of statistically significant morphological change over stratigraphy, an anagenetic model where one taxon evolved into the other could be inferred.

The successively less pronounced posterior parietal embayments of C. russelli, $C$. belli and Vagaceratops irvinensis have been previously attributed to temporal differences, as these taxa were thought to form a faunal succession in the Dinosaur Park Formation, corresponding to Dinosaur Park Faunal Zones 1, 2 and 3 (Fig. 1), respectively (Godfrey and Holmes, 1995; Holmes et al., 2001; Ryan and Evans, 2005). The stratigraphic distribution of the posterior parietal embayment, as well as other cranial 
features, will be re-evaluated in this chapter in light of revised stratigraphic data for specimens (Currie and Russell, 2005), recently collected specimens (i.e., TMP 2009.034.0009, Fig. 16B, and TMP 2011.053.0046, Figs. 18-21), the rediscovery of the C. russelli holotype (CMN 8800) quarry below the Lethbridge Coal Zone (Fig. 2), and the reassignment of the Chasmosaurus skulls AMNH 5402 and YPM 2016 to Vagaceratops.

Directed patterns of morphological change over stratigraphy have been documented in Scannella and Horner's (2010) proposed Triceratops-Torosaurus synonymy. Scannella et al. (2013) noted that certain cranial features present in mature specimens at the base of the Hell Creek Formation of Montana are also present higher in section, but only in immature specimens. They attributed these stratigraphic patterns to heterochrony, in which the expression of these features becomes restricted to progressively earlier stages of ontogeny over time. Scannella and Horner (2011) also suggested that the differing epiparietal count between Torosaurus (10 and 12) and Triceratops (six, excluding midline epiparietal) may be attributable to temporal differences, as the former count is restricted to the base of the Hell Creek Formation, and the latter count occurs higher in section. Their hypothesis has been supported by detailed stratigraphic work on the Hell Creek Formation (Scannella and Fowler, 2014), although this work is ongoing. The differing epiparietal counts of Chasmosaurus (three per side) and Vagaceratops (four to five per side) have likewise, albeit implicitly, been attributed to temporal differences, based on their purported stratigraphic segregation (Holmes et al., 2001; Ryan and Evans, 2005). This latter potential stratigraphic trend will be re-evaluated in this chapter. 
The stratigraphic occurrences of taxa have been used to help reconstruct their phylogenetic relationships, with the implication that earlier taxa are less derived, later taxa are more derived, and taxa with similar or overlapping temporal ranges are more closely related than those separated by relatively large time gaps. In some phylogenetic (e.g., Geisler et al., 2007) and cladistic (e.g., Bloch et al., 2004) studies, the collective stratigraphic range of taxa being examined has been subdivided into chronostratigraphic intervals, and those intervals used as states in an ordered stratigraphic character. A stratophylogenetic analysis is conducted in this chapter, using the 153 unordered morphological characters used in the phylogenetic analysis (Chapter 6), and an ordered stratigraphic character whose states correspond to the three Dinosaur Park faunal zones (Fig. 1).

Morphological variation in Chasmosaurus and Vagaceratops will also be evaluated on the basis of geographic placement. Morphological change over a geographic gradient has been documented in both extant (e.g., Polly, 2007) and extinct (e.g., McGuire, 2010) taxa, and has been largely attributed to adaptations to differing climatic conditions and food resources (i.e., dietary adaptations). In the Dinosaur Provincial Park area, a gradual shift in the makeup of its floral assemblage up section has been documented in the Dinosaur Park Formation, most likely in response to the westward transgression of the Western Interior Seaway (Braman and Koppelhus, 2005). Floral assemblages likely also varied along a west-east transect in what is now southern Alberta, with the number of aquatic taxa increasing in sites sampled closer to the Western Interior Seaway to the east; however, this trend has not been previously demonstrated. Mallon et al. (2013) conducted an evaluation of niche partitioning amongst herbivorous Dinosaur 
Park Formation ornithischians on the basis of morphometric cranial parameters that relate to dietary physiology. Variation in these parameters amongst time-equivalent specimens of Chasmosaurus and of Vagaceratops along a west-east transect, if any, may be attributable to differing dietary adaptations.

Material analyzed:

Although specimen taxonomy will not be fully discussed until Chapter 10, Dinosaur Park Formation chasmosaurine skulls are categorized into two operative taxonomic groupings, corresponding to their inferred phylogenetic relationships given in Chapter 6;

1. Chasmosaurus-like specimens (Figs. 7A and C-Q, 14A-E, 17, 22-32, and 33A-L). Chasmosaurus-like specimens possess, or are inferred as possessing, the following features: parietal fenestrae anteroposteriorly elongate in shape; posterior parietal bar variably embayed; and three epiparietals on each side of the posterior parietal bar.

All specimens currently referred to Chasmosaurus were collected from Alberta (Figs. 3 and 41), except for CMN 8803 that was collected from a bonebed on the north bank of the South Saskatchewan River in Saskatchewan, in present-day Saskatchewan Landing Provincial Park. The known geographic distribution of Chasmosaurus extends from Dinosaur Provincial Park in the north to the southern Milk River region, and east to Saskatchewan Landing Provincial Park, encompassing an area of approximately 22,400 $\mathrm{km}^{2}$. 
2. Vagaceratops-like specimens, which includes Vagaceratops (CMN 41357, TMP 1987.045.0001, TMP 1998.102.0008, and TMP 2009.034.0009), AMNH 5402, TMP 2011.053.0046 and YPM 2016 (Figs. 7B and R, 15-16, 18-21, and 33M-R).

Vagaceratops-like specimens share: anteroposteriorly shortened parietal fenestrae, with the fenestra transverse width equal to or exceeding its anteroposterior length; a straight posterior parietal margin; and five epiossifications on each side of the parietal, with the fifth lateralmost one either articulated entirely with the parietal (epiparietal) or straddling the parietal-squamosal contact (epiparietosquamosal).

All Vagaceratops-like specimens were collected from sediments of the Dinosaur Park Formation or age equivalent strata. Vagaceratops-like specimens have a smaller distribution, encompassing an area of approximately $8,500 \mathrm{~km}^{2}$, extending from Dinosaur Provincial Park and Hilda localities in the north, and south to Irvine and the Milk River region (Figs. 3 and 41).

Stratigraphic and geographic data for all chasmosaurine specimens from the Dinosaur Park Formation, where known, is compiled in Appendix I and summarized in Table 3. The stratigraphic sequence of Dinosaur Park Formation chasmosaurine specimens, as well as their positions relative to the three Dinosaur Park faunal zones identified by Ryan and Evans (2005), is shown in Figure 42. 
Figure 41. Structural contour map of Dinosaur Provincial Park (DPP; shaded area), with contours showing elevation (in metres above sea-level) of regional disconformity between Oldman and Dinosaur Park formations (modified from Eberth, 2005). Thin lines with tick marks represent generalized transect run by David Eberth (Eberth, 2005). Chasmosaurus russelli (red triangle), C. belli (blue inverted triangles), Chasmosaurus sp. (black squares), and Vagaceratops-like specimens (green diamonds) collected from DPP. 
Figure 41.

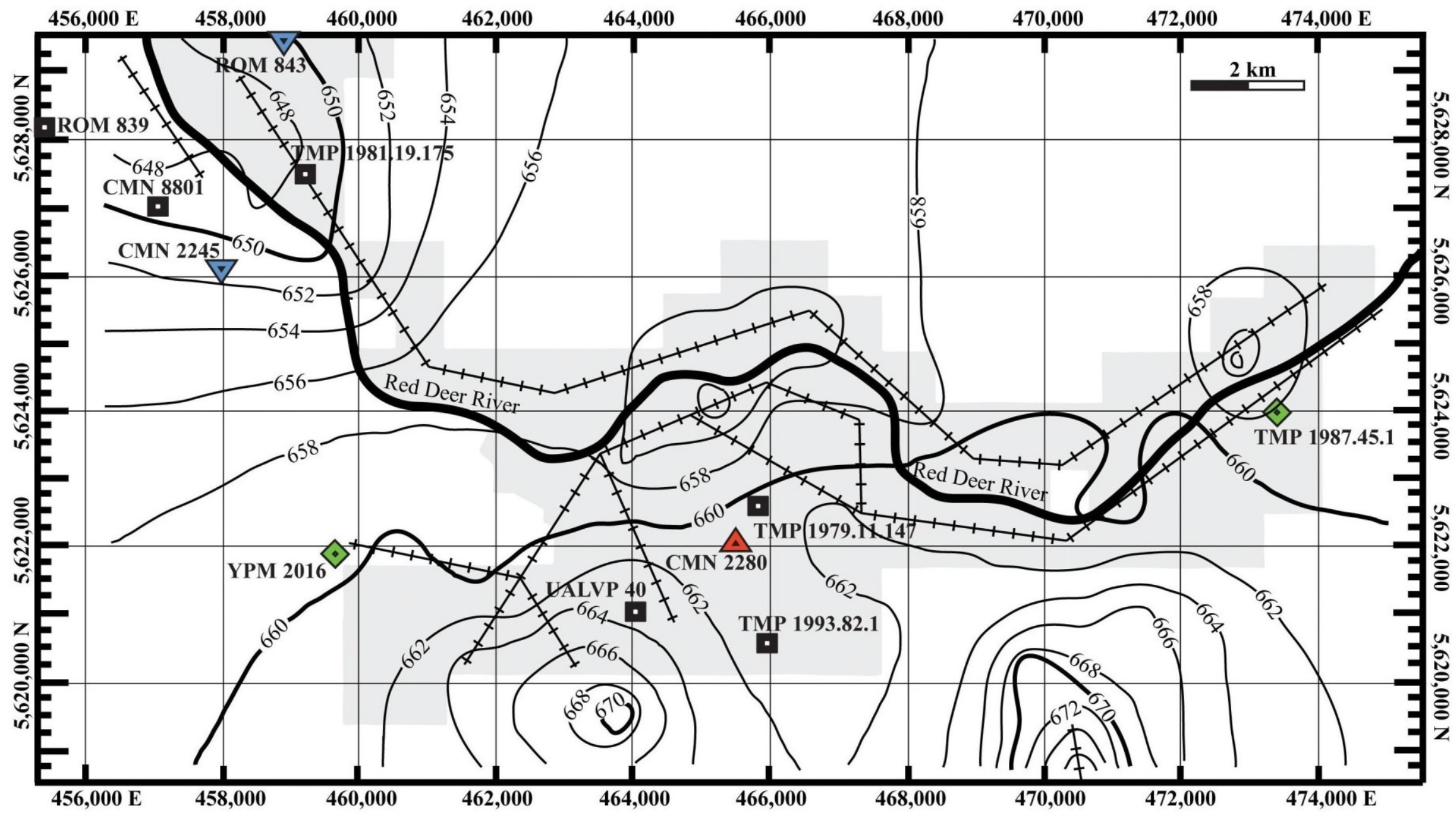


Figure 42. Stratigraphic positions of chasmosaurine specimens from Dinosaur Provincial Park with known data. Specimens above the regional disconformity $(0 \mathrm{~m})$ separating the Dinosaur Park and Oldman formations (error bars $\pm 5 \mathrm{~m}$ ). Specimens are shown on right in ascending stratigraphic order, in dorsal and lateral views. CMN 41357 was collected either at the base of or within the Lethbridge Coal Zone (LCZ), and TMP 1998.102.0008 was collected somewhere within the LCZ, as indicated by the vertical dashed lines. DPFZ 1 = Dinosaur Park Faunal Zone 1 (Centrosaurus-Corythosaurus), DPFZ 2 = Dinosaur Park Faunal Zone 2 (Styracosaurus-Lambeosaurus lambei) and DPFZ 3 = Dinosaur Park Faunal Zone 3 (pachyrhinosaur-Lambeosaurus magnicristatus) (Ryan and Evans, 2005; Ryan et al., 2012); the stratigraphic extent of these faunal zones are shown by horizontal dashed lines on right. Plaster reconstruction shown in grey half-tone.. 
Figure 42.

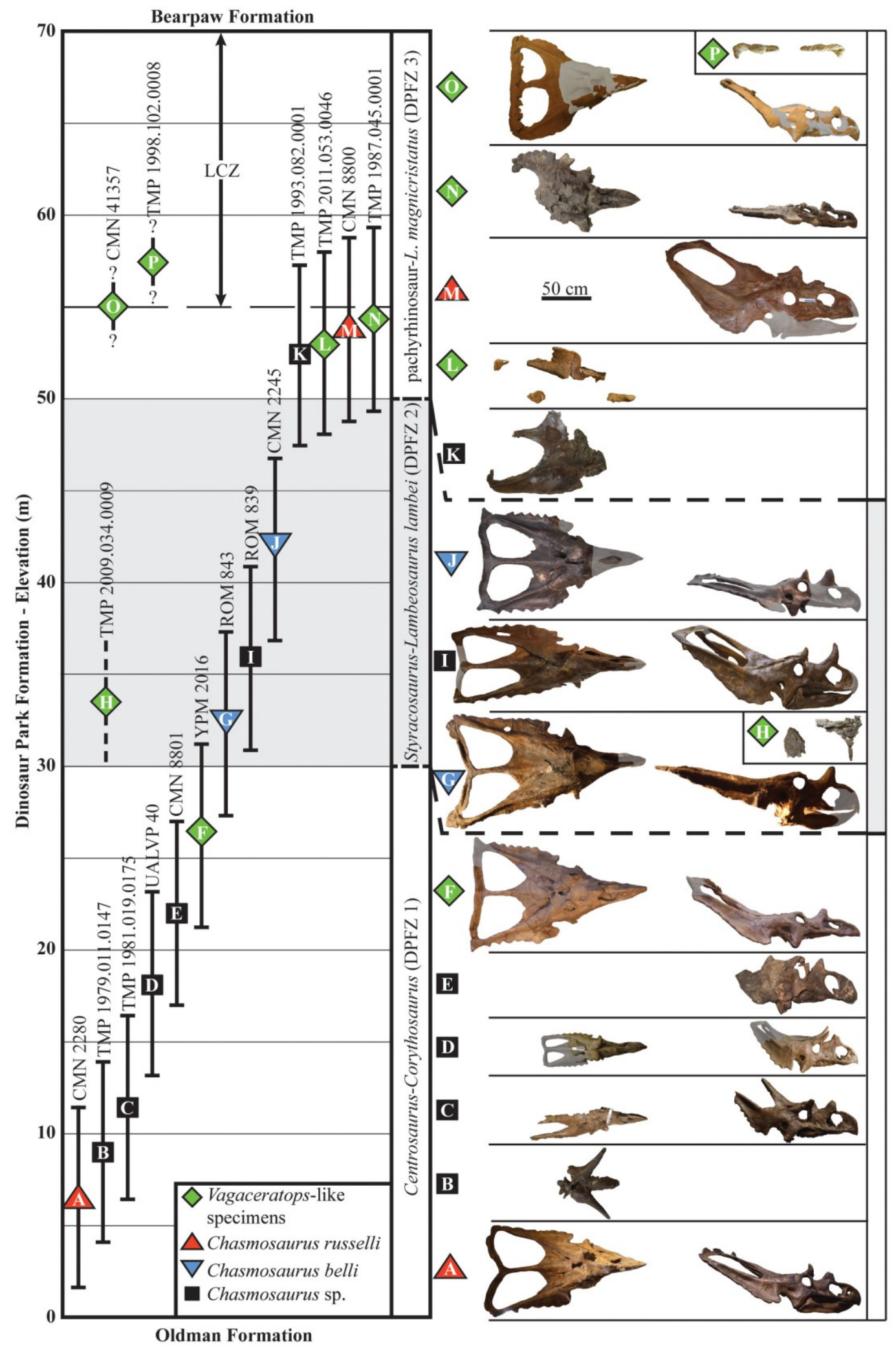


Hypotheses tested:

1. The relationship of stratigraphic position on taxonomic assignment: Using a stratophylogenetic analysis, I test the null hypothesis $\left(\mathrm{H}_{0}\right)$ that Chasmosaurus-like and Vagaceratops-like specimens do not form discrete, non-overlapping stratigraphic intervals in the Dinosaur Park Formation. If the null hypothesis is accepted, this would indicate that Chasmosaurus and Vagaceratops lived contemporaneously (contra Holmes et al., 2001; Ryan and Evans, 2005), and probably evolved via cladogenesis from a common ancestor. If the null hypothesis is rejected, this would support the previously proposed faunal turnover model for Dinosaur Park Formation chasmosaurines (sensu Holmes et al., 2001; Ryan and Evans, 2005) and suggest the scenarios where Chasmosaurus either evolved directly into Vagaceratops (i.e., anagenetic evolution) or was replaced by Vagaceratops over time through habitat tracking as the Western Interior Seaway transgressed across what is now southern Alberta $\left(\mathrm{H}_{1}\right)$. Once these hypotheses have been tested, possible explanations for the determined stratigraphic distribution of Chasmosaurus-like and Vagaceratops-like specimens and associated evolutionary model (cladogenetic vs. anagenetic) are examined.

In the stratophylogenetic analysis, I also test the null hypothesis $\left(\mathrm{H}_{0}\right)$ that specimens of C. belli and C. russelli do not form distinct clades in the resulting strict consensus tree. If the null hypothesis is accepted, this would suggest that Chasmosauruslike specimens correspond to a single evolutionary lineage (i.e., only one valid species is present). If the null hypothesis is rejected, the alternative hypothesis of two evolutionary lineages corresponding to two species $\left(\mathrm{H}_{1}\right)$ will be accepted. If the null hypothesis of one evolutionary lineage is accepted, Chasmosaurus-like specimens will be analyzed to 
determine whether any of the 36 cranial parameters in Figure 5 (measurements given in Appendix II) display a non-random directional change within the Dinosaur Park Formation that would support an anagenetic hypothesis of evolution. These stratigraphic or temporal changes, if any, are inferred as representing evolutionary changes within this group.

2. The effect of geographic position on taxonomic assignment: Closely related extant taxa that are widely separated or separated by barriers are known to exhibit morphological or behavioural differences. An attempt was made to examine the possible effect of geographic locality on morphological variation, i.e., $\mathrm{H}_{0}$; there is no difference; $\mathrm{H}_{1}$, there are differences based on geographic position, with the caveat that this can only be evaluated for specimens from age equivalent, or approximately equivalent, sediments. However, because the majority of specimens (Table 3) available for this study are from Dinosaur Provincial Park, not enough specimens are known from outside Dinosaur Provincial Park to be statistically significant, and the area of Dinosaur Provincial Park $\left(\sim 75 \mathrm{~km}^{2}\right)$ is considered too small to expect variation to be seen within animals of approximately one ton body mass whose post mortem remains may have been moved multiple kilometers. 
Table 3: Taxonomic referrals (bold = holotype), geographic localities and stratigraphic elevations of Chasmosaurus-like and Vagaceratops-like specimens above the Oldman (OF)-Dinosaur Park (DPF) formational contact, as exposed in Dinosaur Provincial Park $(\mathrm{DPP}) ;(\mathrm{NA})=$ not available. See Appendix I for more details.

\begin{tabular}{|c|c|c|c|c|}
\hline \multirow[t]{2}{*}{ Specimen } & \multicolumn{2}{|l|}{ Taxonomic referral } & \multirow{2}{*}{$\begin{array}{l}\text { Geographic } \\
\text { locality }\end{array}$} & \multirow{2}{*}{$\begin{array}{c}\text { Elevation } \\
\text { above OF- } \\
\text { DPF } \\
\text { contact, in } \\
\text { DPP (m) }\end{array}$} \\
\hline & Previous & This study & & \\
\hline AMNH 5401 & $\begin{array}{l}\text { Chasmosaurus kaiseni; } \\
\text { Chasmosaurus sp.; } \\
\text { Mojoceratops perifania }\end{array}$ & Chasmosaurus-like & $\begin{array}{l}\text { Dinosaur } \\
\text { Provincial } \\
\text { Park (DPP) }\end{array}$ & (NA) \\
\hline AMNH 5656 & Chasmosaurus russelli & Chasmosaurus-like & DPP & (NA) \\
\hline CMN 0491 & Chasmosaurus belli & Chasmosaurus-like & DPP & (NA) \\
\hline CMN 1254 & $\begin{array}{l}\text { Eoceratops canadensis; } \\
\text { M. perifania; } \\
\text { Chasmosaurus } \mathrm{sp} .\end{array}$ & Chasmosaurus-like & DPP & (NA) \\
\hline CMN 2245 & C. belli & Chasmosaurus-like & DPP & $42.0 \pm 5.0$ \\
\hline CMN 2280 & C.russelli & Chasmosaurus-like & DPP & $6.6 \pm 5.0$ \\
\hline CMN 8800 & C. russelli & Chasmosaurus-like & Milk River & $54.0 \pm 5.0$ \\
\hline CMN 8801 & Chasmosaurus sp. & Chasmosaurus-like & DPP & $22.0 \pm 5.0$ \\
\hline CMN 8802 & Chasmosaurus sp. & Chasmosaurus-like & Milk River & (NA) \\
\hline CMN 8803 & $\begin{array}{l}\text { C. russelli; } \\
\text { M. perifania }\end{array}$ & Chasmosaurus-like & $\begin{array}{l}\text { Saskatchewan } \\
\text { Landing } \\
\text { Provincial } \\
\text { Park }\end{array}$ & (NA) \\
\hline CMN 34829 & Chasmosaurus sp. & Chasmosaurus-like & DPP & (NA) \\
\hline CMN 34832 & $\begin{array}{l}\text { Chasmosaurus sp.; } \\
\text { M. perifania }\end{array}$ & Chasmosaurus-like & DPP & (NA) \\
\hline NHMUK R4948 & C. belli & Chasmosaurus-like & DPP & (NA) \\
\hline ROM 839 & $\begin{array}{l}\text { Chasmosaurus brevirostris; } \\
\text { Chasmosaurus sp. }\end{array}$ & Chasmosaurus-like & DPP & $36.0 \pm 5.0$ \\
\hline ROM 843 & C. belli & Chasmosaurus-like & DPP & $32.4 \pm 5.0$ \\
\hline
\end{tabular}


Table 3 (continued).

\begin{tabular}{|c|c|c|c|c|}
\hline \multirow[t]{2}{*}{ Specimen } & \multicolumn{2}{|l|}{ Taxonomic referral } & \multirow{2}{*}{$\begin{array}{l}\text { Geographic } \\
\text { locality }\end{array}$} & \multirow{2}{*}{$\begin{array}{c}\text { Elevation } \\
\text { above OF- } \\
\text { DPF } \\
\text { contact, in } \\
\text { DPP (m) }\end{array}$} \\
\hline & Previous & This study & & \\
\hline TMP 1979.011.0147 & $\begin{array}{l}\text { Chasmosaurus sp.; } \\
\text { M. perifania }\end{array}$ & Chasmosaurus-like & DPP & $9.0 \pm 5.0$ \\
\hline TMP 1981.019.0175 & Chasmosaurus sp. & Chasmosaurus-like & DPP & $11.5 \pm 5.0$ \\
\hline TMP 1983.025.0001 & $\begin{array}{l}\text { C. russelli; } \\
\text { M. perifania }\end{array}$ & Chasmosaurus-like & (NA) & (NA) \\
\hline TMP 1993.082.0001 & Chasmosaurus sp. & Chasmosaurus-like & DPP & $53.0 \pm 5.0$ \\
\hline TMP 1997.132.0002 & Chasmosaurus sp. & Chasmosaurus-like & Hilda & (NA) \\
\hline TMP 1999.055.0292 & M. perifania & Chasmosaurus-like & DPP & (NA) \\
\hline TMP 2008.012.0001 & Chasmosaurus sp. & Chasmosaurus-like & DPP & (NA) \\
\hline UALVP 40 & $\begin{array}{l}\text { E. canadensis; } \\
\text { Chasmosaurus sp. }\end{array}$ & Chasmosaurus-like & DPP & $18.2 \pm 5.0$ \\
\hline AMNH 5402 & C. belli & Vagaceratops-like & DPP & (NA) \\
\hline CMN 41357 & Vagaceratops irvinensis & Vagaceratops-like & Irvine & $\sim 55 \pm ?$ \\
\hline TMP 1987.045.0001 & V. irvinensis & Vagaceratops-like & DPP & $54.4 \pm 5.0$ \\
\hline TMP 1998.102.0008 & V. irvinensis & Vagaceratops-like & Milk River & $\geq 55$ \\
\hline TMP 2009.034.0009 & V. irvinensis & Vagaceratops-like & Hilda & $\sim 33.5 \pm ?$ \\
\hline TMP 2011.053.0046 & (NA) & Vagaceratops-like & Milk River & $53.0 \pm 5.0$ \\
\hline YPM 2016 & C. belli & Vagaceratops-like & DPP & $26.5 \pm 5.0$ \\
\hline
\end{tabular}




\subsection{Stratophylogenetic analysis}

\subsubsection{Introduction}

The analysis was conducted using the 153 unordered cranial (131) and postcranial (22) characters as used in the phylogenetic analysis of Chapter 6 (Fig. 37), and one ordered stratigraphic character (character 154) whose states pertain to the Dinosaur Park faunal zones of Ryan and Evans (2005) and Ryan et al. (2012) above the regional disconformity where a specimen is found: Dinosaur Park Faunal Zone $1(0-30 \mathrm{~m})$, state 0; Dinosaur Park Faunal Zone 2 (30-50 m), state 1; and, Dinosaur Park Faunal Zone 3 (5070 m, including Lethbridge Coal Zone), state 2 (Fig. 42).

Only Dinosaur Park Formation chasmosaurine specimens with known stratigraphy $(\mathrm{n}=16)$ were included in this analysis, as they could be coded for character 154. The centrosaurine ceratopsid Centrosaurus apertus, which also occurs in the Dinosaur Park Formation, was selected as the outgroup.

\subsubsection{Results}

Chasmosaurus-like specimens occur within a stratigraphic interval ranging from 6.6 $\pm 5.0 \mathrm{~m}(\mathrm{CMN} 2280$, Dinosaur Park Faunal Zone 1) to approximately 54 $\pm 5.0 \mathrm{~m}(\mathrm{CMN}$ 8800, Dinosaur Park Faunal Zone 3) above the Oldman-Dinosaur Park formational contact, or about $48 \pm 5.0 \mathrm{~m}$ in thickness (Fig. 42). Vagaceratops-like specimens define a shorter interval, ranging from 26.5 \pm 5.0 m (YPM 2016, Dinosaur Park Faunal Zone 1) to at least $55 \mathrm{~m}$ above the Oldman-Dinosaur Park formational contact (TMP 1998.102.0008, Dinosaur Park Faunal Zone 3), or at least 28.5 $\pm 5.0 \mathrm{~m}$ in thickness (Fig. 42).

In the resulting strict consensus tree (495 most parsimonious trees, tree length $=$ 
77 , consistency index $=0.86$, and retention index $=0.74$ ) (not figured), unresolved chasmosaurine specimens formed a polytomy with Centrosaurus apertus. In an attempt to improve resolution, the analysis was re-run using a subset of the 10 most complete specimens (CMN 2245, CMN 2280, CMN 8800, CMN 41357, ROM 839, ROM 843, TMP 1981.019.0175, TMP 1987.045.0001, UALVP 40, and YPM 2016), all of which were used previously in the phylogenetic analysis (Fig. 37). This produced one most parsimonious tree, tree length $=74$, consistency index $=0.88$ and retention index $=0.65$ ) (Fig. 43). In this latter tree, C. apertus remained as an outgroup taxon to chasmosaurine specimens (Node \#1; Fig. 43).

In the lowest faunal zone (Dinosaur Park Faunal Zone 1), the Chasmosaurus-like specimens UALVP 40 and TMP 1981.019.0175 form a sister group (Node \#2) to a clade containing all other Dinosaur Park Formation chasmosaurine specimens (Node \#3; Fig. 43). These latter specimens form two sister clades, corresponding to Vagaceratops-like specimens in Dinosaur Park Faunal Zones 1 and 3 (Node \#4), and Chasmosaurus-like specimens in Dinosaur Park Faunal Zones 1-3 (Node \#5). In the Vagaceratops-like clade, YPM 2016 (Dinosaur Park Faunal Zone 1) is the sister operative taxonomic unit to the group containing the diagnostic Vagaceratops specimens CMN 41357 and TMP 1987.045.0001 (Dinosaur Park Faunal Zone 3). In the Chasmosaurus-like clade, CMN 2280 (Dinosaur Park Faunal Zone 1), and ROM 843 (Dinosaur Park Faunal Zone 2) are successive sister operative taxonomic units to the remaining Chasmosaurus-like specimens ROM 839, CMN 2245 (both Dinosaur Park Faunal Zone 2) and CMN 8800 (Dinosaur Park Faunal Zone 3) which form a polytomy. 
Figure 43. Stratophylogenetic analysis using ceratopsid taxa from Dinosaur Park Formation (DPF) that were included in the phylogenetic analysis (Fig. 37): Centrosaurus apertus, Vagaceratops-like specimens, Chasmosaurus russelli, C. belli, and Chasmosaurus sp.; Chasmosaurine specimens included were those which possess documented stratigraphic positions in the DPF. Strict consensus of one mostparsimonious tree $(\mathrm{TL}=74$ steps, $\mathrm{CI}=0.88, \mathrm{RI}=0.65)$ using $153 \operatorname{cranial}(131)$ and postcranial (22) characters, and 1 stratigraphic character (character 154 - Dinosaur Park Faunal Zone 1 (DPFZ 1; state 0), Dinosaur Park Faunal Zone 2 (DPFZ 2; state 1) and Dinosaur Park Faunal Zone 3 (DPFZ 3; state 2)). Bootstrap replicate frequency values are shown to the left of each node (only Bootstrap values of $50 \%$ or higher are given); Bremer values are shown to the left of each node, in italics. DPF and composite faunal zones are shown to left in ascending stratigraphic order; faunal zones are not to scale with respect to each other. Branch lengths on strict consensus have been adjusted such that all specimens in a given faunal zone are aligned horizontally for convenience; this alignment does not imply that all specimens in a given zone occur at the exact same stratigraphic elevation within that zone. 
Figure 43.

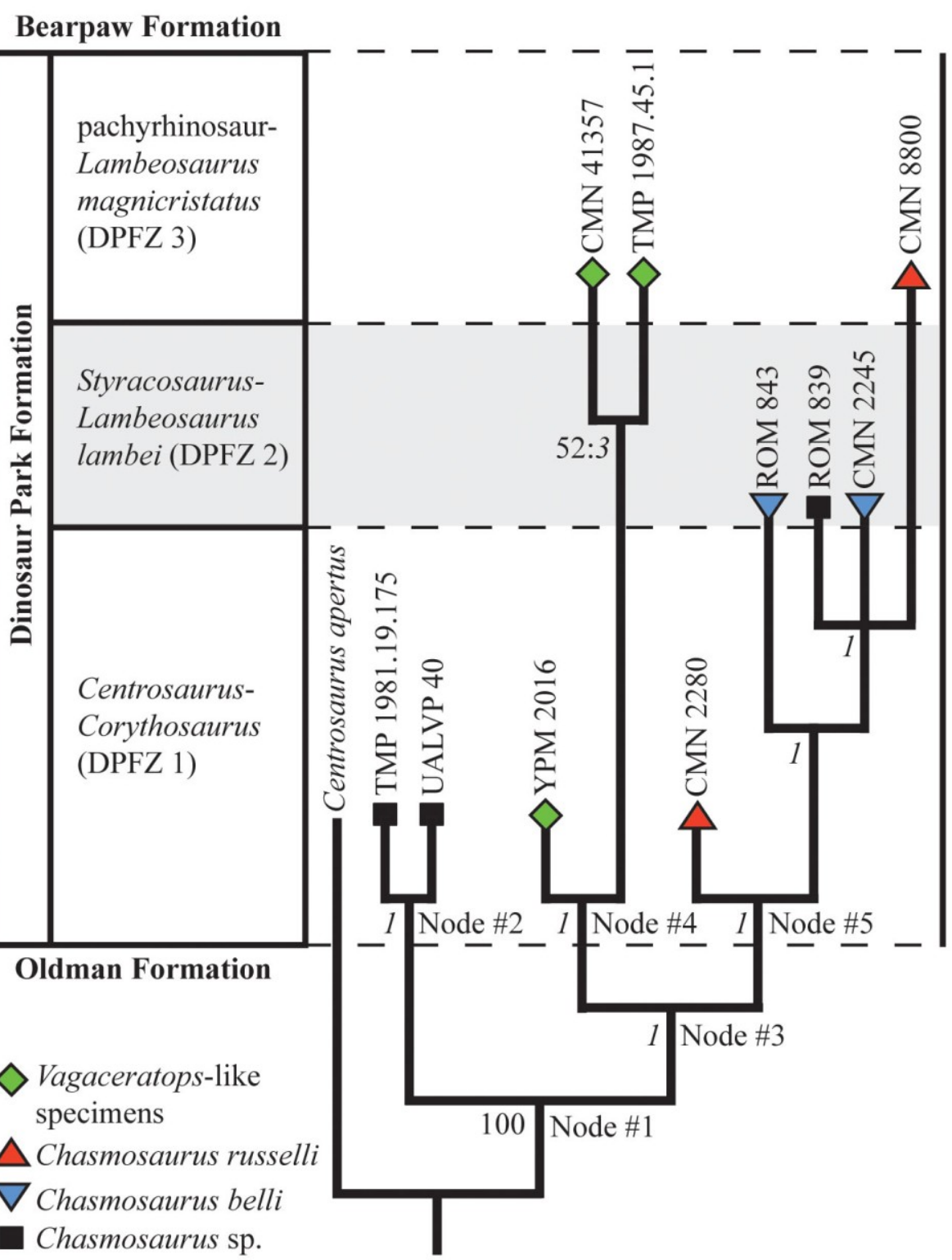




\subsubsection{Discussion}

\subsubsection{Stratophylogenetic analysis}

The stratigraphic occurrences of Chasmosaurus-like and Vagaceratops-like specimens support the null hypothesis that these two operative taxonomic groupings overlap stratigraphically, and thus temporally (Figs. 42-43).

The lack of discrete clades composed of specimens corresponding to Chasmosaurus belli and Chasmosaurus russelli, respectively (Fig. 43), supports the null hypothesis of one species of Chasmosaurus. The clade of Chasmosaurus-like specimens in Figure 43 (Node \#5) is interpreted here as representing the evolutionary lineage of a single taxon, $C$. belli, because it has historical taxonomic priority over C. russelli. These two previously referred species also do not form an anagenetic sequence, as they do not have discrete, non-overlapping stratigraphic occurrences (Figs. 42-43). The basal position of the Chasmosaurus-like specimens TMP 1981.019.0175 and UALVP 40 in Figure 43 (Node \#2) is likely due to the fact that the posterior half of the frill is not preserved in these specimens, preventing their assignment to one of the two more derived clades (Vagaceratops-like clade, Node \#4, and Chasmosaurus-like clade, Node \#5) that are largely distinguished by posterior frill characters (e.g., parietal fenestra length/width ratio, shape of posterior parietal margin and epiparietal count).

The Vagaceratops-like clade in Figure 43 is also inferred here as representing an evolutionary lineage of a single species, with YPM 2016 representing a less derived member and CMN 41357 and TMP 1987.045.0001 representing more derived members of that species. The recovery of YPM 2016 as a basal member of the Vagaceratops-like specimens may be explained by the low stratigraphic position of this specimen (Dinosaur 
Park Faunal Zone 1) relative to other members of this OTU (CMN 41357 and TMP 1987.045.0001, both Dinosaur Park Faunal Zone 3), and the large stratigraphic gap (approximately $28 \pm 5 \mathrm{~m}$ ) between them (Fig. 42). This stratigraphic gap represents a period of approximately 300,000 years (David Eberth, pers. comm.), an interval of time that may reflect the rate of ceratopsid evolution in the Dinosaur Park Formation. A total of nine centrosaurine taxa (Xenoceratops foremostensis; Albertaceratops nesmoi, Spinops sternbergorum, Centrosaurus apertus, Coronosaurus brinkmani, Styracosaurus albertensis, unnamed Pachyrhinosaurus species from Dinosaur Provincial Park, Pachyrhinosaurus canadensis, and Pachyrhinosaurus lakustai) are known from the Campanian to Maastrichtian of Alberta, spanning approximately 5.5 million years (Xenoceratops foremostensis $=78 \mathrm{Ma}($ Ryan et al., 2013a) to $72.5 \mathrm{Ma}=$ Pachyrhinosaurus lakustai (Sampson and Loewen, 2010)), suggesting an average turnover rate of approximately 610,000 years for these taxa.

Evolutionary changes within the Vagaceratops-like operative taxonomic unit are inferred to have taken place between Dinosaur Park Faunal Zones 1 and 3, including a reduction in the parietal fenestra length-width ratio, a lengthening of the epiparietals, and a coalescence of epiparietals at their bases (Figs. 34B-C and 42). These inferred evolutionary changes are difficult to test, however, given the scarcity of Vagaceratopslike specimens between these two faunal zones.

The stratigraphic sequence of chasmosaurine specimens in the Dinosaur Park Formation (Fig. 42) cannot be interpreted as representing an example of sequential faunal turnover in southern Alberta (contra Godfrey and Holmes, 1995; Holmes et al., 2001; Ryan and Evans, 2005). Previous statements of stratigraphic segregation between C. belli 
and C. russelli, and between Chasmosaurus and Vagaceratops (Godfrey and Holmes, 1995; Holmes et al., 2001; Ryan and Evans, 2005) were based on inferences derived from a small sample size of specimens with accurate geographic and stratigraphic coordinate data, and the misplacement of the CMN 8800 (C. russelli holotype) quarry in section.

An anagenetic model where the Chasmosaurus-like group evolved into the Vagaceratops-like operative taxonomic unit over time also cannot be used to explain the stratigraphic distributions of these two operative taxonomic groupings; the evolutionary alternative model, cladogenesis, must therefore be accepted. The lack of stratigraphic segregation between these two operative taxonomic groupings indicates that epiparietal count does not change from three (Chasmosaurus) to four or five (Vagaceratops) over time, as was originally implied by Holmes et al. (2001) and Ryan and Evans (2005). The lack of a stratigraphic pattern in epiparietal count in Dinosaur Park Formation chasmosaurines is unlike what Scannella and Horner (2011) proposed for the Hell Creek Formation of Montana, changing from 10 or 12 (Torosaurus) to six (Triceratops) over time.

The previous referral of the Vagaceratops-like YPM 2016 to Chasmosaurus (Godfrey and Holmes, 1995; Maidment and Barrett, 2011) suggests that their close morphological similarity may be the result of temporal proximity to an inferred common ancestor. The previous inference that Vagaceratops originated in southern Laramidia (Utah; Sampson et al., 2010) is not supported because Vagaceratops shares a common ancestor with Chasmosaurus from northern Laramidia (Alberta). Although this has important implications for the evolution of this branch of Chasmosaurinae, this is outside of the scope of this project. 
The inference that the clade of Chasmosaurus +Vagaceratops evolved from an ancestor that possessed three epiparietals is supported by the fact the outgroups for this clade (e.g., Pentaceratops and Utahceratops; Fig. 37) also have three epiparietals. Assuming that the Chasmosaurus-like and Vagaceratops-like specimens evolved from a common ancestor inhabiting northern Laramidia, it is difficult to determine what event or mechanism caused their speciation, and when this occurred. It is possible that some geographic barrier resulting from e.g., rising water levels in the adjoining Western Interior Seaway divided the parent population in two, leading to two independently evolving subpopulations (Sampson and Loewen, 2010), but there is presently no supporting geological data for this hypothesis.

The co-existence of two chasmosaurine taxa in the same geographic area is relatively rare. Apart from the coeval Chasmosaurus and Vagaceratops-like taxa, the only known co-occurring chasmosaurines are Kosmoceratops and Utahceratops in the Kaiparowits Formation of Utah (Sampson et al., 2010), and Triceratops and Torosaurus in the Hell Creek Formation and age equivalent sediments of Montana and neighbouring states and provinces (Sampson and Loewen, 2010; Longrich and Field, 2012). However, this phenomenon may be even less common if Scannella and Horner's (2010) Triceratops-Torosaurus synonymy is validated.

The rarity of co-occurring chasmosaurine taxa in a given region may be due to insufficient fossil sampling, resulting in poorly established stratigraphic ranges for these taxa. Perhaps more likely, this rarity is due to a large overlap in morphology, physiology and dietary requirements between co-occurring chasmosaurine taxa, resulting in interspecific competition for resources (Sampson and Loewen, 2010). It is likely that the 
Chasmosaurus-like and Vagaceratops-like groups had similar resource requirements, but it is difficult to determine what differing physiological, dietary, or perhaps behavioural mechanism permitted their co-existence between the top of Dinosaur Park Faunal Zone 1 and the base of the Lethbridge Coal Zone (Dinosaur Park Faunal Zone 3; Figs. 42-43), for approximately 300,000 years (David Eberth, pers. comm.). More work needs to be done on differences in skull and beak shape (Henderson, 2010, Mallon and Anderson, 2014), snout morphology and jaw mechanics between these two taxa before hypotheses of habitat preference can be fully explored.

\subsubsection{Morphological change over time}

Elevation is directly related to time as the average sedimentation rate of the Dinosaur Park Formation can be determined (Eberth, 2005; David Eberth, pers. comm.). In Dinosaur Provincial Park, bentonites derived from the top of the Oldman Formation and near the base of the Bearpaw Formation yielded ${ }^{40} \mathrm{Ar} /{ }^{39} \mathrm{Ar}$ dates of $77.0 \pm 0.5 \mathrm{Ma}$ and 75.5 Ma, respectively (David Eberth, pers. comm.; Fig. 1). The Dinosaur Park Formation, therefore, spans approximately 1.44 Ma. Recently completed laser ablation-inductively coupled plasma-mass spectrometry (LA-ICP-MS) analyses of zircons from these same bentonites have yielded ${ }^{206} \mathrm{~Pb} /{ }^{238} \mathrm{U}$ dates which suggest that the Dinosaur Park Formation may have spanned an even shorter time interval (approximately 1.26 Ma; David Eberth, pers. comm.).

By examining Dinosaur Park Formation chasmosaurine specimens in a stratigraphic sequence, morphological change over time can be tested. A line-of-best-fit was assigned to each parameter-elevation plot. Only parameters with a significant 
$(p<0.05)$ relationship with stratigraphy are reported. An analysis using only Vagaceratops-like specimens of known stratigraphy $(\mathrm{n}=6)$ could not be performed due to an insufficient number of specimens of known stratigraphy; three of these six specimens (TMP 1998.102.0008, TMP 2009.034.0009 and TMP 2011.053.0046) are too fragmentary to record any of the 36 parameter measurements.

Of the 10 Chasmosaurus-like specimens of known stratigraphy (Table 3), only one (UALVP 40), has a relatively small rostral-to-epijugal length $(628 \mathrm{~mm}$; parameter 16, Fig. 5) compared to the other nine specimens ( $\geq 750 \mathrm{~mm}, \mathrm{CMN} 2280$ ), and likely represents a relatively immature individual. Ideally, analyses investigating morphometric change over stratigraphy should be limited to specimens of approximately equal stages of maturity to remove the confounding effects of ontogenetic differences. However, given the already small sample size of stratigraphically-documented Chasmosaurus-like specimens, and the fact that the relatively large and putatively mature specimens of this group likely vary ontogenetically as well (based on varying skull sizes), I chose to include UALVP 40 in the morphometric analysis.

Amongst the 10 Chasmosaurus-like specimens examined, only four cranial parameters $(19,20,21$, and 28; Figs. 44-47) of the 36 examined had a significant $(p<0.05)$ relationship with stratigraphy (Table 4$)$, and were positively correlated with elevation. These four parameters, all relating to aspects of the parietosquamosal frill, are: 1, squamosal length (parameter 19; Fig. 44); 2, distance between the anterior margin of the dorsal temporal fenestra and parietal fenestra (parameter 20; Fig. 45); 3, distance between the jugal notch and parietal fenestra (parameter 21; Fig. 46); and 4, distance between the jugal notch and back of frill (parameter 28; Fig. 47). 
Table 4: Cranial parameters with relationship to stratigraphy. Significant results $(p<0.05)$ in bold.

\begin{tabular}{|c|c|c|c|}
\hline $\begin{array}{l}\text { Cranial } \\
\text { parameter }\end{array}$ & $p$ & $\begin{array}{l}\text { Cranial } \\
\text { parameter }\end{array}$ & $p$ \\
\hline 1 & 0.1693 & 16 & 0.1583 \\
\hline 2 & 0.2670 & 17 & 0.3089 \\
\hline 3 & 0.7793 & 18 & 0.6320 \\
\hline 4 & 0.5243 & 19 & 0.0135 \\
\hline 5 & 0.3832 & 20 & 0.0409 \\
\hline $5 a$ & 0.3159 & 21 & 0.0038 \\
\hline $5 b$ & 0.1463 & 22 & 0.9017 \\
\hline $5 c$ & 0.3268 & 23 & 0.1168 \\
\hline 6 & 0.2799 & 24 & 0.4756 \\
\hline 7 & 0.3343 & 25 & 0.1293 \\
\hline 8 & 0.5984 & 26 & 0.1952 \\
\hline 9 & 0.2941 & 27 & 0.5107 \\
\hline 10 & 0.2263 & 28 & 0.0383 \\
\hline 11 & 0.1858 & 29 & 0.2302 \\
\hline 12 & 0.7646 & 30 & 0.9332 \\
\hline 13 & 0.9471 & 31 & 0.9203 \\
\hline 14 & 0.4806 & 32 & 0.5962 \\
\hline 15 & 0.5687 & 33 & 0.7286 \\
\hline
\end{tabular}




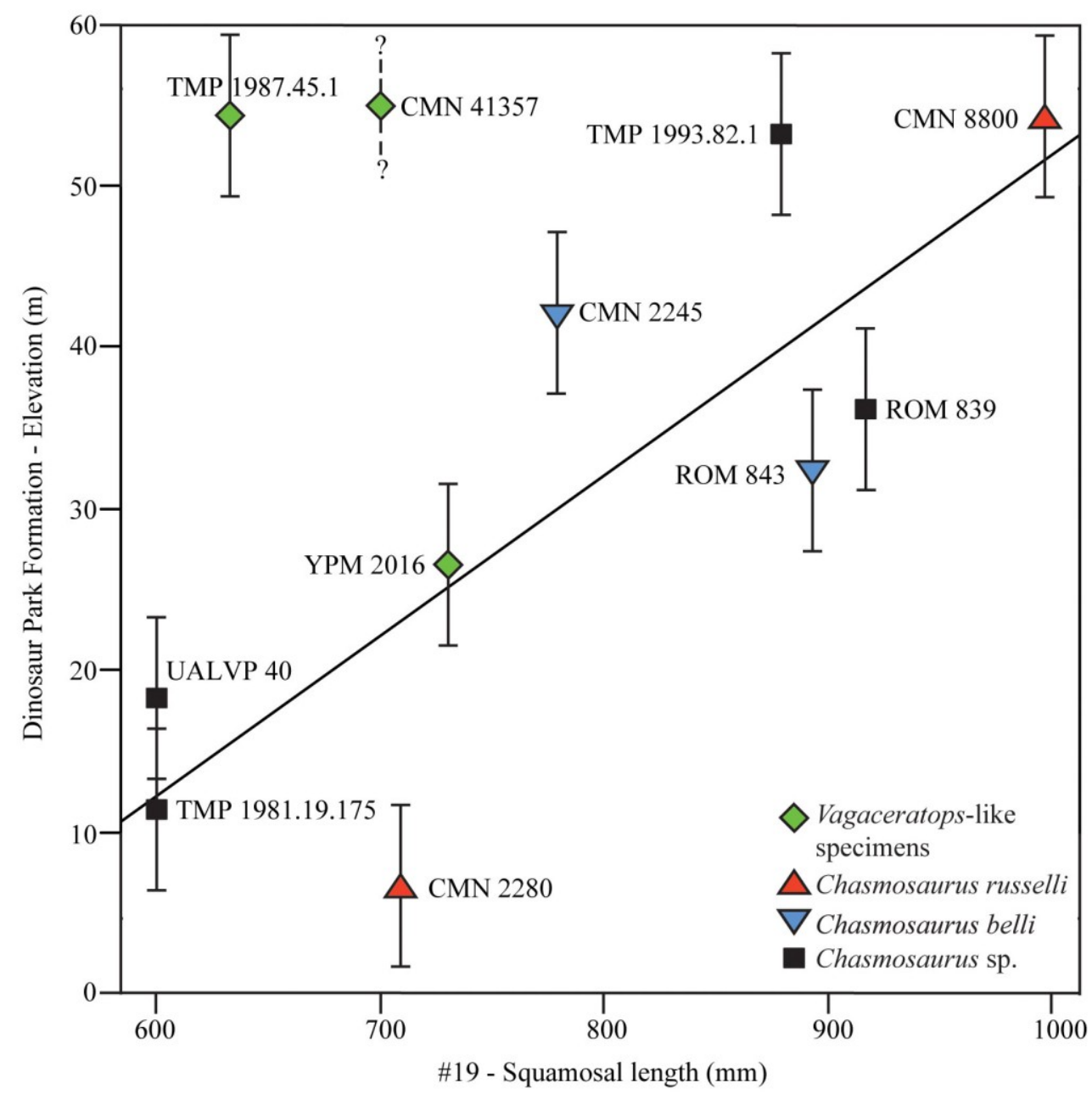

Figure 44. Scatter plot showing squamosal length (parameter 19 in Fig. 5) of

Vagaceratops-like specimens, Chasmosaurus russelli, C. belli, and Chasmosaurus sp. and their respective elevation above the regional disconformity $(0 \mathrm{~m})$ separating the Dinosaur Park and Oldman formations. Line-of-best-fit through Chasmosaurus-like specimens $(p=0.0135)$. 


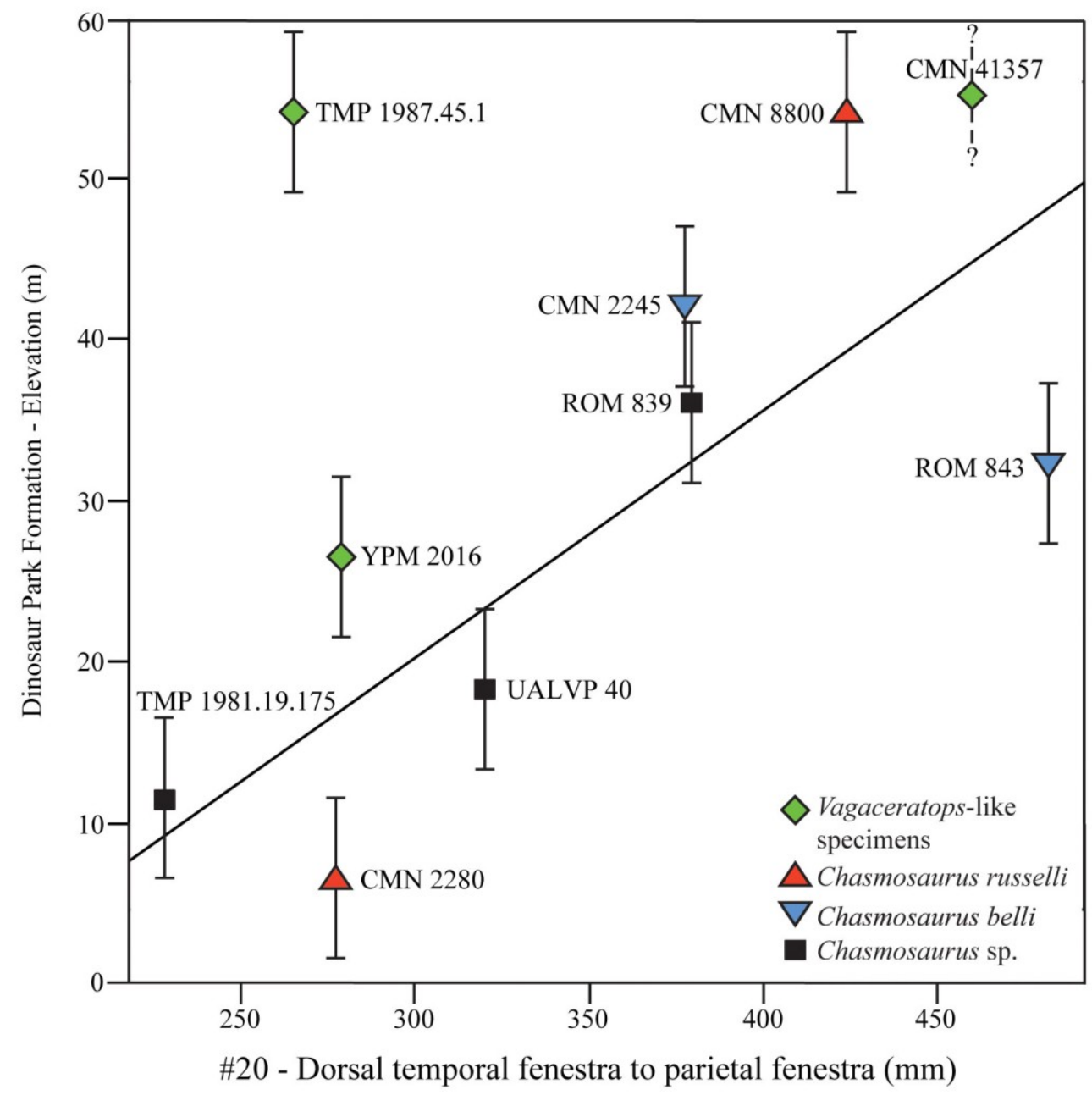

Figure 45. Scatter plot showing dorsal temporal fenestra to parietal fenestra length (parameter 20 in Fig. 5) of Vagaceratops-like specimens, Chasmosaurus russelli, C. belli, and Chasmosaurus sp. and their respective elevation above the regional disconformity ( 0 m) separating the Dinosaur Park and Oldman formations. Line-of-best-fit through Chasmosaurus-like specimens $(p=0.0409)$. 


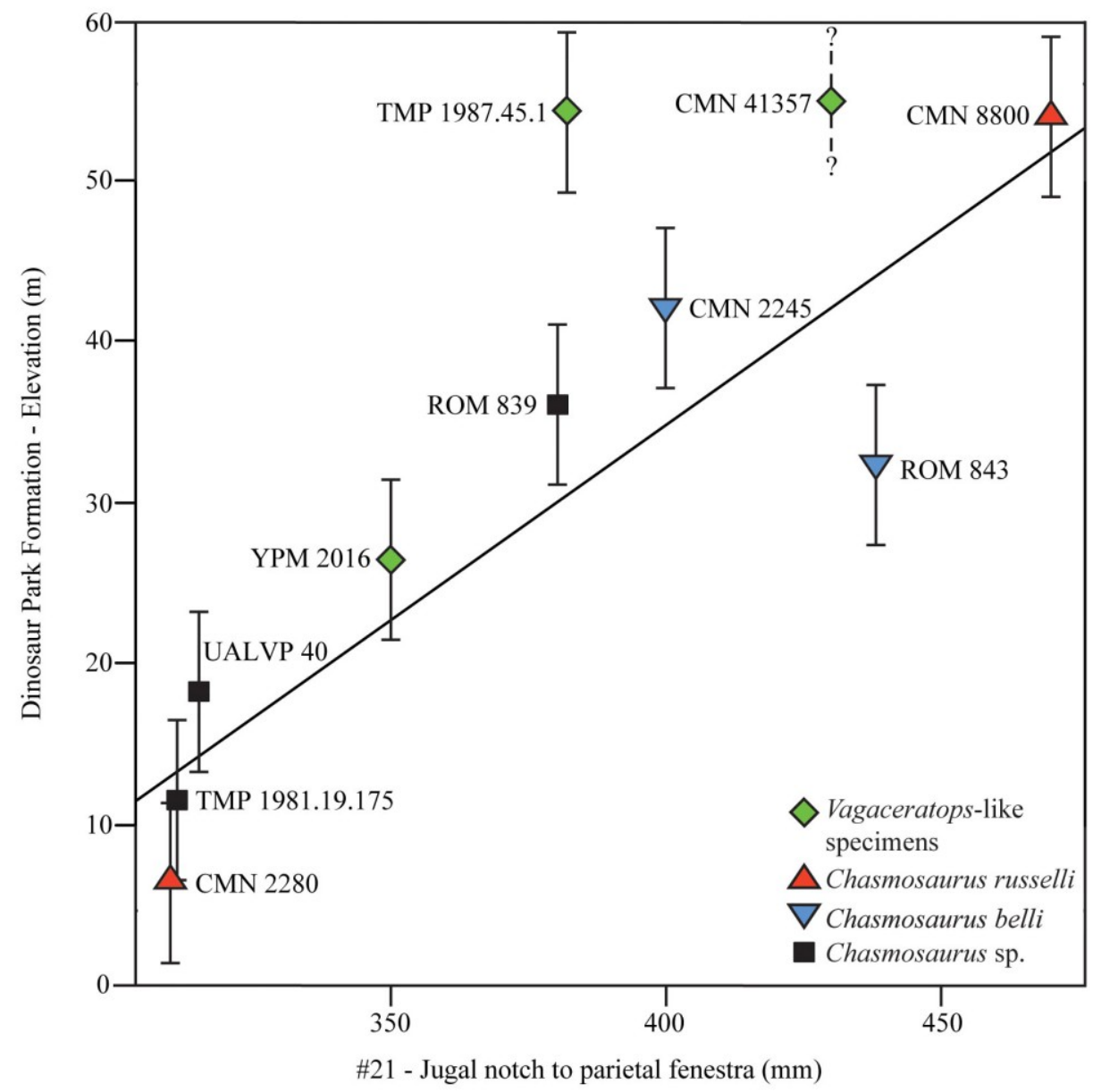

Figure 46. Scatter plot showing jugal notch to parietal fenestra length (parameter 21 in Fig. 5) of Vagaceratops-like specimens, Chasmosaurus russelli, C. belli, and Chasmosaurus sp. and their respective elevation above the regional disconformity $(0 \mathrm{~m})$ separating the Dinosaur Park and Oldman formations. Line-of-best-fit through Chasmosaurus-like specimens $(p=0.0038)$. 


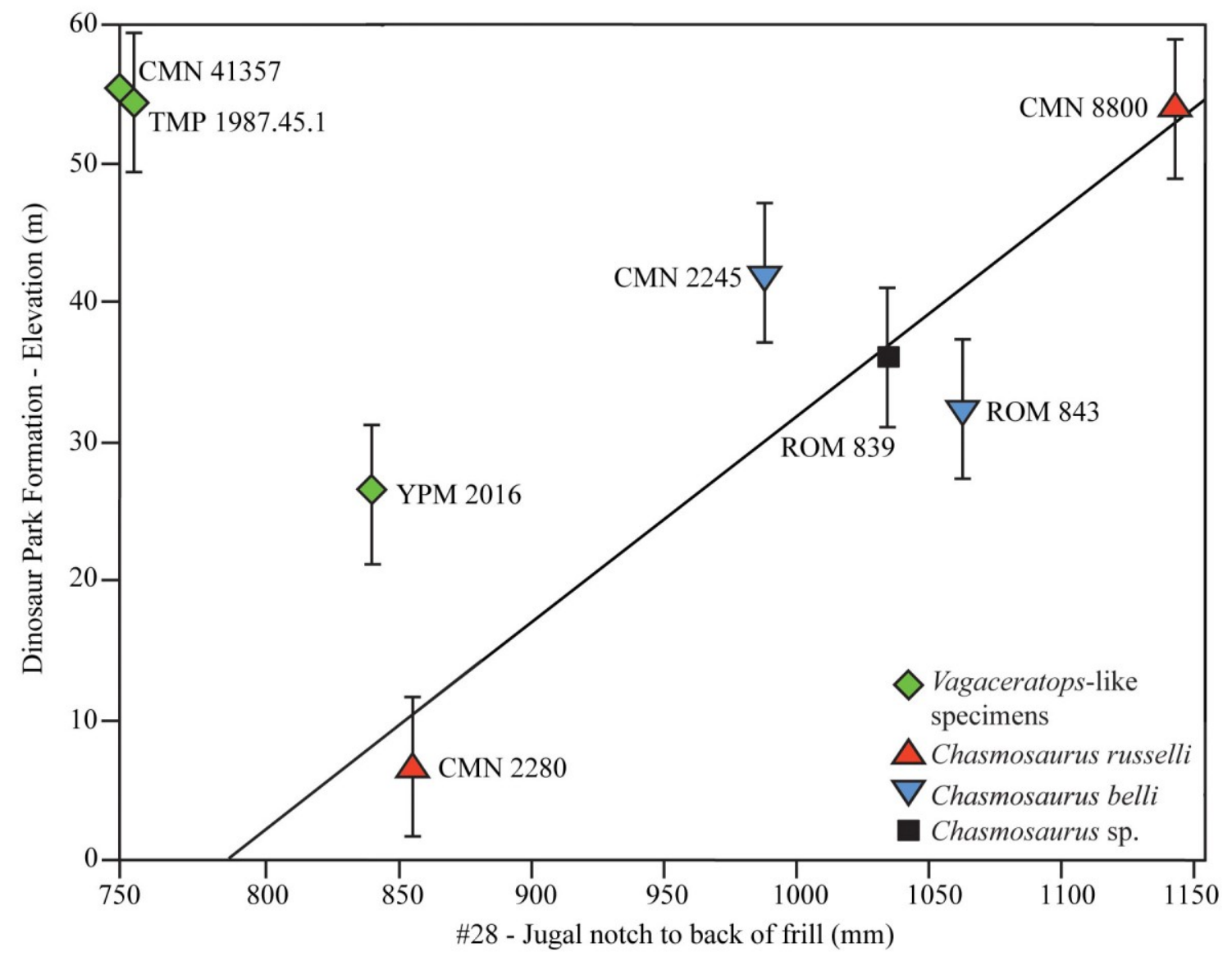

Figure 47. Scatter plot showing jugal notch to back of frill (parameter 28 in Fig. 5) of Vagaceratops-like specimens, Chasmosaurus russelli, C. belli, and Chasmosaurus sp. and their respective elevation above the regional disconformity $(0 \mathrm{~m})$ separating the Dinosaur Park and Oldman formations. Line-of-best-fit through Chasmosaurus-like specimens $(p=0.0383)$. 
These results are indicative of increasing frill length in Chasmosaurus over the time of deposition of the Dinosaur Park Formation and appear to represent directed evolutionary changes within this taxon, although a more robust sample size is required to test this. The lengthening of the lateral margins of the frill (parameters 19 and 28) over time does not correlate with directional change in the angle of embayment on the posterior parietal bar (parameter 33), which is rather randomly distributed throughout the Dinosaur Park Formation. However, before stratigraphic trends amongst Chasmosauruslike specimens can be attributed to evolutionary changes in a single taxon, further testing of the null hypothesis of one species of Chasmosaurus on the basis of ontogeny (Chapter 8) and morphometrics (Chapter 9) is required.

The scarcity of relatively immature Chasmosaurus-like specimens makes it difficult to determine whether this operative taxonomic grouping underwent heterochronic changes over time, as has been proposed in the Triceratops-Torosaurus synonymy of Scannella and Horner, (2010).

\subsubsection{Morphological variation due to geographic distribution}

Inter-regional morphological differences

Morphological differences amongst approximately time equivalent Dinosaur Park Formation chasmosaurine specimens from differing geographic regions are shown in Table 5. Time equivalent groups of specimens are categorized by their taxonomic assignment (Chasmosaurus-like and Vagaceratops-like). The known geographic range of these groups during each stratigraphic or temporal 'snapshot' is also discussed in this section. 
Table 5: Potential regional differences amongst Chasmosaurus-like and Vagaceratopslike specimens; P2 = epiparietal 2, P3 = epiparietal 3, P4 = epiparietal 4; (NA) = not available.

\begin{tabular}{|c|c|c|c|c|c|c|}
\hline \multirow{2}{*}{\multicolumn{3}{|c|}{$\begin{array}{l}\text { Taxonomic assignment and stratigraphic } \\
\text { interval (metres above Oldman-Dinosaur } \\
\text { Park formational contact) }\end{array}$}} & \multicolumn{4}{|c|}{ Geographic region } \\
\hline & & & Dinosaur & Hilda & Irvine & Milk River \\
\hline \multirow{5}{*}{$\begin{array}{l}\text { Vagaceratops- } \\
\text { like; } \sim 54.4 \pm 5.0 \\
\text { to } \geq 55\end{array}$} & \multicolumn{2}{|c|}{ Specimen(s) } & & \multirow[t]{5}{*}{ (NA) } & CMN & TMP \\
\hline & \multirow[t]{4}{*}{$\begin{array}{c}\text { Skull } \\
\text { parameter }\end{array}$} & $\begin{array}{l}\text { P2 } \\
\text { length } \\
(\mathrm{mm})\end{array}$ & (NA) & & $\sim 140$ (left) & $\sim 70$ (A, right) \\
\hline & & $\begin{array}{l}\text { P3 } \\
\text { length } \\
(\mathrm{mm})\end{array}$ & (NA) & & $\sim 140$ (left) & $\sim 50$ (A, left) \\
\hline & & $\begin{array}{c}\mathrm{P} 4 \\
\text { length } \\
(\mathrm{mm})\end{array}$ & $\sim 100$ (left) & & $\sim 100$ (left) & $\begin{array}{l}\sim 100(\mathrm{~A}, \text { left }) \\
\sim 25 \text { (B, left) }\end{array}$ \\
\hline & & $\begin{array}{c}\text { Parietal } \\
\text { fenestra } \\
\text { length/ } \\
\text { width } \\
\text { ratio }\end{array}$ & 0.87 (left) & & 0.72 (left) & (NA) \\
\hline \multirow{2}{*}{$\begin{array}{l}\text { Chasmosaurus- } \\
\text { like; } \sim 53.0 \pm 5.0 \\
\text { to } 54.0 \pm 5.0\end{array}$} & \multicolumn{2}{|c|}{ Specimen } & $\begin{array}{c}\text { TMP } 1993 . \\
082.0001\end{array}$ & (NA) & (NA) & CMN 8800 \\
\hline & \multicolumn{2}{|c|}{ Skull parameter } & \multicolumn{4}{|c|}{$\begin{array}{l}\text { (no distinguishable differences between specimens; limited } \\
\text { overlap in cranial material) }\end{array}$} \\
\hline
\end{tabular}


The precise stratigraphic position of the easternmost Chasmosaurus-like specimen, CMN 8803 (Saskatchewan Landing Provincial Park; Fig. 3), in the Dinosaur Park Formation is not known. However, given the time-transgressive nature of the Lethbridge Coal Zone moving east-west (Eberth, 2005), it is likely that this specimen was collected from sediments time equivalent to the lower or middle portion of the Dinosaur Park Formation as exposed in Dinosaur Provincial Park (i.e., Dinosaur Park faunal zones 1 or 2), as noted by Longrich (2010). Given the fact that Chasmosaurus-like specimens are known from every $10 \mathrm{~m}$ increment in the Dinosaur Park Formation of Dinosaur Provincial Park (Fig. 42), it is also likely that this group had a geographic range that spanned at least as far as Saskatchewan Landing Provincial Park from present day Dinosaur Provincial Park at some time during Dinosaur Park faunal zones 1 or 2 . The isolated, eastern occurrence of CMN 8803 relative to other Chasmosaurus-like specimens is probably due to the limited exposure of Dinosaur Park Formation sediments in Saskatchewan.

The stratigraphically higher Chasmosaurus-like specimens TMP 1993.082.0001 and CMN 8800 suggests that Chasmosaurus had a geographic range that spanned at least as far as the Milk River region from Dinosaur Provincial Park during Dinosaur Park Faunal Zone 3 (Fig. 42; Table 5). As the Dinosaur Park Faunal Zone 3 of eastern Alberta is time equivalent to the marine Bearpaw Formation of Saskatchewan Landing Provincial Park in western Saskatchewan (Eberth, 2005), it can be concluded that the geographic range of the Chasmosaurus-like group became more westerly restricted between Dinosaur Park faunal zones 2 and 3. CMN 8800 and TMP 1993.082.0001 have limited overlapping cranial material, of which no obvious morphological differences exist (Table 
$5)$.

The approximately time equivalent Vagaceratops specimens TMP 1987.045.0001, CMN 41357, TMP 1998.102.0008 and TMP 2011.053.0046 suggest that this taxon had a range encompassing Dinosaur Provincial Park, Irvine and the Milk River region during Dinosaur Park Faunal Zone 3 (Table 5). Epiparietals are similar in size between Dinosaur Provincial Park (TMP 1987.045.0001) and Irvine (CMN 41357), but are generally larger than those in the Milk River region (TMP 1998.102.0008 and TMP 2011.053.0046). However, the large variability of epiparietal length in the Milk River region suggests that this feature is highly individually variable. The parietal fenestra length/width ratio is larger in Dinosaur Provincial Park (TMP 1987.045.0001) compared to Irvine (CMN 41357). However, the difference between these ratios is relatively minor ( 0.87 and 0.72 , respectively), and individual variation is to be expected in a sample size of two.

Longitudinally, Vagaceratops-like specimens from the Milk River and Irvine regions are approximately $75 \mathrm{~km}$ east of those from Dinosaur Provincial Park. These former specimens therefore may have lived closer to the Western Interior Seaway and may have consumed different vegetation than specimens further west. However, of the cranial parameters used by Mallon et al. (2013) to infer dietary physiology (distance from jaw joint to: rostral, posterior beak tip, medial end of toothrow, distal end of toothrow, and coronoid process; maximum beak width, mandible depth, paroccipital process breadth, occiput height, depression of snout below occlusal plane, cranial height, and distance between quadrates) preserved in these specimens, none appear to vary. Perhaps Vagaceratops-like specimens had similar dietary adaptations across southern Alberta. 
Morphological differences amongst approximately time equivalent specimens in this study cannot reliably be attributed to geography alone, due to a statistically insignificant sample size for each stratigraphic or temporal snapshot. Morphological differences within Chasmosaurus-like and Vagaceratops-like specimens are unlikely to vary over a geographic gradient even with an adequate sample size, because these two groups are both restricted to relatively small regions. The recent discovery of new ceratopsid species from western North America with latitudinally-disjunct distributions has been used to support the theory of dinosaur provincialism in Late Cretaceous Laramidia, with little evidence for long-distance migration amongst these species (Sampson et al., 2010, 2013). Body mass is positively correlated with home range size in extant mammals, and is related to increasing resource requirements (Kelt and Van Vuren, 2001). Large-bodied ceratopsids may have had smaller geographic ranges than those of modern megaherbivorous mammals, made possible by potentially having had lower metabolic rates, access to larger amounts of high-quality vegetation, or both (Lehman, 2007; Sampson, 2009). Alternatively, further fossil sampling may reveal that ceratopsids had larger ranges than are currently documented.

Intra-regional morphological differences

The Chasmosaurus-like specimens CMN 2280, TMP 1979.011.0147 and TMP 1981.019.0175 provide a unique example of postorbital horncore length variation within an approximately time equivalent $(6.6 \pm 5.0 \mathrm{~m}$ to $11.5 \pm 5.0 \mathrm{~m}$, Dinosaur Park Faunal Zone 1; Table 3, Fig. 42) group in a single region (Dinosaur Provincial Park). CMN 2280 (rostral-to-epijugal length $=750 \mathrm{~mm}$ ) has a relatively short horncore (left side $=132$ 
$\mathrm{mm}$ ), whereas TMP 1981.019.0175 (rostral-to-epijugal length $=768 \mathrm{~mm}$ ) has a horncore that is comparable in size as preserved (right side $=134 \mathrm{~mm}$ ), but has undergone extensive remodeling, and was most likely considerably longer in its original form. The partial skull, TMP 1979.011.0147, has relatively elongate horncores (right side $=298$ $\mathrm{mm})$.

Given the similarity in skull size between the relatively short-horned CMN 2280 (rostral-to-epijugal length $=750 \mathrm{~mm}$ ) and relatively long-horned TMP 1981.019.0175 (rostral-to-epijugal length $=768 \mathrm{~mm}$; the long-horned TMP 1979.011.0147 likely had a similar skull size), it is likely that these specimens represent similar stages of maturity. If ontogenetic differences cannot account for this variation in postorbital horncore length, perhaps this variation is instead attributable to individual differences or sexual dimorphism. Chapter 8 will examine how postorbital horncore length changes over ontogeny and sexual dimorphism will be tested as a potential source of variation in this feature.

\subsection{Conclusion}

No stratigraphic separation exists between Chasmosaurus-like specimens previously referred to Chasmosaurus belli and Chasmosaurus russelli, or between Chasmosaurus-like and Vagaceratops-like specimens $\left(\mathrm{H}_{0}\right)$. These findings do not support the theory that Chasmosaurus and Vagaceratops experienced sequential replacement in the Dinosaur Park Formation (contra Godfrey and Holmes, 1995; Holmes et al., 2001; Ryan and Evans, 2005), but rather appear to have coexisted and evolved in parallel from a common ancestor. 
The stratophylogenetic analysis supports the null hypothesis that Chasmosauruslike specimens define one evolutionary lineage, which in turn supports the null hypothesis of one species of Chasmosaurus. Of the 36 cranial parameters shown in Figure 5, only four (parameters 19, 20,21, and 28) change significantly and positively with time amongst Chasmosaurus-like specimens indicating that the frill lengthened over time. However, this increase in frill length does not affect the relative embayment of the posterior parietal bar, a feature previously used to distinguish $C$. belli and C. russelli. The random distribution of embayment angle over elevation indicates that the deeply embayed C. russelli and shallowly embayed C. belli do not form an anagenetic sequence.

The fossil-rich Dinosaur Park Formation has extensive lateral exposure in Dinosaur Provincial Park that has historically been more heavily sampled relative to Dinosaur Park Formation exposures in other parts of Alberta and Saskatchewan. This collection bias makes it difficult to assess whether regional differences exist amongst Chasmosaurus-like or Vagaceratops-like specimens. The chasmosaurine skulls from Dinosaur Provincial Park express the complete range of cranial morphological variability for both Chasmosaurus and Vagaceratops. Postorbital horncore length is variable amongst Chasmosaurus-like specimens of similar maturity, collected from approximately time equivalent sediments in the same region (Dinosaur Provincial Park). This suggests that postorbital horncore length is at least partly attributable to individual variation or sexual dimorphism. Further sampling outside of Dinosaur Provincial Park is required to properly make regional comparisons for Chasmosaurus and Vagaceratops. Chapter 8 will examine how postorbital horncore length changes over ontogeny and sexual dimorphism will be tested as a potential source of variation in this feature. 


\section{Chapter 8. Ontogenetic Analysis of Chasmosaurus}

\subsection{Introduction}

This chapter examines whether ontogeny can explain the morphological variation seen in the cranial material referred to Chasmosaurus. Ontogenetic studies on other dinosaurs have resulted in: 1, the establishment of age classes within a given taxon, e.g., the tyrannosaur Albertosaurus sarcophagus (Carr, 2010) and the chasmosaurine Agujaceratops mariscalensis (Lehman, 1990); 2, the invalidation of taxa originally based on immature material, e.g., referring the tyrannosaurs Stygivenator molnari and Dinotyrannus megagracilis as juvenile and subadult members, respectively, of Tyrannosaurus rex (Carr and Williamson, 2004), and the referral of the lambeosaurines Procheneosaurus erectofrons and Procheneosaurus cranibrevis to juveniles of Corythosaurus casuarius (Dodson, 1975); or 3, the validation of taxa previously invalidated, e.g., identifying mature and immature individuals of both of the chasmosaurines Triceratops and Torosaurus, reinstating Torosaurus as a distinct genus and not the mature form of Triceratops (Longrich and Field, 2012).

Establishing the absolute age of an individual at the time of its death can be difficult for fossil taxa. Lines of arrested growth (LAGs) are concentrically-arrayed, cyclic markers deposited annually in bones of extant and extinct animals (Lee and Werning, 2008), and have been used to determine the relative ages of individuals in various ceratopsian taxa (Erickson and Tumanova, 2000; Reizner and Horner, 2006; Lee, 2006, 2007; Lee and Werning, 2008; Erickson and Druckenmiller, 2011). These histological data obtained by thin-sectioning elements, coupled with associated limb bone 
circumferential measurements as a proxy for body size, have been used to reconstruct skeletal growth curves for the examined taxa. When appendicular material is scarce and limited to relatively large, adult sized individuals of a given taxon, as is the case in this study, other proxies for age must be utilized.

Skeletal element size can be useful, in part, to establish age classes (Ryan et al., 2001). For ceratopsids, individual elements of a given taxon have been referred to juvenile-, subadult- and adult-sized categories established in relation to the putative largest adult size known for a taxon (Ryan et al., 2001; Horner and Goodwin, 2006; Currie et al., 2008). An overreliance on size as a proxy for maturity, however, can prevent the distinction between juveniles of larger taxa and adults of smaller, related taxa (Tumarkin-Deratzian, 2010). Size-independent proxies are, therefore, also important in defining age classes within a given taxon and have been used in ceratopsid ontogenetic studies. These include the degree of ossification of epiossifications to the skull, development of cranial ornamentation and changes in cranial bone surficial texture (Ryan et al., 2001; Horner and Goodwin, 2006; Brown et al., 2009; Scannella and Horner, 2010).

The attainment of skeletal maturity does not necessarily imply that sexual maturity has been reached. Sexual maturity precedes skeletal maturity in extant crocodylians (Wilkinson and Rhodes, 1997), squamates (Shine and Charnov, 1992) and medium-to-large-sized mammals (Lee and Werning, 2008), whereas the opposite occurs in extant birds (Tumarkin-Deratzian et al., 2006) and some small-sized mammals (Lee and Werning, 2008). Medullary bone is an endosteal tissue in the medullary cavity of limb bones, and forms before ovulation in some extant female birds as a calcium 
reservoir during egg production (Pahl et al., 1997). This tissue is, therefore, a useful skeletal indicator of both gender and sexual maturity (i.e., the individual was reproductive at the time of death) in taxa that produce it. Medullary bone has been discovered in the hind limbs of some non-adult-sized specimens of Tyrannosaurus rex (Schweitzer et al., 2005), Allosaurus fragilis and Tenontosaurus tilletti (Lee and Werning, 2008), suggesting that these specimens reached sexual maturity before skeletal maturity. Unfortunately, medullary bone is poorly known from most dinosaur taxa.

The only chasmosaurine with a well-documented ontogenetic series is Triceratops (Horner and Goodwin, 2006). This series is based on a large dataset that has been gathered over the past decade by the Museum of the Rockies Hell Creek Project (Scannella and Horner, 2010). Horner and Goodwin (2006) grouped specimens into ontogenetic stages ('ontogimorphs') defined by skull size, the relative degree to which epiossifications are fused to the skull, and development of cranial ornamentation; these stages were later corroborated by Scannella and Horner (2010). Horner and Goodwin (2006) assigned Triceratops specimens with the following basal skull lengths (rostral-tooccipital condyle) to the following stages: $280 \mathrm{~mm}$ (estimated), "baby"; 355 to $610 \mathrm{~mm}$, "juvenile"; greater than $610 \mathrm{~mm}$ to less than $940 \mathrm{~mm}$, "subadult"; and $940 \mathrm{~mm}$ and higher, "adult". The maximum basal skull length $(940 \mathrm{~mm})$ for Triceratops is from MOR 004 (Horner and Goodwin, 2006), and falls just outside the range of the largest Chasmosaurus specimen (ROM 843 (rostral-to-epijugal length, parameter $16=897$ $\mathrm{mm})$ ). 
Materials and methodologies used:

Parsimony-based clustering using 18 cranial characters was conducted using ontogenetically-variable characters to create a growth series following the methodologies of Carr and Williamson (2004; Tyrannosaurus), Carr (2010; Albertosaurus) and Longrich and Field (2012; Triceratops and Torosaurus). These characters pertain to the articulation of epiossifications onto the margin of the skull (characters 1-4 and 6), inferred remodelling of bone (characters 5,13-16 and 18), articulation between elements of the skull roof (characters 7-12), and dimensional changes in cranial elements (character 17) that have been suggested to vary with age (Ryan and Russell, 2005; Horner and Goodwin, 2006; Longrich and Field, 2012). In this study, "articulation" refers to two or more cranial elements in direct contact with each other, as preserved. The articulation pattern of the epiossifications (rostral, epinasal, epijugals, episquamosals, and epiparietals) have been demonstrated to display specific ontogenetic sequences in centrosaurines (Sampson et al., 1997) and chasmosaurines (e.g., Triceratops, Horner and Goodwin, 2006).

Fourteen skulls were included in this analysis: AMNH 5401, AMNH 5402, AMNH 5656, CMN 1254, CMN 2245, CMN 2280, CMN 8800, NHMUK R4948, ROM 839, ROM 843, TMP 1981.019.0175, TMP 1983.025.0001, UALVP 40, and YPM 2016. The skulls were chosen based, in part, by their expression of key transitional ontogenetic changes, as well as their relatively complete nature. An artificial embryo, coded " 0 " for all characters, was used as the outgroup to root the tree and polarize the characters (sensu Carr and Williamson, 2004; Carr, 2010). All but two of these specimens (AMNH 5402 and YPM 2016) were recovered as Chasmosaurus-like in Chapter 6 (Fig. 37); these two 
specimens are referred to as Vagaceratops-like in this study.

Ontogenetically-variable characters are inferred in this analysis based on the presence of a particular character state amongst smaller skulls relative to another state of the same character present in larger skulls. This inference is also based on sequences of changes which can only progress in one direction (e.g., articulation of epiossifications or other cranial elements). For any given character (e.g., character 2, articulation of epinasal with nasals), the presence of a particular character state (epinasal disarticulated) in relatively small skulls was considered as the immature/basal condition, and coded as " 0 ". Likewise, the presence of a different state of the same character (epinasal articulated) in larger skulls was considered as the mature condition, and coded as "1". The size-related states in each of the 18 characters used in this analysis have also been shown to be sizerelated in other ceratopsid taxa, where noted. In the case of eight characters (1-6, 14 and 17), a third state (2) was added to capture the range of ontogenetic variation seen amongst the specimens. These eight characters were ordered in the analysis performed in TNT 1.1 (Goloboff et al., 2003) using the tree bisection reconnection swapping algorithm of 1000 replicates, with 10 trees saved per replication, and the maximum number of trees stored set at 10,000. The specimen-character matrix used in this analysis is listed in Table 6 .

Rostral-to-epijugal length (parameter 16) was used as a baseline for skull size in this study as it approximates basal skull length, rostral-to-occipital condyle, as used in other studies (e.g., Dodson, 1976; Mallon et al., 2011), but is an easier measurement to obtain than the latter given the incomplete nature of many of the skulls. Measurements for the currently inaccessible skulls AMNH 5401 and AMNH 5402 were obtained from Godfrey and Holmes (1995); their study included rostral-to-epijugal length, but not 
rostral-to-occipital condyle length. CMN 8800 and CMN 8801 are both partly encased in plaster or matrix, rendering the occipital condyles inaccessible. CMN 41357 does not preserve the braincase, making a rostral-to-occipital condyle measurement impossible. In this study, Chasmosaurus specimens were designated as small or large based on the following criteria: small specimens are skulls with (or estimated as having) rostral-toepijugal lengths of up to $628 \mathrm{~mm}$; e.g., UALVP 40); large skulls have (or are estimated as having) rostral-to-epijugal lengths of $680 \mathrm{~mm}$; e.g., AMNH 5402, or greater.

Articulated fossilized cranial elements can be held together by: 1, sediment, that has sometimes replaced connective tissue which once held the elements together, but decayed after burial; 2, an interdigitating; or, 3, coalesced contact between them. The fact that most Dinosaur Park Formation chasmosaurine crania were discovered as isolated units (e.g., AMNH 5401, AMNH 5402, AMNH 5656, CMN 0491, CMN 8800, CMN 8802, CMN 8803, CMN 34829, TMP 1981.019.0175, TMP 1983.025.0001, TMP 1998.102.0008, TMP 2009.034.0009, and YPM 2016) suggests that connective tissue decay between skull and body occurred largely before burial. A missing rostral or cranial epiossification (i.e., epinasal, epijugal, episquamosal, and epiparietal) was either not present or formed before death, or represents an element that was lost post mortem. As it is difficult to determine if two closely associated elements are truly fused through the comingling of their boney tissues, or simply held in place by minerals or sediment, such elements are referred to here as 'articulated' unless a portion of their contacting surfaces have been obliterated and are obviously fused. Degree of fusion can then be designated on an element-by-element basis based on the degree of obliteration of connection surfaces. Two articulating elements are considered to exhibit an early stage of articulation 
if their sutures are visible or open externally. An advanced stage of articulation is indicated if their sutures are obliterated or closed externally.

Frill epiossifications (episquamosals and epiparietals) generally articulate anterior to posterior over the course of chasmosaurine ontogeny (Sampson et al., 1997) (e.g., Fig. 7E). Epiparietals are numbered sequentially starting at the midline of the posterior parietal and progress towards the squamosal (Sampson et al., 2010). Although episquamosals have been traditionally numbered sequentially starting at the anterior end of the squamosal (e.g., Forster et al., 1993; Wu et al., 2007), I have chosen to follow the methodology of Sampson et al. (2010) because their numbering system, posterior-toanterior, is more consistent with the epiparietal numbering system.

The number of episquamosals or their underlying episquamosal loci on the squamosal of chasmosaurines from the Dinosaur Park Formation can range from seven (e.g., CMN 1254) to 10 (i.e., CMN 8800) per side, and the number of epiparietals/loci can range from three (e.g., CMN 2280) to five (i.e., YPM 2016) per side. An epiossification bridging the parietal-squamosal contact referred to as an epiparietosquamosal is common on ceratopsids, and is present in two specimens (AMNH 5402 and TMP 1987.045.0001).

Characters analyzed

Character 1 (rostral and premaxilla, articulation) (Longrich and Field, 2012; character 23, modified):

(0) rostral disarticulated; (1) articulated, but suture between dorsal process of rostral and 
dorsal margin of premaxillae open in dorsal view; and (2) articulated, with suture between dorsal process of rostral and dorsal margin of premaxillae closed in dorsal view.

The rostral is assumed to be a disarticulated element in immature ceratopsids (0), but is articulated with the premaxillae later in ontogeny (e.g., Triceratops, Horner and Goodwin, 2008; Pachyrhinosaurus lakustai, Currie et al., 2008). The rostral is known to be articulated with the skull in the smallest known ceratopsian specimens, e.g., the hatchling-sized skulls of Psittacosaurus (Sereno, 2010) and Protoceratops (Fastovsky et al., 2011), suggesting that this element articulated during their embryonic development. Early in ontogeny, the suture between these elements remains open when viewed externally (1). The suture between the dorsal process of the rostral and the dorsal margin of the premaxillae closes in advanced stages of articulation (2), although large ceratopsid skulls are known without rostra that never fused.

These character states are exemplified by UALVP 40 (0), AMNH 5402 (1) and YPM 2016 (2) (Fig. 48). In UALVP 40 (Fig. 48A), the rostral is missing (0), although one has been reconstructed in plaster (shown in grey half tone). In AMNH 5402 (Fig. 48B), the rostral is articulated with the premaxillae, but the suture between the dorsal process of the rostral and the dorsal margin of the premaxillae is open in dorsal view (1). In YPM 2016 (Fig. 48C), the rostral is articulated with the premaxillae, with the suture between the dorsal process of the rostral and the dorsal margin of the premaxillae is closed in dorsal view (2).

Character 2 (epinasal and nasals, articulation) (Longrich and Field, 2012; character 15, modified here): 
(0) disarticulated; (1) articulated, with suture open in lateral view; and (2) articulated, with suture closed in lateral view.

The epinasal is a separate, disarticulated element (0) early in ontogeny, but later articulates with the dorsal margin of the nasals, retaining open sutures when viewed laterally (1); these sutures eventually close (2). These character states are exemplified by CMN 1254 (0), CMN 2245 (1) and ROM 843 (2) (Fig. 49). In CMN 1254 (Fig. 49A), the epinasal is preserved, but disarticulated from the underlying nasal (0). In Figure 49A, the epinasal is shown both separate (left) and adjacent (right) to the nasal to demonstrate the ossification process. In CMN 2245 (Fig. 49B), articulation between the epinasal and nasal has taken place, with the suture between them open (1), but closed (2) in ROM 843 (Fig. 49C). Horner and Goodwin (2008) suggest that in Triceratops, the articulation of the epinasal with the nasals occurred at approximately the same time as articulation of the paired nasals. 

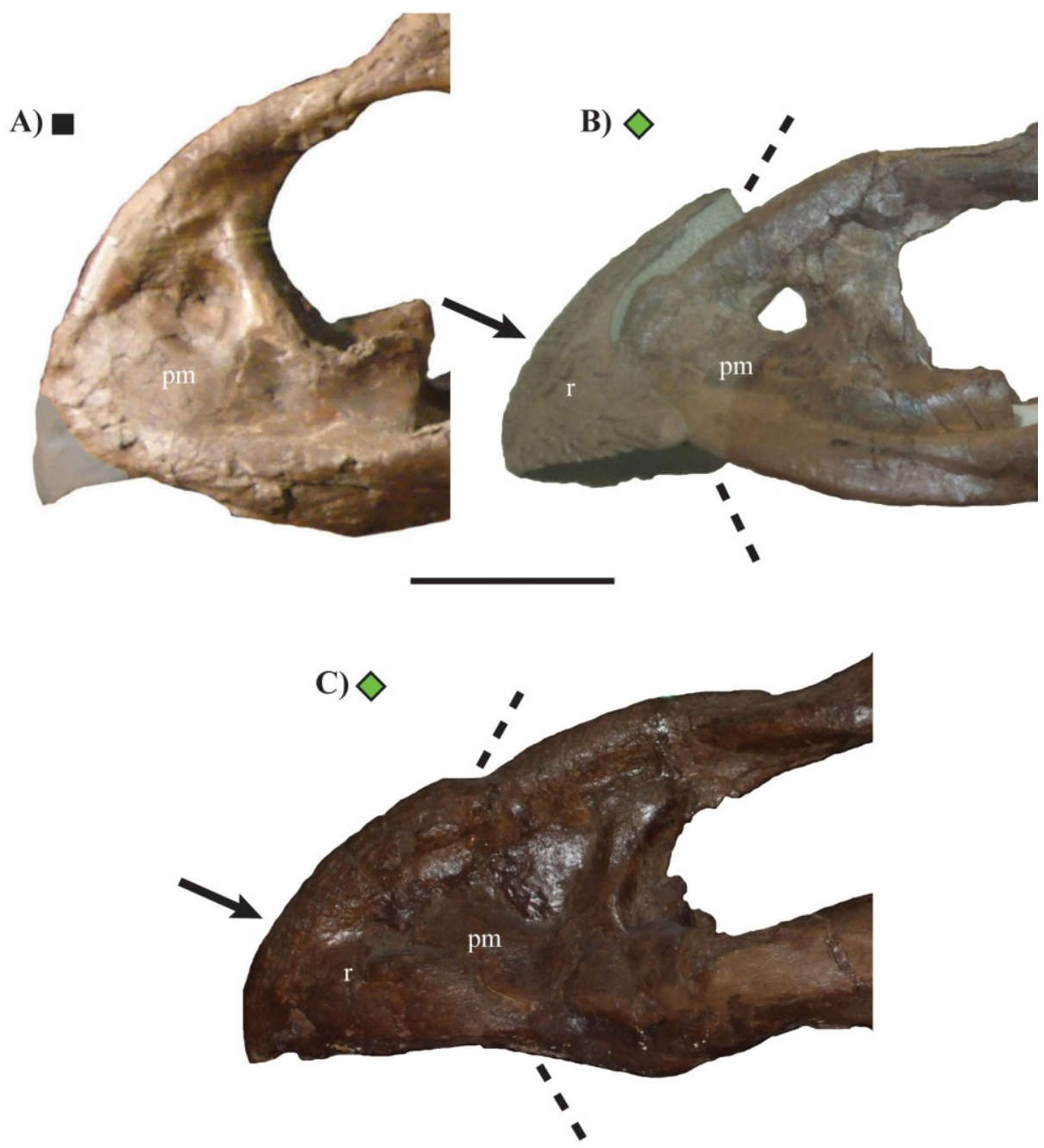

Figure 48. Ontogenetic changes in specimens previously referred to Chasmosaurus articulation of rostral with premaxillae (character 1). A) rostral separate and missing (UALVP 40), B) rostral articulated, but dorsal suture open (AMNH 5402), and C) rostral articulated with dorsal suture closed (YPM 2016); arrows highlight relative degree of articulation. Dashed lines represent sutural contacts. Plaster reconstruction shown in grey half-tone. Chasmosaurus sp. (black square) and Vagaceratops-like specimens (green diamonds). See "List of anatomical abbreviations". Scale bar equals $10 \mathrm{~cm}$. 


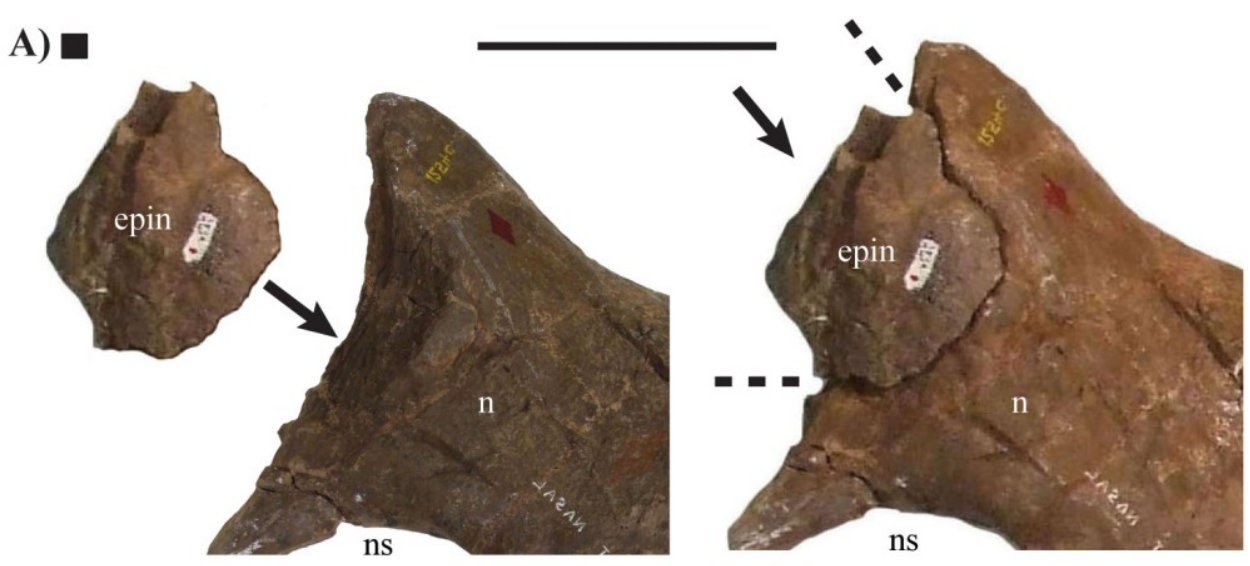

B) $\nabla$

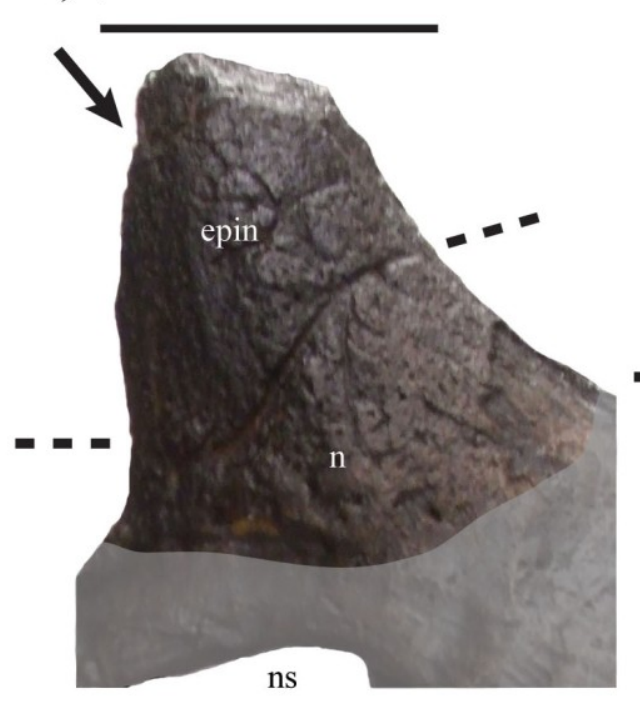

C) $\nabla$

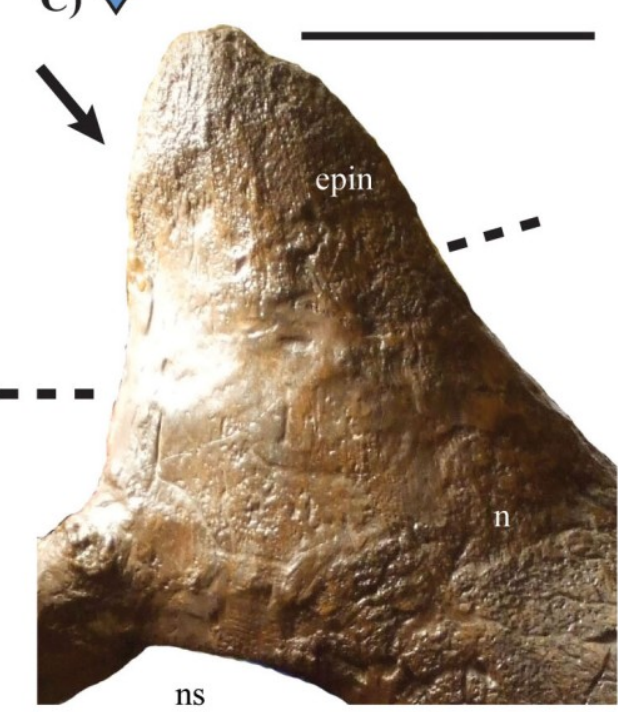

Figure 49. Ontogenetic changes in Chasmosaurus - articulation of epinasal with nasals (character 2). A) Epinasal disarticulated (CMN 1254; flipped), B) epinasal articulated with nasals with suture open (CMN 2245), and C) epinasal articulated with nasals with suture closed (ROM 843). Dashed lines represent sutural contacts. Plaster reconstruction shown in grey half-tone. Chasmosaurus belli (blue inverted triangles) and Chasmosaurus sp. (black square). See "List of anatomical abbreviations". Scale bars equal $10 \mathrm{~cm}$. 
Character 3 (epijugal, articulation with jugal/quadratojugal) (Longrich and Field, 2012; character 22, modified here):

(0) epijugal disarticulated; (1) articulated, with suture open in lateral view; and (2) articulated, with suture closed in lateral view.

The epijugal is a disarticulated element (0) early in ontogeny, but later articulates with the distal ends of the jugal and quadratojugal, retaining open sutures (1); these sutures close over time (2). These character states are exemplified by UALVP 40 (0), ROM 839 (1) and TMP 1981.019.0175 (2) (Fig. 50). In UALVP 40 (Fig. 50A), the epijugal is disarticulated on the right side (0), but articulated with an open suture on the opposite side (1; not shown), resulting in a dimorphic state (0\&1). In Figure 50A, this preserved isolated epijugal is shown both detached (left) and attached, but disarticulated (right) with the jugal and quadratojugal to show the ossification process. In ROM 839 (Fig. 50B), the epijugal is articulated with an open suture on the right side (1), but disarticulated and missing on the opposite side (0; not shown). In TMP 1981.019.0175 (Fig. 50C), the epijugal is articulated with a closed suture (2).

Character 4 (episquamosals, articulation with squamosal) (Longrich and Field, 2012; character 20, modified):

(0) no episquamosals articulated; (1) at least 50\% (but not all) of episquamosals articulated; and (2) all episquamosals articulated. 


\section{A) $\mathbf{a}$}

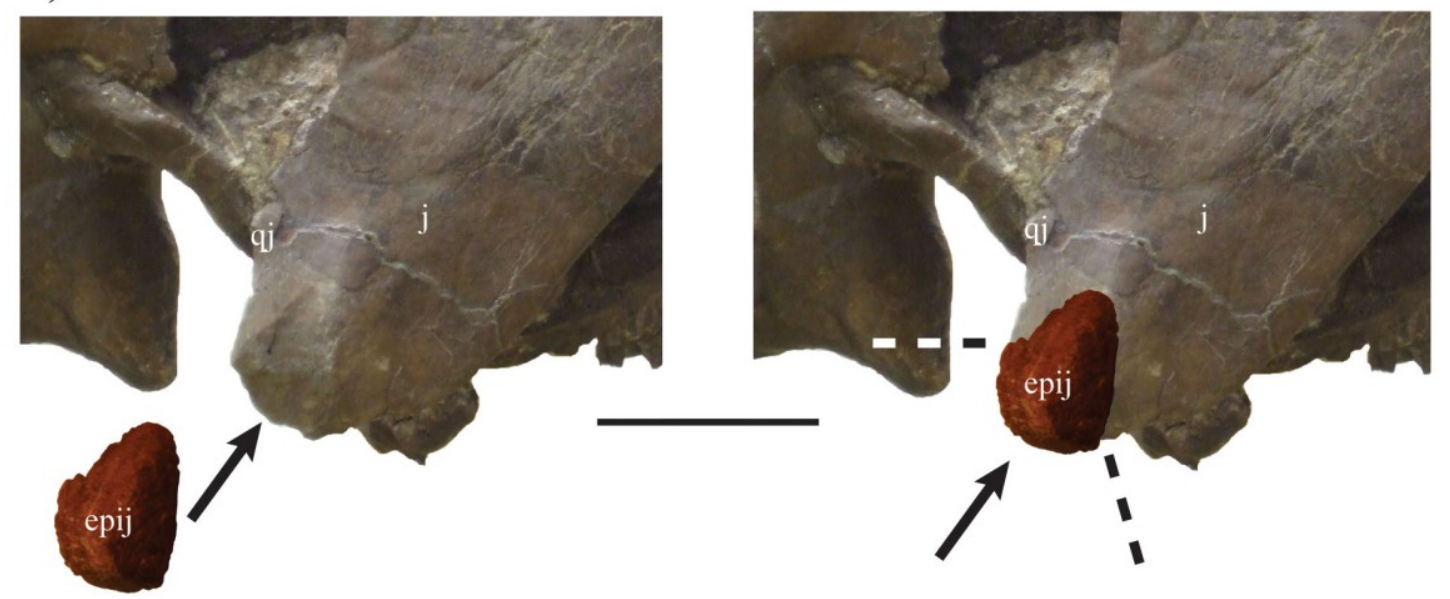

B)

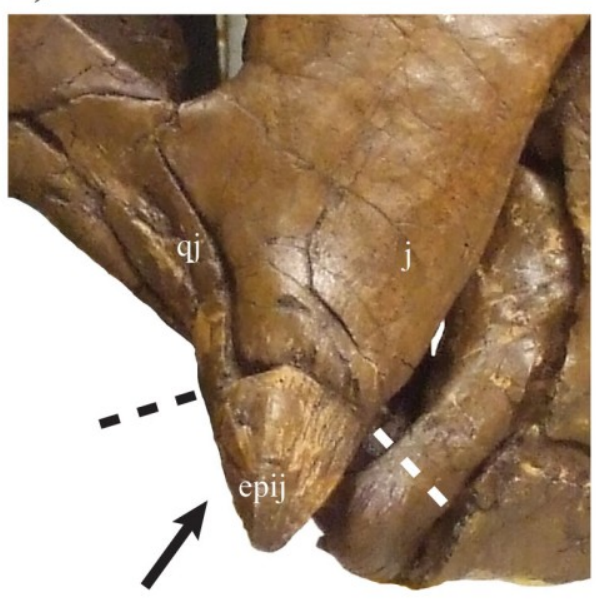

C)

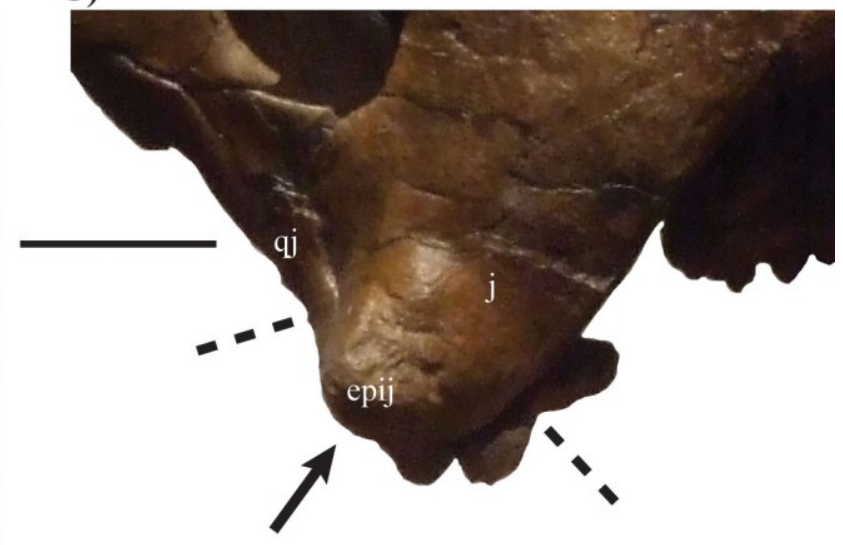

Figure 50. Ontogenetic changes in Chasmosaurus - articulation of epijugal with jugal and quadratojugal (character 3). A) Epijugal disarticulated (UALVP 40), B) epijugal articulated with suture open (ROM 839), and C) epijugal articulated with suture closed (TMP 1981.019.0175). Dashed lines represent sutural contacts. Chasmosaurus sp. (black squares). See "List of anatomical abbreviations". Scale bars equal $10 \mathrm{~cm}$. 
In early ontogeny, episquamosals are disarticulated elements detached from the underlying loci that are located at the apices of the undulations located on the lateral margin of the squamosal (0). As the animal matured, these episquamosals and the squamosal came into contact in a progressive, sequential order, and gradually articulated together (1 and 2). The presence or absence of episquamosals can be difficult to determine as, once they are articulated, they are remodeled throughout ontogeny (character 5) from triangular-profile elements to low, rounded-profile elements whose sutures with the underlying squamosal can become completely obliterated. In almost all Chasmosaurus specimens, however, the complete suture between the episquamosal and squamosal can be discerned, and are coded as 'open'.

Episquamosal remodelling can result in a structure that closely resembles the unadorned loci of a smaller, putatively less mature specimen (e.g., compare the anteriormost loci of CMN 1254, Fig. 7D, with the anteriormost episquamosal of CMN 8800 , Fig. $7 \mathrm{G}$ ). Such a structure in a large, putatively more mature specimen is interpreted as an episquamosal by comparing the structure with more posterior episquamosals in that specimen, whose sutures are more distinct.

Unadorned episquamosal attachment loci can have wide, shallow grooves on the dorsal surface that converge at the apex. These grooves can contribute to a complex attachment site for the episquamosal as in centrosaurines (e.g., P. lakustai; Currie et al., 2008). The number of episquamosals that have articulated with the squamosal (or have undergone remodelling, character 5) cannot be quantified, as episquamosal loci count is not consistent between specimens (e.g., compare CMN 1254 (Fig. 7D) = seven, and CMN 8800 (Fig. 7G) = 10); however, an estimation of none or less than one-half of the 
episquamosals being articulated can be used to differentiate states 0 and 1.

These character states are exemplified by CMN 1254 and TMP 1998.128.0001 (0), AMNH 5401 and AMNH 5656 (1), and NHMUK R4948 (2) (Fig. 51). In both TMP 1998.128.0001 (Fig. 51A) and CMN 1254 (Fig. 51B), no episquamosals are articulated with the squamosal (0). In AMNH 5656 (Fig. 51C) and AMNH 5401 (Fig. 51D), all but the distalmost episquamosal have articulated with the squamosal (1). In NHMUK R4948 (Fig. 51E) and CMN 8800 (Fig. 51F), all episquamosals have articulated with the squamosal (2); however, the distalmost episquamosal is missing on the left squamosal of NHMUK R4948 (1; not shown), resulting in a dimorphic state (0\&1) for this specimen. Although the episquamosals suggest an anterior-to-posterior direction of articulation in specimens, there is a specimen (ROM 839) which is missing episquamosal 5 and 6 (1).

Character 5 (episquamosals, shape) (new character):

(0), all episquamosals triangular in dorsal profile (i.e., unmodified); (1) anteriormost (approximately one-half of total) D-shaped in dorsal profile, posteriormost unmodified; and (2) all episquamosals D-shaped in dorsal profile.

After articulation of the triangular episquamosals (0), they underwent remodeling from anterior to posterior along the margin of the squamosal into a low, "D"-shape on the anterior half (1), and finally the entire margin (2). These character states are exemplified by AMNH 5656 (Fig. 51C) and AMNH 5401 (Fig. 51D) (0), NHMUK R4948 (1; Fig. 51E), and CMN 8800 (2; Fig. 51F).

Horner and Goodwin (2006) demonstrated that during Triceratops ontogeny the episquamosals are laterally compressed, with an approximately triangular profile in 
Figure 51. Ontogenetic changes in Chasmosaurus, squamosal - articulation of episquamosals with squamosal (character 4), remodeling of episquamosals (character 5) changing outline of lateral margin of squamosal (character 16), and increase in squamosal length/width ratio (character 17). A) (TMP 1998.128.0001; flipped), B) (CMN 1254), C) (AMNH 5656), D) (AMNH 5401), E) (NHMUK R4948), and F) (CMN 8800). Blank arrows represent disarticulated episquamosals; solid arrows represent articulated episquamosals. Image of NHMUK R4948 courtesy of Jordan Mallon. Chasmosaurus russelli (red triangles), C. belli (blue inverted triangle) and Chasmosaurus sp. (black squares). Scale bars equal $10 \mathrm{~cm}$. 
Figure 51.

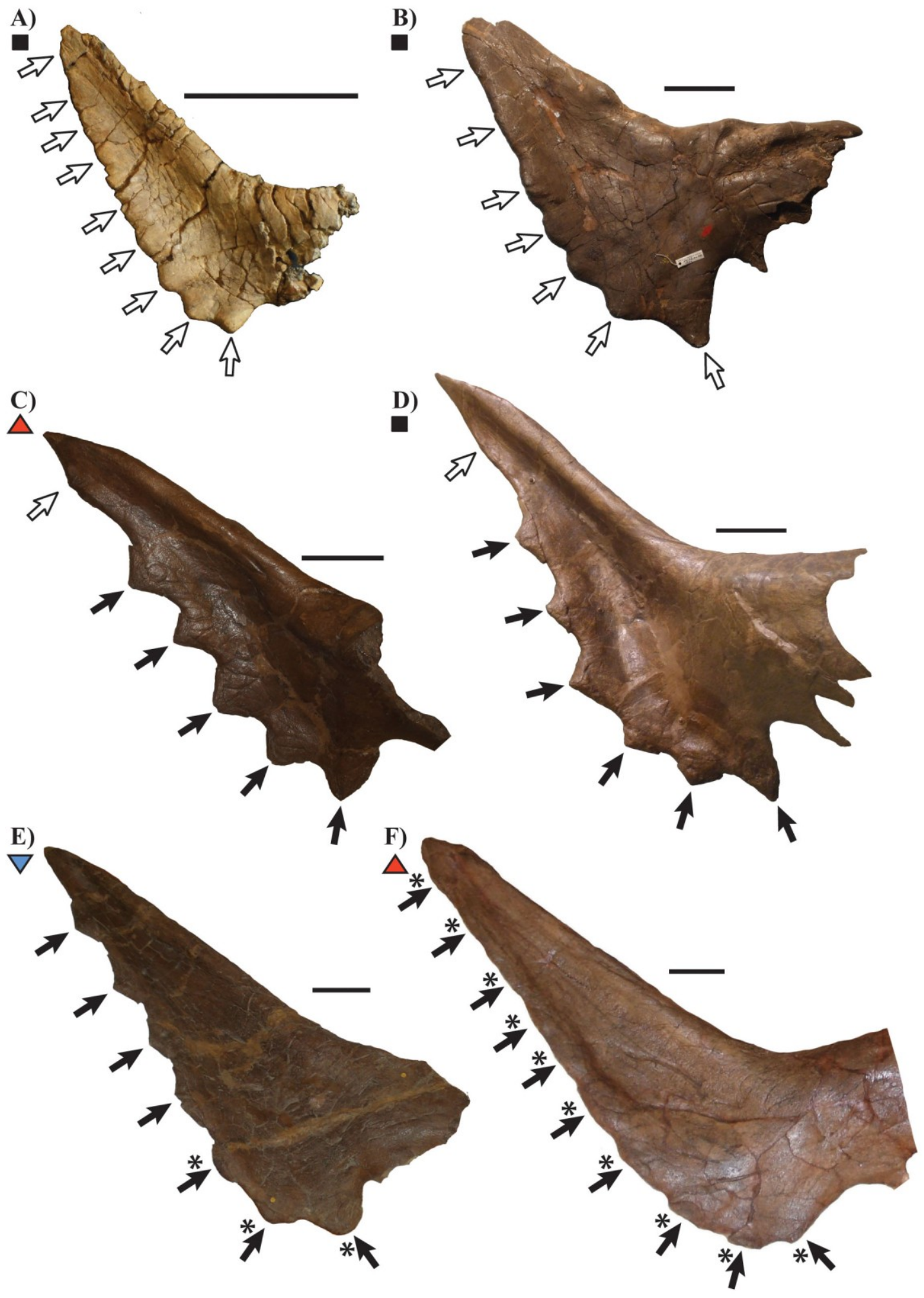


dorsal view in the smallest known specimens (e.g., MOR 1199, UCMP 136306), but become reduced in height proximodistally during maturity, finally completely articulating and being obliterated in putatively mature specimens (e.g., MOR 004, MOR 1120).

Character 6 (epiparietals, articulation with parietal) (Longrich and Field, 2012; character 21, modified):

(0), no epiparietals articulated; (1), at least one (but not all) epiparietal articulated; and (2), all epiparietals articulated.

Epiparietals, like episquamosals, are inferred to have a dermal origin and sequentially articulate with the underlying parietal. As no known specimens express (0) this state is assigned to the artificial embryonic stage. The remaining character states are exemplified by AMNH 5656 (1), and CMN 2280, CMN 8800, ROM 843, and TMP 1983.025.0001 (2) (Fig. 52). The medialmost epiparietals (P1 and 2) are articulated in AMNH 5656 (Fig. 52A), but the lateralmost epiparietal loci (P3) is unoccupied (1). In TMP 1983.025.0001 (Fig. 52B), CMN 2280 (Fig. 52C) and CMN 8800 (Fig. 52F), all epiparietals have articulated with the parietal (2). In ROM 843 (Fig. 52D), the low-relief mound present at the medialmost epiparietal loci (P1) is interpreted as a modified epiparietal; therefore, all three epiparietals are interpreted as articulated, and this specimen was coded as (2).

Character 7 (premaxillae, degree of sutural closure) (new character):

(0) suture between posterodorsal margins of premaxillae open in dorsal view; and (1) suture between posterodorsal margins of premaxillae closed in dorsal view. 
Figure 52. Ontogenetic changes in Chasmosaurus, parietal - articulation of epiparietals with parietal (character 6) and orientation of epiparietals (character 18). A) (AMNH 5656), B) (TMP 1983.025.0001), C) (CMN 2280; flipped), D) (ROM 843), E) (CMN 0491; flipped), and F) (CMN 8800; flipped). Blank triangle represents disarticulated epiparietal; solid triangles represent articulated epiparietals. Long triangles denote epiparietals (or attachment sites) oriented posteriorly in the plane of the frill, and short triangles denote epiparietals oriented anterodorsally. Chasmosaurus russelli (red triangles) and $C$. belli (blue inverted triangles). Scale bars equal $10 \mathrm{~cm}$. 
Figure 52.
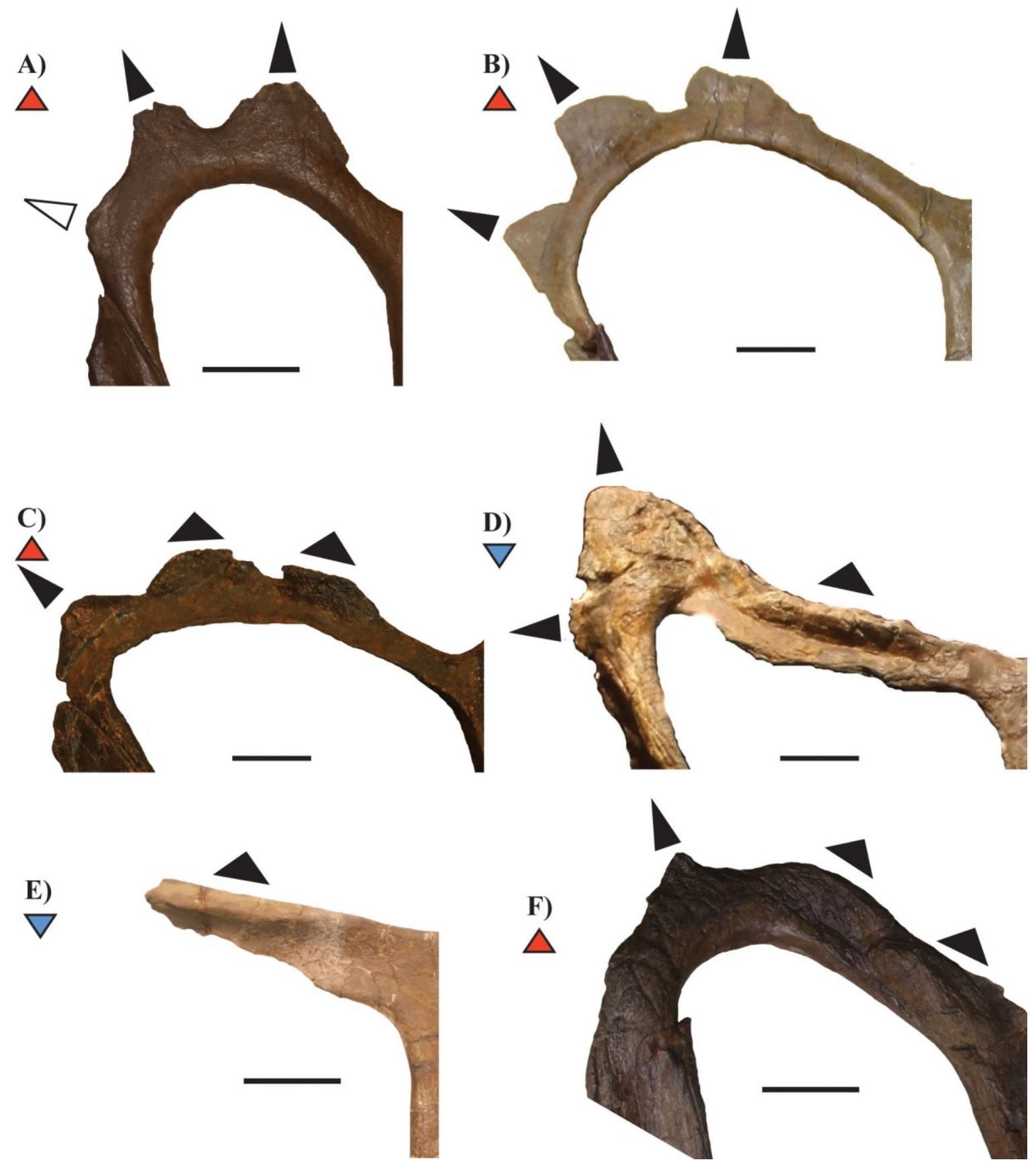
Sutures between elements of the skull roof in ceratopsids close in mature individuals (Sampson et al., 1997; Horner and Goodwin, 2006; Longrich and Field, 2012). Although bone may continue to grow after its sutural contacts are obliterated, growth would be restricted as bone can no longer be deposited between these sutures (Herring, 1974).

The dorsal margin of the premaxillae is covered by the rostral anteriorly, but is exposed posteriorly. The above character states are exemplified by AMNH 5401, AMNH 5402 and UALVP 40 (0), and CMN 2280 (1) (Fig. 53). UALVP 40 (Fig. 53C-D) to AMNH 5402 (Fig. 53E-F) to AMNH 5401 (Fig. 53G-H), illustrate two successively larger specimens where the posterodorsal margins of the premaxillae become in progressively closer contact (0), with the suture between the posterodorsal margins of the premaxillae being closed (1) in CMN 2280 (Fig. 53I-J) .

Character 8 (premaxilla and nasal, degree of sutural closure) (Longrich and Field, 2012; character 24):

(0) suture between dorsal margins of elements open in dorsal view; and (1) suture between dorsal margins of elements closed in dorsal view. The posterodorsal processes of the premaxillae insert between the anterodorsal processes of the nasals, anterior to the nasal horncore.

These character states are exemplified by AMNH 5401, AMNH 5402, CMN 1254, and UALVP 40 (0), and CMN 2280 (1) (Fig. 53). In CMN 1254 (Fig. 53A-B), the sutural contact for the right premaxilla is present on the preserved right nasal, suggesting that sutural closure did not occur between these elements (0). In UALVP 40 (Fig. 53C- 
Figure 53. Ontogenetic changes in Chasmosaurus - articulation between other cranial elements: premaxillae (character 7), premaxilla-nasal (character 8), nasals (character 9), nasal-frontal (character 10), frontals (character 11), and frontal-postorbital (character 12). A-B, CMN 1254; C-D, UALVP 40; E-F, AMNH 5402; G-H, AMNH 5401; and I-J, CMN 2280. Solid line between cranial elements denotes open suture; dotted line is closed suture. Image of AMNH 5401 courtesy of Jordan Mallon. Plaster reconstruction shown in grey. Chasmosaurus russelli (red triangle), Chasmosaurus sp. (black squares) and Vagaceratops-like specimens (green diamond). See "List of anatomical abbreviations". Scale bars equal $10 \mathrm{~cm}$. 
Figure 53.
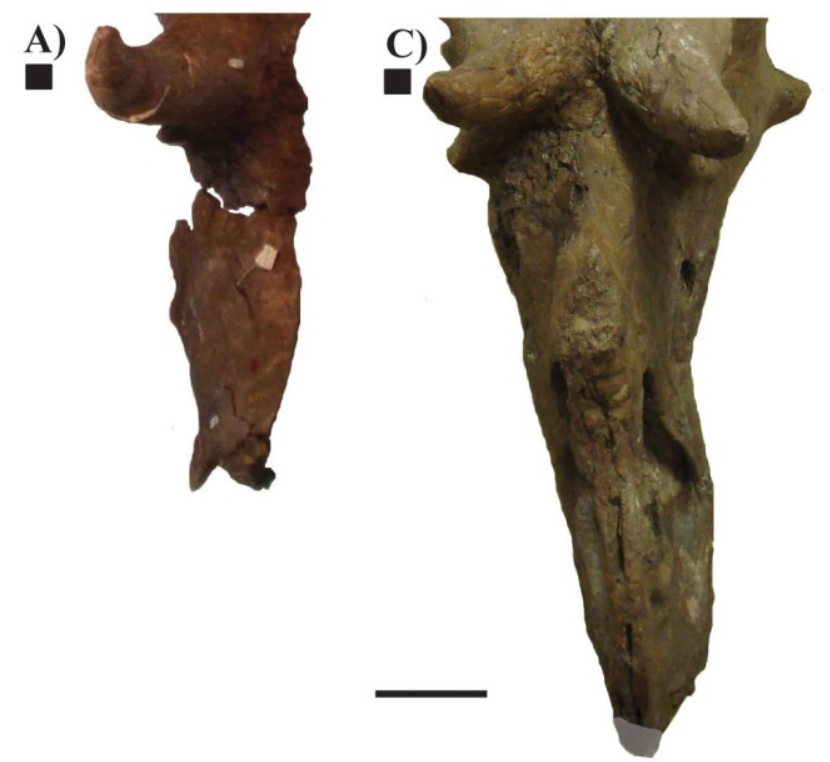

E)

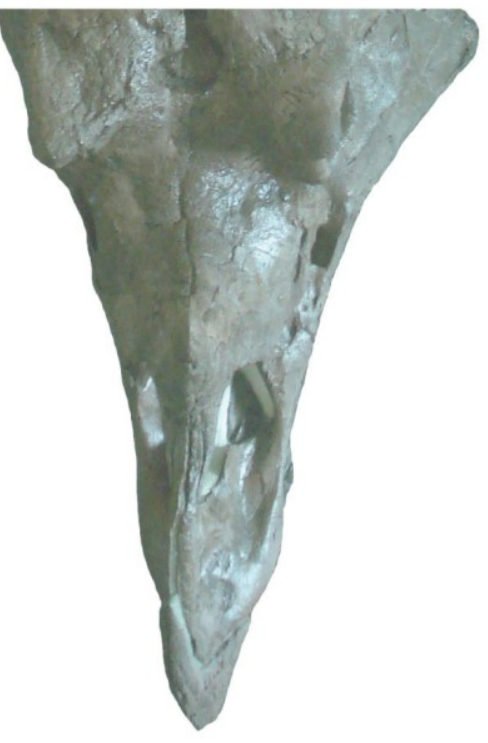

B)

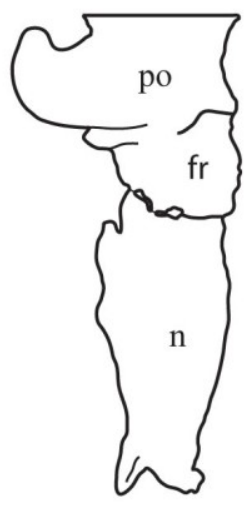

D)

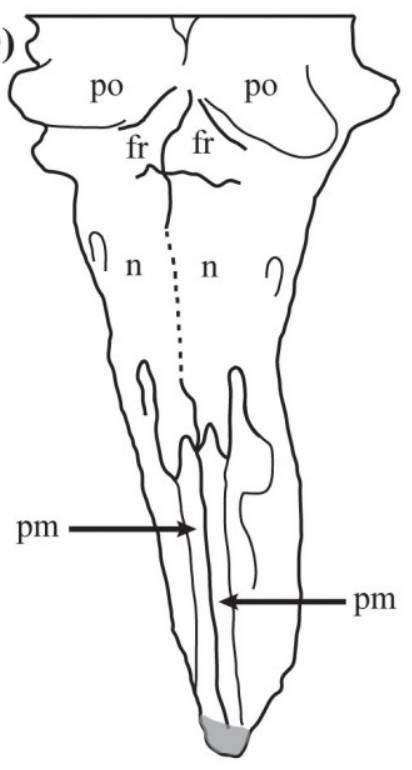

F)

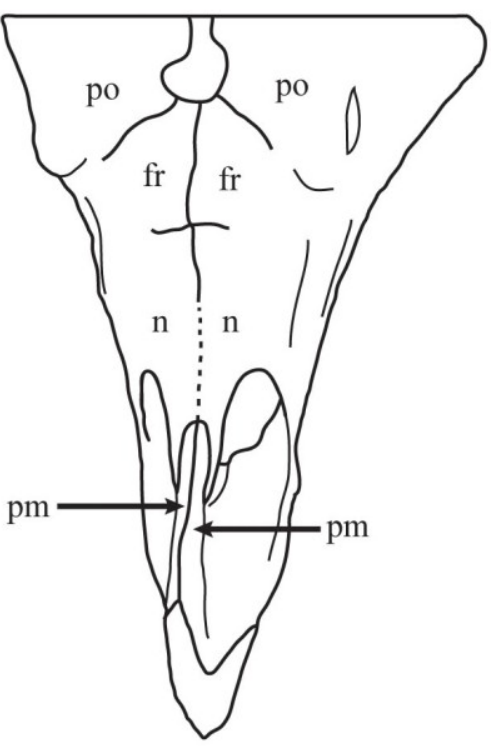


Figure 53. (continued).

G)

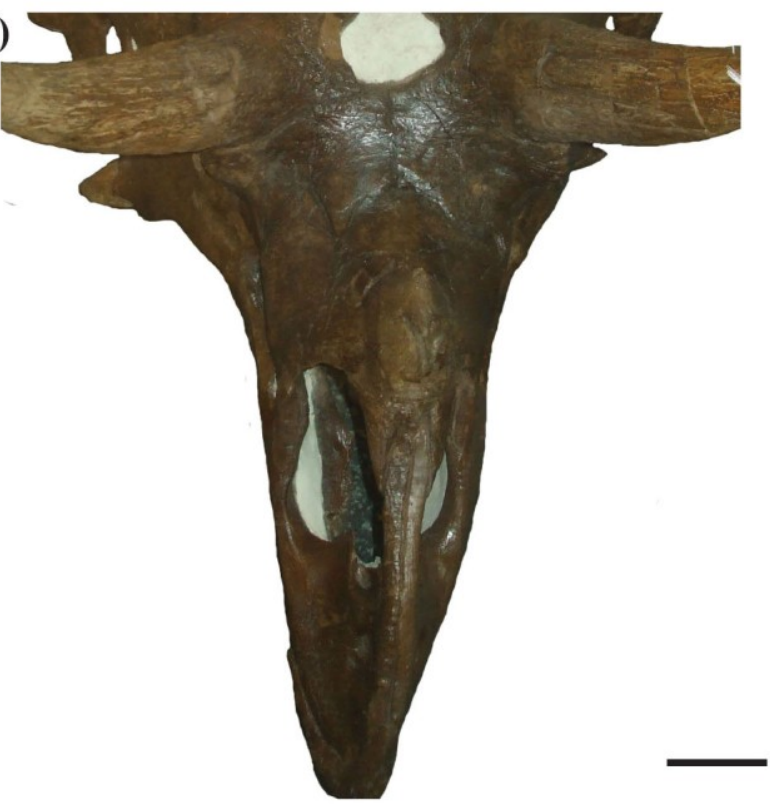

H)

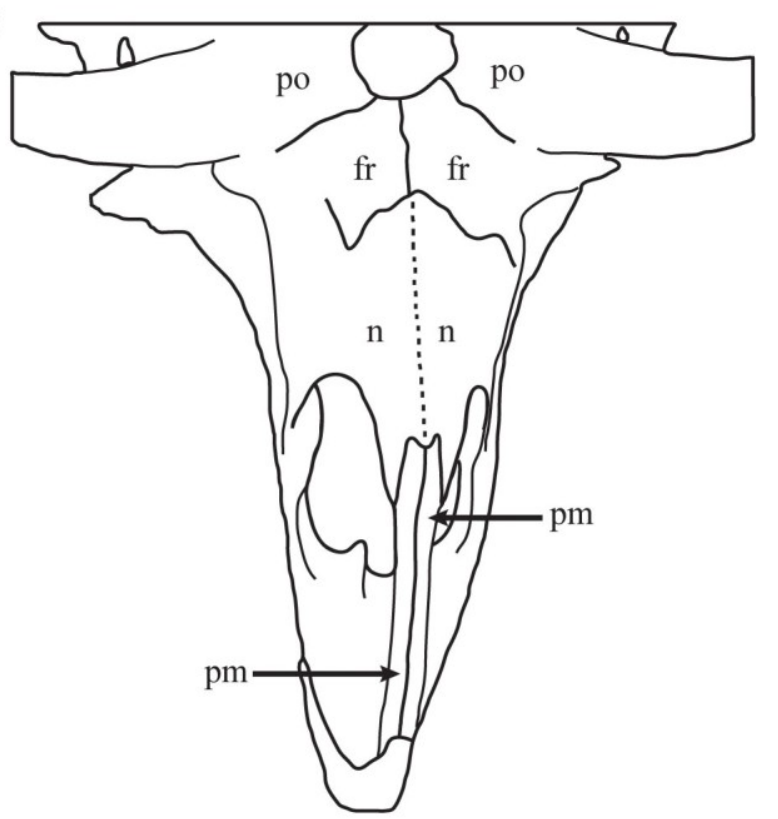

I) $\triangle$

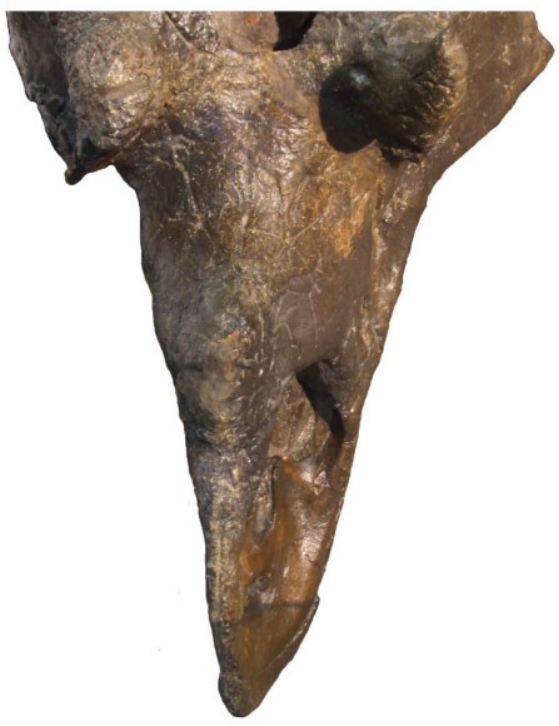

J)

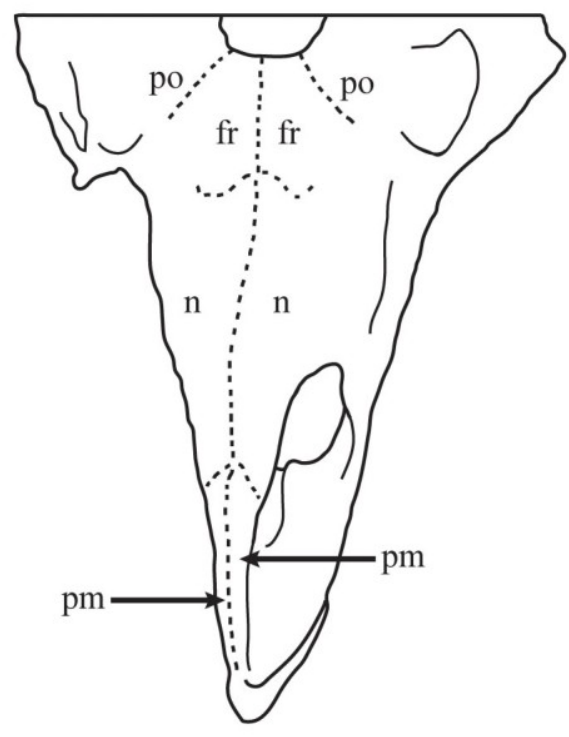


D), AMNH 5402 (Fig. 53E-F) and AMNH 5401 (Fig. 53G-H), the suture between these elements is open in dorsal view (0). In CMN 2280 (Fig. 53I-J), the suture between these elements is closed in dorsal view (1).

Character 9 (nasals, degree of sutural closure) (Longrich and Field, 2012; character 16): (0) suture open in dorsal view; and (1) suture closed in dorsal view.

These character states are exemplified by AMNH 5402, CMN 1254 and UALVP 40 (0), and CMN 2280 (1) (Fig. 53). On the preserved right nasal of CMN 1254 (Fig. 53A-B), the entire midline sutural contact (including the horncore) is present, suggesting that sutural closure did not occur between the nasals before death (0). In UALVP 40 (Fig. 53C-D) and AMNH 5402 (Fig. 53E-F), the nasal sutures are closed adjacent to the nasal horncore, but open further posteriorly (0). In AMNH 5401 (Fig. 53G-H) and CMN 2280 (Fig. 53I-J), the nasal sutures are closed along their entire length (1).

Character 10 (nasal and frontal, degree of sutural closure) (Longrich and Field, 2012; character 19):

(0) suture open in dorsal view; and (1) suture closed in dorsal view.

These character states are exemplified by AMNH 5401, AMNH 5402, CMN 1254, and UALVP 40 (0), and CMN 2280 (1) (Fig. 53). In CMN 1254 (Fig. 53A-B), the nasal and frontal are disarticulated (0). In UALVP 40 (Fig. 53C-D), AMNH 5402 (Fig. 53E-F) and AMNH 5401 (Fig. 53G-H), the nasal and frontal contact each other, but their sutures are still open in dorsal view (0). In CMN 2280 (Fig. 53I-J), the suture between the 
nasal and frontal is closed in dorsal view (1).

Character 11 (frontals, degree of sutural closure) (Longrich and Field, 2012; character 14):

(0) suture open in dorsal view; and (1) suture closed in dorsal view.

These character states are exemplified by AMNH 5401, AMNH 5402, CMN 1254, and UALVP 40 (0), and CMN 2280 (1) (Fig. 53). On the preserved right frontal of CMN 1254 (Fig.53A-B), the entire midline sutural contact for its unpreserved counterpart is present, indicating that sutural closure did not occur between these elements before death (0). In UALVP 40 (Fig. 53C-D), AMNH 5402 (Fig. 53E-F) and AMNH 5401 (Fig. 53G-H), the frontals contact each other, but their sutural contact is still open in dorsal view (0). In CMN 2280 (Fig. 53I-J), the contact between the frontals is closed in dorsal view (1).

Character 12 (frontal and postorbital, degree of sutural closure) (Longrich and Field, 2012; character 17):

(0) suture open in dorsal view; and (1) sutures closed in dorsal view.

These character states are exemplified by AMNH 5401, AMNH 5402, CMN 1254, and UALVP 40 (0), and CMN 2280 (1) (Fig. 53). In CMN 1254 (Fig. 53A-B), UALVP 40 (Fig. 53C-D), AMNH 5402 (Fig. 53E-F), and AMNH 5401 (Fig. 53G-H), the suture between the frontal and postorbital is open in dorsal view (0). In CMN 2280 (Fig. 53I-J), the contact between the frontal and postorbital is closed in dorsal view (1). 
Character 13 (nasal horncore, modification) (new character):

(0) unmodified (smooth surface, comes to a point); and (1) modified (partially resorbed and displaying rugose or pitted texture). The nasal horncore is defined as the articulated nasals and epinasal.

These character states are exemplified by AMNH 5402 (0), and CMN 8800 and YPM 2016 (1) (Fig. 54). In AMNH 5402 (Fig. 54A), the nasal horncore has an unmodified, pointed apex (0). In YPM 2016 (Fig. 54B), the nasal horncore is truncated with a pitted apex (1). In CMN 8800 (Fig. 54C), only the base of the nasal horncore is present, with the rest of the horncore being reduced to a pitted surface (1).

It is proposed here that, as seen on postorbital horncores, the nasal horncore can also be modified by resorption, although the signal and mechanism for this is unknown (possibly as the result of environmental stress or calcium reclamation). Although some extant reptiles (Hoby et al., 2010) and birds (Adkesson and Langan, 2007) have been documented to reduce bone mass as the result of environmental stress, complete remodeling of bony display structures have not been observed in extant vertebrates. Bone resorption has been proposed as a modifying agent in ceratopsids (Sampson, et al., 1997), but the mechanism for this process is unknown. In their study on cranial pitting and lesions in ceratopsids, Tanke and Farke (2007) reasoned that the commonly pitted postorbital horncores were likely the result of nonpathological bone resorption or some sort of pathology resulting from some sort of bone disease. Tanke and Farke (2007) also noted nasal horncore pitting on only one individual (cf. Centrosaurus, TMP 1988.050.0113). 

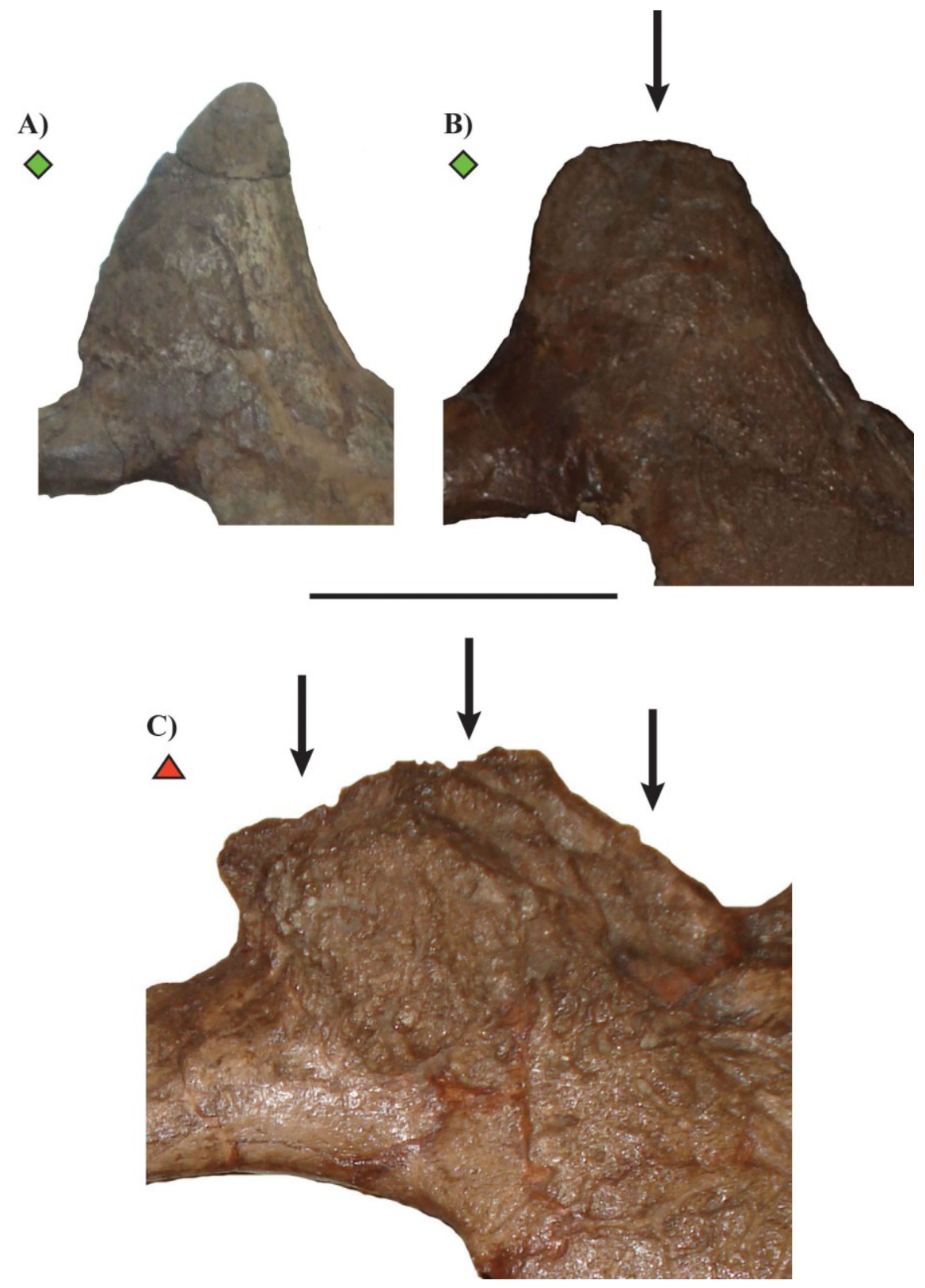

Figure 54. Ontogenetic changes in Chasmosaurus - proposed resorption of nasal horncore (character 13). A) Nasal horncore complete and pointed (AMNH 5402), and B) (YPM 2016) and C) (CMN 8800) nasal horncore partly resorbed. Chasmosaurus russelli (red triangle) and Vagaceratops-like specimens (green diamonds). Scale bar equals 10 $\mathrm{cm}$. 
Character 14 (postorbital horncore, modification) (new character):

(0) unmodified; (1) partly resorbed, horncore between $80 \%$ and $30 \%$ of its original inferred length; and (2) resorbed to base, horncore is less than $20 \%$ its original inferred length, or has been completely lost.

The postorbital horncores have pointed apices (0) in putatively immature specimens; the horncores then underwent resorption starting at the tip and progressing to the base (1 and 2). These character states are exemplified by AMNH 5401 and UALVP 40 (0), TMP 1981.019.0175 (1), and CMN 8800 (1\&2) (Fig. 55). In UALVP 40 (Fig. 55A) and AMNH 5401 (Fig. 55B), the postorbital horncores taper to a point (0). In TMP 1981.019.0175 (Fig. 55C), the tip of the postorbital horncore is truncated and pitted (1). In CMN 8800 (Fig. 55D), both postorbital horncores are reduced and heavily pitted, with more of the right horncore preserved (1), than on the left side (2).

This spectrum of postorbital horncore modification has also been identified in Centrosaurus (Ryan, 1992; Sampson et al., 1997; Ryan et al., 2001; Tanke and Farke, 2007), Einiosaurus (Sampson et al., 1997), Pachyrhinosaurus lakustai (Sampson et al., 1997; Tanke and Farke, 2007), and Styracosaurus (Sampson et al., 1997; Tanke and Farke, 2007) and was found to be size-related, with pitting only occurring in large and, presumably, fully mature adults.

Postorbital horncores are absent in some Chasmosaurus specimens, being replaced with low-relief, rugose surfaces instead. Although these may be interpreted as the pitted remnant of a horncore (as is typically done in the ceratopsian literature), one could also argue that these specimens never developed a horncore. Some ceratopsids (e.g., Pachyrhinosaurus lakustai) only possess postorbital horncores as juveniles in 
Figure 55. Ontogenetic changes in Chasmosaurus - proposed resorption of postorbital horncores (character 14). A) Postorbital horncore complete and pointed (UALVP 40), B) postorbital horncore complete (AMNH 5401; flipped), C) postorbital horncore partly resorbed (TMP 1981.019.0175; flipped), and D) postorbital horncore resorbed to base (CMN 8800). Plaster reconstruction shown in grey half-tone. Chasmosaurus russelli (red triangle) and Chasmosaurus sp. (black squares). Scale bar equals $10 \mathrm{~cm}$. 


\section{Figure 55.}

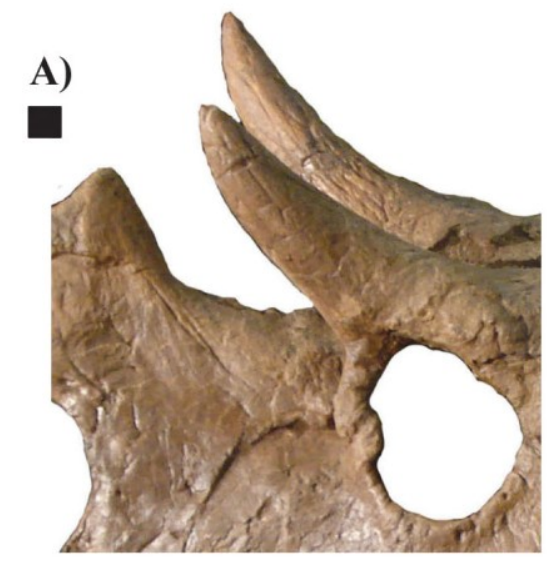

B)
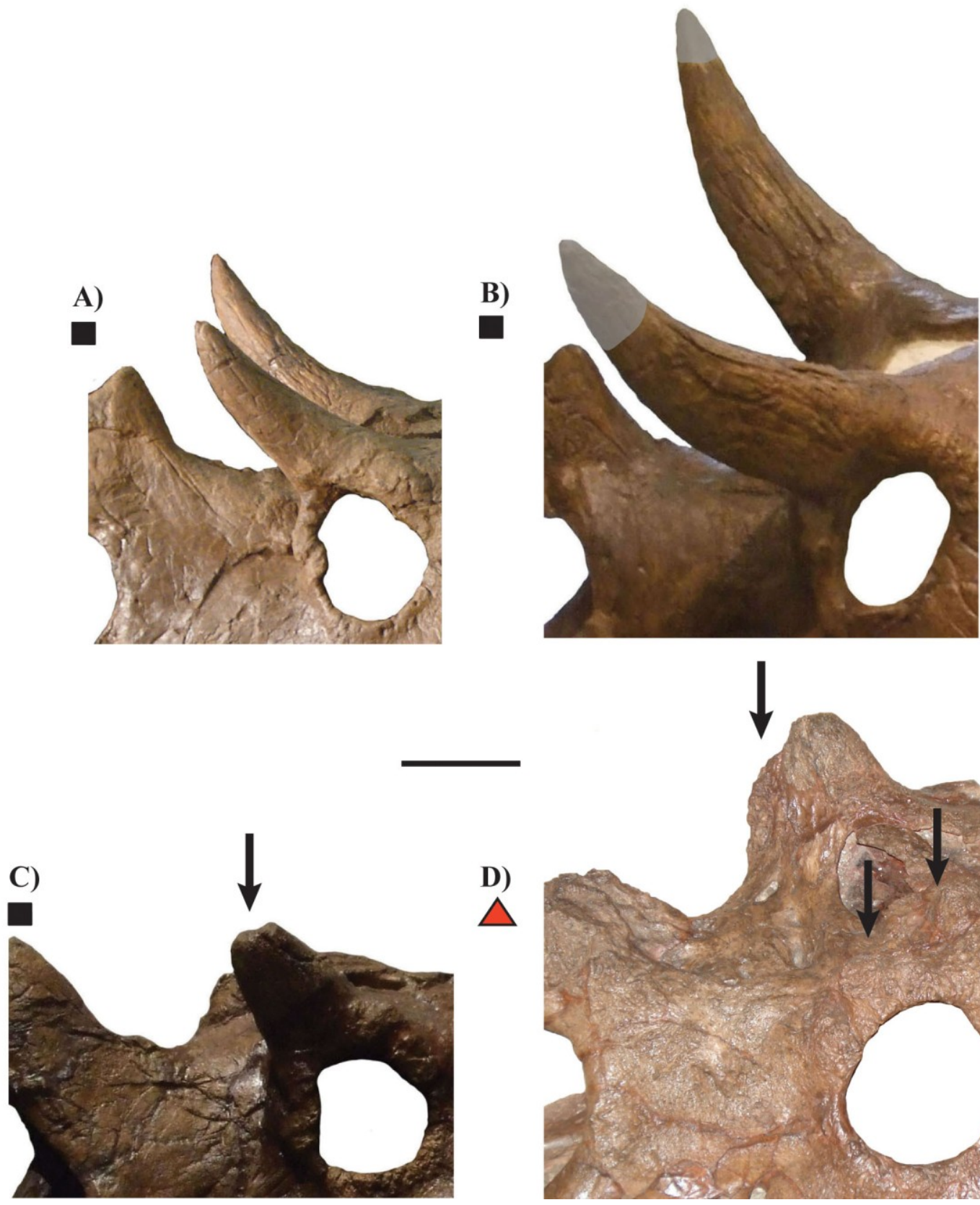

D)
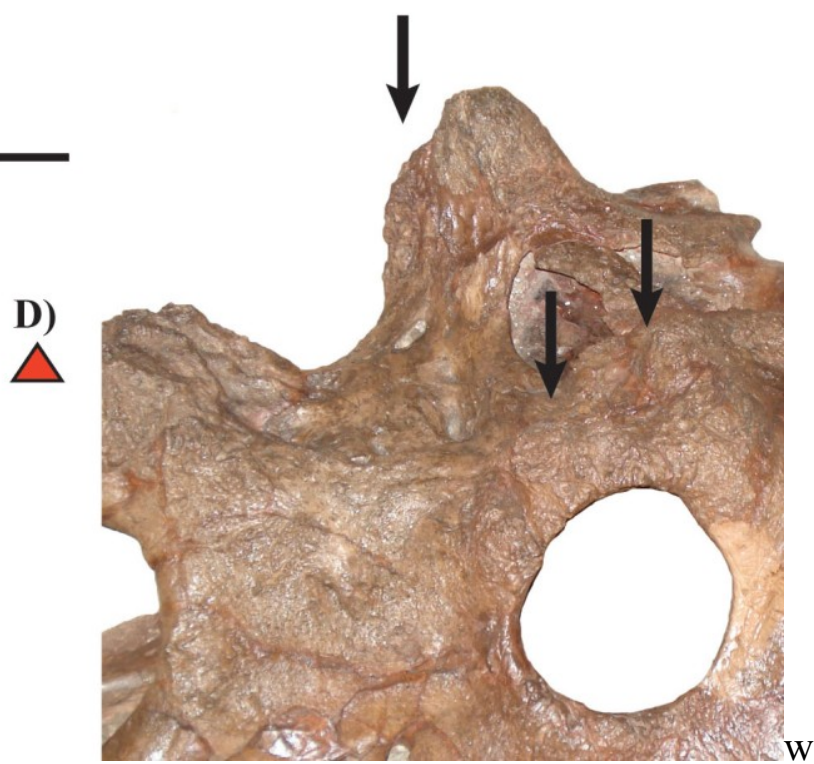

hich they are short and pyramidal-shaped. During ontogeny, this horncore develops into a wide, extensively pitted boss (Currie et al., 2008). The dorsal postorbital surfaces of Vagaceratops irvinensis have low, rugose structures in the place of horncores; Holmes et al. (2001) interpreted this as evidence that the taxon never possessed postorbital 
horncores and incorporated this character into their diagnosis, although they did acknowledge that the pitted surface may represent the base of a resorbed horncore. Recent work by Horner and Goodwin $(2006,2008)$, Scannella and Horner $(2010,2011)$, and Longrich and Field (2012) on Triceratops, and related taxa suggest that the effect of ontogenetic change has been underappreciated in chasmosaurine systematics, and that postorbital horncore modification is a real and significant feature in this clade, just as is it in centrosaurines.

Character 15 (parietal, lateral bar) (new character):

(0) continuous, completely excluding squamosal from parietal fenestra; and (1) discontinuous, allowing squamosal to form part of margin of parietal fenestra.

These character states are exemplified by AMNH 5656 and CMN 1254 (0), and CMN 2280 (1) (Fig. 56). In CMN 2280 (Fig. 56C), the lateral parietal bar tapers medially and becomes discontinuous (1).

The continuity of the lateral parietal bar was previously considered to be a diagnostic character within Chasmosaurus, being continuous in C. belli and discontinuous in C. russelli (Godfrey and Holmes, 1995). However, exceptions are known, e.g., AMNH 5656 (C. russelli) has continuous bars, and YPM 2016 (C. belli) has discontinuous bars. This feature, therefore, does not represent a robust diagnostic feature separating these two species. Instead, this feature appears to be size-related, with a continuous bar occurring in all small and some large-sized Chasmosaurus skulls, and a discontinuous bar occurring in most large skulls (Table 7). This suggests that the lateral bar becomes narrow, and finally discontinuous, with maturity. 
Figure 56. Ontogenetic changes in Chasmosaurus - lateral parietal bar (character 15): A) continuous (CMN 1254), B) continuous (AMNH 5656), and $\mathbf{C}$ ) discontinuous (CMN 2280). Lines represent degree of continuity of lateral parietal bars. Chasmosaurus russelli (red triangles) and Chasmosaurus sp. (black square). 
Figure 56.

A) $\square$
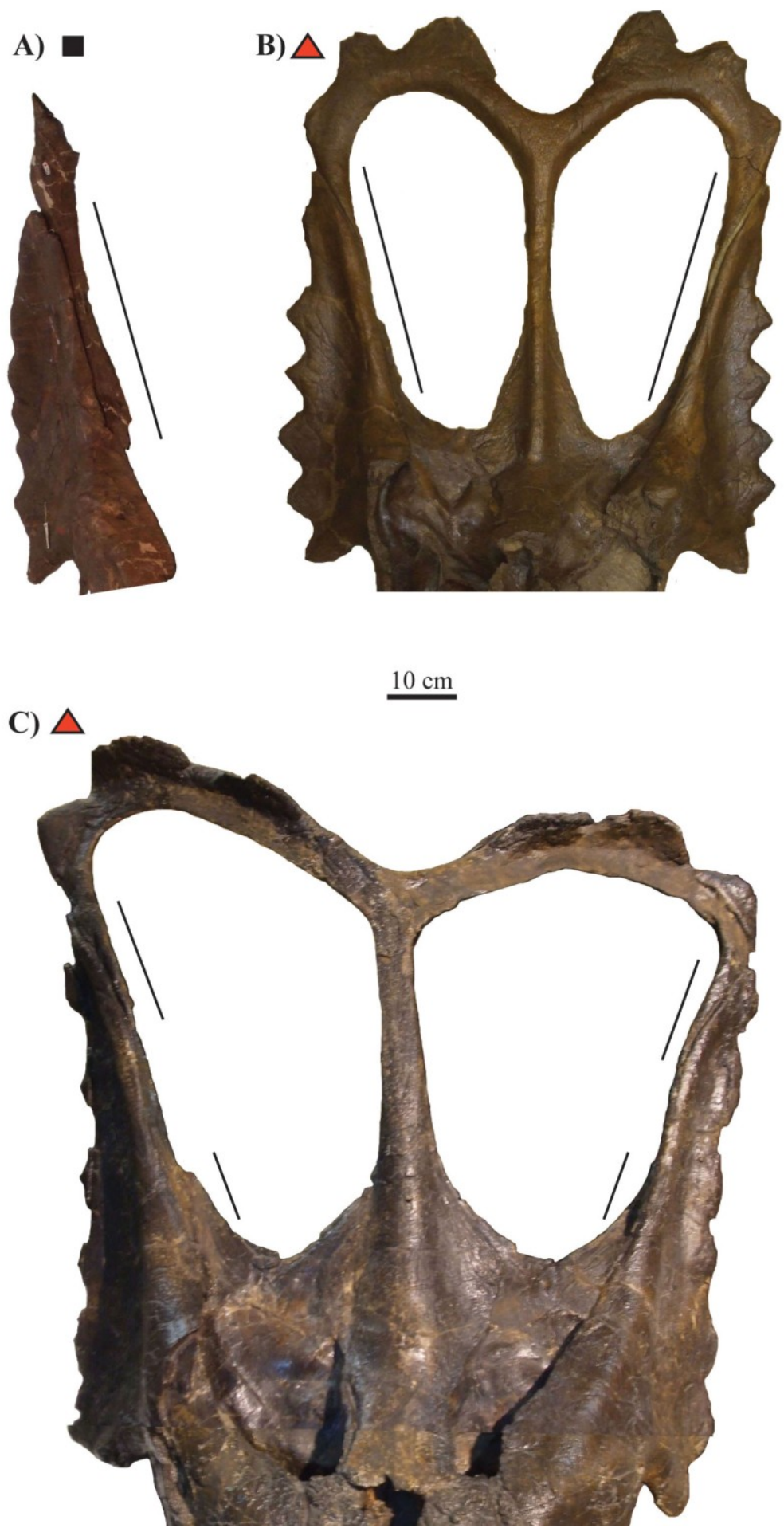
The continuity of the lateral parietal bar also varies in the holotype of $V$. irvinensis (CMN 41357), with only the left side being continuous. Lehman (1989) noted that the continuity of the lateral parietal bar varies with age, and occasionally between sides of the frill in the same individual of Agujaceratops mariscalensis and Pentaceratops sternbergi. It is difficult to assess whether such individuals are consistently more mature than individuals with complete bars due to the small sample sizes within each taxon (in the case of $V$. irvinensis), or insufficient discussion and figuring of specimens with the asymmetric condition in the literature (in the case of $A$. mariscalensis and P. sternbergi, e.g., Lehman, 1989; Forster et al., 1993; Lehman, 1998). Specimens of $A$. mariscalensis and $P$. sternbergi were not personally examined in this thesis.

Character 16 (squamosals, scalloping of lateral margins) (Longrich and Field, 2012; character 6, modified):

(0) strongly scalloped; and (1) weakly scalloped or unscalloped in dorsal view. The lateral margin of the squamosal refers to the margin of the squamosal itself, and not to the episquamosals that variably adorn it.

These character states are exemplified by AMNH 5401, AMNH 5656 and CMN 1254 (0), and CMN 8800 and NHMUK R4948 (1) (Fig. 51). In TMP 1998.128.0001 (Fig. 51A), CMN 1254 (Fig. 51B), AMNH 5656 (Fig. 51C), and AMNH 5401 (Fig. 51D) the lateral margin of the squamosal is strongly scalloped and undulatory (0). In NHMUK R4948 (Fig. 51E) and CMN 8800 (Fig. 51F), the lateral margin of the squamosal is only weakly scalloped (1). 
Character 17 (squamosal, length/width ratio) (Longrich and Field, 2012; character 5 modified and quantified):

$(0)<2.4$; (1) 2.5 to 2.95 ; and (2) $>3.0$.

The squamosal length is defined as the straight distance between the jugal notch and the distal end of the squamosal (parameter 19, Fig. 5). The squamosal width is defined as the minimum distance between the jugal notch and the medial margin of the squamosal (curved measurement, along the surface; parameter 14, Fig. 5). Scannella and Horner (2010) noted that the squamosal width/length ratio increases with skull size in Triceratops, although they defined squamosal width as the maximum width of the element. It is unclear whether Scannella and Horner's (2010) definition of squamosal width includes episquamosals; the presence/absence or degree of remodeling of episquamosals would affect the maximum squamosal width. Here, the jugal notch was chosen as the lateral extent of the squamosal, as it is not affected by the articulation and subsequent remodeling of episquamosals.

The above character states are exemplified by CMN 1254 and TMP 1998.128.0001 (0), AMNH 5401 and AMNH 5656 (1), and CMN 8800 and NHMUK R4948 (2) (Fig. 51). In TMP 1998.128.0001 (Fig. 51A) and CMN 1254 (Fig. 51B), the squamosal has a length/width ratio of less than 2.4 (0). In AMNH 5656 (Fig. 51C) and AMNH 5401 (Fig. 51D), the squamosal has a length/width ratio between 2.5 and 2.95 (1) (Table 7). In NHMUK R4948 (Fig. 51E) and CMN 8800 (Fig. 51F), the squamosal has a length/width ratio of greater than 3.0 (2) (Table 7). 
Character 18 (medial epiparietals/underlying parietal margin, orientation) (new character):

(0) in plane of frill; and (1) anterodorsally recurved.

These character states are exemplified by AMNH 5656 and TMP 1983.025.0001 (0), and CMN 0491, CMN 2280, CMN 8800, and ROM 843 (1) (Fig. 52). In CMN 2280 (Fig. 52C) and CMN 8800 (Fig. 52F), the two medial epiparietals (P1-2) are slightly anterodorsally recurved. In CMN 0491 (Fig. 52E), at least the first medial epiparietal is oriented slightly anterodorsally. In small (AMNH 5656) and some large-sized Chasmosaurus skulls (AMNH 5402 and TMP 1983.025.0001), epiparietals are oriented in the plane of the frill. However, in nearly all large skulls, epiparietals are oriented slightly anterodorsally, as a result of an anterodorsal curving of the posterior margin of the posterior parietal bar. 
Table 6: Specimen-character matrix used in ontogenetic analysis.

\begin{tabular}{lllll} 
Specimen & \multicolumn{1}{c}{5} & 10 & 15 & 18 \\
Embryo & $0000 ?$ & 00000 & 00000 & 000 \\
CMN 1254 & $? 0 ? 0 ?$ & $? ? 000$ & 00000 & $00 ?$ \\
AMNH 5656 & $? ? 010$ & $1 ? ? ? ?$ & $? ? ? ? 0$ & 010 \\
UALVP 40 & $01(01) ? 0$ & $? 0000$ & $0000 ?$ & $01 ?$ \\
AMNH 5401 & 11110 & $? 0010$ & $0000 ?$ & $01 ?$ \\
AMNH 5402 & 12211 & 10000 & 00000 & 120 \\
CMN 2245 & $? 10(12) 1$ & $1 ? ? ? 0$ & 11000 & 021 \\
ROM 839 & $? 2(01) 11$ & $? 0011$ & 10000 & $02 ?$ \\
NHMUK R4948 & $122(12) 1$ & $? 101 ?$ & $? 001 ?$ & 121 \\
ROM 843 & $? 2221$ & 21011 & 11020 & 121 \\
TMP 1981.019.0175 & $222 ? 1$ & $? 1110$ & $000(12) 1$ & $10 ?$ \\
TMP 1983.025.0001 & $? ? 221$ & $2 ? ? ? ?$ & $? ? ? 11$ & 110 \\
YPM 2016 & 22221 & 21111 & 10121 & 111 \\
CMN 2280 & 22221 & 21111 & $111(01) 1$ & 111 \\
CMN 8800 & $? 2 ? 22$ & 21111 & $111(12) 1$ & 121
\end{tabular}


Table 7: Postorbital horncore length (parameter 1, Fig. 5) and basal circumference (parameter 4, Fig. 5), squamosal length/width ratio (parameter 19/14 ratio, Fig. 5) and continuity of lateral parietal bar (cont. $=$ continuous, discont. $=$ discontinuous $)$ in Chasmosaurus specimens (where preserved), arranged from smallest to largest rostral-toepijugal length (parameter 16, Fig. 5); squamosal length (parameter 19, Fig. 5; L = left, R $=$ right side) included as a secondary frame of reference, because not all specimens preserve the snout region of the skull. Symbols: $*=$ estimated measurement; $* *=$ dorsal portion of postorbital horncore missing.

\begin{tabular}{|l|l|l|l|l|}
\hline Specimen & $\begin{array}{c}\text { Rostral-to- } \\
\text { epijugal } \\
\text { length (mm) }\end{array}$ & $\begin{array}{c}\text { Squamosal } \\
\text { length (mm) }\end{array}$ & $\begin{array}{l}\text { Postorbital } \\
\text { horncore } \\
\text { length (mm) }\end{array}$ & $\begin{array}{l}\text { Postorbital } \\
\text { horncore } \\
\text { basal } \\
\text { circum- } \\
\text { ference } \\
\text { (mm) }\end{array}$ \\
\hline TMP 1998.128.0001 & ----- & $208(\mathrm{~L})$ & ----- & ----- \\
\hline CMN 1254 & ----- & $500(\mathrm{R})$ & 216 & 242 \\
\hline AMNH 5656 & ----- & $569(\mathrm{~L})$ & ----- & ----- \\
\hline UALVP 40 & 628 & $600^{*}(\mathrm{~L})$ & 176 & 240 \\
\hline AMNH 5402 & 680 & $685(\mathrm{R})$ & 70 & 190 \\
\hline AMNH 5401 & 730 & $710(\mathrm{~L})$ & 370 & 320 \\
\hline CMN 2245 & ----- & $779(\mathrm{R})$ & 102 & 238 \\
\hline TMP 1983.025.0001 & ----- & $650(\mathrm{R})$ & $142^{* *}$ & 345 \\
\hline NHMUK R4948 & ----- & $840(\mathrm{R})$ & $55^{* *}$ & ----- \\
\hline CMN 2280 & 750 & $709(\mathrm{~L})$ & 132 & 263 \\
\hline TMP 1981.019.0175 & 768 & $600^{*}(\mathrm{R})$ & $134^{* *}$ & 302 \\
\hline ROM 839 & 782 & $917(\mathrm{~L})$ & 107 & 250 \\
\hline YPM 2016 & 800 & $730(\mathrm{R})$ & $41^{* *}$ & 222 \\
\hline CMN 8800 & 880 & $997(\mathrm{~L})$ & $108^{* *}$ & 412 \\
\hline ROM 843 & 897 & $893(\mathrm{~L})$ & $67^{* *}$ & 252 \\
\hline
\end{tabular}


Table 7 (continued).

\begin{tabular}{|l|l|l|l|l|}
\hline Specimen & $\begin{array}{c}\text { Rostral-to- } \\
\text { epijugal } \\
\text { length (mm) }\end{array}$ & $\begin{array}{c}\text { Squamosal } \\
\text { length (mm) }\end{array}$ & $\begin{array}{l}\text { Squamosal } \\
\text { length/width } \\
\text { ratio }\end{array}$ & $\begin{array}{c}\text { Lateral } \\
\text { parietal bar, } \\
\text { continuity }\end{array}$ \\
\hline TMP 1998.128.0001 & ----- & $208(\mathrm{~L})$ & 2.39 & \\
\hline CMN 1254 & ----- & $500(\mathrm{R})$ & 2.00 & cont. \\
\hline AMNH 5656 & ----- & $569(\mathrm{~L})$ & 2.56 & cont. \\
\hline UALVP 40 & 628 & $600^{*}(\mathrm{~L})$ & 2.56 & ----- \\
\hline AMNH 5402 & 680 & $685(\mathrm{R})$ & 3.26 & cont. \\
\hline AMNH 5401 & 730 & $710(\mathrm{~L})$ & 2.63 & ----- \\
\hline CMN 2245 & ----- & $779(\mathrm{R})$ & 3.15 & cont. \\
\hline TMP 1983.025.0001 & ----- & $650(\mathrm{R})$ & 2.65 & discont. \\
\hline NHMUK R4948 & ----- & $840(\mathrm{R})$ & 3.82 & ----- \\
\hline CMN 2280 & 750 & $709(\mathrm{~L})$ & 2.91 & discont. \\
\hline TMP 1981.019.0175 & 768 & $600^{*}(\mathrm{R})$ & 2.38 & discont. \\
\hline ROM 839 & 782 & $917(\mathrm{~L})$ & 3.23 & cont. \\
\hline YPM 2016 & 800 & $730(\mathrm{R})$ & 2.81 & discont. \\
\hline CMN 8800 & 880 & $997(\mathrm{~L})$ & 3.93 & discont. \\
\hline ROM 843 & 897 & $893(\mathrm{~L})$ & 3.03 & cont. \\
\hline
\end{tabular}


Hypotheses tested:

1. The relationship of ontogenetic placement on taxonomic assignment: Using the ontogenetic clustering analysis, I test the null hypothesis $\left(\mathrm{H}_{0}\right)$ that the species Chasmosaurus belli and C. russelli do not form separate, non-overlapping ontogenetic stages. If the null hypothesis is accepted, this would indicate that morphological differences between these two taxa cannot be attributed to ontogeny, and would indicate the presence of two overlapping ontogenetic trajectories corresponding to two valid taxa. If the null hypothesis is rejected, this would indicate that $C$. belli and C. russelli represent separate ontogenetic stages or ontogimorphs of a single taxon $\left(\mathrm{H}_{1}\right)$.

2. The distribution of postorbital horncore size and orientation over ontogeny: Once the ontogenetic analysis has been performed, and specimens ontogeneticallycategorized by size-independent characters, I test the null hypothesis $\left(\mathrm{H}_{0}\right)$ of a single ontogenetic trajectory in Chasmosaurus with respect to postorbital horncore size (length and basal circumference) and orientation. If the null hypothesis is accepted, this would suggest that postorbital horncore size and orientation change consistently over ontogeny (i.e., specimens with small horncores are less mature than specimens with large horncores), and that these features are valid ontogenetically variable characters in Chasmosaurus. If the null hypothesis is rejected, this would indicate that there is a large discrepancy in postorbital horncore size between mature specimens, supporting the presence of two ontogenetic trajectories with respect to this feature $\left(\mathrm{H}_{1}\right)$. The length of the postorbital horncore (parameter 1, Fig. 5) and its basal circumference (parameter 4, Fig. 5; Table 7; Figs. 57-58) are significantly positively correlated $(p<0.05)$ in Dinosaur 


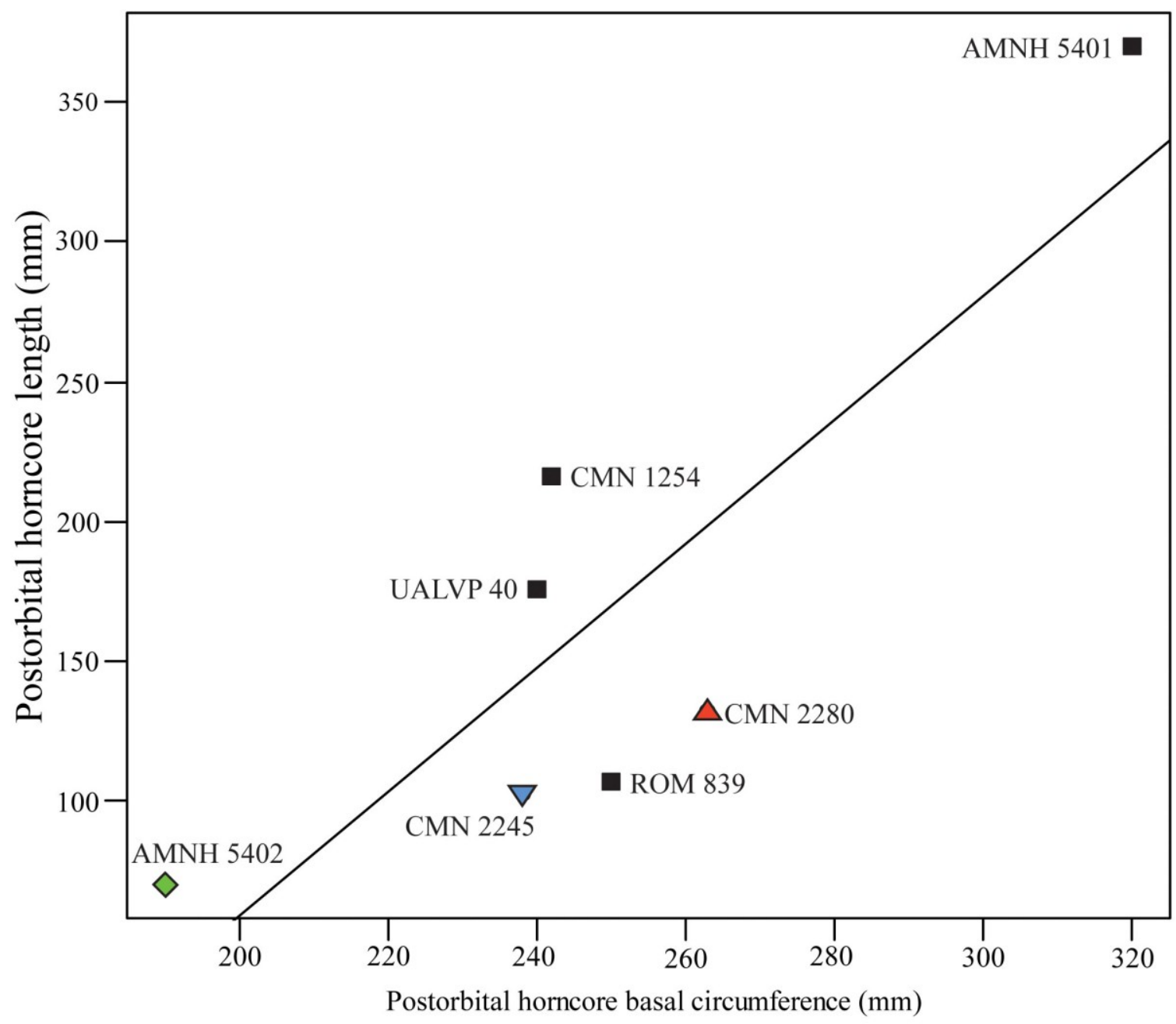

Figure 57. Scatter plot showing postorbital horncore length vs. basal circumference of Chasmosaurus specimens; only complete/pointed horncores included. Line-of-best-fit ( $p$ $=0.0175)$. Chasmosaurus russelli (red triangle), $C$. belli (blue inverted triangle), Chasmosaurus sp. (black squares), and Vagaceratops-like specimens (green diamond). 


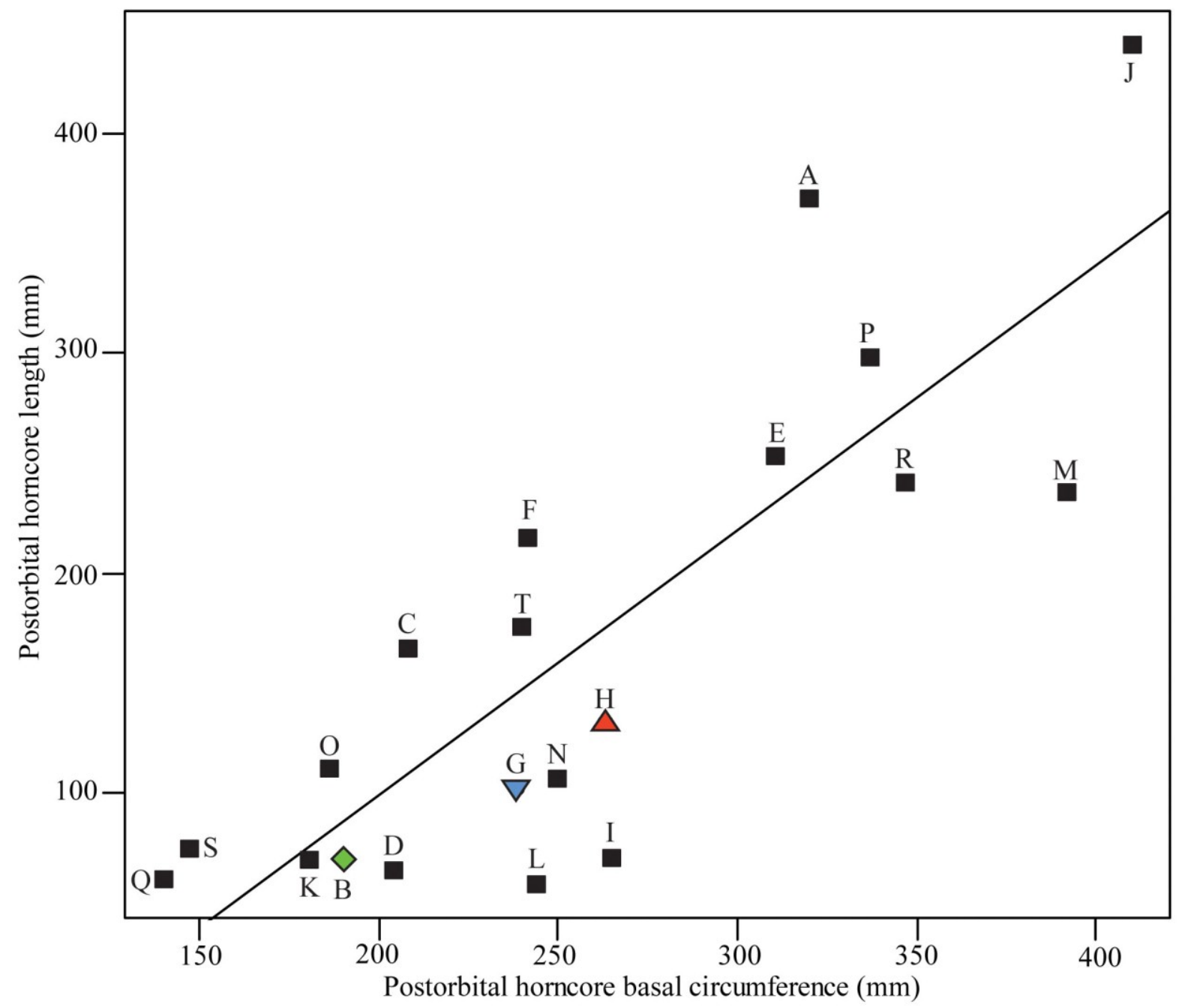

Figure 58. Scatter plot showing postorbital horncore length vs. basal circumference in all

Dinosaur Park Formation chasmosaurine specimens; only complete/pointed horncores included. Specimens: A) AMNH 5401; B) AMNH 5402; C) CMN 0212; D) CMN 0275; E) CMN 0837; F) CMN 1254; G) CMN 2245; H) CMN 2280; I) CMN 8802; J) CMN 9676; K) CMN 9808b; L) CMN 34829; M) CMN 34832; N) ROM 839; O) TMP 1967.017.0005; P) TMP 1979.011.0147; Q) TMP 1979.014.0813; R) TMP 1979.014.0814; S) TMP 2008.012.0074; and T) UALVP 40. Line-of-best-fit ( $p=8.03 \mathrm{x}$ $10^{-6}$ ). Chasmosaurus russelli (red triangle), C. belli (blue inverted triangle), Chasmosaurus sp. (black squares), and Vagaceratops-like specimens (green diamond). 
Park Formation chasmosaurine specimens. This allows for basal circumference to serve as a proxy for estimating the original length of a horncore that has undergone resorption in Chasmosaurus.

3. Distinction between postorbital horncore ontogenetic trajectories: If there is a large discrepancy in postorbital horncore size between mature specimens, suggesting the presence of two ontogenetic trajectories with respect to this feature (i.e., culminates in small and large horncores, respectively), I test the null hypothesis $\left(\mathrm{H}_{0}\right)$ of no significant difference between these two putative trajectories. If the null hypothesis is accepted, this would indicate that the identified trajectories are artificial and instead likely represent individual variation within a single trajectory. If the null hypothesis is rejected, this would indicate that the identified trajectories are distinct and valid $\left(\mathrm{H}_{1}\right)$. This hypothesis will be tested by performing a non-parametric multivariate analysis of variance (NPMANOVA) on the distribution of specimens referred to the two trajectories in Figures 59-62, using the Mahalanobis distance measure. If the null hypothesis is rejected, the specimens comprising each ontogenetic trajectory in Figures 59-62 will be examined to determine whether these trajectories correspond to taxonomic assignments $(C$. belli and C. russelli), or do not. If the trajectories do not correspond to taxonomic assignments, differences in postorbital horncore size and orientation may instead be attributable to sexual dimorphism, as occurs in extant bovids.

NPMANOVA tests for differences between two or more groups of multivariate data and is based on any distance measure. NPMANOVA was conducted in this study because clusters of specimens corresponding to putative postorbital horncore ontogenetic trajectories were small ( $\mathrm{n}=2$ to 6 ) and not normally distributed. The Mahalanobis 
distance measure (Mahalanobis, 1936) was used because it is better suited to smaller, non-spherically symmetric datasets than the traditional Euclidean distance measure. The NPMANOVA was performed in PAST 3.01 (Hammer et al., 2001).

4. Shape of the parietal along the posteromedial margin of the parietal fenestra over ontogeny: Once the ontogenetic analysis has been performed, and specimens ontogenetically-categorized by size-independent characters, I test the null hypothesis $\left(\mathrm{H}_{0}\right)$ that the longitudinal sulcus on the parietal fenestra margin of the medial and posterior parietal bars (Fig. 6) does not develop over ontogeny. If the null hypothesis is accepted, this would suggest that this sulcus is a valid diagnostic character of Mojoceratops perifania, distinct from Chasmosaurus (sensu Longrich, 2010). If the null hypothesis is rejected, this would indicate that this sulcus develops over ontogeny in Dinosaur Park Formation chasmosaurines and that this feature is not diagnostic of $M$. perifania $\left(\mathrm{H}_{1}\right)$.

Variation in postorbital horncores

It is important to note that both postorbital horncore length and orientation were excluded as characters in the ontogenetic analysis. It has been proposed that postorbital horncores lengthened (Agujaceratops, Lehman, 1990; Triceratops, Horner and Goodwin, 2006) and changed their orientation (Triceratops, Horner and Goodwin, 2006; short stubs early in ontogeny, curved posteriorly in juveniles, straightened in subadults, and recurved anteriorly in adults) throughout ceratopsid ontogeny. The ability to recognize both postorbital length and orientation changes throughout Triceratops ontogeny is made possible by its large dataset which encompasses most of its ontogenetic spectrum. The 
length and orientation of postorbital horncores can be documented throughout

Triceratops ontogeny for a large number of specimens, as the former character does not undergo significant modification or resorption.

Prior to conducting the 18 character-based ontogenetic analysis of Chasmosaurus, developmental changes in the size of the postorbital horncore (length and basal circumference - parameters 1 and 4, Fig. 5, respectively) and its orientation were evaluated strictly on the basis of skull size (rostral-to-epijugal length and squamosal length - parameters 16 and 19, Fig. 5, respectively), with larger skulls generally inferred as being more mature than smaller ones. On this basis, it was found that both postorbital horncore length and basal circumference increase with rostral-to-epijugal length (Figs. 59-60) and squamosal length (Figs. 61-62), but do not appear to follow a single ontogenetic trajectory. Instead, there is a large discrepancy in horncore length amongst the largest specimens, if only specimens with unmodified (pointed apex) horncores are considered (e.g., CMN $2280=132 \mathrm{~mm}$ vs. AMNH $5401=370 \mathrm{~mm}$; Figs. 59 and 61). There is a similarly large discrepancy in horncore basal circumference amongst the largest specimens, if specimens with resorbed horncores are also considered (e.g., ROM $843=252 \mathrm{~mm}$ vs. $412 \mathrm{~mm}=\mathrm{CMN} 8800$; Figs. 60 and 62). This discrepancy in postorbital horncore length and basal circumference amongst skulls of putatively similar stages of maturity suggests that differences in these two parameters cannot be explained by a single ontogenetic trajectory. This statement is also supported by the presence of relatively long horncores in some relatively small skulls (e.g., CMN 1254 - squamosal length $=500 \mathrm{~mm}$, horncore length $=216 \mathrm{~mm}$ ) and relatively short horncores in some relatively large skulls (e.g., ROM $839-$ squamosal length $=917 \mathrm{~mm}$, horncore length $=$ 
$107 \mathrm{~mm}$ ) (Fig. 61). Based on the distribution of postorbital horncore sizes in Figures 5962 , there appear to be two ontogenetic trajectories culminating in relatively short, small circumference-based horncores that do not exceed $132 \mathrm{~mm}$ in length (CMN 2280), and relatively long, large circumference-based horncores that do not exceed $370 \mathrm{~mm}$ in length (AMNH 5401), respectively.

The relatively small skull UALVP 40 (rostral-to-epijugal length $=628 \mathrm{~mm}$ ) and large skull AMNH 5401 (rostral-to-epijugal length $=730 \mathrm{~mm}$ ) have postorbital horncore lengths of $176 \mathrm{~mm}$ and $370 \mathrm{~mm}$, respectively, while the larger skull CMN 2280 (rostralto-epijugal length $=750 \mathrm{~mm}$ ) has a horncore only $132 \mathrm{~mm}$ in length (Fig. 59). None of the current hypotheses on postorbital horncore modification during ontogeny predict a model where a shortened horncore can maintain a pointed apex. Taking this into account, it is more likely that UALVP 40 forms an ontogenetic trajectory with AMNH 5401, and not with CMN 2280 (Figs. 59-62). Based on this principal, the two proposed ontogenetic trajectories appear to be composed of the following specimens;

"short" horncore development - AMNH 5402, CMN 2245, CMN 2280, ROM 839, ROM 843, and YPM 2016; and,

“long" horncore development - AMNH 5401, CMN 1254, CMN 8800, TMP

1981.019.0175, TMP 1983.025.0001, and UALVP 40 (Figs. 59-62). 


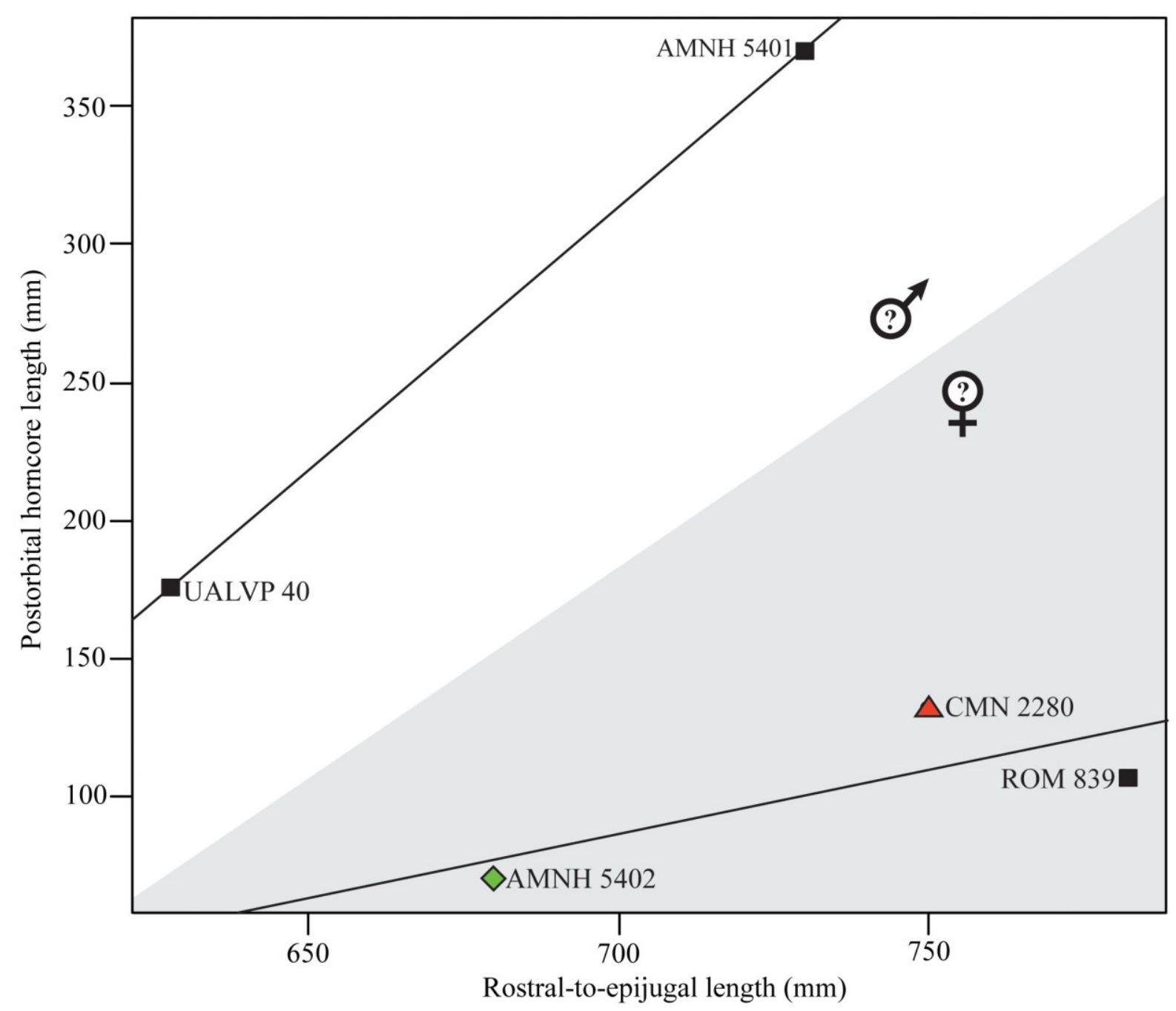

Figure 59. Scatter plot showing postorbital horncore length vs. rostral-to-epijugal length of Chasmosaurus russelli (red triangle), Chasmosaurus sp. (black squares), and Vagaceratops-like specimen (green diamond). Only complete/pointed horncores are included. Proposed "long horncore" (white area) and "short horncore" (shaded area) ontogenetic trajectories. Lines-of-best-fit (solid lines): short horncore trajectory, $p=$ 0.4609. Difference between proposed trajectories: $p=0.1929$. 


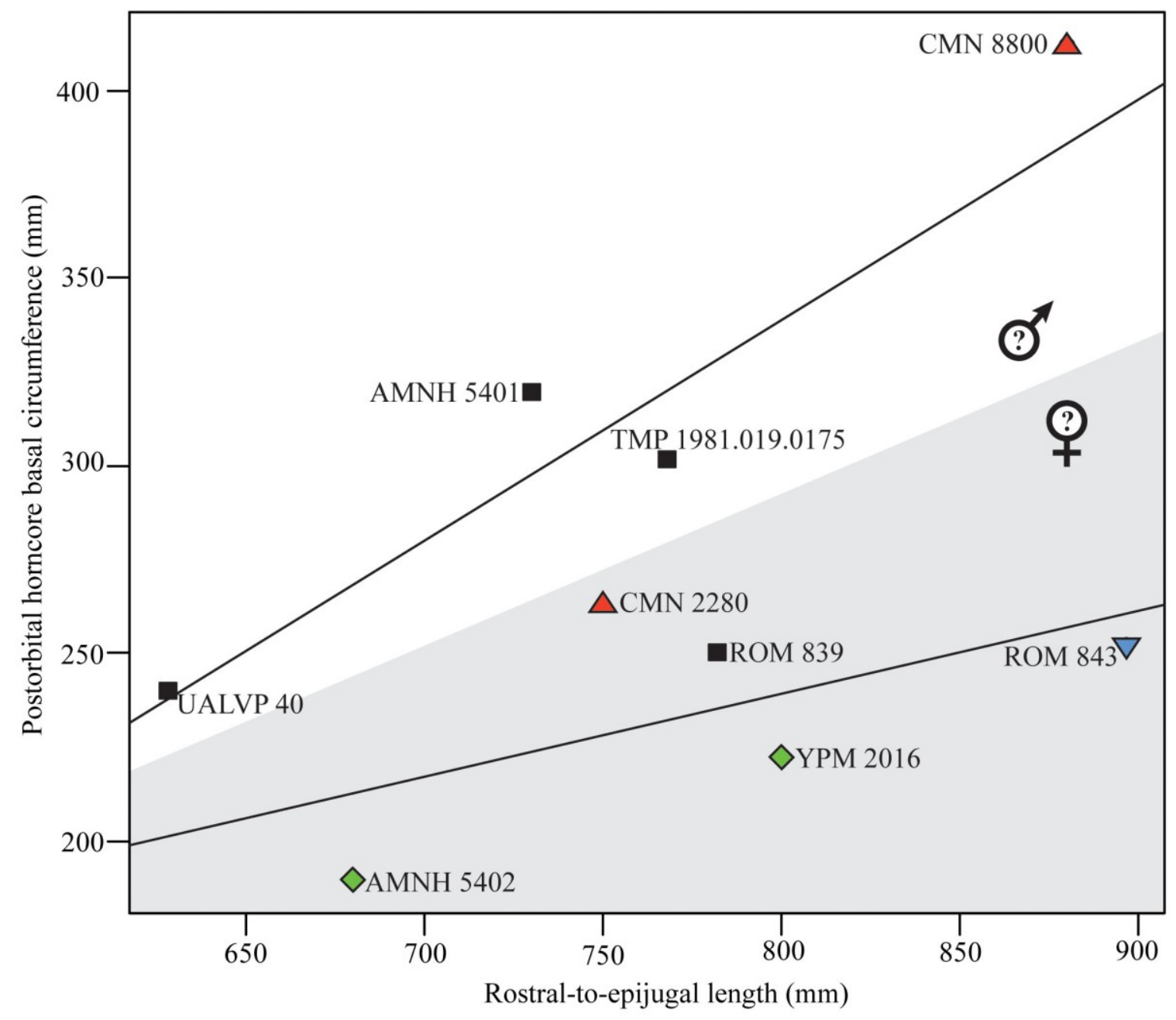

Figure 60. Scatter plot showing postorbital horncore basal circumference vs. rostral-toepijugal length of Vagaceratops-like of specimens (green diamonds), Chasmosaurus russelli (red triangles), C. belli (blue inverted triangle), and Chasmosaurus sp. (black squares). Proposed "long horncore" (white area) and "short horncore" (shaded area) ontogenetic trajectories. Lines-of-best-fit (solid lines): short horncore trajectory, $p=$ 0.3054; long horncore trajectory, $p=0.0363$. Difference between proposed trajectories: $p$ $=0.0136$. 


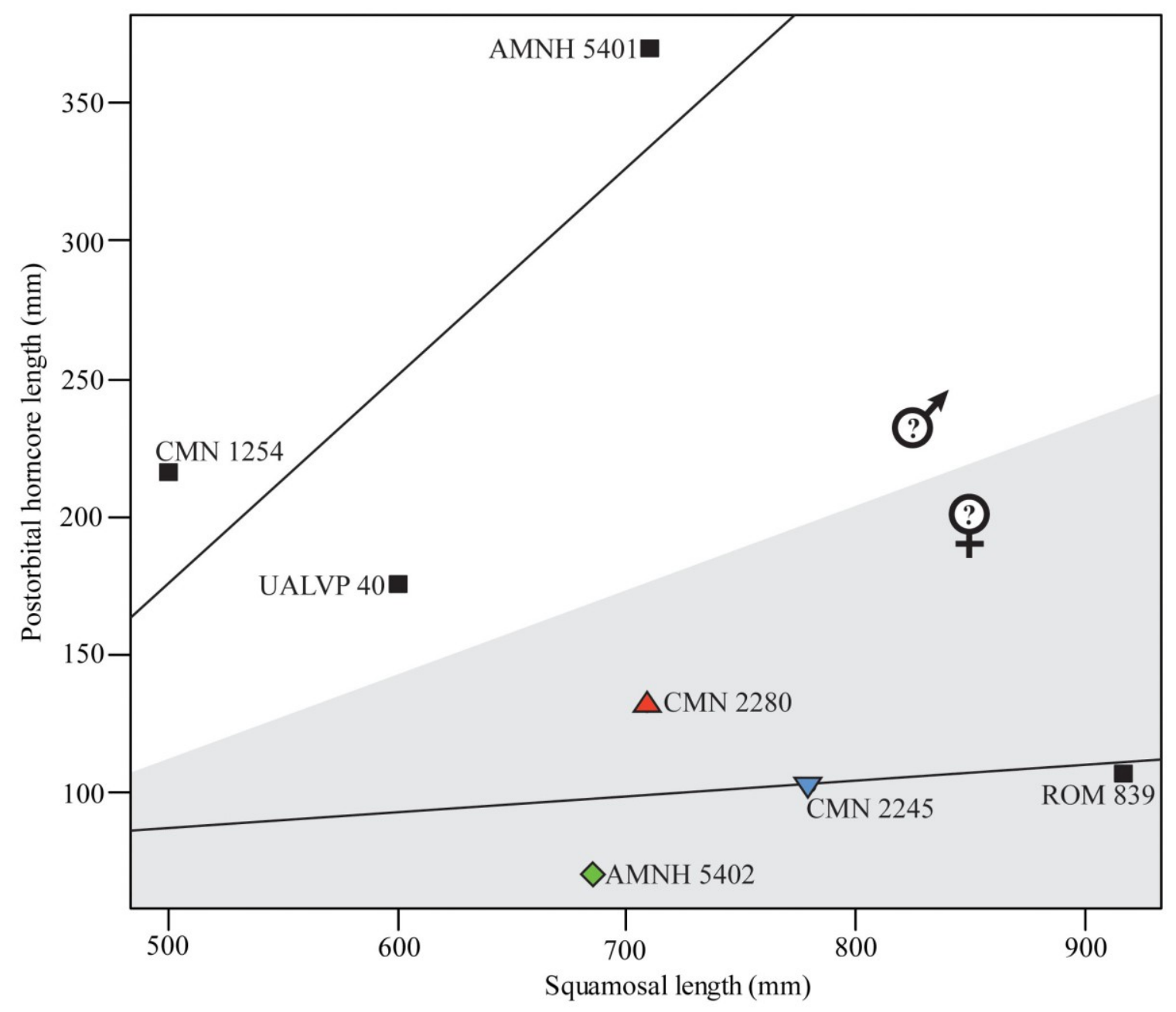

Figure 61. Scatter plot showing postorbital horncore length vs. squamosal length of Vagaceratops-like specimens (green diamond), Chasmosaurus russelli (red triangle), C. belli (blue inverted triangle), and Chasmosaurus sp. (black squares); only complete/pointed horncores are included. Proposed "long horncore" (white area) and "short horncore" (shaded area) ontogenetic trajectories. Lines-of-best-fit (solid lines): short horncore trajectory, $p=0.7970$; long horncore trajectory, $p=0.4409$. Difference between proposed trajectories: $p=0.0291$. 


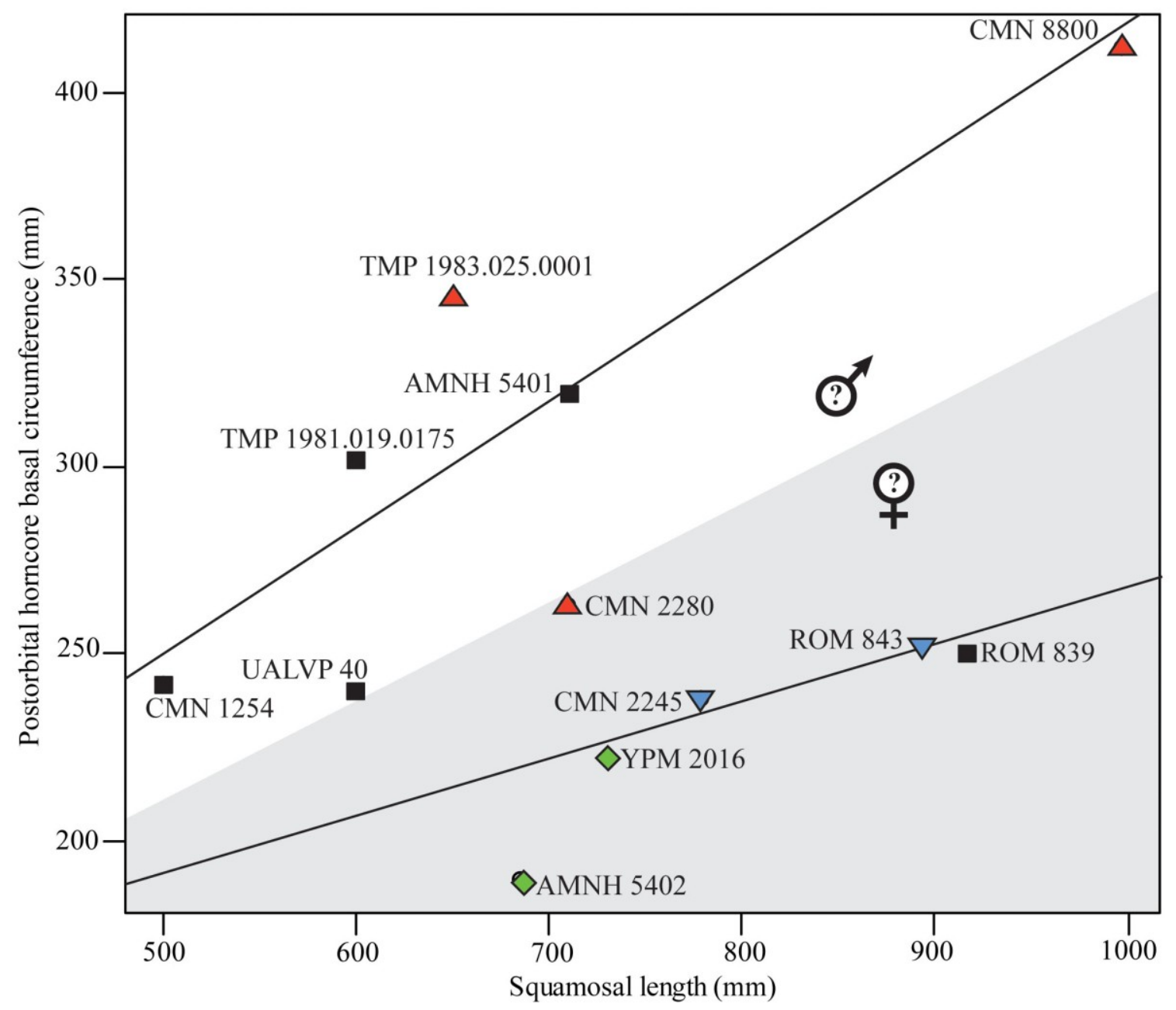

Figure 62. Scatter plot showing postorbital horncore basal circumference vs. squamosal length of Vagaceratops-like specimens (green diamonds), Chasmosaurus russelli (red triangles), C. belli (blue inverted triangles), and Chasmosaurus sp. (black squares). Proposed "long horncore" (white area) and "short horncore" (shaded area) ontogenetic trajectories. Lines-of-best-fit (solid lines): short horncore trajectory, $p=0.2776$; long horncore trajectory, $p=0.0165$.Difference between proposed trajectories: $p=0.0031$. 
The ontogenetic development of postorbital horncore orientation, like length and basal circumference, does not conform to a single ontogenetic trajectory with increasing skull size in Chasmosaurus. Instead, the two ontogenetic trajectories proposed here are each characterized by a unique sequence of horncore orientations. In the "long" horncore trajectory, the relatively small skull CMN 1254 (Fig. 7D) possesses a vertically erect horncore with a slight posterior curvature. The successively larger skulls UALVP 40 (Fig. 7Q) and AMNH 5401 (Fig. 7A) have successively longer and larger circumferencebased horncores that are both more anteriorly and laterally inclined, but retain the posterior curvature seen in CMN 1254. In the "short" horncore trajectory, the relatively large skulls CMN 2245 (Fig. 7E) and CMN 2280 (Fig. 7F) possess vertically erect horncores that are straight and curved posteriorly, respectively. The short-horned skull ROM 839 (Fig. 7K) possesses horncores that are curved slightly posteriorly, which appears to be intermediate to those of CMN 2245 and CMN 2280. The vertically erect, posteriorly-curved horncore of the relatively large skull CMN 2280 is similar to that of the relatively small skull CMN 1254, although the latter is longer $(132 \mathrm{~mm}$ vs. $216 \mathrm{~mm}$, respectively).

The orientation of the postorbital horncore in a given specimen can only be determined if the horncore is unmodified. The orientation of the horncore cannot be determined in six (CMN 8800, NHMUK R4948, ROM 843, TMP 1981.019.0175, TMP 1983.025.0001, and YPM 2016) of the 13 specimens with horncores, as these six specimens are missing the dorsal half or more of the horncore; however the anterolateral inclination of the horncore is still apparent in two (TMP 1981.019.0175 and TMP 1983.025.0001) of these resorbed specimens. 
The two proposed ontogenetic trajectories do not correspond to previous taxonomic groupings, as $C$. russelli specimens occur in both the short (CMN 2280) and long (CMN 8800, TMP 1983.025.0001) horncore trajectories (Figs. 59-62). This suggests that differences in postorbital horncore size in Dinosaur Park Formation chasmosaurines is due to some other, non-taxonomic signal, such as sexual dimorphism.

Bovids (cloven-hoofed ruminant mammals) have been used previously as modern analogues to ceratopsians, on the basis that both groups possess a pair of horncores above or behind the orbits, as well as other morphological factors (Farke, 2006). Ceratopsian horncores, like those of bovids, also possessed an outer (likely keratinous) sheath, as on ceratopsids (Hatcher et al., 1907; Ryan and Russell, 2005; Happ, 2010). Horncores are present in males of all bovids, but are absent in one-third of females (Estes, 1991). In bovids where females possess horncores, they are generally shorter than in males (Estes, 1991; Bro-Jørgensen, 2007). Antlers are another form of cranial ornamentation, but differ from horncores in that they are not permanent structures, and are shed annually (Estes, 1991). Antlers occur in cervids (deer species), but are absent in females except in caribou/reindeer (Holand et al., 2004).

While the sexually dimorphic horncores of bovids may be similar in structure to those of ceratopsians, they are not homologous features, having evolved independently in mammals and reptilians, respectively. To further evaluate the likelihood that ceratopsians possessed sexually dimorphic postorbital horncores, the presence of sexually dimorphic cranial display structures in their closest related extant relatives, birds and crocodylians, must be evaluated; dinosaurs, birds and crocodylians are all archosaurian reptiles.

Sexual dimorphism is well documented in birds, and is expressed in body size, 
body proportions, plumage, and behavioural differences (Selander, 1966; Owens and Hartley, 1998). In terms of cranial display structures, sexual dimorphism has been documented in the shape and size of the beak in some taxa (Selander, 1966). However, visual sexual dimorphism is absent in approximately $30 \%$ of all bird species (Bercovitz et al., 1978), with some taxa possessing monomorphic cranial display structures such as the pronounced head crest in Casuarius casuarius (Dodson, 1975; Bercovitz et al., 1978). Sexual dimorphism is also apparent in crocodylians, with males having larger body sizes and wider, more robust skulls than females (e.g., Crocodylus novaeguineae; Hall and Portier, 1994).

Some extant lizards (non-archosaurian reptiles) also possess cranial ornamentation in the form of horns and spikes, such as species of Phrhynosoma (Powell et al., 2002). This genus possesses horns or spikes along the posterior margin of the parietal and squamosal, and appear to be analogous to epiparietals and episquamosals, respectively, of ceratopsians. This taxon also possesses horny outgrowths of the frontal, similar to postorbital horncores of ceratopsians, or horncores in bovids. An allometric study of members of this genus found that, once ontogenetic differences were factored in, variation in horn length was largely attributable to taxonomic differences, and not sexual dimorphism. While sexual dimorphism cannot account for horn length in this genus, it is important to note that this is a relatively small-bodied taxon compared to large-bodied ceratopsians such as Chasmosaurus.

Sexual dimorphism in terms of cranial display has also been proposed for fossil archosaurian taxa (Borkovic, 2013). Zeigler et al. (2002) attributed the dimorphic skull of the phytosaur Pseudopalatus to sexual dimorphism, with males having more robust 
snouts and more pronounced narial crests. A bimodal distribution has also been documented in winged pterosaurs, with males having pronounced cranial crests and females having rudimentary crests or none at all (e.g., Pteranodon, Bennett, 1992; Darwinopterus, Lü et al., 2011). This hypothesis is compelling, as a non-crested member of Darwinopterus, i.e., the female morph, was found associated with an egg (Lü et al., 2011).

Dinosaurs other than Chasmosaurus have been noted as having bimodal distributions in their cranial display structures. The dimorphic cranial crest of the lambeosaurine hadrosaur Corythosaurus casuarius was once hypothesized as representing sexual dimorphism (Dodson, 1975; Evans, 2003), but subsequently rejected when these two morphs were found to be stratigraphically segregated in the Dinosaur Park Formation (Evans et al., 2006). Dodson (1976) noted a bimodal distribution in Protoceratops andrewsi, and suggested that males had wider frills and cheeks (jugals), greater skull height, more prominent nasal bosses, and greater height as the nasals. A bimodal distribution in postorbital horncore orientation was also noted in Agujaceratops mariscalensis, with longer and more vertically erect horncores considered to be males, and shorter, more anteriorly inclined horncores as females (Lehman, 1990). Carpenter (1990) recognized gracile and robust morphs in the dentaries and maxillae of Tyrannosaurus rex and suggested they represented males and females, respectively. Larson (2008) agreed with Carpenter's (1990) conclusions, based on the discovery of medullary bone in the femur of a robust morph (Schweitzer et al., 2008).

Borkovic (2013) recently conducted an evaluation of sexual dimorphism in the postorbital horncores of Triceratops horridus and Triceratops prorsus and nasal horncore 
of Centrosaurus apertus. He discovered that there was considerable variation in the size and shape of the horncores in these taxa, but concluded that it could not be attributed to sexual dimorphism. He did, however, note an association between anteriorly curving nasal horncores, greater horncore widths, and larger skull size in some adult members of C. apertus, and suggested that sexual dimorphism cannot be ruled out.

Given the sexual dimorphism of cranial ornamentation in extant bovid and cervid mammals, and dimorphism in extinct archosaurian taxa, with males either confirmed or inferred as having more pronounced ornamentation, it is reasonable to suggest that postorbital horncore length was also sexually dimorphic in some ceratopsian taxa. Based on this reasoning, it is possible that the proposed short and long postorbital horncore trajectories in the examined Dinosaur Park Formation chasmosaurine specimens correspond to females and males, respectively.

\subsection{Results}

The analysis produced a strict consensus tree of 32 most parsimonious trees of 40 steps $($ consistency index $=0.65$; retention index $=0.81)$ composed of six clades of successively more ontogenetically-advanced Chasmosaurus skulls (Fig. 63). The first occurrence of a given character state in the tree, as well as character state reversals in specimens, are shown in Figure 63. The artificial embryo is basal to CMN 1254 (Node $\# 1)$ and all other specimens (Nodes \#2-5). CMN 1254 is a relatively fragmentary skull, but possesses several immature characters such as the total lack of episquamosals, a disarticulated epinasal, and a relatively broad squamosal that is short in length $(500 \mathrm{~mm})$. 
Figure 63. Ontogenetic transformation series for Chasmosaurus specimens. Strict consensus of 32 most parsimonious trees $(\mathrm{TL}=40$ steps, $\mathrm{CI}=0.65, \mathrm{RI}=0.81)$ using 18 cranial characters. Bootstrap replicate frequency is shown above each node (only Bootstrap values of $50 \%$ or higher are given); Bremer values are shown above each node, in italics. Ontogenetic characters and associated states (shown in brackets) are shown on left and are mapped at their earliest occurence on the tree. These character state changes represent an inferred sequence of development; however, due to variability in the timing of a given character change amongst specimens, this sequence is an approximation. Below each specimen is the previous taxonomic designation, for reference; Chasmosaurus russelli, C. belli, Chasmosaurus sp., and Vagaceratops-like specimens; character state reversals are listed below each specimen designation. In box to the right of each specimen, rostral-to-epijugal length (top, bold, in mm; parameter 16, Fig. 5) and squamosal length (bottom, in mm; parameter 19, Fig. 5) are given for skull size reference, where preserved. Although the isolated squamosal TMP 1998.128.0001 was not included in the analysis, its basal position on the tree is indicated by a dashed line, based on the specimen's small size and possession of immature character states (4(0), 16(0) and 17(0)). Boxes at bottom delimit age classes - "baby", "juvenile", "subadult", and "adult" (see text for definitions); numbers below each box represent rostral-to-epijugal lengths (in $\mathrm{mm}$ ) for each age class, which generally increases moving up tree (est. = estimated). 
Figure 63.

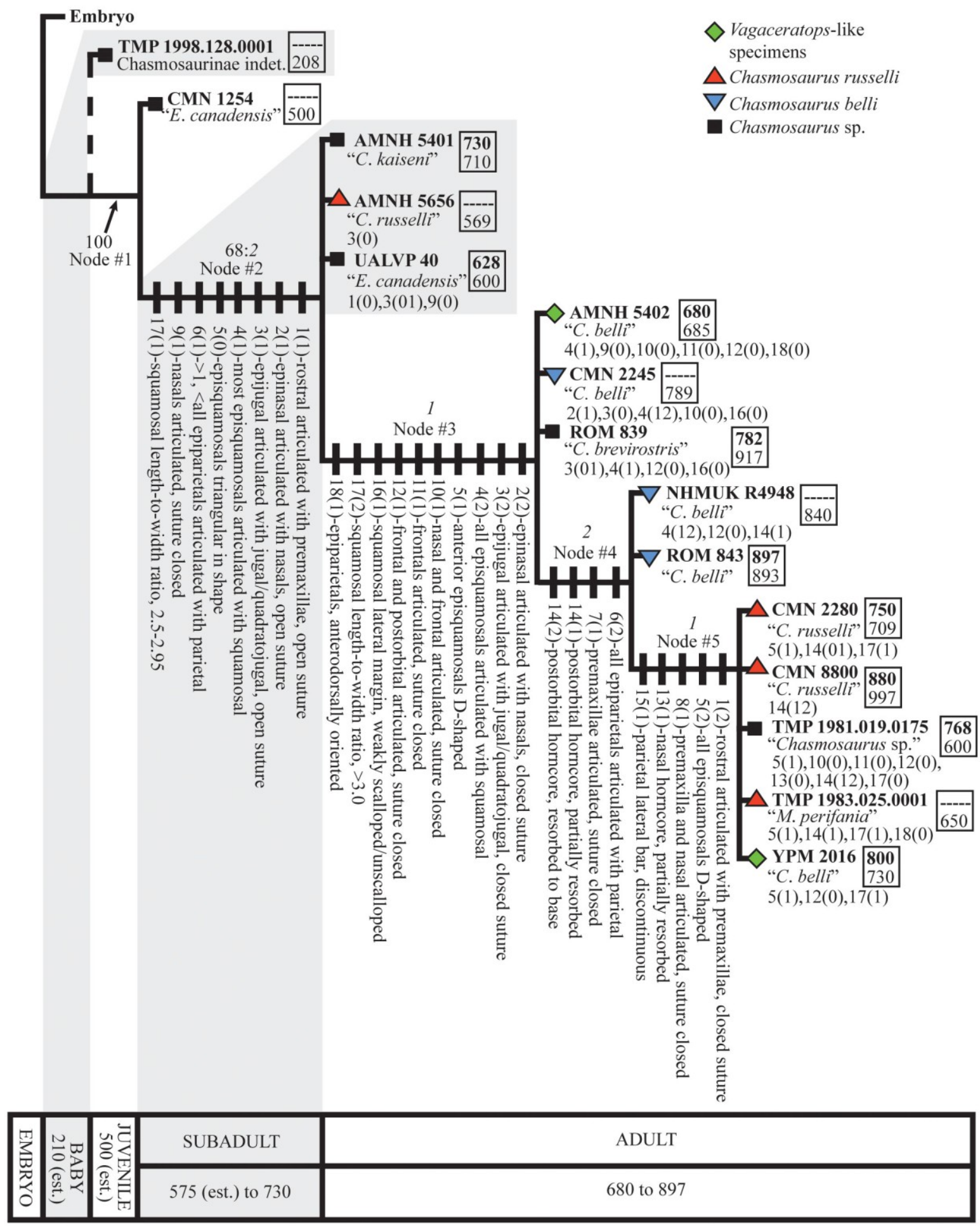


The isolated squamosal TMP 1998.128.0001 was not included in the analysis, but it most likely would have been situated between the artificial embryo and CMN 1254 (shown by dashed line in Fig. 63). TMP 1998.128.0001 exhibits numerous features suggestive of immaturity (lack of episquamosals, strongly scalloped margin, and relatively small squamosal length/width ratio $=2.0)$, and the squamosal is less than half the length $(208$ $\mathrm{mm})$ of CMN 1254 (500 mm).

At Node \#2, AMNH 5401 forms an unresolved relationship with AMNH 5656, UALVP 40 and a clade containing all remaining, more derived specimens. Both epijugals are disarticulated (character 3(0) = reversal) in AMNH 5656, while one is articulated in UALVP 40 (character $3(01)=$ reversal on right side). The posteriormost episquamosal is disarticulated in AMNH 5656; the distal half of the squamosals are not preserved in UALVP 40, making an original episquamosal count impossible. AMNH 5656 has a slightly shorter squamosal (length $=569 \mathrm{~mm})$ than UALVP $40(600 \mathrm{~mm}$, estimated $)$. Given these factors, it is possible that UALVP 40 is more mature than AMNH 5656. The rostral is articulated with the premaxillae with open sutures in AMNH 5401 (character 1(1)); the rostral is disarticulated in UALVP $40(1(0))$. The nasals are articulated with closed sutures (character 9(1) in AMNH 5401; these sutures are still open in UALVP 40 (9(0)). AMNH 5401 has a longer rostral-to-epijugal $(730 \mathrm{~mm})$ and squamosal length $(710$ $\mathrm{mm}$ ) than UALVP 40 (628 and $600 \mathrm{~mm}$, respectively). Given these factors, it is likely that AMNH 5401 is more mature than UALVP 40 and AMNH 5656.

At Node \#3, AMNH 5402 forms an unresolved relationship with CMN 2245, ROM 839, and a clade containing all remaining, more derived specimens. At Node \#4, NHMUK R4948 forms an unresolved relationship with ROM 843 and a clade containing 
all remaining, more derived specimens. At Node \#5, CMN 2280, CMN 8800, TMP 1981.019.0175, TMP 1983.025.0001, and YPM 2016 form a polytomy and cannot be distinguished from each other based on the characters used in this analysis.

The non-parametric multivariate analysis of variance of postorbital horncore ontogenetic trajectories showed significant differences $(p<0.05)$ between clusters of specimens assigned to each trajectory in Figures 60 (rostral-to-epijugal length versus postorbital horncore basal circumference), 61 (squamosal length vs. postorbital horncore length) and 62 (squamosal length vs. postorbital horncore basal circumference), but no significant differences in Figure 59 (rostral-to-epijugal length versus postorbital horncore length) (Table 8).

The posterior end of the medial parietal bar generally thickens during ontogeny and a shallow, longitudinal sulcus develops along the parietal fenestra margin (Fig. 6; Table 9). The sulcus is deeper in the partial parietal CMN 8803 (Fig. 14C); the ontogenetic status of this specimen cannot be determined, due to insufficient preservation. 
Table 8: Results of NPMANOVA of postorbital horncore (small and large) ontogenetic trajectories (Figs. 59-62). Posthoc pairwise comparisons shown in centre (uncorrected $p$ values). $F$ and $p$-values for total analysis shown on right. Significant results $(p<0.05)$ shown in bold.

\begin{tabular}{|c|c|c|c|c|c|c|}
\hline \multirow[t]{2}{*}{$\begin{array}{l}\text { Proxies for skull and } \\
\text { postorbital horncore } \\
\text { sizes, and associated } \\
\text { figure }\end{array}$} & \multicolumn{3}{|c|}{$\begin{array}{l}\text { Ontogenetic trajectory, } \\
\text { postorbital horncore } \\
\text { size (number of } \\
\text { specimens) }\end{array}$} & \multirow[t]{2}{*}{$\begin{array}{c}\text { Posthoc } \\
\text { pairwise } \\
\text { comparisons }\end{array}$} & \multicolumn{2}{|c|}{ Total analysis } \\
\hline & Small & Large & Total & & $F$ & $p$ \\
\hline $\begin{array}{l}\text { Rostral-to-epijugal } \\
\text { length (parameter } \\
\text { 16) and postorbital } \\
\text { horncore length } \\
\text { (parameter 1) (Fig. } \\
\text { 59) }\end{array}$ & 3 & 2 & 5 & 0.1986 & 2.533 & 0.1929 \\
\hline $\begin{array}{l}\text { Rostral-to-epijugal } \\
\text { length (parameter } \\
\text { 16) vs. postorbital } \\
\text { horncore basal } \\
\text { circumference } \\
\text { (parameter 4) (Fig. } \\
60 \text { ) }\end{array}$ & 5 & 4 & 9 & 0.0165 & 4.431 & 0.0136 \\
\hline $\begin{array}{l}\text { Squamosal length } \\
\text { (parameter 19) vs. } \\
\text { postorbital horncore } \\
\text { length (parameter 1) } \\
\text { (Fig. 61) }\end{array}$ & 4 & 3 & 7 & 0.0264 & 3.624 & 0.0291 \\
\hline $\begin{array}{l}\text { Squamosal length } \\
\text { (parameter 19) vs. } \\
\text { postorbital horncore } \\
\text { basal circumference } \\
\text { (parameter 4) (Fig. } \\
62 \text { ) }\end{array}$ & 6 & 6 & 12 & 0.0029 & 6.829 & $\overline{0.0031}$ \\
\hline
\end{tabular}


Table 9: Thickness of posterior end of medial parietal bar (parameter 31), and presence/absence of a shallow, longitudinal sulcus on the parietal fenestra margin (Fig. 6). Specimens arranged in order of increasing maturity, as given in Figure 63.

\begin{tabular}{|l|l|l|}
\hline Specimen & \multicolumn{1}{|c|}{$\begin{array}{c}\text { Medial parietal bar } \\
\text { thickness (mm; parameter } \\
\text { 31) }\end{array}$} & \multicolumn{1}{|c|}{$\begin{array}{c}\text { Shallow, longitudinal } \\
\text { sulcus }\end{array}$} \\
\hline AMNH 5656 & 27 & absent \\
\hline AMNH 5402 & ---- & absent \\
\hline CMN 2245 & 32 & absent \\
\hline ROM 839 & 26 & absent \\
\hline NHMUK R4948 & ---- & absent \\
\hline ROM 843 & 30 & absent \\
\hline CMN 2280 & 34 & present \\
\hline CMN 8800 & 36 & present \\
\hline TMP 1983.025.0001 & 45 & present \\
\hline YPM 2016 & 34 & present \\
\hline
\end{tabular}




\subsection{Discussion}

In general, the 14 skulls representing the ontogenetic transformation in Chasmosaurus increase in size through maturity (Fig. 63), with rostral-to-epijugal length (parameter 16, Fig. 4) ranging from $628 \mathrm{~mm}$ (UALVP 40) to $897 \mathrm{~mm}$ (ROM 843). If the isolated squamosal TMP 1998.128.0001 (squamosal length $=208 \mathrm{~mm}$; parameter 16, Fig. 5) is also considered, this would extend that range down to approximately $210 \mathrm{~mm}$, as squamosal length and rostral-to-epijugal length scale nearly one-to-one (Fig. 64). The scarcity of relatively small and immature Chasmosaurus specimens such as TMP 1998.128.0001, CMN 1254, AMNH 5656, and UALVP 40 is similar to that for Triceratops (Horner and Goodwin, 2006), Agujaceratops (Lehman, 2007), Centrosaurus apertus (Ryan et al., 2001), and Pachyrhinosaurus lakustai (Currie et al., 2008), despite the relatively large datasets of these four latter taxa. The scarcity of such small-bodied and relatively delicate individuals is likely a result of taphonomic size bias (Brown et al., 2013).

CMN 1254 and UALVP 40, both historically referred to Eoceratops canadensis, are among the ontogenetically least derived (CMN 1254, Node \#1; UALVP 40, Node \#2) specimens examined. The larger skull AMNH 5401 (historically Chasmosaurus kaiseni) retains a number of immature traits (Table 6) (Node \#2). ROM 839 (historically Chasmosaurus brevirostris) retains several immature traits, such as incomplete episquamosal articulation (character 4(1)), a disarticulated epijugal (character 3(0)) on the left side of the skull, and some disarticulated cranial elements, despite its relatively large skull size (Node \#3). TMP 1983.025.0001 (Mojoceratops perifania) is recovered in the polytomy of most ontogenetically advanced specimens (Node \#5), but the medialmost 


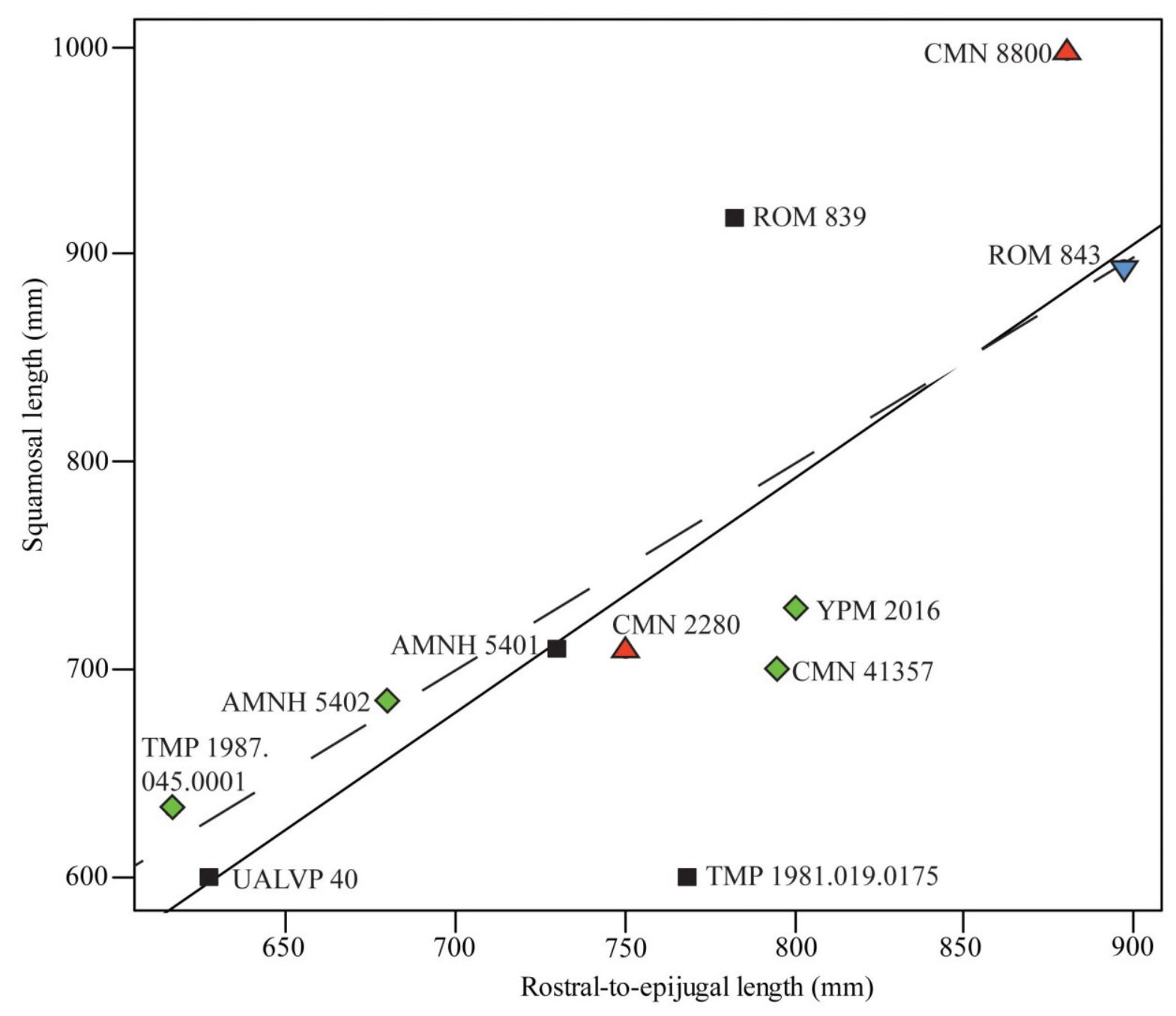

Figure 64. Squamosal length (parameter 19) vs. rostral-to-epijugal length (parameter 16) of Dinosaur Park Formation chasmosaurine skulls. Line-of-best-fit (solid line): $p=$ 0.00606; scaling relationship $=1.13$. Dashed line represents one-to-one scaling relationship, for reference. Chasmosaurus russelli (red triangles), C. belli (blue inverted triangle), Chasmosaurus sp. (black squares), and Vagaceratops-like specimens (green diamonds). 
epiparietals (P1 and 2) remain oriented in the plane of the frill (character 18(0)) - a trait typical of more immature specimens such as AMNH 5656 and AMNH 5402.

Although skull size (rostral-to-epijugal length, parameter 16, Fig. 5) generally increases in more advanced ontogenetic stages (Fig. 63), it is not a perfect relationship. For example, although CMN 2280 and CMN 8800 are sister ontogimorphs, there is a discrepancy in their rostral-to-epijugal lengths $(750 \mathrm{~mm}$ and $880 \mathrm{~mm}$, respectively). Considering the close taxonomic relationship of these two skulls (Fig. 37), and their previous referral to the same species (Chasmosaurus russelli, Godfrey and Holmes, 1995; Maidment and Barrett, 2011), it is possible that their difference in size is due to individual variation.

Ontogenetic recognition of Chasmosaurus belli and Chasmosaurus russelli:

Specimens referred to $C$. belli and $C$. russelli are distributed randomly across the tree (Fig. 63) suggesting that features previously used to distinguish the two species (i.e., embayment of the posterior parietal bar and the relative size of the epiparietals) are not ontogenetically integrated. Thus, the null hypothesis of no ontogenetic separation between these two taxa cannot be rejected, indicating that $C$. belli and C. russelli have considerable ontogenetic overlap. This lack of ontogenetic separation between C. belli and $C$. russelli could be used to support the validity of these two taxa. 
Ontogenetic development of postorbital horncores:

The ontogenetic series given in Figure 63 is generally correlated with skull size, and therefore generally supports the inferred ontogenetic sequence of specimens shown in Figures 59-62. The large discrepancy in postorbital horncore size between mature specimens indicates that the null hypothesis that postorbital horncore size and orientation change consistently over ontogeny (i.e., specimens with small horncores are less mature than specimens with large horncores) must be rejected. This supports the proposed presence of two ontogenetic trajectories culminating in relatively short, small circumference-based horncores that do not exceed $132 \mathrm{~mm}$ in length (CMN 2280), and relatively long, large circumference-based horncores that do not exceed $370 \mathrm{~mm}$ in length (AMNH 5401), respectively.

The significant differences between clusters of specimens assigned to each postorbital horncore ontogenetic trajectory (small and large horncores) in Figures 60-62 (Table 8) indicates that the null hypothesis of one ontogenetic trajectory with respect to postorbital horncore size must be rejected. The insignificant differences in clusters of specimens in Figure 59 is likely due to the lack of long-horned specimens with postorbital horncore lengths and rostral-to-epijugal lengths intermediate to those of AMNH 5401 and UALVP 40. Specimens corresponding to the two inferred ontogenetic trajectories are shown in Figure 65, categorized according to the age classes given in Figure 63. 
Figure 65. Postorbital horncores of Dinosaur Park Formation chasmosaurine skulls arranged by ontogenetic stage; age classes and node numbers on left correspond to those given in the ontogenetic tree (Fig. 63). Proposed "long horncore" (left, white area) and "short horncore" (right, shaded area) ontogenetic trajectories as shown in Figures 59-62. Arrows denote postorbital horncores that have undergone resorption. Plaster reconstruction shown in grey half-tone. Image of ROM 843 courtesy of Jordan Mallon; image of NHMUK R4948 modified from Maidment and Barrett (2011: Fig. 4); images of CMN 1254, NHMUK R4948, ROM 839, and TMP 1981.019.0175 flipped. All specimens to scale. 
Figure 65.

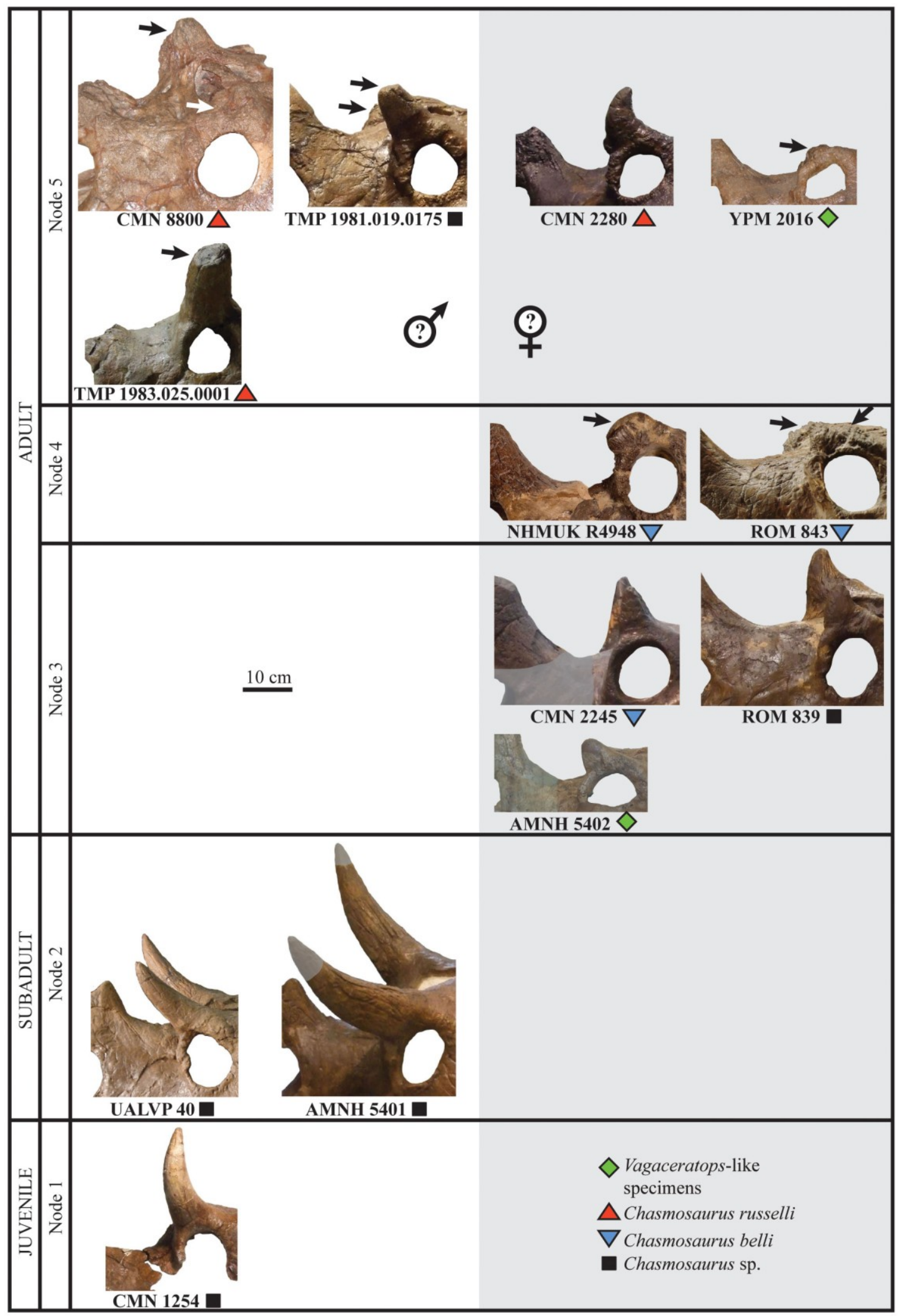


The "short" postorbital horncore ontogenetic trajectory encompasses all Chasmosaurus-like specimens previously referred to C. belli (CMN 2245 and ROM 843), all Vagaceratops-like specimens previously referred to C. belli (AMNH 5402 and YPM 2016), one Chasmosaurus-like specimen previously referred to C. russelli (CMN 2280); and one Chasmosaurus sp. specimen (ROM 839). The "long" postorbital horncore trajectory encompasses the remaining Chasmosaurus-like specimens previously referred to C. russelli (CMN 8800 and TMP 1983.025.0001) and remaining Chasmosaurus sp. specimens (AMNH 5401, CMN 1254, TMP 1981.019.0175, and UALVP 40). The variable distribution of $C$. russelli across both of these proposed trajectories suggests that these trajectories cannot be explained by taxonomic differences. Given the close phylogenetic relationship of all of these skulls (Fig. 37), it is possible that these two postorbital horncore ontogenetic trajectories instead correspond to sexual dimorphism.

The bimodal distribution of cranial display structure (i.e., postorbital horncore) size in Chasmosaurus may be analogous to that of extant bovids, which is due to sexual dimorphism (Estes, 1991). In extant birds and crocodiles, cranial sexual dimorphism, where present, is generally less pronounced and usually restricted to differing skull dimensions (e.g., gracile versus robust skulls in crocodiles, Hall and Portier, 1994; differing beak length and shape in birds, Selander, 1966). Dimorphic cranial display structures have also been noted in numerous extinct archosaurian taxa, such as cranial crest size in pterosaurs (Pteranodon, Bennett, 1992; Darwinopterus, Lü et al., 2011), narial crest size in phytosaurs (Pseudopalatus, Zeigler et al., 2002), and postorbital horncore size (Agujaceratops, Lehman, 1990) and skull proportions (Protoceratops, Dodson, 1976) in ceratopsians. The referral of these dimorphic structures to sexual 
differences is compelling, as both morphs of a given taxon occur in the same fossil assemblage, as do the two postorbital horncore morphs (small and large) in

\section{Chasmosaurus.}

Paedomorphism or paedogenesis is the retention of juvenile traits in sexually mature adults (Geist, 1966). In bighorn sheep, females are considered to be paedomorphic with respect to horncore length as adults have horncores that are comparable in size to those of juvenile males (rams), but are short relative to those of adult rams (Geist, 1966). In such species where horncore length is sexually dimorphic, the presence of relatively long horncores in males has been attributed to female sexual selection (Jarman, 1983). Horncores are weapons and, like other secondary sexual features, are metabolically burdensome. The possession of well-developed horncores in certain adult males is a signal to females that these individuals are more capable of survival and can suppress other adult males possessing lesser-developed horncores (Jarman, 1983). The development of large horncores in adult males also serves to suppress the reproductive efforts of younger males (Jarman, 1983).

The evolution of relatively long postorbital horncores in some Chasmosaurus specimens, herein referred to as males, may have likewise been driven by female sexual selection. Furthermore, the fact that postorbital horncores in adult putative "females" (e.g., CMN 2280) are more similar in size and orientation to those of juvenile "males" (e.g., CMN 1254) than those of larger subadult and adult "males" (e.g., UALVP 40 and AMNH 5401, and TMP 1981.019.0175, respectively), may suggest that "females" were paedomorphic with respect to horncore length (Fig. 65). Vertically erect horncores occur in juvenile "males" and all "female" specimens (adults), while anterolaterally inclined 
horncores are restricted to subadult and adult "males" (Fig. 65). Longer, anterolaterally inclined horncores would seem to be more conducive to combative or defensive behaviour than would shorter, vertically erect horncores with a slightly posterior curvature, as the former project forwards and towards oncoming opponents. The restriction of these putatively more combative horncores to older (subadult and adult) males may suggest a relatively late onset of male-to-male combative behaviour, and limit potentially lethal interactions between sexually immature and mature males.

The reorientation of the postorbital horncore over putative male ontogeny (Fig. 65) is similar to that proposed for Triceratops (Horner and Goodwin, 2006), in that both transition from shorter, vertically erect horncores with a slight posterior curvature, to longer, more anterolaterally inclined horncores that retain the slight posterior curvature. In Triceratops, however, the horncores undergo further remodeling and develop an anterior curvature. It cannot be determined whether this later transformation also occurred in older adult "males" of Chasmosaurus, as the upper halves of the horncores are lost to resorption.

Shape of the parietal along the posteromedial margin of the parietal fenestra over ontogeny:

The posterior end of the medial parietal bar generally thickens during ontogeny and a shallow, longitudinal sulcus develops along the parietal fenestra margin (Fig. 6; Table 9). The null hypothesis of no ontogenetic change in this feature must therefore be 
rejected. This indicates that the sulcus is an invalid diagnostic character of Mojoceratops perifania (contra Longrich (2010).

Other ontogenetic trends:

Rostral and epiossification articulation pattern

The following epiossification patterns occur consistently over Chasmosaurus ontogeny (Fig. 63):

1. Rostral-premaxillae articulation (suture open, character 1(1); e.g., AMNH 5401) occurs after some episquamosals have articulated (e.g., UALVP 40, total episquamosal count cannot be determined), but before they have all articulated (character 4(2); e.g., CMN 2245; right side of skull) with the squamosal, and before all epiparietals have articulated (character 6(2); e.g., ROM 843) with the parietal.

2. Epinasal articulation (open suture, character 2(1)) occurs before epijugal (open suture, character 3(1)), epiparietals (character 6(1)), episquamosals (character 4(1)), and rostral (open suture, character 1(1)) have articulated.

3. The timing of epijugal articulation is quite variable amongst specimens; e.g., UALVP 40 has one (character 3(01), AMNH 5401 has both (3(1)), and CMN 2245 has none (3(0)) articulated. 
4. The anterior episquamosals (up to half of all episquamosals) undergo modification and become D-shaped (character 5(1)) before all episquamosals have articulated with the squamosal (character 4(2)) (e.g., AMNH 5402).

5. Epiparietals articulate concurrently with episquamosals (e.g., AMNH 5656), although total episquamosal articulation (character 4(2)) occurs before total epiparietal articulation (character 6(2)); in CMN 2245, all episquamosals are articulated with the right squamosal, but only one epiparietal is articulated with each side of the parietal.

Triceratops is the only known ceratopsid taxon whose specimens document a complete ontogenetic spectrum, and thus represents the best available model for categorizing specimens of Chasmosaurus on an ontogenetic basis. Horner and Goodwin (2008) noted in their study on Triceratops ontogeny that the timing of epiossification articulation followed a specific sequence; however, the timing of articulation was found to be variable between individuals. They concluded that the epinasal likely articulates first, followed by the rostral, the epijugals, the episquamosals, and finally the epiparietals. In centrosaurines, the posterior to anterior direction of articulation of frill epiossifications (articulation of epiparietals before episquamosals) is predictable (Sampson et al., 1997), but the timing of articulation of the epijugals and rostral is more variable (Sampson et al., 1997; Currie et al., 2008). Because of insufficient preservation amongst Chasmosaurus specimens, as well as the lack of smaller skulls that would express key ontogenetic changes, the ossification sequence of this genus cannot be directly compared with that of Triceratops. 
It is also worth noting that the Vagaceratops-like skull YPM 2016 is recovered as ontogenetically derived, and cannot be ontogenetically distinguished from the Chasmosaurus-like skulls CMN 2280, CMN 8800, TMP 1981.019.0175, and TMP 1983.025.00001 (Node \#5, Fig. 63). Given that YPM 2016 is a sister ontogimorph to these four latter specimens, this suggests that a three-epiparietal-bearing individual does not 'mature' into a five-bearing one, especially considering that there is no room to add two more epiparietals onto the posterior parietal bar (e.g., see AMNH 5656 and CMN 2280). The recovery of AMNH 5402 as immature relative to YPM 2016 supports the recovery of this former specimen outside of the Vagaceratops-like clade (YPM 2016+(CMN 41357+TMP 1987.045.0001)) in Figure 37. Had the individual AMNH 5402 lived longer, it would most likely have attained the five epiossification count (four epiparietals and one epiparietosquamosal) on each side, as inferred in Chapter 5 (Fig. 8).

Cranial suture closure:

The following sequence of cranial element sutural closure (in dorsal view) occurs consistently in Chasmosaurus ontogeny (Fig. 63):

1. Nasals (character 9(1)), premaxillae (character 7(1)) and premaxilla-nasal (character $8(1))$.

2. Nasal-frontal (character 10(1)), frontals (character 11(1)) and frontal-postorbital (character 12(1)), however, are variable in their timing of sutural closure.

Skull size continues to increase following the sutural closure of elements of the skull roof and snout. This may be due to the continued growth of other cranial elements 
such as the squamosal and parietal, whose sutures do not appear to close over ontogeny in Chasmosaurus and other ceratopsids (e.g., Triceratops, Horner and Goodwin, 2006; Pachyrhinosaurus lakustai, Currie et al., 2008).

Bone remodeling

All three cases of bone pitting, thinning and remodelling - pitted nasal horncores, pitted postorbital horncores and discontinuous lateral parietal bars - occur in larger and putatively more mature specimens (Node \#5, \#4, and \#5, respectively). The pitting of the postorbital and nasal horncores represents the first occurence of actual bone loss in the proposed ontogenetic sequence. If bone loss is extended to include accessory squamosal fenestrae, then bone loss first occurs in ROM 839 (Node \#3). Accessory squamosal fenestrae are also known in NHMUK R4948 and ROM 843 at Node \#4, and TMP 1983.025.0001 at Node \#5.

Putative bone resorption (i.e., nasal and postorbital horncore pitting, accessory squamosal and parietal fenestrae, lateral parietal bar thinning, and pitting of epijugals, episquamosals and epiparietals) is widespread across Chasmosaurus-like and Vagaceratops-like (AMNH 5402 and YPM 2016) specimens, becoming increasingly common moving up the tree in Figure 63. Sampson et al. (1997) suggested that postorbital horncore resorption may be explained by a redirection of calcium towards egg production in females. However, they noted that if this were the case, this would result in a pattern of sexual dimorphism in the presence or absence of postorbital horncores. Tanke and Farke (2007) suggested that the same reproductive stresses may also account for 
accessory squamosal fenestrae, but reasoned that it would be more likely for bone density to decrease across the entire skeleton instead of only in a few cranial elements. The widespread occurrence of pitted horncores and, to a lesser extent, accessory squamosal and parietal fenestrae, in adult chasmosaurine specimens from the Dinosaur Park Formation suggests that such bone resorption occurred in both males and females. Sampson et al. (1997) also suggested that resorption of postorbital horncores was not gender-specific, and that these structures instead atrophied in both genders due to a lack of use in old age. Calcium reclamation may also be caused by environmental stress or weight reduction, although Tanke and Farke (2007) reasoned that the latter would have a negligible effect on the weight of the ceratopsid skull.

Histological analysis of ceratopsid cranial ornamentation has revealed that horncores are characterized by fibrolamellar bone - indicative of rapid growth - in early ontogeny, and that they remodel into structures largely composed of trabecular bone later in ontogeny (Kruk et al., 2013). Further histological studies of Dinosaur Park Formation chasmosaurine horncores are recommended, and could test whether bone resorption (external pitting) occurs during horncore growth (i.e., in horncores dominated by fibrolamellar bone) or once horncore growth has diminished or ceased (i.e., in horncores dominated by trabecular bone).

Parietal - lateral and posterior bars:

As the parietosquamosal frill lengthens in life, it is possible that the lateral bar either atrophies or bone deposition ceases as the squamosal, or unpreserved soft tissues, 
take on more of a structural role in supporting the frill. Discontinuous lateral bars are found in all skulls with a rostral-to-epijugal length of $750 \mathrm{~mm}$ or greater, except for ROM $839(782 \mathrm{~mm})$ and ROM $843(897 \mathrm{~mm})$.

In their biomechanical study of Triceratops, Farke et al. (2010) found that the mediolaterally and anteroposteriorly arched frill was effective at confining applied stresses to the squamosals. The squamosals continue to serve an important structural role later in Triceratops ontogeny as the parietal thins, and, in the Scannella and Horner's (2010) Triceratops-Torosaurus synonymy hypothesis, eventually develop parietal fenestrae. Ceratopsids other than Triceratops, Albertaceratops and Diabloceratops possess a relatively flatter and less arched frill, and possess parietal fenestrae of variable extent. Farke et al. (2010) suggested that the thickened medial margin of the squamosal in some of these latter taxa (e.g., Chasmosaurus) may serve to strengthen the frill and prevent applied stresses from propagating into the more delicate, fenestrated parietal.

The first occurrence of an anterodorsally-recurved posterior margin of the posterior parietal bar (character 18(1)) appears in CMN 2245 (Node \#3, Fig. 63). In this skull, the absence of medial epiparietals on the anterodorsally-recurved ridge indicates that the parietal is indeed responsible for the anterodorsal curvature, and not the epiparietals themselves; this has been previously demonstrated in centrosaurine ontogeny (Ryan et al., 2001). Where preserved, this anterodorsal curvature is present in all more mature specimens, with the exception of AMNH 5402 and TMP 1983.025.0001. The presence of the putatively immature feature - posterior parietal margin oriented in plane of frill (18(0)) - in the relatively mature AMNH 5402 and TMP 1983.025.0001 may suggest that the timing of the anterodorsal recurvature of the posterior parietal margin is 
variable amongst specimens.

Chasmosaurus growth stages:

In their study on Triceratops ontogeny, Horner and Goodwin (2006) assigned the smallest skull of that taxon (UCMP 154452; rostral-to-occipital condyle length $=280$ mm, estimated) to their "baby" category. The term "baby" has a variable meaning in the dinosaur literature, having been defined as the embryonic or hatchling stage (Sankey, 2010:528) or as the post-neonate (post-birth) stage (Goodwin and Horner, 2010:551) of development. The Triceratops skull UCMP 154452 is undoubtedly too large to be a hatchling, because the specimen's size would have greatly exceeded the size of the egg it hatched from, as inferred from the known hatchling/adult size ratio in the basal ceratopsians Psittacosaurus and Protoceratops (Coombs, 1982; Barsbold and Perle, 1983; Carpenter, 1999:206). The boundary between the "baby" and older "juvenile" stages is also not well defined in the dinosaur literature; Horner and Goodwin (2006) used the categories "baby" and "juvenile" for convenience. If the "baby" stage starts at birth, then UCMP 154452 most likely represents a relatively large and mature representative of that stage. Alternatively, it could be argued that this specimen instead represents a relatively small and immature representative of the "juvenile" stage. With the lack of direct evidence to the contrary (i.e., "juvenile" stage), I agree with Horner and Goodwin's (2006) assignment of UCMP 154452 to the "baby" stage. Based on their proposed ontogenetic growth stages for Triceratops I recognize the following ontogenetic stage classifications for Chasmosaurus: 
Baby: This is the ontogenetically least developed and smallest size category.

Characteristics include: rostral, epinasal, epijugals, episquamosals, and epiparietals disarticulated from skull; premaxillae, premaxilla-nasal, nasals, nasal-frontal, frontals, and frontal-postorbital sutures open in dorsal view; postorbital horncore unmodified by resorption; lateral parietal bar continuous; squamosal length/width ratio less than 2.4; lateral margin of squamosal strongly scalloped; and posterior margin of the parietal oriented in the plane of the frill. The rostral-to-epijugal length ranges up to approximately $250 \mathrm{~mm}$.

This category is represented by the isolated squamosal TMP 1998.128.0001. It measures $208 \mathrm{~mm}$ in length and represents the smallest individual examined in this study. The rostral-to-epijugal length is estimated to be approximately $210 \mathrm{~mm}$, as this parameter scales nearly one-to-one with squamosal length (Fig. 64).

Juvenile: Juveniles have the same ontogenetic character states as the baby category, but skull size is larger, with a rostral-to-epijugal length between approximately $250 \mathrm{~mm}$ and $550 \mathrm{~mm}$. This category is represented by the partial skull CMN 1254, which has a squamosal length of $500 \mathrm{~mm}$. Using the same rationale as for TMP 1998.128.0001, the rostral-to-epijugal length is estimated to be approximately $500 \mathrm{~mm}$ for CMN 1254 .

Subadult: These specimens are characterized by a rostral-to-epijugal length between approximately $550 \mathrm{~mm}$ and $750 \mathrm{~mm}$, and otherwise can show a mosaic of juvenile and adult characteristics, e.g., rostral either disarticulated or articulated (open suture) with premaxillae; epinasal articulated with open suture; epijugals range from neither to both 
articulated with open suture; some episquamosals and epiparietals articulated; episquamosals triangular in dorsal view; premaxillae, premaxilla-nasal, nasal-frontal, frontals, and frontal-postorbital sutures open in dorsal view; suture between nasals either open or closed in dorsal view; nasal and postorbital horncores unmodified by resorption; continuous lateral parietal bars; lateral margin of squamosal strongly scalloped; squamosal length/width ratio 2.5 to 2.95 ; and posterior margin of parietal (and epiparietals) oriented in the plane of the frill. This category is represented by AMNH 5656, UALVP 40 and AMNH 5401.

Adult: Adult specimens are considered to be the largest and most ontogenetically derived. Adult ontogimorphs would be expected to show all of the derived advanced characters including complete articulation of all epiossifications and obliteration of all sutures; however, some of the specimens examined here still include some subadult characters. Observed characters include: rostral and epinasal each articulated, with suture open or closed; epijugals ranging from neither to both articulated with suture closed; either some or all episquamosals and epiparietals articulated; episquamosals ranging from D-shaped on anterior half to entire length of squamosal; premaxillae, premaxilla-nasal, nasalfrontal, frontals, and frontal-postorbital sutures either open or closed in dorsal view; suture between nasals closed in dorsal view; nasal and postorbital horncores range from being unmodified to resorbed; lateral parietal bar ranging from continuous to discontinuous; lateral margin of squamosal ranging from strongly scalloped to weakly scalloped/unscalloped; squamosal length/width ratio 2.38 (e.g., TMP 1981.019.0175) or higher; and posterior margin of parietal (and epiparietals) oriented either in the plane of 
the frill or anterodorsally. This category is represented by AMNH 5402, CMN 2245, ROM 839, NHMUK R4948, ROM 843, CMN 2280, CMN 8800, TMP 1981.019.0175, TMP 1983.025.0001, and YPM 2016.

\subsection{Conclusion}

Based on the analysis presented here, specimens referred to Chasmosaurus belli and Chasmosaurus russelli are not recognized as belonging to discrete, non-overlapping ontogenetic stages of Chasmosaurus, and thus cannot be referred to separate ontogimorphs of one taxon. This ontogenetic analysis does not support the hypothesis that only one species of Chasmosaurus is present in the Dinosaur Park Formation; however, individual variation or sexual dimorphism cannot be ruled out as possible factors accounting for differences between specimens representing the two putative species C. belli and C. russelli.

The two Vagaceratops-like skulls, AMNH 5402 and YPM 2016, interpreted as having eight and 10 epiparietals, respectively, plot out as relatively immature and mature adults, respectively. The immature status of AMNH 5402 relative to YPM 2016 may account for the recovery of AMNH 5402 outside of the Vagaceratops-like clade in Figure 37 , as this specimen had not attained its inferred complete epiparietal count at the time of death.

Postorbital horncores increase in size and change their orientation over ontogeny, but follows one of two ontogenetic trajectories, culminating in relatively long, large circumference-based horncores that do not exceed $370 \mathrm{~mm}$ in length (AMNH 5401) or relatively short, small circumference-based horncores that do not exceed $132 \mathrm{~mm}$ in 
length (CMN 2280) (Figs. 59-62 and 65). It is possible that postorbital horncore length is sexually dimorphic, and that these "long" and "short" horncore trajectories correspond to males and females, respectively, as occurs in extant bovids and cervids. Dimorphic cranial display structures in extinct archosaurian taxa have likewise been attributed to sexual differences (e.g., Dodson, 1976; Lehman, 1990; Bennett, 1992; Zeigler et al., 2002; Lü et al., 2011). The sexual dimorphism hypothesis for Chasmosaurus will be tested statistically in Chapter 9.

Chasmosaurus specimens in this study are predominately large, adult-sized skulls, with few specimens from the smaller (and putatively younger) size stages. This reflects the taphonomic bias against the preservation of small specimens in the fossil record of the Dinosaur Park Formation as quantified by Brown et al. (2013). Based on the analysis in this study, the following specimens of previously referred taxa are here considered to be Chasmosaurus sp., and can be assigned to the following Chasmosaurus growth stages: Eoceratops canadensis - juvenile (CMN 1254) and subadult (UALVP 40); Chasmosaurus kaiseni (AMNH 5401) - subadult; Chasmosaurus brevirostris (ROM 839) - adult; and Mojoceratops perifania - subadult (AMNH 5656) and adult (TMP 1983.025.0001). 


\section{Chapter 9. Morphometric Analysis of Chasmosaurus and Vagaceratops Specimens}

\subsection{Introduction}

This chapter examines whether previously diagnosed chasmosaurine taxa from the Dinosaur Park Formation can be distinguished on a morphometric basis. Previous statistical morphometric studies on other Late Cretaceous ornithischian taxa have been conducted, resulting in: 1, the synonymy of morphometrically indistinguishable taxa, e.g., referring the chasmosaurine Anchiceratops longirostris to Anchiceratops ornatus (Mallon et al., 2011), and referring the hadrosaurs Anatotitan copei and Edmontosaurus saskatchewanensis to Edmontosaurus annectens and Thespesius edmontoni to Edmontosaurus regalis (Campione and Evans, 2011); and 2, the validation of morphometrically distinct taxa, e.g., Edmontosaurus annectens and Edmontosaurus regalis (Campione and Evans, 2011). The conclusions of Mallon et al. (2011) and Campione et al. (2011) were made, in part, using principal component analysis (PCA). PCA reduces a multivariate dataset into a smaller number of principal components (PCs), which account for the greatest source of variation in that dataset.

Missing data is an unfortunate reality in fossil morphometric studies, resulting from the variable preservation of specimens being examined (e.g., Mallon et al., 2011; Campione et al., 2011; Brown et al., 2012). Bayesian principal component analysis (BPCA) has been used in previous morphometric studies (Campione and Evans, 2011; Brown et al., 2012), and was recently shown to be the most reliable method for estimating missing morphometric data (Brown et al., 2012). 
Materials and methodologies used:

Principal component analysis was conducted using 25 cranial parameters, indicated by asterisks in Figure 5. These parameters consist of 24 linear measurements and one angular measurement (emargination angle of posterior parietal bar). As these parameters were measured in different units, the PCA was conducted using the correlation matrix, and not the variance-covariance matrix. The estimated, complete datasets generated by BPCA and subsequently used in the PCAs in this study are given in Tables A1-A3 (Appendix IV).

Due to the high level of heteroscedasticity (difference in variance) in the dataset, all parameters were log transformed and scaled. This ensured that all parameter variances were approximately equal, such that one parameter does not swamp the signal of another. Log transformation improved the normality of the dataset (Shapiro-Wilks Test: $p>0.05$ ), and ensured that all parameters are linearly related to each other, and not along a curve.

Three principal component analyses were conducted:

PCA 1 analyzed 18 skulls including the Vagaceratops-like specimens (AMNH 5402, CMN 41357, TMP 1987.045.0001, and YPM 2016), Chasmosaurus belli (CMN 2245, NHMUK R4948 and ROM 843), C. russelli (AMNH 5656, CMN 2280, CMN 8800, and TMP 1983.025.0001), and Chasmosaurus sp. (AMNH 5401, CMN 1254, CMN 8801, CMN 34829, ROM 839, TMP 1981.019.0175, and UALVP 40), the latter of which have incomplete posterior frill margins; PCA 2 analyzed a subset of PCA 1, excluding the Chasmosaurus sp. specimens; and, PCA 3 analyzed a subset of PCA 2, using only the seven skulls referred to $C$. belli and $C$. russelli. 
PCA 2 was performed to determine whether the removal of skulls missing their posterior frill margin (i.e., Chasmosaurus sp. specimens) had an effect on the clustering of specimens corresponding to C. belli, C. russelli and the Vagaceratops-like specimens. PCA 3 was performed to determine whether the removal of Vagaceratops-like specimens had an effect on the clustering of specimens of C. belli and C. russelli.

NPMANOVA was conducted on each PCA in this study because taxonomic clusters of specimens (i.e., species) were small ( $n=3$ or 4 ) and not normally distributed. The Mahalanobis distance measure (Mahalanobis, 1936) was used because it is better suited to smaller, non-spherically symmetric datasets than the traditional Euclidean distance measure. An increasing number of PCs, collectively accounting for an increasing proportion of the total variance in the dataset, were included in each NPMANOVA to determine whether significant differences between taxonomic clusters were robust.

Posthoc pairwise comparisons were also conducted using NPMANOVA using Bonferroni correction to determine which particular pairs of taxonomic clusters are significantly different, if any. Bonferroni correction was designed to reduce the probability of committing a Type I error (i.e., rejecting $\mathrm{H}_{0}$ when it should be accepted), which increases with the number of simultaneous pairwise comparisons being made (Sokal and Rohlf, 1995). This correction works by multiplying the $p$-value by the number of pairwise comparisons, and effectively lowering the significance level. However, because Bonferroni correction reduces the statistical power of the test and increases the probability of committing a Type II error (i.e., accepting $\mathrm{H}_{0}$ when it should be rejected) (Nakagawa, 2004), uncorrected probabilities are also reported.

A discriminant function analysis (DFA) was also performed on each PCA to 
determine which parameters best distinguish taxonomic clusters. DFA projects two or more multivariate groups of data (e.g., species), specified a priori, onto a reduced set of discriminant function (DF) axes. For N groups, there are N-1 DF axes accounting for a diminishing proportion of the total between-group variance. DFA operates by maximizing the ratio of between-group variance to within-group variance, and showing the relative contribution or loading of each PC on each DF axis. Of the highest loading PCs on a given DF axis, the highest loading parameters on those PCs are taken to be the parameters that best distinguish the groups along that DF axis.

Of the 18 chasmosaurine skulls included in these analyses, all but four (AMNH 5401, AMNH 5656, CMN 1254, and UALVP 40) have been referred to the adult age class (Fig. 63). In the ontogenetic analysis (Fig. 63), CMN 1254 was recovered as a juvenile, and AMNH 5401, AMNH 5656 and UALVP 40 were recovered as subadults; AMNH 5401 is, however, comparable in size to adult specimens. Given that most $(\mathrm{n}=$ 15) of these skulls are of similar size (rostral-to-epijugal length, AMNH $5402=685 \mathrm{~mm}$ to $897 \mathrm{~mm}=$ ROM 843), with the remaining few $(\mathrm{n}=3)$ not considerably smaller (rostral-to-epijugal length, CMN $1254=500 \mathrm{~mm}$ (estimated) to $628 \mathrm{~mm}=\mathrm{UALVP} 40$ ), most of the morphometric differences amongst these specimens cannot be attributed to ontogenetic variation.

Given the issue of putative bone resorption and its effect on the length of horncores, specimens with inferred resorbed nasal (CMN 2280, CMN 8800 and YPM 2016) and postorbital horncores (CMN 8800, CMN 8801, CMN 41357, NHMUK R4948, ROM 843, TMP 1981.019.0175, TMP 1983.025.0001, TMP 1987.045.0001, and YPM 2016) were coded as missing data for parameters 5 and 1, respectively. 
Hypotheses tested:

1. The validity of $C$. belli and $C$. russelli: $\mathrm{I}$ test the null hypothesis $\left(\mathrm{H}_{0}\right)$ that specimens assigned a priori to $C$. belli and C. russelli do not form statistically different clusters in morphospace. If the null hypothesis is accepted, this would indicate that $C$. belli and C. russelli are morphometrically indistinct, supporting the validity of a single taxon, $C$. belli, as it has historical taxonomic priority. If the null hypothesis is rejected, $C$. belli and C. russelli are morphometrically distinguishable, supporting their validities as distinct taxa $\left(\mathrm{H}_{1}\right)$.

2. Utility of the relative embayment of the posterior parietal margin in distinguishing C. belli and C. russelli: I test the null hypothesis $\left(\mathrm{H}_{0}\right)$ that there is no significant difference in the degree to which the posterior parietal margin is embayed (parameter 33, Fig. 5) between specimens assigned a priori to C. belli and C. russelli. If the null hypothesis is accepted, this would indicate that the posterior embayment cannot be used to distinguish these two taxa, and would support the validity of only one species. If the null hypothesis is rejected, this would support the existence of two morphotypes, which may represent two species or two genders of a single species $\left(\mathrm{H}_{1}\right)$. This hypothesis was tested using a Mann-Whitney U test, which is a two sample (e.g., species), univariate, non-parametric test of whether the medians of such samples are significantly different. This test was chosen because each species sample was small ( $\mathrm{n}=2$ to 5$)$ and not normally distributed. The posterior parietal embayment (parameter 33) is also included in the preceding PCAs. 
3. The relationship between postorbital horncore size and taxonomic assignment: It was postulated earlier in this study (Chapter 8) that the two ontogenetic trajectories in postorbital horncore size (length, parameter 1; basal circumference, parameter 4) are gender-related, with putative males having relatively long and large circumference-based horncores, and females having relatively short and small circumference-based horncores, as occurs in extant bovids. Using a Mann-Whitney U test, I test the null hypothesis $\left(\mathrm{H}_{0}\right)$ that large and small circumference-based postorbital horncores do not correspond to each species of Chasmosaurus. Note that postorbital horncore basal circumference is used as a proxy for horncore length because only two specimens of Chasmosaurus (CMN 2245, C. belli, and CMN 2280, C. russelli) preserve a complete horncore. If the null hypothesis is accepted, this would indicate that postorbital horncore basal circumference (and therefore horncore length) is not taxonomically informative and may represent a sexually dimorphic character within a single taxon. If the null hypothesis is rejected, this would suggest that postorbital horncore size is taxonomically informative $\left(\mathrm{H}_{1}\right)$ and the two taxa can be distinguished by their postorbital horncores. If the sexual dimorphism model for postorbital horncore basal circumference is supported, then other cranial features belonging to each putative gender of Chasmosaurus (i.e., relative degree to which the posterior parietal margin is embayed) will be reassessed in the context of this model.

\subsection{Results}

The results of each PCA (and associated non-parametric multivariate analysis of variance (NPMANOVA) and discriminant function analysis (DFA)) are presented in sequential order, followed by the results of the first Mann-Whitney U test (difference in 
posterior parietal embayment between Chasmosaurus belli and Chasmosaurus russelli), and second Mann-Whitney $\mathrm{U}$ test (difference in postorbital horncore size between $C$. belli and C. russelli).

In PCA 1 of Vagaceratops-like, C. belli, C. russelli, and Chasmosaurus sp. specimens $(\mathrm{n}=18)$, the first 10 PCs account for $95.3 \%$ of the total variance (Fig. 66A-F; Table A4, Appendix IV). In PC1, most parameters are subequal and load negatively, indicating that skull size is a major influence on the variation in this axis (Table A4, Appendix IV); parameters 1, 31 and 33 load positively. Variation along the remaining PC axes is largely independent of size. Of the first 10 PCs, only three (PCs 2, 4 and 5) show separation between taxa; however, the within-taxon variance significantly exceeds the between-taxon variance in each of these axes. The Vagaceratops-like specimens score on the positive side of PC2 (13.4\% of variance; Fig. 66A-B), while Chasmosaurus-like specimens (C. belli, C. russelli and Chasmosaurus sp.) score mostly on the negative side. The highest loading parameters on $\mathrm{PC} 2$, in descending order of importance, indicate that the Vagaceratops-like specimens differs from C. belli and C. russelli in having a relatively wider jugal notch (parameter 22) and anteroposteriorly shorter parietal fenestrae (parameter 30).

Chasmosaurus belli scores on the negative side of PC4 (9.4\% of variance; Fig. 66C), while C. russelli scores on the positive side, and the Vagaceratops-like specimens score between these two clusters. The highest loading parameters on PC4, in descending order of importance, indicate that C. belli differs from C. russelli and, to a lesser extent, the Vagaceratops-like specimens, in having a relatively shallow embayment on the posterior parietal margin (parameter 33) and a smaller postorbital horncore basal 
Figure 66. Principal component analysis (PCA 1) of 18 specimens of the Vagaceratopslike specimens $(\mathrm{n}=4)$, Chasmosaurus russelli $(\mathrm{n}=4)$, C. belli $(\mathrm{n}=3)$, and Chasmosaurus sp. $(\mathrm{n}=7)$ : A), PC1 vs. PC2, cumulatively accounting for $48.5 \%$ of the total variance; B), PC2 vs. PC3, cumulatively accounting for $24.6 \%$ of the total variance; C), PC3 vs. PC4, cumulatively accounting for $20.6 \%$ of the total variance; D), PC5 vs. PC6, cumulatively accounting for $14.0 \%$ of the total variance; E), PC7 vs. PC8, cumulatively accounting for $8.9 \%$ of the total variance; and F), PC9 vs. PC10, cumulatively accounting for $4.0 \%$ of the total variance. 
Figure 66.

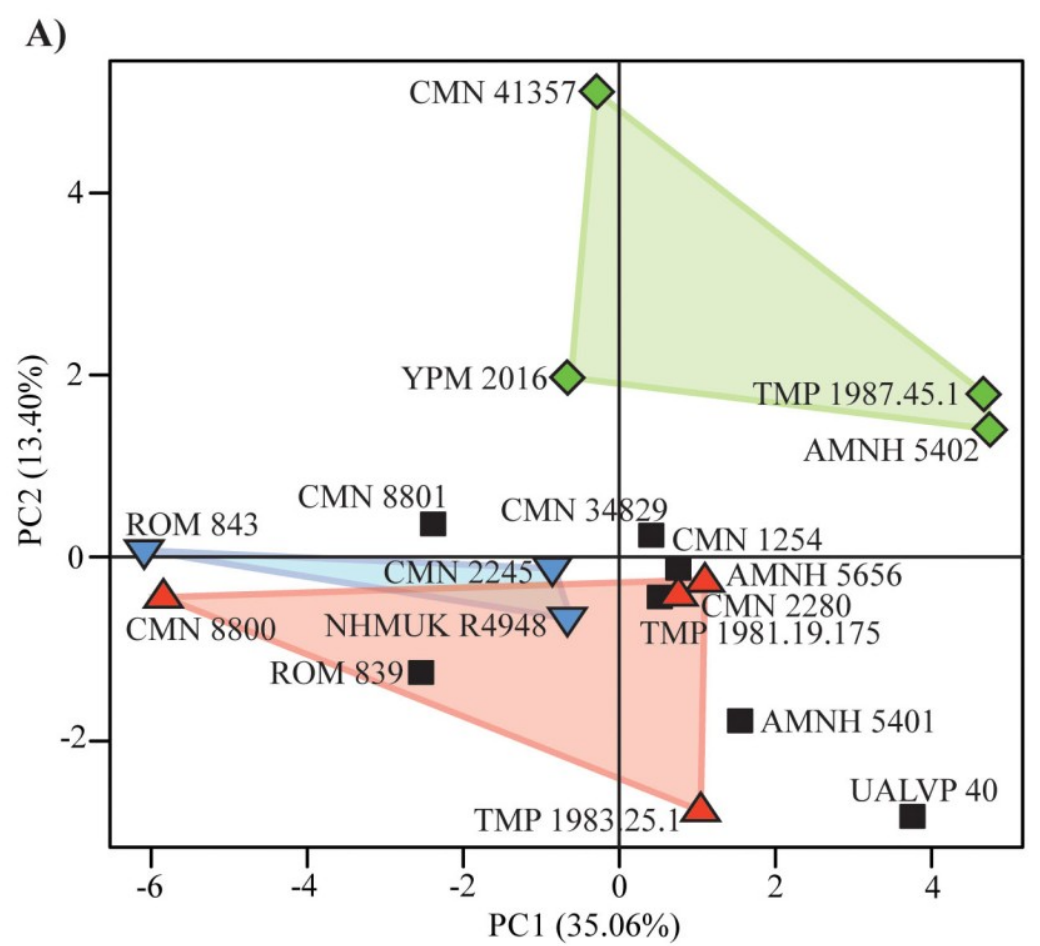

Vagaceratops-like specimens

$\triangle$ Chasmosaurus russelli

$\nabla$ Chasmosaurus belli

B) Chasmosaurus sp.

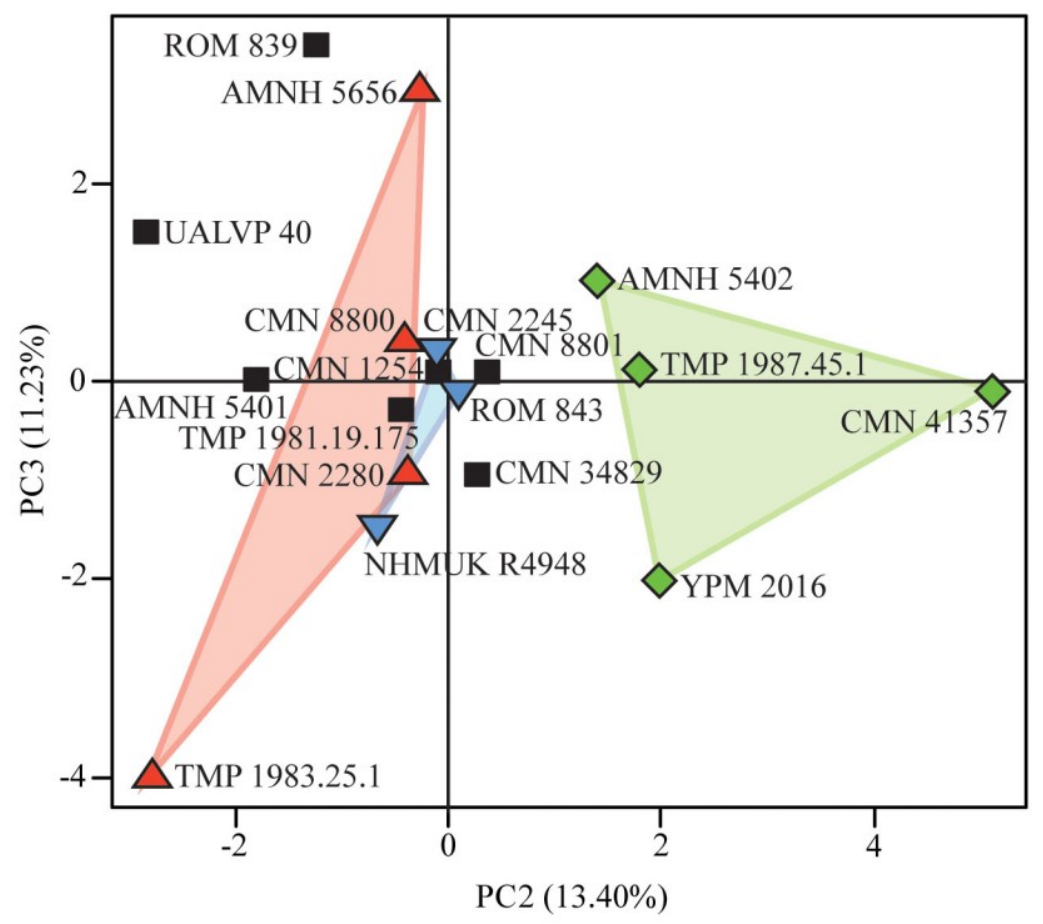


Figure 66. (continued).

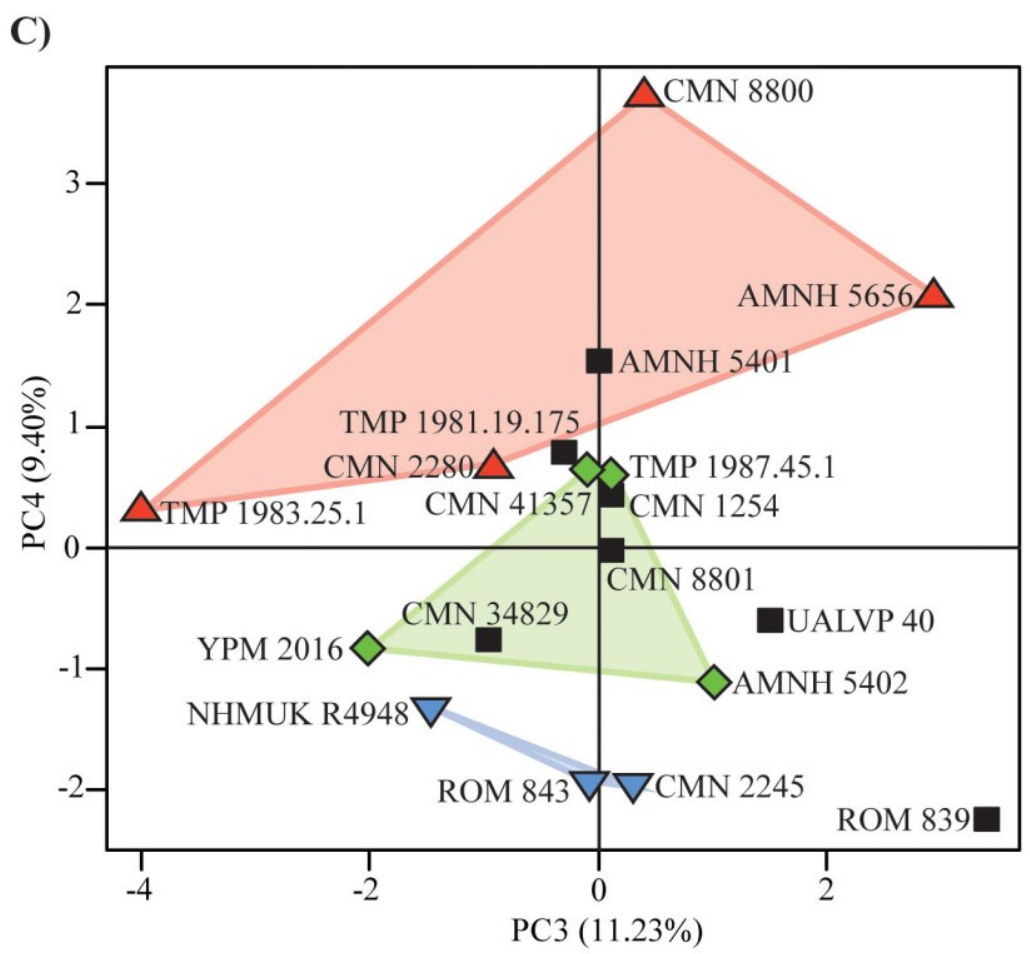

D)

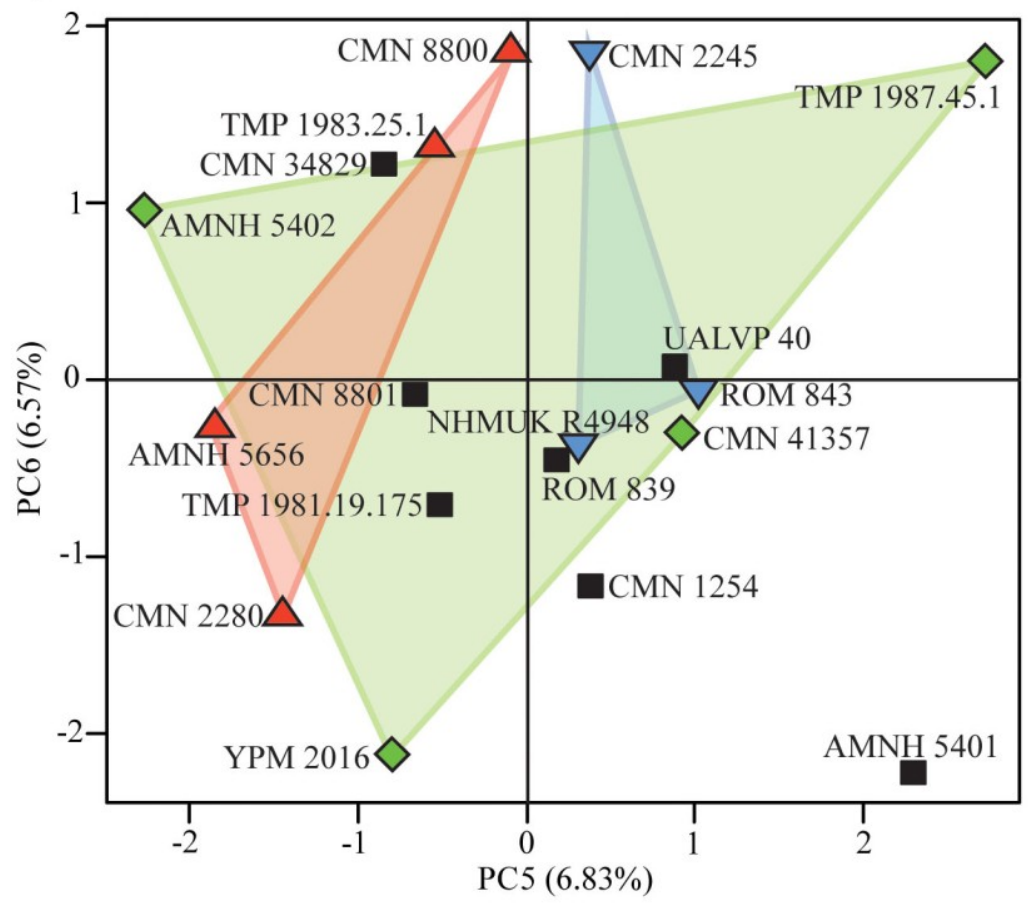


Figure 66. (continued).
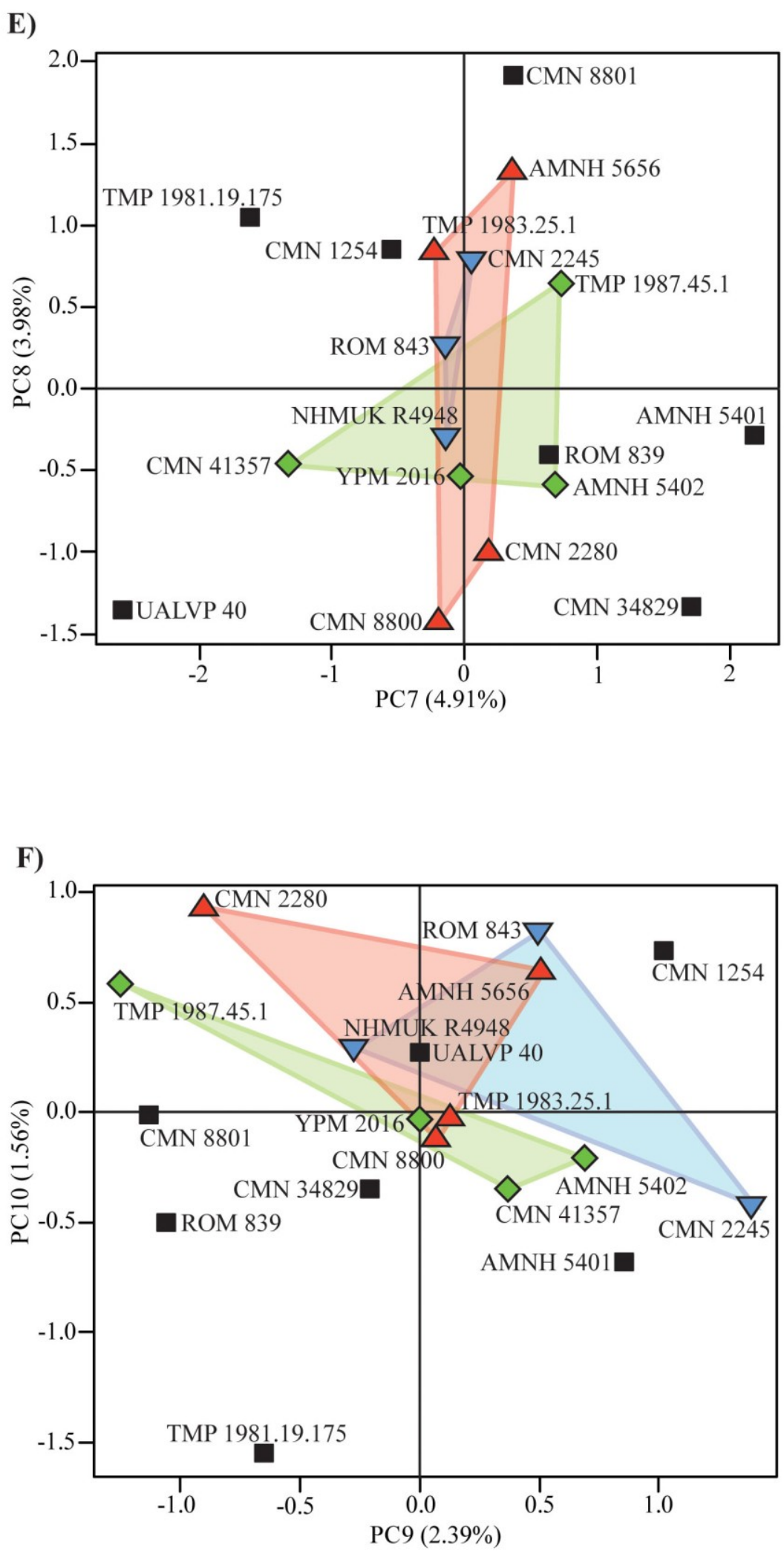
circumference (parameter 4). Chasmosaurus belli scores on the positive side of PC5 (6.8\% of variance; Fig. 66D), while C. russelli scores on the negative side. The highest loading parameters on PC5, in descending order of importance, indicate that $C$. belli differs from C. russelli in having longer postorbital horncores (parameter 1), a greater jugal notch to parietal fenestra length (parameter 21) and a greater maximal squamosal width (parameter 15).

In NPMANOVA 1 , significant differences $(p<0.01)$ amongst the Vagaceratopslike, Chasmosaurus russelli, and C. belli specimens occurred when principal components (PCs) 1-7 were included (Table 10); the $p$-value generally increases with number of PCs used. Posthoc pairwise comparisons reveal that there are no significant differences between any two of these taxa, regardless of the number of PCs used.

The corresponding DFA (DFA 1; Fig. 67; Table A5, Appendix IV) has a 100\% successful classification rate. The first discriminant function (DF1) axis accounts for $74.7 \%$ of the total between-group variance. The Vagaceratops-like specimens score positively on this axis, while C. russelli scores negatively, and C. belli scores in between. PC2 loads strongly and positively on DF1, followed by PC1 (positively). This indicates that the Vagaceratops-like specimens differs primarily from C. russelli and, to a lesser extent, C. belli, in having: PC2, a relatively wide jugal notch (parameter 22), anteroposteriorly shortened parietal fenestrae (parameter 30), and a short distance between the jugal notch and the back of the frill (parameter 28); and PC1, a relatively short rostral-to-epijugal length (parameter 16), rostral-to-maxillary toothrow length (parameter 17) and skull length (parameter 26).

The DF2 axis accounts for the remaining between-group variance. Chasmosaurus 
Table 10: Results of NPMANOVA 1; comparisons between Vagaceratops-like, Chasmosaurus russelli, and C. belli specimens; principal component (PC) scores and specimens $(\mathrm{n}=11)$ taken from PCA 1 (18 specimens). Results of NPMANOVA using the first three, four, five, six, and seven PCs are given. Posthoc pairwise comparisons shown in centre; uncorrected $p$-values shown in upper, white cells, and Bonferroni corrected $p$ values shown in lower, grey cells. $F$ and $p$-values for total analysis shown on right. Significant results $(p<0.05)$ shown in bold.

\begin{tabular}{|c|c|c|c|c|c|c|}
\hline \multirow{2}{*}{\multicolumn{2}{|c|}{$\begin{array}{l}\text { PCs included in } \\
\text { NPMANOVA; variance } \\
\text { accounted by PCs }(\%)\end{array}$}} & \multicolumn{3}{|c|}{ Posthoc pairwise comparisons } & \multicolumn{2}{|c|}{ Total analysis } \\
\hline & & \multirow{2}{*}{$\begin{array}{l}\text { Vagaceratops- } \\
\text { like specimens } \\
(\mathrm{n}=4)\end{array}$} & \multirow{2}{*}{$\begin{array}{l}\text { C. russelli } \\
(\mathrm{n}=4)\end{array}$} & \multirow{2}{*}{$\begin{array}{l}\text { C. belli } \\
(\mathrm{n}=3)\end{array}$} & \multirow{2}{*}{$\begin{array}{c}F \\
\\
1.787\end{array}$} & \multirow{2}{*}{$\begin{array}{c}p \\
\\
0.1042\end{array}$} \\
\hline $\begin{array}{l}\mathrm{PC} \\
1-3\end{array}$ & $\begin{array}{l}\text { Vagaceratops-like } \\
\text { specimens }(n=4)\end{array}$ & & & & & \\
\hline \multirow[t]{2}{*}{59.7} & C. russelli $(\mathrm{n}=4)$ & 0.174 & & 0.6296 & & \\
\hline & C. belli $(\mathrm{n}=3)$ & 0.2712 & 1 & & & \\
\hline $\begin{array}{l}\mathrm{PC} \\
1-4\end{array}$ & $\begin{array}{l}\text { Vagaceratops-like } \\
\text { specimens }(\mathrm{n}=4)\end{array}$ & & 0.0905 & 0.2278 & 2.83 & 0.0004 \\
\hline \multirow[t]{2}{*}{69.1} & C. russelli $(\mathrm{n}=4)$ & 0.2715 & & 0.1965 & & \\
\hline & C. belli $(\mathrm{n}=3)$ & 0.6834 & 0.5895 & & & \\
\hline $\begin{array}{l}\mathrm{PC} \\
1-5\end{array}$ & $\begin{array}{l}\text { Vagaceratops-like } \\
\text { specimens }(n=4)\end{array}$ & & 0.0871 & 0.598 & 2.169 & 0.0006 \\
\hline \multirow[t]{2}{*}{75.9} & C. russelli $(\mathrm{n}=4)$ & 0.2613 & & 0.281 & & \\
\hline & C. belli $(\mathrm{n}=3)$ & 1 & 0.843 & & & \\
\hline $\begin{array}{l}\mathrm{PC} \\
1-6\end{array}$ & $\begin{array}{l}\text { Vagaceratops-like } \\
\text { specimens }(\mathrm{n}=4)\end{array}$ & & 0.0617 & 0.9712 & 1.676 & 0.0053 \\
\hline \multirow[t]{2}{*}{82.5} & C. russelli $(\mathrm{n}=4)$ & 0.1851 & & 0.8319 & & \\
\hline & C. belli $(\mathrm{n}=3)$ & 1 & 1 & & & \\
\hline $\begin{array}{l}\mathrm{PC} \\
1-7\end{array}$ & $\begin{array}{l}\text { Vagaceratops-like } \\
\text { specimens }(\mathrm{n}=4)\end{array}$ & & 0.7396 & 1 & 1.466 & 0.0056 \\
\hline \multirow[t]{2}{*}{87.4} & C. russelli $(\mathrm{n}=4)$ & 1 & & 0.5359 & & \\
\hline & C. belli $(\mathrm{n}=3)$ & 1 & 1 & & & \\
\hline
\end{tabular}




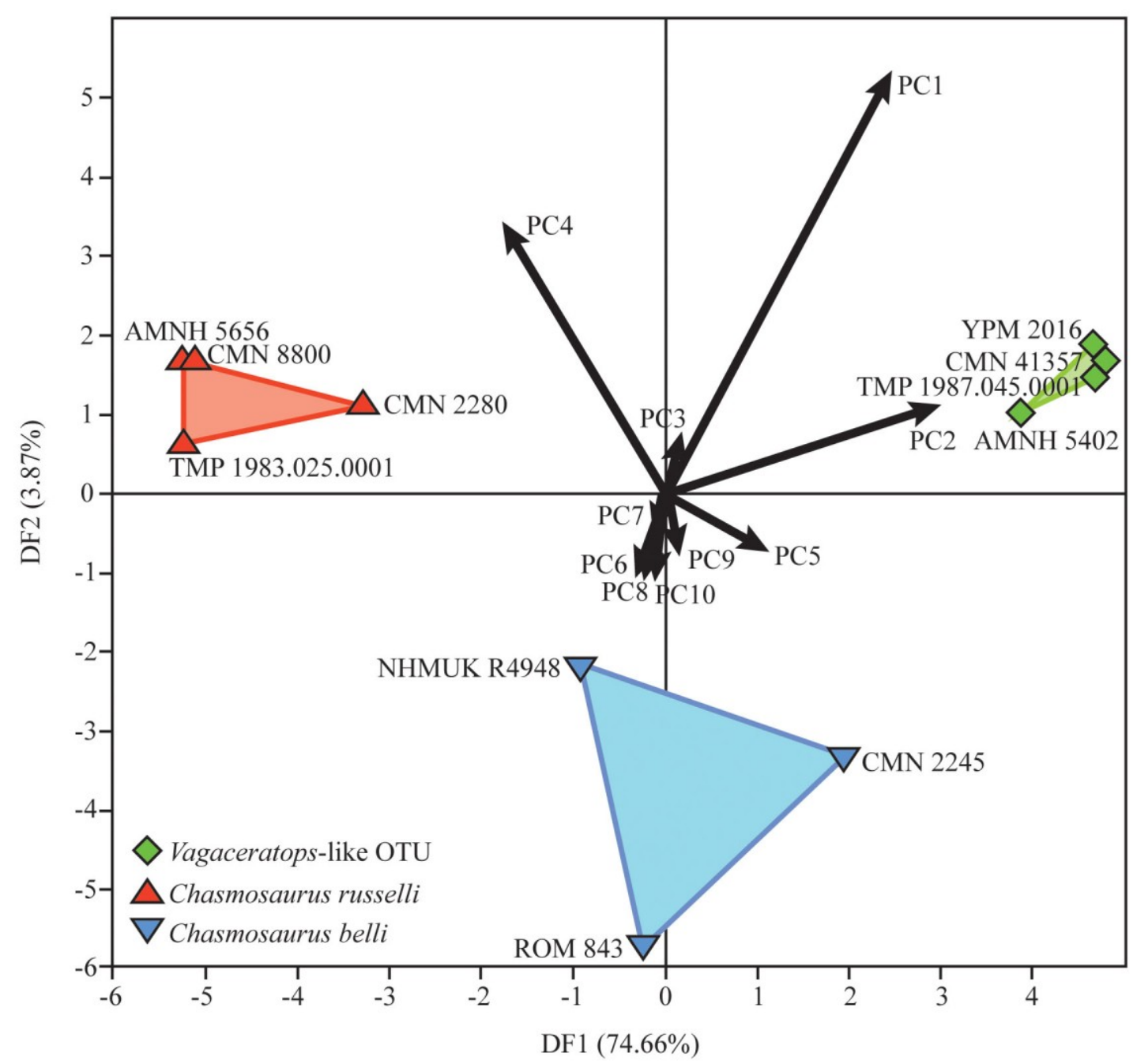

Figure 67. Discriminant function analysis (DFA 1) of 11 specimens of the Vagaceratopslike specimens $(\mathrm{n}=4)$, Chasmosaurus russelli $(\mathrm{n}=4)$, and C. belli $(\mathrm{n}=3)$. Loadings of first 10 principal components (PCs; collectively accounting for $95.3 \%$ of the total variance), on the two discriminant function (DF) axes shown by black arrows. PC scores and specimens taken from PCA1. 
belli scores negatively on this axis, while the Vagaceratops-like and C. russelli specimens both score positively and show little separation. PC1 loads strongly and positively on DF2, followed by PC4 (positively). This indicates that $C$. belli differs primarily from the Vagaceratops-like and C. russelli specimens in having: PC1, a large rostral-to-epijugal length (parameter 16), rostral-to-maxillary toothrow length (parameter 17) and skull length (parameter 26); and PC4, relatively shallow embayment on posterior margin of parietal (parameter 33) and small postorbital horncore basal circumference (parameter 4).

In PCA 2 of Vagaceratops-like, C. belli and C. russelli specimens $(\mathrm{n}=11)$, the first seven PCs account for 96.1\% of the total variance (Fig. 68A-D; Table A6, Appendix IV). In PC1, most parameters are subequal and load positively, indicating that skull size is a major influence on the variation in this axis (Table A6, Appendix IV); parameter 33 loads negatively. Variation along the remaining PC axes is largely independent of size. Of the first seven PCs, only PC1 (47.6\% of variance) shows separation between any two of these taxa, Vagaceratops-like specimens and C. belli (Fig. 68A); however, the withintaxon variance significantly exceeds the between-taxon variance. The Vagaceratops-like specimens score mostly on the negative side of this axis, while $C$. belli scores positively. The highest loading parameters on this axis, in descending order of importance, indicate that the Vagaceratops-like specimens differ from C. belli in having a relatively small skull length (parameter 26), rostral-to-epijugal length (parameter 16) and rostral-to-back of maxillary toothrow length (parameter 17).

In NPMANOVA 2 , significant differences $(p<0.05)$ amongst the Vagaceratopslike, C. russelli, and C. belli specimens occurred when PCs 1-6 were included (Table 11); 
Figure 68. Principal component analysis (PCA 2) of 11 specimens of the Vagaceratopslike specimens $(\mathrm{n}=4)$, Chasmosaurus russelli $(\mathrm{n}=4)$ and C. belli $(\mathrm{n}=3)$ : A), PC1 vs. PC2, cumulatively accounting for $62.9 \%$ of the total variance; B), PC2 vs. PC3, cumulatively accounting for $26.0 \%$ of the total variance; C), PC3 vs. PC4, cumulatively accounting for $19.7 \%$ of the total variance; D), PC5 vs. PC6, cumulatively accounting for $10.9 \%$ of the total variance; and E), PC6 vs. PC7, cumulatively accounting for $6.1 \%$ of the total variance. 
Figure 68.

A)

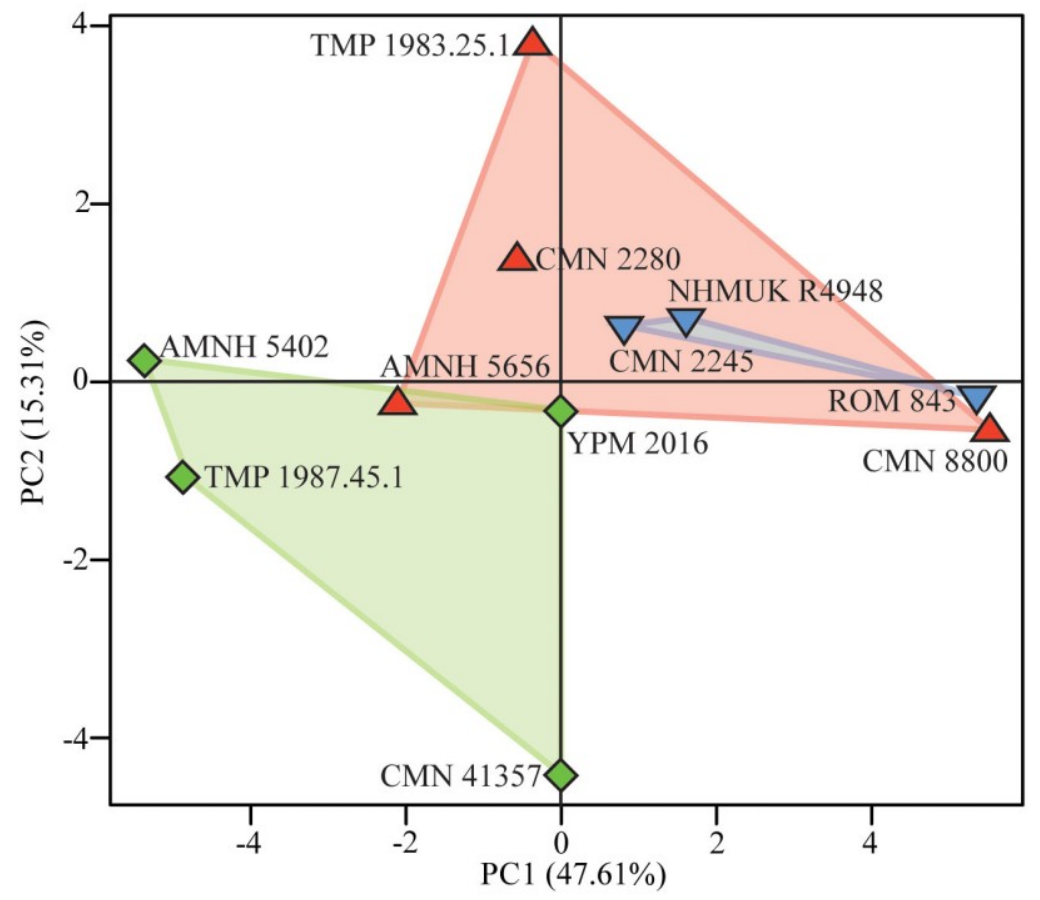

Vagaceratops-like specimens $\triangle$ Chasmosaurus russelli

B) $\nabla$ Chasmosaurus belli

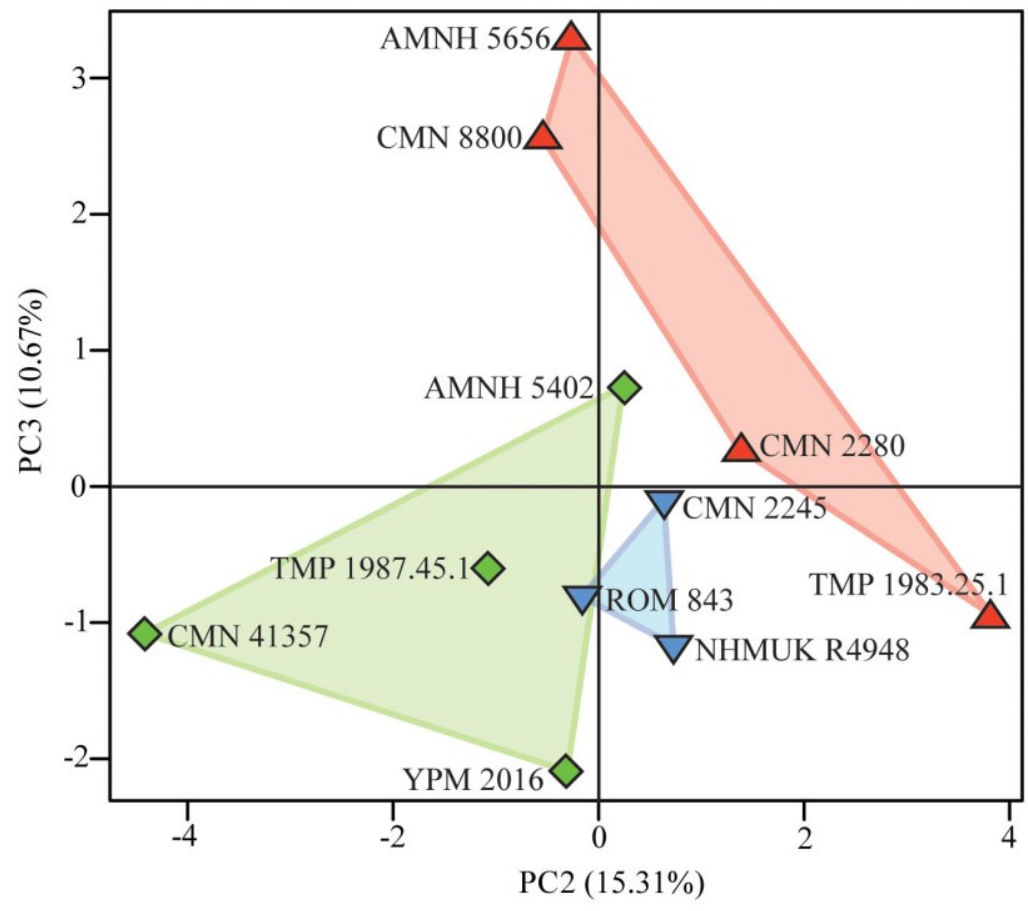


Figure 68. (continued).
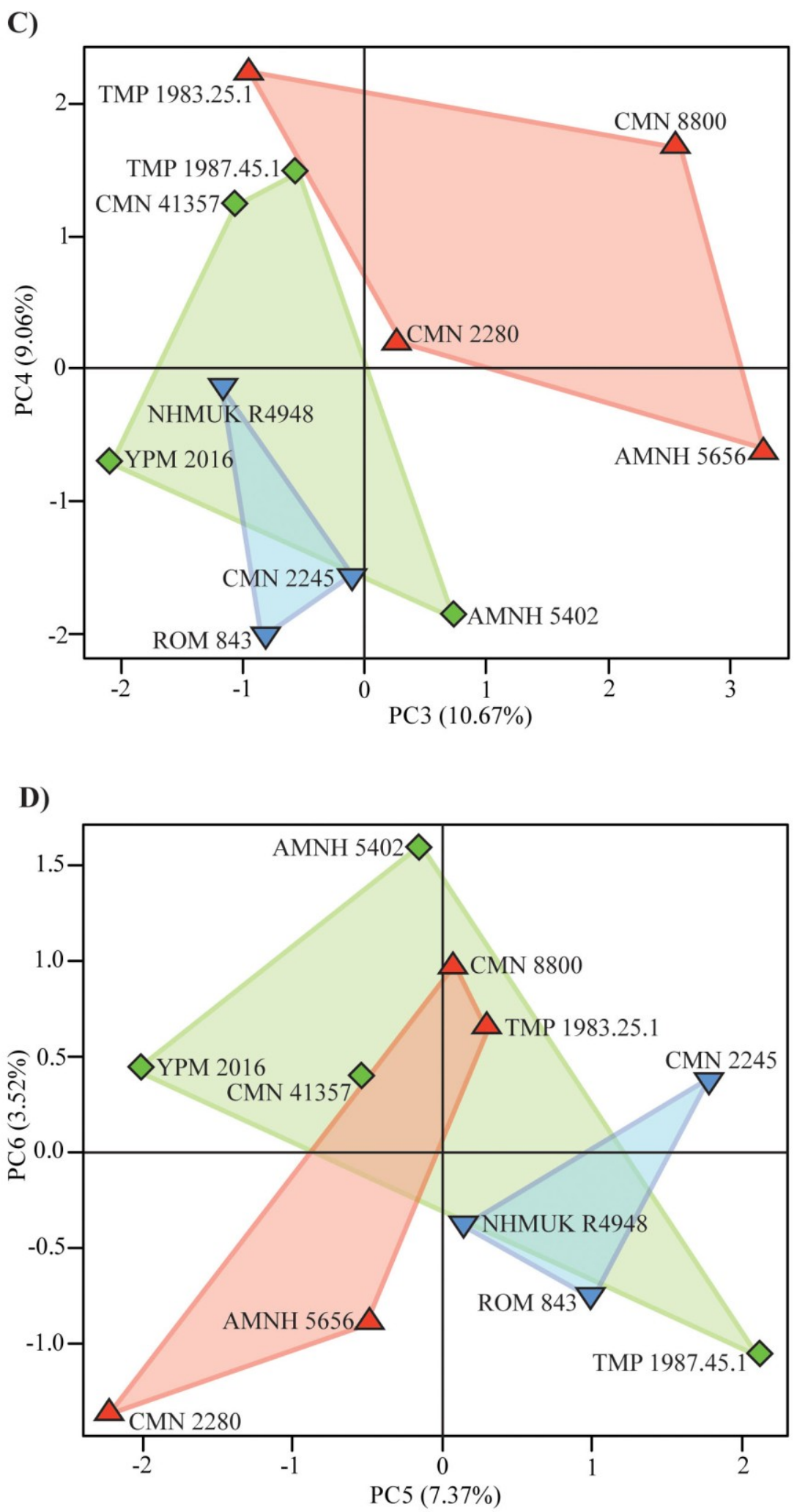
Figure 68. (continued).

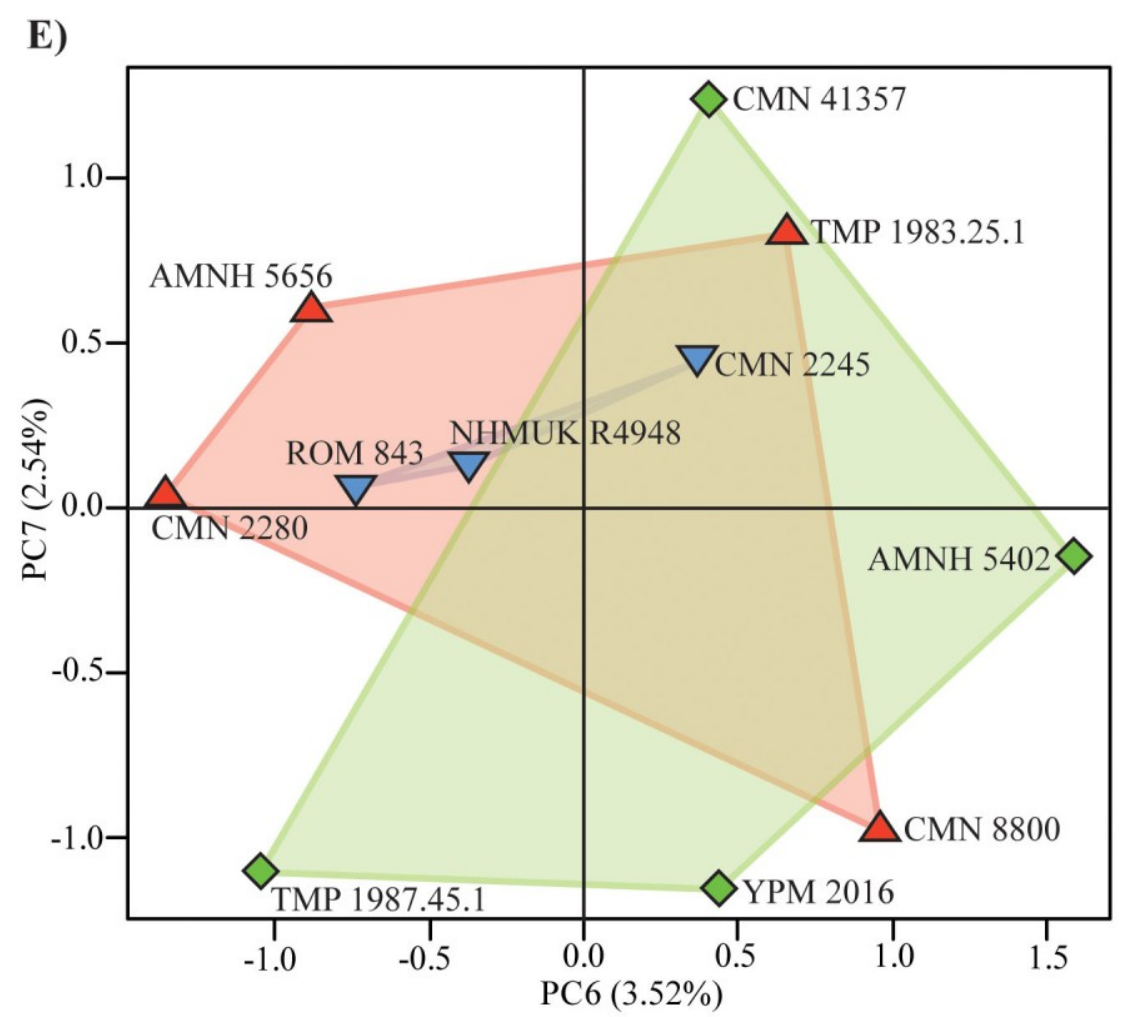


Table 11: Results of NPMANOVA 2; comparisons between Vagaceratops-like, Chasmosaurus russelli, and C. belli specimens; principal component (PC) scores and specimens $(\mathrm{n}=11)$ taken from PCA 2 (11 specimens). Results of NPMANOVA using the first three, four, five, and six PCs are given. Posthoc pairwise comparisons shown in centre; uncorrected $p$-values shown in upper, white cells, and Bonferroni corrected $p$ values shown in lower, grey cells. $F$ and $p$-values for total analysis shown on right. Significant results $(p<0.05)$ shown in bold.

\begin{tabular}{|c|c|c|c|c|c|c|}
\hline \multirow{2}{*}{\multicolumn{2}{|c|}{$\begin{array}{l}\text { PCs included in } \\
\text { NPMANOVA; variance } \\
\text { accounted by PCs }(\%)\end{array}$}} & \multicolumn{3}{|c|}{ Posthoc pairwise comparisons } & \multicolumn{2}{|c|}{ Total analysis } \\
\hline & & \multirow{2}{*}{$\begin{array}{l}\text { Vagaceratops- } \\
\text { like specimens } \\
(\mathrm{n}=4)\end{array}$} & \multirow{2}{*}{$\begin{array}{l}\text { C. russelli } \\
(\mathrm{n}=4)\end{array}$} & \multirow{2}{*}{$\begin{array}{l}\text { C. belli } \\
(\mathrm{n}=3)\end{array}$} & \multirow{2}{*}{$\begin{array}{c}F \\
\\
2.398\end{array}$} & \multirow{2}{*}{$\begin{array}{c}p \\
\mathbf{0 . 0 2 3 3}\end{array}$} \\
\hline $\begin{array}{l}\mathrm{PC} \\
1-3\end{array}$ & $\begin{array}{l}\text { Vagaceratops-like } \\
\text { specimens }(\mathrm{n}=4)\end{array}$ & & & & & \\
\hline \multirow[t]{2}{*}{73.6} & C. russelli $(\mathrm{n}=4)$ & 0.1848 & & 0.0274 & & \\
\hline & C. belli $(\mathrm{n}=3)$ & 0.261 & 0.0822 & & & \\
\hline $\begin{array}{l}\mathrm{PC} \\
1-4\end{array}$ & $\begin{array}{l}\text { Vagaceratops-like } \\
\text { specimens }(\mathrm{n}=4)\end{array}$ & & 0.122 & 0.0306 & 2.314 & 0.0033 \\
\hline \multirow[t]{2}{*}{82.7} & C. russelli $(\mathrm{n}=4)$ & 0.366 & & 0.1701 & & \\
\hline & C. belli $(\mathrm{n}=3)$ & 0.0918 & 0.5103 & & & \\
\hline $\begin{array}{l}\mathrm{PC} \\
1-5\end{array}$ & $\begin{array}{l}\text { Vagaceratops-like } \\
\text { specimens }(\mathrm{n}=4)\end{array}$ & & 0.0311 & 0.2261 & 2.059 & 0.0042 \\
\hline \multirow[t]{2}{*}{90.0} & C. russelli $(\mathrm{n}=4)$ & 0.0933 & & 0.0851 & & \\
\hline & C. belli $(\mathrm{n}=3)$ & 0.6783 & 0.2553 & & & \\
\hline $\begin{array}{l}\mathrm{PC} \\
1-6\end{array}$ & $\begin{array}{l}\text { Vagaceratops-like } \\
\text { specimens }(\mathrm{n}=4)\end{array}$ & & 0.15 & 1 & 1.695 & 0.005 \\
\hline \multirow[t]{2}{*}{93.5} & C. russelli $(\mathrm{n}=4)$ & 0.45 & & 0.2317 & & \\
\hline & C. belli $(\mathrm{n}=3)$ & 1 & 0.6951 & & & \\
\hline
\end{tabular}


the $p$-value generally decreases as more PCs are included. Posthoc pairwise comparisons reveal significant differences $(p<0.05)$ between: $C$. russelli and $C$. belli using the first three PCs; Vagaceratops-like specimens and C. belli using the first four PCs; and Vagaceratops-like specimens and C. russelli using the first five PCs (Table 11).

The corresponding DFA 2 (Fig. 69; Table A7, Appendix IV) has a 100\% successful classification rate. The DF1 axis accounts for $96.1 \%$ of the total betweengroup variance. The Vagaceratops-like specimens scores negatively on this axis, while $C$. russelli scores positively, and C. belli scores in between. PC1 loads strongly and positively on DF1, followed by PC2 (positively). This indicates that the Vagaceratopslike specimens differs primarily from C. russelli and, to a lesser extent, C. belli, in having: PC1, a relatively short skull length (parameter 26), rostral-to-epijugal length (parameter 16) and rostral-to-maxillary toothrow length (parameter 17); and PC2, a relatively wide jugal notch (parameter 22), anteroposteriorly shortened parietal fenestrae (parameter 30) and large distance between the anterior margin of the dorsal temporal fenestra and parietal fenestra (parameter 20).

The DF2 axis accounts for the remaining between-group variance. Chasmosaurus belli scores negatively on this axis, while the Vagaceratops-like and C. russelli specimens both score positively and show little separation. PC1 loads strongly and negatively on DF2. This indicates that $C$. belli differs primarily from the Vagaceratopslike and C. russelli specimens in having a relatively large skull length (parameter 26), rostral-to-epijugal length (parameter 16) and rostral-to-maxillary toothrow length (parameter 17). 


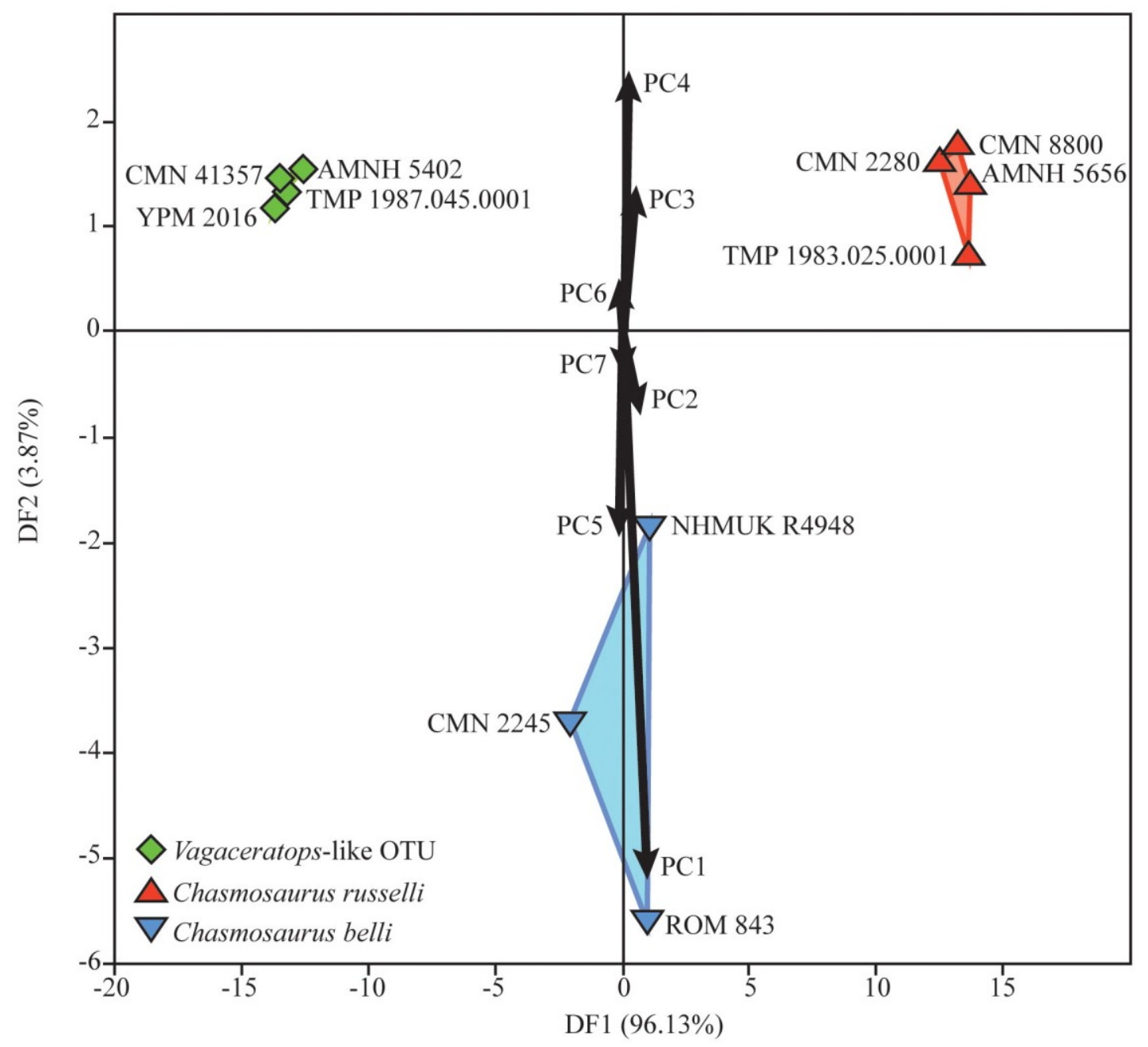

Figure 69. Discriminant function analysis (DFA 2) of 11 specimens of the Vagaceratopslike specimens $(\mathrm{n}=4)$, Chasmosaurus russelli $(\mathrm{n}=4)$, and C. belli $(\mathrm{n}=3)$. Loadings of first seven principal components (PCs; collectively accounting for $96.1 \%$ of the total variance), on the two discriminant function (DF) axes shown by black arrows. PC scores and specimens taken from PCA 2. 
In PCA 3 of $C$. belli and C. russelli specimens ( $(\mathrm{n}=7)$, the first five PCs account for $95.8 \%$ of the total variance (Fig. 70A-D; Table A8, Appendix IV). In PC1, most parameters are subequal and load positively, indicating that skull size is a major influence on the variation in this axis (Table A6, Appendix IV); parameters 1, 25, 27, 30, 31, and 33 load negatively. Variation along the remaining PC axes is largely independent of size. Of the first five PCs, only PC2 (22.2\% of variance) shows separation between these two taxa (Fig. 70A); however, the within-taxon variance significantly exceeds the betweentaxon variance. Chasmosaurus russelli scores mostly on the negative side of this axis, while $C$. belli scores positively. The highest loading parameters on this axis, in descending order of importance, indicate that C. russelli differs from C. belli in having a relatively large postorbital horncore basal circumference (parameter 4), short rostral-toback of maxillary toothrow length (parameter 17) and deeply embayed posterior parietal margin (parameter 33).

In NPMANOVA 3, there are no significant differences between $C$. russelli and $C$. belli, regardless of whether the first three, four or five PCs were used (Table 12); the $p$ value increases as more PCs are included. The corresponding DFA 3 (Fig. 71; Table A9, Appendix IV) has a 100\% successful classification rate. C. belli scores negatively on the sole DF axis, while C. russelli scores positively. PC2 loads strongly and negatively on this axis, followed by PC1 (negatively). This indicates that $C$. belli differs primarily from C. russelli in having: PC2, a relatively small postorbital horncore basal circumference (parameter 4), large rostral-to-maxillary toothrow length (parameter 17) and a shallowly embayed posterior parietal margin (parameter 33); and $\mathrm{PC1}$, a relatively large distance 
Figure 70. Principal component analysis (PCA 3) of seven specimens of Chasmosaurus russelli $(\mathrm{n}=4)$ and $C$. belli $(\mathrm{n}=3)$ : A), PC1 vs. PC2, cumulatively accounting for $62.7 \%$ of the total variance; B), PC2 vs. PC3, cumulatively accounting for $38.3 \%$ of the total variance; $\mathbf{C}$ ), PC3 vs. PC4, cumulatively accounting for $27.7 \%$ of the total variance; and D), PC4 vs. PC5, cumulatively accounting for $17.0 \%$ of the total variance. 
Figure 70.

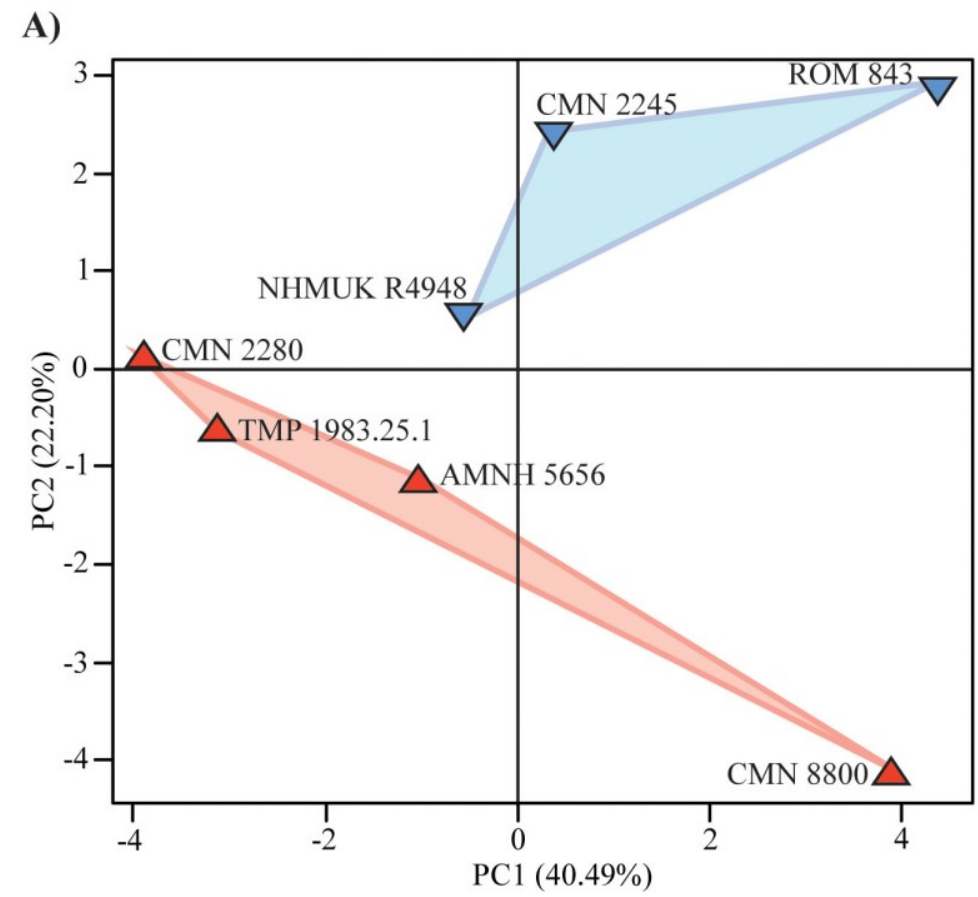

$\triangle$ Chasmosaurus russelli

$\nabla$ Chasmosaurus belli

B)

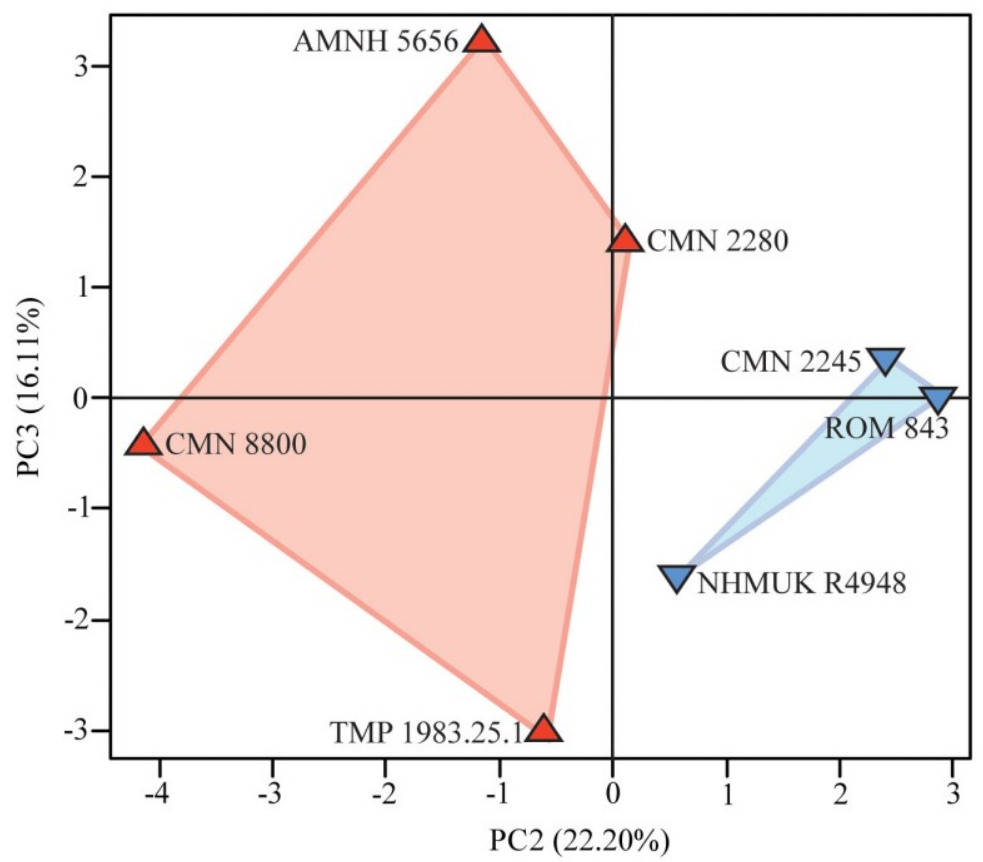


Figure 70. (continued).
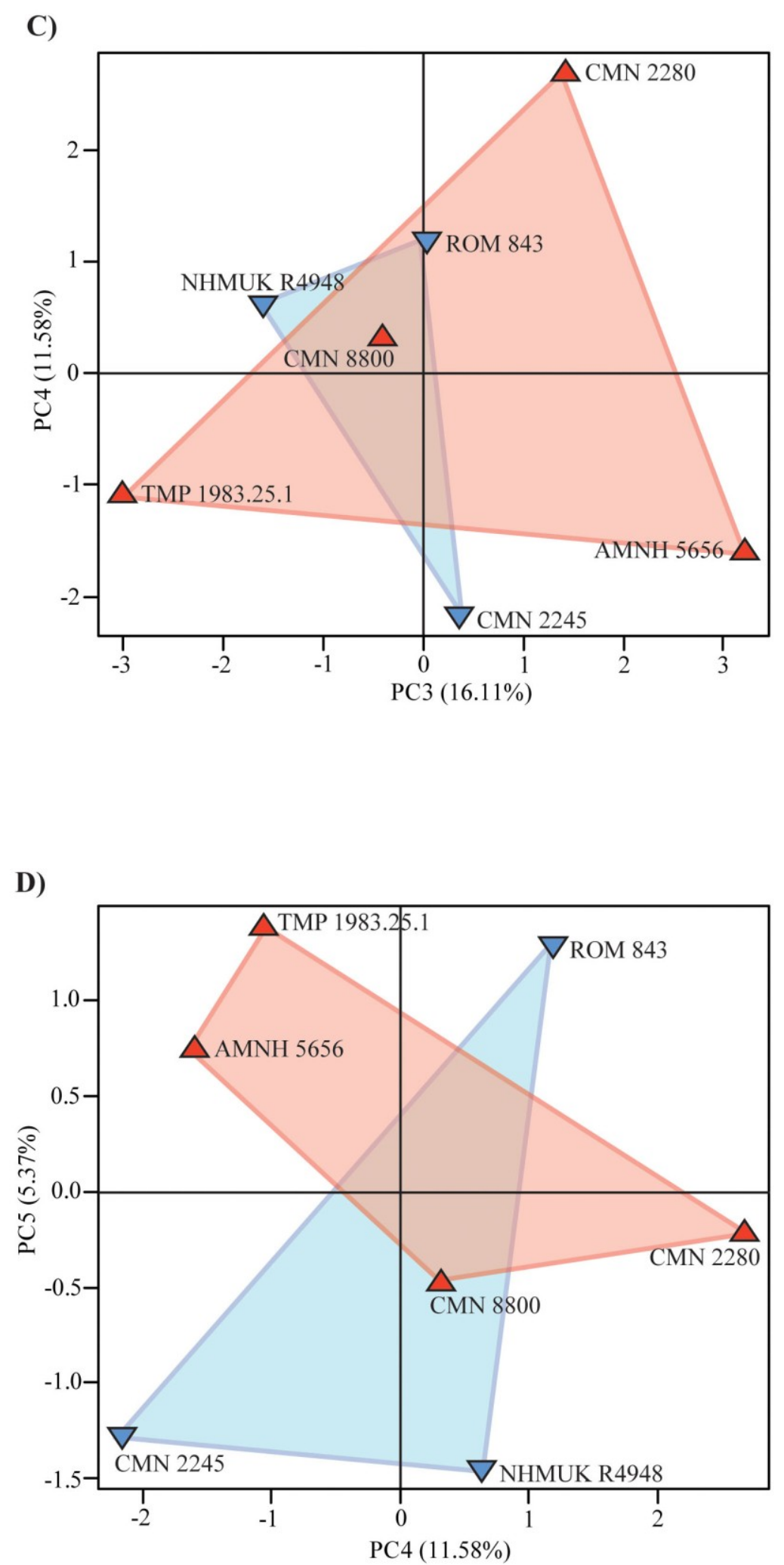
Table 12: Results of NPMANOVA 3; comparisons between Chasmosaurus russelli and C. belli; principal component (PC) scores and specimens $(\mathrm{n}=7)$ taken from PCA 3. Results of NPMANOVA using the first three, four, and five PCs are given. Posthoc pairwise comparisons shown in centre (uncorrected $p$-values). $F$ and $p$-values for total analysis shown on right.

\begin{tabular}{|l|l|l|c|}
\hline $\begin{array}{l}\text { PCs included in } \\
\text { NPMANOVA; } \\
\text { variance accounted by }\end{array}$ & \multirow{2}{*}{$\begin{array}{c}\text { Posthoc pairwise } \\
\text { comparisons }\end{array}$} & \multicolumn{2}{|c|}{ Total analysis } \\
\cline { 3 - 4 } PCs (\%) & & $F$ & $p$ \\
\hline PC 1-3 (78.8) & 0.1111 & 1.824 & 0.1162 \\
\hline PC 1-4 (90.4) & 0.3469 & 1.261 & 0.333 \\
\hline PC 1-5 (95.8) & 0.3738 & 1.179 & 0.3657 \\
\hline
\end{tabular}




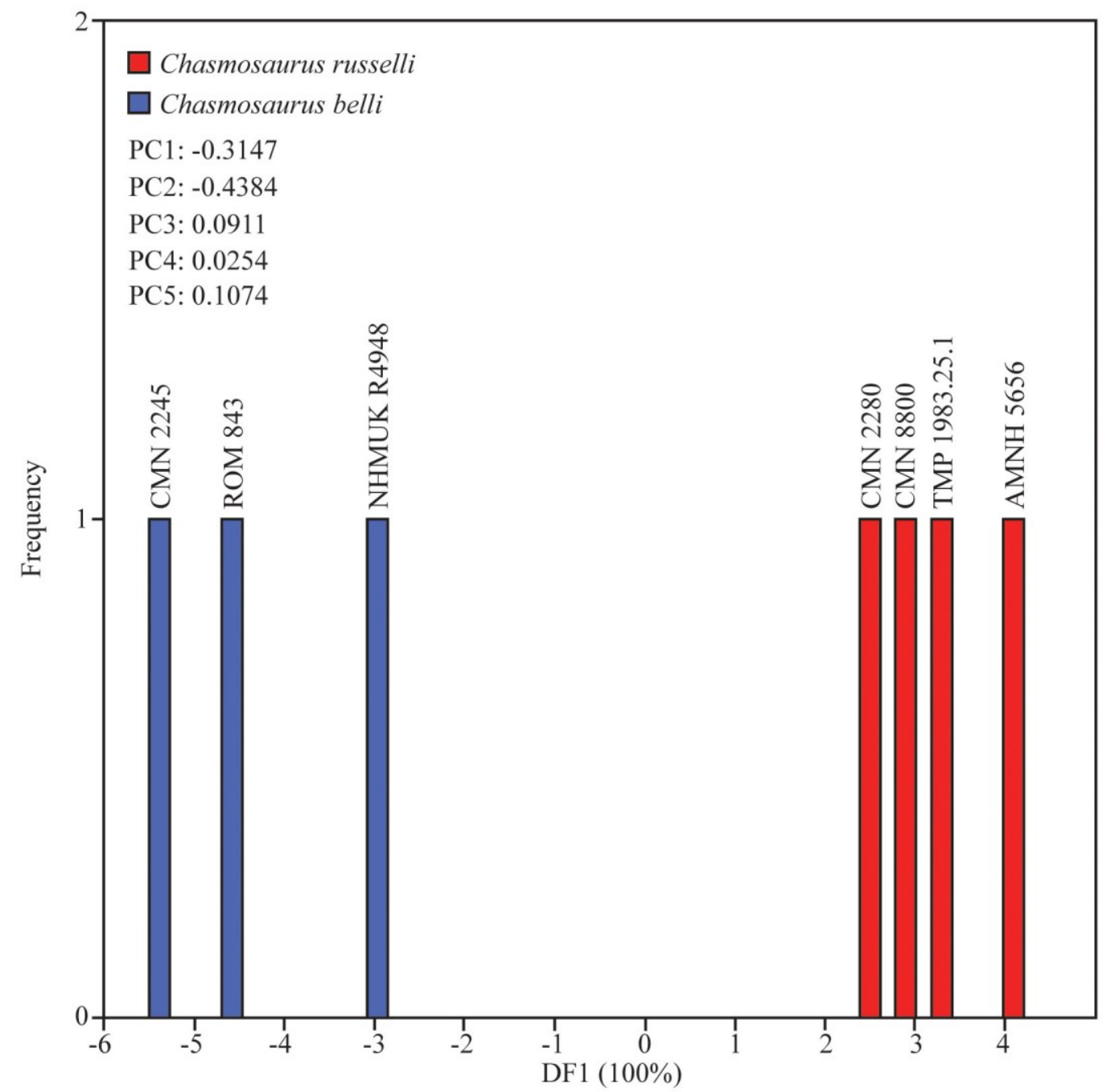

Figure 71. Discriminant function analysis (DFA 3) of seven specimens of Chasmosaurus russelli $(\mathrm{n}=4)$ and $C$. belli $(\mathrm{n}=3)$. Loadings of first five principal components (PCs; collectively accounting for $95.8 \%$ of the total variance) are given. PC scores and specimens taken from PCA 3. 
between the anterior margin of the dorsal temporal fenestra and parietal fenestra (parameter 20) and distance between the anterior margin of frontoparietal fontanelle to the back of the medial parietal bar (parameter 23).

The Mann-Whitney $\mathrm{U}$ test of a median difference between specimens of $C$. belli and C. russelli indicate that there is a significant difference $(p<0.05)$ in the relative degree to which the posterior parietal margin is embayed (parameter 33) (Table 13). The MannWhitney $\mathrm{U}$ test of a median difference between specimens of $C$. belli and C. russelli indicate that there is no significant difference in postorbital horncore basal circumference (Table 14). 
Table 13: Results of Mann-Whitney U test, testing for median differences in the relative embayment of the posterior parietal margin (parameter 33) between Chasmosaurus belli and $C$. russelli. Significant results $(p<0.05)$ shown in bold.

\begin{tabular}{|c|c|c|}
\hline Taxon & Specimen & $\begin{array}{l}\text { Embayment angle } \\
\left({ }^{\circ}\right) \text { of posterior } \\
\text { parietal margin } \\
\text { (parameter } 33 \text { ) }\end{array}$ \\
\hline \multirow{4}{*}{$\begin{array}{l}\text { Chasmosaurus } \\
\text { belli }\end{array}$} & NHMUK R4948 & 162 \\
\hline & CMN 0491 & 158 \\
\hline & CMN 2245 & 156 \\
\hline & ROM 843 & 136 \\
\hline \multirow{5}{*}{$\begin{array}{l}\text { Chasmosaurus } \\
\text { russelli }\end{array}$} & TMP 1983.025.0001 & 128 \\
\hline & CMN 2280 & 124 \\
\hline & AMNH 5656 & 115 \\
\hline & CMN 8803 & 101 \\
\hline & CMN 8800 & 89 \\
\hline \multicolumn{2}{|c|}{$\begin{array}{l}\text { Mann-Whitney } \mathrm{U} \text { test ( } p \text {, exact } \\
\text { permutation) }\end{array}$} & 0.0159 \\
\hline
\end{tabular}


Table 14: Results of Mann-Whitney U test, testing for median differences in postorbital horncore basal circumference (parameter 4) between Chasmosaurus belli and C. russelli.

\begin{tabular}{|l|l|l|}
\hline Taxon & \multicolumn{1}{|c|}{ Specimen } & \multicolumn{1}{c|}{$\begin{array}{c}\text { Postorbital } \\
\text { horncore basal } \\
\text { circumference } \\
\text { (mm; parameter 4) }\end{array}$} \\
\hline $\begin{array}{l}\text { Chasmosaurus } \\
\text { belli }\end{array}$ & CMN 2245 & 238 \\
\cline { 2 - 3 } & ROM 843 & 252 \\
\hline $\begin{array}{l}\text { Chasmosaurus } \\
\text { russelli }\end{array}$ & TMP 1983.025.0001 & 345 \\
\cline { 2 - 3 } & CMN 2280 & 263 \\
\cline { 2 - 3 } & CMN 8800 & 412 \\
\hline \multirow{2}{*}{$\begin{array}{l}\text { Mann-Whitney U test }(p, \text { exact } \\
\text { permutation })\end{array}$} & 0.2 \\
\hline
\end{tabular}




\subsection{Discussion}

The three principal component analyses (PCAs) were performed to determine whether clusters of specimens referred to previously diagnosed Dinosaur Park Formation chasmosaurine taxa are significantly different in morphospace, and whether they remain distinct when differing subsets of specimens are included. The significant differences $(p<0.01)$ in specimen clusters in the first non-parametric multivariate analysis of variance (NPMANOVA 1; Table 10) are largely attributable to the considerable degree of separation between Vagaceratops-like and Chasmosaurus-like (Chasmosaurus belli, Chasmosaurus russelli and Chasmosaurus sp.) specimens along the PC2 axis of PCA 1 (Fig. 66A-B). The recovery of AMNH 5402 and YPM 2016 outside of the Chasmosaurus-like cluster on the PC2 axis supports the referral of these specimens to the Vagaceratops-like operative taxonomic grouping, as recommended in this study. Chasmosaurus belli and $C$. russelli are not significantly different in NPMANOVA 1 (Table 10).

The exclusion of the seven Chasmosaurus-like specimens missing the posterior margin of the frill (AMNH 5401, CMN 1254, CMN 8801, CMN 34829, ROM 839, TMP 1981.019.0175, and UALVP 40) in PCA 2 resulted in significantly different clusters corresponding to previously named taxa, C. belli, C. russelli and the Vagaceratops-like specimens (Table 11). However, in PCA 2, significant differences between taxa were inconsistent across number of principal components (PCs) used in NPMANOVA 2 (Table 11). The anomalously and inconsistently significant differences recovered between $C$. belli and C. russelli in PCA 2 amongst the three PCAs conducted in this study suggests that these putative taxa are morphometrically poorly distinguishable. This is 
supported by the lack of significant differences between C. belli and C. russelli specimens in PCA 3, which only included specimens of these taxa. Therefore, the null hypothesis ( $\mathrm{H} 0)$ of no morphometric difference between C. belli and C. russelli cannot be rejected. Differences between the results of the three PCAs and their respective NPMANOVAs and DFAs may be due to differing proportions of missing data estimation (Tables A1-3, Appendix IV).

Specimens previously referred to Chasmosaurus brevirostris (ROM 839; Lull, 1933), Chasmosaurus kaiseni (AMNH 5401; Brown, 1933), Eoceratops canadensis (CMN 1254 and UALVP 40; Tyson, 1977) and Mojoceratops perifania (AMNH 5401, AMNH 5656 and TMP 1983.025.0001; Longrich, 2010) cannot be distinguished morphometrically from other specimens previously referred to C. belli (CMN 2245, NHMUK R4948 and ROM 843) or C. russelli (CMN 2280 and CMN 8800) (Figs. 66, 68 and 70).

The discriminant function analyses (DFAs) were performed to determine which cranial parameters best distinguish C. belli, C. russelli and Vagaceratops-like specimens. Five of the six highest loading parameters differentiating Vagaceratops-like specimens from specimens of C. belli and C. russelli are shared between DFA 1 and 2. This suggests that Vagaceratops-like specimens differ primarily from C. belli and C. russelli specimens in having a relatively short rostral-to-epijugal length (parameter 16) and rostral-to-back of maxillary toothrow length (parameter 17), wide jugal notch (parameter 22), short skull length (parameter 26), and anteroposteriorly shortened parietal fenestrae (parameter 30). Two of these features, relatively wide jugal notches (parameter 22) and anteroposteriorly shortened parietal fenestrae (parameter 30), have been previously used in diagnosing 
Vagaceratops irvinensis (Holmes et al., 2001; Sampson et al., 2010). The anteroposterior length of the Vagaceratops-like skull YPM 2016 (463 mm) does, however, overlap with that of $C$. belli $(\mathrm{CMN} 2245=452 \mathrm{~mm}$ to $627 \mathrm{~mm}=$ NHMUK R4948). The other three features (parameters 16,17 and 26) indicate that Vagaceratops-like skulls are generally smaller than $C$. belli and C.russelli skulls.

The difference in skull size between Vagaceratops-like skulls and skulls of $C$. belli and C. russelli is unlikely to be ontogenetic because 10 of the 11 skulls tested are adult-sized, except for one C. russelli skull (AMNH 5656, subadult) (Fig. 63); the Vagaceratops skulls CMN 41357 and TMP 1987.045.0001 are both inferred as being adult sized based on their complete articulation of epiossifications and skull sizes that are similar to the other adult-sized Vagaceratops-like skulls AMNH 5402 and YPM 2016. It cannot be determined whether the skulls of adult sized Vagaceratops-like specimens are consistently smaller than adult sized skulls of $C$. belli and $C$. russelli due to the small sample size.

The DFA of C. belli and C. russelli specimens (DFA 3) indicates that the former differs primarily from the latter in having, in descending order of importance, a relatively small postorbital horncore basal circumference (parameter 4), large rostral-to-maxillary toothrow length (parameter 17) and shallowly embayed posterior parietal margin (parameter 33) (Fig. 71). The relative degree to which the posterior parietal margin is embayed (parameter 33) is the only one of the above differences that has been previously used in distinguishing C. belli and C. russelli (Maidment and Barrett, 2011). The relatively high loadings of parameters 4 and 17 are likely a product of missing data estimation and should be treated with caution. Of the seven specimens of $C$. belli and $C$. 
russelli, only three (CMN 2280, CMN 8800 and ROM 843) possess a complete, articulated anterior half of the skull and hence measurement for parameter 17. In two of these seven specimens, postorbital horncores are either not preserved (AMNH 5656) or could not be measured firsthand (NHMUK R4948), preventing a parameter 4 measurement for these specimens.

Chasmosaurus belli and C. russelli differ significantly in the relative degree to which their posterior parietal margins are embayed (Table 13), supporting the alternative hypothesis (H1) of two morphotypes within Chasmosaurus. Although these two morphotypes can be distinguished on the basis of embayment angle, this feature is highly gradational, spanning $26^{\circ}$ in $C$. belli $\left(\right.$ NHMUK R4948 $=162^{\circ}$ to $136^{\circ}=$ ROM 843) and $39^{\circ}$ in C. russelli (TMP $1983.025 .0001=128^{\circ}$ to $89^{\circ}=\mathrm{CMN} 8800$ ), with only $8^{\circ}$ separating these putative taxa (Table 13).

There is no significant difference in postorbital horncore basal circumference between adult specimens previously assigned to C. belli and C. russelli (Table 14), supporting the null hypothesis $\left(\mathrm{H}_{0}\right)$ that this feature, and by extension, postorbital horncore length, is not taxonomically significant within Chasmosaurus. However, the previous findings that there are two statistically significant, discrete clusters of specimens corresponding to the two postorbital horncore ontogenetic trajectories (NPMANOVA; Table 8) inferred in Chapter 8 indicates that postorbital horncore size is instead attributable to some other factor, possibly individual variation or sexual dimorphism. Given that postorbital horncore growth follows one of two ontogenetic trajectories culminating in either relatively short, small circumference-based horncores, or long, large 
circumference-based horncores (Figs. 59-62 and 65), it is likely that these two growth trajectories are the result of sexual dimorphism. It is proposed in this study that short and long postorbital horncores correspond to females and males, respectively, as generally occurs in extant bovids (Estes, 1991). Sexual dimorphism has also been invoked to account for the bimodal distribution of cranial display structures in extinct archosaurian taxa (e.g., Dodson, 1976; Lehman, 1990; Bennett, 1992; Zeigler et al., 2002; Lü et al., 2011).

The range of postorbital horncore basal circumferences within the Vagaceratopslike specimens (AMNH $5402=190 \mathrm{~mm}$ to $250 \mathrm{~mm}=\mathrm{CMN} 41357)$ all fall within the smaller of the two groups for Chasmosaurus-like specimens (Figs. 60, 62, 65, and 72). However, the sample size of Vagaceratops-like specimens is too small to speculate on the possible range of variation within the taxon.

Chasmosaurus-like specimens with relatively small postorbital horncore basal circumferences and inferred short horncores (i.e., females) encompass all C. belli specimens possessing horncores (CMN 2245 and ROM 843; likely NHMUK R4948 as well, but basal circumference could not be measured) and one specimen of C. russelli (CMN 2280) (Fig. 72). The remaining C. russelli specimens possessing horncores (CMN 8800 and TMP 1983.025.0001) have relatively large basal circumferences and inferred long horncores (i.e., males) (Fig. 72). This distribution of postorbital horncore sizes across Chasmosaurus-like specimens suggests that putative females and males generally possess relatively shallowly (NHMUK R $4948=162^{\circ}$ to $124^{\circ}=\mathrm{CMN} 2280$ ) and deeply (TMP 1983.025.0001 $=128^{\circ}$ to $89^{\circ}=\mathrm{CMN} 8800$ ) embayed posterior parietal margins, respectively (Fig. 72). Following this model, males have deeper and more pronounced 


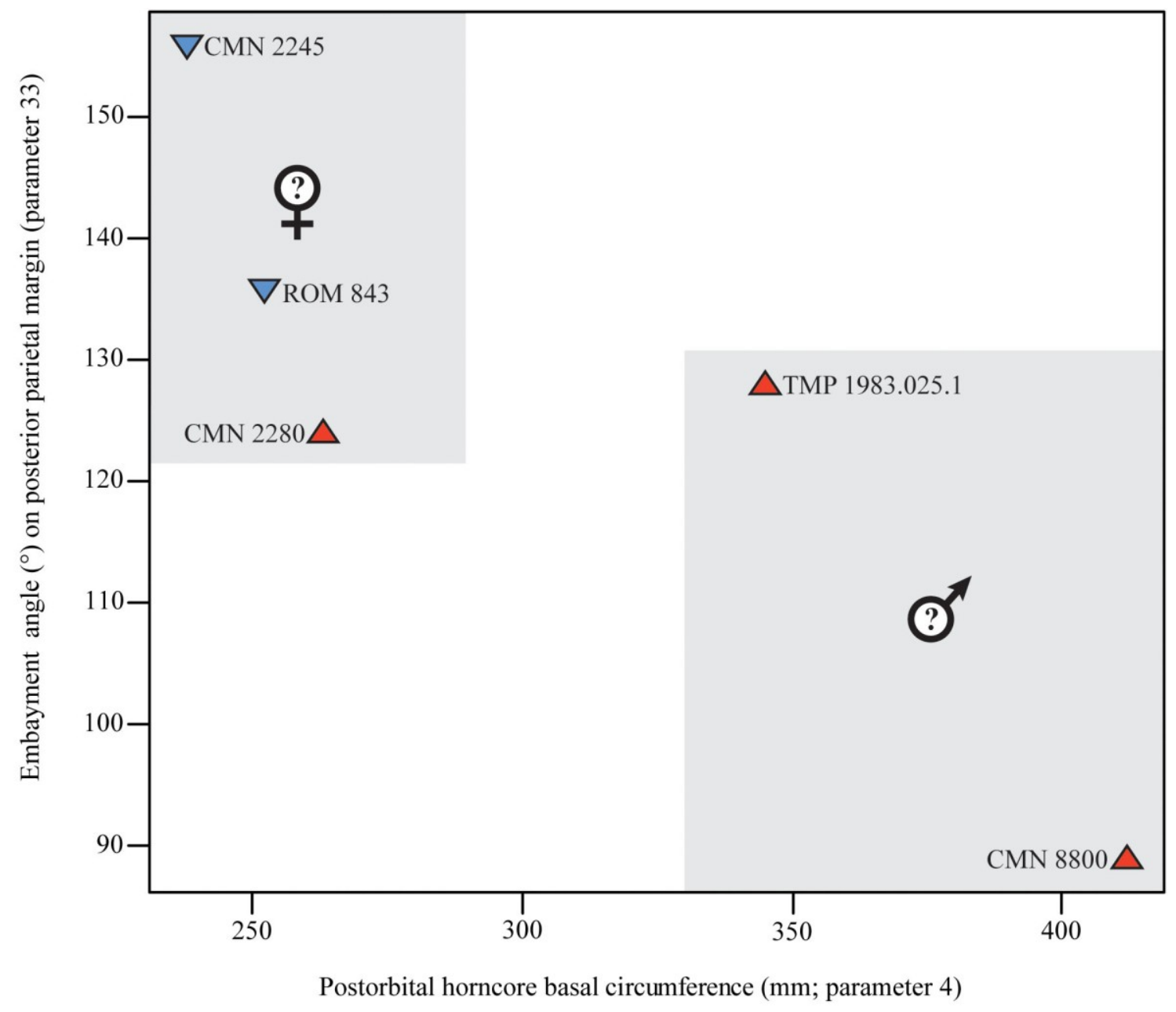

Figure 72. Scatter plot of postorbital horncore basal circumference (parameter 4, Fig. 5) vs. embayment angle on posterior parietal margin (parameter 33, Fig. 5) in specimens of Chasmosaurus belli (blue inverted triangles) and C. russelli (red triangles). Shaded boxes delimit the range of postorbital horncore sizes (see Figures 59-62 and 65) and embayment angles in putative males and females; "females" = short horncores, relatively shallowly embayed margins, and "males" = long horncores, relatively deeply embayed margins. 
posterior embayments, and consequently parietal fenestrae that were more posteriorly expanded relative to the midline of the frill (Fig. 33A-F). Conversely, females have shallower and less pronounced posterior embayments, with parietal fenestrae that do not extend as far posteriorly relative to the midline of the frill (Fig. 33G-L). Differences between inferred male and female specimens, with respect to postorbital horncore basal circumferences and posterior parietal embayments, in Figure 72 are unlikely attributable to ontogenetic differences, as all of these specimens were recovered as adults in Figure 63.

Assuming that the above interpretations and model are correct, and that the parietosquamosal frill served, in part, as a secondary sexual display feature in ceratopsians (Dodson, 1976; Lehman, 1990), it is reasonable that the relative embayment of the posterior parietal margin, as previously used to distinguish C. belli and C. russelli, is instead largely attributable to sexual dimorphism within a single taxon (contra Godfrey and Holmes, 1995; Maidment and Barrett, 2011; sensu Lehman, 1990). Differences within each putative gender with respect to embayment angle are here attributed to individual variation.

\subsection{Conclusion}

The findings indicate that Vagaceratops-like specimens are distinguishable from Chasmosaurus-like specimens. Chasmosaurus belli and Chasmosaurus russelli specimens are distinguishable, but only by the relative embayment of the posterior parietal margin. Vagaceratops-like specimens differ from Chasmosaurus-like specimens in the anteroposterior length of the parietal fenestrae (parameter 30), the width of the 
jugal notch (parameter 22), and the length of the skull (parameters 16, 17 and 26). The relative embayment of the posterior parietal margin is significantly different between $C$. belli and C. russelli, but the amount of intraspecific variation $\left(26^{\circ}\right.$ in C. belli, $39^{\circ}$ in $C$. russelli) is relatively large compared to its interspecific variation ( $8^{\circ}$; compare ROM 843 , C. belli $=136^{\circ}$ with $128^{\circ}=$ TMP 1983.025.0001, C. russelli).

The two significantly different ontogenetic trajectories in postorbital horncore size (short, small circumference-based horncores and long, large circumference-based horncores) discussed in Chapter 8 do not correspond to C. belli and C. russelli, indicating that postorbital horncore size does not have taxonomic utility within Chasmosaurus. Postorbital horncore size may instead represent a sexually dimorphic character, as is the case in extant bovids. In general, short-horned members (putative females) possess relatively shallowly embayed posterior parietal margins, whereas long-horned members (putative males) possess relatively deep embayments. This may suggest that the relative degree to which the posterior parietal margin is embayed - as previously used in distinguishing $C$. belli and C. russelli - instead represents sexual dimorphism within one species of Chasmosaurus (sensu Lehman, 1990). The degree of dimorphism in cranial display structures (i.e., postorbital horncore size and relative posterior embayment) in Chasmosaurus is similar to that of other extinct archosaurs, the latter of which has been suggested as representing sexual differences (e.g., Dodson, 1976; Lehman, 1990; Bennett, 1992; Zeigler et al., 2002; Lü et al., 2011). 


\subsection{Addendum: revised morphometric analyses}

\subsubsection{Introduction}

An additional principal component analysis (PCA 4) was performed, using the 18 Vagaceratops-like $(\mathrm{n}=4)$, Chasmosaurus belli $(\mathrm{n}=3)$, Chasmosaurus russelli $(\mathrm{n}=4)$, and Chasmosaurus sp. $(\mathrm{n}=7)$ specimens. All of these specimens were included in order to maximize the predictability power of missing data estimation, which was conducted using Bayesian principal component analysis (BPCA). However, of the 25 cranial parameters used in the first three PCAs, only 12 were included in PCA 4. This reduction was done, as the number of parameters should not exceed the number of specimens in a PCA. If the number of principal components (PCs; i.e., the number of parameters) exceeds the number of specimens, the surplus PCs will not account for any of the total variance in the dataset (Hammer and Harper, 2006). The 12 parameters included are as follows: postorbital horncore length (parameter 1) and basal circumference (parameter 4), nasal horncore length (parameter 5), distance between rostral and epijugal (parameter 16), distance between orbits (parameter 18), distance between jugal notch and distal end of squamosal (parameter 19), width of jugal notch (parameter 22), width of frill (parameter 27), distance between jugal notch and back of frill (parameter 28), anteroposterior length of parietal fenestra (parameter 30), and thickness (parameter 31) and width (parameter 32) of posterior end of medial parietal bar (Fig. 5).

The remaining 13 parameters were excluded from PCA 4, as they were either significantly correlated with the above 12 parameters, or had not been previously used in diagnosing chasmosaurine species from the Dinosaur Park Formation. The relative embayment of the posterior parietal bar (parameter 33) was excluded from PCA 4, as it is 
a cyclic measurement (i.e., $0^{\circ}=360^{\circ}$ ); this exclusion was also made to determine whether $C$. belli and $C$. russelli are significantly different in terms of cranial parameters other than parameter 33. As all parameters included in PCA 4 were measured using the same unit (mm), this PCA was conducted using the variance-covariance matrix. The estimated, complete dataset generated by BPCA, and subsequently used in PCA 4, is given in Table A10 (Appendix IV).

The scores of each PC axis in PCA 4 were then plotted against parameter 17 (rostral to back of maxillary toothrow), which is a skull size-related parameter. This was done to determine whether any of the PCs are strongly correlated with skull size. Significant differences between taxonomic clusters of specimens along skull size-related PC axes should be treated with caution, as these differences are strongly influenced by ontogeny.

An attempt was also made to reduce the confounding effects of variable skull sizes as a result of ontogenetic differences. This was done by plotting each of the 12 complete, estimated cranial parameters used in PCA 4 against the skull size-related parameter 17 (rostral to back of maxillary toothrow), and assigning a line-of-best-fit. The parameter residuals obtained from the line-of-best-fit were then included in an additional PCA (PCA 5). PCA 4 and 5 were both conducted using the R statistical programming language software (R Development Core Team, 2011).

An analysis of variance (ANOVA) was performed on taxonomic clusters of specimens that showed separation along a given PC axis; posthoc Mann-Whitney pairwise comparisons were also conducted, using both Bonferroni correction and uncorrected probabilities. A non-parametric multivariate analysis of variance 
(NPMANOVA) was also performed on taxonomic clusters that showed separation along pairs of PC axes, using the Mahalanobis distance measure (Mahalanobis, 1936). An ANOVA was also performed to determine whether the relative embayment of the posterior parietal bar is significantly different between Vagaceratops-like, C. belli and $C$. russelli specimens. ANOVAs and NPMANOVAs were both conducted using PAST 3.01 (Hammer et al., 2001).

\subsubsection{Results}

The results of each PCA (and associated ANOVA) are presented in sequential order, followed by the results of the ANOVA testing for differences in the posterior parietal embayment between Vagaceratops-like, C. belli and C. russelli specimens.

In PCA 4, the first five PCs account for $86.0 \%$ of the total variance (Fig. 73A-D;

Table A11, Appendix IV). In PC1 and PC2, most parameters are subequal, and load negatively and positively, respectively, indicating that skull size is a major influence on the variation in these axes (Table A11, Appendix IV); parameters 18, 22 and 31 load positively in PC1, and parameters 1, 30 and 32 load negatively in PC2. Variation along the remaining PC axes is largely independent of size. The Vagaceratops-like specimens score on the positive side of PC1 (32.3\% of variance; Fig. 73A), while Chasmosauruslike specimens (C. belli, C. russelli and Chasmosaurus sp.) score mostly on the negative side; the recovery of the Chasmosaurus sp. specimen, CMN 34829, in the Vagaceratopslike cluster is likely attributable to missing data estimation, as the anteroposterior length of the parietal fenestrae in this specimen is more consistent with that of Chasmosaurus than with Vagaceratops. The highest loading parameters on PC1, in descending order of 
importance, indicate that the Vagaceratops-like specimens differ from C. russelli in having a relatively longer distance between the jugal notch and the back of the frill (parameter 28), anteroposteriorly shorter parietal fenestrae (parameter 30) and shorter postorbital horncores (parameter 1) (Table A11, Appendix IV).

In PCA 4, an ANOVA revealed significant differences $(p<0.01)$ amongst the Vagaceratops-like, C. russelli, and C. belli specimens along the PC1 axis (Table 15). Posthoc pairwise comparisons reveal that there is a significant difference $(p<0.05)$ between Vagaceratops-like and C. russelli specimens along this axis. The PC1 and PC2 axes in PCA 4 are significantly correlated ( $p<0.05$ and 0.01 , respectively) with parameter 17 (rostral to back of maxillary toothrow), and hence skull size (Fig. 74A-B). 
Figure 73. Principal component analysis (PCA 4) of 18 specimens of the Vagaceratopslike specimens $(\mathrm{n}=4)$, Chasmosaurus russelli $(\mathrm{n}=4)$, C. belli $(\mathrm{n}=3)$, and Chasmosaurus sp. $(\mathrm{n}=7)$ : A), PC1 vs. PC2, cumulatively accounting for $52.5 \%$ of the total variance; B), PC2 vs. PC3, cumulatively accounting for $35.4 \%$ of the total variance; C), PC3 vs. PC4, cumulatively accounting for $27.6 \%$ of the total variance; D), PC4 vs. PC5, cumulatively accounting for $18.3 \%$ of the total variance. 


\section{Figure 73.}

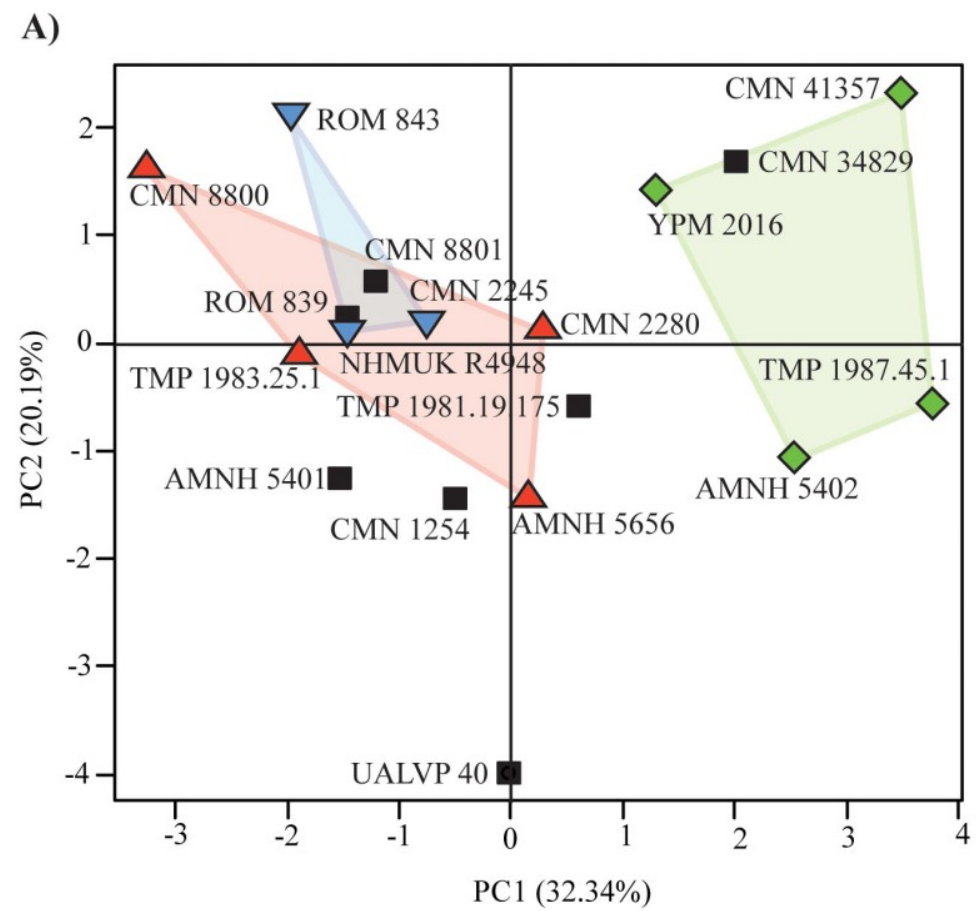

\footnotetext{
$\checkmark$ Vagaceratops-like specimens

$\triangle$ Chasmosaurus russelli

$\nabla$ Chasmosaurus belli

- Chasmosaurus sp.
}

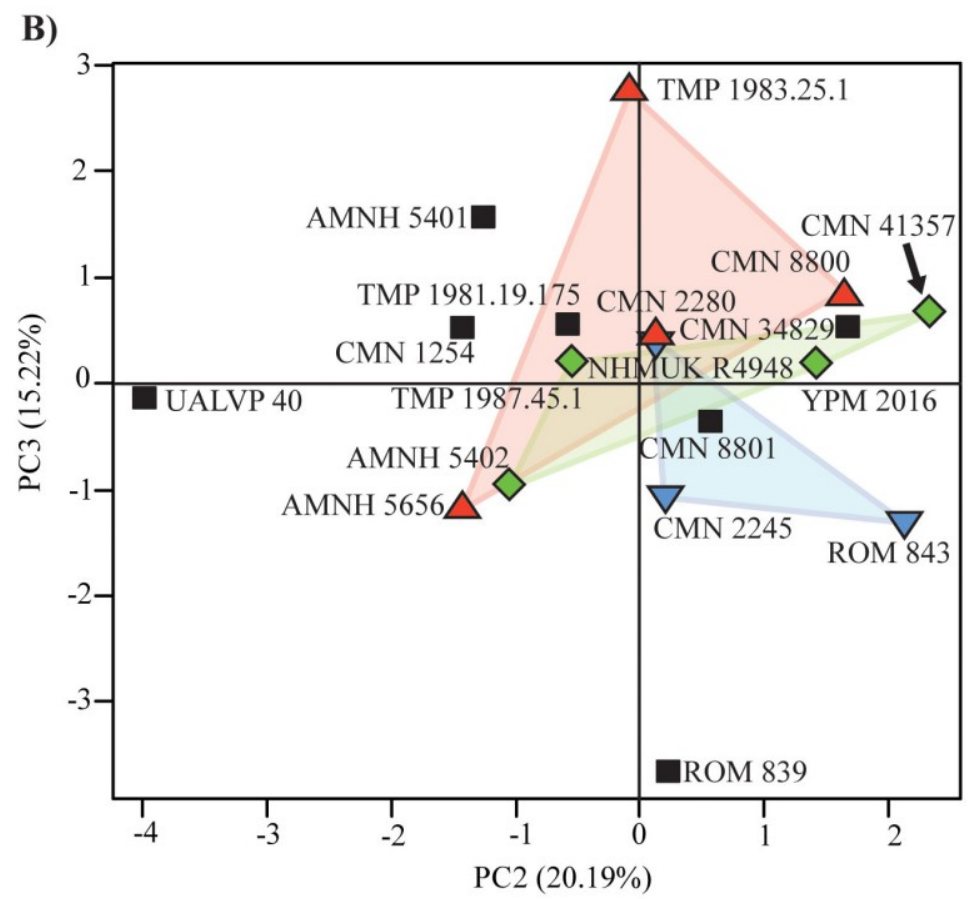


Figure 73. (continued).

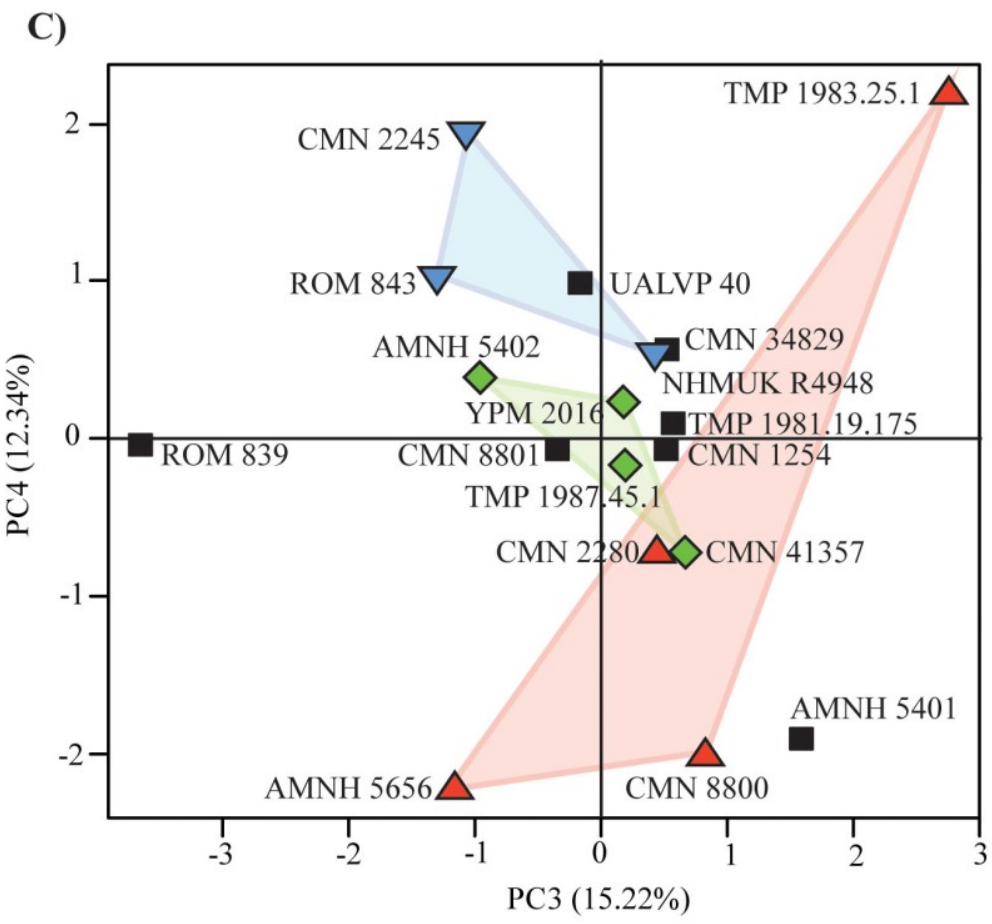

D)

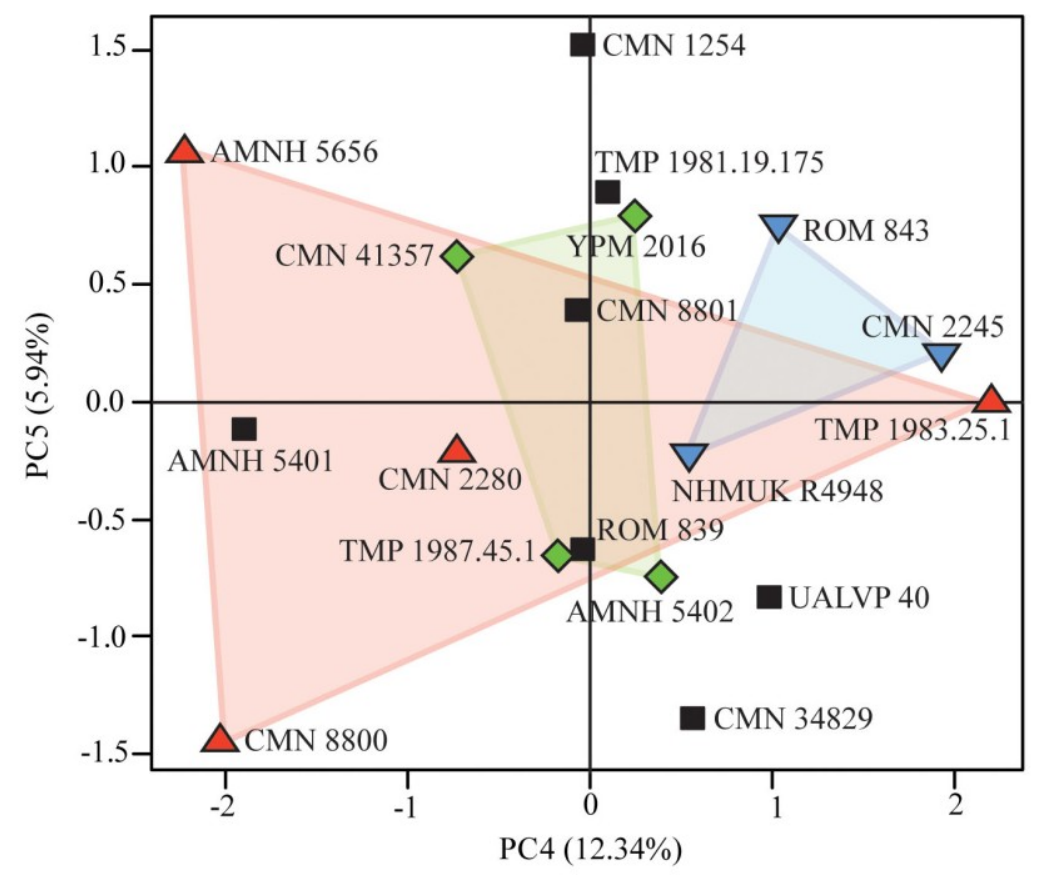


Table 15: Results of ANOVA of Vagaceratops-like, Chasmosaurus russelli, and C. belli specimens along principal component axis 1 of PCA 4. Posthoc pairwise comparisons shown in centre; uncorrected $p$-values shown in upper, white cells, and Bonferroni corrected $p$-values shown in lower, grey cells. $F$ and $p$-values for total analysis shown on right. Significant results $(p<0.05)$ shown in bold.

\begin{tabular}{|c|c|c|c|c|c|c|}
\hline \multirow{2}{*}{\multicolumn{2}{|c|}{$\begin{array}{l}\text { PC included in ANOVA; } \\
\text { variance accounted by PC } \\
(\%)\end{array}$}} & \multicolumn{3}{|c|}{ Posthoc pairwise comparisons } & \multicolumn{2}{|c|}{ Total analysis } \\
\hline & & Vagaceratops- & C. russelli & C. belli & $F$ & $p$ \\
\hline \multirow{3}{*}{$\begin{array}{l}\mathrm{PC} 1 \\
(32.3)\end{array}$} & $\begin{array}{l}\text { Vagaceratops-like } \\
\text { specimens }(\mathrm{n}=4)\end{array}$ & & 0.0304 & 0.0518 & \multirow[t]{3}{*}{18.37} & \multirow[t]{3}{*}{0.0045} \\
\hline & C. russelli $(\mathrm{n}=4)$ & 0.0912 & & 0.8597 & & \\
\hline & C. belli $(\mathrm{n}=3)$ & 0.1555 & 1 & & & \\
\hline
\end{tabular}


Figure 74. Scatter plot showing parameter 17 (rostral to back of maxillary toothrow) vs. principal component axis 1 (A) and 2 (B) of principal component analysis (PCA 4) of 18 specimens of the Vagaceratops-like specimens $(\mathrm{n}=4)$, Chasmosaurus russelli $(\mathrm{n}=4), C$. belli $(\mathrm{n}=3)$, and Chasmosaurus sp. $(\mathrm{n}=7)$; lines-of-best-fit are $p=0.0279$ and 0.0029 , respectively. 


\section{Figure 74.}
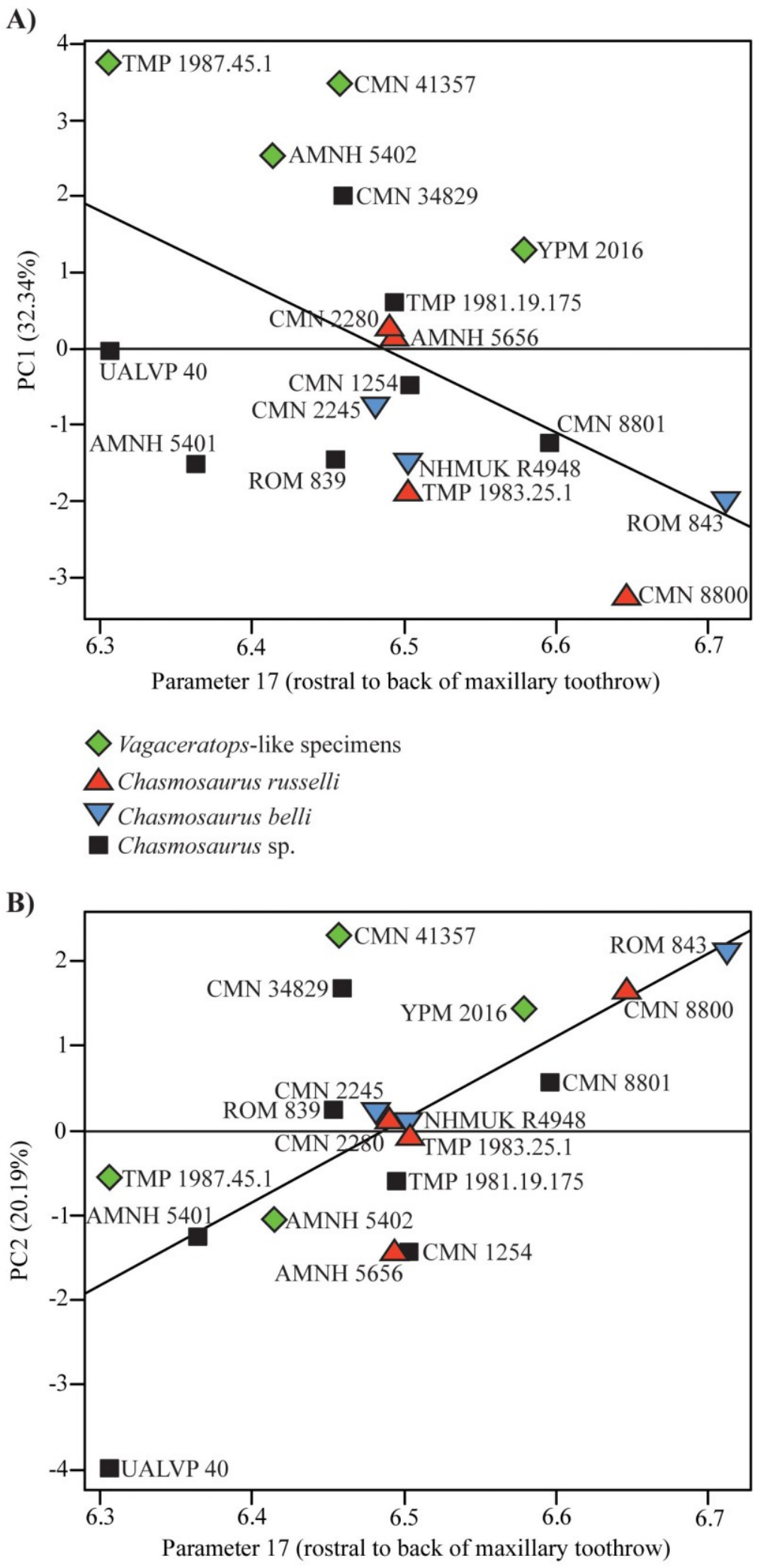
In PCA 5, the first four PCs account for 90.2\% of the total variance (Fig. 75A-C; Table A12, Appendix IV). There is considerable variation in parameter loadings along each of these axes, indicating that skull size is not a major influence on the variation in this axes. The Vagaceratops-like specimens score on the positive side of $\mathrm{PC} 1$ (66.8\% of variance; Fig. 75A), while Chasmosaurus-like specimens (C. belli, C. russelli and Chasmosaurus sp.) score mostly on the negative side; again, the recovery of the Chasmosaurus sp. specimen, CMN 34829, in the Vagaceratops-like cluster is likely attributable to missing data estimation. The highest loading parameters on PC1, in descending order of importance, indicate that the Vagaceratops-like specimens differ from C. russelli in having relatively shorter postorbital horncores (parameter 1), anteroposteriorly shorter parietal fenestrae (parameter 30) and a wider jugal notch (parameter 22) (Table A12, Appendix IV).

In PCA 5, an ANOVA indicates significant differences $(p<0.01)$ amongst the Vagaceratops-like, C. russelli, and C. belli specimens along the PC1 axis (Table 16). Posthoc pairwise comparisons reveal that there is a significant difference $(p<0.05)$ between Vagaceratops-like and C. russelli specimens along this axis.

An ANOVA of Vagaceratops-like, C. belli and C. russelli specimens indicates that these taxa are significantly different with regards to the relative embayment of the posterior parietal bar (Table 17). 
Figure 75. Principal component analysis (PCA 5) of 18 specimens of the Vagaceratopslike specimens $(\mathrm{n}=4)$, Chasmosaurus russelli $(\mathrm{n}=4)$, C. belli $(\mathrm{n}=3)$, and Chasmosaurus sp. $(\mathrm{n}=7)$ : A), PC1 vs. PC2, cumulatively accounting for $77.2 \%$ of the total variance; B), PC2 vs. PC3, cumulatively accounting for $17.2 \%$ of the total variance; C), PC3 vs. PC4, cumulatively accounting for $12.9 \%$ of the total variance. 
Figure 75.

A)

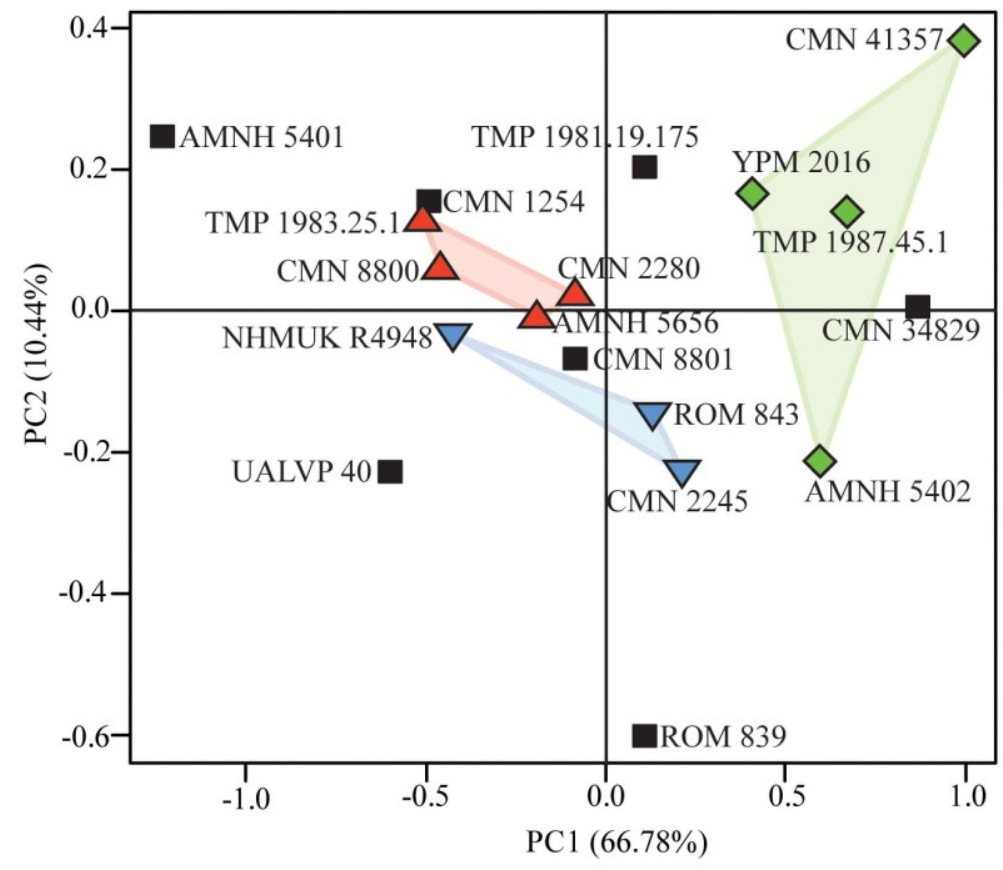

Vagaceratops-like specimens

$\triangle$ Chasmosaurus russelli

$\nabla$ Chasmosaurus belli

- Chasmosaurus sp.

B)

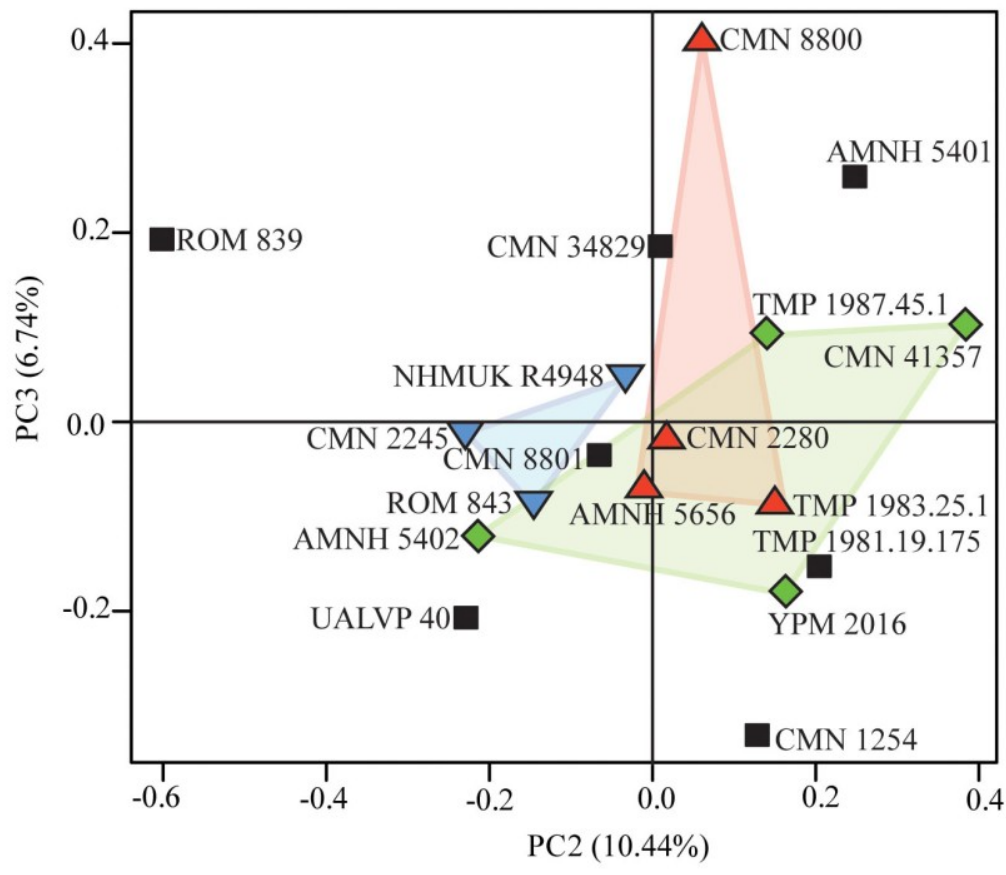


Figure 75. (continued).

\section{C)}

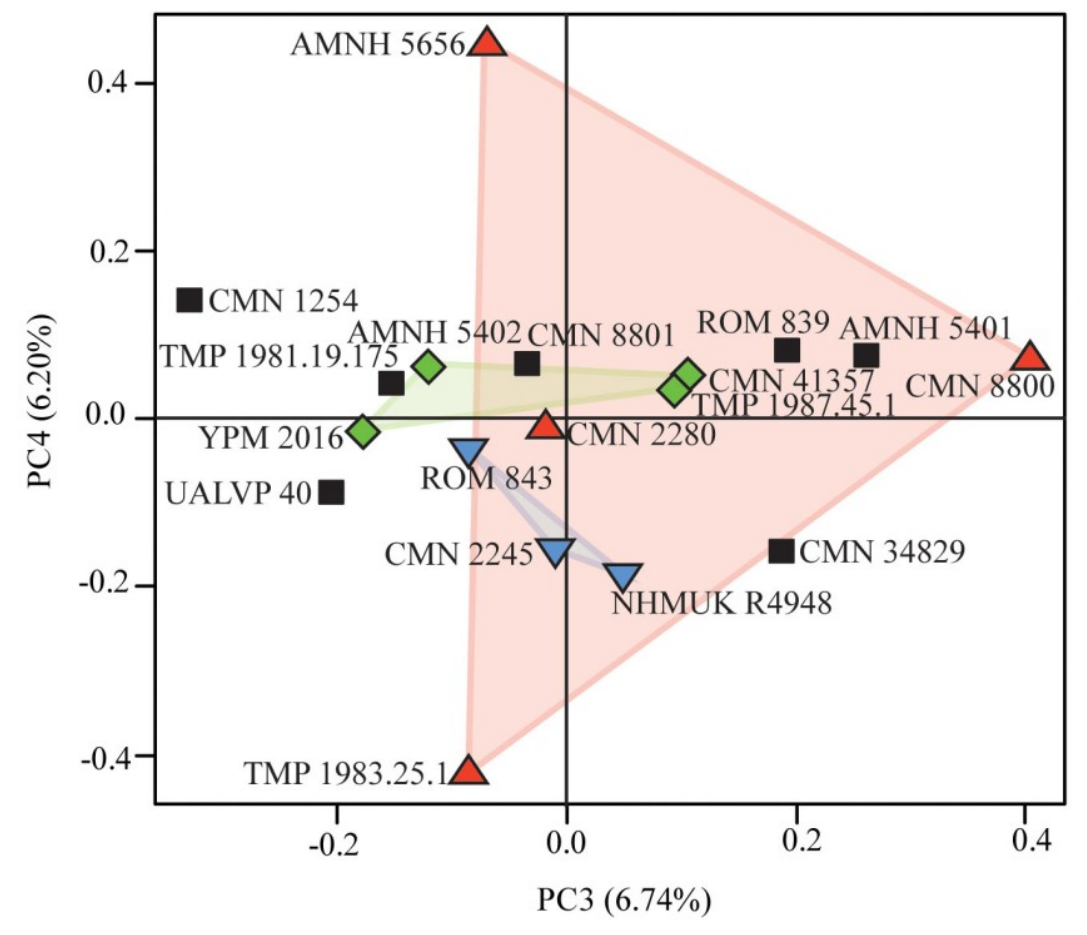


Table 16: Results of ANOVA of Vagaceratops-like, Chasmosaurus russelli, and C. belli specimens along principal component axis 1 of PCA 5. Posthoc pairwise comparisons shown in centre; uncorrected $p$-values shown in upper, white cells, and Bonferroni corrected $p$-values shown in lower, grey cells. $F$ and $p$-values for total analysis shown on right. Significant results $(p<0.05)$ shown in bold.

\begin{tabular}{|l|l|l|l|l|l|c|}
\hline \multirow{2}{*}{$\begin{array}{l}\text { PC included in ANOVA; } \\
\text { variance accounted by PC } \\
(\%)\end{array}$} & \multicolumn{2}{|l|}{ Posthoc pairwise comparisons } & \multicolumn{2}{l|}{ Total analysis } \\
\cline { 2 - 6 } & $\begin{array}{l}\text { Vagaceratops- } \\
\text { like specimens } \\
(\mathrm{n}=4)\end{array}$ & $\begin{array}{l}\text { C. russelli } \\
(\mathrm{n}=4)\end{array}$ & $\begin{array}{l}\text { C. belli } \\
(\mathrm{n}=3)\end{array}$ & $F$ & $p$ \\
\hline \multirow{2}{*}{ PC1 } & $\begin{array}{l}\text { Vagaceratops-like } \\
\text { specimens (n=4) }\end{array}$ & & $\mathbf{0 . 0 3 0 4}$ & 0.0518 & 16.06 & $\mathbf{0 . 0 0 9 0}$ \\
\cline { 2 - 5 } & C. russelli $(\mathrm{n}=4)$ & 0.0912 & 0.6478 & & 0.2159 & \\
\cline { 2 - 5 } & C. belli $(\mathrm{n}=3)$ & 0.1555 & & & \\
\hline
\end{tabular}


Table 17: Results of ANOVA, testing for median differences in the relative embayment of the posterior parietal margin (parameter 33) between Vagaceratops-like specimens, Chasmosaurus russelli and C. belli. Significant results $(p<0.05)$ shown in bold. Posthoc pairwise comparisons shown in centre; uncorrected $p$-values shown in upper, white cells, and Bonferroni corrected $p$-values shown in lower, grey cells. $F$ and $p$-values for total analysis shown on right. Significant results $(p<0.05)$ shown in bold.

\begin{tabular}{|l|l|l|l|c|c|}
\hline Taxon & \multicolumn{2}{|l|}{ Posthoc pairwise comparisons } & \multicolumn{2}{l|}{ Total analysis } \\
\cline { 2 - 5 } & $\begin{array}{l}\text { Vagaceratops- } \\
\text { like specimens } \\
(\mathrm{n}=4)\end{array}$ & $\begin{array}{c}\text { C. russelli } \\
(\mathrm{n}=4)\end{array}$ & $\begin{array}{l}\text { C. belli } \\
(\mathrm{n}=3)\end{array}$ & $F$ & $p$ \\
\hline $\begin{array}{l}\text { Vagaceratops-like } \\
\text { specimens (n=4) }\end{array}$ & & $\mathbf{0 . 0 3 5 8}$ & $\mathbf{0 . 0 4 9 8}$ & 33.45 & $\mathbf{0 . 0 0 0 7}$ \\
\cline { 1 - 4 } C. russelli $(\mathrm{n}=4)$ & 0.1073 & 0.0599 & $\mathbf{0 . 0 2 0 0}$ & \\
\cline { 1 - 4 } C. belli $(\mathrm{n}=3)$ & 0.1492 & & & \\
\hline
\end{tabular}




\subsubsection{Discussion and conclusion}

The results indicate that $C$. belli and $C$. russelli do not differ significantly, except in terms of the relative embayment of the posterior parietal bar (parameter 33), which can be used to easily distinguish these taxa (Fig. 33). The relatively poor morphological distinction between $C$. belli and C. russelli, expect for the parietal embayment angle, supports the null hypothesis of a single species of Chasmosaurus. Vagaceratops-like specimens are morphometrically well-resolved, having a significantly shallower posterior parietal embayment than Chasmosaurus (as well as four or five epiparietals versus three), and shorter postorbital horncores, parietal fenestra anteroposterior length, and wider jugal notches than C. russelli. The relatively anteroposteriorly short parietal fenestrae and wide jugal notches of Vagaceratops-like specimens have been previously used in diagnosing Vagaceratops (Holmes et al., 2001; Sampson et al., 2010). 


\section{Chapter 10. Conclusions}

The number of valid species of Chasmosaurus: This thesis tested several hypotheses concerning the number of valid species that can be diagnosed within the genus Chasmosaurus, a chasmosaurine ceratopsid known exclusively from the Dinosaur Park Formation (Campanian) of the Late Cretaceous of Alberta. A specimen-based phylogenetic analysis produced a polytomic strict consensus tree that did not cluster any of the specimens of the two currently recognized species, Chasmosaurus belli and Chasmosaurus russelli, together. A stratophylogenetic analysis (run with the addition of precise stratigraphic placement data as an additional character) indicated that the specimens referred to $C$. belli and $C$. russelli were coeval and not separated stratigraphically, supporting the referral of all examined specimens to the taxon with historical precedence, Chasmosaurus belli. These two putative taxa are also known from the same geographic regions; however, it is not known whether they occupied these regions at the same time (i.e., sympatric taxa), due to the absence of approximately timeequivalent specimens of each taxon.

The mosaic ontogenetic distribution of Chasmosaurus-like specimens previously referred to $C$. belli and $C$. russelli indicates that these two putative species do not represent separate age classes of a single species, as has been postulated for Triceratops and Torosaurus (Scannella and Horner, 2010). Specimens historically referred to Eoceratops canadensis (CMN 1254 and UALVP 40) cannot be differentiated from other Chasmosaurus-like specimens, and are here considered to be relatively immature members (juvenile and subadult, respectively) of the latter taxon. The relatively elongate 
postorbital horncores used to diagnose Chasmosaurus kaiseni (AMNH 5401) are not unique to this taxon, as other Chasmosaurus-like specimens possess large circumferencebased horncores with truncated apices (e.g., CMN 8800, TMP 1981.019.0175 and TMP 1983.025.0001) that are interpreted as having been removed by bone resorption; $C$. kaiseni is here considered to be a relatively immature member (large subadult) of this latter taxon.

The relatively short snout of Chasmosaurus brevirostris (ROM 839) is not unique to this taxon, and is within the range of variation of other Chasmosaurus-like specimens. Snout length is also variable amongst other chasmosaurines such as Triceratops (Horner and Goodwin, 2006) and Anchiceratops (Mallon et al., 2011). The relatively long snout of Anchiceratops longirostris was originally used to diagnose this taxon (Sternberg, 1929), but was later attributed to individual variation within another species, Anchiceratops ornatus (Mallon et al., 2011). C. brevirostris is therefore not considered to be a valid species in this study.

Mojoceratops perifania (TMP 1983.025.0001) was originally diagnosed by Longrich (2010) for its elongate postorbital horncores, anteroposteriorly elongate parietal fenestrae, pronounced sulcus on the parietal along the posteromedial margin of the parietal fenestrae, relatively deeply embayed posterior parietal margin, and epiparietals oriented in the plane of the frill. All of the above features in TMP 1983.025.0001, and in other referred specimens of M. perifania (AMNH 5401, AMNH 5656, CMN 8803, TMP 1979.011.0147, and TMP 1999.055.0292), occur in other Chasmosaurus-like specimens and do not represent robust diagnostic features of this former taxon. The parietal sulcus described above was found to be ontogenetic in Chasmosaurus-like specimens, becoming 
more pronounced in relatively mature specimens. The ontogenetic reorientation of the posterior parietal margin and epiparietals from plane-of-frill to anterodorsal suggests that the condition in M. perifania is ontogenetic and not taxonomic. In the absence of diagnostic features, M. perifania is regarded as an invalid taxon in this study.

Postorbital horncore size increases over the course of Chasmosaurus ontogeny, but follows two developmental trajectories culminating in relatively short, small circumference-based horncores and long, large circumference-based horncores, respectively. C. belli and C. russelli do not correspond to these two trajectories, suggesting that postorbital horncore size is not taxonomic within Chasmosaurus-like specimens. Representative specimens of each trajectory overlap stratigraphically, indicating that postorbital horncore size does not change over evolutionary time in Chasmosaurus-like specimens. These two trajectories are instead attributed to sexual dimorphism in this study, with females having relatively short, small circumferencebased horncores, and males having relatively long, large circumference-based horncores for mate competition purposes, as occurs in extant bovids. Sexual dimorphism has also been invoked to account for the bimodal distribution of cranial display structures in extinct archosaurian taxa (e.g., Dodson, 1976; Lehman, 1990; Bennett, 1992; Zeigler et al., 2002; Lü et al., 2011).

The inconsistent recovery of Chasmosaurus-like specimens previously referred to C. belli and C. russelli as distinct morphotypes within the morphometric analyses conducted suggests that these two putative taxa are poorly distinguished from each other. However, C. belli and C. russelli do differ significantly in regards to the relative embayment of the posterior parietal margin, the main feature previously used in 
distinguishing these two putative taxa (Godfrey and Holmes, 1995; Maidment and Barrett, 2011). In general, specimens with relatively short postorbital horncores have relatively shallowly embayed posterior parietal margins (i.e., all Chasmosaurus-like specimens previously referred to $C$. belli and one relatively shallowly embayed specimen of $C$. russelli), whereas those with long horncores have deeply embayed margins (i.e., other specimens previously referred to C. russelli). The association between embayment angle and putatively sexually dimorphic postorbital horncore size suggests that this former feature is also sexually dimorphic.

Based on the above findings, Chasmosaurus is most likely represented by a single, sexually dimorphic species, C. belli, as it has historical taxonomic priority.

Vagaceratops-like specimens: Vagaceratops irvinensis was originally diagnosed by Holmes et al. (2001) as having anteroposteriorly shortened parietal fenestrae with length/width ratios ranging from 0.72 (CMN 41357) to 0.87 (TMP 1987.045.0001). The assignment of the previously referred Chasmosaurus specimens AMNH 5402 (1.04) and YPM 2016 (1.03) to the Vagaceratops-like operative taxonomic unit expands the Vagaceratops parietal fenestrae length/width ratio range to $0.72-1.04$. Chasmosaurus-like specimens have a relatively wide range of parietal fenestra length/width ratios, spanning 1.09 (ROM 843) to 1.89 (AMNH 5656). Given the large range of length/width ratios amongst Chasmosaurus-like specimens, and that the Vagaceratops-like operative taxonomic unit is represented by relatively few specimens, it is plausible that Vagaceratops possessed a larger range of parietal fenestra ratios than was previously reported. The presence of postorbital horncores in AMNH 5402 suggests that the 
similarly positioned rugose mounds present in other Vagaceratops-like specimens (CMN 41357, TMP 1987.045.0001 and YPM 2016) represent the bases of remodeled horncores (sensu Holmes et al., 2001).

As pointed out by Mayr (1969), the stratigraphic delimitation of a given species is rather artificial, due to evolutionary continuity. Rather than one discrete morphotype or species replacing another over time, the morphological change is more gradual and may not always be preserved in the fossil record; therefore, intermediate forms between two species may not be preserved. The notion that a Vagaceratops-like chasmosaurine, represented by YPM 2016, with the rare 10 epiparietal condition went extinct, and was replaced stratigraphically by a modified but similar chasmosaurine also with the rare 10 epiparietal condition ( $V$. irvinensis) is unlikely. The Vagaceratops-like operative taxonomic unit is therefore considered to be a single species, $V$. irvinensis, that underwent morphological change over its inferred evolutionary history. The following evolutionary changes are inferred to have taken place: a reduction in the parietal fenestra length/width ratio, and an elongation and anterodorsal recurvature of the body of epiparietals 1-4. However, the scarcity of Vagaceratops-like specimens from Dinosaur Park Faunal Zone 2 (i.e., TMP 2009.034.0009) makes it difficult to test whether these proposed morphological changes occurred over time.

When Chasmosaurus irvinensis was first described by Holmes et al. (2001), this species was recovered in the Chasmosaurus clade, as a sister taxon to C. belli. As $C$. irvinensis was well-nested within the Chasmosaurus clade, Holmes et al. (2001) referred this species to Chasmosaurus while recognizing it probably represented a new taxon. Sampson et al. (2010), who first recovered the C. irvinensis-Kosmoceratops richardsoni 
clade in their analysis, used this as the basis for moving $C$. irvinensis into the new genus Vagaceratops, forming $V$. irvinensis. The restoration of Vagaceratops to its original sister taxon relationship with Chasmosaurus (sensu Holmes et al., 2001) in this study could be used as support for reinstating $V$. irvinensis as a species of Chasmosaurus. However, given that epiparietal count is relatively conservative amongst chasmosaurine taxa (generally three per side), and that Chasmosaurus and Vagaceratops differ in their count (three vs. four or five, respectively), the assignment of $C$. irvinensis to its own genus, Vagaceratops, seems warranted.

The recovery of Vagaceratops as a sister taxon to Chasmosaurus of northern Laramidia (Fig. 37) and not to Kosmoceratops of southern Laramidia (Utah) suggests that the ancestor of Vagaceratops originated in northern Laramidia, and did not originate or "wander" (vagus = Latin for wanderer) from the south (contra Sampson et al., 2010); in this sense, the name "Vagaceratops" is a misnomer. Although this has important implications for the evolution of this branch of Chasmosaurinae, this is outside of the scope of this project.

In Dinosaur Provincial Park, bentonites derived from the top of the Oldman Formation (5.5 m below the Dinosaur Park Formation), the middle of the Dinosaur Park Formation (36.0 $\mathrm{m}$ above the Oldman Formation) and near the base of the Lethbridge Coal Zone (61.5 m above the Oldman Formation) yielded ${ }^{40} \mathrm{Ar} /{ }^{39} \mathrm{Ar}$ dates of $77.0 \pm 0.5$ Ma, 76.4 $\pm 0.4 \mathrm{Ma}$ and 76.1 $\pm 0.5 \mathrm{Ma}$, respectively (David Eberth, pers. comm.; Fig. 1). Assuming a constant sedimentation rate in Dinosaur Provincial Park, the lower (0.0-36.0 m) and upper (36.0-61.5) halves of the Dinosaur Park Formation experienced rates of approximately $6.9 \mathrm{~cm} / 1000$ years and $8.5 \mathrm{~cm} / 1000$ years, respectively. The stratigraphic 
ranges of Chasmosaurus-like (i.e., C. belli $-\mathrm{CMN} 2280=6.6 \mathrm{~m}$ to approximately $54 \mathrm{~m}$ above the Oldman Formation $=\mathrm{CMN} 8800$ ) and Vagaceratops-like (i.e., $V$. irvinensis YPM $2016=29.5 \mathrm{~m}$ to $\geq 55 \mathrm{~m}=$ TMP 1998.128.0008) specimens therefore represent approximately 638,000 years and at least 318,000 years, respectively, with approximately 300,000 years of overlap between these two operative taxonomic groupings. The temporal ranges of Chasmosaurus-like (i.e., C. belli) and Vagaceratops-like (i.e., V. irvinensis) specimens are within the range of variation of other chasmosaurines. Pentaceratops sternbergi has a reported temporal range of 2.5 million years, from 75.072.5 Ma (Lucas et al., 2006), while that of Agujaceratops mariscalensis is 1.0 million years, from 71.5-70.5 Ma (Sampson and Loewen, 2010), and 1.5 million years for Triceratops horridus, from 67.0-65.5 Ma (Sampson and Loewen, 2010). Centrosaurines, ones which are particularly well-sampled from the Dinosaur Park Formation such as Centrosaurus apertus and Styracosaurus albertensis, have relatively shorter temporal ranges, at approximately 700,000 and 500,000 years, respectively (Sampson and Loewen, 2010).

The lack of stratigraphic separation between Chasmosaurus and Vagaceratops contradicts previous reports that these two taxa formed a faunal succession, corresponding to the lower to upper (Dinosaur Park faunal zones 1 and 2), and uppermost (Dinosaur Park Faunal Zone 3) units of the Dinosaur Park Formation, respectively (Holmes et al., 2001; Ryan and Evans, 2005). The stratigraphic ranges of these two taxa cannot be explained by an anagenetic model, whereby Chasmosaurus evolved into Vagaceratops over time. The alternative evolutionary model of cladogenesis, whereby Chasmosaurus and Vagaceratops specimens both evolved from a common ancestor, must 
therefore be accepted.

Ontogenetically variable phylogenetic characters: The morphological characters used in the phylogenetic analysis (Fig. 37) are useful in distinguishing taxa from one another. However, some of these characters have states that change ontogenetically within taxa. A relatively immature ceratopsid specimen lacking features expressed later in life (e.g., epiparietals) could hypothetically plot out as a less derived species on a phylogenetic tree than would a more mature representative of that taxon (Evans et al., 2013). Therefore, character codings for a given taxon should be derived from relatively mature representative specimens. The ontogenetic nature of some morphological characters does not justify their removal from the phylogenetic analysis; rather, these characters should be included, but their ontogenetic nature should be noted. Phylogenetic characters whose states change ontogenetically are listed and discussed in Appendix V.

In conclusion, this study recognizes two chasmosaurine species from the Dinosaur Park Formation, Chasmosaurus belli and Vagaceratops irvinensis. Revised taxonomic diagnoses for these species are given in Appendix VI. 
Appendix I: Dinosaur Park Formation chasmosaurine data 
Appendix I (continued).

\begin{tabular}{|c|c|c|c|c|}
\hline \multirow[t]{2}{*}{ Specimen } & \multicolumn{3}{|c|}{ Taxonomic assignment } & \multirow[t]{2}{*}{ Specimen description } \\
\hline & Genus & Species & Type & \\
\hline \multirow[t]{4}{*}{ AMNH 5401} & Chasmosaurus & kaiseni & holotype & \multirow[t]{4}{*}{ Skull, mostly complete (missing back of frill) } \\
\hline & Chasmosaurus & canadensis & & \\
\hline & Chasmosaurus & belli & & \\
\hline & Mojoceratops & perifania & provisionally referred & \\
\hline AMNH 5402 & Chasmosaurus & belli & & Skull, complete; right lower jaw \\
\hline \multirow[t]{2}{*}{ AMNH 5656} & Chasmosaurus & russelli & & \multirow[t]{2}{*}{ Frill and most of braincase } \\
\hline & Mojoceratops & perifania & provisionally referred & \\
\hline CMN 0187 & Chasmosaurus (?) & & & Mandibular tooth \\
\hline CMN 0212 & Chasmosaurus (?) & & & Orbital horn and part of orbit \\
\hline CMN 0275 & Chasmosaurus (?) & & & Orbital horn \\
\hline CMN 0284 & Chasmosaurus (?) & & & Lower jaw \\
\hline CMN 0285 & Chasmosaurus (?) & & & Maxilla \\
\hline \multirow[t]{4}{*}{ CMN 0491} & Monoclonius & belli & holotype & \multirow[t]{4}{*}{ Parietal (most of posterior and medial bars) } \\
\hline & Ceratops & belli & holotype & \\
\hline & Protorosaurus & belli & holotype & \\
\hline & Chasmosaurus & belli & holotype & \\
\hline CMN 0837 & Chasmosaurus (?) & & & Orbital horn \\
\hline CMN 0838 & Chasmosaurus (?) & & & Orbital horn and part of orbit \\
\hline CMN 0841 & Chasmosaurus (?) & & & Orbital horn and part of orbit \\
\hline \multirow[t]{6}{*}{ CMN 1254} & Monoclonius & canadensis & holotype & \multirow{6}{*}{$\begin{array}{l}\text { Squamosal; lateral parietal bar; circumorbital region; } \\
\text { nasal; lower jaw; dorsal vertebra }\end{array}$} \\
\hline & Ceratops & canadensis & holotype & \\
\hline & Eoceratops & canadensis & holotype & \\
\hline & Chasmosaurus & canadensis & holotype & \\
\hline & Chasmosaurus & sp. & & \\
\hline & Mojoceratops & perifania & Provisionally referred & \\
\hline CMN 2245 & Chasmosaurus & belli & plesiotype & $\begin{array}{l}\text { Skull, mostly complete (missing snout); lower jaws; most } \\
\text { of skeleton }\end{array}$ \\
\hline \multirow[t]{2}{*}{ CMN 2280} & Chasmosaurus & belli & & \multirow[t]{2}{*}{ Skull, complete; lower jaws; most of skeleton } \\
\hline & Chasmosaurus & russelli & & \\
\hline CMN 8800 & Chasmosaurus & russelli & holotype & Skull, mostly complete (missing jugal) \\
\hline
\end{tabular}


Appendix I (continued).

\begin{tabular}{|c|c|c|c|c|}
\hline Specimen & Locality & Quarry & Formation & Additional locality info \\
\hline AMNH 5401 & Dinosaur Provincial Park, Alberta & & Dinosaur Park Fm. & 12 miles below Steveville \\
\hline AMNH 5402 & Dinosaur Provincial Park, Alberta & & Dinosaur Park Fm. & $\begin{array}{l}\text { Township 21, Range 12, about } 1 \text { mile below Steveville, on the } \\
\text { left bank of the river }\end{array}$ \\
\hline AMNH 5656 & $\begin{array}{l}\text { Sand Creek (near Dinosaur Provincial } \\
\text { Park), Alberta }\end{array}$ & & Dinosaur Park Fm. & Sand Creek \\
\hline CMN 0187 & & & Dinosaur Park Fm. & P-1898 \\
\hline CMN 0212 & Near Dinosaur Provincial Park, Alberta & & Dinosaur Park Fm. & P-189704 -E side of Red Deer River at mouth of Berry Creek. \\
\hline CMN 0275 & $\begin{array}{l}\text { Berry Creek (near Dinosaur Provincial } \\
\text { Park), Alberta }\end{array}$ & & Dinosaur Park Fm. & $\begin{array}{l}\text { P-1301 -This is a general locality number assigned to specimens } \\
\text { collected by C.H. Sternberg in } 1913 \text {. He prospected on the W } \\
\text { side of the Red Deer River in Sections } 9-10,15-17,20,21,28 \text {, } \\
29,32,33, \text { T. 20, R. 12, W of 4th Meridian. This is the area S of } \\
\text { Steveville ferry landing. }\end{array}$ \\
\hline CMN 0284 & $\begin{array}{l}\text { Berry Creek (near Dinosaur Provincial } \\
\text { Park), Alberta }\end{array}$ & & Dinosaur Park Fm. & $\begin{array}{l}\text { P-189703 -Three miles below mouth of Berry Creek, Steveville } \\
\text { area; "both sides of river" }\end{array}$ \\
\hline CMN 0285 & Near Dinosaur Provincial Park, Alberta & & Dinosaur Park Fm. & P-189703 (see above) \\
\hline CMN 0491 & $\begin{array}{l}\text { Berry Creek (near Dinosaur Provincial } \\
\text { Park), Alberta }\end{array}$ & & Dinosaur Park Fm. & $\begin{array}{l}\text { P-189803 -E side of Red Deer River, below mouth of Berry } \\
\text { Creek }\end{array}$ \\
\hline CMN 0837 & Alberta & & Dinosaur Park Fm. & $\begin{array}{l}\text { P-1401 -This is a general locality number assigned to specimens } \\
\text { obtained by C.H. Sternberg in } 1914 \text {. He explored (1) Sections } 6 \\
\text { and 7, T. 21, R. } 11 \text { (?), W of 4th Meridian \& (2) the E half of } \\
\text { Section 36, T. 20, R. } 11 \text {, W of 4th Meridian. This is the area S of } \\
\text { "Apple Jack Ferry", Red Deer River, about } 10 \text { miles SE of } \\
\text { Steveville, Alberta. }\end{array}$ \\
\hline CMN 0838 & Alberta & & Dinosaur Park Fm. & P-1401 (see above) \\
\hline CMN 0841 & Alberta & & Dinosaur Park Fm. & P-1401 (see above) \\
\hline CMN 1254 & Red Deer River, Alberta & & Dinosaur Park Fm. & P-0105-E side of Red Deer River "lower beds exposed here" \\
\hline CMN 2245 & Dinosaur Provincial Park, Alberta & 37 & Dinosaur Park Fm. & P-1301 (see above) \\
\hline CMN 2280 & Dinosaur Provincial Park, Alberta & 10 & Dinosaur Park Fm. & P-1401 (see above) \\
\hline CMN 8800 & Manyberries, Alberta & & Dinosaur Park Fm. & $\begin{array}{l}\text { P-3619 -N of centre of NE } 1 / 4 \text { of Sec. } 21 \text {, Twp. 2, R. 4, W. of } \\
\text { 4th Meridian (or approx. } 17.5 \text { miles S and } 9 \text { miles East of } \\
\text { Manyberries, Alta.) }\end{array}$ \\
\hline
\end{tabular}


Appendix I (continued).

\begin{tabular}{|c|c|c|c|c|c|c|c|}
\hline Specimen & UTM & $\begin{array}{c}\text { Elevation } \\
\text { above sea } \\
\text { level }\end{array}$ & $\begin{array}{c}\text { Elevation above } \\
\text { Oldman Fm. (in } \\
\text { Dinosaur } \\
\text { Provincial Park) } \\
\text { (m) }\end{array}$ & $\begin{array}{l}\text { Date } \\
\text { collected }\end{array}$ & Collector & Comments & Reference \\
\hline AMNH 5401 & & & & 1913 & B. Brown, P.C. Kaisen & & $\begin{array}{l}\text { Brown (1933); Lehman } \\
\text { (1989); Godfrey and Holmes } \\
\text { (1995); Longrich (2010) }\end{array}$ \\
\hline AMNH 5402 & & & & 1913 & B. Brown, P.C. Kaisen & & $\begin{array}{l}\text { Lull (1933); Godfrey and } \\
\text { Holmes (1995) }\end{array}$ \\
\hline AMNH 5656 & & & & 1917 & C.H. Sternberg & & $\begin{array}{l}\text { Godfrey and Holmes (1995); } \\
\text { Longrich (2010) }\end{array}$ \\
\hline CMN 0187 & & & & 1897 & L.M. Lambe & & \\
\hline CMN 0212 & & & & 1897 & L.M. Lambe & & Lambe (1902) \\
\hline CMN 0275 & & & & 1913 & C.H. Sternberg & & \\
\hline CMN 0284 & & & & 1897 & L.M. Lambe & & \\
\hline CMN 0285 & & & & 1897 & L.M. Lambe & & \\
\hline CMN 0491 & & & & 1898 & L.M. Lambe & & $\begin{array}{l}\text { Lambe (1902; 1914a, } \\
\text { 1914b); Hatcher et al. (1907) }\end{array}$ \\
\hline CMN 0837 & & & & 1914 & C.H. Sternberg & & \\
\hline CMN 0838 & & & & 1914 & C.H. Sternberg & & \\
\hline CMN 0841 & & & & 1914 & C.H. Sternberg & & \\
\hline CMN 1254 & & & & 1901 & L.M. Lambe & & $\begin{array}{l}\text { Lambe (1902, 1914a, 1915); } \\
\text { Lehman (1989); Godfrey and } \\
\text { Holmes (1995): Longrich } \\
\text { (2010) }\end{array}$ \\
\hline CMN 2245 & $\begin{array}{l}\text { 12U 457984.408E; } \\
5626105.901 \mathrm{~N}\end{array}$ & 692 & 42 & 1913 & C.H. Sternberg & & Lambe (1914) \\
\hline CMN 2280 & $\begin{array}{l}12 \mathrm{U} 465526.191 \mathrm{E} ; \\
5622005.303 \mathrm{~N}\end{array}$ & 666 & 6.6 & 1914 & $\begin{array}{l}\text { C.H. Sternberg, G.F. } \\
\text { Sternberg }\end{array}$ & & $\begin{array}{l}\text { Sternberg (1927); Godfrey } \\
\text { and Holmes (1995) }\end{array}$ \\
\hline CMN 8800 & $\begin{array}{l}\text { 12U 537901E; } \\
5443334 \mathrm{~N}\end{array}$ & 971 & $\begin{array}{l}\sim 54(1 \mathrm{~m} \text { below } \\
\text { Lethbridge Coal } \\
\text { Zone) }\end{array}$ & 1936 & L.S. Russell, H. Johnsen & & Sternberg (1940) \\
\hline
\end{tabular}


Appendix I (continued).

\begin{tabular}{|l|l|l|l|l|}
\hline \multirow{2}{*}{ Specimen } & \multicolumn{2}{|l|}{ Taxonomic assignment } & \multirow{2}{*}{ Type } & \multirow{2}{*}{ Specimen description } \\
\cline { 2 - 4 } & Genus & Species & \multicolumn{1}{|c|}{ Skull (missing frill) } \\
\hline & Chasmosaurus & russelli & paratype & \\
\hline & Chasmosaurus & sp. & & Anterior half of frill, braincase, part of snout, and lower jaws \\
\hline CMN 8802 & Chasmosaurus & russelli & paratype & Parietal (most of posterior and medial bars) \\
\hline CMN 8896 & Chasmosaurus & russelli & paratype & Some postcrania \\
\hline CMN 9673 & Chasmosaurus (?) & & & Orbital horn and part of orbit \\
\hline CMN 9676 & Chasmosaurus (?) & & & Orbital horn and part of orbit \\
\hline CMN 9808a & Chasmosaurus (?) & & & Orbital horn and part of orbit \\
\hline CMN 9808b & Chasmosaurus (?) & & & Orbital horn and part of orbit \\
\hline CMN 9808c & Chasmosaurus (?) & & & Orbital horn and part of orbit \\
\hline CMN 9808d & Chasmosaurus (?) & & & Epijugal \\
\hline CMN 9809 & Chasmosaurus (?) & & & Orbital horn and part of orbit \\
\hline CMN 9813 & Anchiceratops (?) & & & \\
\hline
\end{tabular}


Appendix I (continued).

\begin{tabular}{|c|c|c|c|c|}
\hline Specimen & Locality & Quarry & Formation & Additional locality info \\
\hline CMN 8801 & $\begin{array}{l}\text { Dinosaur } \\
\text { Provincial } \\
\text { Park, Alberta }\end{array}$ & 63 & Dinosaur Park Fm. & $\begin{array}{l}\text { P-2804 -S side of Red Deer River, about } 5 \text { miles SE of Steveville; at western boundary of } \\
\text { SW1/4 of Section 5, T. 21, R. 11, west of 4th meridian. } 120 \text { feet above the river, in middle of } \\
\text { section. }\end{array}$ \\
\hline CMN 8802 & $\begin{array}{l}\text { Manyberries, } \\
\text { Alberta }\end{array}$ & & Dinosaur Park Fm. & $\begin{array}{l}\text { P-3708 -NE } 1 / 4 \text {, Sec. 10, T. 2, R. 5, W of 4th Meridian, or about } 13^{1 / 2} \text { miles south and } 3 \frac{1}{4} 4 \\
\text { miles east of Manyberries. } \\
\text { Conflict.... } \\
\text { Leg. subdiv. 14, in N.W. } 1 / 4 \text { of Sec. 10, Twp. 3, R. 5, W. of 4th Meridian, on south side of } \\
\text { west branch of creek that runs past Joe Gilchrist's Ranch House, about } 10 \text { feet below prairie } \\
\text { (marker \# } 118 \text { in quarry). --> this is thought to be the correct one (David Evans, pers. comm.) }\end{array}$ \\
\hline CMN 8803 & $\begin{array}{l}\text { South } \\
\text { Saskatchewan } \\
\text { River, } \\
\text { Saskatchewan }\end{array}$ & & Dinosaur Park Fm. & $\begin{array}{l}\text { P-3501 -North side of South Saskatchewan River, in NW 1/4 of Section 12, T. 20, R.16, west } \\
\text { of 3rd Meridian. In a bone bed located by Roy Graham in } 1935 \text {. }\end{array}$ \\
\hline CMN 8896 & Alberta & & Dinosaur Park Fm. & P-191905 \\
\hline CMN 9673 & $\begin{array}{l}\text { South } \\
\text { Saskatchewan } \\
\text { River, } \\
\text { Saskatchewan }\end{array}$ & & Dinosaur Park Fm. & P-3501 (see above) \\
\hline CMN 9676 & $\begin{array}{l}\text { Near Dinosaur } \\
\text { Provincial } \\
\text { Park, Alberta }\end{array}$ & & Dinosaur Park Fm. & P-0106 -E side of Red Deer River; extreme SE area. \\
\hline CMN 9808a & Alberta & & Dinosaur Park Fm. & \\
\hline CMN 9808b & Alberta & & Dinosaur Park Fm. & \\
\hline CMN 9808c & Alberta & & Dinosaur Park Fm. & \\
\hline CMN 9808d & Alberta & & Dinosaur Park Fm. & \\
\hline CMN 9809 & Alberta & & Dinosaur Park Fm. & P-189804 -Red Deer River, opposite mouth of Berry Creek. \\
\hline CMN 9813 & Alberta & & Dinosaur Park Fm. & $\begin{array}{l}\text { P-3706 -"about } 12 \text { feet above a light gray sandstone bed," near the top of the Oldman } \\
\text { formation as exposed in legal subdivision 14, Sec. 24, Tp. 2, Rge. 4, west of the Fourth } \\
\text { Meridian (Sectional Map 16, Milk River, Alberta). This locality (P-3706) is about } 22 \text { miles } \\
\text { southeast of the town of Manyberries. An outlier of marine Bearpaw shale occurs } \\
\text { conformably above Oldman beds a few hundred yards to the northeast and stratigraphically } \\
\text { but a few feet above the fossiliferous horizon. }\end{array}$ \\
\hline
\end{tabular}


Appendix I (continued).

\begin{tabular}{|c|c|c|c|c|c|c|c|}
\hline Specimen & UTM & $\begin{array}{l}\text { Elevation above } \\
\text { sea level }\end{array}$ & $\begin{array}{l}\text { Elevation above Oldman } \\
\text { Fm. (in Dinosaur } \\
\text { Provincial Park) (m) }\end{array}$ & $\begin{array}{c}\text { Date } \\
\text { collected }\end{array}$ & Collector & Comments & Reference \\
\hline CMN 8801 & $\begin{array}{l}\text { 12U 457078.232E; } \\
5627018.862 \mathrm{~N}\end{array}$ & 670 & 22 & 1928 & $\begin{array}{l}\text { C.M. } \\
\text { Sternberg, } \\
\text { H.D.R. Lowe }\end{array}$ & Not diagnostic to species & $\begin{array}{l}\text { Sternberg } \\
(1940) \text {; Godfrey } \\
\text { and Holmes } \\
(1995)\end{array}$ \\
\hline CMN 8802 & & & $\begin{array}{l}\text { Top of Oldman } \\
\text { Formation, immediately } \\
\text { below LCZ; no overlying } \\
\text { sediments of the Dinosaur } \\
\text { Park Formation exposed, } \\
\text { however (David Evans, } \\
\text { pers. comm.) }\end{array}$ & 1937 & C.M. Sternberg & $\begin{array}{l}\text { Skull was largely } \\
\text { destroyed during move } \\
\text { from "Motor Building.", } \\
\text { 1941-09. }\end{array}$ & $\begin{array}{l}\text { Sternberg } \\
(1940)\end{array}$ \\
\hline CMN 8803 & & & & 1935 & C.M. Sternberg & & $\begin{array}{l}\text { Sternberg } \\
(1940) ; \\
\text { Longrich (2010) }\end{array}$ \\
\hline \multicolumn{8}{|l|}{ CMN 8896} \\
\hline CMN 9673 & & & & 1935 & $\begin{array}{l}\text { C.M. } \\
\text { Sternberg, R. } \\
\text { Graham }\end{array}$ & & \\
\hline CMN 9676 & & & & & L.M. Lambe & & \\
\hline \multicolumn{8}{|l|}{ CMN 9808a } \\
\hline \multirow{2}{*}{\multicolumn{8}{|c|}{$\begin{array}{l}\text { CMN 9808b } \\
\text { CMN 9808c }\end{array}$}} \\
\hline & & & & & & & \\
\hline \multicolumn{8}{|l|}{ CMN 9808d } \\
\hline CMN 9809 & & & & 1898 & L.M. Lambe & & \\
\hline CMN 9813 & & & & & & & \\
\hline
\end{tabular}


Appendix I (continued).

\begin{tabular}{|c|c|c|c|c|}
\hline \multirow[t]{2}{*}{ Specimen } & \multicolumn{3}{|c|}{ Taxonomic assignment } & \multirow[t]{2}{*}{ Specimen description } \\
\hline & Genus & Species & Type & \\
\hline CMN 9814 & Anchiceratops (?) & & & Caudal parietal with two large epiparietals \\
\hline CMN 34829 & Chasmosaurus & & & Skull (missing snout and back of frill) \\
\hline \multirow[t]{2}{*}{ CMN 34832} & Chasmosaurus & & & \multirow[t]{2}{*}{ Partial skull consisting of the skull roof, postorbital horns, and squamosals } \\
\hline & Mojoceratops & perifania & $\begin{array}{l}\text { provisionally } \\
\text { referred }\end{array}$ & \\
\hline \multirow[t]{2}{*}{ CMN 41357} & Chasmosaurus & irvinensis & holotype & \multirow{2}{*}{$\begin{array}{l}\text { Skull, mostly complete (missing part of braincase and part of facial region); } \\
\text { postcrania }\end{array}$} \\
\hline & Vagaceratops & irvinensis & holotype & \\
\hline CMN 41933 & Chasmosaurus & russelli & $\begin{array}{l}\text { referred } \\
\text { specimen }\end{array}$ & Parietal (posterolateral corner) \\
\hline NHMUK R4948 & Chasmosaurus & belli & & Skull, mostly complete (missing top of braincase); partial skeleton \\
\hline ROM 632 & Chasmosaurus (?) & & & \\
\hline \multirow[t]{3}{*}{ ROM $839(=5436)$} & Chasmosaurus & brevirostris & holotype & \multirow[t]{3}{*}{ Skull, mostly complete (missing back of frill and tip of snout); some postcrania } \\
\hline & Chasmosaurus & belli & & \\
\hline & Chasmosaurus & sp. & & \\
\hline ROM 843 (=5499) & Chasmosaurus & belli & & Skull, mostly complete (missing tip of snout); partial skeleton \\
\hline TMP 1967.017.0005 & Chasmosaurus (?) & & & Orbital horn and part of orbit \\
\hline
\end{tabular}


Appendix I (continued).

\begin{tabular}{|c|c|c|c|c|}
\hline Specimen & Locality & Quarry & Formation & Additional locality info \\
\hline CMN 9814 & $\begin{array}{l}\text { Lost River, Alberta; } \\
\text { P-3706 }\end{array}$ & & & $\begin{array}{l}\text { P-3705 -This locality is on the line between sections } 10 \text { and } 15 \text { just east of the } \\
\text { quarter-section postin Tp. } 3 \text {, Rge. } 5 \text {, west of the Fourth Meridian. This is about } 14 \\
\text { miles south-southeast of Manyberries and } 10 \text { miles northeast of the other } \\
\text { Anchiceratops locality. In view of the prevailingly slight regional dip, the fact that } \\
\text { both localities are near the } 3200 \text {-foot contour line (Map } 567 \mathrm{~A} \text {, Dunmore, Alberta) } \\
\text { suggests a similar stratigraphic position within the upper fifty feet of the Oldman } \\
\text { formation as developed here. }\end{array}$ \\
\hline CMN 34829 & $\begin{array}{l}\text { Near Dinosaur } \\
\text { Provincial Park, } \\
\text { Alberta }\end{array}$ & & $\begin{array}{l}\text { Dinosaur Park } \\
\text { Fm. }\end{array}$ & $\begin{array}{l}\text { P-1532 -Near lower end of Deadlodge Canyon, on S side of Red Deer River, almost } \\
\text { E of the Cravath Corners Post Office (n.b. This hamlet has since been abandoned fidé } \\
\text { R. Day). }\end{array}$ \\
\hline CMN 34832 & $\begin{array}{l}\text { Near Dinosaur } \\
\text { Provincial Park, } \\
\text { Alberta }\end{array}$ & & $\begin{array}{l}\text { Dinosaur Park } \\
\text { Fm. }\end{array}$ & P-5307 -On Red Deer River, approx. 1 mile SE of Steveville Ferry. \\
\hline CMN 41357 & Near Irvine, Alberta & & $\begin{array}{l}\text { Dinosaur Park } \\
\text { Fm. }\end{array}$ & $\begin{array}{l}\text { P-5809 -4. } 2 \text { km SW of Irvine, Alta., in NE } 1 / 4 \text { of Sec. } 23 \text {, Twp. 11, R. 3, W of } 4^{\text {th }} \\
\text { meridian, in upper Dinosaur Park Fm. }\end{array}$ \\
\hline CMN 41933 & & & $\begin{array}{l}\text { Dinosaur Park } \\
\text { Fm. }\end{array}$ & \\
\hline NHMUK R4948 & $\begin{array}{l}\text { Dinosaur Provincial } \\
\text { Park, Alberta }\end{array}$ & 78 & $\begin{array}{l}\text { Dinosaur Park } \\
\text { Fm. }\end{array}$ & \\
\hline ROM 632 & $\begin{array}{l}\text { Dinosaur Provincial } \\
\text { Park, Alberta }\end{array}$ & & $\begin{array}{l}\text { Dinosaur Park } \\
\text { Fm. }\end{array}$ & \\
\hline ROM $839(=5436)$ & $\begin{array}{l}\text { Dinosaur Provincial } \\
\text { Park, Alberta }\end{array}$ & 25 & $\begin{array}{l}\text { Dinosaur Park } \\
\text { Fm. }\end{array}$ & 3 miles south of Steveville on the west side of the river \\
\hline ROM $843(=5499)$ & $\begin{array}{l}\text { Dinosaur Provincial } \\
\text { Park, Alberta }\end{array}$ & 19 & $\begin{array}{l}\text { Dinosaur Park } \\
\text { Fm. }\end{array}$ & 2 miles south of Steveville on the southeast side of the river \\
\hline TMP 1967.017.0005 & $\begin{array}{l}\text { Dinosaur Provincial } \\
\text { Park, Alberta }\end{array}$ & & $\begin{array}{l}\text { Dinosaur Park } \\
\text { Fm. }\end{array}$ & \\
\hline
\end{tabular}


Appendix I (continued).

\begin{tabular}{|c|c|c|c|c|c|c|c|}
\hline Specimen & UTM & $\begin{array}{l}\text { Elevation } \\
\text { above } \\
\text { sea level }\end{array}$ & $\begin{array}{c}\text { Elevation above } \\
\text { Oldman Fm. (in } \\
\text { Dinosaur Provincial } \\
\text { Park) (m) }\end{array}$ & $\begin{array}{c}\text { Date } \\
\text { collected }\end{array}$ & Collector & Comments & Reference \\
\hline CMN 9814 & & & & 1937 & C.M. Sternberg & 'Pentaceratops'-like & \\
\hline CMN 34829 & & & & 1915 & C.H. Sternberg & $\begin{array}{l}\text { During excavation, } \\
\text { was in a slide, crest } \\
\text { was shaken by fall } \\
\text { and scattered }\end{array}$ & \\
\hline CMN 34832 & & & & 1953 & G.E. Lindblad & & Longrich (2010) \\
\hline CMN 41357 & & 795 & & 1958 & $\begin{array}{l}\text { W. Langston, } \\
\text { H.L. Shearman, } \\
\text { H.J. Champagne }\end{array}$ & $\begin{array}{l}\text { Just below or } \\
\text { possibly in } \\
\text { Lethbridge Coal } \\
\text { Zone (Michael } \\
\text { Ryan, pers. comm.) }\end{array}$ & \\
\hline CMN 41933 & & & & & & & $\begin{array}{l}\text { Godfrey and } \\
\text { Holmes (1995) }\end{array}$ \\
\hline NHMUK R4948 & & & & $\begin{array}{l}1919- \\
1920\end{array}$ & W.E. Cutler & & $\begin{array}{l}\text { Maidment and } \\
\text { Barrett (2011) }\end{array}$ \\
\hline ROM 632 & & & & & & $\begin{array}{l}\text { Long since } \\
\text { discarded (David } \\
\text { Evans, pers.comm.) }\end{array}$ & \\
\hline ROM 839 (=5436) & $\begin{array}{l}12 \mathrm{U} \\
455597.974 \mathrm{E} ; \\
5628163.908 \mathrm{~N}\end{array}$ & 684 & 36 & $\begin{array}{l}1920 \\
1926\end{array}$ & L. Sternberg & & $\begin{array}{l}\text { Lull (1933); } \\
\text { Godfrey and } \\
\text { Holmes (1995); } \\
\text { Maidment and } \\
\text { Barrett (2011) }\end{array}$ \\
\hline ROM 843 (=5499) & $\begin{array}{l}12 \mathrm{U} \\
458798.830 \mathrm{E} ; \\
5629388.700 \mathrm{~N} \\
\end{array}$ & 682 & 32.376 & 1926 & L. Sternberg & & Lull (1933) \\
\hline TMP 1967.017.0005 & & & & & & not diagnostic & $\begin{array}{l}\text { Godfrey and } \\
\text { Holmes (1995) }\end{array}$ \\
\hline
\end{tabular}


Appendix I (continued).

\begin{tabular}{|c|c|c|c|c|}
\hline \multirow[t]{2}{*}{ Specimen } & \multicolumn{3}{|c|}{ Taxonomic assignment } & \multirow[t]{2}{*}{ Specimen description } \\
\hline & Genus & Species & Type & \\
\hline TMP 1979.011.0147 & Mojoceratops & perifania & $\begin{array}{l}\text { provisionally } \\
\text { referred }\end{array}$ & Braincase, partial \\
\hline TMP 1979.014.0813 & Chasmosaurus (?) & & & Orbital horn and part of orbit \\
\hline TMP 1979.014.0814 & Chasmosaurus (?) & & & Orbital horn and part of orbit \\
\hline TMP 1980.016.1111 & Chasmosaurus (?) & & & Episquamosal (?) \\
\hline \multicolumn{5}{|l|}{ TMP 1981.003.0004 } \\
\hline TMP 1981.016.0284 & Chasmosaurus (?) & & & Orbital horn (?) \\
\hline TMP 1981.016.0499 & Chasmosaurus (?) & & & Orbital horn and part of orbit \\
\hline TMP 1981.018.0231 & Chasmosaurus (?) & & & Parietal (junction between posterior and medial bars) \\
\hline TMP 1981.019.0017 & Chasmosaurus (?) & & & Quadrate, partial \\
\hline TMP 1981.019.0175 & Chasmosaurus & russelli & & Skull, mostly complete (missing back of frill and left side of skull) \\
\hline TMP 1981.023 .0024 & Chasmosaurus (?) & & & Parietal, partial (anterior half of medial bar) \\
\hline TMP 1981.030.0001 & Chasmosaurus (?) & & & Parietal, partial (posterolateral corner) \\
\hline TMP 1981.041.0116 & Chasmosaurus (?) & & & Orbital horncore, partial \\
\hline TMP 1982.016.0022 & & & & Partial skull, not prepared \\
\hline TMP 1982.016.0215 & Chasmosaurus (?) & & & Parietal, partial (part of medial platform) \\
\hline \multirow[t]{2}{*}{ TMP 1983.025.0001 } & Chasmosaurus & russelli & referred specimen & \multirow[t]{2}{*}{ Frill and braincase } \\
\hline & Mojoceratops & perifania & holotype & \\
\hline TMP 1984.163.0041 & Chasmosaurus (?) & & & Nasal, partial \\
\hline
\end{tabular}


Appendix I (continued).

\begin{tabular}{|c|c|c|c|c|}
\hline Specimen & Locality & Quarry & Formation & Additional locality info \\
\hline TMP 1979.011.0147 & $\begin{array}{l}\text { Dinosaur Provincial } \\
\text { Park, Alberta }\end{array}$ & 143 & Dinosaur Park Fm. & \\
\hline TMP 1979.014.0813 & $\begin{array}{l}\text { Dinosaur Provincial } \\
\text { Park, Alberta }\end{array}$ & & Dinosaur Park Fm. & \\
\hline TMP 1979.014.0814 & $\begin{array}{l}\text { Dinosaur Provincial } \\
\text { Park, Alberta }\end{array}$ & & Dinosaur Park Fm. & \\
\hline \multicolumn{5}{|l|}{ TMP 1980.016.1111 } \\
\hline TMP 1981.003.0004 & & & Dinosaur Park Fm. & \\
\hline TMP 1981.016.0284 & $\begin{array}{l}\text { Dinosaur Provincial } \\
\text { Park, Alberta }\end{array}$ & & Dinosaur Park Fm. & \\
\hline TMP 1981.016.0499 & $\begin{array}{l}\text { Dinosaur Provincial } \\
\text { Park, Alberta }\end{array}$ & & Dinosaur Park Fm. & \\
\hline TMP 1981.018.0231 & $\begin{array}{l}\text { Dinosaur Provincial } \\
\text { Park, Alberta }\end{array}$ & & Dinosaur Park Fm. & \\
\hline TMP 1981.019.0017 & $\begin{array}{l}\text { Dinosaur Provincial } \\
\text { Park, Alberta }\end{array}$ & & Dinosaur Park Fm. & \\
\hline TMP 1981.019.0175 & $\begin{array}{l}\text { Dinosaur Provincial } \\
\text { Park, Alberta }\end{array}$ & 164 & Dinosaur Park Fm. & \\
\hline TMP 1981.023.0024 & $\begin{array}{l}\text { Dinosaur Provincial } \\
\text { Park, Alberta }\end{array}$ & & Dinosaur Park Fm. & \\
\hline TMP 1981.030.0001 & $\begin{array}{l}\text { Dinosaur Provincial } \\
\text { Park, Alberta }\end{array}$ & & Dinosaur Park Fm. & \\
\hline TMP 1981.041.0116 & $\begin{array}{l}\text { Dinosaur Provincial } \\
\text { Park, Alberta }\end{array}$ & & Dinosaur Park Fm. & Steveville \\
\hline TMP 1982.016.0022 & $\begin{array}{l}\text { Dinosaur Provincial } \\
\text { Park, Alberta }\end{array}$ & & Dinosaur Park Fm. & \\
\hline TMP 1982.016.0215 & $\begin{array}{l}\text { Dinosaur Provincial } \\
\text { Park, Alberta }\end{array}$ & & Dinosaur Park Fm. & \\
\hline TMP 1983.025.0001 & Alberta & & Dinosaur Park Fm. & \\
\hline TMP 1984.163.0041 & Alberta & & Dinosaur Park Fm. & \\
\hline
\end{tabular}


Appendix I (continued).

\begin{tabular}{|c|c|c|c|c|c|c|c|}
\hline Specimen & UTM & $\begin{array}{l}\text { Elevation } \\
\text { above } \\
\text { sea level }\end{array}$ & $\begin{array}{c}\text { Elevation above } \\
\text { Oldman Fm. (in } \\
\text { Dinosaur Provincial } \\
\text { Park) (m) }\end{array}$ & $\begin{array}{c}\text { Date } \\
\text { collected }\end{array}$ & Collector & Comments & Reference \\
\hline TMP 1979.011.0147 & $\begin{array}{l}12 \mathrm{U} 465886 \mathrm{E} \\
5622564 \mathrm{~N}\end{array}$ & 669 & 9 & & & & $\begin{array}{l}\text { Ryan (1992); } \\
\text { Longrich (2010) }\end{array}$ \\
\hline TMP 1979.014.0813 & & & & & & not diagnostic & $\begin{array}{l}\text { Godfrey and } \\
\text { Holmes (1995) }\end{array}$ \\
\hline TMP 1979.014.0814 & & & & & & not diagnostic & $\begin{array}{l}\text { Godfrey and } \\
\text { Holmes (1995) }\end{array}$ \\
\hline \multicolumn{8}{|l|}{ TMP 1980.016.1111 } \\
\hline TMP 1981.003.0004 & & & & & & $\begin{array}{l}\text { (in Red Deer warehouse } \\
\text { - Graeme Housego, pers. } \\
\text { comm..) }\end{array}$ & \\
\hline TMP 1981.016.0284 & & & & & & Not diagnostic & $\begin{array}{l}\text { Godfrey and } \\
\text { Holmes (1995) }\end{array}$ \\
\hline TMP 1981.016.0499 & & & & & & Not diagnostic & $\begin{array}{l}\text { Godfrey and } \\
\text { Holmes (1995) }\end{array}$ \\
\hline \multicolumn{8}{|l|}{ TMP 1981.018.0231 } \\
\hline \multicolumn{8}{|l|}{ TMP 1981.019.0017 } \\
\hline TMP 1981.019.0175 & $\begin{array}{l}\text { 12U 459201.791E; } \\
5627476.707 \mathrm{~N}\end{array}$ & 659 & 11.468 & 1981 & $\begin{array}{l}\text { P.J. } \\
\text { Currie }\end{array}$ & & \\
\hline TMP 1981.023.0024 & & & & & & & $\begin{array}{l}\text { Godfrey and } \\
\text { Holmes (1995) }\end{array}$ \\
\hline \multicolumn{8}{|l|}{ TMP 1981.030.0001 } \\
\hline \multicolumn{8}{|l|}{ TMP 1981.041 .0116} \\
\hline TMP 1982.016.0022 & & & & & & Unprepared specimen & \\
\hline \multicolumn{8}{|l|}{ TMP 1982.016.0215 } \\
\hline TMP 1983.025.0001 & & & & & & $\begin{array}{l}\text { Collected by amateurs in } \\
\text { Alberta, site unknown }\end{array}$ & $\begin{array}{l}\text { Godfrey and } \\
\text { Holmes (1995); } \\
\text { Longrich (2010) }\end{array}$ \\
\hline TMP 1984.163.0041 & & & & & & & \\
\hline
\end{tabular}


Appendix I (continued).

\begin{tabular}{|c|c|c|c|c|}
\hline \multirow[t]{2}{*}{ Specimen } & \multicolumn{3}{|c|}{ Taxonomic assignment } & \multirow[t]{2}{*}{ Specimen description } \\
\hline & Genus & Species & Type & \\
\hline TMP 1986.036.0530 & Chasmosaurus (?) & & & Episquamosal (?) \\
\hline TMP 1987.045.0001 & Vagaceratops & irvinensis & referred specimen & Skull, mostly complete (missing most of frill) \\
\hline TMP 1988.036 .0050 & & & & Horncore \\
\hline TMP 1989.007.0006 & Chasmosaurus (?) & & & \\
\hline TMP 1991.046.0004 & Chasmosaurus (?) & & & Squamosal, partial (distal tip) \\
\hline TMP 1992.053.0005 & Chasmosaurus (?) & & & Quadratojugal \\
\hline TMP 1993.075.0005 & Chasmosaurus (?) & & & Epiparietal and attached parietal \\
\hline TMP 1993.082.0001 & Chasmosaurus & sp. & & Skull, partial (missing back of frill, part of braincase and snout) \\
\hline TMP 1993.086.0001 & Chasmosaurus (?) & & & Epiparietal and attached parietal \\
\hline TMP 1994.012.0402 & Chasmosaurus (?) & & & Parietal \\
\hline TMP 1994.012.0778 & Chasmosaurus (?) & & & Parietal, partial (posterolateral corner) \\
\hline TMP 1996.029.0004 & Chasmosaurus (?) & & & Epiparietal \\
\hline TMP 1997.132.0002 & Chasmosaurus (?) & & & Parietal, partial (posterolateral corner) \\
\hline TMP 1998.093.0010 & Chasmosaurus (?) & & & Episquamosal (?) \\
\hline TMP 1998.102.0008 & Vagaceratops & irvinensis & referred specimen & Parietal, partial (most of posterior bar) \\
\hline TMP 1998.128.0001 & Chasmosaurus (?) & & & Squamosal, complete \\
\hline
\end{tabular}


Appendix I (continued).

\begin{tabular}{|c|c|c|c|c|}
\hline Specimen & Locality & Quarry & Formation & Additional locality info \\
\hline TMP 1986.036.0530 & $\begin{array}{l}\text { Dinosaur Provincial } \\
\text { Park, Alberta }\end{array}$ & & $\begin{array}{l}\text { Dinosaur Park } \\
\text { Fm. }\end{array}$ & \\
\hline TMP 1987.045.0001 & $\begin{array}{l}\text { Dinosaur Provincial } \\
\text { Park, Alberta }\end{array}$ & 184 & $\begin{array}{l}\text { Dinosaur Park } \\
\text { Fm. }\end{array}$ & Iddesleigh, Alberta \\
\hline TMP 1988.036.0050 & $\begin{array}{l}\text { Dinosaur Provincial } \\
\text { Park, Alberta }\end{array}$ & & $\begin{array}{l}\text { Dinosaur Park } \\
\text { Fm. }\end{array}$ & \\
\hline TMP 1989.007.0006 & & & $\begin{array}{l}\text { Dinosaur Park } \\
\text { Fm. }\end{array}$ & \\
\hline TMP 1991.046.0004 & $\begin{array}{l}\text { Sandy Point, } \\
\text { Alberta }\end{array}$ & & $\begin{array}{l}\text { Dinosaur Park } \\
\text { Fm. }\end{array}$ & \\
\hline TMP 1992.053.0005 & $\begin{array}{l}\text { Dinosaur Provincial } \\
\text { Park, Alberta }\end{array}$ & & $\begin{array}{l}\text { Dinosaur Park } \\
\text { Fm. }\end{array}$ & \\
\hline TMP 1993.075.0005 & $\begin{array}{l}\text { Dinosaur Provincial } \\
\text { Park, Alberta }\end{array}$ & & $\begin{array}{l}\text { Dinosaur Park } \\
\text { Fm. }\end{array}$ & \\
\hline TMP 1993.082.0001 & $\begin{array}{l}\text { Dinosaur Provincial } \\
\text { Park, Alberta }\end{array}$ & 212 & $\begin{array}{l}\text { Dinosaur Park } \\
\text { Fm. }\end{array}$ & \\
\hline TMP 1993.086.0001 & $\begin{array}{l}\text { Dinosaur Provincial } \\
\text { Park, Alberta }\end{array}$ & & $\begin{array}{l}\text { Dinosaur Park } \\
\text { Fm. }\end{array}$ & \\
\hline TMP 1994.012.0402 & $\begin{array}{l}\text { Dinosaur Provincial } \\
\text { Park, Alberta }\end{array}$ & & $\begin{array}{l}\text { Dinosaur Park } \\
\text { Fm. }\end{array}$ & \\
\hline TMP 1994.012.0778 & $\begin{array}{l}\text { Dinosaur Provincial } \\
\text { Park, Alberta }\end{array}$ & & $\begin{array}{l}\text { Dinosaur Park } \\
\text { Fm. }\end{array}$ & \\
\hline TMP 1996.029.0004 & $\begin{array}{l}\text { Horsethief Canyon, } \\
\text { Alberta }\end{array}$ & & $\begin{array}{l}\text { Dinosaur Park } \\
\text { Fm. }\end{array}$ & \\
\hline TMP 1997.132.0002 & Hilda, Alberta & & $\begin{array}{l}\text { Dinosaur Park } \\
\text { Fm. }\end{array}$ & \\
\hline TMP 1998.093.0010 & $\begin{array}{l}\text { Dinosaur Provincial } \\
\text { Park, Alberta }\end{array}$ & & $\begin{array}{l}\text { Dinosaur Park } \\
\text { Fm. }\end{array}$ & \\
\hline TMP 1998.102.0008 & Onefour, Alberta & & $\begin{array}{l}\text { Dinosaur Park } \\
\text { Fm. }\end{array}$ & Near village of Onefour, southeastern Alberta \\
\hline TMP 1998.128.0001 & Onefour, Alberta & & $\begin{array}{l}\text { Dinosaur Park } \\
\text { Fm. }\end{array}$ & \\
\hline
\end{tabular}


Appendix I (continued).

\begin{tabular}{|c|c|c|c|c|c|c|c|}
\hline Specimen & UTM & $\begin{array}{l}\text { Elevation } \\
\text { above } \\
\text { sea level }\end{array}$ & $\begin{array}{c}\text { Elevation above } \\
\text { Oldman Fm. (in } \\
\text { Dinosaur Provincial } \\
\text { Park) (m) }\end{array}$ & $\begin{array}{c}\text { Date } \\
\text { collected }\end{array}$ & Collector & Comments & Reference \\
\hline \multicolumn{8}{|l|}{ TMP 1986.036.0530 } \\
\hline TMP 1987.045.0001 & $\begin{array}{l}12 \mathrm{U} \\
473422.430 \mathrm{E} ; \\
5623949.690 \mathrm{~N}\end{array}$ & 712 & 54.357 & 1987 & L.J. Strong & & $\begin{array}{l}\text { Holmes et al. } \\
(2001)\end{array}$ \\
\hline TMP 1988.036.0050 & & & & & & Not diagnostic & $\begin{array}{l}\text { Godfrey and } \\
\text { Holmes (1995) }\end{array}$ \\
\hline TMP 1989.007.0006 & & & & & & $\begin{array}{l}\text { On loan from RTMP } \\
\text { (Graeme Housego, pers. } \\
\text { comm..) }\end{array}$ & \\
\hline \multicolumn{8}{|l|}{ TMP 1991.046.0004 } \\
\hline \multicolumn{8}{|l|}{ TMP 1992.053.0005 } \\
\hline \multicolumn{8}{|l|}{ TMP 1993.075.0005 } \\
\hline TMP 1993.082.0001 & $\begin{array}{l}12 \mathrm{U} \\
465961.720 \mathrm{E} ; \\
5620515.130 \mathrm{~N} \\
\end{array}$ & 713 & 53.044 & 1993 & $\begin{array}{l}\text { W. } \\
\text { Sloboda }\end{array}$ & & $\begin{array}{l}\text { Currie and Russell } \\
\text { (2005) }\end{array}$ \\
\hline \multicolumn{8}{|l|}{ TMP 1993.086.0001 } \\
\hline \multicolumn{8}{|l|}{ TMP 1994.012.0402 } \\
\hline \multicolumn{8}{|l|}{ TMP 1994.012.0778 } \\
\hline \multicolumn{8}{|l|}{ TMP 1996.029.0004 } \\
\hline \multicolumn{8}{|l|}{ TMP 1997.132.0002 } \\
\hline \multicolumn{8}{|l|}{ TMP 1998.093.0010 } \\
\hline TMP 1998.102.0008 & $\begin{array}{l}12 \mathrm{U} 543354 \mathrm{E} ; \\
5444609 \mathrm{~N}\end{array}$ & 982 & & & & $\begin{array}{l}\text { Collected in mud-filled } \\
\text { incised valley; therefore, } \\
\text { from somewhere in the } \\
\text { Lethbridge Coal Zone } \\
\text { (Michael Ryan, pers. } \\
\text { comm.) }\end{array}$ & \\
\hline TMP 1998.128.0001 & & & & & & & \\
\hline
\end{tabular}


Appendix I (continued).

\begin{tabular}{|c|c|c|c|c|}
\hline \multirow[t]{2}{*}{ Specimen } & \multicolumn{3}{|c|}{ Taxonomic assignment } & \multirow[t]{2}{*}{ Specimen description } \\
\hline & Genus & Species & Type & \\
\hline TMP 1999.055.0274 & Chasmosaurus (?) & & & Parietal, partial (part of medial platform and medial bar) \\
\hline TMP 1999.055.0292 & Mojoceratops & perifania & referred specimen & Parietal, partial (part of posterior bar) \\
\hline TMP 1999.055.0298 & Chasmosaurus (?) & & & Nasal, partial \\
\hline TMP 2000.057.0009 & Chasmosaurus (?) & & & Squamosal, partial \\
\hline TMP 2005.061.0002 & Chasmosaurus (?) & & & Dentary, phalanx, parietal with epiparietal \\
\hline TMP 2007.020 .0017 & Chasmosaurus (?) & & & Surangular; articular \\
\hline TMP 2007.020.0039 & Chasmosaurus (?) & & & Parietal, partial (medial bar) \\
\hline TMP 2007.020.0103 & Chasmosaurus (?) & & & Parietal, partial (junction between posterior and medial bars) \\
\hline TMP 2008.012.0001 & Chasmosaurus (?) & & & Parietal, partial (medial bar and part of posterior bar) \\
\hline TMP 2008.012.0074 & Chasmosaurus (?) & & & Orbital horncore \\
\hline TMP 2009.032.0008 & Chasmosaurus (?) & & & Squamosal \\
\hline TMP 2009.032.0011 & Chasmosaurus (?) & & & Parietal, partial (medial bar) \\
\hline TMP 2009.034.0009 & Vagaceratops & irvinensis & & Braincase; part of frill (including part of posterior parietal bar) \\
\hline TMP 2011.053.0046 & Chasmosaurus (?) & & & Frill, partial; quadrate, nasal \\
\hline TMP 2011.053 .0056 & Chasmosaurus (?) & & & Epoccipital \\
\hline \multirow[t]{3}{*}{ UALVP 40} & Eoceratops & canadensis & $\begin{array}{l}\text { provisionally } \\
\text { referred }\end{array}$ & \multirow[t]{3}{*}{ Skull, mostly complete (missing back of frill) } \\
\hline & Chasmosaurus & canadensis & & \\
\hline & Chasmosaurus & sp. & & \\
\hline UALVP 52613 & Chasmosaurus & & & \\
\hline YPM 2016 & Chasmosaurus & belli & & Skull, mostly complete (missing posterolateral corner of frill) \\
\hline
\end{tabular}


Appendix I (continued).

\begin{tabular}{|c|c|c|c|c|}
\hline Specimen & Locality & Quarry & Formation & Additional locality info \\
\hline TMP 1999.055.0274 & Dinosaur Provincial Park, Alberta & & Dinosaur Park Fm. & \\
\hline TMP 1999.055.0292 & Dinosaur Provincial Park, Alberta & & Dinosaur Park Fm. & \\
\hline TMP 1999.055.0298 & Dinosaur Provincial Park, Alberta & & Dinosaur Park Fm. & \\
\hline TMP 2000.057.0009 & Onefour, Alberta & & Dinosaur Park Fm. & \\
\hline TMP 2005.061.0002 & Dinosaur Provincial Park, Alberta & & Dinosaur Park Fm. & \\
\hline TMP 2007.020.0017 & Dinosaur Provincial Park, Alberta & & Dinosaur Park Fm. & \\
\hline TMP 2007.020.0039 & Dinosaur Provincial Park, Alberta & & Dinosaur Park Fm. & \\
\hline TMP 2007.020.0103 & Dinosaur Provincial Park, Alberta & & Dinosaur Park Fm. & \\
\hline TMP 2008.012.0001 & Dinosaur Provincial Park, Alberta & & Dinosaur Park Fm. & \\
\hline TMP 2008.012.0074 & Dinosaur Provincial Park, Alberta & & Dinosaur Park Fm. & \\
\hline TMP 2009.032.0008 & $\begin{array}{l}\text { South Saskatchewan River, } \\
\text { Alberta }\end{array}$ & & Dinosaur Park Fm. & \\
\hline TMP 2009.032.0011 & $\begin{array}{l}\text { South Saskatchewan River, } \\
\text { Alberta }\end{array}$ & & Dinosaur Park Fm. & \\
\hline TMP 2009.034.0009 & $\begin{array}{l}\text { South Saskatchewan River, Hilda, } \\
\text { Alberta }\end{array}$ & & Dinosaur Park Fm. & Collected along southeast side of South Saskatchewan River \\
\hline TMP 2011.053.0046 & Manyberries, Alberta & & Dinosaur Park Fm. & Kerry Ranch, Manyberries Pocket \\
\hline TMP 2011.053 .0056 & Sage Creek, Alberta & & Dinosaur Park Fm. & \\
\hline UALVP 40 & Dinosaur Provincial Park, Alberta & 237 & Dinosaur Park Fm. & $\begin{array}{l}2 \text { miles southeast and } 1 \text { mile east of the mouth of Sand } \\
\text { Creek }\end{array}$ \\
\hline UALVP 52613 & Dinosaur Provincial Park, Alberta & 255 & Dinosaur Park Fm. & \\
\hline YPM 2016 & Dinosaur Provincial Park, Alberta & 110 & Dinosaur Park Fm. & $\begin{array}{l}\text { On south side of the west branch of Little Sandhill Creek, } 60 \\
\text { feet below the overlying Pierre shales. }\end{array}$ \\
\hline
\end{tabular}


Appendix I (continued).

\begin{tabular}{|c|c|c|c|c|c|c|c|}
\hline Specimen & UTM & $\begin{array}{c}\text { Elevation } \\
\text { above } \\
\text { sea level }\end{array}$ & $\begin{array}{l}\text { Elevation above Oldman Fm. } \\
\text { (in Dinosaur Provincial Park) } \\
(\mathrm{m})\end{array}$ & $\begin{array}{c}\text { Date } \\
\text { collected }\end{array}$ & Collector & Comments & Reference \\
\hline \multicolumn{8}{|l|}{ TMP 1999.055.0274 } \\
\hline TMP 1999.055.0292 & & & & & & & Longrich (2010) \\
\hline \multicolumn{8}{|l|}{ TMP 1999.055.0298 } \\
\hline \multicolumn{8}{|l|}{ TMP 2000.057.0009 } \\
\hline \multicolumn{8}{|l|}{ TMP 2005.061.0002 } \\
\hline \multicolumn{8}{|l|}{ TMP 2007.020.0017 } \\
\hline \multicolumn{8}{|l|}{ TMP 2007.020.0039 } \\
\hline \multicolumn{8}{|l|}{ TMP 2007.020.0103 } \\
\hline \multicolumn{8}{|l|}{ TMP 2008.012.0001 } \\
\hline \multicolumn{8}{|l|}{ TMP 2008.012.0074 } \\
\hline TMP 2009.032.0008 & & & & & & Still jacketed & \\
\hline \multicolumn{8}{|l|}{ TMP 2009.032.0011 } \\
\hline TMP 2009.034.0009 & $\begin{array}{l}12 \mathrm{U} 559293 \mathrm{E} \\
5611197 \mathrm{~N}\end{array}$ & & $\begin{array}{l}33.5(33.5 \mathrm{~m} \text { above contact at } \\
\text { specimen locality; David } \\
\text { Eberth, pers. comm.) }\end{array}$ & 2009 & $\begin{array}{l}\text { D. Eberth, } \\
\text { N. Longrich, } \\
\text { M. Mitchell } \\
\end{array}$ & & Longrich (2010) \\
\hline TMP 2011.053 .0046 & $\begin{array}{l}12 \mathrm{U} 534562 \mathrm{E} \\
5463885 \mathrm{~N}\end{array}$ & 973 & $\begin{array}{l}\text { 53 ( } 2 \text { m below Lethbridge } \\
\text { Coal Zone) }\end{array}$ & 2011 & D.C. Evans & & \\
\hline TMP 2011.053.0056 & & & & & & $\begin{array}{l}\text { Unprepared } \\
\text { specimen }\end{array}$ & \\
\hline UALVP 40 & $\begin{array}{l}12 \mathrm{U} \\
464079.566 \mathrm{E} ; \\
5621054.664 \mathrm{~N}\end{array}$ & 680 & 18.187 & 1920 & G. Sternberg & $\begin{array}{l}\text { Not } \\
\text { diagnostic to } \\
\text { species }\end{array}$ & $\begin{array}{l}\text { Gilmore (1923); } \\
\text { Tyson (1977); } \\
\text { Lehman (1989); } \\
\text { Godfrey and } \\
\text { Holmes (1995) }\end{array}$ \\
\hline \multicolumn{8}{|l|}{ UALVP 52613} \\
\hline YPM 2016 & $\begin{array}{l}12 \mathrm{U} \\
459708.501 \mathrm{E} ; \\
5621811.713 \mathrm{~N} \\
\end{array}$ & 684 & 26.464 & 1919 & $\begin{array}{l}\text { C.M. } \\
\text { Sternberg }\end{array}$ & & Lull (1933) \\
\hline
\end{tabular}




\section{Appendix II: Cranial parameter data}

List of cranial parameters (see Fig. 5 for visualization):

(1) Postorbital horncore length ( $\mathrm{mm}$ )

(2) Postorbital horncore anteroposterior length (mm)

(3) Postorbital horncore mediolateral width ( $\mathrm{mm}$ )

(4) Postorbital horncore basal circumference (mm)

(5) Nasal horncore length (mm)

(5a) Nasal horncore anteroposterior length ( $\mathrm{mm})$

(5b) Nasal horncore mediolateral width ( $\mathrm{mm})$

(5c) Nasal horncore basal circumference ( $\mathrm{mm})$

(6) Rostral to orbit length (mm)

(7) Posterior margin of external naris to orbit length (mm)

(8) Rostral to posterior margin of nasal horncore (mm)

(9) Epijugal to orbit length (curved surface) (mm)

(10) Lateral temporal fenestra to orbit length (mm)

(11) Orbit length (mm)

(12) Orbit height (mm) 
(13) Maximum orbit diameter (mm)

(14) Minimum distance from jugal notch to medial margin of squamosal ( $\mathrm{mm}$ )

(15) Minimum distance from lateral margin of first episquamosal to medial margin of squamosal (mm)

(16) Rostral to epijugal length (mm)

(17) Rostral to back of maxillary toothrow (mm)

(18) Distance between orbits ( $\mathrm{mm}$ )

(19) Length of squamosal from jugal notch to its distal end (mm)

(20) Anterior margin of dorsal temporal fenestra to parietal fenestra ( $\mathrm{mm}$ )

(21) Jugal notch to parietal fenestra (curved measurement) (mm)

(22) Epijugal to squamosal length (across jugal notch) (mm)

(23) Anterior margin of frontoparietal fontanelle to back of medial parietal bar (curved surface) $(\mathrm{mm})$

(24) Maximum length of parietal fenestra (mm)

(25) Maximum width of parietal fenestra ( $\mathrm{mm})$

(26) Skull length (straight line, maximum distance, excluding epiparietals) (mm)

(27) Maximum width of parietal frill (mm)

(28) Jugal notch (squamosal lateral corner) to back of frill (mm) 
(29) Occipital condyle to back of frill (back of medial bar) length (mm)

(30) Anteroposterior length of parietal fenestra (mm)

(31) Thickness of medial parietal bar (at maximum curvature of posteromedial margin of parietal fenestra) $(\mathrm{mm})$

(32) Width of medial parietal bar (at maximum curvature of posteromedial margin of parietal fenestra) $(\mathrm{mm})$

(33) Emargination angle of posterior parietal bar $\left(^{\circ}\right)$

Measurements taken off best preserved side of skull are shown in bold, and are those used in this study. 
Appendix II (continued).

\begin{tabular}{|c|c|c|c|c|c|}
\hline \multirow{2}{*}{$\begin{array}{l}\text { Para- } \\
\text { meter }\end{array}$} & \multicolumn{2}{|c|}{ Specimen } & \multirow{2}{*}{$\begin{array}{l}\text { Para- } \\
\text { meter }\end{array}$} & \multicolumn{2}{|c|}{ Specimen } \\
\hline & AMNH 5401 & AMNH 5402 & & AMNH 5656 & $\mathrm{CMN} 0212$ \\
\hline 1 & $350 / 370$ & 70/65 & 1 & -----/----- & $-----/ 166$ \\
\hline 2 & $112 / \mathbf{1 1 0}$ & $64 / 56$ & 2 & ------/----- & $-----/ 75$ \\
\hline 3 & $82 / 82$ & $\mathbf{5 8} / 72$ & 3 & ------/----- & $-----/ 61$ \\
\hline 4 & $320 / 320$ & $190 / 170$ & 4 & ------/----- & $-----/ 208$ \\
\hline 5 & 130 & 144 & 5 & ------/----- & ------/----- \\
\hline $5 \mathrm{a}$ & 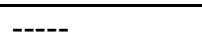 & 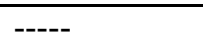 & $5 a$ & ----- & ----- \\
\hline $5 b$ & $\begin{array}{ll}---- \\
--1\end{array}$ & $\begin{array}{ll}---- \\
--1\end{array}$ & $5 b$ & $\begin{array}{ll}---- \\
--1\end{array}$ & $\begin{array}{ll}---- \\
--1\end{array}$ \\
\hline $5 \mathrm{c}$ & ----- & ----- & $5 \mathrm{c}$ & $\begin{array}{cc}---- \\
\end{array}$ & ----- \\
\hline 6 & $570 / \mathbf{5 5 0}$ & $495 / \mathbf{5 2 5}$ & 6 & -----/----- & -----/----- \\
\hline 7 & $215 / 215$ & $180 / \mathbf{2 0 0}$ & 7 & ------/----- & -----/----- \\
\hline 8 & 463 & 400 & 8 & ----- & ----- \\
\hline 9 & $290 / 280$ & 235/----- & 9 & ------/----- & |-----/----- \\
\hline 10 & $110 / \mathbf{1 1 5}$ & $125 / \mathbf{1 1 0}$ & 10 & -----/----- & ----------- \\
\hline 11 & -----/75 & $90 / 85$ & 11 & ------/----- & ------/----- \\
\hline 12 & $125 / 104$ & $66 / 64$ & 12 & -----/----- & ------/----- \\
\hline 13 & $128 / 115$ & $93 / 85$ & 13 & ------/----- & -----/----- \\
\hline 14 & $-----/ 270$ & $210 / \mathbf{2 1 0}$ & 14 & 222/----- & ------/----- \\
\hline 15 & $365 / 380$ & $320 / 300$ & 15 & 304/286 & ------/----- \\
\hline 16 & $670 / 730$ & 680/----- & 16 & ------/----- & -----/----- \\
\hline 17 & $615 / 580$ & $610 / 610$ & 17 & ------/----- & ------/----- \\
\hline 18 & 315 & 250 & 18 & ----- & ----- \\
\hline 19 & $710 / 695$ & $640 / 685$ & 19 & $569 / 560$ & ------/----- \\
\hline 20 & 215/190 & $290 / 290$ & 20 & -----/----- & -----/----- \\
\hline 21 & $315 / 330$ & $325 / 285$ & 21 & $257 / 259$ & ------/----- \\
\hline 22 & $90 / \mathbf{1 1 5}$ & $120 /-----$ & 22 & 129/----- & ------/----- \\
\hline 23 & ----- & 830 & 23 & ----- & ----- \\
\hline 24 & ------/----- & $435 / 500$ & 24 & $513 / 503$ & ------/----- \\
\hline 25 & -----/----- & $340 / \mathbf{2 6 0}$ & 25 & $266 / 264$ & -----/----- \\
\hline 26 & |-----/----- & $1460 / \mathbf{1 5 4 0}$ & 26 & ------/----- & ------/----- \\
\hline 27 & 820 & 920 & 27 & 697 & ----- \\
\hline 28 & $\mathbf{8 7 5} / 875$ & $750 / 780$ & 28 & 854/826 & ------/----- \\
\hline 29 & 710 & 665 & 29 & ----- & ----- \\
\hline 30 & -----/----- & 354/----- & 30 & 503/----- & ------/----- \\
\hline 31 & ----- & ----- & 31 & 27 & ----- \\
\hline 32 & $\begin{array}{l}---- \\
--\end{array}$ & ----- & 32 & 86 & $\begin{array}{l}---- \\
\end{array}$ \\
\hline 33 & ----- & 180 (est.) & 33 & 115 & ----- \\
\hline
\end{tabular}


Appendix II (continued).

\begin{tabular}{|c|c|c|c|c|c|}
\hline \multirow{2}{*}{$\begin{array}{l}\text { Para- } \\
\text { meter }\end{array}$} & \multicolumn{2}{|c|}{ Specimen } & \multirow{2}{*}{$\begin{array}{l}\text { Para- } \\
\text { meter }\end{array}$} & \multicolumn{2}{|c|}{ Specimen } \\
\hline & CMN 0275 & CMN 0491 & & CMN 0837 & $\mathrm{CMN} 0838$ \\
\hline 1 & 65/----- & -----/----- & 1 & $-----/ 253$ & -----/----- \\
\hline 2 & 65/----- & -----/----- & 2 & $-----/ 101$ & 66/----- \\
\hline 3 & 56/----- & -----/----- & 3 & $-----/ 85$ & 54/----- \\
\hline 4 & 204/----- & -----/----- & 4 & $-----/ 311$ & 189/----- \\
\hline 5 & -----/----- & -----/----- & 5 & -----/----- & -----/----- \\
\hline $5 \mathrm{a}$ & ----- & ----- & $5 \mathrm{a}$ & ----- & ----- \\
\hline $5 b$ & $\begin{array}{ll}---- \\
---\end{array}$ & 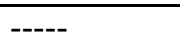 & $5 b$ & $\begin{array}{ll}---- \\
---\end{array}$ & $\begin{array}{ll}---- \\
---1\end{array}$ \\
\hline $5 c$ & ----- & ----- & $5 c$ & ----- & ----- \\
\hline 6 & -----/----- & -----/----- & 6 & ------/----- & -----/----- \\
\hline 7 & -----/----- & -----/----- & 7 & -----/----- & -----/----- \\
\hline 8 & ----- & ----- & 8 & ----- & ----- \\
\hline 9 & ------/----- & ------/----- & 9 & ------/----- & -----/----- \\
\hline 10 & ------/----- & ------/----- & 10 & ------/----- & ------/----- \\
\hline 11 & ------/----- & ------/----- & 11 & ------/----- & ------/----- \\
\hline 12 & -----/----- & -----/----- & 12 & -----/----- & -----/----- \\
\hline 13 & -----/----- & -----/----- & 13 & -----/----- & -----/----- \\
\hline 14 & -----/----- & -----/----- & 14 & -----/----- & -----/----- \\
\hline 15 & -----/----- & -----/----- & 15 & -----/----- & -----/----- \\
\hline 16 & ----------- & ------/----- & 16 & -----/----- & ------/----- \\
\hline 17 & ------/----- & ------/----- & 17 & ------/----- & ------/----- \\
\hline 18 & ----- & ----- & 18 & ----- & ----- \\
\hline 19 & ------/----- & ------/----- & 19 & ------/----- & ------/----- \\
\hline 20 & -----/----- & -----/----- & 20 & ------/----- & -----/------ \\
\hline 21 & ------/----- & ------/----- & 21 & -----/----- & ------/----- \\
\hline 22 & ------/----- & ------/----- & 22 & ------/----- & ------/----- \\
\hline 23 & ----- & ----- & 23 & ----- & ----- \\
\hline 24 & -----/----- & -----/----- & 24 & -----/----- & -----/----- \\
\hline 25 & -----/----- & -----/----- & 25 & -----/----- & -----/----- \\
\hline 26 & -----/----- & -----/----- & 26 & -----/----- & -----/----- \\
\hline 27 & ----- & ----- & 27 & ----- & ----- \\
\hline 28 & ------/----- & ------/----- & 28 & ------/----- & ------/----- \\
\hline 29 & ----- & ----- & 29 & ----- & ----- \\
\hline 30 & -----/----- & -----/----- & 30 & -----/----- & -----/----- \\
\hline 31 & ----- & 37 & 31 & ----- & ----- \\
\hline 32 & ----- & 81 & 32 & ----- & $\begin{array}{l}---- \\
--\end{array}$ \\
\hline 33 & ----- & 158 & 33 & ----- & ----- \\
\hline
\end{tabular}


Appendix II (continued).

\begin{tabular}{|c|c|c|c|c|c|}
\hline \multirow{2}{*}{$\begin{array}{l}\text { Para- } \\
\text { meter }\end{array}$} & \multicolumn{2}{|c|}{ Specimen } & \multirow{2}{*}{$\begin{array}{l}\text { Para- } \\
\text { meter }\end{array}$} & \multicolumn{2}{|c|}{ Specimen } \\
\hline & CMN 0841 & $\overline{C M N} 1254$ & & CMN 2245 & CMN 2280 \\
\hline 1 & $-----/ 81$ & $-----/ 216$ & 1 & $102 / 100$ & 132/88 \\
\hline 2 & $-----/ 89$ & $-----/ 105$ & 2 & 79/74 & $\mathbf{8 8} / 87$ \\
\hline 3 & $-----/ 52$ & $-----/ 74$ & 3 & $63 / 58$ & $76 / 84$ \\
\hline 4 & $-----/ 203$ & $-----/ 242$ & 4 & 238/233 & $263 / 262$ \\
\hline 5 & ------/----- & $-----/ 150$ & 5 & $-----/ 176$ & $141 / 140$ \\
\hline $5 \mathrm{a}$ & ----- & ----- & $5 a$ & 151 & 155 \\
\hline $5 b$ & $\begin{array}{ll}---- \\
--1\end{array}$ & 70 & $5 b$ & 83 & 81 \\
\hline $5 \mathrm{c}$ & ----- & ----- & $5 \mathrm{c}$ & 388 & 372 \\
\hline 6 & ------/----- & ------/----- & 6 & ------/----- & $563 / 559$ \\
\hline 7 & ------/----- & ------/----- & 7 & ------/----- & $208 / 190$ \\
\hline 8 & ----- & ----- & 8 & ----- & 478 \\
\hline 9 & |-----/----- & |-----/----- & 9 & $-----/ 283$ & $274 / 242$ \\
\hline 10 & -----/----- & -----/----- & 10 & $-----/ 127$ & $132 / 105$ \\
\hline 11 & ------/----- & -----/89 & 11 & $101 / 98$ & $90 / 79$ \\
\hline 12 & ------/----- & ------/----- & 12 & $105 / 117$ & $102 / 81$ \\
\hline 13 & ------/----- & ------/----- & 13 & 113/119 & $126 / 105$ \\
\hline 14 & ------/----- & $-----/ 250$ & 14 & $-----/ 247$ & $244 / 214$ \\
\hline 15 & -----/----- & $-----/ 334$ & 15 & $-----/ 345$ & 326/328 \\
\hline 16 & ------/----- & ------/----- & 16 & ------/----- & $750 / 700$ \\
\hline 17 & ------/----- & ------/----- & 17 & ------/----- & ------/----- \\
\hline 18 & ----- & ----- & 18 & 269 & 278 \\
\hline 19 & ------/----- & $-----/ 500$ & 19 & -----/779 & 709/668 \\
\hline 20 & -----/----- & -----/----- & 20 & $363 / 377$ & $277 / 268$ \\
\hline 21 & -----/----- & ------/----- & 21 & $-----/ 400$ & 310/337 \\
\hline 22 & ------/----- & ------/----- & 22 & $-----/ 140$ & 132/179 \\
\hline 23 & ----- & ----- & 23 & ----- & 876 \\
\hline 24 & -----/----- & -----/----- & 24 & $510 / 516$ & $556 / 596$ \\
\hline 25 & ------/----- & -----/----- & 25 & $323 / 346$ & $442 / 370$ \\
\hline 26 & ------/---- & ------/----- & 26 & ------/----- & $1662 / 1657$ \\
\hline 27 & ----- & ----- & 27 & 1078 & 1043 \\
\hline 28 & ------/----- & ------/----- & 28 & -----/988 & $\mathbf{8 5 5} / 865$ \\
\hline 29 & ----- & ----- & 29 & 821 & 740 \\
\hline 30 & -----/----- & -----/----- & 30 & -----/452 & 557/----- \\
\hline 31 & ----- & ----- & 31 & 32 & 34 \\
\hline 32 & ----- & $\begin{array}{l}---- \\
--\end{array}$ & 32 & 116 & 72 \\
\hline 33 & ----- & ----- & 33 & 156 & 124 \\
\hline
\end{tabular}


Appendix II (continued).

\begin{tabular}{|c|c|c|c|c|c|}
\hline \multirow{2}{*}{$\begin{array}{l}\text { Para- } \\
\text { meter }\end{array}$} & \multicolumn{2}{|c|}{ Specimen } & \multirow{2}{*}{$\begin{array}{l}\text { Para- } \\
\text { meter }\end{array}$} & \multicolumn{2}{|c|}{ Specimen } \\
\hline & CMN 8800 & CMN 8801 & & CMN 8802 & CMN 8803 \\
\hline 1 & $40 / \mathbf{1 0 8}$ & $-----/ 57$ & 1 & 71/----- & -----/----- \\
\hline 2 & $140 / 171$ & $-----/ 98$ & 2 & 85/----- & ------/----- \\
\hline 3 & $96 / 115$ & $-----/ 80$ & 3 & 73/----- & ------/----- \\
\hline 4 & 412/----- & $-----/ 300$ & 4 & 265/----- & -----/----- \\
\hline 5 & 122/----- & -----/194 & 5 & ------/----- & ------/----- \\
\hline $5 a$ & 159 & 147 & $5 a$ & ----- & ----- \\
\hline $5 b$ & 95 & 88 & $5 b$ & ----- & ----- \\
\hline $5 \mathrm{c}$ & ----- & ----- & $5 c$ & ----- & ----- \\
\hline 6 & 663/----- & $-----/ 677$ & 6 & ------/----- & ------/----- \\
\hline 7 & 218/----- & $-----/ 238$ & 7 & ------/----- & -----/----- \\
\hline 8 & 552 & 637 & 8 & ----- & ----- \\
\hline 9 & |-----/----- & -----/334 & 9 & ------/----- & |-----/----- \\
\hline 10 & 161/----- & -----/----- & 10 & -----/----- & ----------- \\
\hline 11 & 123/----- & -----/116 & 11 & ------/----- & ------/----- \\
\hline 12 & 128/----- & $-----/ 178$ & 12 & -----/----- & ------/----- \\
\hline 13 & $130 /-----$ & $-----/ 181$ & 13 & -----/----- & -----/----- \\
\hline 14 & 254/----- & ------/----- & 14 & -----/290 & ------/----- \\
\hline 15 & 332/----- & -----/----- & 15 & $-----/ 436$ & -----/----- \\
\hline 16 & 880/----- & $\mathbf{8 3 5} / 802$ & 16 & ------/----- & -----/----- \\
\hline 17 & 770/----- & -----/732 & 17 & ------/----- & ------/----- \\
\hline 18 & 309 & ----- & 18 & ----- & ----- \\
\hline 19 & 997/----- & ------/----- & 19 & ------/----- & ------/----- \\
\hline 20 & 424/----- & -----/----- & 20 & -----/----- & -----/----- \\
\hline 21 & 470/----- & -----/----- & 21 & -----/----- & ------/----- \\
\hline 22 & ------/----- & ------/----- & 22 & ------/----- & ------/----- \\
\hline 23 & 1080 & ----- & 23 & ----- & ----- \\
\hline 24 & 621/----- & -----/----- & 24 & ------/----- & ------/----- \\
\hline 25 & 322/----- & -----/----- & 25 & -----/----- & ------/----- \\
\hline 26 & 2019/----- & -----/----- & 26 & ------/----- & ------/----- \\
\hline 27 & 848 & ----- & 27 & ----- & ----- \\
\hline 28 & 1143/----- & ------/----- & 28 & ------/----- & ------/----- \\
\hline 29 & ----- & ----- & 29 & ----- & ----- \\
\hline 30 & 584/----- & -----/----- & 30 & -----/----- & ------/----- \\
\hline 31 & 36 & ----- & 31 & ----- & 65 \\
\hline 32 & 79 & ----- & 32 & ----- & 72 \\
\hline 33 & 89 & ----- & 33 & ----- & 101 \\
\hline
\end{tabular}


Appendix II (continued).

\begin{tabular}{|c|c|c|c|c|c|}
\hline \multirow{2}{*}{$\begin{array}{l}\text { Para- } \\
\text { meter }\end{array}$} & \multicolumn{2}{|c|}{ Specimen } & \multirow{2}{*}{$\begin{array}{l}\text { Para- } \\
\text { meter }\end{array}$} & \multicolumn{2}{|c|}{ Specimen } \\
\hline & CMN 9673 & CMN 9676 & & CMN 9808b & CMN 9808c \\
\hline 1 & 80/----- & $-----/ 440$ & 1 & 70/----- & $-----/ 86$ \\
\hline 2 & $150 /-----$ & $-----/ 142$ & 2 & 65/----- & ------/----- \\
\hline 3 & 99/----- & -----/104 & 3 & 61/----- & $-----/ 53$ \\
\hline 4 & 405/----- & $-----/ 410$ & 4 & 181/----- & -----/----- \\
\hline 5 & -----/----- & -----/----- & 5 & ------/----- & ------/----- \\
\hline $5 a$ & ----- & ----- & $5 a$ & ----- & ----- \\
\hline $5 b$ & ----- & $\begin{array}{l}---- \\
--1\end{array}$ & $5 b$ & ----- & ----- \\
\hline $5 c$ & $\begin{array}{l}---- \\
\end{array}$ & ----- & $5 c$ & $\begin{array}{l}---- \\
\end{array}$ & $\begin{array}{l}---- \\
\end{array}$ \\
\hline 6 & ------/----- & ------/----- & 6 & ------/----- & ------/----- \\
\hline 7 & ------/----- & ------/----- & 7 & ------/----- & ------/----- \\
\hline 8 & ----- & ----- & 8 & ----- & ----- \\
\hline 9 & ----------- & -----/----- & 9 & -----/----- & -----/----- \\
\hline 10 & ------/----- & ------/----- & 10 & ------/----- & ------/----- \\
\hline 11 & ------/----- & -----/----- & 11 & -----/----- & -----/----- \\
\hline 12 & ------/----- & ------/----- & 12 & ------/----- & ------/----- \\
\hline 13 & ------/----- & ------/----- & 13 & ------/----- & ------/----- \\
\hline 14 & -----/----- & -----/----- & 14 & -----/----- & -----/----- \\
\hline 15 & ------/----- & -----/----- & 15 & -----/----- & -----/----- \\
\hline 16 & ------/----- & ------/----- & 16 & -----/----- & -----/----- \\
\hline 17 & ------/----- & ------/----- & 17 & -----/----- & ------/----- \\
\hline 18 & ----- & ----- & 18 & ----- & ----- \\
\hline 19 & -----/----- & -----/----- & 19 & -----/----- & -----/----- \\
\hline 20 & -----/----- & -----/----- & 20 & -----/----- & -----/----- \\
\hline 21 & -----/----- & -----/----- & 21 & ------/----- & -----/----- \\
\hline 22 & ------/----- & ------/----- & 22 & -----/----- & ------/----- \\
\hline 23 & ----- & ----- & 23 & ----- & ----- \\
\hline 24 & ------/----- & ------/----- & 24 & ------/----- & ------/----- \\
\hline 25 & -----/----- & -----/----- & 25 & -----/----- & -----/----- \\
\hline 26 & -----/----- & -----/----- & 26 & -----/----- & -----/----- \\
\hline 27 & ----- & ----- & 27 & ----- & ----- \\
\hline 28 & ------/----- & ------/----- & 28 & -----/----- & -----/----- \\
\hline 29 & ----- & ----- & 29 & ----- & ----- \\
\hline 30 & ------/----- & ------/----- & 30 & ------/----- & ------/----- \\
\hline 31 & ----- & ----- & 31 & ----- & ----- \\
\hline 32 & ----- & ----- & 32 & ----- & ----- \\
\hline 33 & ----- & ----- & 33 & ----- & ----- \\
\hline
\end{tabular}


Appendix II (continued).

\begin{tabular}{|c|c|c|c|c|c|}
\hline \multirow{2}{*}{$\begin{array}{l}\text { Para- } \\
\text { meter }\end{array}$} & \multicolumn{2}{|c|}{ Specimen } & \multirow{2}{*}{$\begin{array}{l}\text { Para- } \\
\text { meter }\end{array}$} & \multicolumn{2}{|c|}{ Specimen } \\
\hline & CMN 9808d & CMN 9809 & & CMN 34829 & CMN 34832 \\
\hline 1 & -----/----- & 93/----- & 1 & $56 / \mathbf{5 9}$ & $236 / 237$ \\
\hline 2 & ------/----- & ------/----- & 2 & $75 / 74$ & $112 / \mathbf{1 1 1}$ \\
\hline 3 & $-----/ 45$ & ------/----- & 3 & $66 / 67$ & $108 / \mathbf{1 0 7}$ \\
\hline 4 & ------/----- & -----/----- & 4 & $227 / 244$ & 392/----- \\
\hline 5 & ------/----- & -----/----- & 5 & ------/----- & ------/----- \\
\hline $5 a$ & ----- & ----- & $5 a$ & ----- & ----- \\
\hline $5 b$ & ----- & $\begin{array}{l}---- \\
--1\end{array}$ & $5 b$ & ----- & ----- \\
\hline $5 c$ & $\begin{array}{l}---- \\
\end{array}$ & ----- & $5 c$ & $\begin{array}{l}---- \\
\end{array}$ & $\begin{array}{l}---- \\
\end{array}$ \\
\hline 6 & ------/----- & ------/----- & 6 & ------/----- & ------/----- \\
\hline 7 & ------/----- & ------/----- & 7 & ------/----- & ------/----- \\
\hline 8 & ----- & ----- & 8 & ----- & ----- \\
\hline 9 & ----------- & -----/----- & 9 & -----/----- & -----/----- \\
\hline 10 & ------/----- & ------/----- & 10 & $-----/ 115$ & ------/----- \\
\hline 11 & -----/----- & -----/----- & 11 & $-----/ 82$ & -----/----- \\
\hline 12 & ------/----- & ------/----- & 12 & $-----/ 99$ & ------/----- \\
\hline 13 & ------/----- & ------/----- & 13 & ------/----- & ------/----- \\
\hline 14 & -----/----- & -----/----- & 14 & $-----/ 287$ & ------/----- \\
\hline 15 & ------/----- & -----/----- & 15 & $-----/ 345$ & ----------- \\
\hline 16 & ------/----- & ------/----- & 16 & -----/----- & ------/----- \\
\hline 17 & ------/----- & ------/----- & 17 & -----/----- & ------/----- \\
\hline 18 & ----- & ----- & 18 & 299 & 377 \\
\hline 19 & -----/----- & -----/----- & 19 & $\mathbf{8 5 0} / 810$ & 682/----- \\
\hline 20 & -----/----- & -----/----- & 20 & 308/----- & ------"---- \\
\hline 21 & -----/----- & ------/----- & 21 & -----/----- & ------/----- \\
\hline 22 & ------/----- & ------/----- & 22 & ------/----- & ------/----- \\
\hline 23 & ----- & ----- & 23 & 920 & ----- \\
\hline 24 & ------/----- & ------/----- & 24 & ------/----- & ------/----- \\
\hline 25 & -----/----- & -----/----- & 25 & -----/----- & -----/----- \\
\hline 26 & -----/----- & -----/------ & 26 & -----/----- & ------/----- \\
\hline 27 & ----- & ----- & 27 & ----- & ----- \\
\hline 28 & ------/----- & ------/----- & 28 & ------/----- & ------/----- \\
\hline 29 & ----- & ----- & 29 & ----- & ----- \\
\hline 30 & ------/----- & ------/----- & 30 & ------/----- & ------/----- \\
\hline 31 & ----- & ----- & 31 & 40 & ----- \\
\hline 32 & ----- & ----- & 32 & ----- & ----- \\
\hline 33 & ----- & ----- & 33 & ----- & ----- \\
\hline
\end{tabular}


Appendix II (continued).

\begin{tabular}{|c|c|c|c|c|c|}
\hline \multirow{2}{*}{$\begin{array}{l}\text { Para- } \\
\text { meter }\end{array}$} & \multicolumn{2}{|c|}{ Specimen } & \multirow{2}{*}{$\begin{array}{l}\text { Para- } \\
\text { meter }\end{array}$} & \multicolumn{2}{|c|}{ Specimen } \\
\hline & CMN 41357 & $\begin{array}{l}\text { NHMUK } \\
\text { R4948 }\end{array}$ & & ROM 839 & ROM 843 \\
\hline 1 & $-----/ 36$ & 55 & 1 & $107 / 93$ & $67 / 55$ \\
\hline 2 & $-----/ 99$ & 100 & 2 & $\mathbf{8 5} / 84$ & $73 / 78$ \\
\hline 3 & $-----/ 65$ & -----/----- & 3 & $62 / 64$ & $72 / 66$ \\
\hline 4 & $-----/ 250$ & ------/----- & 4 & $250 / 250$ & 252/247 \\
\hline 5 & $123 / 115$ & 155 & 5 & $227 / \mathbf{2 3 7}$ & $199 / 198$ \\
\hline $5 a$ & 127 & 160 & $5 a$ & 122 & 170 \\
\hline $5 b$ & 88 & 90 & $5 b$ & 75 & 95 \\
\hline $5 c$ & 352 & $\begin{array}{ll}---- \\
--\end{array}$ & $5 c$ & 332 & 445 \\
\hline 6 & $-----/ 600$ & ------/----- & 6 & $545 / 555$ & 652/632 \\
\hline 7 & $-----/ 220$ & ------/----- & 7 & $224 / 234$ & $\mathbf{2 4 5} / 225$ \\
\hline 8 & 463 & ----- & 8 & 427 & 518 \\
\hline 9 & $-----/ 279$ & 285 & 9 & $-----/ 355$ & 328/303 \\
\hline 10 & $-----/ 138$ & 120 & 10 & $134 / \mathbf{1 4 3}$ & $144 / 134$ \\
\hline 11 & $-----/ 107$ & 100 & 11 & $98 / 90$ & $109 / 122$ \\
\hline 12 & -----/95 & ------/----- & 12 & $128 / \mathbf{1 2 3}$ & 115/126 \\
\hline 13 & -----/107 & 135 & 13 & $138 / \mathbf{1 2 6}$ & 131/129 \\
\hline 14 & $-----/ 275$ & 220 & 14 & $284 / 285$ & $295 / 335$ \\
\hline 15 & $293 / 295$ & 360 & 15 & $368 / 375$ & 395/417 \\
\hline 16 & -----/795 & ------/----- & 16 & $-----/ 782$ & $\mathbf{8 9 7 / 8 5 2}$ \\
\hline 17 & -----/----- & -----/----- & 17 & 635/667 & 822/----- \\
\hline 18 & 320 & ----- & 18 & 231 & 290 \\
\hline 19 & $680 / 700$ & 840 & 19 & $917 / 955$ & 893/965 \\
\hline 20 & 460 & ------/----- & 20 & -----/379 & 482/472 \\
\hline 21 & $435 / 430$ & ------/----- & 21 & $-----/ 380$ & $438 / 503$ \\
\hline 22 & $-----/ 225$ & ------/----- & 22 & $-----/ 115$ & 151/198 \\
\hline 23 & 1020 & ----- & 23 & 1020 & 1082 \\
\hline 24 & $360 / 405$ & 710/----- & 24 & $-----/ 635$ & $600 / 668$ \\
\hline 25 & $370 / \mathbf{3 8 0}$ & 490/----- & 25 & $287 / 347$ & $457 / 458$ \\
\hline 26 & $1632 / \mathbf{1 6 6 2}$ & ------/----- & 26 & $\mathbf{1 8 0 0} / 1868$ & $\mathbf{2 0 2 3} / 2115$ \\
\hline 27 & 1047 & 1160 & 27 & 893 & 1227 \\
\hline 28 & $740 / 753$ & ------/----- & 28 & $\mathbf{1 0 3 4} / 1082$ & $\mathbf{1 0 6 3} / 1180$ \\
\hline 29 & ----- & ----- & 29 & 900 & 817 \\
\hline 30 & 265/----- & 627/----- & 30 & ------/----- & 498/----- \\
\hline 31 & 33 & ----- & 31 & 26 & 30 \\
\hline 32 & 76 & 90 & 32 & ----- & 94 \\
\hline 33 & 180 & 162 & 33 & ----- & 136 \\
\hline
\end{tabular}


Appendix II (continued).

\begin{tabular}{|c|c|c|c|c|c|}
\hline \multirow{2}{*}{$\begin{array}{l}\text { Para- } \\
\text { meter }\end{array}$} & \multicolumn{2}{|c|}{ Specimen } & \multirow{2}{*}{$\begin{array}{l}\text { Para- } \\
\text { meter }\end{array}$} & \multicolumn{2}{|c|}{ Specimen } \\
\hline & $\begin{array}{l}\text { TMP } 1967 . \\
017.0005\end{array}$ & $\begin{array}{l}\text { TMP } 1979 . \\
011.0147\end{array}$ & & $\begin{array}{l}\text { TMP } 1979 . \\
014.0813\end{array}$ & $\begin{array}{l}\text { TMP } 1979 . \\
014.0814\end{array}$ \\
\hline 1 & 111/----- & $263 / \mathbf{2 9 8}$ & 1 & 61/----- & $-----/ 241$ \\
\hline 2 & 60/----- & $123 / \mathbf{1 1 8}$ & 2 & $56 /-----$ & $-----/ 116$ \\
\hline 3 & 49/----- & $101 / 98$ & 3 & 37/----- & -----/88 \\
\hline 4 & 186/----- & $353 / 337$ & 4 & $140 /-----$ & $-----/ 347$ \\
\hline 5 & ------/----- & 114/108 & 5 & ------/----- & ------/----- \\
\hline $5 a$ & ----- & 128 & $5 a$ & ----- & ----- \\
\hline $5 b$ & ----- & 75 & $5 b$ & ----- & ----- \\
\hline $5 c$ & ----- & 334 & $5 c$ & ----- & ----- \\
\hline 6 & -----/----- & -----/----- & 6 & -----/----- & -----/----- \\
\hline 7 & ------/----- & ------/----- & 7 & ------/----- & ------/----- \\
\hline 8 & ----- & ----- & 8 & ----- & ----- \\
\hline 9 & ------/----- & ------/----- & 9 & ------/----- & ------/----- \\
\hline 10 & -----/----- & -----/----- & 10 & -----/----- & -----/----- \\
\hline 11 & -----/----- & -----/----- & 11 & -----/----- & -----/----- \\
\hline 12 & -----/----- & -----/----- & 12 & -----/----- & -----/----- \\
\hline 13 & -----/----- & -----/----- & 13 & -----/----- & -----/----- \\
\hline 14 & ------/----- & ------/----- & 14 & ------/----- & ------/----- \\
\hline 15 & ------/----- & ------/----- & 15 & ------/----- & ------/----- \\
\hline 16 & -----/----- & -----/----- & 16 & -----/----- & -----/----- \\
\hline 17 & -----/----- & -----/----- & 17 & -----/----- & -----/----- \\
\hline 18 & ----- & 325 & 18 & ----- & ----- \\
\hline 19 & -----/----- & -----/----- & 19 & -----/----- & -----/----- \\
\hline 20 & ------/----- & ------/----- & 20 & ------/----- & ------/----- \\
\hline 21 & -----/----- & ------/----- & 21 & -----/----- & ------/----- \\
\hline 22 & -----/----- & -----/----- & 22 & -----/----- & -----/----- \\
\hline 23 & ----- & ----- & 23 & ----- & ----- \\
\hline 24 & -----/----- & -----/----- & 24 & -----/----- & -----/----- \\
\hline 25 & ------/----- & ------/----- & 25 & ------/----- & ------/----- \\
\hline 26 & ------/----- & ------/----- & 26 & ------/----- & ------/----- \\
\hline 27 & ----- & ----- & 27 & ----- & ----- \\
\hline 28 & ------/----- & ------/----- & 28 & ------/----- & ------/----- \\
\hline 29 & ----- & ----- & 29 & ----- & ----- \\
\hline 30 & ------/----- & ------/----- & 30 & ------/----- & ------/----- \\
\hline 31 & ----- & ----- & 31 & ----- & ----- \\
\hline 32 & $\begin{array}{l}---- \\
\end{array}$ & ----- & 32 & ----- & ----- \\
\hline 33 & ----- & ----- & 33 & $\begin{array}{l}---- \\
\end{array}$ & $\begin{array}{l}---- \\
\end{array}$ \\
\hline
\end{tabular}


Appendix II (continued).

\begin{tabular}{|c|c|c|c|c|c|}
\hline \multirow{2}{*}{$\begin{array}{l}\text { Para- } \\
\text { meter }\end{array}$} & \multicolumn{2}{|c|}{ Specimen } & \multirow{2}{*}{$\begin{array}{l}\text { Para- } \\
\text { meter }\end{array}$} & \multicolumn{2}{|c|}{ Specimen } \\
\hline & $\begin{array}{l}\text { TMP } 1981 . \\
018.0231\end{array}$ & $\begin{array}{l}\text { TMP } 1981 . \\
019.0175\end{array}$ & & $\begin{array}{l}\text { TMP } 1981 . \\
041.0116\end{array}$ & $\begin{array}{l}\text { TMP } 1983 . \\
025.0001\end{array}$ \\
\hline 1 & -----/------ & 64/134 & 1 & $-----/ 187$ & 142/----- \\
\hline 2 & -----/----- & $113 / 85$ & 2 & -----152 & 107/----- \\
\hline 3 & -----/----- & $70 / 86$ & 3 & $-----/ 116$ & 101/----- \\
\hline 4 & -----/----- & $338 / \mathbf{3 0 2}$ & 4 & -----/439 & 345/----- \\
\hline 5 & ------/----- & $140 / 134$ & 5 & ------/----- & ------/----- \\
\hline $5 a$ & ----- & 132 & $5 a$ & ----- & ----- \\
\hline $5 b$ & ----- & 62 & $5 b$ & ----- & ----- \\
\hline $5 c$ & ----- & 322 & $5 c$ & ----- & ----- \\
\hline 6 & -----/----- & $556 / 558$ & 6 & -----/----- & -----/----- \\
\hline 7 & ------/----- & $187 / \mathbf{2 0 0}$ & 7 & ------/----- & 171/----- \\
\hline 8 & ----- & 475 & 8 & ----- & ----- \\
\hline 9 & ------/----- & $-----/ 294$ & 9 & ------/----- & 256/----- \\
\hline 10 & -----/----- & $----/ 142$ & 10 & -----/----- & 113/----- \\
\hline 11 & -----/----- & -----/103 & 11 & -----/----- & 98/----- \\
\hline 12 & -----/----- & -----/107 & 12 & -----/----- & 91/----- \\
\hline 13 & -----/----- & -----/109 & 13 & -----/----- & 110/----- \\
\hline 14 & ------/----- & -----/252 & 14 & ------/----- & $245 / 271$ \\
\hline 15 & ------/----- & ------/337 & 15 & ------/----- & $352 / 363$ \\
\hline 16 & -----/----- & $----/ 768$ & 16 & -----/----- & -----/----- \\
\hline 17 & -----/----- & $-----/ 661$ & 17 & -----/----- & ------/----- \\
\hline 18 & ----- & 217 & 18 & ----- & ----- \\
\hline 19 & -----/----- & -----/600 & 19 & -----/----- & $-----/ 650$ \\
\hline 20 & ------/----- & $-----/ 228$ & 20 & ------/----- & 227/----- \\
\hline 21 & -----/----- & $-----/ 311$ & 21 & ------/----- & $290 / 323$ \\
\hline 22 & -----/----- & $-----/ 171$ & 22 & -----/----- & 125/----- \\
\hline 23 & ----- & ----- & 23 & ----- & 868 \\
\hline 24 & -----/----- & -----/----- & 24 & ------/----- & -----/658 \\
\hline 25 & ------/----- & ------/----- & 25 & ------/----- & -----/450 \\
\hline 26 & ------/----- & ------/----- & 26 & ------/----- & ------/----- \\
\hline 27 & ----- & ----- & 27 & ----- & 1373 \\
\hline 28 & ------/----- & ------/----- & 28 & ------/----- & -----/952 \\
\hline 29 & ----- & ----- & 29 & ----- & ----- \\
\hline 30 & ------/----- & ------/----- & 30 & -----/----- & -----/592 \\
\hline 31 & 56 & ----- & 31 & ----- & 45 \\
\hline 32 & $\begin{array}{l}---- \\
\end{array}$ & ----- & 32 & ----- & 102 \\
\hline 33 & ----- & ----- & 33 & ----- & 128 \\
\hline
\end{tabular}


Appendix II (continued).

\begin{tabular}{|c|c|c|c|c|c|}
\hline \multirow{2}{*}{$\begin{array}{l}\text { Para- } \\
\text { meter }\end{array}$} & \multicolumn{2}{|c|}{ Specimen } & \multirow{2}{*}{$\begin{array}{l}\text { Para- } \\
\text { meter }\end{array}$} & \multicolumn{2}{|c|}{ Specimen } \\
\hline & $\begin{array}{l}\text { TMP } 1984 . \\
163.0041\end{array}$ & $\begin{array}{l}\text { TMP } 1987 . \\
045.0001\end{array}$ & & $\begin{array}{l}\text { TMP } 1993 . \\
082.0001\end{array}$ & $\begin{array}{l}\text { TMP } 1998 . \\
128.0001\end{array}$ \\
\hline 1 & -----/------ & $44 / 25$ & 1 & ------/----- & ------/----- \\
\hline 2 & -----/----- & $69 / 54$ & 2 & -----/----- & ------/----- \\
\hline 3 & -----/----- & $57 / 66$ & 3 & -----/----- & -----/----- \\
\hline 4 & ------/----- & 233/207 & 4 & -----/----- & -----/------ \\
\hline 5 & 131/----- & $124 / 108$ & 5 & ------/----- & ------/----- \\
\hline $5 a$ & ----- & 124 & $5 a$ & ----- & ----- \\
\hline $5 b$ & ----- & 86 & $5 b$ & ----- & ----- \\
\hline $5 c$ & ----- & 366 & $5 c$ & ----- & ----- \\
\hline 6 & -----/----- & $583 / 595$ & 6 & -----/----- & -----/----- \\
\hline 7 & ------/----- & $158 / 182$ & 7 & ------/----- & ------/----- \\
\hline 8 & ----- & 485 & 8 & ----- & ----- \\
\hline 9 & ------/----- & 258/------ & 9 & ------/----- & ------/----- \\
\hline 10 & -----/----- & $86 / 70$ & 10 & -----/----- & -----/----- \\
\hline 11 & -----/----- & ------/----- & 11 & -----/----- & -----/----- \\
\hline 12 & -----/----- & -----/------ & 12 & -----/----- & -----/----- \\
\hline 13 & -----/----- & -----/----- & 13 & -----/----- & -----/----- \\
\hline 14 & ------/----- & 265/----- & 14 & ------/----- & 87/----- \\
\hline 15 & ------/----- & 352/----- & 15 & ------/----- & 104/----- \\
\hline 16 & -----/----- & $617 / 637$ & 16 & -----/----- & -----/----- \\
\hline 17 & -----/----- & $548 / 558$ & 17 & -----/----- & -----/----- \\
\hline 18 & ----- & 285 & 18 & ----- & ----- \\
\hline 19 & -----/----- & 633/----- & 19 & 879/----- & 208/----- \\
\hline 20 & ------/----- & $265 /-----$ & 20 & ------/----- & ------/----- \\
\hline 21 & -----/----- & 382/----- & 21 & -----/----- & ------/----- \\
\hline 22 & -----/----- & $157 /-----$ & 22 & -----/----- & -----/----- \\
\hline 23 & ----- & ----- & 23 & ----- & ----- \\
\hline 24 & -----/----- & 338/----- & 24 & 611/----- & -----/----- \\
\hline 25 & ------/----- & 300/----- & 25 & ------/----- & ------/----- \\
\hline 26 & ------/----- & 1450/----- & 26 & ------/----- & ------/----- \\
\hline 27 & ----- & 940 & 27 & ----- & ----- \\
\hline 28 & ------/----- & 755/----- & 28 & ------/----- & ------/----- \\
\hline 29 & ----- & ----- & 29 & ----- & ----- \\
\hline 30 & ------/----- & $260 /-----$ & 30 & -----/----- & -----/----- \\
\hline 31 & ----- & ----- & 31 & ----- & ----- \\
\hline 32 & $\begin{array}{l}---- \\
\end{array}$ & ----- & 32 & ----- & ----- \\
\hline 33 & ----- & ----- & 33 & ----- & ----- \\
\hline
\end{tabular}


Appendix II (continued).

\begin{tabular}{|c|c|c|c|c|c|}
\hline \multirow{2}{*}{$\begin{array}{l}\text { Para- } \\
\text { meter }\end{array}$} & \multicolumn{2}{|c|}{ Specimen } & \multirow{2}{*}{$\begin{array}{l}\text { Para- } \\
\text { meter }\end{array}$} & \multicolumn{2}{|c|}{ Specimen } \\
\hline & $\begin{array}{l}\text { TMP } 2007 . \\
020.0103\end{array}$ & $\begin{array}{l}\text { TMP } 2008 . \\
012.0001\end{array}$ & & $\begin{array}{l}\text { TMP } 2008 . \\
012.0074\end{array}$ & $\begin{array}{l}\text { TMP } 2009 . \\
032.0011\end{array}$ \\
\hline 1 & -----/----- & -----/----- & 1 & 75/----- & ------/----- \\
\hline 2 & -----/----- & -----/----- & 2 & -----/----- & -----/----- \\
\hline 3 & -----/----- & -----/----- & 3 & 35/----- & -----/----- \\
\hline 4 & -----/----- & ------/----- & 4 & $147 /----$ & ------/----- \\
\hline 5 & -----/----- & -----/----- & 5 & -----/---- & -----/----- \\
\hline $5 a$ & ----- & ---- & $5 a$ & ----- & ----- \\
\hline $5 b$ & ----- & ----- & $5 b$ & ----- & ----- \\
\hline $5 c$ & ----- & ----- & $5 c$ & ----- & ----- \\
\hline 6 & -----/----- & -----/----- & 6 & $-----/----$ & -----/----- \\
\hline 7 & -----/----- & -----/----- & 7 & -----/----- & -----/----- \\
\hline 8 & ----- & ----- & 8 & ----- & ----- \\
\hline 9 & -----/----- & -----/----- & 9 & -----/----- & -----/----- \\
\hline 10 & -----/----- & ------/----- & 10 & -----/----- & ------/----- \\
\hline 11 & ------/----- & ------/----- & 11 & ------/------ & ------/------ \\
\hline 12 & ------/----- & ------/----- & 12 & ------/------ & ------/----- \\
\hline 13 & ------/----- & ------/----- & 13 & -----/------ & ------/----- \\
\hline 14 & -----/----- & -----/----- & 14 & -----/----- & -----/----- \\
\hline 15 & -----/------ & ------/----- & 15 & -----/------ & -----/------ \\
\hline 16 & -----/----- & -----/----- & 16 & -----/----- & -----/----- \\
\hline 17 & -----/----- & ------/----- & 17 & ------/----- & -----/----- \\
\hline 18 & ----- & ----- & 18 & ----- & ----- \\
\hline 19 & ------/----- & ------/----- & 19 & ------/----- & ------/----- \\
\hline 20 & -----/----- & -----/----- & 20 & -----/----- & -----/----- \\
\hline 21 & ------/----- & ------/----- & 21 & ------/----- & ------/----- \\
\hline 22 & ------/----- & ------/------ & 22 & ------/------ & ------/------ \\
\hline 23 & ----- & ----- & 23 & ----- & ----- \\
\hline 24 & -----/----- & -----/----- & 24 & -----/----- & -----/----- \\
\hline 25 & ------/----- & ------/----- & 25 & ------/----- & ------/----- \\
\hline 26 & ------/----- & ------/----- & 26 & ------/----- & ------/----- \\
\hline 27 & ---- & ----- & 27 & ----- & ----- \\
\hline 28 & ------/----- & ------/----- & 28 & ------/------ & ------/----- \\
\hline 29 & ----- & ----- & 29 & ----- & ----- \\
\hline 30 & -----/------ & -----/------ & 30 & -----/------ & -----/----- \\
\hline 31 & 37 & 27 & 31 & ---- & 34 \\
\hline 32 & 88 & 62 & 32 & ----- & 70 \\
\hline 33 & ----- & 171 & 33 & ----- & ----- \\
\hline
\end{tabular}


Appendix II (continued).

\begin{tabular}{|c|c|c|}
\hline \multirow{2}{*}{$\begin{array}{l}\text { Para- } \\
\text { meter }\end{array}$} & \multicolumn{2}{|c|}{ Specimen } \\
\hline & UALVP 40 & YPM 2016 \\
\hline 1 & $164 / \mathbf{1 7 6}$ & $37 / 41$ \\
\hline 2 & $75 / 72$ & $72 / 71$ \\
\hline 3 & $75 / 69$ & $53 / 64$ \\
\hline 4 & $254 / \mathbf{2 4 0}$ & $217 / \mathbf{2 2 2}$ \\
\hline 5 & $131 / 120$ & $123 / 113$ \\
\hline $5 a$ & 114 & 118 \\
\hline $5 b$ & 48 & 67 \\
\hline $5 c$ & 305 & 300 \\
\hline 6 & 440/442 & $592 / 637$ \\
\hline 7 & $174 / 177$ & $205 / 235$ \\
\hline 8 & 385 & 477 \\
\hline 9 & 270/----- & 243/----- \\
\hline 10 & 114/109 & $119 / \mathbf{1 5 0}$ \\
\hline 11 & 90/92 & $78 / 93$ \\
\hline 12 & $\mathbf{1 0 8} / 96$ & $82 / 96$ \\
\hline 13 & 108/103 & 96/103 \\
\hline 14 & 234/----- & $270 / \mathbf{2 6 0}$ \\
\hline 15 & 337/----- & 360/----- \\
\hline 16 & 628/645 & $735 / 800$ \\
\hline 17 & $548 / 570$ & $660 / 720$ \\
\hline 18 & 180 & 271 \\
\hline 19 & 600/----- & $750 / 730$ \\
\hline 20 & 320/----- & $330 / 279$ \\
\hline 21 & 315/----- & $385 / 350$ \\
\hline 22 & 88/----- & 193/----- \\
\hline 23 & ----- & 940 \\
\hline 24 & ------/----- & $515 / 575$ \\
\hline 25 & -----/----- & $410 / 450$ \\
\hline 26 & -----/------ & $1668 / \mathbf{1 6 9 0}$ \\
\hline 27 & ----- & 1162 \\
\hline 28 & ------/----- & $855 / 840$ \\
\hline 29 & ----- & 775 \\
\hline 30 & ------/----- & -----/463 \\
\hline 31 & ----- & 34 \\
\hline 32 & $\begin{array}{l}---- \\
--\end{array}$ & 75 \\
\hline 33 & ----- & 171 \\
\hline
\end{tabular}




\section{Appendix III: List of characters used in specimen-based phylogenetic analysis}

\section{Anatomical terminology modifications}

For the sake of using consistent terminology, the following descriptive changes were made to the following characters: "supraorbital" changed to "postorbital" (35-41); "laterotemporal fenestra" (42 and 52) changed to "lateral temporal fenestra"; "infratemporal process" changed to "lateral temporal process" (47); "dorsotemporal fenestra" (54, 62 and 74) changed to "dorsal temporal fenestra"; "frontal fontanelle" (55 and 56) changed to "frontoparietal fontanelle"; "transverse parietal bar" (67, 76 and 77) and "caudal ramus of parietal" (68) changed to "posterior parietal bar"; and "rostral or rostro-" changed to "anterior or antero-" and "caudal or caudo-" changed to "posterior or postero-““ $(1,4,7,9,17-19,24,25,29,32,34,37,38,40,41,46,52,57,62,66,68,70$, $72,76,77,86,98,103,111,118,119,128,142$, and 152). The above replaced terms are well established in the literature, but were changed to be more consistent with the terminology used in the rest of this study.

Epiparietals are numbered sequentially starting at the midline of the posterior parietal and progress towards the squamosal (Sampson et al., 2010). Although episquamosals have been traditionally numbered sequentially starting at the anterior end of the squamosal, I have chosen to follow the methodology of Sampson et al. (2010), in which episquamosals are numbered sequentially starting at the posterior end of the squamosal. 


\section{List of characters}

Taxa scored differently from Mallon et al. (2011) are shown below, organized by characters; rescored codings for Bravoceratops polyphemus are also given, modified from Wick and Lehman (2013). Some characters in this study describe the presence or absence of a feature, and are followed by other characters that describe that feature in species or specimens that possess that feature. Species or specimens coded as 'absent' for the former characters were coded as '?' in the latter characters. Species or specimens in which a given feature is not preserved were also coded as'?' for characters relating to that feature. Therefore, species or specimens in which a given feature is either absent or not preserved were coded as “?”.

It could be argued that characters $18,22,84,114$, and 116 are not independent of other characters $(19,21,82,113$, and 115 , respectively); however, these characters were not excluded from the analysis as they have been used in previous phylogenetic studies (e.g., Forster et al., 1993; Sampson et al., 2010; Mallon et al., 2011; Wick and Lehman, 2013). For each of the 153 character descriptions, the terms "outgroup", "centrosaurine", "chasmosaurine", "ceratopsid", and "neoceratopsian" taxa refer strictly to the relevant taxa included in the analysis, and not necessarily to these groups in their entirety.

\section{Dermal skull roof}

(1) Rostral, extent of dorsal and ventral processes (Dodson et al., 2004, character 1):

(0) - triangular in lateral view, with short dorsal and ventral processes.

(1) - elongate, with deeply concave posterior margin and hypertrophied dorsal and ventral processes. 
The dorsal and ventral processes of the rostral (Fig. 35) are elongate in Leptoceratops, Zuniceratops and chasmosaurines. These processes are short in Protoceratops and in centrosaurines.

(2) Nares, size and position (Sampson et al., 2010, character 2):

(0) - small, restricted to dorsal $1 / 3$ of premaxilla, undifferentiated, $10 \%$ or less that of basal skull length.

(1) - large, expanded to occupy most of the depth of the premaxilla, $15 \%$ or greater than basal skull length.

The naris is relatively large in Zuniceratops, and in ceratopsids. The naris is relatively small in Leptoceratops and Protoceratops.

(3) Premaxillary septum (Chinnery and Weishampel, 1998, character 10):

(0) - absent.

(1) - present.

The premaxillary septum is a relatively thin, medial wall of bone formed by both premaxillae (Fig. 35). This septum is present in Zuniceratops and in ceratopsids. The septum is absent in Leptoceratops and Protoceratops.

(4) Premaxillary septum, shape (Dodson et al., 2004, character 4):

(0) - anteriorly elongate.

(1) - hemicircular. 
The premaxillary septum is anteriorly elongate in Zuniceratops and in chasmosaurines, but hemicircular in centrosaurines.

(5) Premaxillary septum, nasal contribution (Sampson et al., 2010, character 5):

(0) - septum formed by premaxilla only.

(1) - septum formed by premaxilla and nasal.

The premaxillary septum is formed only by the premaxilla in Zuniceratops and in chasmosaurines. The septum is formed by the premaxilla and nasal in centrosaurines.

(6) Premaxilla, narial strut (Holmes et al., 2001, character 1):

(0) - absent.

(1) - present.

The narial strut is a narrow strut situated towards the posterior margin of the premaxillary septum (Fig. 35), and is sub-vertically oriented. This strut is present in chasmosaurines, but absent in centrosaurines and outgroup taxa.

(7) Premaxilla, narial strut orientation (Dodson et al., 2004, character 6):

(0) - anteriorly inclined.

(1) - posteriorly inclined.

The narial strut of the premaxilla is anteriorly inclined in most Chasmosaurus specimens (except for CMN 8801 and YPM 2016) and other 
chasmosaurines, but is posteriorly inclined in Kosmoceratops, Coahuilaceratops, Anchiceratops, and Arrhinoceratops.

(8) Premaxilla, septal flange (Holmes et al., 2001, character 2):

(0) - absent.

(1) - present.

The septal flange is a thin flange of bone on the posterior margin of the premaxillary septum, and projects towards the naris (Fig. 35). This flange is present in Chasmosaurus, Vagaceratops, Kosmoceratops, Agujaceratops, Utahceratops, Pentaceratops, and dimorphic for Anchiceratops. The flange is absent in other chasmosaurines and in centrosaurines.

Centrosaurus apertus and Pachyrhinosaurus lakustai were both recoded from ? to 0 because the premaxilla septal flange is absent.

(9) Premaxilla, septal flange length (Forster et al., 1993, character 1):

(0) - spans entire posterior margin of narial strut.

(1) - restricted to ventral portion of narial strut.

The septal flange of the premaxilla spans the entire posterior margin of the narial strut in Chasmosaurus, Vagaceratops and Agujaceratops. The flange is restricted to the ventral portion of the narial strut in Kosmoceratops, Utahceratops, Pentaceratops, and Anchiceratops. 
(10) Premaxilla, septal fossa (Holmes et al., 2001, character 4):

(0) - absent.

(1) - present.

The septal fossa is a subcircular fossa that indents the premaxillary septum (Fig. 35). This fossa is present in chasmosaurines, but absent in centrosaurines and outgroup taxa.

(11) Premaxilla, subordinate fossa in premaxillary septum (Dodson et al., 2004, character 8 , modified here)

(0) - absent.

(1) - present.

This character originally read "interpremaxillary fossa", but was changed to "subordinate fossa". The "interpremaxillary fossa" of Dodson et al. (2004) and "septal fossa" of Holmes et al. (2001; and character 10 in this study) both refer to the same feature; the "premaxillary fossa" of Forster (1996) also refers to the same feature. Sampson et al. (2010) and Mallon et al. (2011), however, used the term "interpremaxillary fossa" to refer to the smaller fossa situated anterior to the septal fossa (Fig. 35). Lehman (1998:902) refers to this smaller fossa as the "subordinate fossa" of the premaxilla. The term "subordinate fossa" was used in this study to avoid confusion, as its name indicates that it is subordinate in size compared to the septal fossa. The subordinate fossa is present in Agujaceratops, Pentaceratops, Eotriceratops, Nedoceratops, Torosaurus latus, and both species 


\section{of Triceratops.}

Agujaceratops mariscalensis and Pentaceratops sternbergi were both recoded from 0 to 1 because the subordinate fossa of the premaxilla is present.

(12) Premaxilla, accessory strut in septal fossa (Sampson et al., 2010, character 12):

(0) - no accessory strut.

(1) - strut present.

This accessory strut is situated in the septal fossa of the premaxilla, anterior to the narial strut (Fig. 35). This accessory strut is absent in Chasmosaurus, Vagaceratops, Kosmoceratops, Agujaceratops, Anchiceratops, Arrhinoceratops, and Eotriceratops. The accessory strut is present in other chasmosaurines.

(13) Premaxilla, triangular process (Forster, 1990, character 21):

(0) - absent.

(1)- present .

The triangular process is a process on the posteroventral margin of the premaxillary septum, and it projects into the naris (Fig. 35). This process is present in chasmosaurines, but absent in centrosaurines and outgroup taxa.

(14) Premaxilla, triangular process shape (Sampson et al., 2010, character 14): (0) - square. 
(1) - pinched and triangular with concave facets.

The triangular process of the premaxilla is square in Chasmosaurus, Vagaceratops and Agujaceratops, but triangular in other chasmosaurines.

(15) Premaxilla, triangular process recess (Dodson et al., 2004, character 12):

(0) - absent.

(1) - present.

This recess is a pronounced concave surface on the lateral margin of the triangular process of the premaxilla (Fig. 35). This recess is absent in chasmosaurines, except for Nedoceratops and both species of Triceratops.

(16) Premaxilla, recess along ventral portion of septum (Dodson et al., 2004, character 9): (0) - absent. (1) - present.

The recess along the ventral portion of the premaxillary septum is absent in chasmosaurines and outgroup taxa, but present in centrosaurines (Fig. 35).

(17) Premaxilla, posteroventral expansion of oral margin (Forster, 1990, character 6): (0) - absent. (1) - present.

The oral margin of the premaxilla is not posteroventrally expanded in chasmosaurines, but is expanded in centrosaurines and outgroup taxa (Fig. 35). 
(18) Premaxilla, position of posterior tip of posteroventral process (Forster et al., 1993, character 7):

(0) - inserts into an embayment in the nasal.

(1) - intervenes between nasal and maxilla.

Character 18 does not appear to be independent of 19. The posterior tip of the posteroventral process of the premaxilla inserts between the nasal and maxilla in ceratopsids. Of the ceratopsids that possess a forked posterior tip, the upper part of the fork inserts into an embayment in the nasal. Therefore, 18(0) always pairs with 19(1), and 18(1) always pairs with 19(0) (Robert Holmes and Andrew Farke, pers. comm.).

(19) Premaxilla, distal end of posteroventral process forked (Forster, 1990, character 14; and Forster et al., 1993, character 7, modified here):

(0) - absent.

(1) - present, with upper half of fork inserting into an embayment in the nasal.

"With upper half of fork inserting into an embayment in the nasal" was added to state (1). This was done to accommodate pairing with character 18 (Robert Holmes and Andrew Farke, pers. comm.). The process is forked in Zuniceratops, Chasmosaurus, Vagaceratops, Kosmoceratops, Agujaceratops, Utahceratops, and Pentaceratops (Fig. 35). The fork is absent in Leptoceratops, Protoceratops, centrosaurines, and other chasmosaurines. 
(20) Premaxilla-nasal contact in dorsal view (Sampson et al., 2010, character 20):

(0) - premaxillae insert between nasal.

(1) - nasals insert between premaxillae.

The nasals insert between the premaxillae in dorsal view in Agujaceratops, Utahceratops, Pentaceratops and Coahuilaceratops. In other chasmosaurines, centrosaurines and outgroup taxa, the premaxillae insert between the nasals (Fig. 35).

(21) Accessory antorbital fenestra (Forster, 1990, character 15):

(0) - present.

(1) - absent.

The accessory antorbital fenestra is a perforation situated anterior to the antorbital fenestra, at the junction between the premaxilla, maxilla and nasal. This feature was scored as present only in taxa possessing a large, distinct perforation. Taxa possessing either a small and indistinct perforation or no perforation were scored as absent. This was done because a small perforation could actually represent breakage, or its apparent absence could be attributed to plaster reconstruction. This fenestra is absent in Chasmosaurus, except for AMNH 5401 and CMN 8800. The fenestra is present in Zuniceratops, and in the chasmosaurines Kosmoceratops, Utahceratops and Pentaceratops (absent in other chasmosaurines); this fenestra is absent in centrosaurines (except for Diabloceratops - not included in this analysis). 
Protoceratops andrewsi was recoded from 0 to 1 because it lacks a large, distinct accessory antorbital fenestra.

(22) Accessory antorbital fenestra size (Sampson et al., 2010, character 22):

(0) - pronounced, penetration of nasal cavity visible in lateral view.

(1) - slight penetration, nasal cavity not visible in lateral view.

Character 22 does not appear to be independent of 21 . In taxa possessing a large and distinct accessory antorbital fenestra (21(0) - Zuniceratops, Kosmoceratops, Utahceratops, Pentaceratops, and the Chasmosaurus specimens AMNH 5401 and CMN 8800), the nasal cavity is visible in lateral view (22(0)). Therefore, 21(0) always pairs with 22(0).

Kosmoceratops richardsoni was recoded from 1 to 0, Utahceratops gettyi ? to 0 , and Pentaceratops sternbergi 1 to 0 because the nasal cavity is visible through the accessory antorbital fenestra.

(23) External antorbital fossa, size (Forster, 1990, character 44):

(0) - large, $20 \%$ or more length of body of maxilla.

(1) - greatly reduced or absent, less than $10 \%$ length of body of maxilla.

The external antorbital fossa is a shallow, funnel-like depression on the side of the snout that leads into the antorbital fenestra. This fossa is relatively large in outgroup taxa, but relatively small in ceratopsids.

Turanoceratops tardabilis was recoded from 0 to ? because the maxilla is 
fragmentary and does not preserve the entire external antorbital fossa.

(24) Maxilla, relation of alveolar margin to anterior edentulous margin (Sampson et al., 2010, character 24):

(0) - edentulous portion maxilla elevated above level of alveoli.

(1) - at same level.

The alveolar and edentulous margins of the maxilla are at the same level in chasmosaurines, Centrosaurus and Pachyrhinosaurus. The edentulous margin is above the alveolar margin in Albertaceratops and outgroup taxa.

(25) Maxilla and premaxilla, diastema on anterior maxilla (Sampson et al., 2010, character 25, modified here):

(0) - present.

(1) - absent.

This character was changed from "maxilla" to "maxilla and premaxilla", as it pertains to both elements. The anterior end of the maxilla is edentulous in ceratopsians. However, premaxillary teeth are present in some taxa, making the edentulous part of the maxilla a diastema (Robert Holmes and Andrew Farke, pers. comm.). This diastema is present in Protoceratops, but absent in Leptoceratops, Zuniceratops and ceratopsids.

Leptoceratops gracilis and Zuniceratops christopheri were both recoded from 0 to 1 because they lack premaxillary teeth, and therefore lack a diastema 
between the premaxilla and maxilla. Turanoceratops tardabilis was recoded from 0 to ? because the premaxilla is not preserved, and the presence of a diastema cannot be determined.

(26) Maxilla, maxillary cavity (Sampson et al., character 26):

(0) - absent.

(1) - present.

The maxillary cavity is a large cavity on the medial margin of the maxilla. This cavity is present in ceratopsids, but absent in outgroup taxa.

(27) Nasal, ornamentation in adult (Sampson et al., 2010, character 27):

(0) - absent.

(1) - present.

Nasal ornamentation is present in Protoceratops and ceratopsids, but absent in Leptoceratops and Zuniceratops.

(28) Nasal, ornamentation type in adult (Sampson et al., 2010, character 28, modified here):

(0) - non-pronounced.

(1) - distinct and/or forming a horncore.

(2) - pachyostotic boss.

State (1) was modified from "distinct horncore" to "distinct and/or 
forming a horncore", as Albertaceratops (and Medusaceratops - not included in this analysis) have pronounced nasal ornamentation but not quite a horncore. The nasals form a horncore in chasmosaurines and in most centrosaurines except for Pachyrhinosaurus (pachyostotic boss). The nasal ornamentation is nonpronounced in Protoceratops.

Albertaceratops nesmoi was recoded from 0 to 1 because it has pronounced nasal ornamentation (although it is not technically a horncore).

(29) Nasal, ornamentation position, measured perpendicular to horizontal toothrow (Sampson et al., 2010, character 29):

(0) - centred dorsal to or posterior to centre of endonaris.

(1) - centred anterior to centre of endonaris.

Nasal ornamentation is centered dorsal to or posterior to the centre of the endonaris in Protoceratops, centrosaurines, and chasmosaurines except for Coahuilaceratops, Ojoceratops, Torosaurus, Nedoceratops, Triceratops, and Bravoceratops.

(30) Nasal, narial spine (Forster, 1990, character 22):

(0) - absent.

(1) - present.

The narial spine of the nasal is a finger-like process on the rim of the naris 
that projects into the narial opening (Fig. 35). This spine of the nasal is present in centrosaurines, but absent in chasmosaurines and outgroup taxa.

(31) Posterior end of maxillary alveolar process, visibility in lateral view (Sampson et al., 2010, character 31, modified here):

(0) - deep, alveolar process of maxilla entirely visible.

(1) - shallow, alveolar process of maxilla obscured by jugal.

This character phrasing replaces the original "facial skeleton, dorsoventral depth in orbital region", as the former better explains this character. The jugal obscures the alveolar process of the maxilla in lateral view in Chasmosaurus and Vagaceratops. In other chasmosaurines, centrosaurines and outgroup taxa, the alveolar process is entirely visible in lateral view.

(32) Orbit, orientation (Sampson et al., 2010, character 32):

(0) - directed anterolaterally.

(1) - directed laterally.

The orbit is laterally-oriented in ceratopsids and Zuniceratops, but anterolaterally-oriented in Leptoceratops and Protoceratops.

(33) Orbit diameter (Sampson et al., 2010, character 33, modified here):

(0) - more than $20 \%$ of basal skull length.

(1) - less than $15 \%$ of basal skull length. 
In character 33, "skull length" actually refers to basal skull length (Robert Holmes and Andrew Farke, pers. comm.); the change has been made here. Basal skull length is defined as the distance between the rostral and occipital condyle, as measured along the midline. The orbit diameter is relatively small in ceratopsids and Zuniceratops but relatively large in Leptoceratops and Protoceratops.

Turanoceratops tardabilis was recoded from 0 to ? because the orbit is not preserved.

(34) Lacrimal size (Chinnery and Weishampel, 1998, character 1):

(0) - large, forms $50 \%$ or more of the anterior orbital margin.

(1) - small, forms $40 \%$ or less of the anterior orbital margin.

The lacrimal is relatively small in ceratopsids and Zuniceratops, but relatively large in Leptoceratops and Protoceratops.

(35) Postorbital, postorbital ornamentation in adult (Sampson et al., 2010, character 35):

(0) - absent.

(1) - present.

Postorbital ornamentation is present in ceratopsids, Zuniceratops and Turanoceratops, but absent in Leptoceratops and Protoceratops. Vagaceratops (CMN 41357 and TMP 1987.045.0001) were scored as “?”, because they possess a low-relief mound above each orbit. These mounds are difficult to interpret, and it cannot be determined whether they represent the bases of longer horncores 
removed by bone resorption, or if horncores were never present in life. A welldeveloped pit is present in the preserved right mound of CMN 41357

(Vagaceratops), which may suggest that this individual had horncores in life.

(36) Postorbital, extent of cornual sinuses in base of postorbital ornamentation (Sampson et al., 2010, character 36):

(0) - sinus space invades frontal and parietal.

(1) - sinus space enters postorbital.

The cornual sinus is a large cavity in the dorsal roof of the skull, situated between the orbits. This sinus enters the postorbital in chasmosaurines and Pachyrhinosaurus. The sinus enters the frontal and parietal in Zuniceratops and Centrosaurus.

(37) Postorbital, position of postorbital horncore (Lehman, 1996, character 9):

(0) - centred anterodorsal or dorsal to orbit, narrow base with posterior margin of postorbital horncore extending to or only slightly behind posterior margin of orbit.

(1) - centred posterodorsal to orbit, broad base with posterior margin of postorbital horncore extending well behind posterior orbit.

The postorbital horncore is centred anterodorsal/dorsal to the orbit in Chasmosaurus, Kosmoceratops, Agujaceratops, Pentaceratops, Judiceratops, Centrosaurus, Albertaceratops, and outgroup taxa. The horncore is centred 
posterodorsal to orbit in other chasmosaurines and in Pachyrhinosaurus.

Vagaceratops (CMN 41357 and TMP 1987.045.0001) was scored as “?”, because its possible horncores are low-relief mounds.

(38) Postorbital, orientation of postorbital horncore base (Sampson et al., 2010, character 38, modified here):

(0) - dorsally directed.

(1) - anterodorsolaterally directed.

State (1) was changed from "dorsolaterally" to "anterodorsolaterally", as laterally directed postorbital horncores typically have a slight anterior orientation as well. In Chasmosaurus, the postorbital horncore is oriented both dorsally (AMNH 5402, CMN 1254, CMN 2245, CMN 2280, CMN 34829, and ROM 839) and anterodorsolaterally (AMNH 5401, CMN 34832, TMP 1979.011.0147, TMP 1981.019.0175, TMP 1983.025.0001, and UALVP 40). Horncores are dorsallyoriented in centrosaurines (but anterodorsolaterally oriented in Coronosaurus not included in analysis) and outgroup taxa (but not Turanoceratops anterodorsolaterally oriented), as well as some chasmosaurines (Pentaceratops, Torosaurus utahensis, Nedoceratops, and Triceratops). Other chasmosaurines have anterodorsolaterally-oriented horncores, although Torosaurus latus is dimorphic.

Turanoceratops tardabilis was recoded from 0 to 1 because the postorbital horncore is anterodorsolaterally oriented. Bravoceratops polyphemus was recoded 
from 0 to ? because the postorbital horncore is fragmentary and its orientation cannot be determined.

(39) Postorbital, length of unmodified postorbital horncore (Sampson et al., 2010, character 39, modified here):

(0) - short, less than $15 \%$ basal skull length.

(1) - present, elongate, greater than $35 \%$ basal skull length.

The term "unmodified" was added to this character description, as this character describes the length of a horncore unmodified by subsequent breakage or potential resorption of bone - both of which artificially shorten the original horncore. In Chasmosaurus, postorbital horncores are both relatively short (AMNH 5402, CMN 2245, CMN 2280, CMN 8802, CMN 34829, ROM 839, and UALVP 40; inferred for TMP 1981.019.0175 and UALVP 40) and long (AMNH 5401, CMN 1254, CMN 34832, and TMP 1979.011.0147; inferred for TMP 1983.025.0001). Postorbital horncores are relatively long in other chasmosaurines (except for Utahceratops), and relatively short in centrosaurines (except for Albertaceratops, Diabloceratops and Xenoceratops), and short in outgroup taxa except for Zuniceratops and Turanoceratops.

(40) Postorbital, curvature of postorbital horncore in lateral view (Sampson et al., 2010, character 40):

(0) - posteriorly recurved. 
(1) - anteriorly curved.

(2) - straight.

In Chasmosaurus, postorbital horncores are both posteriorly recurved (AMNH 5401, CMN 1254, CMN 2280, ROM 839, and UALVP 40) and straight (CMN 2245, CMN 8802, CMN 34832, and TMP 1979.011.0147) in lateral view. Posteriorly recurved horncores are also present in Agujaceratops, Judiceratops, Zuniceratops and Turanoceratops. Anteriorly curved horncores are present in other chasmosaurines except for Utahceratops (straight), and in Albertaceratops. Straight horncores are present in Centrosaurus.

Bravoceratops polyphemus was recoded from 1 to ? because the postorbital horncore is fragmentary and its orientation in lateral view cannot be determined.

(41) Postorbital, curvature of postorbital horncore in anterior view (Sampson et al., 2010, character 41):

(0) - medially recurved.

(1) - laterally curved.

(2) - straight.

The postorbital horncore is straight in anterior view in chasmosaurines except for Agujaceratops (medially-recurved), Anchiceratops, Arrhinoceratops and Kosmoceratops (laterally curved), as well as the centrosaurines Albertaceratops and Centrosaurus (but laterally curved in Coronosaurus - not included in analysis), and in the outgroup taxa Zuniceratops and Turanoceratops. 
Turanoceratops tardabilis and Centrosaurus apertus were both recoded from 1 to 2 because their postorbital horncores are straight in anterior view. Kosmoceratops richardsoni was recoded from 2 to 1 because the postorbital horncores are laterally curved in anterior view.

(42) Postorbital, separation from lateral temporal fenestra (Sampson et al., 2010, character 42):

(0) - narrowly excluded from fenestra by narrow strip of jugal.

(1) - broadly excluded from fenestra by a substantial jugal-squamosal contact.

The postorbital is broadly excluded from the lateral temporal fenestra in ceratopsids and Zuniceratops, but narrowly excluded in Leptoceratops and Protoceratops.

Bravoceratops polyphemus was recoded from 1 to ? because the portion of the jugal dorsal to the lateral temporal fenestra is not preserved, and the degree to which the jugal separates the postorbital from the lateral temporal fenestra cannot be determined.

(43) Palpebral, shape (Sampson et al., 2010, character 43):

(0) - rod-like, articulates with prefrontal only at its base and projects across dorsal orbit, ligamentous attachment.

(1) - blocky, fully fused into dorsal orbital margin, sutural articulation with prefrontal and frontal. 
The palpebral is blocky in ceratopsids and Zuniceratops, but rod-like in Leptoceratops and Protoceratops.

Turanoceratops tardabilis was recoded from 1 to ? because the palpebral is not completely preserved.

(44) Palpebral, antorbital buttress (Sampson et al., 2010, character 44):

(0) - absent.

(1) - present.

The palpebral occasionally forms a thick, antorbital buttress. This buttress is present in chasmosaurines. In centrosaurines, the buttress is either present (Pachyrhinosaurus; and Diabloceratops - not included in this analysis), or absent (Centrosaurus and Albertaceratops; Coronosaurus and Styracosaurus as well not included in this analysis). In outgroup taxa, the buttress is either present (Zuniceratops) or absent (Leptoceratops and Protoceratops).

Centrosaurus apertus and Albertaceratops nesmoi were both recoded from 1 to 0 because the palpebral does not form an antorbital buttress.

Pachyrhinosaurus lakustai was recoded from 0 to 1 because the palpebral does form an antorbital buttress.

(45) Palpebral, extent of antorbital buttress (Sampson et al., 2010, character 45, modified here):

(0) - present along only anterodorsal portion of orbit. 
(1) - present along entire or nearly entire anterior portion of orbit.

State (1) was changed from "entire" to "entire or nearly entire", as the ventral extent of the buttress differs subtly. The buttress, where present, forms the entire/nearly anterior portion of the orbit in ceratopsids. The buttress forms only the anterodorsal portion of the orbit in Zuniceratops.

Centrosaurus apertus and Albertaceratops nesmoi were both recoded from 1 to ? because the palpebral does not form an antorbital buttress.

Pachyrhinosaurus lakustai was recoded from ? to 1 because the palpebral does form an antorbital buttress, which forms most of the anterior margin of the orbit.

(46) Jugal, size and orientation of jugal body (Makovicky, 2001, character 22):

(0) - projects strongly posteroventrally, does not extend below the level of the maxillary tooth row.

(1) - projects nearly ventrally, elongated to extend below the level of the maxillary tooth row.

The jugal projects nearly ventrally in ceratopsids and Zuniceratops. The jugal projects strongly posteroventrally in Leptoceratops and Protoceratops. Bravoceratops polyphemus was recoded from 1 to ? because the maxillary tooth row is not preserved and its ventral extent cannot be compared with that of the jugal.

(47) Jugal lateral temporal process (Sampson et al., 2010, character 47): 
(0) - absent.

(1) - present, contacts or nearly contacts lateral temporal process of squamosal.

The jugal lateral temporal process on the posterior margin of the jugal projects posteriorly to contact or nearly contact the lateral temporal process of the squamosal, and forms the anteroventral margin of the lateral temporal fenestra. The jugal lateral temporal process is present in Chasmosaurus, Vagaceratops and Judiceratops, dimorphic in Pentaceratops (compare AMNH 1624 and AMNH 6325 - Lehman, 1998: Fig. 1), but absent in other chasmosaurines, Leptoceratops and Protoceratops. The process is present in the Albertaceratops, Centrosaurus, Pachyrhinosaurus, and Zuniceratops.

Pachyrhinosaurus lakustai was recoded from ? to 1 because the jugal lateral temporal process is present. Pentaceratops sternbergi was recoded from 0 to $0 \& 1)$ because the jugal lateral temporal process is present in at least one specimen (AMNH 1624 - Lehman, 1998: Fig. 1).

(48) Jugal-lacrimal contact (Makovicky and Norell, 2006, character 26):

(0) - reduced.

(1) - expanded.

The jugal-lacrimal contact is expanded in ceratopsids, but reduced in outgroup taxa.

(49) Epijugal attachment scar, shape (Sampson et al., 2010, character 49, modified here): 
(0) - large, obtuse isosceles triangle with obtuse angle oriented towards quadratojugal.

(1) - scar roughly equilateral in shape.

The word "shape" was added to the character description. State (0) was changed from "blade like triangle" to "obtuse isosceles triangle", as the latter better describes the shape of the epijugal attachment scar. The epijugal attachment scar is roughly equilateral in shape in ceratopsids, but obtuse isosceles shaped in outgroup taxa.

(50) Epijugal length (Sampson et al., 2010, character 50, quantified):

(0) - long, proximodistal length approximately equal to anteroposterior basal width.

(1) - hyperlong, proximodistal length $>1.25$ anteroposterior basal width (2) - short, proximodistal length $<0.75$ anteroposterior basal width.

States (0), (1) and (2) originally read "long", "hyperlong" and "short", but were changed to the above; these changes were made to better quantify the relative elongation of the epijugal. In Chasmosaurus, the epijugal is either long (AMNH 5401, ROM 839 and ROM 843) or short (all other specimens). The epijugal is hyperlong in Pentaceratops and Bravoceratops, long in Utahceratops, Agujaceratops, Kosmoceratops, Anchiceratops, Arrhinoceratops, and Eotriceratops, but short in other chasmosaurines. The epijugal is long in Leptoceratops and Protoceratops.

Kosmoceratops richardsoni, Agujaceratops mariscalensis, Utahceratops 
gettyi and Anchiceratops ornatus were all recoded from 1 to 0 , and Arrhinoceratops brachyops and Eotriceratops xerinsularis were recoded from 2 to 0 because their epijugals are long (proximodistal length approximately equal to anteroposterior basal width).

(51) Quadratojugal-squamosal contact (Sampson et al., 2010, character 51):

(0) - absent.

(1) - present.

The quadratojugal-squamosal contact is present in ceratopsids, but absent in outgroup taxa.

(52) Lateral temporal fenestra, size and position (Chinnery and Weishampel, 1998, character 7):

(0) - relatively large, diameter $20 \%$ or greater that of basal skull length, positioned posterior to orbit.

(1) - greatly reduced, diameter $10 \%$ or less that of basal skull length, positioned entirely below ventral limit of orbit.

The lateral temporal fenestra is relatively small in ceratopsids and Zuniceratops. The fenestra is relatively large in Leptoceratops and Protoceratops.

(53) Frontal, contribution to orbital margin (Forster, 1990, character 51):

(0) - present. 
(1) - absent.

The frontal is excluded from the orbital margin in ceratopsids, Zuniceratops and Turanoceratops. The frontal forms part of the orbital margin in Leptoceratops and Protoceratops.

(54) Frontal, contribution to dorsal temporal fenestra (Sampson et al., 2010, character 54):

(0) - present.

(1) - absent.

The frontal is excluded from the margin of the dorsal temporal fenestra in ceratopsids. The frontal forms part of this margin in outgroup taxa.

(55) Frontoparietal fontanelle leading into supracranial cavity complex (Sampson et al., 2010, character 55):

(0) - absent.

(1) - present.

The frontoparietal fontanelle, which leads into the supracranial cavity complex, is present in most chasmosaurines (except for Arrhinoceratops, Kosmoceratops and Triceratops prorsus; Torosaurus latus is dimorphic), and centrosaurines. The above chasmosaurines without a frontoparietal fontanelle do possess a supracranial sinus, but is roofed over by bone over ontogeny. Outgroup taxa were coded as absent, as they do not possess a supracranial cavity, but a 
frontoparietal depression instead. This latter feature is a depression and not a sinus, as it is not partially enclosed by bone, and is not as deeply excavated as the supracranial sinus; however, the frontoparietal depression is interpreted as being the evolutionary precursor to the supracranial sinus (Farke, 2010).

Kosmoceratops richardsoni was recoded from 1 to 0 because it does not possess a frontoparietal fontanelle.

(56) Frontoparietal fontanelle, shape (Sampson et al., 2010, character 56, modified here): (0) - parallel-sided.

(1) - non-parallel-sided.

State (0) was changed from "transversely narrow, slit-like" to "parallelsided", and state (1) was changed from "key-hole shaped, circular or elongate oval" to "non-parallel-sided". The frontoparietal fontanelle is non-parallel-sided in chasmosaurines, and parallel-sided in centrosaurines.

(57) Parietal, anterior extent on dorsum of skull relative to occipital condyle (Sampson et al., 2010, character 57):

(0) - anterior end of parietal located well in front of occipital condyle.

(1) - anterior end of parietal lies directly over occipital condyle.

The anterior end of the parietal lies directly over the occipital condyle in ceratopsids. This end is located well in front of the condyle in outgroup taxa. 
(58) Squamosal, posterior expansion (Sampson et al., 2010, character 58, operationalized:

(0) - absent or very slight.

(1) - present, anteroposterior distance from the posterior margin (squamosal) of the lateral temporal fenestra to the posterior end of the squamosal equal to or greater than the anteroposterior width of the lateral temporal fenestra.

State (1) originally read "present", but was quantified here as shown above. The squamosal is posteriorly expanded (1) in ceratopsids and Protoceratops. The squamosal exhibits little posterior expansion (0) in Leptoceratops.

(59) Squamosal, shape of expanded blade (Sampson et al., 2010, character 59):

(0) - sub-rectangular in outline.

(1) - triangular in outline, posteriorly narrowed.

The squamosal posterior blade is triangular in chasmosaurines, but subrectangular in centrosaurines and outgroup taxa.

Bravoceratops polyphemus was recoded from 2 to 1 because the squamosal is an elongate triangle (also, there is no 2 state described for this character).

(60) Squamosal, anteroposterior proportion of parietosquamosal frill as measured from posterior margin of lateral temporal fenestra to posterior end of parietal (Sampson et 
al., 2010 , character 60 , modified and quantified):

(0) - squamosal less than $50 \%$ of frill length.

(1) - squamosal greater than $70 \%$ of frill length, but less than total frill length.

(2) - squamosal length equal to total frill length.

"Squamosal, length relative to parietal", was changed to the above description to better quantify the length of the squamosal in relation to the frill. The anterior margin of the frill was here defined as the posterior margin of the lateral temporal fenestra and not the jugal notch, as outgroup taxa do not possess a distinct jugal notch. State (0) originally read "squamosal much shorter than parietal, large portion of posterolateral frill made up of parietal" to the above. State (1) originally read "squamosal slightly shorter than parietal, only posterolateralmost margin of frill formed by the parietal" to the above. State (2) originally read "squamosal and parietal equal in length" to the above. The squamosal is slightly shorter $(>70 \%$ to $<100 \%)$ than the total frill length in chasmosaurines except for Kosmoceratops, Anchiceratops, Arrhinoceratops, and Ojoceratops (squamosal equal to total frill length). The squamosal is much shorter $(<50 \%)$ than the total frill length in centrosaurines and outgroup taxa.

(61) Squamosal forms part of posterior margin of frill (Sampson et al., 2010, character 61):

(0) - present.

(1) - absent.

The squamosal is excluded from the posterior margin of the frill in 
chasmosaurines (except Kosmoceratops and Ojoceratops), centrosaurines and Protoceratops. The squamosal forms part of the posterior margin of the frill in Leptoceratops.

(62) Squamosal, anteromedial lamina forming the posterolateral floor of dorsal temporal fossa (Sampson et al., 2010, character 62):

(0) - absent.

(1) - present.

The anteromedial lamina of the squamosal is situated along the parietalsquamosal contact, laterally adjacent to the dorsal temporal fenestra. This lamina is excluded from the posterolateral floor of the dorsal temporal fossa in chasmosaurines and Leptoceratops. The lamina forms part of the posterolateral floor in centrosaurines and Protoceratops.

(63) Squamosal-quadrate contact, shape (Sampson et al., 2010, character 63):

(0) - socket-like cotylus on ventrolateral squamosal for ball-like quadrate head.

(1) - elongate groove on medial surface of squamosal to receive lamina of quadrate.

The squamosal-quadrate contact is elongate in ceratopsids, but socket-like in outgroup taxa.

(64) Squamosal, thickened, rounded swelling along medial margin (Forster, 1990, 
character 90, modified here):

(0) - absent, dorsal surface of squamosal flat to slightly convex.

(1) - present, dorsal surface of squamosal slightly concave.

The presence of a raised ridge along the medial margin of the squamosal results in the element having a concave dorsal surface. In states (0) and (1), "lateral" was changed to "dorsal", as the concavity is present on the dorsal, and not lateral, surface. This swelling is present in chasmosaurines except for Kosmoceratops, Ojoceratops, Nedoceratops, and Triceratops. The swelling is absent in centrosaurines and outgroup taxa.

(65) Parietosquamosal contact, shape in lateral view (Forster, 1990, character 119, modified here):

(0) - straight.

(1) - curved, laterally concave.

In state (1), "medially" was changed to "laterally", because this character describes the shape of the contact in lateral view (Robert Holmes and Andrew Farke, pers. comm.); the change has been made here. The parietosquamosal contact is curved in lateral view in chasmosaurines, but straight in centrosaurines and outgroup taxa.

(66) Parietal, concave median embayment on posterior margin (Sampson et al., 2010, character 66): 
(0) - absent.

(1) - present.

The term "embayment" refers to the concave medial portion of the posterior parietal bar, as seen in dorsal view. The existence, shape and extent of such an embayment pertains to the posterior margin of the parietal itself and not to the projecting epiparietals; epiparietals are ignored here and in characters $67-$ 68, as they can exaggerate the parietal concavity. In Chasmosaurus, the concave median embayment of the parietal is both absent (i.e., YPM 2016 and AMNH 5402) and present (all other specimens). The embayment is absent in Vagaceratops (CMN 41357) and other chasmosaurines (except for Kosmoceratops, Agujaceratops, Utahceratops, and Pentaceratops), Leptoceratops and Protoceratops. The embayment is present in centrosaurines.

Bravoceratops polyphemus was recoded from 0 to ? because the posterior parietal bar is insufficiently preserved to determine whether an embayment existed or not.

(67) Parietal, shape of concave median embayment (Sampson et al., 2010, character 67, renumbered and modified here):

(0) - shallow, restricted to centre of margin.

(1) - shallow, entire posterior bar is a V-shaped embayment.

(2) - notch-like, restricted to centre of margin.

The states were originally misnumbered as “(0)”, “(2)” and “(3)”, with no “(1)"; they have been changed to “(0)", “(1)” and "(2)”, respectively (Robert 
Holmes and Andrew Farke, pers. comm.), and character codings were changed accordingly. In Chasmosaurus, both V-shaped (AMNH 5656, CMN 0491, CMN 2245, CMN 2280, NHMUK R4948, ROM 843, and TMP 1983.025.0001) and notch-like (CMN 8800 and CMN 8803) embayments are present. Other chasmosaurines that possess an embayment are either V-shaped (Kosmoceratops) or notch-like (Agujaceratops, Utahceratops and Pentaceratops). The embayment is shallow in centrosaurines.

(68) Parietal, location of posteriormost point of posterior bar (Sampson et al., 2010, character 68):

(0) - on midline.

(1) - between midline and lateralmost corner.

(2) - at lateralmost corner adjacent to squamosal.

In Chasmosaurus, the posteriormost point of the posterior bar of the parietal is either between the midline and lateralmost corner (AMNH 5656, CMN 2245, CMN 2280, CMN 8800, CMN 8803, NHMUK R4948, and TMP 1983.025.0001) or the lateralmost corner adjacent to the squamosal (ROM 843). In other chasmosaurines, the posteriormost point is on the midline (Torosaurus utahensis), between the midline and lateralmost corner (Utahceratops, Anchiceratops and Arrhinoceratops), and at the lateralmost corner (Kosmoceratops and Pentaceratops); Torosaurus latus is dimorphic (midline, between midline and lateralmost corner). The posteriormost point is between the midline and lateralmost corner in centrosaurines, and on the midline in 
outgroup taxa.

Bravoceratops polyphemus was recoded from 0 to ? because the posterior parietal bar is insufficiently preserved.

(69) Parietosquamosal frill, midline length relative to basal skull length (Sampson et al., 2010, character 69 , modified here):

(0) - short, 0.70 or less.

(1) - elongate, 0.85 or more.

The anterior extent of the frill - anterior edge of parietal at midline - was better defined here by changing "length" to "midline length"; the posterior extent of the frill is the posteriormost extent of the parietal, and not of the epiparietals. The parietosquamosal frill is relatively elongate in chasmosaurines (except for Nedoceratops and Triceratops) and centrosaurines, but relatively short in outgroup taxa.

(70) Parietosquamosal frill, location of maximum transverse width (Sampson et al., 2010, character 70):

(0) - posteriorly, at rear margin of frill.

(1) - anteriorly, in association with proximal half of frill.

(2) - frill relatively equal in width.

In Chasmosaurus, the parietosquamosal frill is widest posteriorly (CMN 2280, ROM 843, and TMP 1983.025.0001) or approximately equal in width 
(AMNH 5401, AMNH 5402, AMNH 5656, CMN 2245, CMN 8800, NHMUK

R4948, ROM 839, TMP 1993.082.0001, and YPM 2016). In Vagaceratops (CMN

41357 and TMP 1987.045.0001), the frill is approximately equal in width between both margins. In other chasmosaurines, the frill is widest anteriorly (Kosmoceratops, Agujaceratops, Utahceratops, Pentaceratops, Anchiceratops, and Arrhinoceratops) or approximately equal in width (Torosaurus, Nedoceratops and Triceratops). The frill is widest posteriorly in Centrosaurus and Pachyrhinosaurus. The frill is widest posteriorly in Protoceratops and Leptoceratops.

Albertaceratops nesmoi was recoded from 1 to ? because the frill is transversely compressed due to taphonomic compaction, and the widest part of the frill cannot be determined with certainty.

(71) Parietal, parietal sulci (Sampson et al., 2010, character 71):

(0) - absent.

(1) - present.

Refers to the sulci than connect the dorsal temporal fenestra and supracranial sinus complex. These sulci are present in ceratopsids, but absent in outgroup taxa.

(72) Parietal, overall lateral shape (Sampson et al., 2010, character 72):

(0) - nearly straight along midline in lateral view and gently arched from side to 
side.

(1) - "saddle-shaped", dorsally concave in lateral view with upturned posterior margin, and arched strongly from side to side.

The parietal is saddle-shaped in Triceratops; in other neoceratopsians, the parietal is nearly straight along the midline.

(73) Parietal fenestrae (Forster, 1990, character 84):

(0) - absent.

(1) - present.

Parietal fenestrae are present in chasmosaurines (except for Triceratops), centrosaurines, Protoceratops, and Zuniceratops. Parietal fenestrae are absent in Leptoceratops.

(74) Parietal, rim on medial margin of dorsal temporal fenestra (Forster, 1990, character 86):

(0) - absent.

(1) - present, well-defined, laterally projecting rim defines medial margin of fenestra.

"Fenstra" (spelling error) was changed to "fenestra". This rim is a ridge that separates the raised anteromedial platform of the parietal from the relatively low dorsal temporal fenestra. This rim is present in chasmosaurines (except for Torosaurus and Triceratops) and centrosaurines, but absent in outgroup taxa. 
Judiceratops was scored here as "present", because I interpret the convex lateral margin of Longrich's (2013) "medial parietal bar" as representing the laterally projecting rim.

(75) Parietal, sharp median crest (Sampson et al., 2010, character 75):

(0) - present.

(1) -absent.

This crest is a narrow ridge that occurs along the midline of the parietal. This crest is absent in ceratopsids, but present in outgroup taxa.

(76) Parietal, anteroposterior width of posterior bar at narrowest point (Holmes et al., 2001, character 22, modified here):

(0) - narrow and strap-like, less than 10\% total parietal length.

(1) - broad, $20 \%$ or more of total parietal length.

Anteroposterior "thickness" was replaced with "width", as the term thickness implies a dorsoventral measurement. The posterior parietal bar is narrow and strap-like in chasmosaurines with parietal fenestrae, except for Anchiceratops, Arrhinoceratops, Ojoceratops, Torosaurus, and Nedoceratops. The bar is also strap-like in Protoceratops. The bar is broad in centrosaurines and Zuniceratops.

Bravoceratops polyphemus was recoded from 1 to ? because most of the 
posterior parietal bar is missing and the narrowest point of this bar therefore cannot be determined.

(77) Parietal, relative anteroposterior width of broad posterior bar (Sampson et al., 2010, character 77, modified here):

(0) - subequal medial to lateral.

(1) - tapering so that the narrowest point occurs medially.

Anteroposterior "depth" was replaced with "width", as the term depth implies a dorsoventral measurement. In neoceratopsians which possess parietal fenestrae and therefore a posterior parietal bar, this bar is subequal medial to lateral except for Utahceratops and Pentaceratops.

(78) Parietal, cross-sectional shape of median bar (Sampson et al., 2010, character 78):

(0) - diamond-shaped, tapers laterally.

(1) - rectangular or subrectangular, margin facing parietal fenestrae thick and oriented sub-perpendicular to parietal surface.

(2) - round to lenticular.

(3) - v-shaped, opening ventrally.

In Chasmosaurus and Vagaceratops, the medial parietal bar is rectangular to subrectangular in cross-section. In other chasmosaurines, the bar is diamondshaped (Kosmoceratops, Anchiceratops, Arrhinoceratops, and Ojoceratops), round to lenticular (Agujaceratops, Utahceratops, Pentaceratops, 
Coahuilaceratops, and Bravoceratops) and v-shaped (Torosaurus, Nedoceratops).

The bar is diamond-shaped in the centrosaurines Centrosaurus, Pachyrhinosaurus and Albertaceratops, and in the outgroup taxa Protoceratops and Zuniceratops. Judiceratops was originally diagnosed by Longrich (2013) as having a ventrally concave medial bar, but I interpret his "medial bar" as a medial platform instead; therefore, Judiceratops is scored here as "?".

(79) Parietal, median bar, transverse width (Holmes et al., 2001, character 23):

(0) - narrow and strap-like, transverse width less than $10 \%$ total parietal length.

(1) - relatively wide, transverse width, transverse width $15 \%$ or more of total parietal length.

The medial parietal bar is strap-like in chasmosaurines except for Kosmoceratops, Anchiceratops, Arrhinoceratops, Torosaurus, and Nedoceratops. The bar is strap-like in Leptoceratops and Protoceratops. The bar is relatively wide in centrosaurines. Judiceratops was originally diagnosed by Longrich (2013) as having a relatively wide medial bar, but I interpret his "medial bar" as a medial platform instead; therefore, Judiceratops is scored here is "?".

(80) Parietal fenestrae, orientation (Sampson et al., 2010, character 80):

(0) - long axis directed transversely.

(1) - long axis directed axially.

(2) - axial and transverse axes equal. 
In Chasmosaurus, the parietal fenestrae are axially elongate (AMNH 5401, AMNH 5656, CMN 2245, CMN 2280, CMN 8800, NHMUK R4948, ROM 839, TMP 1983.025.0001, and TMP 1993.082.0001) and equal both axially and transversely (AMNH 5402, ROM 843 and YPM 2016). In Vagaceratops and Kosmoceratops, the fenestrae are transversely elongate. The fenestrae are axially elongate in other chasmosaurines (except for Torosaurus - equal axially and transversely) and centrosaurines, but transversely elongate in outgroup taxa.

(81) Parietal fenestrae, maximum proximodistal length (Sampson et al., 2010, character 81):

(0) $-35 \%$ or less total parietal length.

(1) $-45 \%$ or more total parietal length.

The parietal fenestrae are relatively long in chasmosaurines (except for Kosmoceratops, Anchiceratops, Arrhinoceratops and Torosaurus) and centrosaurines, but relatively short in Protoceratops.

(82) Parietosquamosal frill, marginal undulations (Sampson et al., 2010, character 82):

(0) - absent.

(1) - present.

These undulations refer to the scalloped free margin of the parietosquamosal frill, and correspond to epiossification (episquamosal and epiparietal) attachment sites. These undulations are present in ceratopsids, but 
absent in Leptoceratops and Protoceratops.

Turanoceratops tardabilis was recoded from 1 to ? because none of the frill is preserved.

(83) Parietosquamosal frill, imbrications of undulations (Dodson et al., 2004, character 34):

(0) - absent.

(1) - present.

Imbrication of parietosquamosal frill marginal undulations is absent in chasmosaurines (but is dimorphic in Kosmoceratops), Leptoceratops and Protoceratops. Imbricated undulations are present in centrosaurines. Judiceratops was originally diagnosed by Longrich (2013) as possessing imbricated squamosal undulations, but I could not discern this feature on this taxon; therefore, Judiceratops is scored here as "absent".

Epiossifications on parietosquamosal frill

(84) Marginal dermal ossifications on parietosquamosal frill (Sampson et al., 2010, character 84):

(0) - absent.

(1) - present.

Character 84 does not appear to be independent of 82 - in taxa possessing 
marginal undulations on the frill, these undulations are adorned by epiossifications.

(85) Episquamosals on midlateral squamosal margin, basal width (Sampson et al., 2010, character 85, modified here):

(0) - small, less than $50 \mathrm{~mm}$ wide in adults.

(1) - large and wide, greater than $90 \mathrm{~mm}$ wide in adults.

This character refers to the basal width of episquamosals, but was originally written as "length" - the term "width" is used here instead. The midlateral squamosals are here defined as the episquamosals occupying the medial third of the lateral margin of the squamosal. In Chasmosaurus, episquamosals on the midlateral margin of the squamosal are both relatively small (AMNH 5402, CMN 2245, TMP 1981.019.0175, TMP 1983.025.0001, and UALVP 40) and large (AMNH 5401, AMNH 5656, CMN 2280, CMN 8800, CMN 8802, CMN 34829, NHMUK R4948, ROM 839, ROM 843, and YPM 2016). Such episquamosals are large in other chasmosaurines, but small in centrosaurines.

Agujaceratops mariscalensis and Kosmoceratops richardsoni were both recoded from 0 to 1 because the episquamosals on the midlateral margin of the squamosal are large and wide (greater than $90 \mathrm{~mm}$ ).

(86) Episquamosal, location of largest/longest episquamosal (Sampson et al., 2010, 
character 86 , modified here):

(0) - anteriormost episquamosal by far the largest.

(1) - posteriormost episquamosal by far the largest.

This character was modified from Sampson et al. (2010), which originally had three states. The original (0) state, "episquamosals subequal in size", was removed because it does not pertain to the location of the largest episquamosal; the original (1) and (2) were recoded as (0) and (1), respectively. Taxa originally coded as "episquamosals subequal in size" are coded here as “?”. Episquamosals are subequal in size in chasmosaurines, except for Kosmoceratops, Utahceratops, Pentaceratops, and Anchiceratops (posteriormost episquamosal the largest). Judiceratops was originally diagnosed by Longrich (2013) as having a large anterior episquamosal compared to the others - however, it is actually the underlying undulation (loci) of the squamosal that makes the anteriormost episquamosal appear to be the largest of the episquamosals; Judiceratops was thus coded here as "?" (episquamosals subequal in size). Bravoceratops was coded as "?", because the posterior end of the squamosal and episquamosal(s) is not preserved. Episquamosals are subequal in size in the centrosaurines Centrosaurus, Pachyrhinosaurus and Albertaceratops. Albertaceratops nesmoi, Centrosaurus apertus, Arrhinoceratops brachyops, Ojoceratops fowleri, Torosaurus utahensis, Nedoceratops hatcheri, Triceratops horridus, and Triceratops prorsus were all recoded from 0 to ? because the episquamosals are subequal in size. Kosmoceratops richardsoni, Utahceratops gettyi, Pentaceratops sternbergi, and Anchiceratops ornatus were 
all recoded from 2 to 1 because the largest episquamosal occurs on the posterior end of the squamosal. Bravoceratops polyphemus was recoded from 1 to ? because the posterior end of the squamosal is not preserved and the size of the posteriormost episquamosals cannot be determined.

(87) Episquamosal, midlateral, shape (Sampson et al., 2010, character 87):

(0) - crescentic or ellipsoidal.

(1) - triangular or elongate.

Episquamosals on the midlateral margin of the squamosal are triangular in chasmosaurines, but crescentic or ellipsoidal in centrosaurines.

(88) Episquamosal locus ES1 shape (Sampson et al., 2010, character 88):

(0) - small and crescentic.

(1) - low, raised D-shaped process.

(2) - large triangular process.

(3) - elongate hook.

Here, episquamosals are numbered sequentially posterior to anterior on the squamosal. The posteriormost episquamosal is a low raised D-shaped process in chasmosaurines, except for Kosmoceratops (elongate hook), and Utahceratops, Pentaceratops and Anchiceratops (well-developed larger triangular process). The posteriormost episquamosal is small and crescentic-shaped in Centrosaurus. 
(89) Episquamosal ES2 shape (Sampson et al., 2010, character 89, modified here):

(0) - small and crescentic.

(1) - low, raised D-shaped process.

(2) - large triangular process.

For this character, "episquamosal locus S2 shape" was changed to "episquamosal ES2 shape", as this character describes the shape of episquamosal 2, and not of the underlying locus. Episquamosal 2 is a low raised D-shaped process in chasmosaurines, except for Kosmoceratops, Utahceratops, Pentaceratops, and Anchiceratops (well-developed large triangular process). Episquamosal 2 is small and crescentic in Centrosaurus.

Bravoceratops polyphemus was recoded from 1 to ? because episquamosal 2 is not preserved and its shape cannot be determined.

(90) Episquamosal ES2 size relative to other episquamosals (Sampson et al., 2010, character 90, modified here):

(0) - subequal.

(1) - second only to ES1 in size, larger than ES3.

Episquamosal locus S2" was changed to "episquamosal ES2", because a locus is not the same as an epiossification and should not be compared. Episquamosal 2 is subequal relative to other episquamosals in chasmosaurines, except for Kosmoceratops, Utahceratops, Pentaceratops, and Torosaurus utahensis. Episquamosal 2 is smaller than 1 and larger than 3 in Centrosaurus. Bravoceratops polyphemus was recoded from 0 to ? because episquamosal 
2 is not preserved and its size relative to other episquamosals cannot be determined.

(91) Epiparietosquamosal, marginal ossification crossing squamosal-parietal contact (Dodson et al., 2004, character 43):

(0) - absent.

(1) - present.

In Chasmosaurus, a marginal ossification crossing the squamosal-parietal contact is both present (AMNH 5402) and absent (all other specimens). In Vagaceratops, such an ossification is also both present (TMP 1987.045.0001) and absent (CMN 41357). Such an ossification is also present in Kosmoceratops, Torosaurus utahensis, Eotriceratops, Nedoceratops, and Triceratops, and dimorphic in Anchiceratops, and absent in other chasmosaurines. Such an ossification is also present in Centrosaurus, Albertaceratops and Pachyrhinosaurus.

Albertaceratops nesmoi, Centrosaurus apertus and Pachyrhinosaurus lakustai were all recoded from 0 to 1 because they all possess a marginal epiossification crossing the squamosal-parietal contact (epiparietosquamosal).

(92) Epiparietosquamosal, shape (Sampson et al., 2010, character 92):

(0) - small and crescentic.

(1) -strongly recurved process. 
(2) - well-developed triangular process.

Chasmosaurines have a well-developed triangular process, except for Kosmoceratops (strongly recurved process). In centrosaurines, this ossification is small and crescentic.

(93) Epiparietals, number per side (Holmes et al., 2001, character 28, modified here): (0) - three.

(1) - four or more.

State (1) was changed from "five" to "four". In Vagaceratops (TMP 1987.045.0001 and TMP 1998.102.0008), the fifth epiossification from the midline of the parietal is variably co-ossified with the squamosal, making this epiossification an epiparietosquamosal. In the Chasmosaurus skull AMNH 5402, the inferred fifth epiossification from the midline overhangs the posterior end of the squamosal and would most likely have been co-articulated with this element later in life. In this cases, only four epiparietals are entirely articulated with each side of the parietal, necessitating the above change to (1). In Chasmosaurus, epiparietal count is either five (YPM 2016) or three (all other specimens), except for AMNH 5402 (four inferred). Three epiparietals are present in other chasmosaurines, except for Vagaceratops (four to five) and Torosaurus (five to six); Anchiceratops is dimorphic (three to four). Judiceratops is coded here as having three, as originally diagnosed by Longrich (2013), although this cannot be confirmed because the posterior margin of the parietal medial to the preserved three is poorly preserved. Centrosaurines have at least five epiparietals. 
Anchiceratops ornatus was recoded from 0 to $0 \& 1$ because it possesses either three (e.g., UALVP 1618 and AMNH 5273) or four epiparietals (e.g., CMN 8535 and UW 2419) on each side of the posterior parietal bar (Mallon et al., 2011).

(94) Epiparietals, fused to adjacent epiparietal at base (Holmes et al., 2001, character 29): (0) - absent.

(1) - present.

Basal fusion between adjacent epiparietals is absent in neoceratopsians, except for Vagaceratops and Kosmoceratops (and in Coronosaurus - not included in analysis).

(95) Epiparietal P0 (Sampson et al., 2010, character 95):

(0) - absent.

(1) - present.

Epiparietals are numbered in sequential order from the midline of the parietal and progressing anteriorly. An epiparietal on the midline of the parietal is present in Ojoceratops, Torosaurus utahensis and Triceratops, but absent in other ceratopsids (Avaceratops - not included in analysis - has a locus for $\mathrm{P} 0$, but the epiparietal is not present). Judiceratops was coded as “?”, because the medial portion of the posterior parietal margin is poorly preserved. 
(96) Epiparietal at P1 locus (Sampson et al., 2010, character 96, modified here):

(0) - absent.

(1) - present.

This character was changed from "epiparietal locus DPP1" to "epiparietal at P1 locus", because the first parietal process on the posterior surface of the parietal $($ dorsal parietal process $=$ DPP $)$ adjacent to the midline in chasmosaurines is interpreted here as epiparietal 1. All ceratopsids possess a P1 locus that is adorned with an epiparietal; the two exceptions in this analysis are P. lakustai and Albertaceratops, which do not have a P1 epiparietal. The Chasmosaurus skull CMN 2245 does not have a P1 epiparietal as preserved, but is inferred as would having one later in life, based on its well-developed P1 locus that is similar in shape and size to that of its P3 locus which is adorned by an epiparietal.

Judiceratops was coded as “?”, because the medial portion of the posterior parietal margin is poorly preserved.

Pachyrhinosaurus lakustai was recoded from 1 to 0 because it does not possess an epiparietal at its $\mathrm{P} 1$ locus. Kosmoceratops richardsoni, Anchiceratops ornatus, Arrhinoceratops brachyops, Ojoceratops fowleri, Torosaurus latus, Torosaurus utahensis, Triceratops horridus, and Triceratops prorsus were all recoded from 0 to 1, and Agujaceratops mariscalensis and Nedoceratops hatcheri were both recoded from ? to 1 because they all possess an epiparietal at their P1 locus. Bravoceratops polyphemus was recoded from 0 to ? because the posterior 
parietal bar is insufficiently complete to determine whether an epiparietal 1 locus was present.

(97) Epiparietal, P1 shape (Sampson et al., 2010, character 97, modified here):

(0) - low D-shaped process.

(1) - elongate, flattened process or spike.

(2) - strongly recurved triangular or recurved low rugose triangular process.

(3) - well-developed triangular process.

(4) - elongate low process.

"Locus P1 shape" was changed to "P1 shape", as the states refer to the shape of the adorning epiparietal. In state (2), "gnarled" was changed to "rugose", as the latter better describes the irregular shape of such epiparietals. In Chasmosaurus, epiparietal 1 is either low D-shaped (AMNH 5402, CMN 0491, CMN 8800, CMN 8803, ROM 843, and YPM 2016) or a well-developed triangular process (AMNH 5656, CMN 2280 and TMP 1983.025.0001). In Vagaceratops and Kosmoceratops, epiparietal 1 is a strongly recurved process. In other chasmosaurines, epiparietal 1 is an elongate low process, except for Utahceratops, Pentaceratops and Anchiceratops (well-developed triangular process). Judiceratops is coded as "?", because only the lateral portion of the putative epiparietal 1 is preserved. In Centrosaurus, epiparietal 1 is an elongate spike.

Albertaceratops nesmoi and Pachyrhinosaurus lakustai were both recoded 
from 1 to ? because neither of them have epiparietal 1 (the medialmost epiparietal is interpreted here as 3 in $A$. nesmoi and 2 in P. lakustai).

(98) Epiparietal, P1 orientation (Sampson et al., 2010, character 98, modified here):

(0) - epiparietal oriented in the plane of the frill.

(1) - directed anterodorsally.

"Locus P1 orientation" was changed to "P1 orientation", as the states refer to the orientation of the adorning epiparietal. In state (0), "posteriorly" was removed, as not all P1s that are oriented in the plane of the frill are directed posteriorly (some are oriented posteromedially). In Chasmosaurus, epiparietal 1 is oriented either posteriorly (AMNH 5402, AMNH 5656, CMN 8803, and TMP 1983.025.0001) or anterodorsally (all other specimens). In other chasmosaurines, epiparietal 1 is oriented in the plane of the frill, except for Vagaceratops, Kosmoceratops, Utahceratops, Pentaceratops, and Anchiceratops; the preserved lateral portion of the putative epiparietal 1 in Judiceratops is oriented in the plane of the frill and was therefore coded as such. Epiparietal 1 is anterodorsallyoriented in Centrosaurus.

Albertaceratops nesmoi and Pachyrhinosaurus lakustai were both recoded from 0 to ? because neither have epiparietal 1. Bravoceratops polyphemus was recoded from 2 to ? because the existence of epiparietal 1 is unknown.

(99) Epiparietal, P1 curvature (Sampson et al., 2010, character 99, modified here): 
(0) - straight

(1) - laterally curved.

(2) - medially curved.

(3) - anterodorsally curved.

"Locus P1 curvature" was changed to "P1 curvature", as the states refer to the curvature of the body of the epiparietal; state (3) was also changed from "dorsally" curved to "anterodorsally curved", because there are no dorsally curved P1s in this analysis. In Chasmosaurus, epiparietal 1 is straight. Epiparietal 1 is anterodorsally curved in Vagaceratops, Kosmoceratops, Utahceratops, Pentaceratops, and Anchiceratops, but straight in other chasmosaurines. The curvature of the putative epiparietal 1 in Judiceratops was coded as “?” due to incomplete preservation. Epiparietal 1 is anterodorsally curved in Centrosaurus.

Centrosaurus apertus was recoded from 2 to 3 because epiparietal 1 is anterodorsally curved. Albertaceratops nesmoi was recoded from 1 to ?, and Pachyrhinosaurus lakustai from 2 to ? because neither possess epiparietal 1. Bravoceratops polyphemus was recoded from 3 to ? because the existence of epiparietal 1 is unknown.

(100) Epiparietal, P2 shape (Sampson et al., 2010, character 100, modified here):

(0) - low D-shaped process.

(1) - elongate, flattened process or spike.

(2) - strongly recurved triangular or recurved, low rugose triangular process.

(3) - well-developed triangular process. 
(4) - elongate low process.

"Locus P2 shape" was changed to "P2 shape", as the states refer to the shape of the adorning epiparietal. In state (2), "gnarled" was changed to "rugose", as the latter better describes the irregular shape of such epiparietals. In Chasmosaurus, epiparietal 2 is either low D-shaped (CMN 8800 and YPM 2016) or a well-developed triangular process (all other specimens). In other chasmosaurines, epiparietal 2 is variably a strongly recurved triangular (Vagaceratops and Kosmoceratops), a well-developed (Utahceratops, Pentaceratops and Anchiceratops) or an elongate low process (Arrhinoceratops, Torosaurus, Triceratops, and Judiceratops). Epiparietal 2 is a strongly recurved triangular process in Centrosaurus and Pachyrhinosaurus.

Albertaceratops nesmoi was recoded from 3 to ? because it does not possess epiparietal 2. Centrosaurus apertus and Pachyrhinosaurus lakustai were both recoded from 1 to 2 because epiparietal 2 is a recurved triangular process. Ojoceratops fowleri was recoded from 4 to ? because epiparietal 2 is not preserved (but the locus for it is).

(101) Epiparietal P2 curvature (Sampson et al., 2010, character 101, modified here): (0) - straight.

(1) - medially or laterally curved in the plane of the frill.

(2) - recurved onto dorsal surface of frill.

In this character, "locus P2 curvature" was changed to "P2 curvature", as the states refer to the curvature of the adorning epiparietal. Epiparietal 2 is straight 
in Chasmosaurus and other chasmosaurines, except for Vagaceratops and Kosmoceratops (dorsally recurved). Epiparietal 2 is medially curved in Centrosaurus and Pachyrhinosaurus.

Centrosaurus apertus was recoded from 0 to 1 because epiparietal 2 is medially curved in the plane of the frill. Albertaceratops nesmoi was recoded from 0 to ? because it does not possess epiparietal 2. Ojoceratops fowleri was recoded from 0 to ? because epiparietal 2 is not preserved (but the locus for it is).

(102) Epiparietal P3 shape (Sampson et al., 2010, character 102, modified here):

(0) - low raised D-shaped process.

(1) - elongate spike.

(2) - strongly recurved triangular or recurved, low rugose triangular process.

(3) - well-developed triangular process.

(4) - elongate, low process.

"Locus P3 shape" was changed to "P3 shape", as the states refer to the shape of the adorning epiparietal. In state (2), "gnarled" was changed to "rugose", as the latter better describes the irregular shape of such epiparietals. In Chasmosaurus, epiparietal 3 is either a low, raised D-shaped (YPM 2016) or a well-developed triangular (all other specimens) process. In other chasmosaurines, epiparietal 3 is a strongly recurved triangular (Vagaceratops and Kosmoceratops), well-developed triangular (Utahceratops, Pentaceratops and Anchiceratops) or an elongate, low process (Arrhinoceratops, Ojoceratops, Torosaurus, and Triceratops). The putative epiparietal 2 in Judiceratops was coded as “?”, because 
only the medial portion is preserved. Epiparietal 3 is an elongate (modified) spike in Albertaceratops, elongate spike in Pachyrhinosaurus and a low raised Dshaped process in Centrosaurus.

Albertaceratops nesmoi was recoded from 3 to 1, and Pachyrhinosaurus lakustai from 0 to 1 because epiparietal 3 is an elongate spike. Centrosaurus apertus was recoded from 3 to 0 because epiparietal 4 is a low D-shaped process.

(103) Epiparietal, P3 orientation (Sampson et al., 2010, character 103, modified here): (0) - epiparietal oriented in the plane of the frill. (1) - directed anterodorsally. "Locus P3 orientation" was changed to "P3 orientation", as the character refers to the orientation of the adorning epiparietal. In state (0), "posteriorly" was removed, as not all $\mathrm{P} 3 \mathrm{~s}$ that are oriented in the plane of the frill are oriented posteriorly (some are oriented posterolaterally). In Chasmosaurus, epiparietal 3 is either anterodorsally oriented (YPM 2016) or oriented in the plane of the frill (all other specimens). In other chasmosaurines, epiparietal 3 is oriented in the plane of the frill, except for Vagaceratops and Kosmoceratops. The putative epiparietal 3 in Judiceratops was coded as "??" due to incomplete preservation. Epiparietal 3 is oriented in the plane of the frill in Centrosaurus, Albertaceratops and Pachyrhinosaurus; the distal end of epiparietal 3 is twisted dorsally in some specimens of Pachyrhinosaurus, although this epiparietal is largely oriented in the plane of the frill. 
Braincase

(104) Basioccipital, contribution to occipital condyle (Forster, 1990, character 71):

(0) - forms approximately $2 / 3$ of occipital condyle.

(1) - forms $1 / 3$ of the occipital condyle.

The basioccipital forms $1 / 3$ of the occipital condyle in ceratopsids, and 2/3 of the occipital condyle in Protoceratops and Leptoceratops.

Leptoceratops gracilis was recoded from ? to 0 because the basioccipital forms $2 / 3$ of the occipital condyle.

(105) Supraoccipital, contribution to foramen magnum (Forster, 1990, character 63):

(0) - forms dorsal margin of foramen magnum.

(1) - eliminated from margin by exoccipital-exoccipital contact on midline.

The supraoccipital is eliminated from the margin of the foramen magnum in neoceratopsians, except for Pachyrhinosaurus, Leptoceratops and Protoceratops.

(106) Supraoccipital, ventrolateral processes (Sereno, 1999, character 131):

(0) - absent.

(1) - present.

These processes extend ventrolaterally and overlap the exoccipitals. These processes are present in neoceratopsians, except for Leptoceratops and Protoceratops. 
(107) Exoccipital, exits for cranial nerves in exoccipital (Forster, 1990, character 68):

(0) - three foramina.

(1) - two foramina.

There are two foramina for cranial nerve exits in the exoccipital in neoceratopsians, except for Protoceratops.

(108) Paroccipital process, dorsoventral distal expansion (Forster, 1990, character 66):

(0) - distal process only slightly expanded.

(1) - distal process expanded to at least 0.8 two times the depth at its narrowest point.

State (0) is not quantified here, but it should be; this quantification was not done here, as it does not affect the outcome of the analysis. The paroccipital process, a lateral projection of the exoccipital, is greatly expanded distally in neoceratopsians, except for Leptoceratops and Protoceratops.

Palate

(109) Ectopterygoid, contributes to palate and contacts the jugal (Forster, 1990, character 32):

(0) - present.

(1) - absent. 
The ectopterygoid contributes to the palate and contacts the jugal in Leptoceratops and Protoceratops, but does not in other neoceratopsians.

(110) Secondary palate, relative contribution of maxilla (Sampson et al., 2010, character 110):

(0) - maxilla forms at least $45 \%$ of the secondary palate.

(1) - maxilla contributes only to the posterior portion, forms $30 \%$ or less of secondary palate.

The maxilla forms a relatively small portion of the secondary palate in neoceratopsians, except for Leptoceratops and Protoceratops.

(111) Palatine, shape and relationship to maxilla (Sampson et al., 2010, character 111): (0) - palatine contacts nearly the entire medial surface of the maxilla, restricting size of choanae, anterodorsal process embraces posterior end of vomer. (1) - palatine contacts only the posterior one-third of medial surface of maxilla, contact with vomer lost, choanae enlarged.

The palatine contacts only the posterior one-third of the medial surface of the maxilla in neoceratopsians, except for Leptoceratops and Protoceratops.

Lower jaw 
(112) Lower jaw, level of mandibular articulation (Forster, 1990, character 74):

(0) - at or slightly below occlusal surface of tooth row.

(1) - depressed well below level of occlusal surface of tooth row.

The mandibular articulation of the lower jaw is depressed well below the tooth row occlusal surface in neoceratopsians, except for Leptoceratops and Protoceratops.

(113) Predentary, length relative to dentary (Sampson et al., 2010, character 113): (0) - equal to or more than two-thirds of dentary length.

(1) - less than two-thirds of dentary length.

The predentary is less than two-thirds the length of the dentary in neoceratopsians, except for Leptoceratops and Protoceratops.

(114) Predentary, dentary processes (Sampson et al., 2010, character 114): (0) - ventral processes much longer than abbreviated dorsal processes. (1) - dorsal and ventral processes elongate and subequal in length.

Character 114 does not appear to be independent of 113 . The predentary of protoceratopsids is $2 / 3$ the length of the dentary because the ventral process of the predentary is long; therefore, 113(0) always pairs with 114(0), and 113(1) always pairs with 114(1) (Robert Holmes and Andrew Farke, pers. comm.).

(115) Predentary, orientation of triturating surface (Dodson, 2004, character 57): 
(0) - nearly horizontal.

(1) - inclined steeply laterally.

The triturating surface of the predentary is inclined steeply laterally in centrosaurines, but is nearly horizontal in chasmosaurines and outgroup taxa.

(116) Dentary lateral ridge confluent with cutting surface of predentary (Sampson et al., 2010, character 116):

(0) - present.

(1) - absent.

Character 116 does not appear to be independent of 115 . In centrosaurines, the lateral edge of the predentary cutting surface lies ventral to its medial edge, with the lateral edge confluent with the lateral ridge of the dentary, and resulting in a dorsolaterally-facing cutting surface. In chasmosaurines, the lateral and medial edges of the cutting surface are both horizontal, forming a dorsally-facing cutting surface that is above the lateral ridge of the dentary. For these reasons, 115(1) pairs with 116(0), and 115(0) pairs with 116(1), respectively, in ceratopsids (Robert Holmes and Andrew Farke, pers. comm.). The only exceptions to this pairing pattern in this analysis are the outgroup taxa Leptoceratops and Protoceratops, which both code as 115(0) and 116(0).

Protoceratops andrewsi was recoded from ? to 0 because the lateral edge of the dentary is confluent with the cutting surface of the predentary. 
(117) Dentary, shape of ventral margin in adults (Forster, 1990, character 73):

(0) - strongly convexly bowed.

(1) - straight.

The ventral margin of the dentary is straight in neoceratopsians, except for Leptoceratops and Protoceratops; most of the other leptoceratopsids, and psittacosaurs are also variably bowed - not included in analysis.

(118) Dentary, posterior extent of tooth row (Chinnery and Weishampel, 1998, character 18):

(0) - terminates at the centre of the coronoid process.

(1) - terminates posterior to the coronoid process.

The posterior extent of the tooth row on the dentary terminates posterior to the coronoid process in neoceratopsians, except for Leptoceratops, Protoceratops and Zuniceratops.

(119) Dentary, shape of coronoid process (Sampson et al., 2010, character 119):

(0) - short, with gently convex apex, base of ascending ramus anteroposteriorly expanded.

(1) - tall, expanded at apex into anteriorly projecting hook, base of ascending ramus anteroposteriorly restricted. 
The coronoid process of the dentary is tall in neoceratopsians, except for Leptoceratops and Protoceratops.

(120) Dentary, separation of body from ascending ramus of coronoid process (Sampson et al., 2010, character 120):

(0) - absent.

(1) - present.

The coronoid process is separated from the body of the dentary in neoceratopsians, except for Leptoceratops and Protoceratops.

(121) Splenial, shape (Sampson et al., 2010, character 121):

(0) - nearly as deep as the body of the dentary, does not contact articular, angular exposed in medial view.

(1) - shallow, contacts articular, covers angular in medial view.

The splenial is shallow in neoceratopsians, except for Leptoceratops and Protoceratops.

(122) Prearticular-dentary contact (Sampson et al., 2010, character 122):

(0) - absent.

(1) - present.

The prearticular-dentary contact is present in neoceratopsians, except for 
Leptoceratops and Protoceratops.

Dentition

(123) Tooth, number of roots (Forster, 1990, character 34):

(0) - one.

(1) - two.

All ceratopsids have double-rooted teeth; non-ceratopsid teeth are singlerooted.

(124) Tooth, number of replacements per alveolus (Sereno, 1999, character 137):

(0) - one or two replacement teeth.

(1) - three or more replacement teeth.

Alveoli have three or more replacement teeth in ceratopsids, but only one or two in outgroup taxa. Turanoceratops has at least two, possibly three replacement teeth per alveolus, and was scored as dimorphic.

Turanoceratops tardabilis was recoded from 0 to $0 \& 1$ because it has at least two (0), possibly three (1) replacement teeth per alveolus.

(125) Tooth magazine, case-like alveolar slots for vertical tooth families formed by spongy bone (Sampson et al., 2010, character 125):

(0) - absent.

(1) - present. 
The alveolar slots for vertical tooth families is formed by spongy bone in neoceratopsians, except for Leptoceratops and Protoceratops.

(126) Cheek teeth (Forster, 1990, character 37):

(0) - spaced.

(1) - closely packed, roots abut.

All taxa used in this analysis have closely packed teeth; Psittacosaurus has spaced teeth, but this taxon was not included in this analysis. This character is therefore parsimony uninformative.

Leptoceratops gracilis and Protoceratops andrewsi were both recoded from 0 to 1 because the cheek teeth are closely packed.

Axial skeleton

(127) Cervical vertebrae, formation of syncervical (Forster, 1990, character 122):

(0) - C1-3 fused or tightly articulated, atlantal hypocentrum present as a ventrally placed, wedge-like bone.

(1) - C1-3 firmly fused, atlantal hypocentrum forms a complete ring. Cervical vertebrae 1-3 are firmly fused in ceratopsids, but not in Protoceratops.

(128) Axis, neural spine shape and orientation (Sereno, 1999, character 141): (0) - blade-like and nearly vertical, overhangs only anteriormost portion of C3. 
(1) - blade-like morphology lost, spine steeply angled to reach posterior margin of C3.

The axis neural spine is blade-like in Leptoceratops and Protoceratops, but not in ceratopsids.

(129) Atlantal rib (Sampson et al., 2010, character 129):

(0) - present.

(1) - absent.

The atlantal rib for the atlas is present in Leptoceratops and Protoceratops, but absent in ceratopsids.

(130) Dorsal vertebrae, shape of centra (Sampson et al., 2010, character 130):

(0) - relatively axially elongate.

(1) - axially shortened.

The dorsal vertebral centra are axially shortened in neoceratopsians, except for Leptoceratops and Protoceratops.

(131) Sacrum, longitudinal sulcus on ventral surface (Lehman, 1989; Sereno, 1999, character144):

(0) - absent.

(1) - present.

A longitudinal sulcus is present on the ventral surface of the sacrum in 
neoceratopsians, except for Leptoceratops and Protoceratops.

Agujaceratops mariscalensis, Pentaceratops sternbergi and Triceratops

horridus were all recoded from 2 to 1 because the sacrum has a longitudinal

sulcus on the ventral surface (also, there is no 2 state described for this character).

Pectoral girdle and forelimb

(132) Scapula, relative contribution to glenoid fossa (Sereno, 1999, character 145):

(0) - scapula and coracoid contribute equally.

(1) - scapula contributes well over half of the glenoid.

The scapula contributes well over half of the glenoid fossa in ceratopsids, but only half in outgroup taxa.

(133) Scapula, orientation of scapular spine (Sampson et al., 2010, character 133):

(0) - obliquely across blade.

(1) - longitudinally along blade.

The scapular spine is oriented obliquely across the blade of the scapula in neoceratopsians, except for Triceratops.

(134) Olecranon process (Forster, 1990, character 104, modified)

(0) - relatively small.

(1) - enlarged (>one-third of ulnar length).

In chasmosaurines, the olecranon process is either relatively small 
(Chasmosaurus) or large (Triceratops). This process is large in centrosaurines

(Centrosaurus), but small in outgroup taxa.

(135) Clavicle (Sereno, 1999, character 147):

(0) - present.

(1) - absent.

The clavicle is absent in ceratopsids, but present in the outgroup taxa Protoceratops and Leptoceratops (also present in Psittacosaurus - not included in analysis).

(136) Manual and pedal unguals, shape (Chinnery and Weishampel, 1998, character 64):

(0) - taper to distal tip.

(1) - dorsoventrally flattened with blunt and rounded distal tips.

The manual and pedal unguals have blunt and rounded tips in ceratopsids, but taper to the distal tip in the outgroup taxa Leptoceratops and Protoceratops.

(137) Manual and pedal penultimate phalanges, shape (Sampson et al., 2010, character 137):

(0) - length exceeds width.

(1) - width exceeds length.

The manual and pedal penultimate phalanges are wider than long in ceratopsids, but are longer than wide in outgroup taxa. 
Pelvic girdle and hind limb

(138) Ilium, lateral eversion of dorsal margin (Sampson et al., 2010, character 138):

(0) - absent.

(1) - present.

The lateral eversion of the dorsal margin of the ilium is present in ceratopsids, but absent in outgroup taxa.

(139) Ilium, relative lengths of pubic and ischial peduncles (Sampson et al., 2010, character 139):

(0) - pubic and ischial peduncles long, extend well below body of ilium approximately the same distance.

(1) - ischial peduncle reduced along ventral aspect, pubic peduncle projects further ventrally than ischial peduncle.

The pubic peduncle projects further ventrally than the ischial peduncle in ceratopsids, but both peduncles are equal in length in outgroup taxa.

(140) Pubis, prepubic process (Forster, 1990, character 111):

(0) - short and unexpanded distally.

(1) - elongate, distal end greatly expanded dorsoventrally.

The prepubic process is elongate in ceratopsids, but short in outgroup taxa. 
This character was left unquantified, as it does not affect the outcome of the analysis.

(141) Pubis, position and length of postpubic rod (Forster, 1990, character 110):

(0) - relatively short, but extends past ischial peduncle of ilium, arises ventral to acetabulum and lies along ventral and ventromedial margin of ischium.

(1) - very abbreviated, terminates at level of ischial peduncle, arises medial to acetabulum and passes entirely medial to ischium.

The postpubic rod terminates at the level of the ischial peduncle in ceratopsids, but extends past the ischial peduncle in outgroup taxa. This character was left unquantified, as it does not affect the outcome of the analysis.

(142) Pubis and ischium, morphology of contributions to acetabulum (Sampson et al., 2010, character 142):

(0) - pubic acetabular surface faces posterolaterally, pubis and pubic process of ischium contribute equally to ventral margin of acetabulum.

(1) - pubic acetabular surface faces laterally and forms a partial medial wall to the acetabulum, pubic process of ischium elongate and meets pubis close to anterior margin of acetabulum, ventral portion of pubic acetabular surface lies medial to pubic ramus of ischium.

The pubic acetabular surface faces laterally in ceratopsids, but 
posterolaterally in outgroup taxa.

(143) Ischium, cross-sectional shape of shaft (Forster, 1990, character 112):

(0) - thick and ovoid.

(1) - laterally compressed and bladelike, tapered dorsally.

The cross-sectional shape of the ilium shaft is laterally compressed in chasmosaurines, but thick and ovoid in centrosaurines and outgroup taxa.

(144) Ischium, orientation of shaft (Forster, 1990, character 113):

(0) - nearly straight or slightly decurved.

(1) - broadly and continuously curved.

The ischium shaft is broadly curved in neoceratopsians, except for Leptoceratops and Protoceratops.

(145) Femur, morphology of greater and lesser trochanters (Dodson et al., 2004, character 72):

(0) - trochanters distinct and located below the level of the femoral head.

(1) - trochanters coalesced and level with the femoral head.

The greater and lesser trochanters of the femur are coalesced in ceratopsids, but distinct in outgroup taxa.

(146) Femur, size of fourth trochanter (Sereno, 1999, character 154): 
(0) - large and pendant.

(1) - small, reduced to low prominence.

The fourth trochanter of the femur is small in ceratopsids, but large in outgroup taxa.

(147) Femur-tibia proportion (Forster, 1990, character 103):

(0) - tibia longer than femur.

(1) - femur longer than tibia.

The femur is longer than the tibia in ceratopsids, but the tibia is longer than the femur in outgroup taxa.

(148) Pes, metatarsal proportions (Sampson et al., 2010, character 148):

(0) - length of MT I two-thirds the length of MT II.

(1) - MT I reduced to one half or less the length of MT II.

Metatarsal I is half or less the length is metatarsal II in ceratopsids, but metatarsal I is two-thirds the length of metatarsal II in outgroup taxa.

Lower jaw

(149) Lateral ridge of dentary (Mallon et al., 2011, character 149):

(0) - present.

(1) - absent.

The lateral ridge of the dentary is absent in chasmosaurines, except for 
Anchiceratops. This feature is dimorphic in Centrosaurus and Pachyrhinosaurus. In outgroup taxa, it is dimorphic in Leptoceratops, present in Protoceratops, and absent in Zuniceratops.

\section{Dermal Skull Roof}

(150) Quadratojugal-squamosal contact in lateral view (Wick and Lehman, 2013, character 150):

(0) - quadratojugal overlapped by squamosal dorsally.

(1) - quadratojugal bifurcated with processes dorsal and ventral to squamosal.

The quadratojugal is overlapped by the squamosal in most chasmosaurines (but bifurcated in Bravoceratops and dimorphic in Torosaurus utahensis, Triceratops horridus and Triceratops prorsus) and centrosaurines. Taxa either missing (not preserved) the quadratojugal or without a contact between the quadratojugal and squamosal were coded here as “?”.

Epiossifications on parietosquamosal frill

(151) Epiparietal P4 shape (new):

(0) - low raised D-shaped process.

(1) - strongly recurved triangular or recurved low gnarled triangular process.

(2) - well-developed triangular process.

(3) - elongate, low process.

In chasmosaurines, epiparietal 4 is low D-shaped (Chasmosaurus - 
AMNH 5402 and YPM 2016), strongly recurved triangular (Vagaceratops -

CMN 41357 and TMP 1987.045.0001), well-developed triangular

(Anchiceratops), or an elongate low process (Torosaurus). Epiparietal 4 is a low raised D-shaped process in Centrosaurus, Albertaceratops and Pachyrhinosaurus.

(152) Epiparietal, P4 orientation (new):

(0) - epiparietal oriented in the plane of the frill.

(1) - directed anterodorsally.

In Chasmosaurus, epiparietal 4 is either oriented in the plane of the frill (e.g., AMNH 5402) or anterodorsally oriented (e.g., YPM 2016). In other chasmosaurines, epiparietal 4 is oriented in the plane of the frill (Torosaurus and Anchiceratops) or anterodorsally oriented (CMN 41357 and TMP 1987.045.0001, Vagaceratops). Epiparietal 4 is posterolaterally oriented in Centrosaurus, Albertaceratops and Pachyrhinosaurus.

(153) Epiossification in P5 position, shape (new):

(0) - low raised D-shaped process.

(1) - well-developed triangular process.

(2) - elongate low process.

In chasmosaurines, epiparietal 5 is a well-developed triangular process in Chasmosaurus (YPM 2016) and Vagaceratops (CMN 41357), and an elongate low process in Torosaurus. Epiparietal 5 is a low raised D-shaped process in 
Centrosaurus, Albertaceratops and Pachyrhinosaurus. An epiparietosquamosal situated lateral to epiparietal 4 occurs in Chasmosaurus (AMNH 5402) and Vagaceratops (TMP 1987.045.0001). The position of the epiparietosquamosal in relation to the parietal-squamosal suture varies between these two specimens, with either the lateral tip (AMNH 5402) or entire lateral half (TMP 1987.045.0001) overlying the squamosal. In AMNH 5402, the epiparietosquamosal, as preserved, is articulated only with the parietal; however, the lateral end of this epiossification overhangs the posterior end of the squamosal, suggesting that it would have been partly articulated with the squamosal later in life (i.e., an epiparietosquamosal). Mallon et al. (2011) noted that the position of the fourth epiossification from the parietal midline was variable in Anchiceratops ornatus, either bridging the parietal-squamosal contact (epiparietosquamosal $=3$ epiparietals per side; e.g., AMNH 5273 and UALVP 1618) or articulated only with the parietal (epiparietal $=4$ epiparietals per side; e.g., CMN 8535 and UW 2419). They noted that A. ornatus skulls possessing an epiparietosquamosal ( 3 epiparietals per side) are generally smaller and less mature than A. ornatus skulls with an epiparietal (4 epiparietals per side), which are generally larger and more mature. They suggested that the epiparietosquamosal migrates medially over ontogeny, becoming articulated entirely with the parietal. The epiparietosquamosal and epiparietal 4 therefore appear to be homologous in A. ornatus. The epiparietosquamosal and epiparietal 5 are likewise interpreted here as being homologous in Chasmosaurus (AMNH 5402 and YPM 2016, 
respectively) and Vagaceratops (TMP 1987.045.0001 and CMN 41357, respectively), that may or may not be related to ontogeny. 


\section{Appendix IV: Chapter 9 supporting data}

Table A1: Measurement dataset used in PCA 1; 18 specimens of the Vagaceratops-like operative taxonomic unit $(\mathrm{n}=4)$, Chasmosaurus russelli $(\mathrm{n}=4)$, C. belli $(\mathrm{n}=3)$, and Chasmosaurus sp. $(\mathrm{n}=7)$. Estimated missing data shown in bold. All parameter measurements are in mm, except for parameter $33\left(^{\circ}\right)$.

\begin{tabular}{|l|l|l|l|l|l|l|l|l|l|l|l|l|}
\hline Specimen/Parameter & 1 & 4 & 5 & 6 & 7 & 9 & 10 & 11 & 15 & 16 & 17 & 18 \\
\hline AMNH 5401 & 370 & 320 & 130 & 550 & 215 & 280 & 115 & 75 & 380 & 730 & 580 & 315 \\
\hline AMNH 5402 & 70 & 190 & 144 & 525 & 200 & 235 & 110 & 85 & 300 & 680 & 610 & 250 \\
\hline AMNH 5656 & $\mathbf{1 3 0}$ & $\mathbf{2 6 4}$ & $\mathbf{1 5 5}$ & $\mathbf{5 8 0}$ & $\mathbf{2 0 7}$ & $\mathbf{2 8 2}$ & $\mathbf{1 2 6}$ & $\mathbf{9 6}$ & 304 & $\mathbf{7 5 9}$ & $\mathbf{6 5 6}$ & $\mathbf{2 6 7}$ \\
\hline CMN 1254 & 216 & 242 & 150 & $\mathbf{5 8 0}$ & $\mathbf{2 0 7}$ & $\mathbf{2 8 2}$ & $\mathbf{1 2 6}$ & 89 & 334 & $\mathbf{7 5 9}$ & $\mathbf{6 5 6}$ & $\mathbf{2 6 7}$ \\
\hline CMN 2245 & 102 & 238 & 176 & $\mathbf{5 8 0}$ & $\mathbf{2 0 7}$ & 283 & 127 & 101 & 345 & $\mathbf{7 5 9}$ & $\mathbf{6 5 6}$ & 269 \\
\hline CMN 2280 & 132 & 263 & $\mathbf{1 5 5}$ & 563 & 208 & 274 & 132 & 90 & 326 & 750 & $\mathbf{6 5 6}$ & 278 \\
\hline CMN 8800 & $\mathbf{1 3 0}$ & 412 & $\mathbf{1 5 5}$ & 663 & 218 & $\mathbf{2 8 2}$ & 161 & 123 & 332 & 880 & 770 & 309 \\
\hline CMN 8801 & $\mathbf{1 3 0}$ & 300 & 194 & 677 & 238 & 334 & $\mathbf{1 2 6}$ & 116 & $\mathbf{3 4 1}$ & 835 & 732 & $\mathbf{2 6 7}$ \\
\hline CMN 34829 & 59 & 244 & $\mathbf{1 5 5}$ & $\mathbf{5 8 0}$ & $\mathbf{2 0 7}$ & $\mathbf{2 8 2}$ & 115 & 82 & 345 & $\mathbf{7 5 9}$ & $\mathbf{6 5 6}$ & 299 \\
\hline CMN 41357 & $\mathbf{1 3 0}$ & 250 & 123 & 600 & 220 & 279 & 138 & 107 & 295 & 795 & $\mathbf{6 5 6}$ & 320 \\
\hline NHMUK R4948 & $\mathbf{1 3 0}$ & $\mathbf{2 6 4}$ & 155 & $\mathbf{5 8 0}$ & $\mathbf{2 0 7}$ & 285 & 120 & 100 & 360 & $\mathbf{7 5 9}$ & $\mathbf{6 5 6}$ & $\mathbf{2 6 7}$ \\
\hline ROM 839 & 107 & 250 & 237 & 555 & 234 & 355 & 143 & 90 & 368 & 782 & 635 & 231 \\
\hline ROM 843 & $\mathbf{1 3 0}$ & 252 & 199 & 652 & 245 & 328 & 144 & 109 & 395 & 897 & 822 & 290 \\
\hline $\begin{array}{l}\text { TMP } \\
1981.019 .0175\end{array}$ & $\mathbf{1 3 0}$ & 302 & 134 & 558 & 200 & 294 & 142 & 103 & 337 & 768 & 661 & 217 \\
\hline $\begin{array}{l}\text { TMP } \\
1983.025 .0001\end{array}$ & $\mathbf{1 3 0}$ & 345 & $\mathbf{1 5 5}$ & $\mathbf{5 8 0}$ & 171 & 256 & 113 & 98 & 352 & $\mathbf{7 5 9}$ & $\mathbf{6 5 6}$ & $\mathbf{2 6 7}$ \\
\hline $\begin{array}{l}\text { TMP } \\
1987.045 .0001\end{array}$ & $\mathbf{1 3 0}$ & 233 & 124 & 583 & 158 & 258 & 86 & $\mathbf{9 6}$ & 352 & 617 & 548 & 285 \\
\hline UALVP 40 & 176 & 240 & 131 & 440 & 174 & 270 & 114 & 90 & 337 & 628 & 548 & 180 \\
\hline YPM 2016 & $\mathbf{1 3 0}$ & 222 & $\mathbf{1 5 5}$ & 637 & 235 & 243 & 150 & 93 & 360 & 800 & 720 & 271 \\
\hline
\end{tabular}


Table A1 (continued).

\begin{tabular}{|c|c|c|c|c|c|c|c|c|c|c|c|c|c|}
\hline $\begin{array}{l}\text { Speci- } \\
\text { men/Par- } \\
\text { ameter }\end{array}$ & 19 & 20 & 21 & 22 & 23 & 25 & 26 & 27 & 28 & 30 & 31 & 32 & 33 \\
\hline $\begin{array}{l}\text { AMNH } \\
5401\end{array}$ & 710 & 215 & 330 & 115 & 955 & 368 & 1720 & 820 & 875 & 450 & 33 & 87 & 141 \\
\hline $\begin{array}{l}\text { AMNH } \\
5402\end{array}$ & 685 & 290 & 285 & 120 & 830 & 260 & 1540 & 920 & 780 & 354 & 33 & 87 & 180 \\
\hline $\begin{array}{l}\text { AMNH } \\
5656\end{array}$ & 569 & 313 & 257 & 129 & 955 & 266 & 1720 & 697 & 854 & 503 & 27 & 86 & 115 \\
\hline $\begin{array}{l}\text { CMN } \\
1254 \\
\end{array}$ & 500 & 313 & 348 & 139 & 955 & 368 & 1720 & 1000 & 900 & 450 & 33 & 87 & 141 \\
\hline $\begin{array}{l}\mathrm{CMN} \\
2245 \\
\end{array}$ & 779 & 377 & 400 & 140 & 955 & 346 & 1720 & 1078 & 988 & 452 & 32 & 116 & 156 \\
\hline $\begin{array}{l}\mathrm{CMN} \\
2280 \\
\end{array}$ & 709 & 277 & 310 & 132 & 876 & 442 & 1662 & 1043 & 855 & 557 & 34 & 72 & 124 \\
\hline $\begin{array}{l}\text { CMN } \\
8800 \\
\end{array}$ & 997 & 424 & 470 & 139 & 1080 & 322 & 2019 & 848 & 1143 & 584 & 36 & 79 & 89 \\
\hline $\begin{array}{l}\mathrm{CMN} \\
8801 \\
\end{array}$ & 715 & 313 & 348 & 139 & 955 & 368 & 1720 & 1000 & 900 & 450 & 33 & 87 & 141 \\
\hline $\begin{array}{l}\mathrm{CMN} \\
34829 \\
\end{array}$ & 850 & 308 & 348 & 139 & 920 & 368 & 1720 & 1000 & 900 & 450 & 40 & 87 & 141 \\
\hline $\begin{array}{l}\text { CMN } \\
41357\end{array}$ & 700 & 460 & 430 & 225 & 1020 & 380 & 1662 & 1047 & 753 & 265 & 33 & 76 & 180 \\
\hline $\begin{array}{l}\text { NHMU } \\
\text { K R4948 }\end{array}$ & 840 & 313 & 348 & 139 & 955 & 490 & 1720 & 1160 & 900 & 627 & 33 & 90 & 162 \\
\hline $\begin{array}{l}\text { ROM } \\
839 \\
\end{array}$ & 917 & 379 & 380 & 115 & 1020 & 347 & 1800 & 893 & 1034 & 450 & 26 & 87 & 141 \\
\hline $\begin{array}{l}\text { ROM } \\
843 \\
\end{array}$ & 893 & 482 & 438 & 151 & 1082 & 457 & 2023 & 1227 & 1063 & 498 & 30 & 94 & 136 \\
\hline $\begin{array}{l}\text { TMP } 81 . \\
19.175\end{array}$ & 600 & 228 & 311 & 171 & 955 & 368 & 1720 & 1000 & 900 & 450 & 33 & 87 & 141 \\
\hline $\begin{array}{l}\text { TMP } 83 . \\
25.1\end{array}$ & 650 & 227 & 290 & 125 & 868 & 450 & 1720 & 1373 & 952 & 592 & 45 & 102 & 128 \\
\hline $\begin{array}{l}\text { TMP } 87 . \\
45.1\end{array}$ & 633 & 265 & 382 & 157 & 955 & 300 & 1450 & 940 & 755 & 260 & 33 & 87 & 141 \\
\hline $\begin{array}{l}\text { UALVP } \\
40\end{array}$ & 600 & 320 & 315 & 88 & 955 & 368 & 1720 & 1000 & 900 & 450 & 33 & 87 & 141 \\
\hline $\begin{array}{l}\text { YPM } \\
2016\end{array}$ & 730 & 279 & 350 & 193 & 940 & 450 & 1690 & 1162 & 840 & 463 & 34 & 75 & 171 \\
\hline
\end{tabular}


Table A2: Measurement dataset used in PCA 2; 11 specimens of the Vagaceratops-like operative taxonomic unit $(\mathrm{n}=4)$, Chasmosaurus russelli $(\mathrm{n}=4)$ and $C$. belli $(\mathrm{n}=3)$. Estimated missing data shown in bold. All parameter measurements are in mm, except for parameter $33\left(^{\circ}\right)$.

\begin{tabular}{|c|c|c|c|c|c|c|c|c|c|c|c|c|c|}
\hline Sp./par. & 1 & 4 & & 5 & 6 & 7 & 9 & 10 & 11 & 15 & 16 & 17 & 18 \\
\hline $\begin{array}{l}\text { AMNH } \\
5402 \\
\end{array}$ & 70 & 190 & & 144 & 525 & 200 & 235 & 110 & 85 & 300 & 680 & 610 & 250 \\
\hline $\begin{array}{l}\text { AMNH } \\
5656 \\
\end{array}$ & 93 & 247 & & 145 & 591 & 200 & 264 & 121 & 97 & 304 & 742 & 661 & 280 \\
\hline $\begin{array}{l}\text { CMN } \\
2245 \\
\end{array}$ & 102 & 238 & & 176 & 605 & 207 & 283 & 127 & 101 & 345 & 778 & 696 & 269 \\
\hline $\begin{array}{l}\text { CMN } \\
2280 \\
\end{array}$ & 132 & 263 & & 154 & 563 & 208 & 274 & 132 & 90 & 326 & 750 & 701 & 278 \\
\hline $\begin{array}{l}\text { CMN } \\
8800 \\
\end{array}$ & 115 & 412 & & 171 & 663 & 218 & 291 & 161 & 123 & 332 & 880 & 770 & 309 \\
\hline $\begin{array}{l}\text { CMN } \\
41357 \\
\end{array}$ & 93 & 250 & & 123 & 600 & 220 & 279 & 138 & 107 & 295 & 795 & 662 & 320 \\
\hline $\begin{array}{l}\text { NHMU } \\
\text { K R4948 }\end{array}$ & 104 & 278 & & 155 & 616 & 212 & 285 & 120 & 100 & 360 & 803 & 719 & 287 \\
\hline $\begin{array}{l}\text { ROM } \\
843\end{array}$ & 111 & 252 & & 199 & 652 & 245 & 328 & 144 & 109 & 395 & 897 & 822 & 290 \\
\hline $\begin{array}{l}\text { TMP } 83 . \\
25.1\end{array}$ & 101 & 345 & & 154 & 607 & 171 & 256 & 113 & 98 & 352 & 782 & 700 & 285 \\
\hline $\begin{array}{l}\text { TMP } 87 . \\
45.1\end{array}$ & 84 & 233 & & 124 & 583 & 158 & 258 & 86 & 92 & 352 & 617 & 548 & 285 \\
\hline $\begin{array}{l}\text { YPM } \\
2016\end{array}$ & 98 & 222 & & 151 & 637 & 235 & 243 & 150 & 93 & 360 & 800 & 720 & 271 \\
\hline Sp./par. & 19 & 20 & 21 & 22 & 23 & 25 & 26 & 27 & 28 & 30 & 31 & 32 & 33 \\
\hline $\begin{array}{l}\text { AMNH } \\
5402 \\
\end{array}$ & 685 & 290 & 285 & 120 & 830 & 260 & 1540 & 920 & 780 & 354 & 33 & 86 & 180 \\
\hline $\begin{array}{l}\text { AMNH } \\
5656 \\
\end{array}$ & 569 & 318 & 257 & 129 & 931 & 266 & 1652 & 697 & 854 & 503 & 27 & 86 & 115 \\
\hline $\begin{array}{l}\text { CMN } \\
2245 \\
\end{array}$ & 779 & 377 & 400 & 140 & 959 & 346 & 1730 & 1078 & 988 & 452 & 32 & 116 & 156 \\
\hline $\begin{array}{l}\text { CMN } \\
2280 \\
\end{array}$ & 709 & 277 & 310 & 132 & 876 & 442 & 1662 & 1043 & 855 & 557 & 34 & 72 & 124 \\
\hline $\begin{array}{l}\text { CMN } \\
8800 \\
\end{array}$ & 997 & 424 & 470 & 150 & 1080 & 322 & 2019 & 848 & 1143 & 584 & 36 & 79 & 89 \\
\hline $\begin{array}{l}\text { CMN } \\
41357\end{array}$ & 700 & 460 & 430 & 225 & 1020 & 380 & 1662 & 1047 & 753 & 265 & 33 & 76 & 180 \\
\hline $\begin{array}{l}\text { NHMU } \\
\text { K R4948 }\end{array}$ & 840 & 349 & 369 & 149 & 979 & 490 & 1784 & 1160 & 940 & 627 & 34 & 90 & 162 \\
\hline $\begin{array}{l}\text { ROM } \\
843\end{array}$ & 893 & 482 & 438 & 151 & 1082 & 457 & 2023 & 1227 & 1063 & 498 & 30 & 94 & 136 \\
\hline $\begin{array}{l}\text { TMP } 83 . \\
25.1\end{array}$ & 650 & 227 & 290 & 125 & 868 & 450 & 1740 & 1373 & 952 & 592 & 45 & 102 & 128 \\
\hline $\begin{array}{l}\text { TMP } 87 . \\
45.1\end{array}$ & 633 & 265 & 382 & 157 & 886 & 300 & 1450 & 940 & 755 & 260 & 33 & 85 & 164 \\
\hline $\begin{array}{l}\text { YPM } \\
2016 \\
\end{array}$ & 730 & 279 & 350 & 193 & 940 & 450 & 1690 & 1162 & 840 & 463 & 34 & 75 & 171 \\
\hline
\end{tabular}


Table A3: Measurement dataset used in PCA 3; 7 specimens of Chasmosaurus russelli $(\mathrm{n}=4)$ and $C$. belli $(\mathrm{n}=3)$. Estimated missing data shown in bold. All parameter measurements are in $\mathrm{mm}$, except for parameter $33\left(^{\circ}\right)$.

\begin{tabular}{|c|c|c|c|c|c|c|c|c|c|c|c|c|c|}
\hline $\begin{array}{l}\text { Specimen/ } \\
\text { Parameter }\end{array}$ & 1 & 4 & 5 & 6 & 7 & s & & 10 & 11 & 15 & 16 & 17 & 18 \\
\hline $\begin{array}{l}\text { AMNH } \\
5656\end{array}$ & 116 & 295 & 176 & 624 & 20 & & 84 & 132 & 103 & 304 & 840 & 796 & 286 \\
\hline $\begin{array}{l}\text { CMN } \\
2245\end{array}$ & 102 & 238 & 176 & 624 & 20 & & 83 & 127 & 101 & 345 & 840 & 796 & 269 \\
\hline $\begin{array}{l}\text { CMN } \\
2280\end{array}$ & 132 & 263 & 176 & 563 & 20 & & 74 & 132 & 90 & 326 & 750 & 796 & 278 \\
\hline $\begin{array}{l}\text { CMN } \\
8800\end{array}$ & 116 & 412 & 176 & 663 & 21 & & 84 & 161 & 123 & 332 & 880 & 770 & 309 \\
\hline $\begin{array}{l}\text { NHMUK } \\
\text { R4948 }\end{array}$ & 116 & 295 & 155 & 624 & 20 & & 85 & 120 & 100 & 360 & 840 & 796 & 286 \\
\hline ROM 843 & 116 & 252 & 199 & 652 & 24 & & 28 & 144 & 109 & 395 & 897 & 822 & 290 \\
\hline $\begin{array}{l}\text { TMP } \\
1983.025 . \\
0001\end{array}$ & 116 & 345 & 176 & 624 & 17 & & 56 & 113 & 98 & 352 & 840 & 796 & 286 \\
\hline $\begin{array}{l}\text { Specimen/ } \\
\text { Parameter }\end{array}$ & 19 & 20 & 21 & 22 & 23 & 25 & 26 & 27 & 28 & 30 & 31 & 32 & 33 \\
\hline $\begin{array}{l}\text { AMNH } \\
5656\end{array}$ & 569 & 344 & 257 & 129 & 971 & 266 & 1893 & 697 & 854 & 503 & 27 & 86 & 115 \\
\hline $\begin{array}{l}\text { CMN } \\
2245\end{array}$ & 779 & 377 & 400 & 140 & 971 & 346 & 1893 & 1078 & 988 & 452 & 32 & 116 & 156 \\
\hline $\begin{array}{l}\text { CMN } \\
2280\end{array}$ & 709 & 277 & 310 & 132 & 876 & 442 & 1662 & 1043 & 855 & 557 & 34 & 72 & 124 \\
\hline $\begin{array}{l}\text { CMN } \\
8800\end{array}$ & 997 & 424 & 470 & 135 & 1080 & 322 & 2019 & 848 & 1143 & 584 & 36 & 79 & 89 \\
\hline $\begin{array}{l}\text { NHMUK } \\
\text { R4948 }\end{array}$ & 840 & 344 & 352 & 135 & 971 & 490 & 1893 & 1160 & 970 & 627 & 34 & 90 & 162 \\
\hline ROM 843 & 893 & 482 & 438 & 151 & 1082 & 457 & 2023 & 1227 & 1063 & 498 & 30 & 94 & 136 \\
\hline $\begin{array}{l}\text { TMP } \\
1983.025 . \\
0001\end{array}$ & 650 & 227 & 290 & 125 & 868 & 450 & 1893 & 1373 & 952 & 592 & 45 & 102 & 128 \\
\hline
\end{tabular}


Table A4: Results of PCA 1 (18 specimens) - standard deviation, variance and cumulative variance, and parameter loadings on principal component (PC) axes.

\begin{tabular}{|l|l|l|l|l|l|l|l|l|l|l|l|l|l|}
\hline Axis & PC1 & PC2 & PC3 & PC4 & PC5 & PC6 & $\begin{array}{l}\text { Parameters } \\
\text { continued }\end{array}$ & PC1 & PC2 & PC3 & PC4 & PC5 & PC6 \\
\hline $\begin{array}{l}\text { Standard } \\
\text { deviation }\end{array}$ & 2.9606 & 1.8300 & 1.6756 & 1.53253 & 1.30628 & 1.28122 & 18 & -0.1106 & 0.2554 & -0.1832 & 0.2126 & 0.1260 & 0.0365 \\
\hline Variance (\%) & 35.06 & 13.40 & 11.23 & 9.395 & 6.825 & 6.566 & 19 & -0.2304 & 0.0098 & -0.0068 & -0.1426 & 0.0328 & 0.1983 \\
\hline $\begin{array}{l}\text { Cumulative } \\
\text { variance (\%) }\end{array}$ & 35.06 & 48.46 & 59.69 & 69.081 & 75.907 & 82.473 & 20 & -0.2127 & 0.2027 & 0.2198 & -0.1271 & 0.0542 & 0.2079 \\
\hline Parameter 1 & 0.0102 & -0.1633 & -0.0056 & 0.2508 & 0.4280 & -0.4462 & 21 & -0.2193 & 0.1990 & 0.0054 & -0.0413 & 0.4021 & 0.1928 \\
\hline 4 & -0.1586 & -0.2230 & -0.1414 & 0.4265 & 0.0890 & 0.0674 & 22 & -0.0852 & 0.4458 & -0.2010 & 0.0532 & 0.0373 & -0.0851 \\
\hline 5 & -0.2184 & -0.1290 & 0.1451 & -0.3215 & -0.1815 & 0.0286 & 23 & -0.2482 & 0.0702 & 0.1997 & 0.0829 & 0.3746 & 0.0082 \\
\hline 6 & -0.2374 & 0.2186 & -0.1649 & 0.1187 & -0.0394 & 0.0430 & 25 & -0.1227 & -0.0856 & -0.4032 & -0.2009 & 0.1205 & -0.2992 \\
\hline 7 & -0.2487 & 0.1342 & 0.1114 & -0.1124 & -0.1672 & -0.3445 & 26 & -0.2966 & -0.1777 & 0.0388 & 0.0566 & -0.0100 & -0.0231 \\
\hline 9 & -0.2194 & -0.0996 & 0.2442 & -0.1374 & 0.1309 & -0.0837 & 27 & -0.0543 & -0.0018 & -0.4590 & -0.3325 & 0.0449 & 0.0434 \\
\hline 10 & -0.2633 & 0.0453 & 0.0493 & 0.0567 & -0.2281 & -0.2457 & 28 & -0.2608 & -0.3008 & 0.0441 & -0.0224 & 0.0071 & 0.1674 \\
\hline 11 & -0.2127 & 0.1261 & -0.0385 & 0.1351 & -0.0196 & 0.2850 & 30 & -0.1506 & -0.3815 & -0.1473 & 0.0373 & -0.2816 & -0.1052 \\
\hline 15 & -0.1263 & -0.2402 & -0.1399 & -0.2397 & 0.3924 & -0.1349 & 31 & 0.0566 & -0.0681 & -0.4962 & 0.1302 & -0.0456 & 0.2350 \\
\hline 16 & -0.3126 & 0.0686 & -0.0801 & 0.0512 & -0.1649 & -0.1043 & 32 & -0.0034 & -0.2177 & -0.0402 & -0.2719 & 0.1288 & 0.3831 \\
\hline 17 & -0.2957 & 0.0885 & -0.1242 & 0.0121 & -0.2363 & -0.0154 & 33 & 0.1323 & 0.2578 & -0.0770 & -0.4297 & 0.0447 & -0.1891 \\
\hline
\end{tabular}


Table A4 (continued).

\begin{tabular}{|c|c|c|c|c|c|c|c|c|c|c|c|c|c|}
\hline Axis & PC7 & PC8 & PC9 & PC10 & PC11 & PC12 & $\begin{array}{l}\text { Parameters } \\
\text { continued }\end{array}$ & PC7 & PC8 & PC9 & PC10 & PC11 & PC12 \\
\hline $\begin{array}{l}\text { Standard } \\
\text { deviation }\end{array}$ & 1.10823 & 0.99689 & $\begin{array}{l}0.7730 \\
7\end{array}$ & 0.62408 & 0.61762 & 0.51918 & 18 & 0.5294 & -0.0221 & 0.2630 & 0.2258 & 0.2402 & -0.0741 \\
\hline Variance (\%) & 4.913 & 3.975 & 2.391 & 1.558 & 1.526 & 1.078 & 19 & 0.2933 & -0.4596 & -0.1919 & -0.2267 & -0.0110 & -0.4615 \\
\hline $\begin{array}{l}\text { Cumulative } \\
\text { variance }(\%)\end{array}$ & 87.386 & 91.361 & 93.751 & 95.309 & 96.835 & 97.913 & 20 & -0.2112 & -0.2074 & 0.2343 & 0.3850 & 0.2575 & -0.0085 \\
\hline Parameter 1 & -0.0857 & 0.1588 & 0.2550 & 0.1385 & 0.0889 & -0.1722 & 21 & -0.0440 & -0.2329 & 0.0096 & -0.0401 & -0.0319 & 0.0725 \\
\hline 4 & -0.0036 & 0.0831 & -0.1484 & -0.3572 & 0.2567 & -0.1670 & 22 & -0.0863 & 0.1776 & $\begin{array}{l}-0.0598 \\
\end{array}$ & -0.2399 & -0.2494 & 0.0571 \\
\hline 5 & 0.2003 & 0.1856 & -0.1986 & 0.1566 & 0.0576 & 0.0793 & 23 & -0.1723 & -0.0428 & 0.0052 & -0.0083 & -0.1367 & 0.0661 \\
\hline 6 & 0.2609 & 0.3239 & -0.1188 & 0.1581 & -0.1692 & -0.0098 & 25 & -0.1743 & -0.1180 & -0.1553 & 0.1923 & 0.2043 & -0.1243 \\
\hline 7 & 0.1758 & -0.0168 & 0.1407 & -0.1519 & 0.1271 & -0.0240 & 26 & -0.1170 & -0.1455 & 0.2598 & -0.0110 & -0.0044 & 0.1947 \\
\hline 9 & 0.0280 & 0.2645 & -0.3741 & -0.1355 & 0.5087 & 0.2253 & 27 & -0.2698 & 0.0212 & -0.0269 & 0.1461 & 0.0723 & 0.0785 \\
\hline 10 & -0.2420 & -0.1543 & 0.1365 & -0.3241 & -0.1548 & 0.0303 & 28 & -0.0287 & -0.0795 & 0.1257 & -0.0996 & -0.1510 & 0.1503 \\
\hline 11 & -0.3915 & 0.2879 & -0.2339 & 0.0770 & -0.0275 & -0.4144 & 30 & 0.0091 & -0.0623 & 0.0865 & 0.2029 & -0.0716 & -0.3629 \\
\hline 15 & 0.2562 & 0.0288 & -0.1633 & -0.0041 & -0.4451 & 0.1070 & 31 & 0.0103 & -0.1818 & 0.0244 & -0.1751 & 0.2342 & 0.3845 \\
\hline 16 & 0.0473 & 0.1100 & 0.1271 & -0.1081 & 0.1091 & 0.0958 & 32 & 0.0522 & 0.4480 & 0.5096 & -0.2312 & 0.0199 & -0.1415 \\
\hline 17 & -0.0202 & 0.1251 & 0.0952 & 0.1620 & -0.1623 & 0.1383 & 33 & -0.0447 & 0.0353 & 0.1843 & -0.3316 & 0.1487 & -0.2431 \\
\hline
\end{tabular}


Table A4 (continued).

\begin{tabular}{|c|c|c|c|c|c|c|c|c|c|c|c|c|c|}
\hline Axis & PC13 & PC14 & PC15 & PC16 & PC17 & PC18 & $\begin{array}{l}\text { Parameters } \\
\text { continued }\end{array}$ & PC13 & PC14 & PC15 & PC16 & PC17 & PC18 \\
\hline $\begin{array}{l}\text { Standard } \\
\text { deviation }\end{array}$ & 0.47720 & 0.39876 & 0.27768 & 0.20562 & 0.12467 & $\begin{array}{l}5.619 \\
\times 10^{-15} \\
\end{array}$ & 18 & 0.2064 & -0.1478 & -0.1413 & 0.1105 & -0.0919 & 0.1182 \\
\hline Variance (\%) & 0.911 & 0.636 & 0.308 & 0.169 & 0.062 & 0.00 & 19 & -0.0081 & 0.0925 & -0.2033 & 0.0976 & 0.0508 & -0.0790 \\
\hline $\begin{array}{l}\text { Cumulative } \\
\text { variance }(\%)\end{array}$ & 98.824 & 99.460 & 99.769 & 99.938 & 100.000 & 100 & 20 & 0.0435 & 0.0172 & -0.0570 & -0.2049 & 0.1339 & 0.0622 \\
\hline Parameter 1 & -0.3532 & -0.1218 & -0.0693 & 0.0342 & -0.0223 & 0.0368 & 21 & -0.2499 & -0.3057 & 0.4308 & 0.2313 & -0.1303 & -0.0690 \\
\hline 4 & -0.0247 & -0.0526 & -0.2410 & -0.1766 & -0.0757 & 0.0281 & 22 & 0.4168 & -0.2085 & 0.0423 & -0.0476 & -0.0334 & 0.3012 \\
\hline 5 & -0.2384 & -0.3942 & -0.1896 & -0.3964 & 0.0519 & 0.2089 & 23 & 0.2272 & 0.2219 & 0.0177 & -0.4729 & 0.0173 & -0.3443 \\
\hline 6 & -0.1891 & 0.0296 & 0.1876 & -0.2697 & 0.0263 & -0.0814 & 25 & 0.2758 & -0.0752 & 0.0853 & -0.0063 & 0.3190 & 0.1671 \\
\hline 7 & -0.2471 & 0.1912 & 0.1933 & 0.1269 & 0.3584 & -0.0229 & 26 & 0.1043 & 0.3186 & -0.2013 & -0.0101 & -0.0880 & 0.4589 \\
\hline 9 & 0.2299 & 0.0670 & 0.1943 & 0.2503 & -0.1175 & -0.0258 & 27 & -0.0818 & -0.1231 & -0.3424 & 0.1276 & -0.2178 & -0.3309 \\
\hline 10 & -0.0187 & -0.3907 & -0.0930 & 0.0260 & 0.2593 & -0.1163 & 28 & -0.0706 & -0.2312 & 0.1138 & 0.0600 & -0.3347 & 0.2525 \\
\hline 11 & -0.2242 & 0.1662 & 0.0124 & 0.1394 & 0.1057 & 0.2155 & 30 & 0.2427 & -0.0202 & 0.4986 & -0.1437 & -0.2303 & -0.1166 \\
\hline 15 & -0.0214 & 0.2171 & -0.0935 & 0.0272 & 0.1173 & 0.0597 & 31 & -0.2409 & 0.1591 & 0.2445 & -0.2447 & 0.2245 & 0.0521 \\
\hline 16 & 0.0225 & 0.0563 & -0.1477 & -0.0405 & -0.2074 & -0.4116 & 32 & 0.1747 & -0.0560 & 0.0437 & 0.0812 & 0.3185 & -0.1000 \\
\hline 17 & -0.0892 & 0.3003 & -0.0532 & 0.3243 & -0.1219 & -0.0151 & 33 & -0.1737 & 0.2070 & 0.0900 & -0.2847 & -0.4206 & 0.1925 \\
\hline
\end{tabular}


Table A5. Results of DFA 1 - standard deviation, between-group variance and cumulative between-group variance, and principal component (PC) loadings on discriminant function (DF) axes. Discrimination between Vagaceratops-like operative taxonomic unit, Chasmosaurus russelli, and C. belli; PC scores and specimens $(\mathrm{n}=11)$ taken from PCA 1.

\begin{tabular}{|c|c|c|}
\hline Axis & DF1 & DF2 \\
\hline Standard deviation & 21.304 & 7.2317 \\
\hline Variance $(\%)$ & 74.66 & 25.34 \\
\hline Cumulative variance $(\%)$ & 74.66 & 100 \\
\hline PC1 & 0.29506 & 0.64882 \\
\hline PC2 & 0.36712 & 0.13384 \\
\hline PC3 & 0.021189 & 0.091344 \\
\hline PC4 & -0.21595 & 0.41457 \\
\hline PC5 & 0.13758 & -0.088757 \\
\hline PC6 & -0.039626 & -0.12754 \\
\hline PC7 & -0.011172 & -0.044429 \\
\hline PC8 & -0.026932 & -0.13104 \\
\hline PC9 & 0.016924 & -0.09247 \\
\hline PC10 & -0.017822 & -0.13283 \\
\hline
\end{tabular}


Table A6: Results of PCA 2 (11 specimens) - standard deviation, variance and cumulative variance, and parameter loadings on principal component (PC) axes.

\begin{tabular}{|c|c|c|c|c|c|c|c|c|c|c|c|c|c|}
\hline Axis & PC1 & PC2 & PC3 & PC4 & PC5 & PC6 & $\begin{array}{l}\text { Parameters } \\
\text { continued }\end{array}$ & PC1 & PC2 & PC3 & PC4 & PC5 & PC6 \\
\hline $\begin{array}{l}\text { Standard } \\
\text { deviation }\end{array}$ & 3.4501 & 1.9564 & 1.6337 & 1.50468 & 1.35692 & 0.93756 & 18 & 0.1582 & -0.2519 & 0.0097 & 0.4116 & 0.0351 & -0.1628 \\
\hline Variance (\%) & 47.61 & 15.31 & 10.67 & 9.056 & 7.365 & 3.516 & 19 & 0.2424 & -0.0252 & -0.0383 & -0.0717 & 0.0810 & 0.2368 \\
\hline $\begin{array}{l}\text { Cumulative } \\
\text { variance (\%) }\end{array}$ & 47.61 & 62.92 & 73.60 & 82.654 & 90.019 & 93.535 & 20 & 0.1938 & -0.3121 & 0.0710 & -0.1746 & 0.1440 & 0.0467 \\
\hline Parameter 1 & 0.2094 & 0.1222 & 0.0058 & 0.1149 & -0.2194 & -0.4658 & 21 & 0.1897 & -0.2537 & -0.1499 & 0.0756 & 0.0810 & 0.0727 \\
\hline 4 & 0.1833 & 0.1378 & 0.1615 & 0.4438 & 0.0240 & 0.0830 & 22 & 0.0624 & $\begin{array}{l}-0.3940 \\
\end{array}$ & $\begin{array}{l}-0.2983 \\
\end{array}$ & 0.1317 & -0.1188 & 0.0177 \\
\hline 5 & 0.2142 & 0.2267 & 0.0578 & -0.3027 & 0.0917 & 0.0234 & 23 & 0.2517 & -0.2312 & 0.0304 & -0.0157 & 0.1102 & -0.0365 \\
\hline 6 & 0.2484 & -0.0403 & -0.0728 & 0.1130 & 0.0823 & -0.0328 & 25 & 0.1524 & 0.1378 & $\begin{array}{l}-0.4278 \\
\end{array}$ & 0.0635 & -0.2032 & -0.2389 \\
\hline 7 & 0.1886 & -0.1605 & -0.0469 & -0.3513 & $\begin{array}{l}-0.3139 \\
\end{array}$ & 0.1122 & 26 & 0.2811 & 0.0614 & 0.0665 & -0.0480 & -0.0025 & 0.1077 \\
\hline 9 & 0.2356 & -0.0781 & 0.0207 & -0.0700 & 0.2192 & -0.3892 & 27 & 0.0824 & 0.1875 & -0.5227 & 0.0321 & 0.0203 & 0.0893 \\
\hline 10 & 0.2271 & -0.0924 & 0.0471 & -0.1019 & -0.3617 & 0.2365 & 28 & 0.2481 & 0.1982 & 0.1389 & -0.0349 & 0.1555 & 0.1090 \\
\hline 11 & 0.2462 & -0.1426 & 0.1375 & 0.1804 & 0.1670 & 0.1300 & 30 & 0.1628 & 0.3564 & 0.1231 & -0.0399 & -0.2058 & -0.0594 \\
\hline 15 & 0.1421 & 0.1973 & -0.3070 & -0.0817 & 0.2369 & -0.3008 & 31 & 0.0267 & 0.2596 & -0.2216 & 0.4229 & -0.0453 & 0.4407 \\
\hline 16 & 0.2736 & 0.0056 & -0.0173 & -0.0575 & -0.1441 & 0.1578 & 32 & 0.0245 & 0.2309 & -0.0446 & -0.1588 & 0.5352 & 0.1504 \\
\hline 17 & 0.2675 & 0.0990 & -0.0198 & -0.1304 & -0.1710 & 0.0393 & 33 & -0.1489 & -0.1553 & -0.4259 & -0.2112 & 0.0600 & 0.1419 \\
\hline
\end{tabular}


Table A6 (continued).

\begin{tabular}{|c|c|c|c|c|c|c|c|c|c|c|c|}
\hline Axis & PC7 & PC8 & PC9 & PC10 & PC11 & $\begin{array}{l}\text { Parameters } \\
\text { continued }\end{array}$ & PC7 & PC8 & PC9 & PC10 & PC11 \\
\hline $\begin{array}{l}\text { Standard } \\
\text { deviation }\end{array}$ & 0.7969 & 0.76111 & 0.47723 & 0.41744 & $\begin{array}{l}9.63 \\
\times 10^{-15}\end{array}$ & 18 & 0.2520 & -0.0273 & 0.1862 & 0.0785 & 0.0399 \\
\hline Variance $(\%)$ & 2.54 & 2.317 & 0.911 & 0.0697 & 0.00 & 19 & -0.3674 & 0.4412 & 0.1783 & -0.2968 & 0.0463 \\
\hline $\begin{array}{l}\text { Cumulative } \\
\text { variance }(\%)\end{array}$ & 96.07 & 98.392 & 99.303 & 100.000 & 100 & 20 & 0.2370 & 0.1811 & 0.1086 & -0.0186 & -0.2690 \\
\hline Parameter 1 & 0.0512 & 0.2583 & -0.5410 & -0.1665 & -0.0149 & 21 & -0.2902 & 0.2920 & -0.2621 & -0.0807 & 0.2241 \\
\hline 4 & 0.0165 & 0.0231 & 0.0848 & -0.1216 & -0.2104 & 22 & -0.0684 & -0.2572 & -0.2293 & -0.1385 & -0.0275 \\
\hline 5 & -0.0715 & 0.0631 & -0.1723 & 0.2676 & -0.0291 & 23 & -0.0039 & -0.1328 & 0.1241 & -0.0766 & 0.3770 \\
\hline 6 & -0.2461 & -0.5300 & -0.0721 & -0.0650 & -0.3843 & 25 & 0.1507 & 0.0489 & 0.1469 & -0.1879 & 0.1094 \\
\hline 7 & 0.0167 & -0.0076 & 0.0398 & -0.0069 & -0.3042 & 26 & 0.0434 & -0.0300 & 0.2181 & 0.1779 & 0.1058 \\
\hline 9 & 0.2445 & 0.2551 & 0.1147 & 0.1414 & -0.3468 & 27 & 0.2048 & 0.0993 & 0.0034 & 0.2744 & 0.1162 \\
\hline 10 & 0.0032 & -0.0648 & -0.3653 & 0.0827 & 0.0120 & 28 & -0.1014 & -0.0006 & -0.0597 & -0.0591 & -0.1941 \\
\hline 11 & 0.0846 & -0.0998 & 0.0018 & -0.0120 & 0.2203 & 30 & 0.0258 & -0.0965 & 0.2373 & -0.5591 & 0.0701 \\
\hline 15 & -0.4417 & -0.2121 & 0.1492 & 0.1863 & -0.0235 & 31 & 0.0251 & 0.1783 & -0.0542 & 0.1405 & -0.2385 \\
\hline 16 & 0.2049 & -0.1407 & 0.0775 & 0.0780 & 0.0033 & 32 & 0.4207 & -0.2129 & -0.3487 & -0.2725 & 0.0738 \\
\hline 17 & 0.1055 & -0.0780 & 0.0676 & 0.1558 & 0.3239 & 33 & 0.1316 & 0.0311 & 0.1256 & -0.3332 & -0.1709 \\
\hline
\end{tabular}


Table A7. Results of DFA 2 - standard deviation, between-group variance and cumulative between-group variance, and principal component (PC) loadings on discriminant function (DF) axes. Discrimination between Vagaceratops-like operative taxonomic unit, Chasmosaurus russelli, and C. belli; PC scores and specimens $(\mathrm{n}=11)$ taken from PCA 2.

\begin{tabular}{|c|c|c|}
\hline Axis & DF1 & DF2 \\
\hline Standard deviation & 175.67 & 7.0674 \\
\hline Variance (\%) & 96.13 & 3.87 \\
\hline Cumulative variance (\%) & 96.13 & 100 \\
\hline PC1 & 0.11907 & -0.6983 \\
\hline PC2 & 0.093402 & -0.1064 \\
\hline PC3 & 0.077084 & 0.18894 \\
\hline PC4 & 0.031482 & 0.33479 \\
\hline PC5 & -0.016583 & -0.26284 \\
\hline PC6 & -0.01895 & 0.067385 \\
\hline PC7 & 0.015597 & -0.058828 \\
\hline
\end{tabular}


Table A8: Results of PCA 3 (7 specimens) - standard deviation, variance and cumulative variance, and parameter loadings on principal component (PC) axes.

\begin{tabular}{|l|l|l|l|l|l|l|l|l|l|}
\hline Axis & PC1 & PC2 & PC3 & PC4 & $\begin{array}{l}\text { Parameters } \\
\text { continued }\end{array}$ & PC1 & PC2 & PC3 & PC4 \\
\hline $\begin{array}{l}\text { Standard } \\
\text { deviation }\end{array}$ & 3.1818 & 2.3558 & 2.0068 & 1.7011 & 18 & 0.1735 & -0.3062 & -0.0926 & 0.1322 \\
\hline Variance (\%) & 40.49 & 22.20 & 16.11 & 11.58 & 19 & 0.2371 & -0.0182 & -0.1756 & 0.2189 \\
\hline $\begin{array}{l}\text { Cumulative } \\
\text { variance (\%) }\end{array}$ & 40.49 & 62.69 & 78.80 & 90.38 & 20 & 0.2898 & 0.0662 & 0.1463 & 0.0311 \\
\hline Parameter 1 & -0.1213 & -0.1204 & 0.0757 & 0.4812 & 21 & 0.2621 & 0.0287 & -0.1252 & 0.1154 \\
\hline 4 & 0.0408 & -0.3859 & -0.1840 & -0.0273 & 22 & 0.2386 & 0.2487 & 0.0459 & 0.1417 \\
\hline 5 & 0.1417 & 0.1207 & 0.1178 & 0.0527 & 23 & 0.3071 & -0.0241 & 0.0701 & 0.0056 \\
\hline 6 & 0.2613 & -0.0775 & -0.1357 & -0.2276 & 25 & -0.0538 & 0.1928 & -0.3282 & 0.3333 \\
\hline 7 & 0.2344 & 0.1144 & 0.2264 & 0.2218 & 26 & 0.2640 & -0.0406 & -0.1331 & -0.2198 \\
\hline 9 & 0.2439 & 0.1882 & 0.1454 & 0.1518 & 27 & -0.0401 & 0.2298 & -0.3919 & 0.1170 \\
\hline 10 & 0.2367 & -0.1602 & 0.1853 & 0.1773 & 28 & 0.2671 & -0.0630 & -0.2295 & 0.0048 \\
\hline 11 & 0.2752 & -0.1911 & -0.0314 & -0.0938 & 30 & -0.0762 & -0.2255 & -0.2841 & 0.2493 \\
\hline 15 & 0.1421 & 0.2547 & -0.2974 & 0.1415 & 31 & -0.1001 & -0.1227 & -0.4163 & 0.0215 \\
\hline 16 & 0.2641 & -0.0080 & -0.1296 & -0.2112 & 32 & 0.0318 & 0.2333 & -0.1802 & -0.4342 \\
\hline 17 & 0.0152 & 0.3650 & 0.0318 & 0.0858 & 33 & -0.0790 & 0.3599 & -0.1115 & -0.0687 \\
\hline
\end{tabular}


Table A8 (continued).

\begin{tabular}{|l|l|l|l|l|l|l|l|}
\hline Axis & PC5 & PC6 & PC7 & $\begin{array}{l}\text { Parameters } \\
\text { continued }\end{array}$ & PC5 & PC6 & PC7 \\
\hline $\begin{array}{l}\text { Standard } \\
\text { deviation }\end{array}$ & 1.15897 & 1.03057 & $\begin{array}{l}9.799 \\
\mathrm{x} 10^{-15}\end{array}$ & 18 & 0.1961 & -0.1898 & -0.1227 \\
\hline Variance (\%) & 5.373 & 4.248 & 0.00 & 19 & -0.3264 & 0.1471 & -0.1010 \\
\hline $\begin{array}{l}\text { Cumulative } \\
\text { variance (\%) }\end{array}$ & 95.752 & 100.00 & 100 & 20 & -0.1497 & -0.0773 & -0.1424 \\
\hline Parameter 1 & 0.2280 & -0.0850 & 0.2492 & 21 & -0.2702 & 0.3072 & -0.3459 \\
\hline 4 & 0.1018 & -0.0636 & -0.0940 & 22 & -0.0778 & 0.0743 & 0.2361 \\
\hline 5 & 0.5825 & 0.4299 & -0.0300 & 23 & -0.0590 & -0.1271 & -0.1120 \\
\hline 6 & 0.1206 & -0.1724 & 0.1816 & 25 & 0.0030 & -0.0928 & 0.1433 \\
\hline 7 & -0.1031 & -0.0871 & 0.1029 & 26 & 0.1596 & -0.1936 & -0.0521 \\
\hline 9 & 0.0839 & -0.1941 & -0.1402 & 27 & 0.1028 & 0.1313 & -0.0160 \\
\hline 10 & -0.0086 & 0.2401 & -0.1476 & 28 & -0.0866 & 0.1781 & 0.2458 \\
\hline 11 & 0.0244 & -0.0038 & 0.1312 & 30 & -0.0643 & -0.3740 & -0.3215 \\
\hline 15 & 0.1162 & -0.0568 & 0.2457 & 31 & 0.0969 & 0.3112 & -0.1192 \\
\hline 16 & 0.1922 & -0.2105 & 0.2113 & 32 & -0.0163 & 0.1021 & -0.1992 \\
\hline 17 & 0.3762 & -0.2007 & -0.4947 & 33 & -0.2573 & -0.2481 & 0.0402 \\
\hline
\end{tabular}


Table A9. Results of DFA 3 - standard deviation, between-group variance and cumulative between-group variance, and principal component (PC) loadings on discriminant function (DF) axis. Discrimination between Chasmosaurus russelli and $C$. belli; PC scores and specimens $(\mathrm{n}=7)$ taken from PCA 3.

\begin{tabular}{|c|c|}
\hline Axis & DF1 \\
\hline Standard deviation & 20.709 \\
\hline Variance (\%) & 100 \\
\hline Cumulative variance (\%) & 100 \\
\hline PC1 & -0.3147 \\
\hline PC2 & -0.4384 \\
\hline PC3 & 0.0911 \\
\hline PC4 & 0.0254 \\
\hline PC5 & 0.1074 \\
\hline
\end{tabular}


Table A10: Measurement dataset used in PCA 4; 18 specimens of the Vagaceratops-like operative taxonomic unit $(\mathrm{n}=4)$, Chasmosaurus russelli $(\mathrm{n}=4)$, C. belli $(\mathrm{n}=3)$, and Chasmosaurus $\mathrm{sp} .(\mathrm{n}=7)$. Estimated missing data shown in bold. All parameter measurements are in mm.

\begin{tabular}{|l|l|l|l|l|l|l|l|l|l|l|l|l|}
\hline Specimen/Parameter & 1 & 4 & 5 & 16 & 18 & 19 & 22 & 27 & 28 & 30 & 31 & 32 \\
\hline AMNH 5401 & 370 & 320 & 130 & 730 & 315 & 710 & 115 & 820 & 875 & $\mathbf{6 7 8}$ & $\mathbf{3 4}$ & $\mathbf{9 1}$ \\
\hline AMNH 5402 & 70 & 190 & 144 & 680 & 250 & 685 & 120 & 920 & 780 & 354 & $\mathbf{3 3}$ & $\mathbf{8 4}$ \\
\hline AMNH 5656 & $\mathbf{1 5 0}$ & $\mathbf{2 7 2}$ & $\mathbf{1 5 6}$ & $\mathbf{7 6 3}$ & $\mathbf{2 6 6}$ & 569 & 129 & 697 & 854 & 503 & 27 & 86 \\
\hline CMN 1254 & 216 & 242 & 150 & $\mathbf{7 7 3}$ & $\mathbf{2 6 4}$ & 500 & $\mathbf{1 2 6}$ & $\mathbf{9 9 0}$ & $\mathbf{9 4 9}$ & $\mathbf{5 4 6}$ & $\mathbf{3 3}$ & $\mathbf{8 9}$ \\
\hline CMN 2245 & 102 & 238 & 176 & $\mathbf{7 5 3}$ & 269 & 779 & 140 & 1078 & 988 & 452 & 32 & 116 \\
\hline CMN 2280 & 132 & 263 & $\mathbf{1 5 5}$ & 750 & 278 & 709 & 132 & 1043 & 855 & 557 & 34 & 72 \\
\hline CMN 8800 & $\mathbf{2 0 7}$ & 412 & $\mathbf{1 5 8}$ & 880 & 309 & 997 & $\mathbf{1 2 3}$ & 848 & 1143 & 584 & 36 & 79 \\
\hline CMN 8801 & $\mathbf{1 5 2}$ & 300 & 194 & 835 & $\mathbf{2 6 6}$ & $\mathbf{7 1 4}$ & $\mathbf{1 3 4}$ & $\mathbf{9 9 6}$ & $\mathbf{9 1 9}$ & $\mathbf{4 8 6}$ & $\mathbf{3 3}$ & $\mathbf{8 8}$ \\
\hline CMN 34829 & 59 & 244 & $\mathbf{1 5 0}$ & $\mathbf{7 3 6}$ & 299 & 850 & $\mathbf{1 6 4}$ & $\mathbf{1 0 1 7}$ & $\mathbf{8 2 4}$ & $\mathbf{3 2 7}$ & 40 & $\mathbf{8 3}$ \\
\hline CMN 41357 & $\mathbf{6 3}$ & 250 & 123 & 795 & 320 & 700 & 225 & 1047 & 753 & 265 & 33 & 76 \\
\hline NHMUK R4948 & $\mathbf{1 8 2}$ & $\mathbf{2 8 3}$ & 155 & $\mathbf{7 7 1}$ & $\mathbf{2 6 5}$ & 840 & $\mathbf{1 2 8}$ & 1160 & $\mathbf{9 4 2}$ & 627 & $\mathbf{3 3}$ & 90 \\
\hline ROM 839 & 107 & 250 & 237 & 782 & 231 & 917 & 115 & 893 & 1034 & $\mathbf{4 3 1}$ & 26 & $\mathbf{8 6}$ \\
\hline ROM 843 & $\mathbf{1 4 3}$ & 252 & 199 & 897 & 290 & 893 & 151 & 1227 & 1063 & 498 & 30 & 94 \\
\hline $\begin{array}{l}\text { TMP } \\
1981.019 .0175\end{array}$ & $\mathbf{1 2 2}$ & 302 & 134 & 768 & 217 & 600 & 171 & $\mathbf{1 0 0 2}$ & $\mathbf{8 9 1}$ & $\mathbf{4 3 5}$ & $\mathbf{3 3}$ & $\mathbf{8 6}$ \\
\hline $\begin{array}{l}\text { TMP } \\
1983.025 .0001\end{array}$ & $\mathbf{1 9 2}$ & 345 & $\mathbf{1 5 8}$ & $\mathbf{7 7 3}$ & $\mathbf{2 6 4}$ & 650 & 125 & 1373 & 952 & 592 & 45 & 102 \\
\hline $\begin{array}{l}\text { TMP } \\
1987.045 .0001\end{array}$ & $\mathbf{7 1}$ & 233 & 124 & 617 & 285 & 633 & 157 & 940 & 755 & 260 & $\mathbf{3 3}$ & $\mathbf{8 3}$ \\
\hline UALVP 40 & 176 & 240 & 131 & 628 & 180 & 600 & 88 & $\mathbf{9 9 2}$ & $\mathbf{9 3 7}$ & $\mathbf{5 2 1}$ & $\mathbf{3 3}$ & $\mathbf{8 8}$ \\
\hline YPM 2016 & $\mathbf{1 0 6}$ & 222 & $\mathbf{1 5 3}$ & 800 & 271 & 730 & 193 & 1162 & 840 & 463 & 34 & $\mathbf{7 5}$ \\
\hline
\end{tabular}


Table A11: Results of PCA 4 (18 specimens) - standard deviation, variance and cumulative variance, and parameter loadings on principal component (PC) axes.

\begin{tabular}{|c|c|c|c|c|c|c|c|c|c|c|c|c|}
\hline Axis & $\mathrm{PC} 1$ & PC2 & PC3 & $\mathrm{PC} 4$ & PC5 & PC6 & PC7 & PC8 & PC9 & PC410 & PC11 & PC12 \\
\hline $\begin{array}{l}\text { Standard } \\
\text { deviation }\end{array}$ & 1.9699 & 1.5566 & 1.3514 & 1.2171 & 0.84399 & 0.83510 & $\begin{array}{l}0.6553 \\
1\end{array}$ & 0.49698 & 0.36525 & 0.31766 & 0.2019 & 0.16653 \\
\hline Variance $(\%)$ & 32.34 & 20.19 & 15.22 & 12.34 & 5.936 & 5.812 & 3.579 & 2.058 & 1.112 & 0.841 & 0.34 & 0.231 \\
\hline $\begin{array}{l}\text { Cumulative } \\
\text { variance }(\%)\end{array}$ & 32.34 & 52.53 & 67.75 & 80.09 & 86.028 & 91.840 & 95.418 & 97.476 & 98.588 & 99.429 & 99.77 & 100 \\
\hline Parameter 1 & -0.3982 & -0.2378 & 0.2395 & -0.1980 & 0.2128 & -0.0007 & 0.2327 & -0.2025 & 0.0670 & -0.2207 & -0.0150 & 0.7032 \\
\hline 4 & -0.3413 & 0.0959 & 0.3528 & -0.2641 & -0.1333 & -0.0391 & -0.5825 & 0.0544 & 0.4552 & -0.2400 & 0.0603 & -0.2241 \\
\hline 5 & -0.2907 & 0.2006 & -0.4730 & 0.1771 & 0.0416 & 0.0477 & 0.1014 & 0.6089 & 0.3358 & -0.1246 & -0.2812 & 0.1664 \\
\hline 16 & -0.2907 & 0.4462 & -0.0381 & -0.1087 & 0.3963 & 0.1481 & -0.1104 & 0.1466 & -0.2557 & 0.3123 & 0.5690 & 0.0622 \\
\hline 18 & 0.0221 & 0.4437 & 0.2267 & -0.3009 & 0.0107 & -0.5054 & 0.4767 & 0.1263 & -0.1320 & -0.3106 & -0.0607 & -0.2107 \\
\hline 19 & -0.1867 & 0.4234 & -0.1986 & 0.0147 & -0.6415 & -0.0107 & 0.1340 & -0.4312 & 0.1771 & 0.2361 & 0.0776 & 0.1937 \\
\hline 22 & 0.2708 & 0.4578 & 0.1031 & -0.0072 & 0.4184 & 0.0092 & -0.2446 & -0.2684 & 0.1334 & 0.1967 & -0.5555 & 0.1853 \\
\hline 27 & -0.0352 & 0.2216 & 0.2317 & 0.6607 & 0.1553 & 0.2971 & 0.2036 & -0.2211 & 0.1696 & -0.4128 & 0.1810 & -0.1576 \\
\hline 28 & -0.4620 & 0.0566 & -0.1453 & 0.0999 & -0.0833 & 0.0574 & -0.2479 & -0.1656 & -0.6562 & -0.2581 & -0.3723 & -0.1316 \\
\hline 30 & -0.4341 & -0.1739 & 0.1626 & -0.0688 & 0.1537 & 0.1730 & 0.3882 & -0.0935 & 0.1756 & 0.4748 & -0.2723 & -0.4546 \\
\hline 31 & 0.0034 & 0.0834 & 0.6239 & 0.2880 & -0.3373 & 0.0652 & -0.0226 & 0.4417 & -0.2198 & 0.2811 & -0.1466 & 0.2416 \\
\hline 32 & -0.2102 & -0.1403 & -0.0317 & 0.4721 & 0.1519 & -0.7700 & -0.1579 & -0.0980 & 0.0715 & 0.2196 & 0.0896 & 0.0186 \\
\hline
\end{tabular}


Table A12: Results of PCA 5 (18 specimens) - standard deviation, variance and cumulative variance, and parameter loadings on principal component (PC) axes.

\begin{tabular}{|c|c|c|c|c|c|c|c|c|c|c|c|c|}
\hline Axis & PC1 & $\mathrm{PC} 2$ & PC3 & $\mathrm{PC} 4$ & PC5 & PC6 & PC7 & PC8 & PC9 & PC410 & PC11 & PC12 \\
\hline $\begin{array}{l}\text { Standard } \\
\text { deviation }\end{array}$ & 0.5744 & 0.2271 & $\begin{array}{l}0.1824 \\
5 \\
\end{array}$ & 0.17499 & 0.1203 & 0.11079 & $\begin{array}{l}0.0944 \\
5 \\
\end{array}$ & 0.07438 & 0.06797 & 0.03618 & 0.03287 & 0.02127 \\
\hline Variance $(\%)$ & 66.78 & 10.44 & 6.737 & 6.198 & 2.93 & 2.484 & 1.805 & 1.120 & 0.935 & 0.265 & 0.219 & 0.092 \\
\hline $\begin{array}{l}\text { Cumulative } \\
\text { variance }(\%)\end{array}$ & 66.78 & 77.22 & 83.953 & 90.150 & 93.08 & 95.565 & 97.370 & 98.490 & 99.425 & 99.690 & 99.908 & 100 \\
\hline Parameter 1 & -0.8303 & 0.2142 & -0.0033 & 0.0949 & -0.2322 & 0.0872 & -0.0741 & 0.1888 & -0.3056 & 0.1332 & -0.2003 & 0.0413 \\
\hline 4 & -0.1798 & 0.2358 & 0.4685 & -0.1083 & 0.5167 & 0.4075 & -0.1996 & -0.2758 & 0.0462 & -0.3546 & 0.0354 & 0.0528 \\
\hline 5 & -0.0129 & -0.5440 & 0.0688 & -0.0242 & -0.2514 & 0.2839 & -0.0592 & -0.6310 & -0.2874 & 0.2012 & -0.0969 & 0.1433 \\
\hline 16 & -0.0137 & 0.0253 & 0.0916 & 0.0279 & -0.0826 & 0.0950 & -0.1158 & -0.1073 & -0.0148 & 0.1379 & 0.0908 & -0.9601 \\
\hline 18 & 0.0430 & 0.3016 & 0.4143 & 0.0433 & -0.4567 & -0.0703 & 0.5653 & -0.1722 & -0.1175 & -0.1929 & 0.3442 & 0.0381 \\
\hline 19 & 0.0448 & -0.2905 & 0.6955 & -0.2864 & -0.1725 & -0.2914 & -0.2075 & 0.3183 & 0.0829 & 0.0571 & -0.2807 & 0.0056 \\
\hline 22 & 0.2655 & 0.5220 & 0.0326 & 0.0086 & -0.3971 & 0.1995 & -0.4994 & -0.1143 & 0.2313 & 0.3300 & -0.0103 & 0.1830 \\
\hline 27 & 0.0414 & 0.0727 & -0.2876 & -0.7473 & -0.2052 & -0.0109 & -0.2026 & 0.0268 & -0.3254 & -0.3797 & 0.1244 & -0.0476 \\
\hline 28 & -0.1052 & -0.2233 & 0.0993 & -0.1042 & 0.0996 & 0.2154 & -0.1001 & 0.3152 & -0.0358 & 0.3623 & 0.7786 & 0.1179 \\
\hline 30 & -0.4328 & -0.1008 & -0.1006 & -0.1570 & -0.0907 & -0.3957 & -0.0945 & -0.3802 & 0.6285 & -0.0643 & 0.2240 & 0.0141 \\
\hline 31 & -0.0122 & 0.2626 & 0.0224 & -0.4899 & 0.3286 & -0.1279 & 0.3696 & -0.1848 & -0.0544 & 0.6047 & -0.1633 & 0.0024 \\
\hline 32 & -0.0577 & -0.1409 & -0.0885 & -0.2449 & -0.2117 & 0.6226 & 0.3593 & 0.2361 & 0.4923 & -0.0489 & -0.2124 & -0.0431 \\
\hline
\end{tabular}




\section{Appendix V: Ontogenetically variable characters used in phylogenetic analysis}

Character 8 - Premaxilla, septal flange:

The presence of this flange appears to vary ontogenetically in Triceratops, being present (1) in immature specimens, but absent (0) in mature specimens (Scannella et al., 2013).

Character 35 - Postorbital, postorbital ornamentation in adult:

Holmes et al. (2001) originally diagnosed Vagaceratops irvinensis as lacking postorbital horncores (0); however, they left open the possibility that the rugose and pitted mound present on the dorsal surface of the postorbital in CMN 41357 and TMP 1987.045.0001 may represent the base of a remodeled and resorbed horncore (1). The Vagaceratops-like skulls AMNH 5402 and YPM 2016 were recovered in this study as relatively immature and mature adults, respectively, and possess complete postorbital horncores and pitted mounds, respectively. This suggests that Vagaceratops-like specimens possessed postorbital horncores earlier in life, but were subsequently lost through remodeling later in life. Postorbital horncore remodeling also occurs in Chasmosaurus-like specimens, as evidenced by specimens possessing both a complete or partially complete horncore on one side of the skull and a pitted mound on the other (e.g., CMN 2280, CMN 8800 and TMP 1981.019.0175).

Character 36 - Postorbital, extent of cornual sinus in base of postorbital ornamentation:

The extent of the cornual sinus into the postorbital is known to change ontogenetically within Triceratops (Farke, 2006). This sinus expands into the base of the 
postorbital horncore over ontogeny, and eventually excavates the shaft of the horncore.

Character 39 - Postorbital, length of postorbital horncore:

In Triceratops ontogeny, postorbital horncores are relatively short ( 0 , less than $15 \%$ basal skull length) early in life, and lengthen (1, greater than $35 \%$ of basal skull) later on (Horner and Goodwin, 2006).

Character 40 - Postorbital, curvature of postorbital horncore in lateral view:

The orientation of the postorbital horncores in lateral view is known to change ontogenetically in Triceratops, being posteriorly curved (0) early in life, straightening out (2), and becoming anteriorly curved (1) later on (Horner and Goodwin, 2006).

Character 50 - Epijugal length:

A decrease in the length of the epijugal relative to its basal width may be partly ontogenetic in Chasmosaurus-like specimens, with epijugals being relatively long (0) in some relatively less mature specimens (i.e., AMNH 5401, ROM 839 and ROM 843), and relatively short (2) in relatively more mature specimens (e.g., CMN 2280 and TMP 1981.019.0175).

Character 55 - Frontoparietal fontanelle leading into supracranial cavity complex:

The absence (0) and presence (1) of a frontoparietal fontanelle in both Triceratops and Torosaurus is thought to related to ontogeny, with the gradual closure of the fontanelle due to bone overgrowth (Farke, 2010). 
Character 56 - Frontoparietal fontanelle, shape:

The shape of the frontoparietal fontanelle changes as a result of bone overgrowth in some taxa (Triceratops and Torosaurus; Farke, 2010), and is hence related to ontogeny.

Character 73 - Parietal fenestra:

In their Triceratops-Torosaurus synonymy, Scannella and Horner (2010) propose that the formation of parietal fenestrae is ontogenetic with the unfenestrated (0) and putatively immature Triceratops maturing into a fenestrated (1) and putatively mature Torosaurus. The formation of parietal fenestrae is also ontogenetic in the basal ceratopsian Protoceratops (Fastovsky et al., 2011).

Character 75 - Parietal, sharp median crest:

In Triceratops (Goodwin et al., 2006) and Centrosaurus (Ryan et al., 2001;

Brown et al., 2009) ontogeny, this crest is present (0) early in life, but gradually becomes less pronounced (1) later in ontogeny.

Character 82 - Parietosquamosal frill, marginal undulations:

These undulations refer to the scalloped free margin of the parietosquamosal frill, and correspond to epiossification (episquamosal and epiparietal) attachment sites. In ceratopsids, these undulations are most pronounced in relatively immature individuals, but become less pronounced later in maturity and are difficult to discern underneath the 
articulated epiossifications; these undulations do not disappear entirely, however, as they serve as an attachment site for epiossifications throughout maturity (see epiparietalparietal cross-section in Chasmosaurus-like specimen CMN 8802, Figs. 30E-J).

Character 84 - Marginal dermal ossifications on parietosquamosal frill:

In ceratopsids, parietosquamosal frill dermal ossifications (episquamosals and epiparietals) are disarticulated from the frill and absent (0) early in life, but are present (1) and articulated with the frill later on (Horner and Goodwin, 2006).

Character 91 - Epiparietosquamosal, marginal ossification crossing squamosal-parietal contact:

The Vagaceratops-like skull AMNH 5402, the inferred fifth epiossification from the midline of the parietal is fused entirely onto the parietal, although the lateral end of the base of this epiossification overhangs the distal end of the squamosal. In chasmosaurine ontogeny, the entire base of each epiparietal and episquamosal fuses onto the frill margin (Horner and Goodwin, 2006). If the epiossification (epiparietal) in AMNH 5402 had undergone complete articulation with the underlying frill margin, it would have partly articulated with the squamosal as well, making it an epiparietosquamosal. Epiparietosquamosals, where present in Dinosaur Park Formation chasmosaurine skulls (AMNH 5402 and TMP 1987.045.0001, and likely TMP 1998.102.0008), are interpreted as being ontogenetic, going from absent (0) to present (1). 
Character 93 - Epiparietals, number per side:

Epiparietal count is relatively conservative amongst chasmosaurines, with all but three genera having three per side. Anchiceratops has three to four, Vagaceratops-like specimens have four to five, and Torosaurus has five to six per side. Mallon et al. (2011) found that in Anchiceratops, specimens with three epiparietals and one epiparietosquamosal per side were relatively immature and those with four epiparietals per side were more mature. They reasoned that the epiparietosquamosal migrates laterally over ontogeny and becomes articulated exclusively with the parietal, becoming an epiparietal. It is likely that epiparietal count changes from five to four in amongst some members of the Vagaceratops-like operative taxonomic unit, as suggested by AMNH 5402 (see character 91 above).

Characters 97, 100 and 102 - Shape of epiparietal 1, 2 and 3, respectively:

Epiparietal 1, 2 and 3 are well-developed triangular processes (3) in immature specimens of Triceratops, but they remodel into elongate low processes (4) later in maturity (Horner and Goodwin, 2006).

Character 98 - Epiparietal, locus P1 orientation:

P1 is oriented in the plane of the frill (0) in relatively immature Dinosaur Park Formation chasmosaurine specimens, but becomes anterodorsally oriented (1) later in life. This is due to the reorientation of the underlying parietal margin over ontogeny, as it develops into an anterodorsally thickened ridge. 
Character 103 - Epiparietal, locus P3 orientation:

Epiparietal 3 is not preserved in the relatively immature Vagaceratops-like specimen AMNH 5402. However, given that the inferred parietal loci is oriented in the plane of the frill, it is likely that this epiparietal would have also been oriented in the plane of the frill (0). Epiparietal 3 is oriented anterodorsally (1) in the more mature member Vagaceratops-like skull YPM 2016.

Character 105 - Supraoccipital, contribution to foramen magnum:

In Triceratops ontogeny, the supraoccipital forms the dorsal margin of the foramen magnum (0) early in life, but this element is eliminated from the foramen magnum (1) by the exoccipitals later in life (Goodwin et al., 2006).

Character 149 - Lateral ridge of dentary:

The presence of this ridge appears to vary ontogenetically in Triceratops, being present (0) in immature specimens, but absent (1) in mature specimens (Scannella et al., 2013).

Character 152 - Epiparietal, locus P4 orientation:

The ontogenetic nature of this character follows the same reasoning as described above for character 103, involving AMNH 5402 (0) and YPM 2016 (1). 


\title{
Appendix VI: Revised taxonomic diagnoses of Dinosaur Park Formation chasmosaurine taxa considered valid in this study
}

\section{Systematic palaeontology (proposed in this study)}

\author{
Ceratopsia Marsh, 1888
}

Neoceratopsia Sereno, 1986

Ceratopsidae Marsh, 1888

Chasmosaurinae Lambe, 1915

Chasmosaurus Lambe, 1914b

Type and only species. Chasmosaurus belli Lambe, 1902:66

The most recent diagnosis for Chasmosaurus (characters 1-5 and 8) by Maidment and Barrett (2011) - characters 1-3 after Forster et al. (1993). Character 5 has been changed from "rounded or anteroposteriorly longer" to "anteroposteriorly longer" to reflect the removal of YPM 2016 and AMNH 5402 from Chasmosaurus, which have rounded parietal fenestrae, with length/width ratios of 1.03 and 1.04, respectively. Character 8 has been changed from "epiparietals triangular in shape and project posteriorly or dorsally but do not curve anteriorly" to "epiparietals straight and triangular in shape and oriented posteriorly or anterodorsally", as the body of the epiparietal is straight in Chasmosaurus, and its orientation depends on the orientation of the underlying parietal margin (posteriorly or anterodorsally). Characters 6, 7 and 9 have been added to accommodate variation in the type and only species, Chasmosaurus belli. 
Revised diagnosis. "Chasmosaurus is diagnosed based on the following combination of characters: (1) Premaxillary flange along entire anterior margin of external naris; (2) [postorbital horncores], when present, curve posteriorly along their length; (3) frill broadens posteriorly to form triangular shield with maximum width more than twice the skull width at orbits; (4) medial margin of squamosal, where it articulates with the lateral bar of the parietal, straight;" Maidment and Barrett (2011:4); (5) parietal fenestrae large, occupying most of the parietal, and being anteroposteriorly longer than transversely wide; (6) posterior parietal bar bearing a median emargination ranging from deeply embayed to nearly straight; (7) each side of the posterior parietal bar bears three epiparietals that range from being roughly equal-sized to being variable in size relative to each other; (8) epiparietals straight and triangular in shape and oriented posteriorly or anterodorsally; and (9) dorsal margin of the posterior parietal bar underlying epiparietals 1 and variably 2 recurves anterodorsally in mature members, forming a dorsoventrally-thickened 'ridge'. The ridge on each side of the posterior parietal bar are not connected medially. All of these features are seen in other chasmosaurines and do not, therefore, represent autapomorphies, but this combination of features appears to be unique to Chasmosaurus.

\section{Chasmosaurus belli Lambe, 1902}

Monoclonius belli Lambe, 1902:66

Monoclonius canadensis Lambe, 1902:63

Ceratops belli Hatcher, Marsh and Lull, 1907:97

Ceratops canadensis Hatcher, Marsh and Lull, 1907:97

Protorosaurus belli Lambe, 1914:131 
Chasmosaurus belli Lambe, 1914:149

Eoceratops canadensis Lambe, 1915:2

Chasmosaurus kaiseni Brown, 1933:2

Chasmosaurus brevirostris Lull, 1933:94

Chasmosaurus russelli Sternberg, 1940:478

Chasmosaurus canadensis Lehman, 1989:139

Mojoceratops perifania Longrich, 2010:683

Revised diagnosis. As for the genus.

Holotype. CMN 0491, a partial parietal. Although fragmentary, the holotype is diagnostic based on the combination of generic characters $5,6,8$, and 9 , a combination not observed in any other chasmosaurine; the width of the parietal fenestrae in this specimen, however, cannot be determined.

Distribution. Lower to uppermost beds of the Dinosaur Park Formation (Dinosaur Park faunal zones 1 to 3 of Ryan and Evans (2005)) of Alberta (Dinosaur Provincial Park, Hilda, Manyberries, Onefour) and Saskatchewan (Saskatchewan Landing Provincial Park), and age-equivalent sediments of the uppermost Oldman Formation in southern Alberta (Milk River region), Canada.

Referred specimens. AMNH 5401, AMNH 5656, CMN 1254, CMN 2245, CMN 2280, CMN 8800, CMN 8801, CMN 8802, CMN 8803, CMN 34829, CMN 34832, CMN 41933, NHMUK R4948, ROM 839, ROM 843, TMP 1979.011.0147, TMP 1981.019.0175, TMP 1983.025.0001, TMP 1993.082.0001, TMP 1997.132.0002, TMP 1999.055.0292, and UALVP 40. 
Specimens removed from C. belli. AMNH 5402 and YPM 2016.

\section{Vagaceratops Sampson et al., 2010}

Type and only species. Vagaceratops irvinensis Holmes, Forster, Ryan, and Shepherd, 2001:1424

Revised diagnosis. (1) parietal fenestrae ranging from rounded to transversely wider than anteroposteriorly long; (2) posterior parietal bar straight, bearing no median emargination; (3) each side of the posterior parietal bar bears either five epiparietals or four epiparietals and one epiparietosquamosal; (4) epiparietals 1-4 triangular and oriented anterodorsally, and either straight or anterodorsally curved, and epiparietal 5 or epiparietosquamosal triangular, straight and oriented posterolaterally in the plane of the frill; and (5) dorsal margin of the posterior parietal bar underlying epiparietals 1-4 recurves anterodorsally in mature members, forming a dorsoventrally-thickened 'ridge'. The ridge on each side of the posterior parietal bar are connected medially. All of these features are seen in other chasmosaurines and do not, therefore, represent autapomorphies, but this combination of features appears to be unique to Vagaceratops.

Vagaceratops irvinensis Holmes, Forster, Ryan, and Shepherd, 2001

Revised diagnosis. As for the genus.

Holotype. CMN 41357, a mostly complete skull and skeleton.

Distribution. Middle to uppermost beds of the Dinosaur Park Formation (uppermost Dinosaur Park Faunal Zone 1 to Dinosaur Park Faunal Zone 3 of Ryan and Evans 
(2005)); southeastern Alberta, Canada; Dinosaur Provincial Park, Hilda (South Saskatchewan River), Irvine, Manyberries, and Onefour.

Referred specimens. AMNH 5402, TMP 1987.045.0001, TMP 1998.102.0008, TMP 2009.034.0009, TMP 2011.053.0046, and YPM 2016. 


\section{References}

Adkesson, M. J., and J. N. Langan. 2007. Metabolic bone disease in juvenile Humboldt penguins (Spheniscus humboldti): investigation of ionized calcium, parathyroid hormone, and vitamin $\mathrm{D}_{3}$ as diagnostic parameters. Journal of Zoo and Wildlife Medicine 38:85-92.

Barsbold, R., and A. Perle. 1983. On the taphonomy of the joint burial of juvenile dinosaurs and some aspects of their ecology. Transactions of the SovietMongolian Paleontological Expedition 24:121-125.

Bennett, S. C. 1992. Sexual dimorphism of Pteranodon and other pterosaurs, with comments on cranial crests. Journal of Vertebrate Paleontology 12:422-434.

Bercovitz, A. B., N. M. Czekala, and B. L. Lasley. 1978. A new method of sex determination in monomorphic birds. The Journal of Zoo Animal Medicine $9: 114-124$.

Bloch, J. I., R. Secord, and P. D. Gingerich. 2004. Systematics and phylogeny of Late Paleocene and Early Eocene Palaeoryctinae (Mammalia, Insectivora) from the Clarks Fork and Bighorn basins, Wyoming. Contributions from the Museum of Paleontology, University of Michigan 31:119-154. 
Borkovic, B. 2013. Investigating sexual dimorphism in ceratopsid horncores. M.S. thesis, University of Calgary, Calgary, Alberta, $305 \mathrm{pp}$.

Bowen, C. F. 1915. The stratigraphy of the Montana Group with special reference to the position and age of the Judith River Formation in north-central Montana. United States Geological Survey, Professional Paper 90:95-153.

Braman, D. R., and E. B. Koppelhus. 2005. Campanian palynomorphs; pp. 101-130 in P. J. Currie and E. B. Koppelhus (eds.), Dinosaur Provincial Park: A Spectacular Ancient Ecosystem Revealed. Indiana University Press, Bloomington, Indiana.

Bro-Jørgensen, J. 2007. The intensity of sexual selection predicts weapon size in male bovids. Evolution 61:1316-1326.

Brown, B. 1914. Anchiceratops, a new genus of horned dinosaurs from the Edmonton Cretaceous of Alberta. With discussion of the origin of the ceratopsian crest and brain casts of Anchiceratops and Trachodon. Bulletin of the American Museum of Natural History 33:539-548.

Brown, B. 1933. A new longhorned Belly River ceratopsian. American Museum Novitates 669:1-3.

Brown, C. M., A. P. Russell, and M. J. Ryan. 2009. Pattern and transition of surficial 
bone texture of the centrosaurine frill and their ontogenetic and taxonomic implications. Journal of Vertebrate Paleontology 29:132-141.

Brown, C. M., J. H. Arbour, and D. A. Jackson. 2012. Testing of the effect of missing data estimation and distribution in morphometric multivariate data analyses. Systematic Biology doi:10.1093/sysbio/sys047.

Brown, C. M., D. C. Evans, N. E. Campione, L. J. O’Brien, and D. A. Eberth. 2013. Evidence for taphonomic size bias in the Dinosaur Park Formation (Campanian, Alberta), a model Mesozoic terrestrial alluvial-paralic system. Palaeogeography, Palaeoclimatology, Palaeoecology 372:108-122.

Campione, N. E., and D. C. Evans. 2011. Cranial growth and variation in edmontosaurs (Dinosauria: Hadrosauridae): Implications for latest Cretaceous megaherbivore diversity in North America. PLoS ONE 6:e25186.doi:10.1371/journal.pone. 0025186.

Carpenter, K. 1990. Variation in Tyrannosaurus rex; pp. 141-146 in P. Currie and K. Carpenter (eds.), Dinosaur Systematics: Approaches and Perspectives. Cambridge University Press, Cambridge, UK.

Carpenter, K. 1999. Eggs, nests, and baby dinosaurs: a look at dinosaur reproduction. Indiana University Press, Bloomington, Indiana. 
Carr, T. D. 2010. A taxonomic assessment of the type series of Albertosaurus sarcophagus and the identity of Tyrannosauridae (Dinosauria, Coelurosauria) in the Albertosaurus bonebed from the Horseshoe Canyon Formation (CampanianMaastrichtian, Late Cretaceous). Canadian Journal of Earth Sciences 47:12131226.

Carr, T. D., and T. E. Williamson. 2004. Diversity of late Maastrichtian Tyrannosauridae (Dinosauria: Theropoda) from western North America. Zoological Journal of the Linnean Society 142:479-523.

Coombs, W. P. 1982. Juvenile specimens of the ornithischian dinosaur Psittacosaurus. Palaeontology 25:89-107.

Currie, P. J. 2005. History of research; pp. 3-33 in P. J. Currie and E. B. Koppelhus (eds.), Dinosaur Provincial Park: A Spectacular Ancient Ecosystem Revealed. Indiana University Press, Bloomington, Indiana.

Currie, P. J., and D. A. Russell. 2005. The geographic and stratigraphic distribution of articulated and associated dinosaur remains; pp. 537-569 in P. J. Currie and E. B. Koppelhus (eds.), Dinosaur Provincial Park: A Spectacular Ancient Ecosystem Revealed. Indiana University Press, Bloomington, Indiana. 
Currie, P. J., W. Jr. Langston, and D. H. Tanke. 2008. A New Horned Dinosaur from an Upper Cretaceous Bone Bed in Alberta. 144 pp. NRC Research Press, Ottawa.

Dawson, G. M. 1883. Preliminary report on the geology of the Bow and Belly River region, North-west Territory, with special reference to coal deposits. Geological and Natural History Survey and Museum of Canada, Report of Progress, 1880-82, pt. B, pp. 1-23.

Dodson, P. 1975. Taxonomic implications of relative growth in lambeosaurine hadrosaurs. Systematic Zoology 24:37-54.

Dodson, P. 1976. Quantitative aspects of relative growth and sexual dimorphism in Protoceratops. Journal of Paleontology 50:929-940.

Dodson, P., C. A. Forster, and S. D. Sampson. 2004. Ceratopsidae; pp. 494-513 in D. B. Weishampel, P. Dodson, and H. Osmólska (eds.), The Dinosauria, 2nd Edition. University of California Press, Berkeley.

Eberth, D. A. 1996. Origin and significance of mud-filled incised valleys (Upper Cretaceous) in southern Alberta, Canada. Sedimentology 43:459-477.

Eberth, D. A. 2005. The geology; pp. 54-82 in P. J. Currie and E. B. Koppelhus (eds.), Dinosaur Provincial Park: A Spectacular Ancient Ecosystem Revealed. Indiana 
University Press, Bloomington, Indiana.

Eberth, D. A., and A. L. Deino. 1992. A geochronology of the non-marine Judith River Formation of southern Alberta; pp. 24-25 in SEPM 1992 Theme Meeting, Mesozoic of the Western Interior, Ft. Collins, Abstracts with Programs.

Eberth, D. A., and A. P. Hamblin. 1993. Tectonic, stratigraphic, and sedimentologic significance of a regional disconformity in the upper Judith River Group (Belly River wedge) of southern Alberta, Saskatchewan, and northern Montana. Canadian Journal of Earth Sciences 30:174-200.

Erickson G. M., and T. A. Tumanova. 2000. Growth curve of Psittacosaurus mongoliensis Osborn (Ceratopsia: Psittacosauridae) inferred from long bone histology. Zoological Journal of the Linnean Society 130:551-566.

Erickson G. M., and P. S. Druckenmiller. 2011. Longevity and growth rate estimates for a polar dinosaur: a Pachyrhinosaurus (Dinosauria: Neoceratopsia) specimen from the North Slope of Alaska showing a complete developmental record. Historical Biology 23:327-334.

Estes, R. D. 1991. The significance of horns and other male secondary sexual characters in female bovids. Applied Animal Behaviour Science 29:403-451. 
Evans, D. 2003. Cranial Osteology and ontogeny of Corythosaurus (Ornithischia: Hadrosauridae); pp. 48-49 in Journal of Vertebrate Paleontology, Program and Abstracts.

Evans, D., P. Currie, D. Eberth, and M. Ryan. 2006. High-resolution lambeosaurine dinosaur biostratigraphy, Dinosaur Park Formation, Alberta: sexual dimorphism reconsidered; p. 59 in Journal of Vertebrate Paleontology, Program and Abstracts.

Evans, D., N. Campione, K. Brink, R. Schott, and C. Brown. 2013. Wasted youth: the importance of ontogenetically equivalent semaphoronts in dinosaur phylogenetic systematics; p. 123 in Journal of Vertebrate Paleontology, Program and Abstracts.

Farke, A. A. 2006. Morphology and ontogeny of the cornual sinuses in chasmosaurine dinosaurs (Ornithischia: Ceratopsidae). Journal of Paleontology 80:780-785.

Farke, A. A. 2010. Evolution, homology, and function of the supracranial sinuses in ceratopsian dinosaurs. Journal of Vertebrate Paleontology 30:1486-1500.

Farke, A. A. 2011. Anatomy and taxonomic status of the chasmosaurine ceratopsid Nedoceratops hatcheri from the Upper Cretaceous Lance Formation of Wyoming, U.S.A. PLoS ONE 6:e16196.doi:10.1371/journal.pone.0016196.

Farke, A. A., R. E. Chapman, and A. Andersen. 2010. Modeling structural properties of 
the frill of Triceratops; pp. 264-270 in M. J. Ryan, B. J. Chinnery-Allgeier, and D. A. Eberth (eds.), New Perspectives on Horned Dinosaurs: The Royal Tyrrell Museum Ceratopsian Symposium. Indiana University Press, Bloomington, Indiana.

Farke, A. A., M. J. Ryan, P. M. Barrett, D. H. Tanke, D. R. Braman, M. A. Loewen, and M. R. Graham. 2011. A new centrosaurine from the Late Cretaceous of Alberta, and the evolution of parietal ornamentation in horned dinosaurs. Acta Palaeontologica Polonica 56:691-702.

Fastovsky, D. E., D. B. Weishampel, M. Watabe, R. Barsbold, KH. Tsogtbaatar, and P. Narmandakh. 2011. A nest of Protoceratops andrewsi (Dinosauria. Ornithischia). Journal of Paleontology 85:1035-1041.

Foreman, B. Z., R. R. Rogers, A. L. Deino, K. R. Wirth, and J. T. Thole. 2008. Geochemical characterization of bentonite beds in the Two Medicine Formation (Campanian, Montana), including a new ${ }^{40} \mathrm{Ar} /{ }^{39} \mathrm{Ar}$ age. Cretaceous Research 29:373-385.

Forster, C. A. 1990. The cranial morphology and systematics of Triceratops with a preliminary analysis of ceratopsian phylogeny. Ph.D dissertation, Department of Geology, University of Pennsylvania, Philadelphia, 227 pp. 
Forster, C. A. 1996. New information on the skull of Triceratops. Journal of Vertebrate Paleontology 16:246-258.

Forster, C. A., P. C. Sereno, T. W. Evans, and T. Rowe. 1993. A complete skull of Chasmosaurus mariscalensis (Dinosauria: Ceratopsidae) from the Aguja Formation (Late Campanian) of West Texas. Journal of Vertebrate Paleontology 13:161-170.

Geisler, J. H., J. M. Theodor, M. D. Uhen, and S. E. Foss. 2007; pp. 19-31 in D. R. Prothero and S. E. Foss (eds.), Phylogenetic relationships of cetaceans to terrestrial artiodactyls. The Evolution of Artiodactyls. The Johns Hopkins University Press, Baltimore Maryland.

Geist, V. 1966. On the behaviour and evolution of American mountain sheep. Ph.D dissertation, University of British Columbia, Vancouver, British Columbia, 352 pp.

Gilmore, C. W. 1923. A new species of Corythosaurus with notes on other Belly River Dinosauria. Canadian Field-Naturalist 37:46-52.

Gilmore, C. W. 1946. Reptilian fauna of the North Horn Formation of central Utah. U.S. Geological Survey Professional Paper 210C:1-52. 
Godfrey, S. J., and R. Holmes. 1995. Cranial morphology and systematics of Chasmosaurus (Dinosauria: Ceratopsidae) from the Upper Cretaceous of western Canada. Journal of Vertebrate Paleontology 15:726-742.

Goloboff, P. A., J. S. Farris, and K. C. Nixon. 2003. TNT: Tree analysis using new technology. Program and documentation available from the authors and at www.zmuc.dk/public/phylogeny.

Goodwin, M. B., and A. L. Deino. 1989. The first radiometric ages from the Judith River Formation (Upper Cretaceous), Hill County, Montana. Canadian Journal of Earth Sciences 26:1384-1391.

Goodwin, M. B., and J. R. Horner. 2010. Historical collecting bias and the fossil record of Triceratops in Montana; pp. 551-564 in M. J. Ryan, B. J. Chinnery-Allgeier, and D. A. Eberth (eds.), New Perspectives on Horned Dinosaurs: The Royal Tyrrell Museum Ceratopsian Symposium. Indiana University Press, Bloomington, Indiana.

Hall, P. M., and K. M. Portier. 1994. Cranial morphometry of New Guinea crocodiles (Crocodylus novaeguineae): ontogenetic variation in relative growth of the skull and an assessment of its utility as a predictor of the sex and size of individuals. Herpetological Monographs 8:203-225. 
Hamblin, A. P., and B. W. Abrahamson. 1996. Stratigraphic architecture of "Basal Belly River" cycles, Foremost Formation Belly River Group, subsurface of southern Alberta and southwestern Saskatchewan. Bulletin of Canadian Petroleum Geology 44:654-673.

Hammer, Ø., and D. A. T. Harper. 2006. Paleontological Data Analysis. Blackwell Publishing, Malden, Massachusetts, 355 pp.

Hammer, Ø., D. A. T. Harper, and P. D. Ryan. 2001. PAST: Paleontological Statistics software package for education and data analysis. Palaeontologia Electronica 4:9. Available: http://nhm2.uio.no/norlex/past/download.html. Website retrieved January 4, 2014.

Happ, J. W. 2010. New evidence regarding the structure and function of the horns in Triceratops (Dinosauria: Ceratopsidae); pp. 271-281 in M. J. Ryan, B. J. Chinnery-Allgeier, and D. A. Eberth (eds.), New Perspectives on Horned Dinosaurs: The Royal Tyrrell Museum Ceratopsian Symposium. Indiana University Press, Bloomington, Indiana.

Hatcher, J. B., O. C. Marsh, and R. S. Lull. 1907. The Ceratopsia. US Geological Survey Monograph 49:1-300.

He, S., T. K. Kyser, and W. G. E. Caldwell. 2005. Paleoenvironment of the Western Interior Seaway inferred from $\delta^{18} \mathrm{O}$ and $\delta^{13} \mathrm{C}$ values of molluscs from the 
Cretaceous Bearpaw marine cyclothem. Palaeogeography, Palaeoclimatology, Palaeoecology 217:67-85.

Henderson, D. M. 2010. Skull shapes as indicators of niche partitioning by sympatric chasmosaurine and centrosaurine dinosaurs; pp. 293-307 in M. J. Ryan, B. J. Chinnery-Allgeier, and D. A. Eberth (eds.), New Perspectives on Horned Dinosaurs: The Royal Tyrrell Museum Ceratopsian Symposium. Indiana University Press, Bloomington, Indiana.

HeRMIS, 2012. Heritage Resources Management Information System - Royal Tyrrell Museum. Government of Alberta. https://hermis.alberta.ca/rtmp/. Website retrieved September 15, 2012.

Herring, S. W. 1974. A biometric study of suture fusion and skull growth in peccaries. Anatomy and Embryology 146:167-180.

Hoby, S., C. Wenker, N. Robert, T. Jermann, S. Hartnack, H. Segner, C-P. Aebischer, and A. Liesegang. 2010. Nutritional metabolic bone disease in juvenile veiled chameleons (Chamaeleo calyptratus) and its prevention. Journal of Nutrition 140:1923-1931.

Holand, Ø., H. Gjøstein, A. Losvar, J. Kumpala, M. E. Smith, K. H. Røed, M. Nieminen, and R. B. Weladji. 2004. Social rank in female reindeer (Rangifer tarandus): 
effects of body mass, antler size and age. Journal of Zoology 263:365-372.

Holmes, R. B., C. Forster, M. Ryan, and K. M. Shepherd. 2001. A new species of Chasmosaurus (Dinosauria: Ceratopsia) from the Dinosaur Park Formation of southern Alberta. Canadian Journal of Earth Sciences 38:1423-1438.

Horner, J. R., and M. B. Goodwin. 2006. Major cranial changes during Triceratops ontogeny. Proceedings of the Royal Society of London B 273:2757-2761.

Horner, J. R., and M. B. Goodwin. 2008. Ontogeny of cranial epi-ossifications in Triceratops. Journal of Vertebrate Paleontology 28:134-144.

Jarman, P. 1983. Mating system and sexual dimorphism in large, terrestrial, mammalian herbivores. Biological Reviews 58:485-520.

Jerzykiewicz, T., and D. K. Norris. 1994. Stratigraphy, structure and syntectonic sedimentation of the Campanian "Belly River" clastic wedge in the southern Canadian Cordillera. Cretaceous Research 15:367-399.

Kelt, D. A, and D. H. Van Vuren. 2001. The ecology and macroecology of mammalian home range area. The American Naturalist 157:637-645.

Kirkland, J. I., and D. D. Deblieux. 2010. New basal centrosaurine ceratopsian skulls 
from the Wahweap Formation (Middle Campanian), Grand Staircase-Escalante National Monument, southern Utah; pp. 117-140 in M. J. Ryan, B. J. ChinneryAllgeier, and D. A. Eberth (eds.), New Perspectives on Horned Dinosaurs: The Royal Tyrrell Museum Ceratopsian Symposium. Indiana University Press, Bloomington.

Kruk, B., M. E. Burns, and P. J. Currie. 2013. Histological study of cranial elaboration in ceratopsian dinosaurs: functional and developmental implications. Canadian Paleontology Conference and University of Alberta Laboratory for Vertebrate Palaeontology $50^{\text {th }}$ Anniversary, Abstracts. University of Alberta, Edmonton, Alberta, pp. 37.

Lambe, L. M. 1902. On Vertebrata of the Mid-Cretaceous of the Northwest Territory, Part 2. New genera and species from the Belly River Series (Mid-Cretaceous). Canadian Geological Survey, Contributions to Canadian Paleontology 3:25-81.

Lambe, L. M. 1914a. On the fore-limb of a carnivorous dinosaur from the Belly River Formation of Alberta, and a new genus of Ceratopsia from the same horizon, with remarks on the integument of some Cretaceous herbivorous dinosaurs. Ottawa Naturalist 27:129-135.

Lambe, L. M. 1914b. On Gryposaurus notabilis, a new genus and species of trachodont dinosaur from the Belly River Formation of Alberta, with description of the skull 
of Chasmosaurus belli. Ottawa Naturalist 27:145-155.

Lambe, L. M. 1915. On Eoceratops canadensis, gen. nov., with remarks on other genera of Cretaceous horned dinosaurs. Geological Survey of Canada Museum Bulletin 12:1-49.

Larson, P. 2008. Variation and sexual dimorphism in Tyrannosaurus rex; pp. 103-130 in P. Larson and K. Carpenter (eds.), Tyrannosaurus rex, the tyrant king. Indiana University Press, Bloomington, Indiana.

Lee, A. 2006. Evolution of rapid limb growth and vascular channel organization in ceratopsian dinosaurs. Journal of Vertebrate Paleontology 26(3, Suppl.):89A.

Lee, A. 2007. How Centrosaurus (and other ceratopsians) grew to large size; pp. 105106 in D. R. Braman (comp.), Ceratopsian Symposium: Short Papers, Abstracts, and Programs. Drumheller: Royal Tyrrell Museum of Palaeontology.

Lee, A., and S. Werning. 2008. Sexual maturity in growing dinosaurs does not fit reptilian growth models. Proceedings of the National Academy of Sciences 105:582-587.

Lehman, T. M. 1989. Chasmosaurus mariscalensis, sp. nov., a new ceratopsian dinosaur from Texas. Journal of Vertebrate Paleontology 9:137-162. 
Lehman, T. M. 1990. The ceratopsian subfamily Chasmosaurinae: sexual dimorphism and systematics; pp. 211-229 in K. Carpenter and P. Currie (eds.), Dinosaur Systematics: Perspectives and Approaches. Cambridge University Press, New York.

Lehman, T. M. 1993. New data on the ceratopsian dinosaur Pentaceratops sternbergii Osborn from New Mexico. Journal of Paleontology 67:279-288.

Lehman, T. M. 1996. A horned dinosaur from the El Picacho Formation of West Texas, and review of ceratopsian dinosaurs from the American Southwest. Journal of Paleontology 70:494-508.

Lehman, T. M. 1998. A gigantic skull and skeleton of the horned dinosaur Pentaceratops sternbergi from New Mexico. Journal of Paleontology 72:894-906.

Lehman, T. M. 2007. Growth and population age structure in the horned dinosaur Chasmosaurus; pp. 259-317 in K. Carpenter (ed.), Horns and Beaks: Ceratopsian and Ornithopod Dinosaurs. Indiana University Press, Bloomington and Indianapolis, Indiana.

Loewen, M. A., S. D. Sampson, E. K. Lund, A. A. Farke, M. C. Aguillón-Martínez, C. A. De Leon, R. A. Rodríguez de la Rosa, M. A. Getty, and D. A. Eberth. 2010. 
Horned dinosaurs (Ornithischia: Ceratopsidae) from the Upper Cretaceous (Campanian) Cerro del Pueblo Formation, Coahuila, Mexico; pp. 99-116 in M. J. Ryan, B. J. Chinnery-Allgeier, and D. A. Eberth (eds.), New Perspectives on Horned Dinosaurs: The Royal Tyrrell Museum Ceratopsian Symposium. Indiana University Press, Bloomington, Indiana.

Longrich, N. R. 2010. Mojoceratops perifania, a new chasmosaurine ceratopsid from the Late Campanian of western Canada. Journal of Paleontology 84:681-694.

Longrich, N. R. 2011. Titanoceratops ouranos, a giant horned dinosaur from the Late Campanian of New Mexico. Cretaceous Research 32:264-276.

Longrich, N. R., and D. J. Field. 2012. Torosaurus is not Triceratops: ontogeny in chasmosaurine ceratopsids as a case study in dinosaur taxonomy. PLoS ONE 7:e32623.doi:10.1371/journal.pone.0032623.

Longrich, N. R. 2013. Judiceratops tigris, a new horned dinosaur from the Middle Campanian Judith River Formation of Montana. Bulletin of the Peabody Museum of Natural History 54:51-65.

Lü, J., D. M. Unwin, D. C. Deeming, X. Jin, Y. Liu, and Q. Ji. 2011. An egg-adult association, gender, and reproduction in pterosaurs. Science 331:321-324. 
Lucas, S. G., R. M. Sullivan, and A. P. Hunt. 2006. Re-evaluation of Pentaceratops and Chasmosaurus (Ornithischia: Ceratopsidae) in the Upper Cretaceous of the Western Interior. New Mexico Museum of Natural History and Science Bulletin 35:367-370.

Lull, R. S. 1905. Restoration of the horned dinosaur Diceratops. American Journal of Science 20:420-422.

Lull, R. S. 1933. A revision of the Ceratopsia or horned dinosaurs. Memoirs of the Peabody Museum 3:1-175.

Mahalanobis, P. C. 1936. On the generalised distance in statistics. Proceedings National Institute of Science, India 2:49-55.

Maidment, S. C. R., and P. M. Barrett. 2011. A new specimen of Chasmosaurus belli (Ornithischia: Ceratopsidae), a revision of the genus, and the utility of postcrania in the taxonomy and systematics of ceratopsid dinosaurs. Zootaxa 2963:1-47.

Mallon, J. C., R. Holmes, D. A. Eberth, M. J. Ryan, and J. S. Anderson. 2011. Variation in the skull of Anchiceratops (Dinosauria, Ceratopsidae) from the Horseshoe Canyon Formation (Upper Cretaceous) of Alberta. Journal of Vertebrate Paleontology 31:1047-1071. 
Mallon, J. C., and J. S. Anderson. 2013. Skull ecomorphology of megaherbivorous dinosaurs from the Dinosaur Park Formation (Upper Campanian) of Alberta, Canada. PLoS ONE 8: e67182. doi:10.1371/journal.pone.0067182.

Mallon, J. C., and J. S. Anderson. 2014. Implications of beak morphology for the evolutionary paleoecology of the megaherbivorous dinosaurs from the Dinosaur Park Formation (upper Campanian) of Alberta, Canada. Palaeogeography, Palaeoclimatology, Palaeoecology 394:29-41.

Marsh, O. C. 1888. A new family of horned dinosaurs from the Cretaceous. American Journal of Science, series 3, 36:477-478.

Marsh, O. C. 1889. Notice of gigantic horned Dinosauria from the Cretaceous. American Journal of Science, series 3, 38:173-175.

Marsh, O. C. 1890. Description of new dinosaurian reptiles. American Journal of Science, series 3, 39:81-86.

Marsh, O. C. 1891. Notice of new vertebrate fossils. American Journal of Science, series $3,42: 265-269$.

Mayr, E. 1969. Principles of Systematic Zoology. McGraw-Hill. New York, 428 pp. 
McGuire, J. L. 2010. Geometric morphometrics of vole (Microtus californicus) dentition as a new paleoclimate proxy: shape change along geographic and climatic clines. Quaternary International 212:198-205.

McLean, J. R. 1971. Stratigraphy of the Upper Cretaceous Judith River Formation in the Canadian Great Plains. Saskatchewan Research Council, Geology Division, Report 11.

Meng, Q., J.-Y. Liu, D. J. Varricchio, T. Huang, and C.-L. Gao. 2004. Parental care in an ornithischian dinosaur. Nature 431:145-146.

Nakagawa, S. 2004. A farewell to Bonferroni: the problems of low statistical power and publication bias. Behavioral Ecology 15:1044-1045.

Oba, S., M. Sato, I. Takemasa, M. Monden, K. Matsubara, and S. Ishii. 2003. A Bayesian missing value estimation method for gene expression profile data. Bioinformatics 19:2088-2096.

Osborn, H. F. 1923. A new genus and species of Ceratopsia from New Mexico, Pentaceratops sternbergii. American Museum of Natural History Novitates 93:13.

Ostrom, J. H., and P. Wellnhofer. 1990. Triceratops: an example of flawed systematics; 
pp. 245-254 in K. Carpenter and P. J. Currie (eds.), Dinosaur Systematics, Approaches and Perspectives. Cambridge University Press, Cambridge.

Ott, C. J., and P. L. Larson. 2010. A new, small ceratopsian dinosaur from the latest Cretaceous Hell Creek Formation, northwest South Dakota, United States: a preliminary description; pp. 203-220 in M. J. Ryan, B. J. Chinnery-Allgeier, and D. A. Eberth (eds.), New Perspectives on Horned Dinosaurs: The Royal Tyrrell Museum Ceratopsian Symposium. Indiana University Press, Bloomington, Indiana.

Owens, I. P. F., and I. R. Hartley. 1998. Sexual dimorphism in birds: why are there so many different forms of dimorphism? Proceedings of the Royal Society of London B 265:397-407.

Pahl, R., D. W. Winkler, J. Graveland, and B. W. Batterman. 1997. Songbirds do not create long-term stores in their legs prior to laying: results from high-resolution radiography. Proceedings of the Royal Society of London B 264:239-244.

Parks, W. A. 1925. Arrhinoceratops brachyops, a new genus and species of Ceratopsia from the Edmonton Formation of Alberta. University of Toronto Studies, Geology Series 19:1-15.

Polly, P. D. 2007. Phylogeographic differentiation in Sorex araneus: morphology in 
relation to geography and karyotype. Russian Journal of Theriology 6:73-84.

Powell, G. L., A. P. Russell, and M. J. Ryan. 2002. Ontogenetic scaling of the cranial horn array in Phrynosoma orbiculare (Squamata: Phrynosomatidae). Journal of Herpetology 36:578-589.

R Development Core Team. 2011. R: A Language and Environment for Statistical Computing. 2.15.2. ed. Vienna, Austria: R Foundation for Statistical Computing available from: http://www.R-project.org/.

Reizner J., and J. Horner. 2006. An ontogenetic series of the ceratopsid dinosaur Einiosaurus procurvicornis as determined by long bone histology. Journal of Vertebrate Paleontology 26(3, Suppl.):114A.

Rogers, R. R. 1994. Nature and origin of through-going discontinuities in nonmarine foreland basin strata, Upper Cretaceous, Montana: Implications for sequence analysis. Geology 22:1119-1122.

Rogers, R. R. 1995. Sequence stratigraphy and vertebrate taphonomy of the Upper Cretaceous Two Medicine and Judith River formations, Montana. Ph.D. thesis, University of Chicago, Chicago, Illinois, 400 pp.

Rogers, R. R., and C. C. Swisher. 1996. The Claggett and Bearpaw transgressions 
revisited; new ${ }^{40} \mathrm{Ar} /{ }^{39} \mathrm{Ar}$ data and a review of possible drivers; p. 62 in Geological Society of America, Program and Abstracts.

Rogers, R. R., H. C. Fricke, V. Addona, R. R. Canavan, C. N. Dwyer, C. L. Harwood, A. E. Koenig, R. Murray, J. T. Thole, and J. Williams. 2010. Using laser ablationinductively coupled plasma-mass spectrometry (LA-ICP-MS) to explore geochemical taphonomy of vertebrate fossils in the Upper Cretaceous Two Medicine and Judith River formations of Montana. Palaios 25:183-195.

Russell, L. S., and R. W. Landes. 1940. Geology of the southern Alberta plains. Geological Survey of Canada, Memoir 221.

Ryan, M. J. 1992. The taphonomy of a Centrosaurus (Reptilia: Ornithischia) bone bed (Campanian), Dinosaur Provincial Park, Alberta, Canada. M.S. thesis, University of Calgary, Calgary, Alberta, 526 pp.

Ryan, M. J., A. P. Russell, D. A. Eberth, and P. J. Currie. 2001. The taphonomy of a Centrosaurus (Ornithischia: Ceratopsidae) bone bed from the Dinosaur Park Formation (Upper Campanian), Alberta, Canada, with comments on cranial ontogeny. Palaios 16:482-506.

Ryan, M. J. 2003. Taxonomy, systematics and evolution of centrosaurine ceratopsids of the Campanian Western Interior Basin of North America. Ph.D dissertation, 
University of Calgary, Calgary, Alberta, 609 pp.

Ryan, M. J. 2007. A new basal centrosaurine ceratopsid from the Oldman Formation, southeastern Alberta. Journal of Paleontology 81:376-396.

Ryan, M. J., and D. C. Evans. 2005. Ornithischian dinosaurs; pp. 312-348 in P. J. Currie and E. B. Koppelhus (eds.), Dinosaur Provincial Park: A Spectacular Ancient Ecosystem Revealed. Indiana University Press, Bloomington, Indiana.

Ryan, M. J., and A. P. Russell. 2005. A new centrosaurine ceratopsid from the Oldman Formation of Alberta and its implications for centrosaurine taxonomy and systematics. Canadian Journal of Earth Sciences 42:1369-1387.

Ryan, M. J., R. Holmes, and A. P. Russell. 2007. A revision of the Late Campanian centrosaurine genus Styracosaurus from the Western Interior of North America. Journal of Vertebrate Paleontology 27:944-962.

Ryan, M. J., D. A. Eberth, D. B. Brinkman, P. J. Currie, and D. H. Tanke. 2010a. A new Pachyrhinosaurus-like ceratopsid from the Upper Dinosaur Park Formation (Late Campanian) of southern Alberta, Canada; pp. 141-155 in M. J. Ryan, B. J. Chinnery-Allgeier, and D. A. Eberth (eds.), New Perspectives on Horned Dinosaurs: The Royal Tyrrell Museum Ceratopsian Symposium. Indiana University Press, Bloomington, Indiana. 
Ryan, M. J., A. P. Russell, and S. Hartman. 2010b. A new chasmosaurine ceratopsid from the Judith River Formation, Montana; pp. 181-188 in M. J. Ryan, B. J. ChinneryAllgeier, and D. A. Eberth (eds.), New Perspectives on Horned Dinosaurs: The Royal Tyrrell Museum Ceratopsian Symposium. Indiana University Press, Bloomington, Indiana.

Ryan, M. J., D. C. Evans, P. J. Currie, C. M. Brown, and D. B. Brinkman. 2012. New leptoceratopsids from the Upper Cretaceous of Alberta, Canada. Cretaceous Research 35:69-80.

Ryan, M. J., D. C. Evans, and K. M. Shepherd. 2013a. A new ceratopsid from the Foremost Formation (Middle Campanian) of Alberta. Canadian Journal of Earth Sciences 49:1251-1262.

Ryan, M., R. Holmes, and J. Mallon. 2013b. A new relict basal ceratopsid from the Oldman Formation (Campanian) of Alberta with implications for centrosaurine evolution; p. 202 in Journal of Vertebrate Paleontology, Program and Abstracts.

Sampson, S. D. 2009. Dinosaur Odyssey: Fossil Threads in the Web of Life. University of California Press, Berkeley, California, 332 pp.

Sampson, S. D., and M. A. Loewen. 2010. Unraveling a radiation: a review of the 
diversity, stratigraphic distribution, biogeography, and evolution of horned dinosaurs (Ornithischia: Ceratopsidae); pp. 405-427 in M. J. Ryan, B. J. Chinnery-Allgeier, and D. A. Eberth (eds.), New Perspectives on Horned Dinosaurs: The Royal Tyrrell Museum Ceratopsian Symposium. Indiana University Press, Bloomington, Indiana.

Sampson, S. D., M. J. Ryan, and D. H. Tanke. 1997. Craniofacial ontogeny in centrosaurine dinosaurs (Ornithischia: Ceratopsidae): Taxonomic and behavioural implications. Zoological Journal of the Linnean Society 121:293-337.

Sampson, S. D., M. A. Loewen, A. A. Farke, E. M. Roberts, C. A. Forster, J. A. Smith, and A. L. Titus. 2010. New horned dinosaurs from Utah provide evidence for intracontinental dinosaur endemism. PLoS ONE 5:1-12.

Sampson, S. D., Lund, E. K., Loewen, M. A., Farke, A. A., and Clayton, K. E. 2013. A remarkable short-snouted horned dinosaur from the Late Cretaceous (late Campanian) of southern Laramidia. Proceedings of the Royal Society B 280: no.1766; doi:10.1098/rspb.2013.1186.

Sankey, J. T. 2010. Faunal composition and significance of high-diversity, mixed bonebeds containing Agujaceratops mariscalensis and other dinosaurs, Aguja Formation (Upper Cretaceous), Big Bend, Texas; pp. 520-540 in M. J. Ryan, B. J. Chinnery-Allgeier, and D. A. Eberth (eds.), New Perspectives on Horned 
Dinosaurs: The Royal Tyrrell Museum Ceratopsian Symposium. Indiana University Press, Bloomington, Indiana.

Scannella, J. B., and J. R. Horner. 2010. Torosaurus Marsh, 1891, is Triceratops, Marsh, 1889 (Ceratopsidae: Chasmosaurinae): synonymy through ontogeny. Journal of Vertebrate Paleontology 30:1157-1168.

Scannella, J. B., and J. R. Horner. 2011. 'Nedoceratops': an example of a transitional morphology. PLoS ONE 6:e28705.doi:10.1371/journal.pone.0028705.

Scannella, J. B., and D. W. Fowler. 2014. A stratigraphic survey of Triceratops localities in the Hell Creek Formation, northeastern Montana (2006-2010); pp. 313-332 in G. P. Wilson, W. A. Clemens, J. R. Horner, and J. H. Hartman (eds.), Through the End of the Cretaceous in the Type Locality of the Hell Creek Formation in Montana and Adjacent Areas; Geological Society of America Special Paper 503.

Scannella, J., D. Fowler, M. Goodwin, and J. Horner. 2013. The clandestine role of heterochrony in ceratopsian evolution as revealed by juvenile Triceratops; p. 206 in Journal of Vertebrate Paleontology, Program and Abstracts.

Schweitzer, M. H., J. L. Wittmeyer, and J. R. Horner. 2005. Gender-specific reproductive tissue in ratites and Tyrannosaurus rex. Science 308:1456-1460. 
Schweitzer, M. H., J. L. Wittmeyer, and J. R. Horner. 2008. One pretty amazing T. rex; pp. 93-102 in P. Larson and K. Carpenter (eds.), Tyrannosaurus rex, the tyrant king. Indiana University Press, Bloomington, Indiana.

Selander, R. K. 1966. Sexual dimorphism and differential niche utilization in birds. The Condor 68:113-151.

Sereno, P. C. 1986. Phylogeny of the bird-hipped dinosaurs (order Ornithischia). National Geographic Research 2:234-256.

Sereno, P. C. 2010. Taxonomy, cranial morphology, and relationships of parrot-beaked dinosaurs (Ceratopsia: Psittacosaurus); pp. 21-58 in M. J. Ryan, B. J. ChinneryAllgeier, and D. A. Eberth (eds.), New Perspectives on Horned Dinosaurs: The Royal Tyrrell Museum Ceratopsian Symposium. Indiana University Press, Bloomington, Indiana.

Shine, R., and E. L. Charnov. 1992. Patterns of survival, growth, and maturation in snakes and lizards. American Naturalist 139:1257-1269.

Sokal, R. R., and F. J. Rohlf. 1995. Biometry: The Principals and Practice of Statistics in Biological Research, 3rd edition. W. H. Freeman and Company, New York.

Stacklies, W., H. Redestig, M. Scholz, D. Walther, J. Selbig. 2007. pcaMethods - a 
bioconductor package providing PCA methods for incomplete data. Bioinformatics 23:1164-1167.

Sternberg, C. H. 1915. Field notes, 1915 season. Geological Survey of Canada.

Sternberg, C. M. 1927. Horned dinosaur group in the National Museum of Canada. Canadian Field-Naturalist 41:67-73.

Sternberg, C. M. 1929. A new species of horned dinosaur from the Upper Cretaceous of Alberta. Bulletin of the National Museum of Canada 54:34-37.

Sternberg, C. M. 1940. Ceratopsidae from Alberta. Journal of Paleontology 14:468-480.

Sternberg, C. M. 1949-1950. Annual Report of National Museum of Canada.

Sternberg, C. M. 1950. Steveville west of the 4th meridian, with notes on fossil localities. Geological Survey of Canada, Map 969A.

Sullivan, R. M., and S. G. Lucas. 2010. A new chasmosaurine (Ceratopsidae, Dinosauria) from the Upper Cretaceous Ojo Alamo Formation (Naashoibito Member) San Juan Basin, New Mexico; pp. 169-180 in M. J. Ryan, B. J. Chinnery-Allgeier, and D. A. Eberth (eds.), New Perspectives on Horned Dinosaurs: The Royal Tyrrell Museum Ceratopsian Symposium. Indiana University Press, Bloomington, 
Indiana.

Tanke, D. H., and A. A. Farke. 2007. Bone resorption, bone lesions and extra cranial fenestrae in ceratopsid dinosaurs: a preliminary assessment; pp. 319-347 in K. Carpenter (ed.), Horns and Beaks: Ceratopsian and Ornithopod Dinosaurs. Indiana University Press, Bloomington and Indianapolis, Indiana.

Tumarkin-Deratzian, A. R. 2010. Histological evaluation of ontogenetic bone surface texture changes in the frill of Centrosaurus apertus; pp. 251-263 in M. J. Ryan, B. J. Chinnery-Allgeier, and D. A. Eberth (eds.), New Perspectives on Horned Dinosaurs: The Royal Tyrrell Museum Ceratopsian Symposium. Indiana University Press, Bloomington, Indiana.

Tumarkin-Deratzian, A. R., D. R. Vann, and P. Dodson. 2006. Bone surface texture as an ontogenetic indicator in long bones of the Canada goose Branta canadensis (Anseriformes: Anatidae). Zoological Journal of the Linnean Society 148:133168.

Tyson, H. L. 1977. Functional craniology of the Ceratopsia (Reptilia: Ornithischia) with special reference to Eoceratops. M.S. thesis, University of Alberta, Edmonton, Alberta, 339 pp.

Wick, S. L., and T. M. Lehman. 2013. A new ceratopsian dinosaur from the Javelina 
Formation (Maastrichtian) of West Texas and implications for chasmosaurine phylogeny. Naturwissenschaften 100:667-682.

Wilkinson. P. M., and W. E. Rhodes. 1997. Growth rates of American alligators in coastal South Carolina. Journal of Wildlife Management 61:397-402.

Wolfe, D. G., and J. I. Kirkland. 1998. Zuniceratops christopheri n. gen \& n. sp., a ceratopsian dinosaur from the Moreno Hill Formation (Cretaceous, Turonian) of west-central New Mexico; pp. 303-317 in S. G. Lucas, J. I. Kirkland, and J. W. Estep (eds.), Lower and Middle Cretaceous Terrestrial Ecosystems. New Mexico Museum of Natural History and Science Bulletin 14.

Wu, X.-C., D. B. Brinkman, D. A. Eberth, and D.R. Braman. 2007. A new ceratopsid dinosaur (Ornithischia) from the uppermost Horseshoe Canyon Formation (upper Maastrichtian), Alberta, Canada. Canadian Journal of Earth Sciences 44:12431265.

Xu, X., K. Wang, X. Zhao, and D. Li. 2010. First ceratopsid dinosaur from China and its biogeographical implications. Chinese Science Bulletin 55:1631-1635.

Zeigler, K. E., S. G. Lucas, and A. B. Heckert. 2002. The Late Triassic Canjilon Quarry (Upper Chinle Group, New Mexico) phytosaur skulls: evidence of sexual dimorphism in phytosaurs: pp. 179-188 in A. B. Heckert and S. G. Lucas (eds.), 
Upper Triassic Stratigraphy and Paleontology. New Mexico Museum of Natural History and Science Bulletin No. 21. 